Portland State University

PDXScholar

$5-12-2021$

\title{
Technology Management Maturity Assessment Model in Healthcare
}

Amir Shaygan

Portland State University

Follow this and additional works at: https://pdxscholar.library.pdx.edu/open_access_etds

Part of the Health and Medical Administration Commons, and the Technology and Innovation Commons

Let us know how access to this document benefits you.

\section{Recommended Citation}

Shaygan, Amir, "Technology Management Maturity Assessment Model in Healthcare" (2021).

Dissertations and Theses. Paper 5696.

https://doi.org/10.15760/etd.7569

This Dissertation is brought to you for free and open access. It has been accepted for inclusion in Dissertations and Theses by an authorized administrator of PDXScholar. Please contact us if we can make this document more accessible: pdxscholar@pdx.edu. 
Technology Management Maturity Assessment Model in Healthcare

by

Amir Shaygan

A dissertation submitted in partial fulfillment of the requirements for the degree of

\author{
Doctor of Philosophy \\ in \\ Technology Management
}

Dissertation Committee:

Dr. Tugrul Daim, Chair

Dr. Mark Ahn

Dr. Peter Graven

Dr. Kelly Clifton

Portland State University

2021 


\begin{abstract}
Significant gaps in the practical transformation of clinical knowledge into practices, increasing healthcare costs, costly medical errors, healthcare institutions' obligations towards improving safety, clinical outcomes, and efficacy of care from one side; and the rise of disruptive innovations, the adoption of electronic health records and novel diagnostic tools, and the plethora of data from the other side has made the need for a new approach in managing the U.S healthcare systems an imperative. Continuous learning has been utilized to mitigate some of these issues have been in healthcare organizations. Continuous learning is especially important in the research centers that act as innovation hubs within University Hospitals. These centers align with learning and improving current systems and practices in a specific area of healthcare with goals of better serving the population in need of those specific services or treatments. Maturity Models are organizational management tools that have been used as a way of responding to the constant pressure of trying to achieve and maintain competitive advantage through concurrent innovation, quality improvement, and cost reduction. In the context of continuous learning in healthcare organizations, a mature system can be defined as a system that generates timely actions to the information that it derives from internal and external data to create meaningful measurement regarding system learning and increased efficacy and effectiveness in health outcomes. However, there is a lack of a model that provides managers and decision-makers with a systematic, multi-criteria, validated, quantifiable, and repeatable maturity model to assess and enhance health organizations' performance in continuous learning and technology management. This research proposes
\end{abstract}


a multi-criteria model to assess technology management maturity and continuous learning in research centers within university hospitals by using Hierarchical Decision Model (HDM). The model can help these research centers with pinpointing their strengths and opportunities in terms of continuous learning from the data they have access to while giving them organizational self-awareness and guide them in setting their strategies and resource allocation. The model will serve as a much-needed technology management tool for healthcare organizations to assess their technology management maturity and continuous learning efforts and assist them in creating more effective roadmaps. 


\section{Acknowledgments}

Throughout the writing of this dissertation I have received a great deal of support and assistance.

I would first like to thank my adviser, Professor Tugrul Daim, which none of this would have been possible without his guidance and support. Your insightful feedback pushed me to sharpen my thinking and brought my work to a higher level. I am forever grateful for the opportunity you gave me, your support, and mentorship.

I would also like to express my deepest appreciation to my dissertation committee. Dr. Mark Ahn, for believing in me since day one. I have learned so much from you and forever grateful for all your support in the last four years, Dr. Peter Graven for all the great insights and feedback which have improved my research greatly and for all that I have learned from you by working at OHSU. Dr. Kelly Clifton for all the support and the opportunities to hone my analytical skills while writing my dissertation.

I would also like to extend my sincere thanks to all the subject matter experts who participated in my research. Your time, feedback, and insights were vital to the validation, quantification, and application of this research model.

Moreover, my sincere gratitude goes to all the people at Portland State University's Engineering and Technology Management Department (ETM) and Office of Institutional Research and Planning (OIRP), and Oregon Health and Science University's Business Intelligence and Advanced Analytics (BIAA) team who I have learned a lot from and will forever cherish their support. 
Great thank you also goes to my friends and colleagues that have supported and helped me in different ways in different steps of my research. I like to thank Dr. Sanaz Riahi, Anna Steeves-Reece, Dr. Joao Lavoie, Dr. Husam Barham, Dr. Edwin Garces, Sarah Dys, Diane Coward, JoZell Johnson, Marc Abbott, Dr. Loreen Herwaldt, Dr. Noelle Wiggins, and Adi Zweier.

I want to thank my parents Hedieh and Mehrdad for their endless love, support and sacrifices throughout my life. Thank you both for giving me strength to chase my dreams. This would not have been possible without your support.

Great appreciation and gratitude go to my dogs Odin and Eureka who made all the difficult and overwhelming moments easier with their presence and unconditional love.

Last but not least, I would like to thank my partner, Dr. Maral Razmand. Thank you for all the love, patience, and encouragement. You help me be a better version of myself, and I could not have done this without you. 


\section{Table of Contents}

\begin{tabular}{|c|c|}
\hline \\
\hline \\
\hline \multirow{2}{*}{\multicolumn{2}{|c|}{ 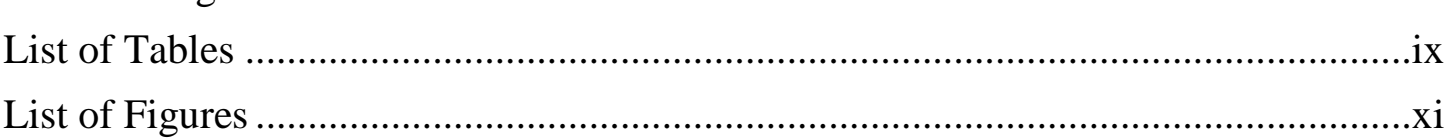 }} \\
\hline & \\
\hline 1. & Introduction ............................ \\
\hline 1.1. & Problem Statement......... \\
\hline 2. & Literature Review ....................................... \\
\hline 2.1 & Healthcare Landscape in United States........................ \\
\hline 2.2 & 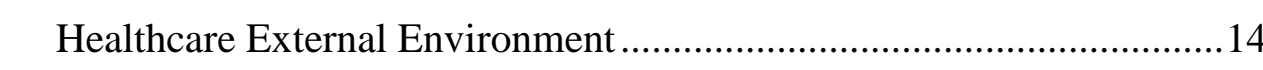 \\
\hline 2.2 .1 & External Change Perspectives ..................................... \\
\hline 2.2.1.1 & 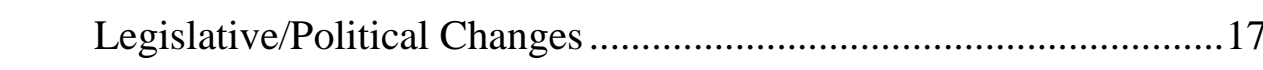 \\
\hline 2.2.1.2 & 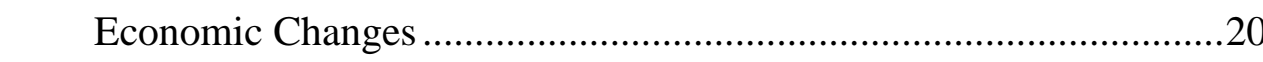 \\
\hline 2.2.1.3 & 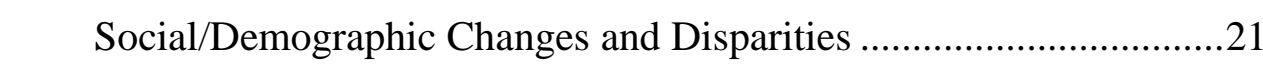 \\
\hline 2.2.1.4 & 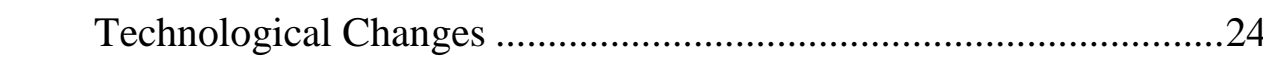 \\
\hline 2.2.1.5 & 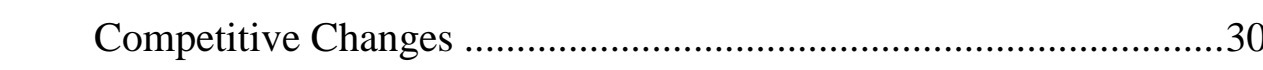 \\
\hline 2.2 .2 & Summary \\
\hline 2.3 & 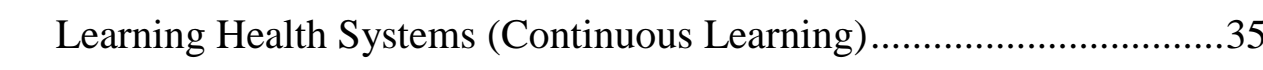 \\
\hline $\begin{array}{l}2.3 .1 \\
\text { Organiz }\end{array}$ & $\begin{array}{l}\text { A Multi-perspective Approach for Continuous Learning in Healthcare } \\
\text { ations }\end{array}$ \\
\hline 2.3.1.1 & 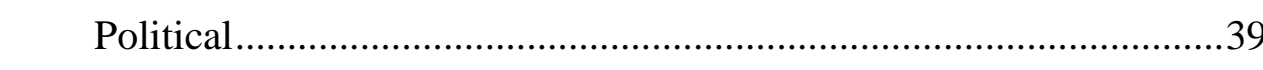 \\
\hline 2.3.1.2 & 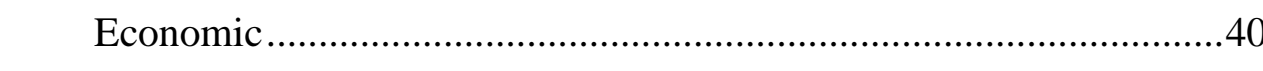 \\
\hline 2.3.1.3 & Social ................. \\
\hline 2.3.1.4 & 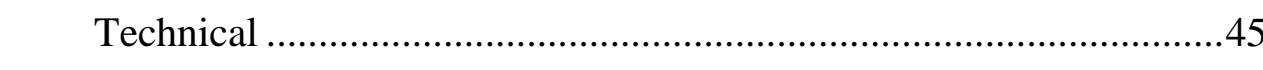 \\
\hline 2.4 & Maturity Models.................................. \\
\hline 2.4 .1 & Technology Management Maturity in Healthcare.................................51 \\
\hline 2.5 & Quadruple Aim of Care \\
\hline 2.6 & 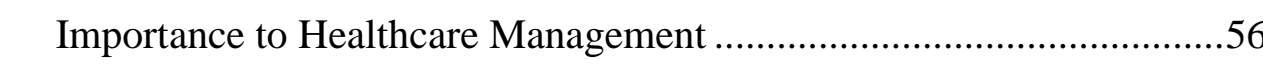 \\
\hline 2.7 & 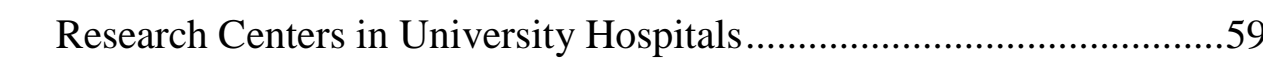 \\
\hline 3. & 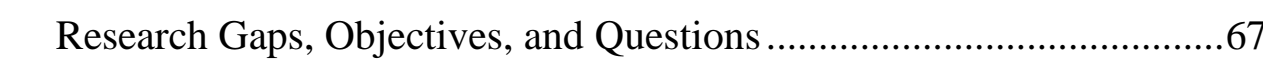 \\
\hline 3.1. & 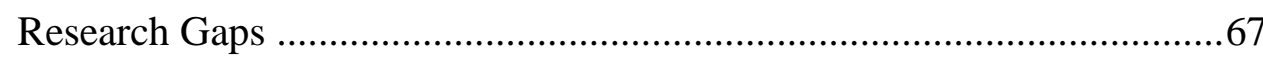 \\
\hline 3.2.1. & 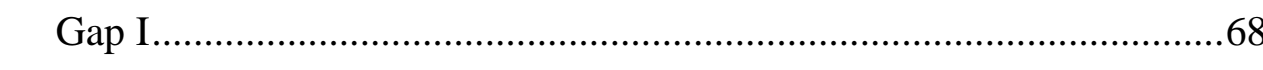 \\
\hline 3.2.2. & 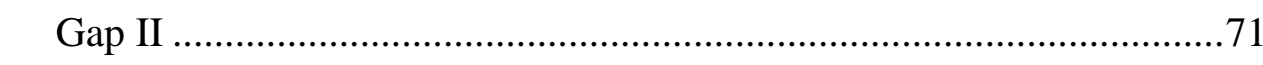 \\
\hline
\end{tabular}




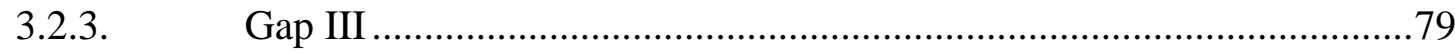

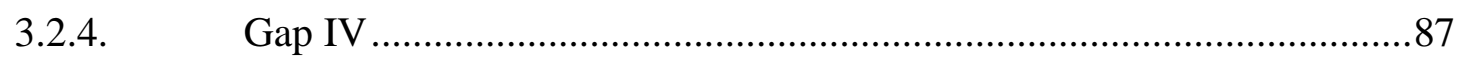

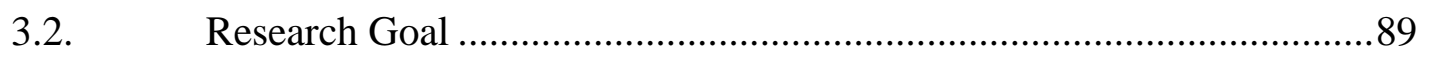

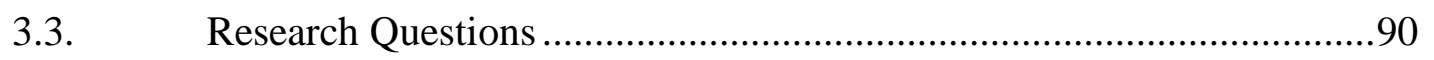

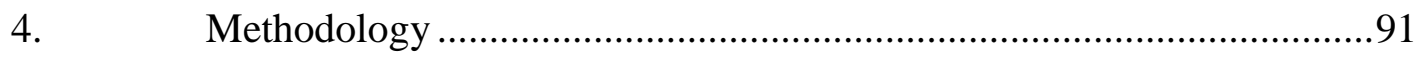

4.1 Hierarchical Decision Model (HDM) Model ........................................91

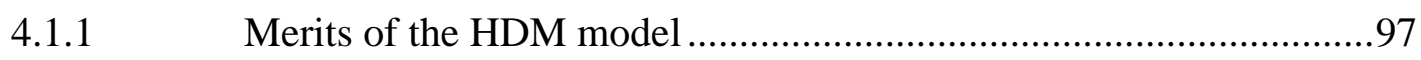

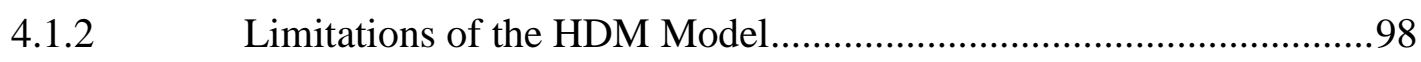

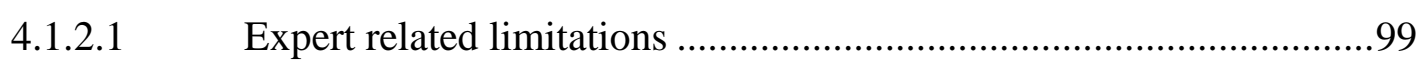

4.1.2.2 Model Sensitivity ....................................................................... 100

4.1.2.3 Criteria Balance among Perspectives/Criteria.....................................101

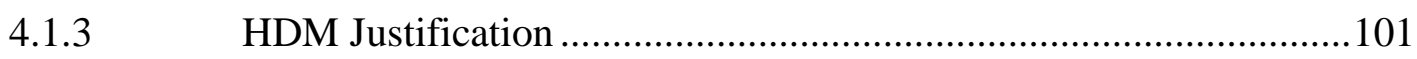

4.2. Critical Issues in Forming an Expert Panel and Selecting the Experts .........105

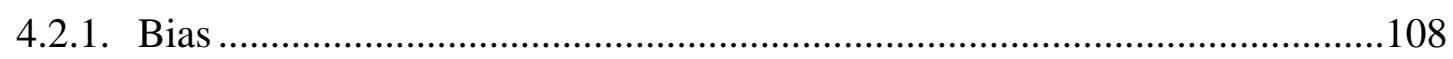

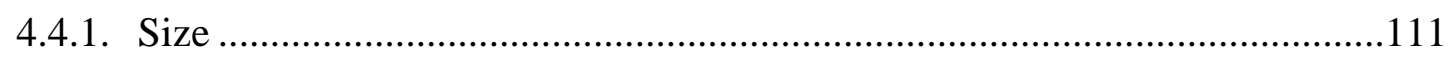

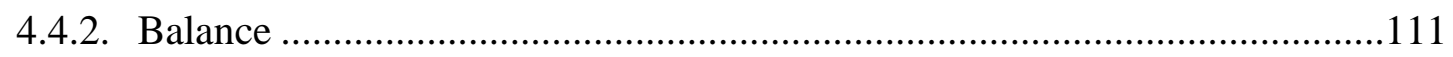

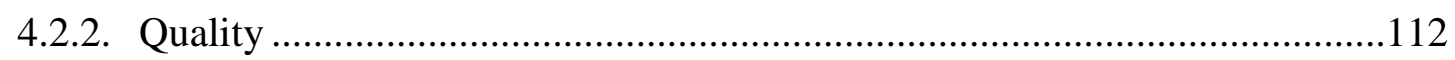

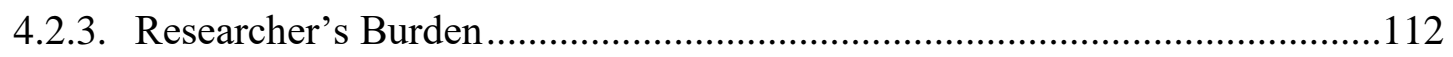

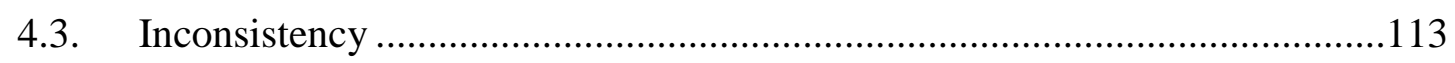

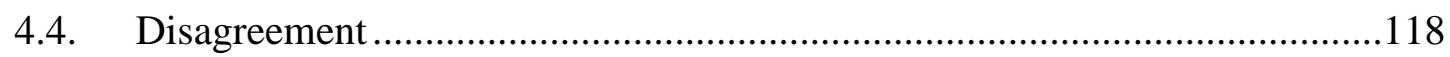

4.4.1. Hierarchical Agglomerative Clustering (HAC),..........................................120

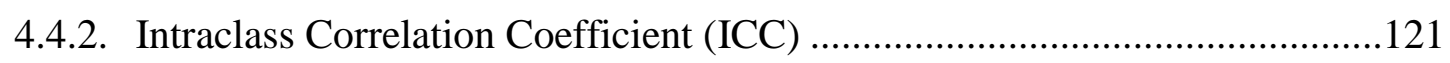

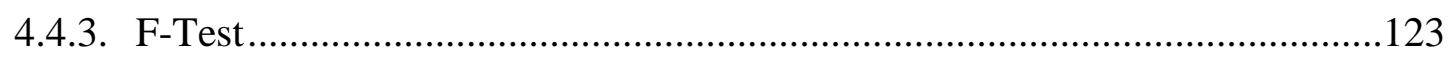

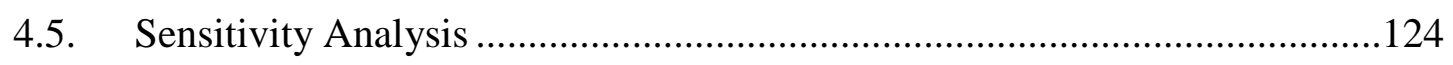

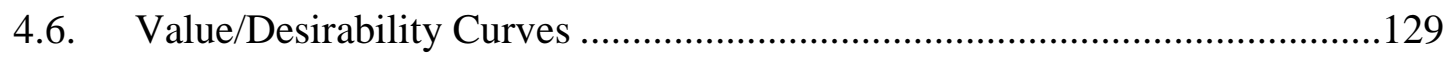

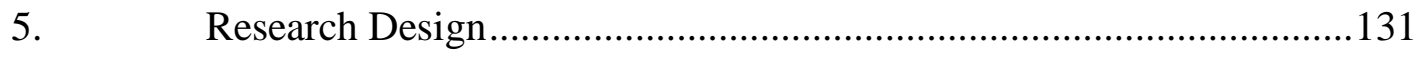

5.1. Perspectives and Criteria.................................................................137

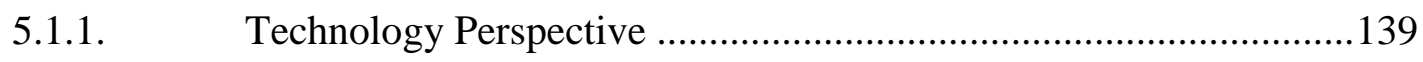

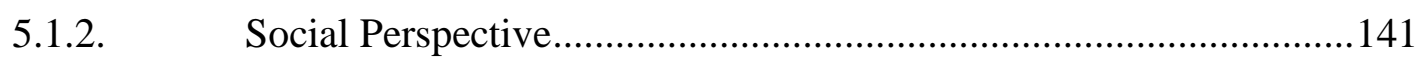

5.1.3. Organizational Perspective ...........................................................143

5.1.4. Regulatory Perspective ............................................................... 144

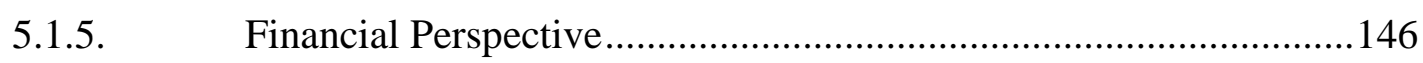

5.2. Initial Identified Perspectives and Criteria............................................ 148 
5.2. Model Description for Data Collection................................................153

5.3. Justification of the Selected Elements .................................................155

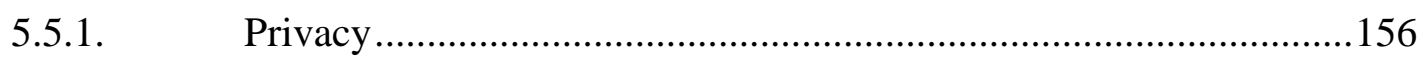

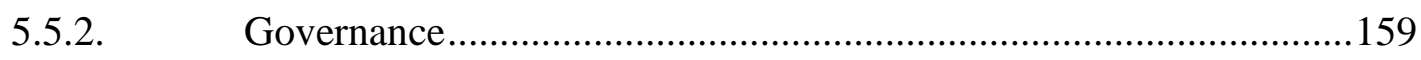

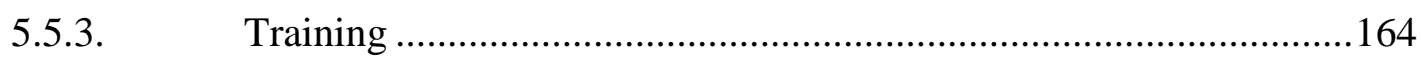

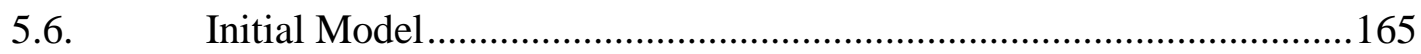

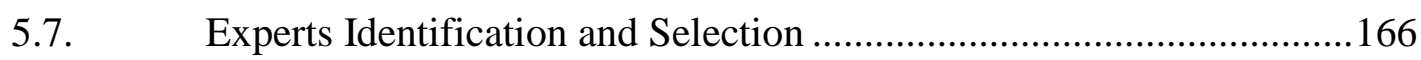

6. Results of Model Validation and Quantification ..................................173

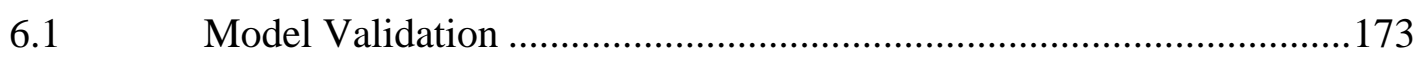

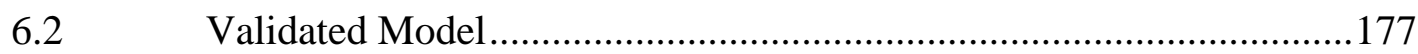

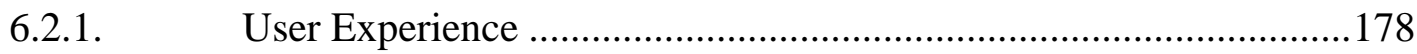

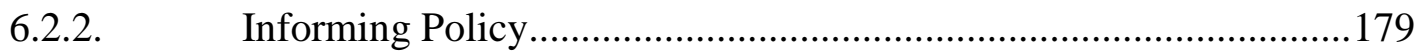

6.2.3. Financial Synergy with Policies and Priorities...................................180

6.2.4. Finalized Model.......................................................................... 181

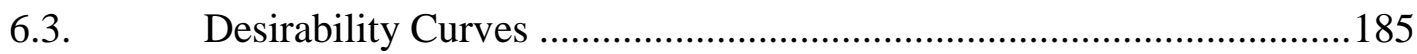

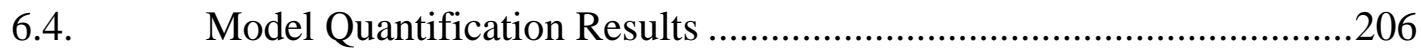

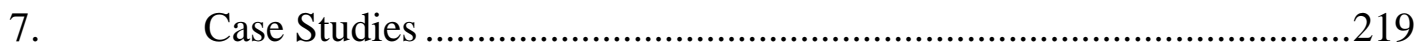

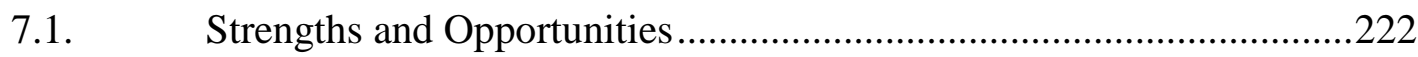

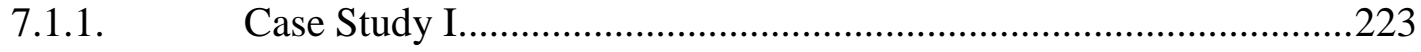

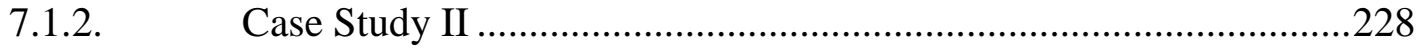

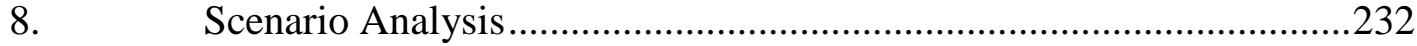

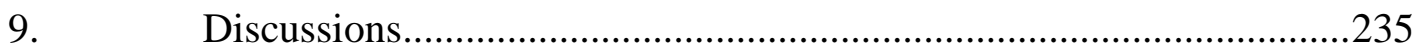

9.1. Research and Practical Implications ..................................................236

9.1.1. Stakeholder Engagement ..........................................................237

9.1.2. Talent Acquisition and Retention..................................................238

9.1.3. Strategic Management .................................................................239

9.1.4. Funding and Mission Alignment ...................................................239

9.1.5. Real-time Knowledge Access and Sharing ……………..................240

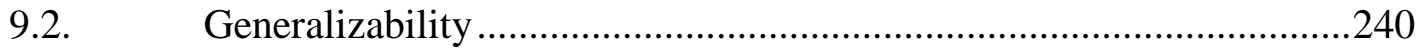

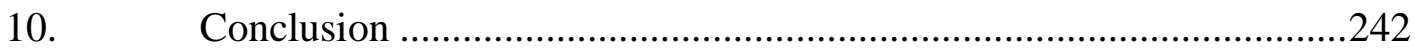

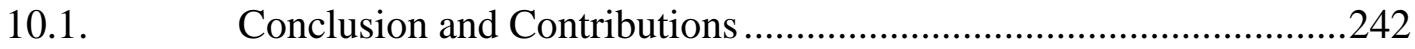

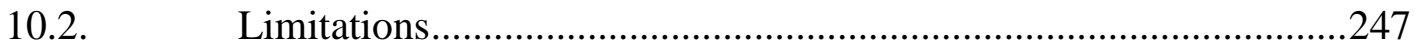

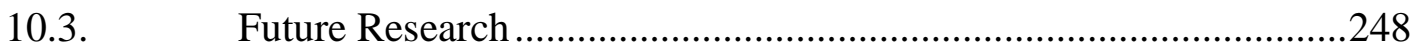




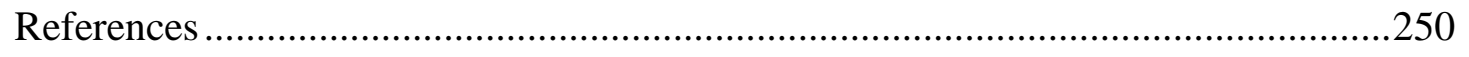

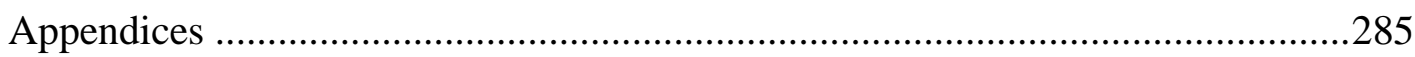

Appendix A- Perspectives and Criteria Definitions ...............................................28

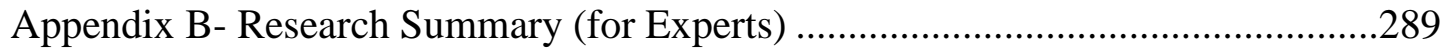

Appendix C- Expert Emails and Instructions......................................................296

Appendix D- Qualtrics Surveys and ETM HDM Software ...................................305 


\section{List of Tables}

TABLE 1: MATURITY ModELS IN DIFFERENT FIELDS...............................................50

TABLE 2: MATURITY ModelS IN HEALTHCARE …………….....................................53

TABLE 3: NIH FUNDING AND THE ChANGE THROUGHOUT THE YEARS .............................63

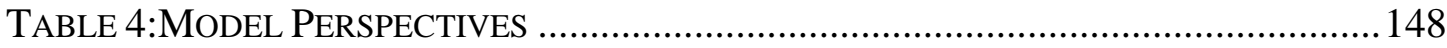

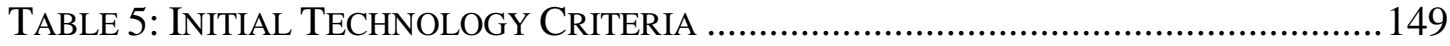

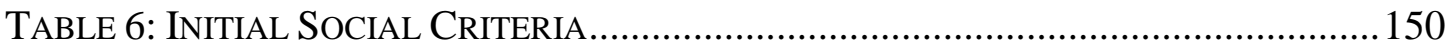

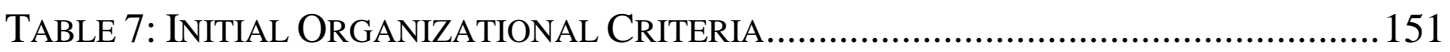

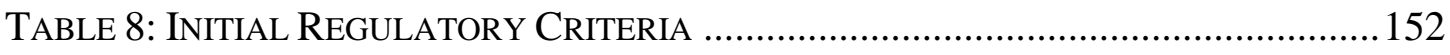

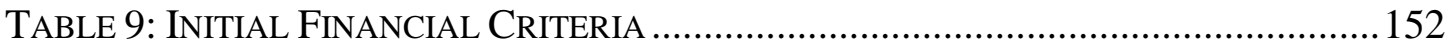

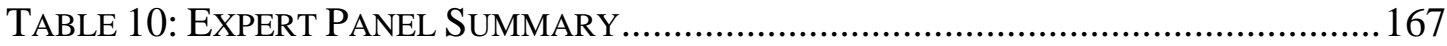

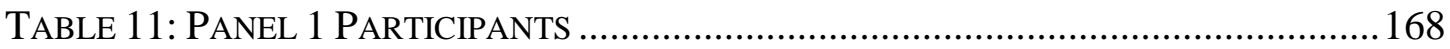

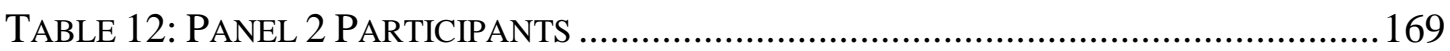

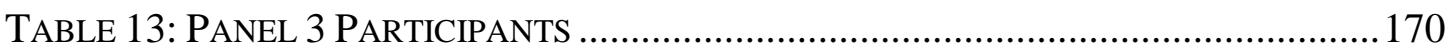

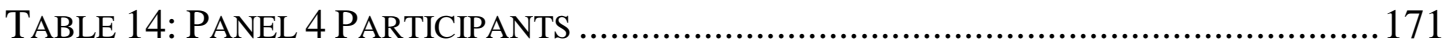

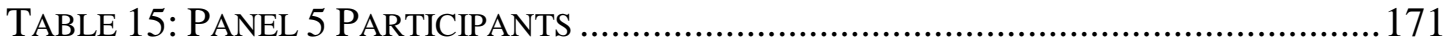

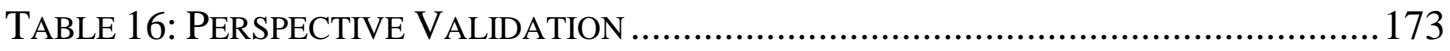

TABLE 17: TECHNOLOGY CRITERIA VALIDATION …………...................................... 174

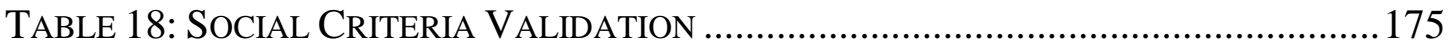

TABLE 19: ORGANIZATIONAL CRITERIA VALIDATION ………......................................176

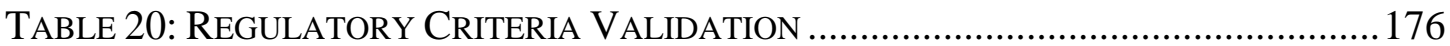

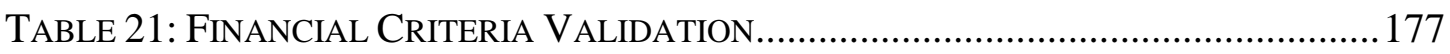

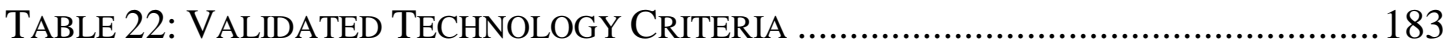

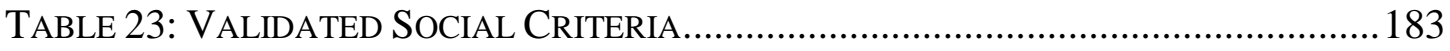

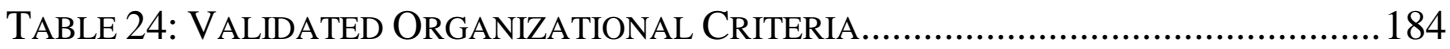

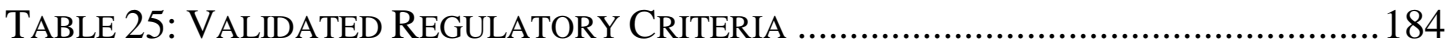

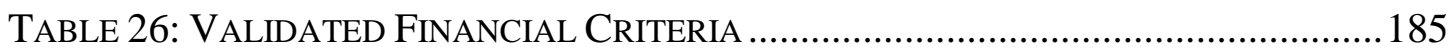

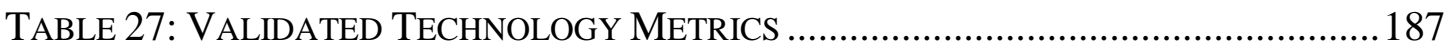

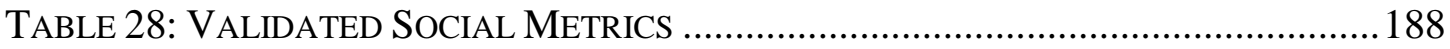

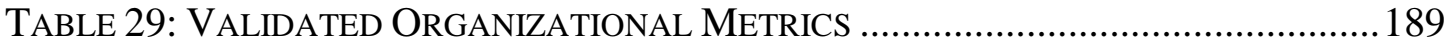

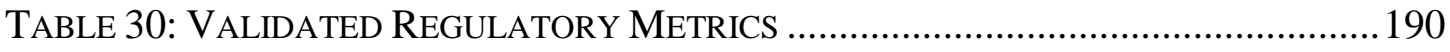

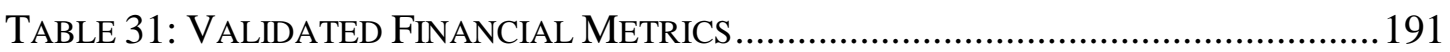

TABLE 32:DATA MANAGEMENT METRICS VALUE.....................................................192

TABLE 33: SUPPORTING INFRASTRUCTURE METRICS VALUE.........................................192

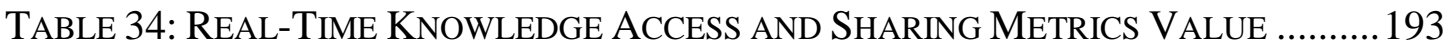

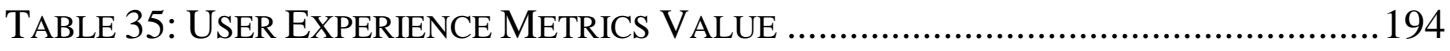

TABLE 36: STAKEHOLDER TRUST Metrics VALUE ..................................................... 194

TABLE 37: ACCESSIBILITY MetRICS VALUE.............................................................. 195

TABLE 38: STAKEHOLDER ENGAGEMENT MetRICS VALUE ..........................................196

TABle 39: TALENT ACQUisition AND Retention Metrics VALUE ...........................196

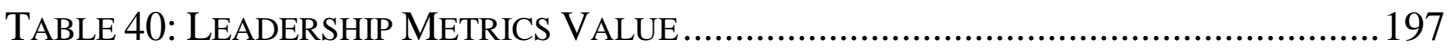

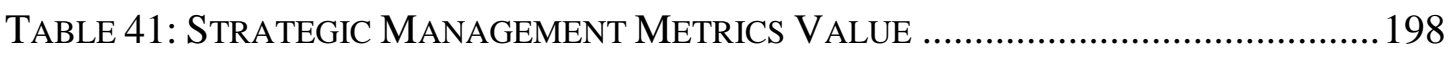

TABle 42: Culture AND TRanSParency Metrics Value .......................................198

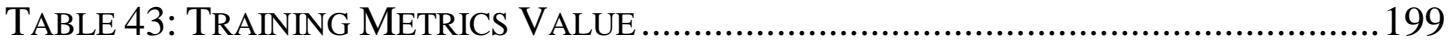




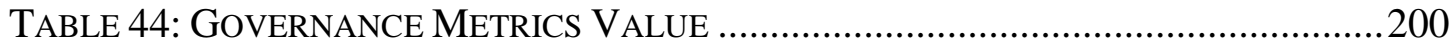

TABLE 45: STANDARDS CoMPLIANCE METRICS VALUE ……………………...............20

Table 46: Policy PREPAREDNESS AND AdAPTATION METRICS VALUE .......................201

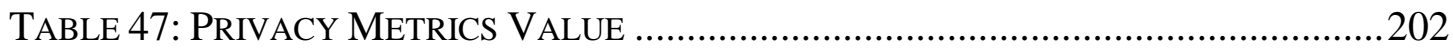

TABLE 48: INFORMING PoliCy Metrics VALUE........................................................202

TABLE 49:ResourCe AlLoCATION METRICS VALUE...................................................2203

TABLE 50: Funding AND Mission ALIGNMENT Metrics VAluE................................2204

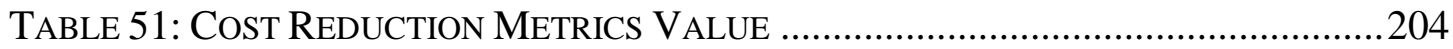

Table 52: Financial Synergy with Policies AND PrioRities Metrics VAlue...205

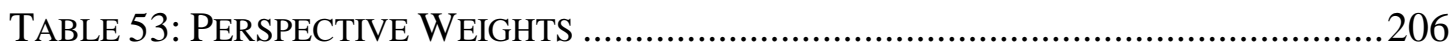

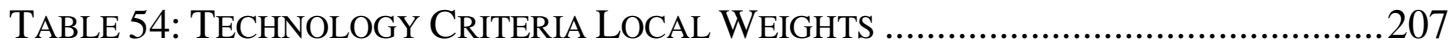

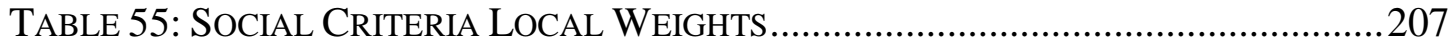

TABLE 56: ORGANIZATIONAL CRITERIA LOCAL WEIGHTS..........................................208

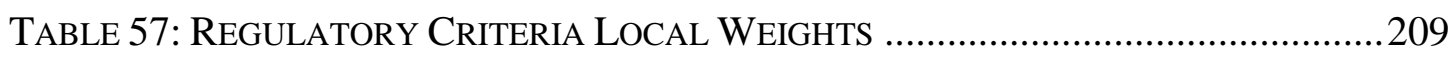

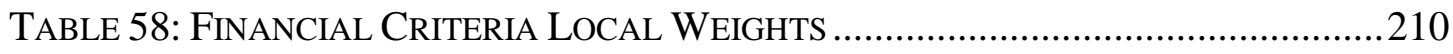

TABLE 59: INCONSISTENCY AND DISAGREEMENT RESULTS …….................................210

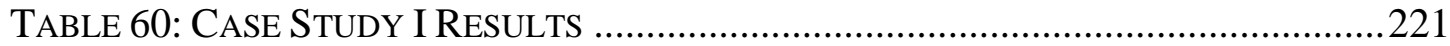

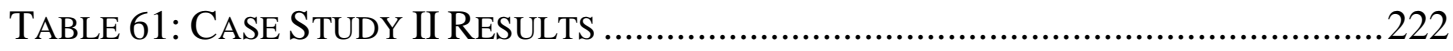

TABLE 62: RECOMMENDED IMPROVEMENTS FOR CASE STUDY I SIMULATION I ..........226

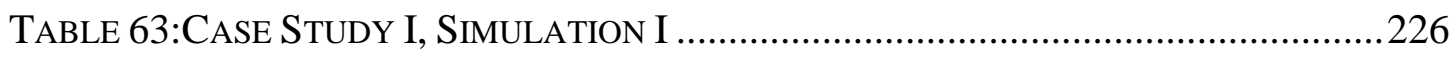

TABLE 64:RECOMMENDED IMPROVEMENTS FOR CASE STUDY I SIMULATION II..........227

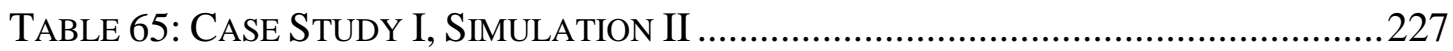

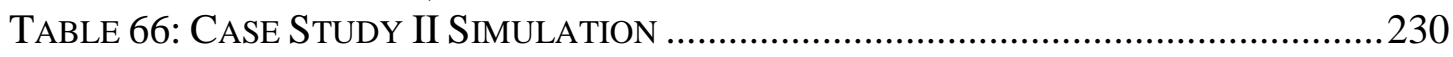

TABLE 67: RECOMMENDED IMPROVEMENTS FOR CASE STUDY II SIMULATION I.........231

TABLE 68: SCENARIO ANALYSIS ALLOCATED WEIGHTS.............................................23

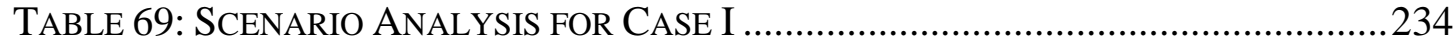

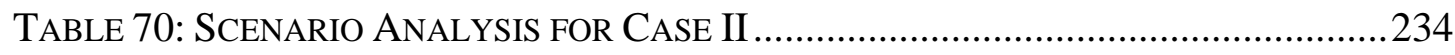

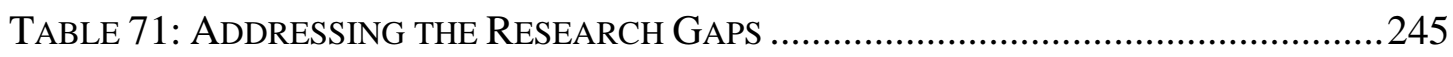

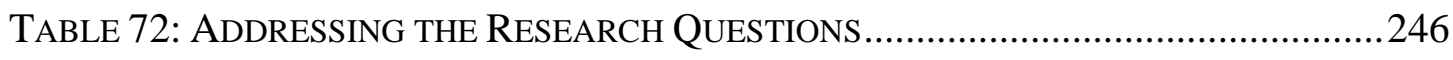




\section{List of Figures}

FigURE 1:EXTERNAL COMPONENTS OF HEALTHCARE ENVIRONMENT .......................... 16

FIGURE 2: CAUSE AND EFFECT DIAGRAM FOR EXTRINSIC CHANGE IN THE HEALTHCARE

ENVIRONMENT

Figure 3: Modified CAUSE AND EFFECT Diagram FOR EXTRINSIC CHANGE IN US

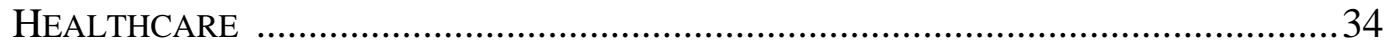

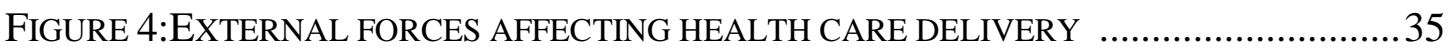

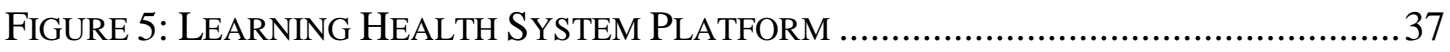

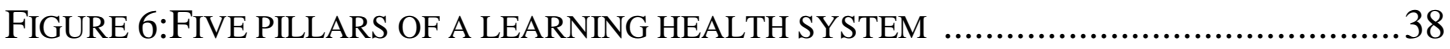

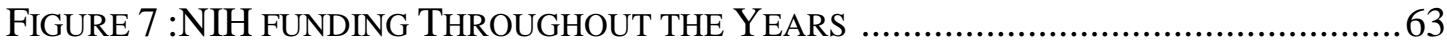

Figure 8: NIH Funding Throughout THE YeARs (PROGRAM LEVEL PROJECTED

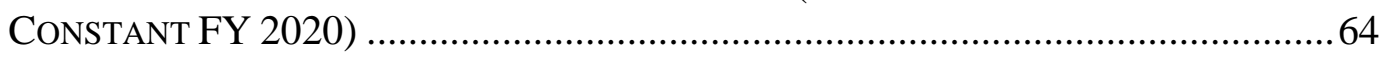

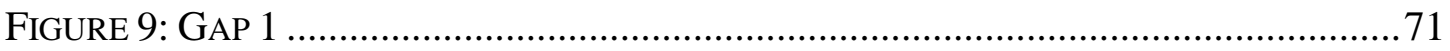

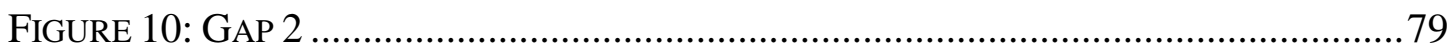

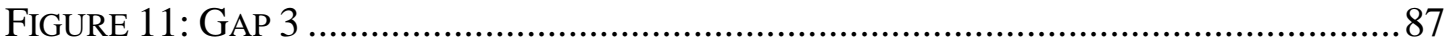

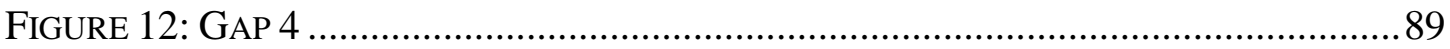

FIGURE 13: RESEARCH GAPS, GOAL, AND QUESTIONS............................................. 90

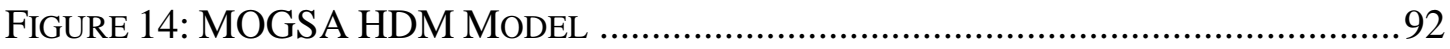

FIGURE 15: MisSION /PERSPECTIVE/ CRITERIA MODEL .............................................. 93

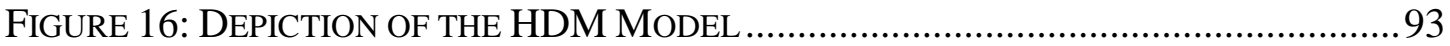

FIGURE 17: RESEARCH GAPS, GOAL, AND QUESTIONS (REPRISE) ............................. 103

FIGURE 18: CATEGORIES OF BIAS IN RESEARCH EVALUATION .................................. 110

FIGURE 19: SCREENSHOT OF THE FINANCIAL PAIRWISE COMPARISONS ...................... 114

FIGURE 20: AN EXAMPLE OF DESIRABILITY CURVES.................................................. 130

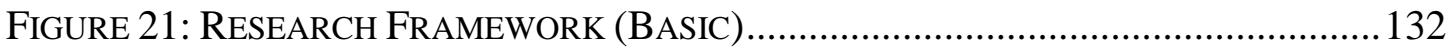

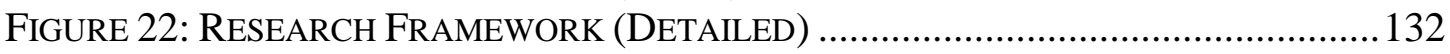

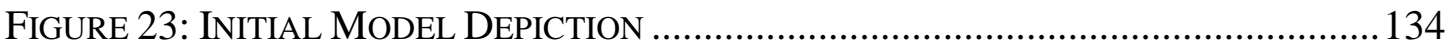

FIGURE 24: SNAPSHOT OF THE INITIAL SELECTED CRITERIA.................................... 139

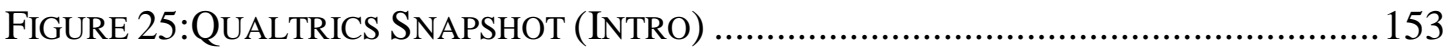

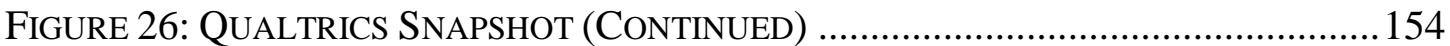

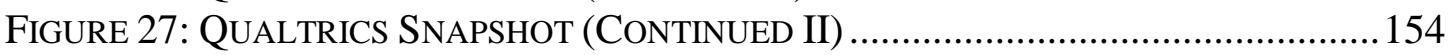

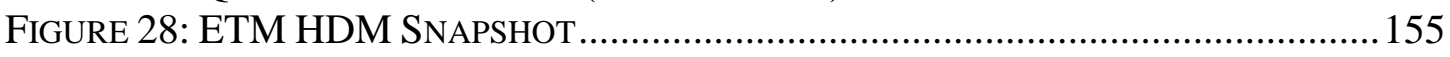

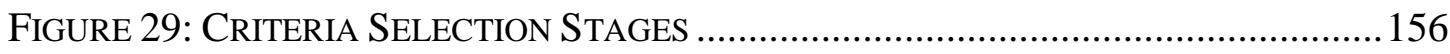

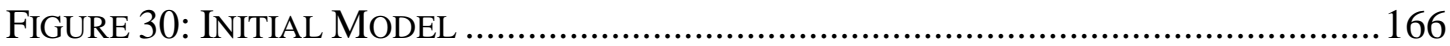

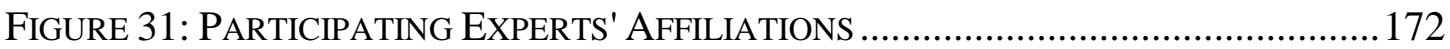

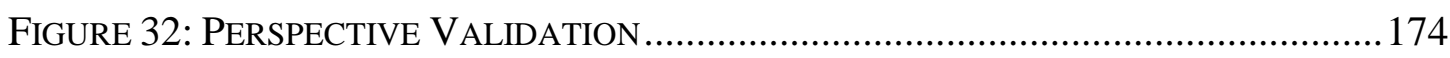

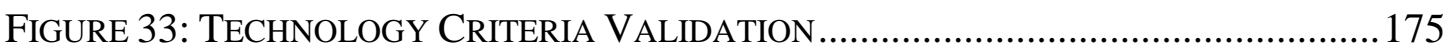

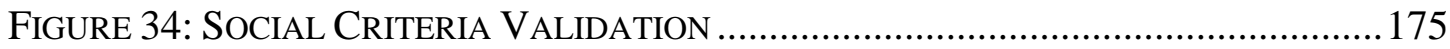

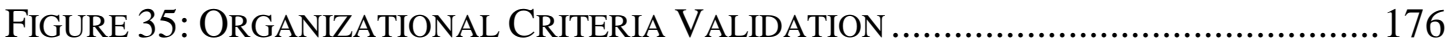

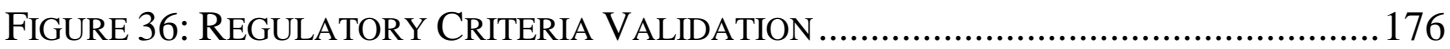

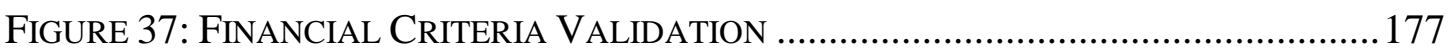

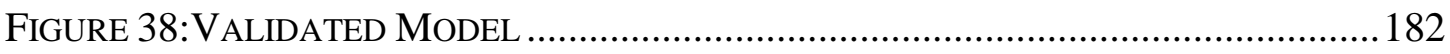

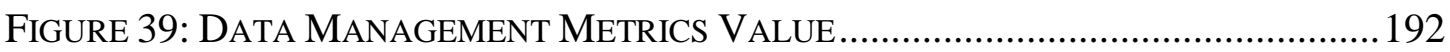

FIGURE 40: SUPPORTING INFRASTRUCTURE METRICS VALUE ................................... 193 
Figure 41: Real-Time Knowledge Access and Sharing Metrics Value .......... 193

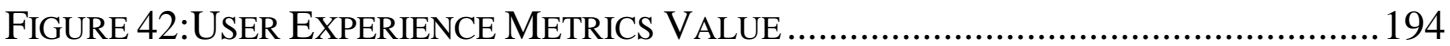

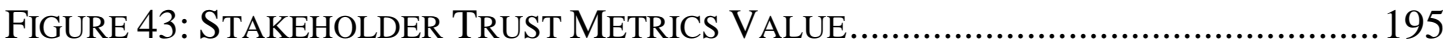

FIGURE 44: ACCESSIBILITY METRICS VALUE.......................................................... 195

FiguRE 45: StakeHolder ENGAGEMENT METRICS VALUE.......................................... 196

FiguRE 46: TALENT ACQUisition AND RETENTION METRICS VALUE ........................... 197

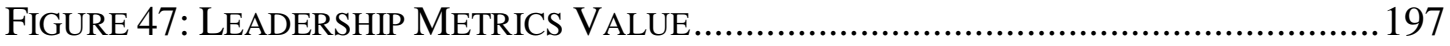

FiguRE 48: StRategic Management Metrics Value .............................................198

FiguRE 49: Culture AND TRANSPARENCY Metrics VALUE ....................................199

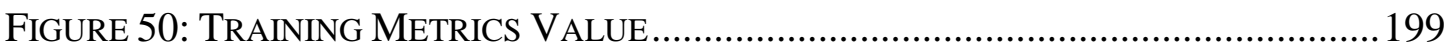

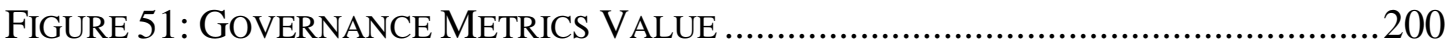

FIGURE 52: STANDARDS COMPLIANCE METRICS VALUE ……………………….........201

FiguRe 53: Policy PREPAREDNESS AND AdaPtation Metrics VAlue ......................201

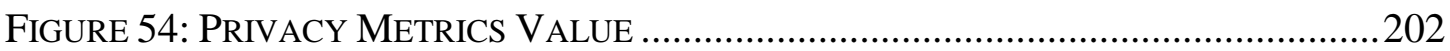

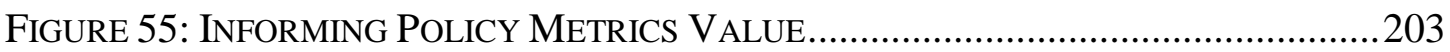

FiguRE 56: ReSOURCE ALLOCATION METRICS VALUE................................................203

FiguRE 57: Funding AND MisSION ALIGNMENT METRICS VALUE ……………….......204

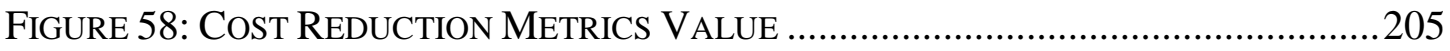

Figure 59: FinANCIAl SyNergy with Policies AND PRIORITIES Metrics VAlue.. 205

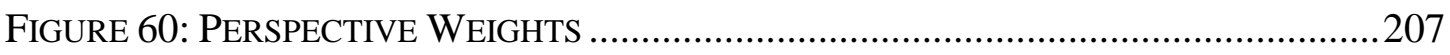

FIGURE 61: TECHNOLOGY CRITERIA LOCAL WEIGHTS ………………………............207

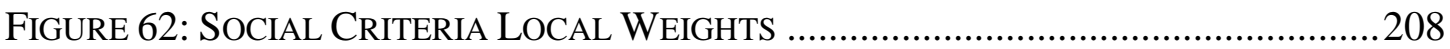

FIGURE 63: ORGANIZATIONAL CRITERIA LOCAL WEIGHTS ………….........................209

FIGURE 64: REGULATORY CRITERIA LOCAL WEIGHTS ................................................20

FigURE 65: FinANCIAL CRITERIA LoCAL WEIGHTS ...................................................210

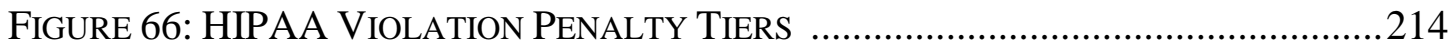

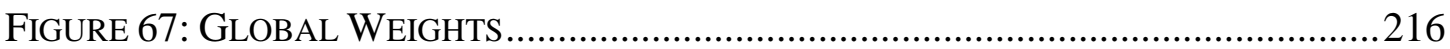

FIGURE 68: THE MODEL WITH GLOBAL WeIGHTS ..................................................217

FIGURE 69: GLOBAL WeIGHTS SORTED ……………….....................................218

FiguRE 70:SNAPSHOT OF THE DESIRABILITY VALUE SELECTION AS A PART OF THE CASE

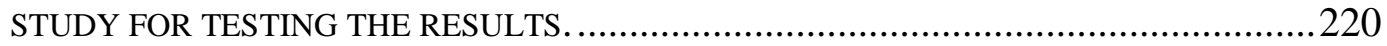




\section{Introduction}

In 1983 the prospective payment system (PPS) was introduced as a part of the Social Security Amendments Act of 1983 with the goals of addressing the high cost of healthcare by establishing fixed fees for provided services which were later modified to include a prospective payment system for Medicaid and Medicare in 2000 (CMS.gov, 2020). These changes have forever changed the way healthcare systems are managed in the United States (Ginter, Duncan and Swayne, 2013). Externally, the healthcare environment has been constantly changing in terms of technology, economy, and socio-political context and with each election (congress, senate, or presidential), different healthcare organizations are faced with new opportunities and risks due to the new political and legislative directions (Santilli and Randy Vogenberg, 2015; Shaygan, 2018). Internally, healthcare practices deal with long delay times for the transfer of clinical knowledge to clinical practice in the US (about 17 years) (Balas and Boren, 2000; Morris, Wooding and Grant, 2011; Kitson and Harvey, 2016). Furthermore, there are also studies indicating that adult patients receive only approximately half of recommended therapies (McGlynn et al., 2003). An Institute of Medicine report refers to this problem as "Too much care that is important is often not delivered, and too much care that is delivered is often not important.”(Institute of Medicine (IOM), 2007; National Health Service (NHS), 2014). To pile on these challenges, medical errors are the third leading cause of death in the US (Makary and Daniel, 2016). The United States consumes more health care services as a proportion of its total economic output than any other country in the world while having the largest economy in the world representing a greater ratio of the country's total 
economic output (Shi and Singh, 2019). The cost of care that has been increasing in US is signified by both per capita payments, and also by measuring health care expenditures as a percentage of the Gross Domestic Product (GDP) (Weiss and Lonnquist, 2000; Conklin, 2002). The US care spending has grown from $\$ 3708$ (per capita) to $\$ 9990$ from 1996 to 2015 (CMS.gov, 2016). Some studies suggest that up to a third of this spending is avoidable waste (IOM, 2011). As researchers form Stanford Medical school point out, in the healthcare system, "we do a lot, without knowing what works" at scientific, medical, and practice levels (Shah, 2016). In his book, Graban also mentions that the US has world-class doctors and treatments parallel to an utterly broken system (Graban, 2016). There is also the public health concern which with all the new technology and methods, the already disadvantaged are going to be even further disadvantaged in their access to the clinical and medicinal innovations (Faden et al., 2013). Moreover, the problem of health disparities based on Racial Ethnicity and Language (REAL) and Sexual Orientation and Gender Identity (SOGI) needs to be addressed. Although the healthcare systems in the United States have the ability to provide some of the best clinical care in the world, they lack delivering equitable services to every American. The healthcare system in the US certainly fails in terms of providing cost-efficient services (Shi and Singh, 2019). Due to the mentioned changes, there have been many attempts at improving managed care systems.

The United States has a unique system of healthcare delivery. The term "delivery" refers to the provision of healthcare services by various providers (Shi and Singh, 2019). Almost all developed countries have national health insurance programs managed and delivered 
by the government and financed through taxes and almost all their citizens are entitled to receive healthcare services. This is not the case in the United States, where Americans are not automatically covered by health insurance (Shi and Singh, 2019). Wolinsky (1988) disagrees with calling the American health care delivery a "system" as a true, cohesive system that does not exist in the US but for the sake of consistency, the research is going to use the word "system" for the US healthcare. One of the other significances of the healthcare system in the United States is that employs a significant amount of people with total employment that exceeded 16.4 million people in 2010 in various health delivery settings. This number included more than 838,000 professionally active Medical Doctors (MDs), 70,480 osteopathic physicians (DOs), and 2.6 million active nurses (U.S. Census Bureau, 2012). The majority of healthcare and services professionals (5.98 million) work in ambulatory health service settings, such as the offices of physicians, dentists, and other health practitioners, medical and diagnostic laboratories, and home health care service locations. Smaller proportions of these professionals are employed by hospitals (4.7 million) and nursing and residential care facilities (3.13 million). The wide spectrum of health care organizations in the United States includes around 5,795 hospitals, 15,700 nursing homes, and 13,337 substance abuse treatment facilities (US Census Bureau, 2012).

There have been some offered solutions for different challenges in US healthcare. Health IT adoption and diffusion of meaningful use can expedite the purgatory time for newly discovered knowledge (CMS.GOV, 2017). As a result of the new technologies and research, health organizations try to become more mature as in an organization that 
generates timely actions to the information that it derives from data to create meaningful measurement regarding systematic learning and increase efficacy and effectiveness in health outcomes. Challenged by internal and external factors, health care organizations need guidance regarding how to achieve improved maturity (Shaygan and Daim, 2019). Continuous learning may be one of the keys leading to more mature systems (Ainsworth and Buchan, 2015; Singer, Benzer and Hamdan, 2015; Priestman et al., 2019).

One of the ways in which health organizations can apply continuous learning into their practices is to use the acquired data beyond its original purpose (delivery of care) to increase the rate of speed from applying knowledge from laboratories to patient's rooms in hospitals (Friedman, Wong and Blumenthal, 2010). This change in looking at data can be implemented in any health system regardless of its level and scale by self-studying and self-improving continuously and routinely (Friedman et al., 2014, 2017). By using the data from drugs, diseases, clinical processes, devices, claims and with the newer data generated from technologies such as genomics and wearable devices, there is a volume of data that health systems can learn from like never before. Continuous feedback of learnings from data to patients, clinicians, managers, and policymakers can act as a catalyst for change while generating evidence and a science base for bolstering clinical practice and quality of care (Deeny and Steventon, 2015; Bhandari et al., 2016)

However, as interesting as the idea of this self-learning system sounds, its practical implementation is indeed a daunting and developing task. Morrain et al. (2016) pinpoint leadership, funding, regulatory and political influences, and company culture as some of the important factors in making a continuously learning health system (Morain, Kass and 
Grossmann, 2017). Friedman et al. (2013) stress the importance of technology, policies, and standards in turning the learning health system into reality (Bloomrosen and Detmer, 2010; Blumenthal, 2010; Friedman, Wong and Blumenthal, 2010).

Continuously learning health systems are being studied from a wide range of different perspectives in terms of adoption and implementation (Kelley et al., 2015; Brooks et al., 2017; Cahan and Cimino, 2017; Daniel et al., 2017; Kraft et al., 2017; Okun and Goodwin, 2017; Rubin, 2017; Tegenege et al., 2017). However, there is a need to look at these perspectives together to gain some insights on the bigger picture opportunities and barriers surrounding these health systems. In other words, although many health organizations are trying to bolster their continuous improvement and consequently their maturity, there is no particular way for them to know or assess if they are on the right path or the extent to which they are adopting or implementing this way of doing things.

With the current unaddressed deficiencies and the new groundbreaking innovations, there are hopes of a paradigm shift in US healthcare systems like never before. Continuous selfstudying and self-improving healthcare systems can be the answer to many of these problems by leveraging many of the discussed technologies and innovations. However, as a substantially complex environment due to the many involved stakeholders, health organizations face many challenges and are provided with many opportunities from different aspects in the area of continuous learning and improvement. Although researchers should keep on studying this topic from detailed and specific perspectives, a multi-perspective study of the different impacting aspects of this promising and nascent technology is vital. The cross-criteria study of the health organizations with the ability of 
continuous learning can help provide a bigger picture of what needs to be considered to initiate the constantly improving and waste eliminating healthcare systems. One of the goals of this study is to act as the first step in the multi-perspective study of this topic. There is imperative to look deeper and with further detail into the political (regulatory, organizational), economic, social (patient, public health), and technical factors contributing to how successful health organizations are in terms of technology management maturity. Challenged by internal and external factors, health organizations need guidance regarding how to achieve improved maturity (Shaygan and Daim, 2019). Continuous learning in different areas specific to the health organizations may be one of the keys to this improvement (Singer, Benzer and Hamdan, 2015; Priestman et al., 2019).

There is also a need for studies on how the new technologies, innovations, and policies affecting healthcare should be managed in order to raise the chances of success for this nascent area. The potential positive results of studies in the field of continuous learning health organizations (in terms of increased quality of care, decreased medical errors, and cost) may encourage knowledge cross-pollination and further adoption and diffusion of the culture of continuous learning across the United States. It should however be understood that becoming a continuously learning health organization is not something that its effects can be felt immediately, and it may take years of cultural adaptation and practice concurrent to the sufficient funding and socio-technical infrastructure to start fulfilling its promises.

From the organizational self-awareness point of view, there is a need for health organizations to assess their performance and maturity from different aspects that 
contribute to the adoption, implementation, and management of the technologies they possess. It is also important to take a deeper look into the multi-perspective management of technology maturity in health organizations. This need for maturity has become more important with advances in technology, the plethora of data, and an increase in the pressure to be more effective and consistent.

\subsection{Problem Statement}

As it was explained in the previous section, significant-gaps in the practical transformation of clinical knowledge into clinical practices ever-increasing healthcare costs, high rates of medical errors, healthcare institutions' obligations towards improving safety, clinical outcomes, and efficacy of care, the extrinsic fluctuant nature of the healthcare industry, and the advances in technology and available information has put more pressure on healthcare organizations to adopt new ways of doing things to cut costs, bolster care in terms of quality, safety, accessibility among other goals. Some of these goals include engaged patients and patient-centered communication, evidence-based care to include clinical trial and comparative effectiveness research, learning health IT system, turning evidence into practice and improvement in performance and quality, and affordable care for all patients (IOM, 2013; Shah et al., 2016). Some of the other goals based on literature include the promotion of improvement science, the impact of research findings, and faster implementation and translation of research findings and evidencebased treatment in practice (Kilbourne et al., 2017).

There has also been research on quality improvement in specific administrative and clinical issues in healthcare organizations. Mottes, Goldstein, and Basu (2019) discuss 
the benefits of adopting quality dashboards and the adoption of continuous quality improvement strategies in adherence to institutional standards and delivery of renal replacement therapy while Fried et al. (2011) applied a lean improvement process to further improve process and outcomes in successful sepsis protocols. Moreover, there has been literature focusing on quality improvement in other clinical and administrative issues such as diabetes care in populations and outpatient clinics (Solberg et al., 1997; Nicolucci et al., 2008), minimizing medical and medication errors (Becher and Chassin, 2001; Lee, 2013), improving care in patients with severe sepsis and septic shocks (Seoane et al., 2013; Armen et al., 2016), reducing avoidable readmissions (Kripalani et al., 2014), nephrology (Nunes et al., 2016; Mottes, Goldstein and Basu, 2019), outpatient cancer surgery centers (French et al., 2019), chemotherapy wait times in pediatric oncology clinics (Elsaid et al., 2019), TNM classification in cancer (Gospodarowicz et al., 2004), management of Chronic Obstructive Pulmonary Disease (COPD) (Tiep et al., 2018), supply chain performance measurement (Swinehart and Smith, 2005), and complications and cost after high-risk surgeries (Dimick et al., 2003).

One of the ways of addressing these changes and moving towards the mentioned goals is to increasing efficiency by generating more timely actions to the information that they derive from the available data to generate meaningful conclusions regarding fields such as systematic learning in order to increase efficacy and effectiveness in health outcomes. The systematic continuous learning by health organizations can be aligned with continuous improvement and innovation from scientific, informatics, incentives, and culture aspects, and knowledge about best practices is consistently captured, assimilated, 
and embedded within the system. Moreover, continuous feedback of learnings from data to patients, clinicians, managers, and policymakers can act as a catalyst for change while generating evidence and a science base for bolstering clinical practice and quality of care (Morain, Kass and Grossmann, 2017). A paradigm shift in looking at data can be implemented in any health system regardless of its level and scale by self-studying and self-improving continuously and routinely (Deeny and Steventon, 2015; Friedman et al., 2017). Health IT adoption and diffusion of meaningful, and using the acquired data (drugs, diseases, clinical processes, devices, claims and with the newer data generated from technologies such as genomics and wearable devices (Shah, 2016)) beyond its original purpose (delivery of care) can expedite knowledge transfer from laboratories to patient's rooms.

With all this promise, this concept mostly remains as a concept than a reality (Budrionis and Bellika, 2016). Many health organizations have some idea of how to become a learning health system or think that they are one while they are not. A lot of these problems are caused due to the lack of a systematic assessment tool for health organizations to measure and assess their maturity in terms of adoption, implementation, and management of technologies and in this case, becoming a continuous learning practicing health organization. Moreover, there needs to be a multi-criteria decisionmaking model for the management of the different aspects of the factors leading to more maturity. 
Therefore, there is a need for a model to give health organizations a sense of selfawareness in terms of adoption, implementation, and management of these new sociotechnological mentalities. This model should be able to:

- Identify potential common factors that have a major impact on the maturity of a health organization's continuous learning approach while considering its complexity and multi-perspectives.

- Assess the health organization's performance against each of those factors potentially leading to important conversations around the next steps and improvement initiatives.

- Help in identifying the areas of strength and where there is a need for corrective/preventive actions based on the assessment, giving the organization a sense of self-awareness and direction.

- Assist decision-makers in their strategic management and leadership and resource allocation.

Health organizations can get a better sense of direction in order to prioritize their improvement paths and agenda while having a better grip on their performance and maturity of the intended socio-technological solutions leading them to be more mature organizations. 


\section{Literature Review}

\subsection{Healthcare Landscape in United States}

Shi and Singh (2019) posit that U.S. health care delivery does not function as a rational and integrated network of components designed to work together coherently. They describe it as a kaleidoscope of financing, insurance, delivery, and payment mechanisms that are not integrated efficiently as they represent a mixture of the public (government) and private sources. The public sector, finance and ensure health care for eligible groups of people while delivering direct care services (less volume) to certain groups such as veterans, military personnel, American Indians/Alaska Natives, and some uninsured people. Outside of this, however, the majority of financing, insurance, payment, and delivery services remain private in the United States. Private financing, mostly via employers, accounts for around 52\% of total health care expenditures while the government finances around 48\% (DeNisco, 2019). Moreover, as the United States possesses a market-driven economy, private entrepreneurs seek to profit from it by improving important functions of the delivery of health. Most people in the United States receive health services through the private sector as the health insurance is bought by the employers while the government provides public insurance for health services through programs like Medicare, Medicaid, and Children's Health Insurance Program (CHIP) for a significant portion of the country's low-income, elderly, disabled, and pediatric populations (although being public, the health services for the enrollees of these programs are provided through private health organizations and providers such as maintenance organizations (HMOs). In general, in this research, the term provider refers to any entity that delivers health care services and either independently bills for those services or is 
supported through tax revenues such as physicians, dentists, optometrists, and therapists in private practices, hospitals, and diagnostic and imaging clinics, and suppliers of medical equipment.

The healthcare delivery system in the US lacks standardization as a system that is functionally not centralized with non-integrated parts since a central agency such as the government does not govern its coordination. This can cause challenges such as duplication, overlap, inadequacy, inconsistency, and waste and consequently a costly and complex system that lacks planning, direction, coordination, and efficiency manipulated by private entrepreneurial system trying to change the monetary incentives towards their goals without worrying about the effects on the system as a whole resulting cost control to be merely a mirage (Shi and Singh, 2019).

Some of the other characteristics of the healthcare delivery system in the United States which makes it different than other countries on top of the absence of the central governing agency are insurance coverage-based access to health, and the presence of third-party insurance companies and multiple payers. Moreover, the US healthcare system deals with legal risks as influencers of the practice behavior or physicians through "defensive medicine" by prescribing additional diagnostic tests, follow-up visits, shy away from using the newer technologies due to lack of experience with those technologies and creating comprehensive documentation to avoid litigation risks. The United States is the world leader in terms of research and development and innovation in the field of medical technology and as helpful as these new technologies are, they create demands while tightening the resources for health organizations to finance the latest and best 
technologies. Therefore, managing these new technologies is a very critical task especially in the health organizations in the United States.

In the last several decades, the healthcare industry in the US has become more complex and difficult to manage (Guterman and Dobson, 1986; Zook, 2007; Ginter, Duncan and Swayne, 2013). Some of the factors influencing this state are the changes in legislative direction with each election, domestic and global economic climate, social changes, and emergence and improvement of related technologies which can affect the health care delivery dramatically. In the light of these changes, the healthcare manager's task of comprehending the surrounding environment and hence, preparing the organization to cope with or strategically take advantage of the changes in it becomes vital. Since the changes in the healthcare industry in the early 80 s, managers have found out that focusing solely on financial aspects of planning is not enough and health organizations should be dynamic in order to deal with the dynamic environment (Zook, 2007).

Taking the made points into account, the U.S. health care delivery system is a gargantuan thing that is almost impossible for any single entity to manage or control (Shi and Singh, 2019). In short, this costly system is not effective in the most important missions of an acceptable health care delivery system which are:

- Enable all citizens to obtain needed health care services.

- Ensure that services are cost-effective and meet certain established standards of quality. 
While the U.S. healthcare delivery system falls short of both these basic ideals, the United States leads the world in providing the latest and the best in medical technology, training, and research. It offers some of the most sophisticated institutions, products, and processes of health care delivery Thus, the constant analysis and assessment of strategy is imperative to healthcare organizations in the US. In order to do this, managers should have a clear understanding of the prospective changes to act proactively and plan. However, since the dynamic healthcare environment is affected by multiple elements, identifying these changes poses a big challenge to organizations.

\subsection{Healthcare External Environment}

In the turbulent environment of US healthcare, like any other business or organization, identifying ways to add more value compared to competitors in order to gain a vying advantage is an important task and challenge (Mallard et al., 2004). This is a big problem for care organizations as value creation is the perceived relationship between contentedness and price (Hart and Milstein, 2003). Going through a smooth appointment and billing system, high quality of professionals and equipment, good insurance alliances are just some of the ways health organizations can create value internally (Mallard et al., 2004; Testik et al., 2017a). However, concurrent to these internal issues and opportunities, care organizations should pay immense attention to the factors happening and changing in their external environment. Out of date management styles, disregarding demographic changes and emerging legislations and technologies are some of the telltales of organizations that are either unable to anticipate changes, ignoring them, or resisting them before their imminent demise. Although, completely predicting the looming 
changes and opportunities is not feasible, health organizations and managers can bolster their chances of dodging or taking advantage of changes by keeping abreast of the possible changes in the healthcare environment. Perera and Peiro (2012) proposes "Analyzing the external environment" as the first stage in strategic planning for health organizations (Rodríguez Perera and Peiró, 2012).

It is important to mention that different entities existing in the healthcare environment have impacts on each other and can affect healthcare organizations both directly and indirectly. Figure 1 shows the interrelationships of different components of healthcare's external environment. As an example of these interrelationships, government institutes regulate laws for businesses and education while funding some research institutions while research institutions provide $\mathrm{R} \& \mathrm{D}$ for businesses and a chance of better quality or access to individuals. Education institutes provide researchers for research institutes and have the power to raise care awareness for individuals. Businesses provide the government with tax money and researchers, hospital and education establishments with real-life data, sponsorship, and funding as well as jobs, products, and services for individuals. Individuals shape the demographic aspect of the environment in general while providing revenues and workforce for business, tax money for governments, individuals for education, and data for research centers. Other than government's regulative impacts (Department of Health and Human Services, Center for Medicare, and Medicaid Services), organizations such as "Public Health Department", "State Health Planning Agency", "Joint Commission on Accreditation of Healthcare Organizations" and "Council on Education for Public Health" have regulatory and planning impacts on 
healthcare organizations. These interrelationships would pose great impacts on hospitals and healthcare organizations in both direct and indirect manners. Hence, a better look into these approaching changes in order to better understand and consequently prepare to mitigate their harms or benefit from them is critical to health organizations. For this purpose, impactful perspectives and their sub-criteria will be explained in the following sections.

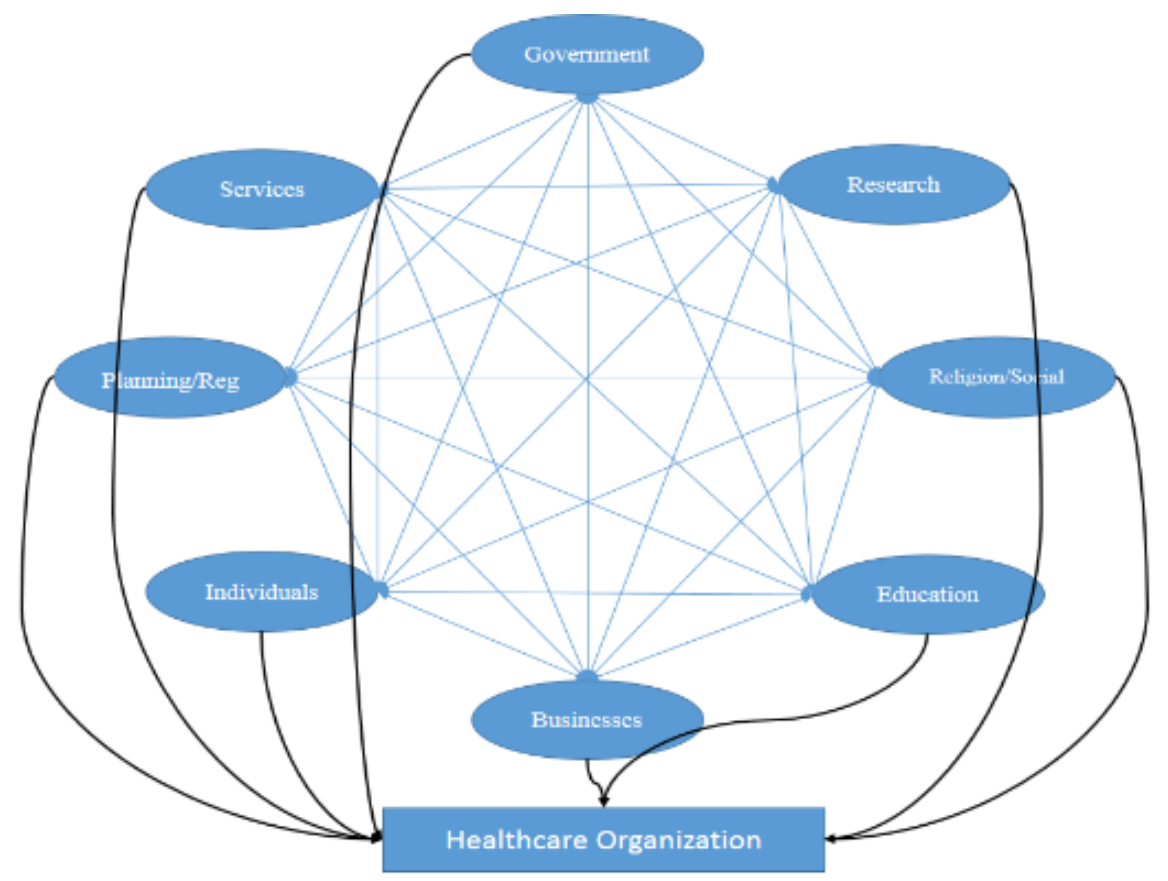

Figure 1:External Components of Healthcare Environment

\subsubsection{External Change Perspectives}

It is a difficult task to categorize the different perspectives affecting healthcare organizations due to the complexity and inter-relationships of different categories and criteria. Schuman (2001) points out that more studies have focused on external perspectives causing concern in health organizations compared to the distress caused by 
the internal environment of the respective organizations (Such as management styles and policies (Suchman, 2001). However, the literature on healthcare external environmental analysis is not an abundant one. Casalino et al. (2003) discuss the effects of incentives, IT, and structured process in order to bolster the quality of care for chronic patients (Casalino et al., 2003). Also Ginter (2013) groups the external change categories as "Legislative", "Economic", "Technological", "Social", and "Competitive" (Ginter, Duncan and Swayne, 2013). Although this book mentions some of the sub-categories relating to the perspectives, it does not offer clear explanations on what those sub-criteria are. Santilli et al. also identify the trends affecting decision-making processes and roles in healthcare organizations in order to bolster firms' ability to act better in market transformations (Guterman and Dobson, 1986). This study continues to use the perspectives from Ginter and Santilli while exploring the current change catalysts falling under each category.

\subsubsection{Legislative/Political Changes}

The legislative and political changes can significantly affect the environment for healthcare organizations and each change in administration or swing in senate power can mean big changes coming healthcare's way. The passing of the Affordable Care Act (ACA) for instance, undoubtedly restructured the role of healthcare stakeholders. Healthcare providers have a more serious role in terms of contributing to savings, risk, and establishing relationships compared to the time before ACA.

Since being passed on March 23, 2010, the Patient Protection and Affordable Care Act has had significant impacts on the healthcare environment in different ways. The bill 
tended to transform hospitals' way of doing things financially, technologically, and clinically in order to achieve a better quality of care and its accessibility at fewer costs. As an example, ACA paved the way for the emergence of accountable care organizations, switching from fee-for-service to bundled payments (CMS.gov, 2019a), Hospital Readmissions Reduction Program (HRRP) (CMS.gov, 2019b), and new insurance standards, among other things. Also, it has had a great impact on the technological structures of hospitals due to cost/quality initiatives in terms of electronic health/medical records. In 2013, about 93\% of hospitals had certified EHR technology, increasing by 29\% since 2011 (Charles, Gabriel and Searcy, 2015). This number rose to $96 \%$ percent by 2015 in terms of EHR systems with functionality, capability, and security measures of the Department of Health and Human Services (Henry et al., 2014). The Medicare and Medicaid EHR Incentive programs incentivize professionals, eligible hospitals, and critical access hospitals to adopt and implement meaningful use of certified EHR technology. As mentioned in the previous section, ACA rebuilt the role of healthcare organizations by giving them a bigger role in terms of contributing to savings, risk, and establishing relationships. A more efficient system would allow hospitals to cut costs by focusing on preventive care and wellness in long term. ACA has also paved the way for more trends such as consumers with stronger roles, more structured quality measures, and healthcare consolidations which will be discussed in the next sections (Santilli and Vogenberg, 2015). Also, post-ACA, the attention has switched to outcomes as quality metrics such as the success rate of treatments, morbidity rate, length of hospital stays, and patient satisfaction, among others. 
Although ACA has had many great impacts on the way healthcare is provided in the United States and its shift from transaction-based rewards to outcome-based ones, it is not a panacea for the problems in the healthcare structure and needs reforms. However, like insurance and financing for healthcare is mostly employment-based in the United States, it has left some employed individuals uninsured due to some small businesses' inability to afford group insurance (therefore not offering insurance to their employees) and/or voluntary participation in health insurance programs in some work settings (Shi and Singh, 2019). Some employees choose not to sign up, mainly because they cannot afford the cost of health insurance premiums. Moreover, some individuals cannot afford the premium cost-sharing provided by their employees (paying a portion of the cost to their employers) and some have to deal with higher individual rates as self-employed people and other individuals who are not covered by employer-based plans.

Although ACA greatly has somewhat mitigated the rise in the percentage of healthcare in US GDP, it has been the constant repealing by the republican party. This kind of volatility poses a great uncertainty for healthcare organizations in terms of strategic management and finding ways of achieving competitive advantage in a highly volatile unstable environment. The changes inherent in legislative reforms offer stakeholders numerous opportunities and threats that have to be analyzed and ways to prepare for them or take advantage of them thought through. Executive, legislative, and judicial branches of the US government will all have effects on future bill changes, and companies should pay a great amount of attention to changes and prepare themselves in terms of strategies for different outcomes. What makes a change in legislation such as ACA so important is the 
inevitable effects it will have in different change perspectives for healthcare companies in terms of the external environment (Shaygan, 2018).

Politics have significant effects on the lack of balance socioeconomic health as well (Borrell et al., 2007). Changes brought up by the elections and union densities have the power to affect labor markets and welfare states resulting in income and socioeconomic disparities and consequently impacting healthcare and the organizations in that area. This means that not only legislations and politics can have direct effects on healthcare, but they can also have indirect impacts by influencing other change perspectives such as social/cultural.

\subsubsection{Economic Changes}

Issues such as increasing average age of the population, health care, and treatment costs, and changes in the number of insured people can have effects on the country's economy and certainly be affected by it. Many of the changes that have led to a managed care system can be traced back to economic changes. The increasing cost of health care in the US is signified by both per capita payments, and also by measuring health care expenditures as a percentage of the Gross Domestic Product (GDP) (Weiss and Lonnquist, 2000; Conklin, 2002). The US care spending has grown from $\$ 3708$ (per capita) to $\$ 9990$ from 1996 to 2015 which is about 70 times bigger than the per capita health spending of \$141 in 196 (CMS.gov, 2016). Moreover, faster growth in total health care spending in 2015 was caused by bigger growth in private health insurance, hospital care, physician and clinical services expenditures, and the growth in Medicaid and retail prescription drug expenditures. 
Many of the factors that have had impacts on this growth can be rooted back to other change perspectives such as social, technological, and legislative. US population has had an increase of $79.25 \%$ between 1960 and 2015 (Bureau, no date), and based on Kinsella and Gist (1995), the percentage aged 65 and older in the United States will be tripled from 1944 to 2033 (Kinsella and Gist, 1995; Shaygan, 2018). Some of the other factors which affect the economic perspective and consequently the health care environment are the rise in the cost of insurance, increase in the alliance of health care organizations, improvements in technology, and rise in malpractice insurance and case settlements (Weiss and Lonnquist, 2000).

\subsubsection{Social/Demographic Changes and Disparities}

In 1991 Stromborg pointed out the dramatic change that is going to happen in the balance of the American population (Frank-Stromborg, 1991). This change in the composition of the population in terms of Social and demographic diversification is happening much faster today. As an example, the Hispanic population has gone from around 6 million to 52 million from 1960 to 2012 and Asian and Pacific Islander population from 980 thousand to 15 million in the same time frame (US Census Bureau Public Information Office, 2012). From the age aspect, based on Day (1992), from 2010 to 2030 the population of 65 and over is estimated to go from about 40 million to 70 million accounting for more than 20 percent of the population (Day, 1992). With the increase in the number of insured people in the Obama administration, many people who were added to the insurance pool had a lot of health conditions due to avoiding medical care for the sake of their costs. Some people also needed time to adapt to and learn the system and 
this experience curve costs money and time. Although in short term the number of increased insured people has had dramatic effect on the cost of care, it will become smoother as time goes by. These shifts will pose great demands on US health-care organizations. In a 2010 report issued by the Institute of Medicine, the healthcare workforce is estimated to be too small and under-equipped to satisfy the demands of the rising and aging population (Stall, 2010).

Parallel to these changes, health organizations need to address the racial and ethnic disparities that are extremely prevalent today. Racial disparities in health care delivery and patient outcomes exist and persist in the health care system in the United States (Haider and Pronovost, 2011). Minorities tend to receive lower-quality health care even when insurance status and income are controlled (Baker, 2001). Fiscella et al. (2000) discuss that Black patients receive less intensive hospital care such as receiving fewer cardiac procedures, lung resections for cancer, and kidney and bone marrow transplants (Fiscella et al., 2000). Similarly, Haider et al. (2012) argue that minority patients tend to seek care at a relatively small number of lower-performing institutions (sometimes due to location) and due to that, are disproportionately affected by the suboptimal quality of care by looking at 434 hospitals and concluding that trauma patients seen at hospitals with more than $50 \%$ minority patients have $37 \%$ higher adjusted odds of death compared to similarly injured patients treated at hospitals with less than $25 \%$ minority patients (Haider et al., 2012).

During the 2020 COVID-19 pandemic, based on the Center for Disease Control's data, the cases, hospitalization, and death for American Indian or Alaska Native on-Hispanic 
persons were 2.8, 5.3, and 1.4 times higher compared to white, non-Hispanic persons, respectively. The numbers for Black or African American, Non-Hispanic persons were 2.6,4.7, and 2.1 times higher for the same metrics and 2.8, 4.6, and 1.1 times higher for Hispanic or Latinx persons (COVID-19 Hospitalization and Death by Race/Ethnicity | $C D C$, no date). To better serve their community, health organizations need to address things such as engineered inequality, default discrimination, and the misconception that all technologies are always benevolent for everyone. Creating roadmaps for establishing the systems needed to support health organization's path towards addressing health disparities can increase the patients' trust in them which can include investigation of racial and ethnic disparities faced by their population, learning where to start with strategies in order to achieve a reliable collection of race and ethnicity data in electronic health systems and consequently taking the needs of the community population into account and stratifying quality measures based patient demographics. It should be noted that the path towards addressing disparities should include efforts by health organizations' information technology groups to build systems that support those initiatives. These efforts can be in the forms of (but not limited to) mapping legacy data to CDC criteria, adopting standards, developing business intelligence data infrastructure, and stratifying artificial intelligence quality measures based on Racial Ethnicity and Language (REAL) and Sexual Orientation and Gender Identity (SOGI). Improvement of data governance, using analytical tools to identify data quality improvement opportunities, and addressing data anomalies can be named as big opportunities for improvement in this area for healthcare organizations. 


\subsubsection{Technological Changes}

With all the changes in population and demand, healthcare organizations are in necessary need of leveraging the value of technology advancements in order to be cost-effective, competitive, and responsive. Technological tools assisting decision-makers in Analyzing data, leveraging the power of big data, and information technology systems have become pillars of improving quality of care, identifying trends, anticipating changes, and controlling costs. Electronic Health Records (EHR) have shown to have great benefits in increasing outcomes for healthcare organizations in $92 \%$ of studies in the literature despite low patient engagement numbers (Buntin et al., 2011; Furukawa et al., 2014).

Healthcare organizations should leverage the developments in technology and computing in order to increase patient engagement and satisfaction, reduce costs, anticipate changes, and learn to develop intangible assets in orders to integrate and intelligently use their resources. Based on a report published by the Institute of Medicine (IOM) of the National Academy of Sciences, the quality of health care in the United States is weaker compared to biomedical knowledge, and that this gap in quality due to organizational incompetency, rather than of individual physicians'(Beckles et al., 1998; Chassin, Galvin and Quality, 1998; Corrigan, 2001).

Every year new technologies are emerging focused on empowering patients and providers in order for organizations to better manage changes and costs. Furthermore, innovation in biotech and pharmaceutical industries has led to faster market entry and stronger research and development pipeline. As an example, a sudden change of speed in cost per genome has occurred in 2008 reflecting the transition from Sanger-based sequencing to 
next-generation genome sequencing technologies (Mardis, 2008). Besides, rare diseases have gained more attention from pharma companies due to significantly less time needed in terms of patient testing, government financial incentives, and higher approval rates from the US Food and Drug Administration (FDA) (Seoane-Vazquez et al., 2008).

It is healthcare managers' and organizations' duty to leverage, coordinate, and manage emerging technology tools to bolster increased data transparency, patient involvement. This perspective can be affected by and affect other change perspectives such as legislative, social, competitive, and the economy in a great way as mentioned before.

Innovations in computing and big data services are causing a transformation in the manner that health data is stored and transferred among patients and providers. Healthcare organizations are embracing technologies and innovations such as EHRs and EMRs, clinical documentation tools, big data, and telemedicine devices in order to improve the process of health information collection and consumption. Wearable technology, mobile health, and big data analytics are becoming progressively valuable in healthcare delivery systems. Although these innovations and technologies will significantly facilitate diagnosis, prevention, and treatment more efficiently, they dramatically increase the need for organizations to care about the security of their data (Shaygan, 2018). However, Lyon et al. (2014) suggest that as more patients adopt new information technologies, the importance of data analysis for organizations surpasses privacy and security concerns.

As for big data analytics, the healthcare environment is one of the areas which is going to be most affected by it. The cost of healthcare in the US is undeniably high (approximately 
$17.6 \%$ of the nation's GDP increasing from $8.9 \%$ in 1980) (Nambiar et al., 2013; Christopher G. Worley and Mohrman, 2014). It is also estimated that one-third of that spending is due to waste caused by (as listed by the Institute of Medicine) unnecessary services, administrative waste including unproductive and duplicate documentation, inefficiently delivered services, high prices, fraud and missed prevention opportunities (Nambiar et al., 2013). In addition to that, the current reimbursement model favors the number of patients over treatment effectiveness: "physicians have been compensated under a fee-for-service system that only considers treatment volume, not outcomes" and patients have "little responsibility for the cost of the health care services they demand" (Kayyali, Van Kuiken and Knott, 2013). On top of the financial loss, other consequences of this problematic system include statistics such as: "one out of five elderly patients are readmitted within 30 days of discharge for no known reason" (Nambiar et al., 2013). Big Data analytics may hold the key to solving some of these issues. Barham (2017) conducts a literature review on how big data can create value for organizations and discusses the challenges hindering its adoption. Health organizations to leverage the power of big data in order to gain competitive advantage in terms of being more cost and time effective and improve their quality and experience of care for their patients. Barham (2017) recommends companies to acquire experienced data scientist, learn from successes and failures of other organizations, and embrace the benefits of data integration and sharing with partners, and finally working closely with software developers to develop more userfriendly applications for patients and professionals. 
Like in other developed countries, the public sector of the U.S. has started to act concerning big data in order to leverage its potential in overcoming various complex challenges. In 2012, for instance, the Obama Administration invested \$200 million in the "Big Data Research and Development Initiative", with goals including the advancement of state-of-the-art core technologies of the big data era, acceleration of the pace of discovery in science and engineering, strengthen national security, and transform teaching and learning and to expanding the workforce needed to develop and use big data technologies (Jee and Kim, 2013).

Social media is increasingly being used as a tool by governments, communities, and organizations for a range of purposes in disaster preparedness in areas such as disaster management. In recent years, the government is bolstering the ease of data release and accessibility, which enables better access to and standardization of public data of patients, clinical trials, and health insurance (Christopher G. Worley and Mohrman, 2014). Moreover, ACA has also started the process of fundamentally re-shaping the industry and the interrelationships between healthcare entities. The private sector is also being affected. Hospitals, providers, clinics, insurance companies, pharmaceutical companies, researchers, physicians, nurses, and patients are all impacted by the changes that the use of big data is bringing into the industry. Meanwhile, traditional pharmaceutical retailers such as CVS Health are developing internal Digital Innovation Labs, that are rolling out Apple-Watch apps and facilitating the process of filling medications remotely (CVS Health, 2015). 
All in all, new IT innovations can give health organizations insight and advantage. However, managers and organizations leveraging the fruits of these innovations should manage the ethical, security, and privacy risks that come along with them. The use of data and analytics in patient care opens up new opportunities for boosting care effectiveness and efficiency even though the full realization of the importance of data-driven insights has been clouded by some barriers. United States' health information technology website lists some of these barriers as current data input and output limitations of medical record systems, scarcity of robust business models for interoperable data exchange across organizations, and wider organizational barriers that require coordinated solutions across stakeholders (HealthIT.gov, 2018).

In the second decade of the 21 st century, social media has become a significant part of many industries and organizations, and the healthcare environment is not an exception. Vance et al. (2009) hint at the increasing evidence showing that social media use among patients and health professionals is rising significantly. Personal social networks use between health professionals and physicians-in-training reflect the general trend in the environment and as for the Patient-doctor interactions, most of them are initiated by patients which demonstrates the level of awareness and interest from patients' perspective.

There is also a growth in online social media awareness in Western European hospitals, but different countries have applied social media to address different issues. Van der Belt et al.(2012) mentions that other than the Netherlands and UK, there is a small proportion of hospitals using social media among European countries. As an example, hospitals use 
LinkedIn to hire professionals. Again, there is a need for further research to define metrics and connect the effects of social media in healthcare quality improvement. Chou et al. (2009) suggest that new technologies such as social media are reshaping the patterns in communication in the United States.

A very important issue in leaning towards social media for health organizations is the consideration of the targeted group's age, socioeconomic status, and racial ethnicity in order to assure enough awareness and interest among that specific target. In more rural structured areas, there may be less inclination, awareness, and confidence by patients to use social media. This inertia, however, can be mitigated by education and promotion of the benefits and conveniences of using social media. Clinicians also use online social networks, specifically the newer generation of clinicians for both personal and reference aims.

However, a big portion of respondents have a cynical attitude towards online interactions and see them as being ethically problematic (Bosslet et al., 2011). Quantification of social media effects is a nascent area of research due to the lack of used terminology and research methods (von Muhlen and Ohno-Machado, 2012). Antheunis et al. (2013) argue that the literature has mostly focused on the benefits of social media on healthcare and suggests that their study has found dis-concordance in patients' and professionals' motives, barriers, and expectations regarding health-related social media use. Hawn (2009) discusses the impact of social media on health care and its benefits such as "Improving quality through "better communication" and "Empowering Patients" 
resulting in happier patients. The study also pinpoints the privacy, standard, and cost downsides of social media in healthcare.

In sum, health organizations and managers should carefully monitor the potential effects and implications of social media in the environment and industry to be prepared for the challenges and clinch opportunities in order to reach or manage competitive advantage.

\subsubsection{Competitive Changes}

The last change perspective reviewed in this section is the competitive changes perspective. As the healthcare environment is becoming a harsher vying one for providers, there is significant pressure for providing a better quality of care at a lower cost. As mentioned before, these changes are causing a paradigm shift from the conventional fee-for-service models to value-based ones and focus on the quality of care as supposed to the number of services provided. This pressure is stronger in areas with more demand which there is buyer power. According to a report by Stanford Medicine, Physician practices in less vying areas cost more for office visits compared to more competitive areas of care (Baker, 2014). From another aspect, these pressures are leading to the consolidation of healthcare entities to increase efficiency, cost-effectiveness, and share risks. Although there is research evidence for the benefits of consolidation of healthcare entities in terms of information sharing and flow, there is not much supporting the same effect for cost-effectiveness. Dranove and Lindrooth (2003) Find robust, significant, and persistent results showing that consolidations into systems do not create savings whereas acquisitions and mergers generate savings from $3^{\text {rd }}$ and $2^{\text {nd }}$ consolidation years respectively (Dranove and Lindrooth, 2003).

However, if these consolidations lead to prevention of medical errors, avoidable hospital admissions, and readmissions and/or improve hospital efficacy, an increase of shareddecision making, and improvement of targeting costly services, achieving better quality at lower costs may be possible (Olsen, Saunders and Yong, 2010). Other change sub- 
criteria such as healthcare networks becoming narrower and health quality measures becoming tighter have been discussed in the literature (Santilli and Vogenberg, 2015; Shaygan, 2018).

\subsubsection{Summary}

Accumulation of information is becoming more significant for health organizations (Sutcliffe and Weber, 2003). With the methodical study of adaptive systems, healthcare organizations can provide managers with great insights and assistance in the preparation for emerging issues within the organization as well as healthcare delivery management (Shaygan, 2018). In this sense, being familiar with the external environment can be the key to leading a successful and competitive health system (Ginter, Duncan and Swayne, 2013).

Due to the involvement of several volatile change perspectives, the healthcare environment is currently a very erratic one making the extrinsic environmental analysis a daunting task for health organizations and managers. This study aims at contemplating emerging extrinsic changes happening in the United States healthcare environment in different areas. A literature review is performed to define and identify the different current change perspectives and their subcategories. To better illustrate these issues, the Ishikawa diagram (Cause and effect diagram) is used in this study as shown in Figure 2. Five perspectives were identified (Political/Legislative, Economic, Social/ Demographic, Technological, and Competitive) and each of their sub-criteria was studied. 


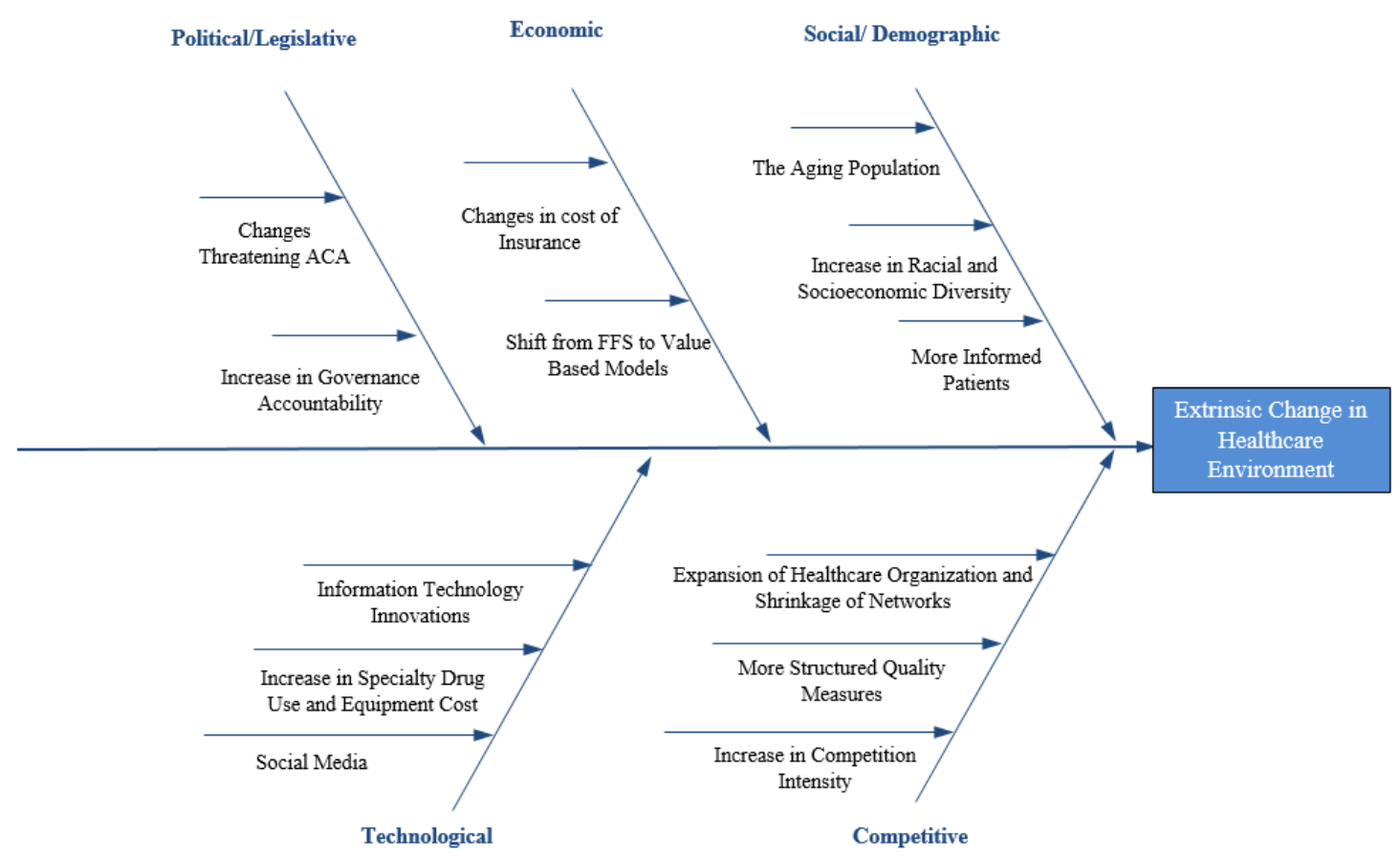

Figure 2: Cause and effect diagram for extrinsic change in the healthcare environment (Shaygan, 2018)

An interesting finding of this study is the inter-dependence of different change perspectives through literature and to better reflect that finding some modifications were applied to the cause-and-effect diagram with the aims of better showing the effectiveness of change perspectives on each other.

The Healthcare industry, in general, is very complicated and dynamic as many nascent trends and potential political changes are emerging and happening nationally and globally. This is even a bigger issue in the United States which is facing waves of political, social, economic, competitive, and technological changes. Healthcare organizations need to be better prepared in predicating, identifying, adapting, being proactive and taking advantage of these changes, and cope with the potential harms coming their way. They should be vigilant in sensing valuable tangible and intangible assets, efficiently seizing 
them, and finally be flexible enough as an organization to transform and mold these resources to their advantage (Teece, 2007).

In the same sense, healthcare organizations should sense the changes, seize the opportunities, or dodge the threats, and develop organizational flexibility to reconfigure themselves and gain a competitive advantage in the industry through learning mechanisms, alliances, innovation, and being cognizant of surrounding changes on national and global levels. As Eisendhardt (2000) suggests, in the highly dynamic environments (such as healthcare) no matter how valuable, rare, inimitable, and nonsubstitutable the resources (such as tacit and explicit knowledge), they are going to give companies a fleeting and finite competitive advantage as opposed to a sustained one and the important thing is how firms reconfigure their "best practices" in unique ways (Eisenhardt and Martin, 2000). Health managers need to sense new opportunities and threats, seize them for the advantage of the company and reconfigure them in order to provide the organization with a sustained competitive advantage (Teece, 2007). 


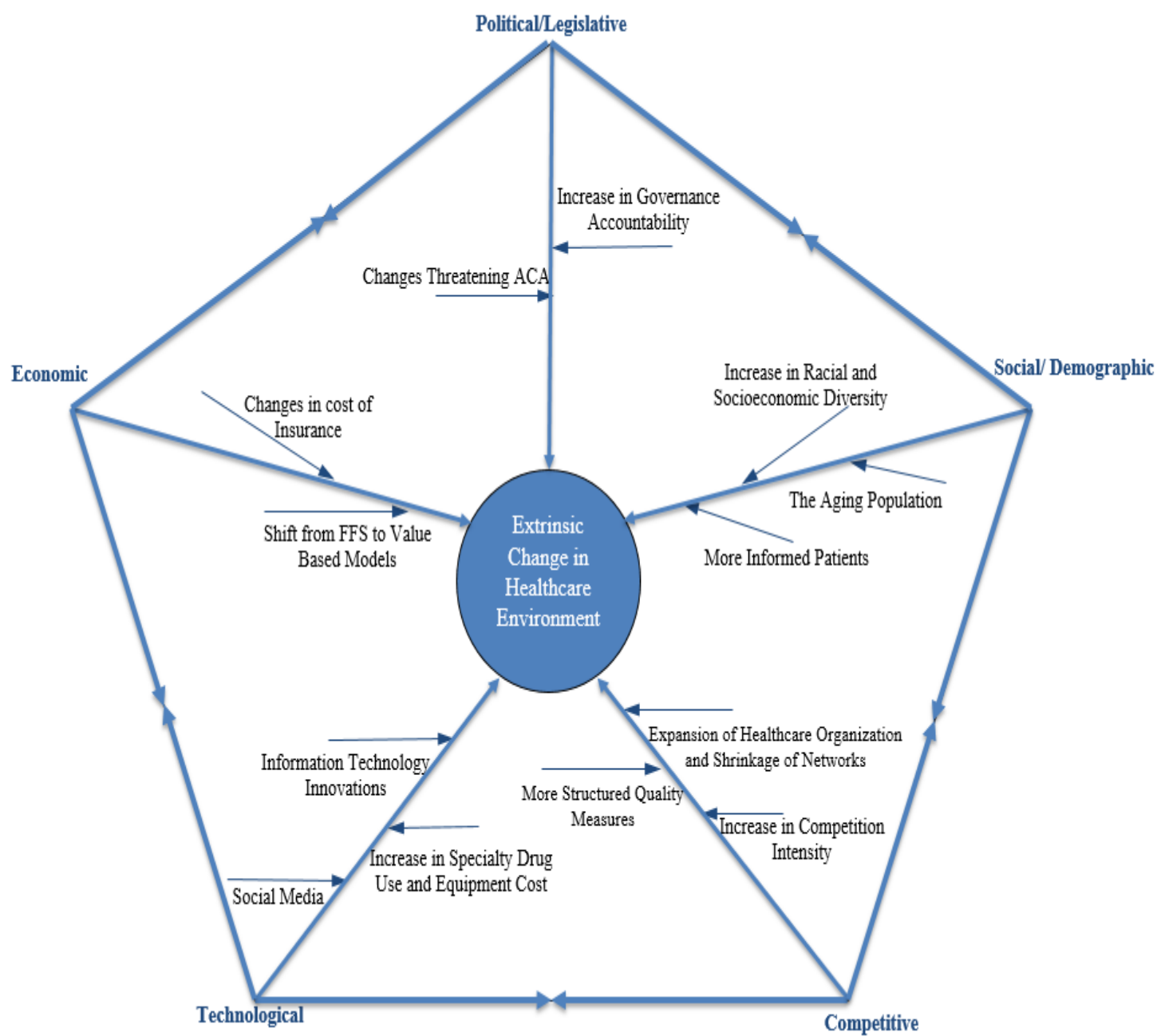

Figure 3: Modified Cause and Effect Diagram for Extrinsic Change in US Healthcare (Shaygan, 2018)

Shi and Singh (2019) discuss the external forces on the United States healthcare delivery system as being national political climate, economic development, technological progress, social and cultural values, physical environment, population characteristics (i.e., demographic and health trends), and global influences which can be a great complement to aspects mentioned in this sections. 


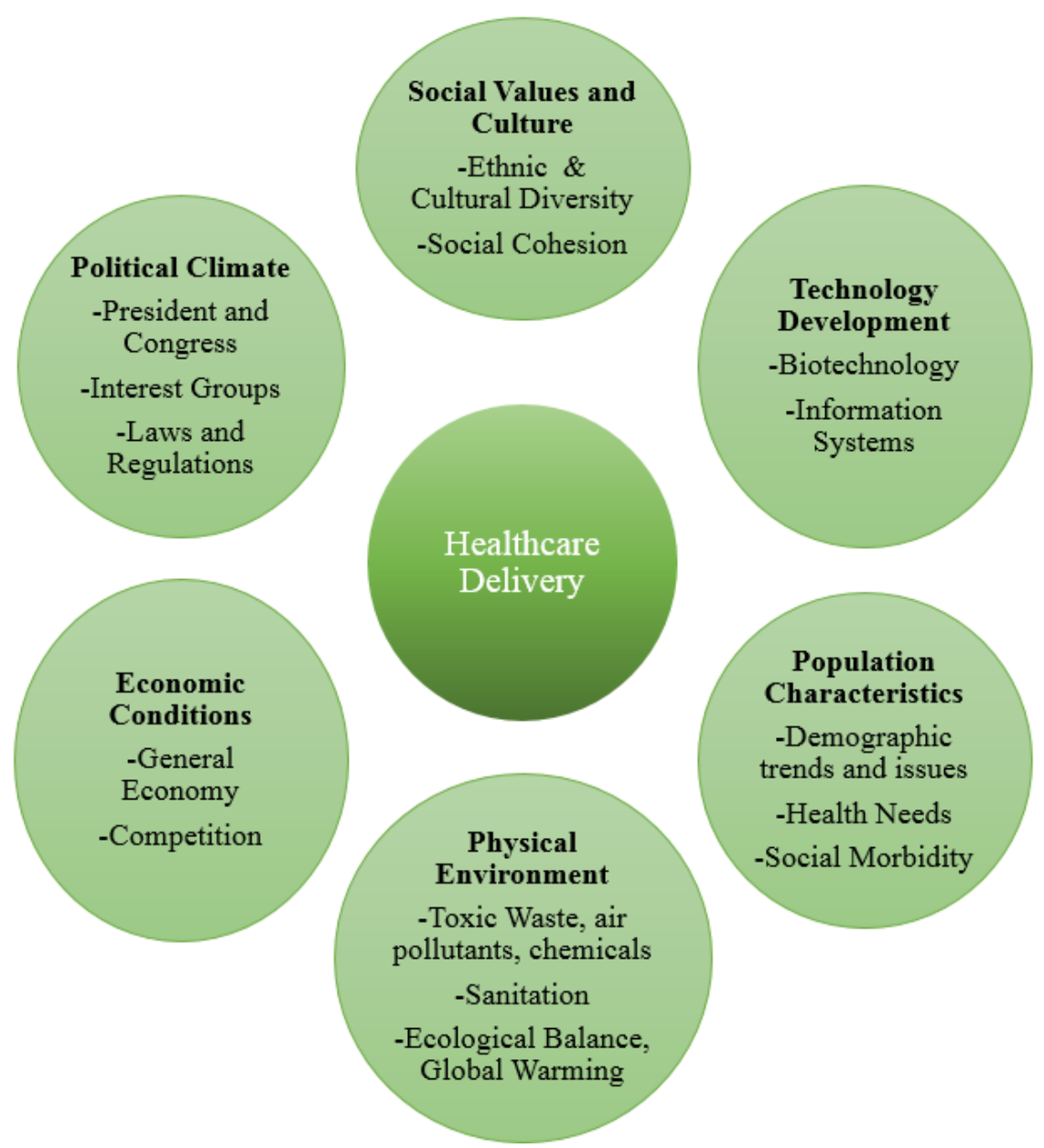

Figure 4:External forces affecting health care delivery (Shi and Singh, 2019)

\subsection{Learning Health Systems (Continuous Learning)}

The term Learning Health Systems (LHS) was first used in a 2007 report by the National Academies of Medicine (formerly known as the Institute of Medicine) as a system with some distinguishable characteristics (Institute of Medicine (IOM), 2007). The patient information, experience, and characteristics of every patient (consenting ones) is an opportunity for the health system to learn from. Moreover, evidence and science-based knowledge regarding best practices are instantly available to bolster the decision-making processes in a continuous and iterative quality improvement manner. Finally, the LHS 
way of thinking becomes a piece of the employees' and stakeholder's culture through appropriate leadership as a part of a socio-technical infrastructure (Friedman et al., 2017). Milstein (2013) emphasizes that adopting LHS is one of the three things that the US should pursue in order to cut down care costs as a percentage of its GDP (Milstein, 2013).

However, there is an imperative for infrastructure for continuous improvement which will result in cost decrease through learning and economies of scale. In a perpetual cycle, the ongoing accumulation and analysis of data lead to result interpretations which assist decision-makers in taking actions and deciding what to study next. The continuous quality improvement has the potential to not only bolster the clinical evidence and research-based data and update the best practice in real-time but also will be able to address a lot of administrative and practice wastes which their elimination can cut care costs without the need for layoffs as the lean and continuous improvement philosophy is parallel with respect for people (Graban, 2016). Concurrent to this waste reduction, the accumulation of research and practice-based knowledge can take a substantially shorter time in terms of a transitional gap. 


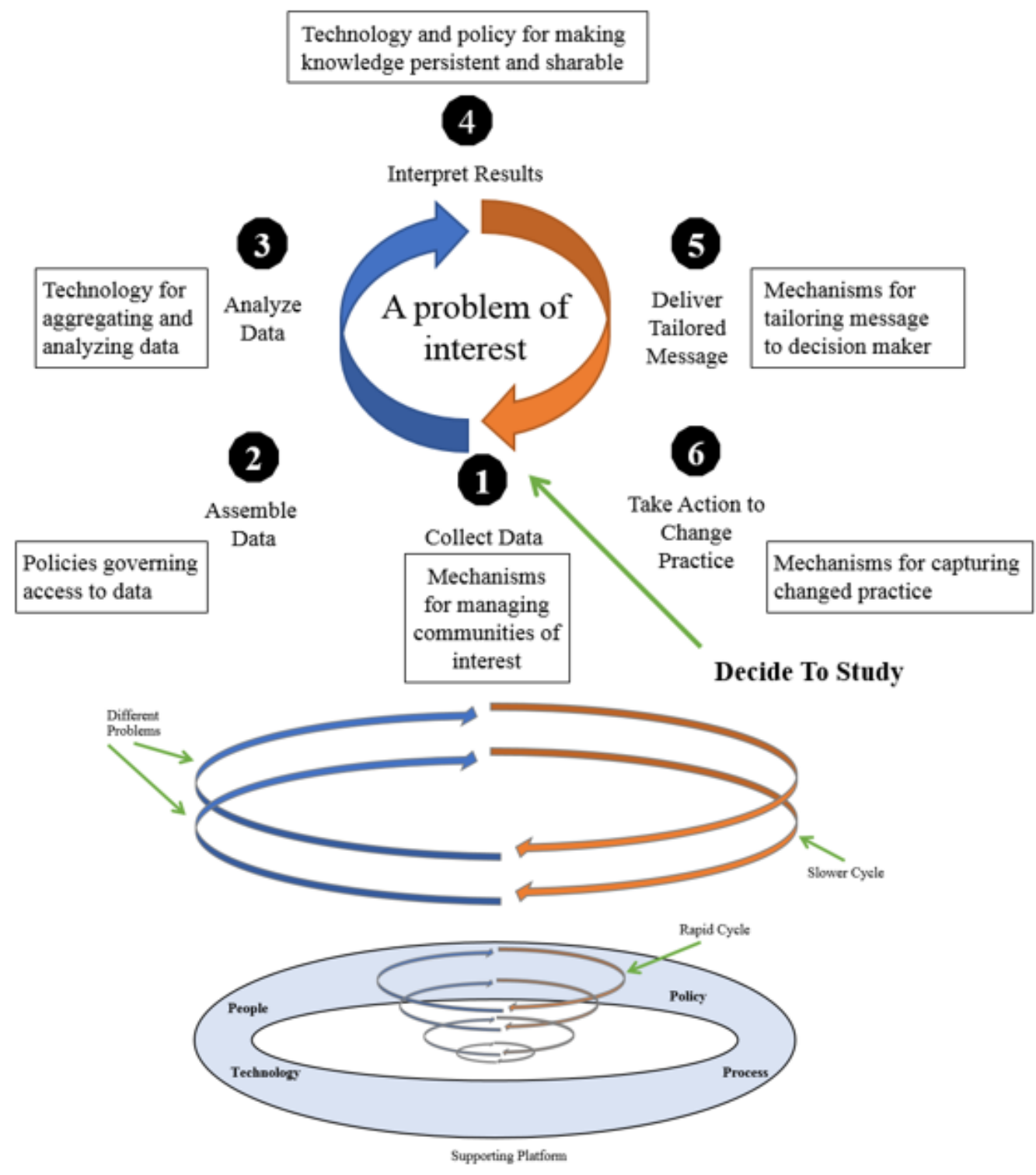

Figure 5: Learning Health System Platform (Friedman et al., 2017)

As much as health systems need to assimilate and gain knowledge, they must jettison the knowledge and resources that are impeding quality progress or have become obsolete due to innovation. Coicera (2017) stresses the importance of forgetting in LHSs as a procedure of programmatically decommissioning obsolete data and practices which their existence hinders change and lead to unnecessary complexity (Coiera, 2017). 
Taking all these different benefits and challenges into account points out that there are many players and impacting the continuous learning and consequently the maturity of health organizations. These factors can be government legislations, funds, and initiatives concurrent to the acceptance of its culture in terms of organizational and personal levels. The role of community and socio-technical infrastructure among many other aspects cannot be underestimated either.

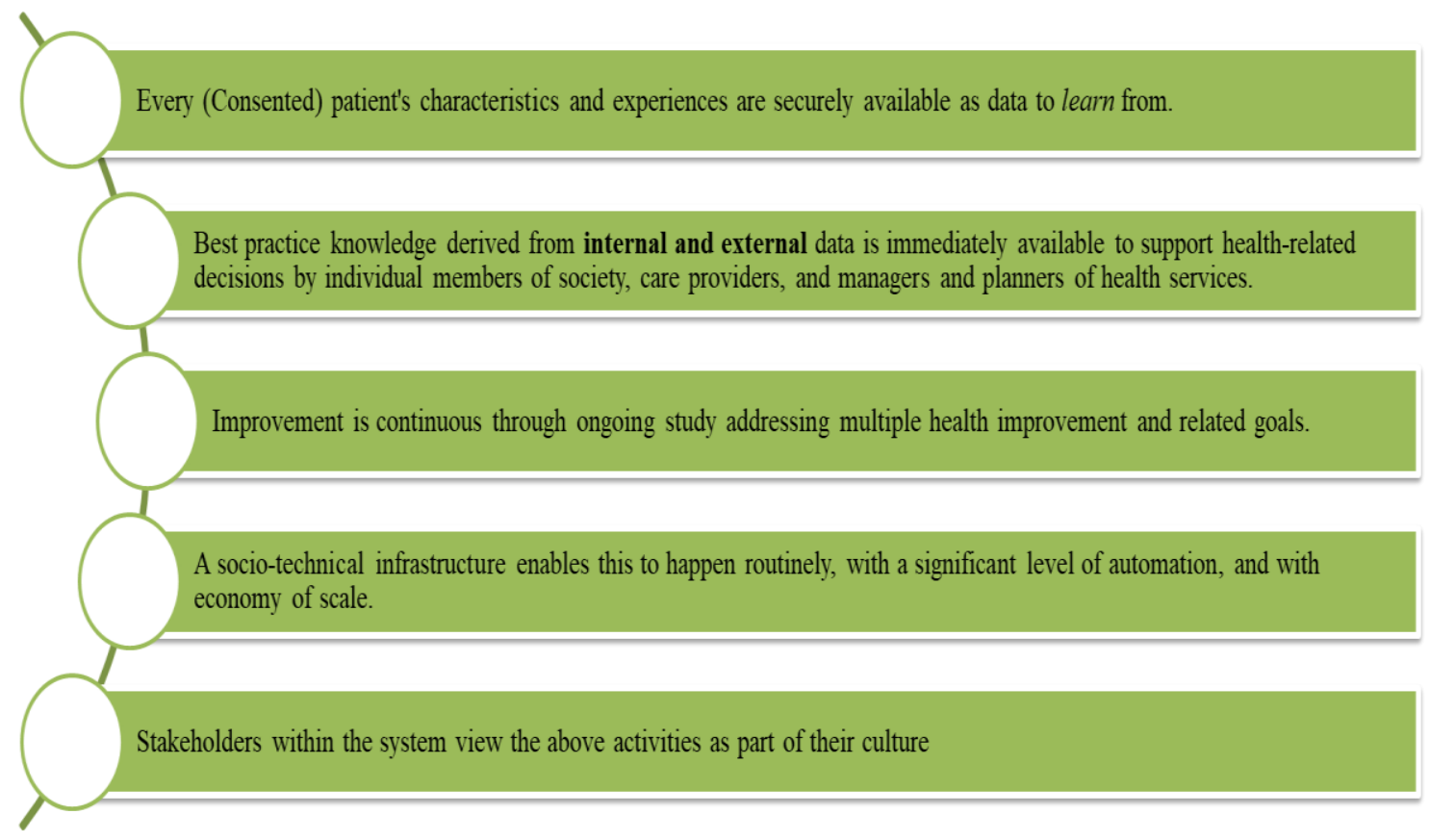

Figure 6:Five pillars of a learning health system (Institute of Medicine (IOM), 2007; Milstein, 2013)

It should be noted that in the second characteristic of continuous learning maturity, the data is referring to the internal data generated by the health organization. However, it can be argued that health organizations can and should use the data generated externally (by other organizations) concurrent to the ones they generate themselves. This may lead to a significantly higher amount of data and a better understanding of some issues that they 
may not have enough data regarding themselves and eventually more mature health organizations.

\subsubsection{A Multi-perspective Approach for Continuous Learning in Healthcare Organizations}

\subsubsection{Political}

The road for aligning care with individual patient needs has been paved in recent years more than ever with goals of population and patient outcome betterment (Epstein et al., 2010). One of the areas that can benefit from the patient input and proliferation of clinical data is the federal and state organizations in terms of regulatory decision-making. Historically, the actions of the regulatory departments such as the FDA have been more reactive than overactive (The increased speed of approvals for specific drugs in times of crisis and rare disease-related and orphan drugs). The new paradigm means that regulatory decision-makers such as FDA can reflect patient needs better and more proactive in their actions. There have been studies regarding topics such as the incorporation of the needs of patients into the risk-benefit evaluation of the medical device and the effects of patient-generated data in systems such as LHS on regulatory decision making (Levitan, Phillips and Walker, 2014; Irony et al., 2016; Tegenege et al., 2017). In addition to the patient preferences and needs, the continuous improvement mindset will help demonstrate the benefits and challenges of LHS implementation and adoption in a clearer way and as a result, enables the more efficient allocation of federal and state funds into the needed areas. As Travis et al. (2004) discuss, in many countries, despite the decrease in prices and increase in funds, the move towards healthcare goals remains tedious and slow. 
Some of the other challenges based on the mentioned study lie in the delay in knowledge and practice and the weak evidence-based information (Travis et al., 2004). Continuous learning can mitigate the weak data and significantly shorten the delay times between clinical research and practice. All in all, although there have been studies on the political and regulatory aspects of the adoption and assessment of learning health systems, more research can paint a clearer picture on how this health paradigm shift can impact the regulatory players with goals of increasing the quality of public health.

\subsubsection{Economic}

One of the main drivers behind the endeavors for new healthcare systems and management is the current high costs of US healthcare. Although the Affordable Care Act (ACA) has positively impacted the way healthcare is provided in the US through actions such as shifting from transaction-based rewards to outcome-based ones, it is not a panacea for driving down the healthcare costs. Although ACA has toned down the increasing percentage of healthcare costs in United States GDP (0.124\% increase from 2010 to 2014 compared to $1.863 \%$ from 2005 to 2010), about $18 \%$ of the GDP is still a staggering figure (compared to $8.9 \%$ in 1980) (McKinsey\&Comapny, 2014.; The World Bank, 2014.; Worley \& Mohrman, 2014). The desire to drive down the costs of care merged with the surfacing of translational research's importance and the adoption of EHRs has proliferated the calls for the development of systems that can drive down the costs and increase the quality of care (Lowes et al., 2017).

As LHSs follow the continuous quality improvement philosophy they are aligned with the practices of some of the existing lean hospitals. In lean hospitals, it is important to 
know that employee lay-offs are not the solution and hospitals focus on eliminating the different types of waste that are going on in their organization. This is especially important since up to a third of care spending in the US is considered as avoidable waste (unnecessary services, administrative waste including unproductive and duplicate documentation, inefficiently delivered services, high prices, fraud, and missed prevention opportunities) (Institute of Medicine (IOM), 2007). Lowes et al. (2016), study the impact of the learning health systems implementation called "Learning From Every Patient (LFEP)" on 131 children with cerebral palsy which resulted in a 43\% decrease in inpatient days, $27 \%$ fall in inpatient admission, $30 \%$ decrease in emergency room admissions, and $210 \%$ and $176 \%$ cost reduction compared to time control group and program activities control group respectively (Lowes et al., 2017). Even if the costs aspect of the care would play second fiddle to the important topic of quality of care and its betterment, cost cuts would happen as a result of improved quality and decreased waste. There is an opportunity for research on how the LHS would affect the costs in hospitals in order to generate more evidence-based data on the challenges, benefits, and cost-cutting capabilities of continuous learning.

\subsubsection{Social}

One of the main drivers behind the emergence of LHSs is the need to deliver safe and efficient care to patients (Kelley et al., 2015). The need for clinical care to be both evidence and science-based concurrent to the increased pressure on quality improvement in care systems, the data and knowledge accumulation in practice and research can complement each other more than ever (Altman et al., 2013; Faden et al., 2013; Kass et 
al., 2013). In researching to improve the quality of care, however, some social and patientrelated points should be considered. Firstly, although some patients welcome the increased convergence in research and practice, many others would not want themselves or their loved ones to take part in randomized clinical trials for comparative-effectiveness research. One of the concerns is about the proliferation of paper works, consent forms needed due to the increase in connected clinical research and practice adding to the organizational wastes. However, conditioned on robust practices and transparent mechanisms, many low-risk randomized clinical trials would not need any patient consent while the higher-risk procedures would get their needed concentration resulting in a safer system (Faden et al., 2014).

Faden et al. (2014) also stress the importance of the thorough participation of patients and other stakeholders for mining the suitable specification of the institutional implications of the LHS decision making frameworks Faden et al. (2014) proposes an ethics framework for an LHS which is iconoclastic to traditional held thought about the separation in research and clinical ethics. This framework includes seven pillars for the ethical implementation of LHSs which are: 1-Respect the right and dignity of patients, 2Respect clinician judgments, 3- provide optimal clinical care to patients, 4- Avoid imposing non-clinical risks and burdens on patients, 5- Address health inequalities, 6Conduct continuous learning in order to improve the quality of clinical care and health care systems, and 7-Contribute to the common purpose of improving the quality and value of clinical care and health care systems (Faden et al., 2013). The mentioned paper also discusses the issue that patient protection actions can sometimes hinder the improvement 
of the healthcare system. Although protecting patients is a very important subject, several hundred thousand people die needlessly each year from medical mistakes which can be avoided through quality improvement (Kohn, Corrigan and Donaldson, 2002). One of the important factors in the adoption of LHSs from the patient perspective is the clinician. As patients have more trust in their doctors compared to the researchers, there should be collaboration and teamwork between the clinicians and researchers in order to bolster the acceptance of LHSs and increase patients' participation through shared decision making (Barry and Edgman-Levitan, 2012). Patients, for their good, should feel the need to contribute to and participate in the learning process just as health professionals and systems are obligated to learn. Kelley et al. (2015) points out that patients' interest in individualized care correlated with the value they put on the relationship they have with their physician. So, the adoption of LHSs not only does not undermine the role of physicians but also highlights their role as an influential part of the system (Kelley et al., 2015). This element turns clinicians' attitude towards LHSs, quality improvement, and comparative efficiency research into an important factor. Health systems should involve clinicians more effectively in quality improvement in order to boost their attitudes and the diffusion of LHSs (Butler et al., 2017). Wolf et al. (2009) emphasize the importance of patients' "health learning capacity" that should be considered. This capacity includes a wide spectrum of cognitive and psychosocial skills assisting patients or family members in effectively promoting, protecting, and managing their own or a loved ones' health (Wolf et al., 2009). The need for this capacity shows that the education and structure of LHS culture should not be limited to the health system employees and educating patients on these matters can be critical in the adoption and acceptance of these systems. Roth et 
al. (2016) hint at the reciprocal benefits that LHS and human factors can have on each other through gaining access to new research fields and adoption of a range of tools to pinpoint and address the implementation challenges respectively (Roth et al., 2016). To conclude, the social and patient side of LHSs is a very crucial one which includes many other aspects that should be looked into in terms of patient privacy, inclusiveness, transparency, accessibility, adaptability, governance among others (Rubin, 2017).

One of the other areas that have been the focus of some research papers for LHS, is the area of public health and equity. Many are concerned that the improved quality and outcomes in healthcare would be limited to premium health services and people who can pay for them by themselves or through their insurances. Faden et al (2013) focus on the societal goal of a just healthcare system with the objective of high-quality care and economic welfare through LHSs (Faden et al., 2013). With the continuation of care quality disparities for racial and ethnic minorities, LHS can potentially bolster the care quality through applications such as data analysis, continuous quality improvement, and patient-centered care (Brooks et al., 2017). Brooks et al. (2017) posit a framework for integrating health equity into LHSs. This method includes prioritizing health equities, engaging the community, targeting health disparities, acting on data, learning, and improving. Some of the other factors that are important in the implementation of LHSs are the type of city and community that they are going to be structured in. As Khurshid (2016) puts it, working with champions and backbone organizations in the community, not being limited with the legacy systems, and looking at data sharing (among different levels of healthcare employees) as a tool for transforming healthcare and not as a health 
solution (Khurshid, 2017). Through literature, there are also examples of data-fueled learning health community constantly powered by the people and for the people (Okun and Goodwin, 2017). With the rise of new technologies such as genomics, the opportunity of gaining so much data from a wide range of different people is within reach. However, there are still barriers that hinder this access. Blizinsky and Bonham (2017) identify three barriers that face the LHS data that are provided by genomics. The challenges are inequality in the use of genomic medicine, lack of pharmacogenomics access in clinical care, and insufficient integration of social and environmental data into EHRs (Blizinsky and Bonham, 2017). Integration of technologies such as genomics and wearables as feeding factors for the data in LHS can play a vital role in LHSs' adoption and their impact on public health through gaining more data about racial and ethnic, socio-economic, economic backgrounds, and age of people and the health issues they deal with. As the racial diversity is increasing in the US and by 2050 it is estimated that about one-fifth of the US population is going to be over 65, it is imperative to use opportunities like LHS to promote and improve public health and equity and deal with these extrinsic changes (Shaygan, 2018).

\subsubsection{Technical}

As the implementation of LHS needs a socio-technical infrastructure, the technical side of its adoption of assessment has significant importance. The adoption of EHRs in the US from one side and the development in fields such as genomics and wearable devices from the other side are paving the path for the collection of great amounts of data for health systems. Especially with all the demographic and demand changes, healthcare 
organizations are forced to leverage the value of technology advancements to be costeffective, competitive, and responsive. Concurrent to the mentioned advances, acquiring experienced data scientists and leveraging the benefits of data integration, sharing with different stakeholders, and acquisition and curation of data are some of the areas that can help healthcare organizations with their goals of increasing quality care, patient satisfaction, and public health while driving down the costs (Krumholz, Terry and Waldstreicher, 2016; Barham, 2017). The ongoing adoption of smart wearable devices is an important part of contributing to the body of patient-data in general and specific fields (such as diabetes, surgeries, and user empowerment and health promotion) (Lukowicz, Kirstein and Tröster, 2004; Kolodzey et al., 2017; Shaygan, Ozdemir-Gungor, D. Kutgun and Daneshi, 2017). The shift to LHS can provide the needed infrastructure for the development of new generation learning decision support tools in order to mitigate the diagnostic errors (Corrigan et al., 2017). As mentioned before, the adoption of EHRs can play a vital role in clinical research and consequently smoother implementation of LHSs and there are already new studies trying to build upon what has been done it terms of EHRs showing further interest in LHSs. A study by Daniel et al. (2016) tries to build on a platform called EHR4CR which is developed in order to provide communication, security, and semantic interoperability services between twenty-one European hospitals and pharmaceutical companies in five countries by proposing the need for cross border semantic interoperability for LHSs (De Moor et al., 2015; Daniel et al., 2017). There are also studies, seminars, and conference talks on the connections of LHSs, precision medicine, and implementation sciences. Chambers et al. (2016) stresses the importance of LHS and investing in its intellectual and physical infrastructure in achieving high 
quality and efficient healthcare system on pillars of precision medicine (Chambers, Feero and Khoury, 2016). This study goes on to talk about the synergy areas between LHS and implementation science in terms of improving the implementation of effective practices. Machine learning is one of the main enabling technologies behind LHS in terms of enabling and improving pattern recognition. Translation of the textual knowledge into machine-learned knowledge will enable the integration of the knowledge with a specific patient. Cahan and Cimino (2017) propose a self-growing and self-maintaining patient knowledge base that can be in reach of health professionals worldwide which can benefit low resource health systems, practitioners, and researchers concurrently (Cahan and Cimino, 2017). A 2017 report by the Office of National Coordinator for Health IT (ONC) and Agency for Healthcare Research and Quality (AHRQ) discusses the convergence of factors such as being fed up with the legacy systems, widespread adoption of networked devices, and increased public acceptance and exposure to systems like Amazon is paving the way for the increased integration of artificial intelligence in Healthcare (Derrington, 2017).

More evidence and research studies into the outcomes of the implemented LHS can provide insights into technical and infrastructural needs aligned with bolstering the systems and mitigating their shortcomings. There are studies in the literature which discuss the implementation of LHS cluster for specific diseases and areas (Hobbs, 2012; Kwon et al., 2012; Mandl et al., 2014; Shah et al., 2016; Turley, 2016; Johnson et al., 2017; Pronovost et al., 2017; Schmittdiel et al., 2017). 


\subsection{Maturity Models}

Maturity models are instruments to facilitate organizational management (Vidal Carvalho, Rocha and Abreu, 2017). These models have been used with different goals and purposes for benchmarking system development or organizational management for years. They are built on entities such as people, organizations, functional areas, processes among many others (Rocha, 2011). Maturity models normally are used to evaluate these entities in terms of predicted patterns in the form of stages or levels.

Maturity models have been proven to be useful in a myriad of different fields such as software, system engineering, project, and program management, energy management, technology road mapping, healthcare technology management, and other areas with goals of facilitating process improvement (Kappel, 2001; Kerzner, 2001; Paulk, 2002, 2009; Garets and Mike, 2006; Dunbrack and Hand, 2013; Introna et al., 2014; Sanders and Burton, 2016).

In the field of software, Paulk et al. (1993) came up with the Capability Maturity Model (CMM) which could be used as a maturity framework for bolstering organizational processes with goals of development and management of software and it encompasses five levels of maturity including initial, repeatable, defined, managed, and finally, optimizing (Paulk, 2002). The Software CMM has been replaced by CMM Integration (CMMI) since 1997, which integrates System Engineering with Software Engineering and Integrated Product Development in a single model (Paulk, 2009). Also, on the project management side, there are maturity models such as OPM3, P3M3, and the project management maturity model (PMMM). The P3M3 has the same levels compared to the 
CMM model with the exception that the first step in P3M3 is awareness instead of initial. The project management maturity model (PMMM) includes a common language, common processes, singular methodology, benchmarking, and continuous improvement as its maturity levels (Kerzner, 2001; AXELOS, 2018). Demir and Kocabas applied the PMMM in education with the same maturity levels (Demir and Kocabas, 2010). In 2002, Knowledge Process Quality Model (KPQM), was introduced to aid companies to evaluate their knowledge management structures and finding ways for future improvements (Paulzen et al., 2002). Many current maturity models have different levels of maturity in order to describe the current state of the measured entity. Most of these models include 5 levels and are based on CMM or CMMI which were discussed earlier with the level labels including but not limited to:

Level 1 -getting started/awareness/initial/ common language.

Level 2 -developing/focusing/repeatable/knowledge/ common process/ basic knowledge.

Level 3 -complying/practicing/competence/defined/singular methodology/process definition.

Level 4 -sustaining/exploiting/managed/excellence /benchmarking/ process control.

Level 5 -advocating/transforming/optimized/ optimizing/mature/continuous improvement/process improvement.

There are studies that have revised the CMM model and added some stages to the conventional maturity models. Galliers and Sutherland propose a seven-stage maturity 
model for information system management and strategy (Galliers and Sutherland, 1991). These levels include Ad hocacy, foundations, centralized, cooperation, entrepreneurial, and harmonious. This research evaluates maturity for strategy, structure, systems, staff, style, skills, and superordinate goals.

As it can be seen, maturity models have been proposed to address different issues, in different aspects of different areas and industries. The Healthcare industry has been no exception in using maturity models as models have been created to address specific parts and dimensions of the healthcare industry. The healthcare maturity models will be discussed next. Table 1 presents a myriad of maturity models in areas such as software, energy, systems engineering, project management, road-mapping, safety, knowledge and change management, risk and capability management, social media, manufacturing engineering, organizational design, quality engineering, education, and their number of stages in terms of maturity.

Table 1: Maturity Models in Different Fields

\begin{tabular}{|c|c|c|c|}
\hline Model & Area & Stages & Reference \\
\hline $\begin{array}{l}\text { Capability Maturity Model } \\
\text { (CMM/CMMI/QMMG) }\end{array}$ & $\begin{array}{l}\text { System Engineering Capability, } \\
\text { Software Engineering, and } \\
\text { Integrated Product Development }\end{array}$ & 5 & (Paulk, 2002, 2009) \\
\hline $\begin{array}{c}\text { Complex Product Systems (CoPS) } \\
\text { Maturity Model }\end{array}$ & Project Risk Management & 5 & (Yeo and Ren, 2008) \\
\hline $\begin{array}{c}\text { Knowledge Process Quality Model } \\
\text { (KPQM) }\end{array}$ & $\begin{array}{c}\text { Knowledge Management, Quality } \\
\text { Improvement }\end{array}$ & 5 & $\begin{array}{l}\text { (Paulzen et al., } \\
\text { 2002) }\end{array}$ \\
\hline OPM3 & Organizational Project Management & Binary & $\begin{array}{c}\text { (Project } \\
\text { Management } \\
\text { Institute (PMI), } \\
\text { 2013) }\end{array}$ \\
\hline $\begin{array}{l}\text { Project Management Maturity Model } \\
\text { (PMMM) in Education }\end{array}$ & $\begin{array}{c}\text { Project Management in Educational } \\
\text { Organizations }\end{array}$ & 5 & $\begin{array}{c}(\text { Demir and } \\
\text { Kocabas, 2010) }\end{array}$ \\
\hline
\end{tabular}




\begin{tabular}{|c|c|c|c|}
\hline Model & Area & Stages & Reference \\
\hline P3M3 & Portfolio, Program, and Project & 5 & (AXELOS, 2018) \\
\hline Project Management Maturity Model & Project Management & 5 & (Kerzner, 2001) \\
\hline Energy Management Maturity Model & Energy Management & 5 & (Introna et al., 2014) \\
\hline Roadmapping Maturity Model & Roadmapping & 6 & (Petrick, 2008) \\
\hline Roadmapping Influence model & Roadmapping & 4 & (Kappel, 2001) \\
\hline Social Media Maturity Model & Social Media & 5 & $\begin{array}{c}\text { (Thomas and } \\
\text { Woodside, 2016) }\end{array}$ \\
\hline Service Systems Maturity Model & Manufacturing Enterprises & 5 & (Neff et al., 2014) \\
\hline Maturity Models and safety culture & Safety Culture & Lit Rev & $\begin{array}{l}\text { (Goncalves Filho } \\
\text { and Waterson, 2018) }\end{array}$ \\
\hline $\begin{array}{c}\text { Organizational Design Maturity } \\
\text { Model }\end{array}$ & $\begin{array}{c}\text { Organizational Design, Design } \\
\text { Research }\end{array}$ & 4 & $\begin{array}{l}\text { (Mettler and } \\
\text { Rohner, 2009) }\end{array}$ \\
\hline Cognizant Enterprise Maturity Model & Knowledge Management & 5 & $\begin{array}{l}\text { (Harigopal and } \\
\text { Satyadas, 2001) }\end{array}$ \\
\hline $\begin{array}{c}\text { Information Quality } \\
\text { Management Maturity Model (IQM3) }\end{array}$ & Quality Management & 5 & $\begin{array}{l}\text { (Caballero et al., } \\
\text { 2008) }\end{array}$ \\
\hline $\begin{array}{c}\text { Organizational Change Readiness } \\
\text { MM }\end{array}$ & Change Management & 6 & $\begin{array}{l}\text { (Zephir, Minel and } \\
\text { Chapotot, 2011) }\end{array}$ \\
\hline $\begin{array}{c}\text { Capability Maturity Model for } \\
\text { Business Intelligence }\end{array}$ & Business Intelligence & 5 & $\begin{array}{l}\text { (Raber, Winter and } \\
\text { Wortmann, 2012) }\end{array}$ \\
\hline $\begin{array}{l}\text { Business Process Maturity Model } \\
\text { (BPMM) }\end{array}$ & Business Processes & 5 & $\begin{array}{l}\text { (Lee, Lee and Kang, } \\
\text { 2007) }\end{array}$ \\
\hline
\end{tabular}

\subsubsection{Technology Management Maturity in Healthcare}

Maturity models have also been used in the healthcare domain specifically in the information system technology sector (Vidal Carvalho, Rocha and Abreu, 2017). Tarhan et al (2015) argue that only a scarce number of maturity models have been proposed with varied focus and depth for business processes in healthcare before the last decade (Tarhan, 
Turetken and van den Biggelaar, 2015). However, there has been a myriad of studies in the field of maturity models in healthcare in recent years.

There are models such as IDC's mobility maturity model for mhealth and models such as HIMSS maturity model for electronic medical records (EMRAM), patient record/content management maturity model (Forrester model), and maturity model for electronic patient record (EPRMM) for the field of electronic medical records (Garets and Mike, 2006; Priestman, 2007; Clair, Brown and Moore, 2010; Dunbrack and Hand, 2013). There have been other maturity models designed for different fields in healthcare such as interoperability, infrastructure IT, data warehousing, analysis networking, telemedicine, networkability, and usability among other (Nehta, 2007; Sharma, 2008; Fitterer and Rohner, 2010; NHS, 2011; Zephir, Minel and Chapotot, 2011; Brooks, El-Gayar and Sarnikar, 2013, 2015; Van Dyk and Schutte, 2013; Sanders and Burton, 2016; HIMSS Analytics, 2018). As for organizations with goals of continuous learning, a mature system is defined as a system that generates timely actions to the information that it derives (or it can be derived) from data in order to create meaningful measurement regarding system learning (Ainsworth and Buchan, 2015). In this research, a mature health organization (system) in terms of technology management is defined as:

"A system that generates timely actions to the information that it derives from data in order to create meaningful measurement regarding systematic learning and increased efficacy and effectiveness in health outcomes."

As an initial part of this research, Shaygan \& Daim (2019) proposed an exploratory model to assess technology management maturity in healthcare organizations by using Hierarchical 
Decision Model (HDM). The model can help health organizations with pinpointing their strengths and weaknesses in the adoption and implementation of new technologies and socio-technological approaches such as Learning Health Systems (LHS) and their infrastructure while giving them organizational and competitive self-awareness and guide them in setting their strategies and resource allocation. The mentioned study was done to create an exploratory model to serve as a much-needed technology management tool for health organizations to assess their technology management maturity for both public and organization's advantage in a more effective way.

A list of maturity models proposed in healthcare is shown in Table 2.

Table 2: Maturity Models in Healthcare

\begin{tabular}{|c|c|c|c|}
\hline Model & Area & Stages & Reference \\
\hline $\begin{array}{c}\text { Governance, Risk, and Compliance } \\
\text { MM (GRC) }\end{array}$ & Hospital Management & 5 & $\begin{array}{c}\text { (Batenburg, } \\
\text { Neppelenbroek } \\
\text { and Shahim, } \\
\text { 2014) }\end{array}$ \\
\hline $\begin{array}{c}\text { Hospital Cooperation Maturity } \\
\text { Model (HCMM) }\end{array}$ & Hospital Corporation Quality & 4 & $\begin{array}{c}\text { (Mettler and } \\
\text { Blondiau, 2012) }\end{array}$ \\
\hline $\begin{array}{l}\text { Quintegra Maturity Model for } \\
\text { Electronic Healthcare (eHMM) }\end{array}$ & $\begin{array}{l}\text { Healthcare Information } \\
\text { System Technology }\end{array}$ & 7 & (Sharma, 2008) \\
\hline $\begin{array}{l}\text { Healthcare Data Quality Maturity } \\
\text { Model (HDQM2) }\end{array}$ & Healthcare Data Quality & 5 & $\begin{array}{l}\text { (Pinto-valverde } \\
\text { et al., 2013) }\end{array}$ \\
\hline $\begin{array}{c}\text { IDC Healthcare IT (HIT) Maturity } \\
\text { Model }\end{array}$ & $\begin{array}{l}\text { Healthcare Information } \\
\text { System Technology }\end{array}$ & 5 & $\begin{array}{l}\text { (Dunbrack and } \\
\text { Hand, 2013) }\end{array}$ \\
\hline IDC's mobility maturity model & Healthcare, mHealth & 5 & $\begin{array}{l}\text { (Dunbrack and } \\
\text { Hand, 2013) }\end{array}$ \\
\hline PACS Maturity Model & $\begin{array}{l}\text { Picture Archiving and } \\
\text { Communication Systems in } \\
\text { Healthcare }\end{array}$ & 6 & $\begin{array}{l}\text { (van de } \\
\text { Wetering and } \\
\text { Batenburg, } \\
\text { 2009) }\end{array}$ \\
\hline
\end{tabular}




\begin{tabular}{|c|c|c|c|}
\hline Model & Area & Stages & Reference \\
\hline $\begin{array}{c}\text { Networkability of Health Care } \\
\text { Providers }\end{array}$ & $\begin{array}{l}\text { Healthcare Networkability } \\
\text { and Interoperability }\end{array}$ & 5 & $\begin{array}{l}\text { (Fitterer and } \\
\text { Rohner, 2010) }\end{array}$ \\
\hline $\begin{array}{c}\text { Electronic Healthcare Maturity } \\
\text { Model (eHMM) }\end{array}$ & EHRs & 7 & $\begin{array}{c}\text { (Quintegra } \\
\text { Solutions } \\
\text { Limited, 2008) }\end{array}$ \\
\hline NHS Maturity Model & Healthcare & 5 & (NHS, 2011) \\
\hline Process Management in Hospitals & $\begin{array}{c}\text { Diagnosis- } \\
\text { Related Groups (DRG) }\end{array}$ & 5 & $\begin{array}{l}\text { (Cleven } \text { et al., } \\
\text { 2014) }\end{array}$ \\
\hline $\begin{array}{l}\text { HIMSS Maturity Model for } \\
\text { Electronic Medical Record } \\
\text { (EMRAM) }\end{array}$ & Healthcare, EMR & 8 & $\begin{array}{c}\text { (HIMSS } \\
\text { Analytics, } \\
\text { 2018)(Garets } \\
\text { and Mike, } \\
\text { 2006) }\end{array}$ \\
\hline $\begin{array}{l}\text { HIMSS Continuity of care maturity } \\
\text { model (CCMM) }\end{array}$ & $\begin{array}{c}\text { Healthcare, Healthcare } \\
\text { Information System } \\
\text { Technology }\end{array}$ & 8 & $\begin{array}{c}\text { (Carvalho, } \\
\text { Rocha and } \\
\text { Abreu, 2016) }\end{array}$ \\
\hline $\begin{array}{l}\text { Patient records/content management } \\
\text { maturity model (Forrester Model) }\end{array}$ & Healthcare, EMR & 3 & $\begin{array}{l}\text { (Clair, Brown } \\
\text { and Moore, } \\
\text { 2010) }\end{array}$ \\
\hline $\begin{array}{l}\text { Maturity Model for Electronic } \\
\text { Patient Record (EPRMM) }\end{array}$ & Healthcare, EMR & 6 & $\begin{array}{l}\text { (Priestman, } \\
\text { 2007) }\end{array}$ \\
\hline $\begin{array}{c}\text { NEHTA Interoperability Maturity } \\
\text { Model (IMM) }\end{array}$ & Healthcare, Interoperability & 5 & (Nehta, 2007) \\
\hline $\begin{array}{l}\text { NHS Infrastructure Maturity Model } \\
\text { (NIMM) }\end{array}$ & Healthcare, Infrastructure IT & 5 & (NHS, 2011) \\
\hline $\begin{array}{c}\text { Healthcare Analysis Adoption } \\
\text { Model (HAAM) }\end{array}$ & $\begin{array}{c}\text { Healthcare, Data Warehouse, } \\
\text { Analysis }\end{array}$ & 9 & $\begin{array}{l}\text { (Sanders and } \\
\text { Burton, 2016) }\end{array}$ \\
\hline $\begin{array}{l}\text { Hospital Cooperation Maturity } \\
\text { Model (HCMM) }\end{array}$ & $\begin{array}{l}\text { Healthcare, Networking, } \\
\text { Cooperation }\end{array}$ & 4 & $\begin{array}{c}\text { (Mettler and } \\
\text { Blondiau, 2012) }\end{array}$ \\
\hline PACS Maturity Model (PMM) & Healthcare & 5 & \\
\hline $\begin{array}{c}\text { Telemedicine Service Maturity } \\
\text { Model }\end{array}$ & Healthcare, Telemedicine & 5 & $\begin{array}{l}\text { (Van Dyk and } \\
\text { Schutte, 2013) }\end{array}$ \\
\hline $\begin{array}{c}\text { Healthcare Usability Maturity } \\
\text { Model }\end{array}$ & Healthcare, Usability & 5 & (HIMSS, 2018.) \\
\hline
\end{tabular}




\begin{tabular}{|c|c|c|c|}
\hline Model & Area & Stages & Reference \\
\hline $\begin{array}{c}\text { Healthcare Business Intelligence } \\
\text { Maturity Model }\end{array}$ & Healthcare BI & $\begin{array}{c}9 \\
\text { Success } \\
\text { Factors }\end{array}$ & $\begin{array}{c}\text { (Brooks, El- } \\
\text { Gayar and } \\
\text { Sarnikar, 2015) }\end{array}$ \\
\hline $\begin{array}{c}\text { Healthcare Information System } \\
\text { Maturity Model (HISMM) }\end{array}$ & $\begin{array}{c}\text { Healthcare Information } \\
\text { System }\end{array}$ & 7 & $\begin{array}{c}\text { (Carvalho } \text { et al. } \\
\text { 2019) }\end{array}$ \\
\hline
\end{tabular}

\subsection{Quadruple Aim of Care}

In 2008, Donald Berwick et al. provided a framework for the delivery of high-value care in the United States, revolving around three main goals: improving the individual experience of care; improving the health of populations; and reducing the per capita cost of healthcare also known as the triple aim of care (Berwick, Nolan and Whittington, 2008). This framework discusses that and healthcare organizations that deliver individual care and population-based interventions that are evidence-based and highly reliable can achieve these three qualities. The enablers of this achievement in a care system are access to up-to-date medical knowledge, standardized definitions of quality and cost, and evidence and measurement collected and distributed by a thoroughly trustworthy body as indicators of the "state of the system" by its stakeholders. This state is assessed through its reliability, adherence to evidence, cost, and progress in improvement. These goals would ideally result in care that produces the best outcomes at the lowest cost over time (Porter and Teisberg, 2006).

The important enablers for the positioning of the health organization for a successful pursuit of these aims are recognition of a population as the unit of concern, externally supplied policy constraints (such as a total budget limit or the requirement that all 
subgroups be treated equitably), and the existence of an "integrator" with the duty of focusing and coordinating services to assist the population on all three dimensions at once. Donald Berwick et al. (2008) define the roles of the integrators or health organizations pushing for the achievement of the triple aim of care are partnership with individuals and families, redesign of primary care, population health management, financial management, and macro system integration.

As, in recent years, Physician well-being has become an increasing concern, due to increasing evidence of an epidemic of burnout and stress among medical professionals, the goal of improving the work-life of health care providers was proposed to be added to the triple aim of care by Bodenheimer and Sinsky in 2014 (Shanafelt et al., 2012; Bodenheimer and Sinsky, 2014; West, 2016). In other words, as the Triple Aim of care focuses on improving the patient experience of care, improving the health of populations, and reducing the per capita cost of healthcare will benefit from the expansion, the Quadruple Aim of care also includes the goal of improving the well-being of health professionals. The fourth aim can be addressed through different channels such as the implementation of a documentation team (to lessen the burden from physicians, Use of pre-visit/lab/follow up planning, expanding the role of nurses and medical assistants, standardization and coordination of workflows, and more effective training among others (Bodenheimer and Sinsky, 2014).

\subsection{Importance to Healthcare Management}

As the healthcare landscape and environment in the United States remains a volatile and dynamic one, managers and decision-makers in both private and public healthcare 
organizations need to have an understanding of the healthcare system and its landscape as they need to comprehend the bigger picture of the environment which they try to plan and manage decision and strategies. This knowledge (or lack thereof) can significantly affect the viability, success, quality, and efficiency of the delivered care in results of impacting the timeliness of decision-making actions. Shi and Singh (2019) discuss this significance for care manages from different aspects such as positioning and understanding, handling threats and opportunities, evaluating implications, complying with regulations, planning, capturing new markets, and following the organizational mission. Moreover, pursuing goals of continuous improvement and learning in the dynamic healthcare environment can change the roles of managers from managing processes to people (leadership, motivation,...) (Poksinska, Swartling and Drotz, 2013). This necessitates a continuous effort from the managers' side to balance the new organizational, technological, social, financial, and regulatory changes with the organization's culture, goals, and resources.

In their book, Hegarty, Amoore, Blackett, McCarthy, \& Scott (2017) divide healthcare technology management into two groups; Supporting and Advancing Care and Equipment Management. The former includes topics such as clinical supporting, teaching and training, clinical information, regulatory and standards issues, quality and risk management, adverse event investigation, and support and innovation in care processes. The second group, however, focuses on data management, scheduled maintenance, medical equipment, and systems support, technology assessment, performance verification, and corrective actions. This research and the continuous learning it is 
referring to and the assessment of technology management maturity it is trying to evaluate includes many elements from both remits of technology management in healthcare.

Knowledge of the health care system and its development is critical for the effective management of healthcare organizations. Therefore, health organizations should pay attention to community needs, technological progress, consumer demand, and economic prospects, to be better positioned to achieve and maintain their organizational missions and technology management maturity as an organization. This maturity can bolster access, service quality, and efficiency in the delivery of services. Health organizations should address the need to take into account which technological, social, organizational, regulatory, and financial might affect their organization's long-term stability. This knowledge should enable healthcare organization's decision-makers and managers to identify strengths, weaknesses, opportunities, and threats in a more effective and timely manner. The same thing goes for effectively allocating resources to manage changes (both taking advantage of them or mitigating their unwanted results). The action taken can address certain learning and improvement areas as well in terms of what should be learned, added, modified, jettisoned, and what sort of organizations training, technological infrastructure, public trust, or financial/regulatory need should be conformed with aligned with organizations goals and mission. In conclusion, knowledge of the health care system and its development is significantly important for the effective management of healthcare organizations. Factors such as requirements, technological advancements, stakeholder needs, and financial prospects, the decision-makers in healthcare organizations can be better equipped to achieve and maintain organizational 
missions and the goals of healthcare (quadruple aims of care (Bodenheimer and Sinsky, 2014)).

\subsection{Research Centers in University Hospitals}

Healthcare organizations and hospitals (including university hospitals) are large institutions and consist of a very complex structure of management. Usually, different organizations within a hospital may have different management styles and smaller governance silos with certain guidelines being the same across these organizations. For this reason, this research will focus on a smaller entity inside university hospitals which are University Hospital Research Centers (UHRC) as the unit of study for this research. These centers focus on specific areas within a bigger health organization and have their directors, managers, and staff with different budget sources such as grants and donations, among others. Although they operate under the guidelines and goals of the university hospital, they have their own goals and missions which are aligned with their parent organization but more specific. These units also have a certain amount of autonomy that allows them to operate aligned with their mission and goals.

A university hospital is an institution that combines the services of a hospital with the education of medical students and with medical research. Van der Meulen, 1994 describes support from the federal government and the introduction of faculty practice plans in medical schools as major growth factors for these academic health centers (Van Der Meulen, 1994). The mentioned research goes on to discuss the high cost of healthcare as an external force in the growth of university hospitals and increasing concern about the number of people who were not covered by insurance. University hospitals undergo 
significant cost to develop the ability to treat serious illnesses at the high end and in doing so they need to cope with a larger population with needs around different disorders as more and more types of patients are now being treated in more specialized care centers (Christensen, Bohmer and Kenagy, 2000).

University hospitals are typically affiliated with a medical school or university. Most of these university hospitals have research and innovation centers as compartmentalized units within them that may focus on one or several specific topics. These units have different ways of financing their budgets and have certain amounts of autonomy in the way they are managed. These units can lead to breakthrough research for new cures, new standards of care, and better understanding of different issues in specified areas of care. In the United States, most of the funding for the research institutes and centers comes from publicly funded grants (such as NIH grants), private grants, industry partnerships, foundations (benefactor), endowments, state and federal customers, and donations. These centers focus on topics such as imaging, cancer, vaccine and gene therapy, medicine, public health, system effectiveness, Alzheimer's, cardiovascular, occupational health, evidence-based practice, neuroscience, aging, immunology, orphan diseases, and innovation, among others. Each of these centers may focus on one or several topics within their field. One factor that may be decisive in the direction of the research in the center can be publicly or privately funded grants. In general, funding for science has changed throughout time and moved from largely being supported through private patronage (the backing of a prominent person or family), church sponsorship, or simply paid by research to a combination of grants from various government agencies, institutions, and 
foundations (Understanding Science at Berkeley, 2020). As an example, Oregon Health and Science University (OHSU), located in Portland, Oregon, is a university hospital established in 1887. OHSU has around 33 research centers and institutes focusing on topics such as oncology, public health, imaging, aging, neuroscience, and systems effectiveness among many others. Some of these centers are created aligned with federal or state-level initiatives for specific needs.

The U.S. Department of Health and Human Services (HHS) has a mission of enhancing the health and well-being of Americans by providing for effective health and human services and by fostering sound, sustained advances in the sciences underlying medicine, public health, and social services (U.S. Department of Health and Human Services (HHS), 2020). This department has different grants under programs like Administration for Children \& Families, Agency for Healthcare Research and Quality, Centers for Disease Control and Prevention, Food and Drug Administration, and National Institutes of Health. The National Institutes of Health (NIH) is the primary federal agency for medical, health, and behavioral research. It is the largest of the eight health-related agencies within the Public Health Service (PHS) within the HHS consisting of the Office of the Director and 27 Institutes and Centers (ICs) that focus on aspects of health, human development, and biomedical science (Sekar, 2020). NIH activities span from basic, clinical, and translational research, focused on particular diseases, areas of human health and development, to more fundamental parts of biology and behavior. Research training and health information collection and the spread of knowledge are among the mission of this agency and over $80 \%$ of its budget funds outside research in form of grants, contracts, 
and other awards (HHS.gov, 2018). This allocated amount can fund more than 300,000 researchers working at over 2,500 hospitals, medical schools, universities, and other research institutions in the United States (Sekar, 2020). Table 3 shows the NIH funding and the change for each year while demonstrating the change in funding compared to the peak year which was 2003. Figure 7 and Figure 8 demonstrate the change in NIH funding throughout the years in current and projected constant FY2020 values respectively (Sekar, 2020). Research funded by the NIH has led to saving lives while increasing longevity and quality of life for people in the United States and around the world. Glass et al., 2020 points at success stories such as The Framingham Heart Study, development of childhood vaccines, and the rapid decrease in morbidity and mortality from human immunodeficiency virus as some success stories among the countless success stories of the results of grants fund by the NIH in different areas such as disease-specific initiatives and various specialties (Jacob and Lefgren, 2011; Colaco et al., 2013; Blume-Kohout and Adhikari, 2016; Glass et al., 2020; National Institutes of Health (NIH), 2020).

Carline (2014) explores grants on the medical education and educational innovation, as an example, discussing grants such as the Edward J. Stemmler, MD Medical Education Research Fund Awards from the National Board of Medical Examiners or awards from the Fund for Improvement of Post-Secondary Education for the smaller research while discussing the Undergraduate Medical Education for the 21st Century project with goals of medical education reform and training (innovation and research) grants from the Bureau of Health Professions in NIH (Carline, 2004). 
Table 3: NIH funding and the Change Throughout the Years (Sekar, 2020)

\begin{tabular}{|c|cc|c|cc|c|}
\hline Fiscal Year & \multicolumn{2}{|c|}{ Funding (B) } & Change & \multicolumn{2}{|l|}{ Funding in 2020 values } & \% Below 2003 Funding \\
\hline 1994 & $\$$ & 10.90 & & $\$$ & 23.80 & \\
\hline 1995 & $\$$ & 11.30 & $3.1 \%$ & $\$$ & 23.74 & \\
\hline 1996 & $\$$ & 11.90 & $5.6 \%$ & $\$$ & 24.40 & \\
\hline 1997 & $\$$ & 12.70 & $6.8 \%$ & $\$$ & 25.30 & \\
\hline 1998 & $\$$ & 13.60 & $7.3 \%$ & $\$$ & 26.30 & \\
\hline 1999 & $\$$ & 15.60 & $14.3 \%$ & $\$$ & 29.20 & \\
\hline 2000 & $\$$ & 17.80 & $14.1 \%$ & $\$$ & 32.10 & \\
\hline 2001 & $\$$ & 20.40 & $14.7 \%$ & $\$$ & 35.60 & \\
\hline 2002 & $\$$ & 23.30 & $14.0 \%$ & $\$$ & 39.30 & \\
\hline 2003 & $\$$ & 27.10 & $16.5 \%$ & $\$$ & 44.20 & \\
\hline 2004 & $\$$ & 28.00 & $3.2 \%$ & $\$$ & 44.00 & $-0.5 \%$ \\
\hline 2005 & $\$$ & 28.50 & $2.0 \%$ & $\$$ & 43.20 & $-2.3 \%$ \\
\hline 2006 & $\$$ & 28.50 & $-0.1 \%$ & $\$$ & 41.20 & $-6.8 \%$ \\
\hline 2007 & $\$$ & 29.10 & $2.2 \%$ & $\$$ & 40.60 & $-8.2 \%$ \\
\hline 2008 & $\$$ & 29.60 & $1.5 \%$ & $\$$ & 39.40 & $-11.0 \%$ \\
\hline 2009 & $\$$ & 30.50 & $3.2 \%$ & $\$$ & 39.40 & $-10.8 \%$ \\
\hline 2010 & $\$$ & 31.20 & $2.3 \%$ & $\$$ & 39.10 & $-11.5 \%$ \\
\hline 2011 & $\$$ & 30.90 & $-1.0 \%$ & $\$$ & 37.70 & $-14.8 \%$ \\
\hline 2012 & $\$$ & 30.80 & $-0.2 \%$ & $\$$ & 37.10 & $-16.1 \%$ \\
\hline 2013 & $\$$ & 29.30 & $-5.0 \%$ & $\$$ & 34.60 & $-21.7 \%$ \\
\hline 2014 & $\$$ & 30.10 & $2.8 \%$ & $\$$ & 34.80 & $-21.2 \%$ \\
\hline 2015 & $\$$ & 30.30 & $0.6 \%$ & $\$$ & 34.30 & $-22.4 \%$ \\
\hline 2016 & $\$$ & 32.30 & $6.6 \%$ & $\$$ & 35.80 & $-19.0 \%$ \\
\hline 2017 & $\$$ & 34.30 & $6.2 \%$ & $\$$ & 37.10 & $-16.2 \%$ \\
\hline 2018 & $\$$ & 37.30 & $8.8 \%$ & $\$$ & 39.30 & $-11.2 \%$ \\
\hline 2019 & $\$$ & 39.30 & $5.4 \%$ & $\$$ & 40.30 & $-8.8 \%$ \\
\hline 2020 & $\$$ & 41.90 & $6.6 \%$ & $\$$ & 41.90 & $-5.4 \%$ \\
\hline & & & & & & \\
\hline
\end{tabular}

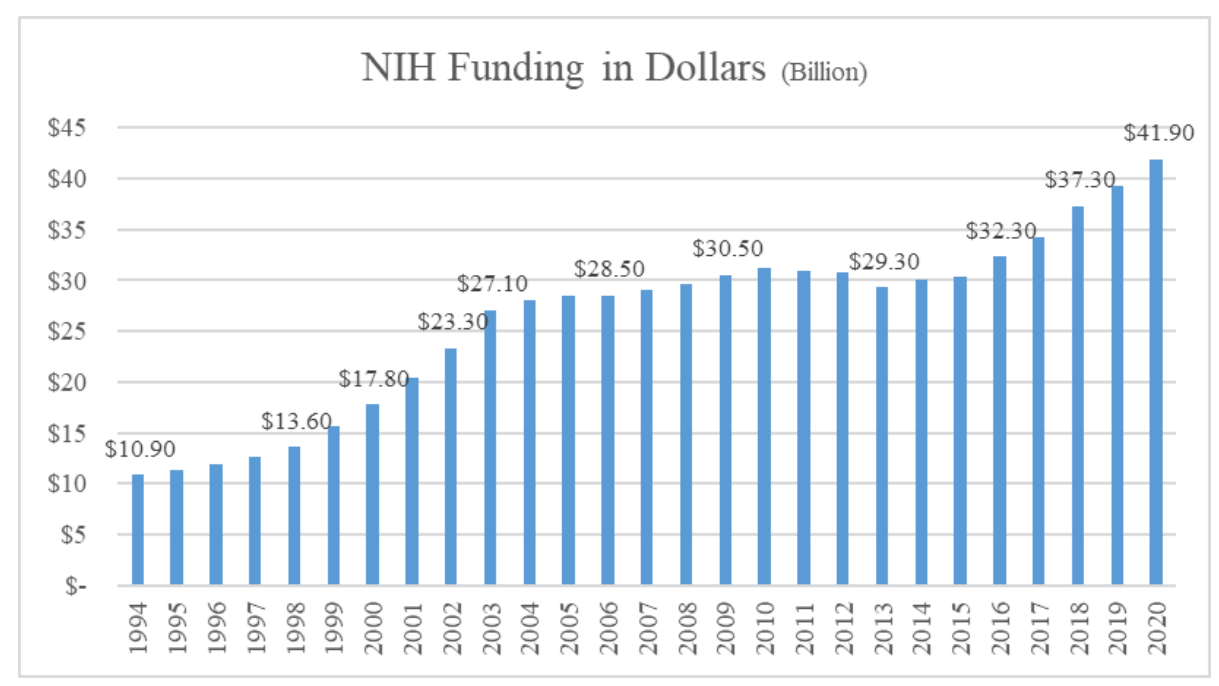

Figure 7 :NIH funding Throughout the Years (Sekar, 2020) 


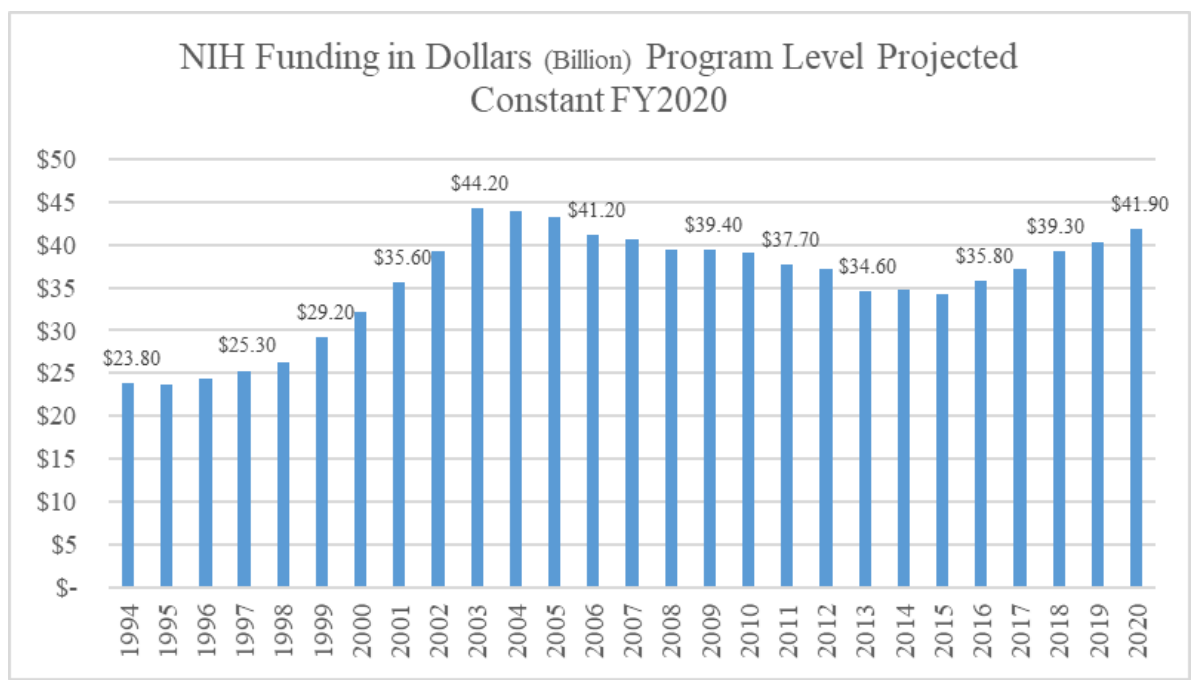

Figure 8: NIH Funding Throughout the Years (Program Level Projected Constant FY 2020) (Sekar, 2020)

Consoli and Mina, 2009 present a sketch for health innovation systems and the building blocks, interactions, and feedback between them (Consoli and Ramlogan, 2008; Consoli and Mina, 2009). This sketch consists of different elements around a problem and the factors it affects in the individual sphere (patient and practitioner), service provision (training, experience, therapy, and diagnosis), technology market (drugs and devices), regulations, and scientific community (publications).

One of the reasons behind the realization of the need for health centers within university hospitals can be traced to the rise of evidence-based care and the opportunity for different centers with different focuses to collect data and perform research in the respective area of research. The paradigm shift in evidence-based healthcare was multi-perspective. The research strategy was shifting from lack of national leadership with scattered funding without effective communication and coordination to improve strategic nationwide leadership with improved communications between funders and researchers which led to bolstered coherence in the research agenda (Walshe and Rundall, 2001). Furthermore, the 64 
research direction shifted from being research-led to needs-led while better research programs paved the way for higher research quality. This shift also led to improved research methods (more appropriate use of research methods), research outputs (improvement of clinical practice being set as the primary goal rather than publications), dissemination, mode of access to research findings (from innovation pull to innovation push to clinicians in a proactive manner), and practitioner understanding of research findings (more focus on metal analyses and systematic reviews as opposed to reports of individual research (Walshe and Rundall, 2001).

In the United States, health systems are trying to shift from decades of operational systems designed to deliver health care as a reimbursable service and morph into systems that deliver health as a population goal (Goldman, Kumanyika and Shah, 2016). Parallel to these changes is the constant quality improvement efforts in areas such as reducing hospital-acquired infections and readmissions for heart failure. In this direction, both transformation and incremental improvement are called for the learning health system, which can constantly improve itself in different areas using the data generated internally and externally meaning clinicians use each patient encounter as an opportunity to make the next one better (Krumholz, Terry and Waldstreicher, 2016). This is especially important in the research centers within health organizations as their goal and mission are to learn and improve in a specific area of healthcare to be able to serve the population in need of that specific service or treatment better.

As entities within university hospitals, health centers need to manage their technology, knowledge, and resources to ensure continuous improvement and efficient learning and innovation. These centers need to deal with factors in different areas such as technology 
(such as devices, data, access), social (patients, stakeholders, workforce), regulations (keeping tabs on the changes in regulations and healthcare structure), organizational (quality improvement, training, governance, management), and financials. 


\section{Research Gaps, Objectives, and Questions}

As the result of the literature review provided in the previous section, this part focuses on clarifying the research gaps, research questions, and research goals.

\subsection{Research Gaps}

As the result of the literature review, several gaps were identified which this research will attempt to bridge. The themes of the gaps range from the lack of a structured assessment tool for the technology management to the need for organized and classified categories of dimensions and criteria and emphasis on the much-needed focus on the technology management side of maturity in health organizations (Grossmann, Goolsby, Olsen and Michael, 2011; Hobbs, 2012; Grant et al., 2016; Brooks et al., 2017; Foley and Vale, 2017). Some of the existing research in the literature also calls for the need for a model to include the main influence factors and their importance levels regarding maturity models in healthcare technologies and information systems (Carvalho, Rocha and Abreu, 2016; Vidal Carvalho, Rocha and Abreu, 2017).

As for continuous learning, its adoption, and implementation, there is a need for a multicriteria approach in studying this topic in the context of technology management maturity of health organizations. Most of the existing research in the literature focuses on healthcare maturity and continuous learning in healthcare organizations only through the specific lens based on the respective research field (Shaygan, Lavoie and Daim, 2018). Therefore, these studies may not address the complexity of health care organizations' maturity and the multi-perspective critical issues around it. 
This section will go through each of these gaps by summarizing the existing literature around these talking points and clarifying the underlying gaps within those areas. Furthermore, in the research goal's section, the ways in which the model will help mitigate and bridge these gaps will be explained.

\subsubsection{Gap I}

According to the literature and as discussed in the literature review in this research, the adoption and implementation of technology management maturity models in healthcare and continuous learning have not been studied in a multi-perspective way. In other words, through literature, each domain of research only talks about these topics from their lens. Research in public health has been focusing on the equity and social aspects (There's a further need to strategically align maturity and learning with health equity in terms of addressing health disparities) (Bauer et al., 2015; Brooks et al., 2017) while technical and technological research focuses on the EHR, EMR, and machine learning aspects and technological infrastructure (Shah, 2016; Kraft et al., 2017; Malenfant et al., 2019; Nordo et al., 2019; Takenouchi et al., 2019). Furthermore, on the maturity models side, studies focus on healthcare information technology and systems or business intelligence exclusively (Brooks, El-Gayar and Sarnikar, 2013, 2015; Carvalho, Rocha and Abreu, 2016; Carvalho et al., 2019). As an example (Brooks et al., 2017) proposes a practical framework to incorporate health equity into a developing continuous learning system and make sure that health disparities are considered in the development of such healthcare systems. Bauer et al. (2015) discuss the importance of learning and implementation

science for psychosocial and medical interventions for mental and physical health 
concerns (Bauer et al., 2015). Brooks et al (2013) and (2015) proposes a review of existing business intelligence maturity models to determine their adequacy for use in healthcare while Carvalho et al. (2016,2018,2019) develop a maturity model for healthcare information systems (Carvalho et al., 2018). Zephir et al. in 2011 propose a methodology that revolves around coping with organizational readiness to achieve business goals through technological and structural improvements bringing technical and human capabilities together with goals of measuring organizational development maturity (Zephir, Minel and Chapotot, 2011). Again, in this research, only two general dimensions of maturity in healthcare are integrated to determine maturity with a focus only on change management. Batenburg et al. propose a preliminary maturity model to evaluate and monitor factors including Governance, risk and compliance (GRC) and GRC maturity in Dutch hospitals (Batenburg, Neppelenbroek and Shahim, 2014).

In general Most of the existing literature focuses on one aspect of maturity in healthcare (machine learning, public health, leadership, political, regulatory, organizational, economic, technical) (Olsen, Saunders and Mcginnis, no date; Ren, Pazzi and Boukerche, 2010; Lambin et al., 2013; Morain and Kass, 2016; Butler et al., 2017; Finkelstein and Jeong, 2017; Malenfant et al., 2019; Takenouchi et al., 2019). According to some authors the drivers of healthcare maturity and continuous learning adoption and maturity are multifaceted and need to be studied that way) (Hobbs, 2012; Schmittdiel et al., 2017; Shellum et al., 2017). Furthermore, there are no systematic attempts to investigate the potential impacts of different aspects of maturity and continuous learning on the quality of healthcare organizations (Foley and Vale, 2017). Brooks et al. (2013) stress the need 
for a validated maturity model by defining healthcare processes, maturity levels, and functionality or capability at each perspective at each maturity level. Carvalho et al. (2016) reviews a wide variety of different healthcare maturity models through a literature review and concludes that none of the identified models has a sufficiently broad scope covering all areas and subsystems of health care organizations (Carvalho, Rocha and Abreu, 2016). One of the other challenges that the existing models face is regarding important factors for maturity. Carvalho et al. (2016) points out the existence of entries with the same name in different maturity models and entries with different names but with the same meaning or interpretation due to selected terminologies. This can also be due to the fact that as criteria are not grouped and organized in different categories and perspectives, lack of context would potentially cause confusion, duplication, and inconsistency.

All in all, the criteria for assessing technology management maturity models in healthcare in general and adoption and implementation of areas such as continuous learning in health organizations are not organized and classified into perspectives. 


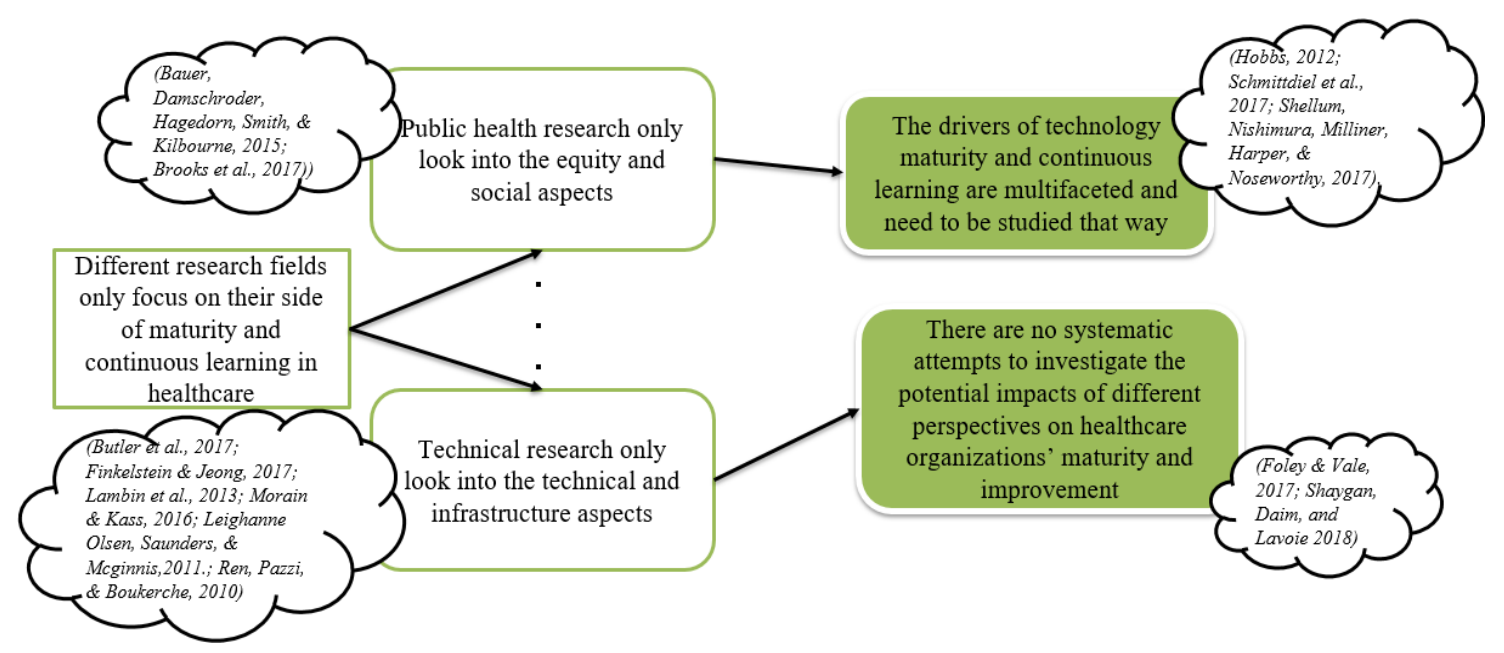

Figure 9: Gap 1

Gap 1: The criteria for assessing technology maturity models in healthcare in terms of management of technology and continuous learning are not organized and classified into perspectives.

\subsubsection{Gap II}

There's a need for a framework to better assess the technology management maturity in terms of implemented and adopted technologies across all aspects of care (Grossmann, Goolsby, Olsen and Michael, 2011; Foley and Vale, 2017). In other words, there is no structured way of assessing the current state of the organization in terms of adoption and implementation of different aspects of technology maturity. There is a need for analysis to be conducted, evidence to be gathered, arguments to be constructed maintained over time around development, design, and implementation of continuous learning across all levels of care (Friedman et al., 2014). Moreover, there's a need for tools for addressing the different aspects of the enterprise (Rouse, Johns and Pepe, 2017). There is also a need 
for a tool to assist health organizations in decision-making and assessment of the adoption and implementation of systematic learning and its socio-technological infrastructure. Budrionis and Bellika (2016) argue that there is a lack of focus on the evaluation of the impact of learning health systems on service quality and patient outcomes (Budrionis and Bellika, 2016).

Carvalho et al.(2016; 2015) discuss the need for a model including the main ingredient factors of healthcare technology maturity and the potential benefits of assigning weights to them to understand their relative importance:

"A maturity model with a holistic approach including a comprehensive set of influencing factors is missing. In this perspective, a new model to fill the gap should be designed. This new model should include the main influence factors with different weights depending on their relative importance and its development should be supported by rigorous scientific methods of conceptualization and validation.” [106, Page 131]

Budrionis and Bellika (2016) also stress the increased pressure for the need for impact evaluation of continuous learning in healthcare organizations due to becoming more mature as a novel care delivery paradigm and point out that this is not a major focus for researchers yet. In other words, although research exists around these ideas, there is a lack of assessment tools and research in this area and more focus is being put towards the study of the feasibility of continuous learning rather than their assessment and their impact. The creation of learning-related methodologies may also encourage further studies about this topic and boost its adoption speed on different scales. Furthermore, many of the existing healthcare maturity models in healthcare merely provide the health organization with 
scales and levels and do not provide a score or quantification of important factors regarding maturity in the organization. Lin et al. (2011) explore the status of e-healthcare maturity in Taiwan in light of a nationwide investigation using Nolan's stage model (Nolan, 1973) among others. The mentioned study offers a multi-perspective model to better understanding the current status of e-healthcare maturity in hospitals with goals of better formulating e-healthcare policies to encourage the adoption of electronic medical records (Liu, Hwang and Chang, 2011). However, this model only uses surveys and interviews and does not include any quantification of this perspective and their respective criteria for topics such as application portfolio, integration, IT infrastructure, user awareness, information system staff, and planning. Brooks et al. (2013) conduct a literature review of existing business intelligence maturity models to determine if they will be useful in healthcare organizations (Brooks, El-Gayar and Sarnikar, 2013). A maturity model can provide a readiness assessment and plan for a business intelligence strategy by providing insight into the important checklists and processes necessary to achieve the desired level in business intelligence maturity. Again none of the studied models include quantification of important factors for maturity in healthcare organizations (Watson, Ariyachandra and Matyska, 2001; Fisher, 2005; Min-Hooi Chuah and Wong, 2012; Raber, Winter and Wortmann, 2012; Halper and Stodder, 2014). Brooks et al. (2013) stress the need for a validated maturity model by defining healthcare processes, maturity levels, and functionality or capability at each perspective at each maturity level. The validation factor is critical to confirm the accuracy and completeness of the proposed model in the healthcare environment. Brooks et al. continued their research in 2015 and identified the need for research into a healthcare case study to create 
a business intelligence maturity model assessment tool (Brooks, El-Gayar and Sarnikar, 2015). This potential model needs to serve as a guidance tool for business intelligence deployment initiatives and assess hospitals' readiness to go to the next maturity level and eventually bolster information management's control and comprehension across the organization. Raber, Winter, and Wortmann proposed a business intelligence maturity model built based on fifty-eight capabilities across five levels ranging from initiate to perpetuate with goals of identifying business intelligence weaknesses and strengths (Raber, Winter and Wortmann, 2012).

Pak and Song proposed a capability maturity model (HCMM) in 2016 with the goals of improving an individual's capability to manage their health by using personal health records (Pak and Song, 2016). This model was created based on Capability Maturity Model Integration (CMMI) (Paulk, 2002; Caballero et al., 2008), which helps developers in selecting software improvement strategies by determining their current maturity and pinpointing the issues needed for improvement which did not include any quantification. Pak and Song identified a lack of statistically and empirically validation test in their study as a limitation despite the fact that it was grounded on the theoretical and empirical models and called for the need for a validated framework which takes different dimensions into account (Mettler and Blondiau, 2012).

Similarly, Zephir et al. (2011) build on CMM to assess the organizational maturity with goals of integrating new practices in times of structural or technological change (Zephir, Minel and Chapotot, 2011). This methodology revolves around coping with organizational readiness to achieve business goals through technological and structural 
improvements. This research integrated technical and human capabilities with the goals of measuring organizational development maturity. Batenburg et al. propose a preliminary maturity model to evaluate and monitor Governance, risk, and compliance (GRC) and GRC maturity in Dutch hospitals (Batenburg, Neppelenbroek, and Shahim, 2014). This model contains five maturity levels ranging from forming to optimized and contains definitions or characteristics for four different criteria in each of the governance, compliance, and risk areas across all five maturity levels. This model, however, is not quantitatively addressing the maturity assessment and does not have validation as a part of its procedure and discusses a need for a statistical method, such as factor analysis, or path analysis (using Structural Equation Modelling, SEM) to be used to find unknown factors that may have influenced the results. Knowledge Process Quality Model (KPQM) used to evaluate their knowledge management structures in companies pinpoints the needs for further test into their model including analysis of suitable measures for model validation (Paulzen et al., 2002). Fitterer and Rohner (2010) identify a future step for their research as a longitudinal model involving before/after evaluation of organizations regarding networkability advancing projects with analysis of reliability and validity of the prospective maturity model (Fitterer and Rohner, 2010).

Carvalho et al. (2016) reviews a wide variety of different healthcare maturity models through a literature review and concludes that none of the identified models has a sufficiently broad scope covering all areas and subsystems of health care organizations and therefore, stresses the need for a new model to encompass main maturity factors with different importance based on their relative weights through a rigorous scientific method 
and subsequent validation (Carvalho, Rocha and Abreu, 2016). One of the other gaps mentioned in the discussed study is that maturity models in the healthcare setting are either highly specialized or too general in sense of healthcare information systems such as eHMM, IDC HIT, and CCMM. Moreover, most of these models do not discuss the design or validation for these models (Mettler and Blondiau, 2012). As for the other gaps identified in similar research, not of the studied research included any weights assignment or factors quantifications. Lastly, this paper points out that many of these models do not necessarily provide organizations with an improvement roadmap of the desired maturity through a systematized process serving as a maturity ladder. In 2018, the next level of Carvalho et al. research was published and identified the need for involve the development of an automatic tool for assessing hospital information system maturity based on the important influencing factors as a future research (Carvalho et al., 2018). As a continuation of their study Carvalho et al. proposed their model in 2019 named the hospital information system maturity model or HISMM for short (Carvalho et al., 2019). This model is a classic maturity model including a matrix made of different maturity stages and six important typified factors affecting maturity in hospital information systems. This model defines the current maturity stage, the next maturity stage and identifies the attributes that must be met to reach a new maturity stage. These six factors include people, data analysis, strategy, information security, infrastructure, and electronic medical records. Each of the six stages have certain characteristics that need to be achieved to in order for the hospital to reach that level of maturity in a certain factor. The literature review in this study was followed by surveys, creation of the initial maturity model, interviews, and generation of the final maturity model. However, this study the 
improvement roadmap cannot be specifically pinpointed, and the maturity model measurements is not related to hospital performance within the hospital and does not address the optimal diffusion of this kind of maturity within the organization (van de Wetering, 2016). Moreover, similar to prior models, there is still no room for quantification of the model and subsequently creation of a quantified, validated, assessment tool for maturity in healthcare organizations. Finally, without quantification or a resulting maturity score, comparing hospitals' performances against their competitors which was identified as a gap was not addressed.

Furthermore, based on the literature many maturity models are created by health national and corporations, which advocate technological developments (IDC Health Insights and HIMSS or even by national health organizations as the National Health Service or National Electronic Health Transition Authority). This poses a challenge to look deeper into the methodology, validation, and development of these models. Besides, many of the existing healthcare maturity models are not published in academic journals and only a small number have gone through a peer-reviewed process (van de Wetering and Batenburg, 2009; Mettler and Blondiau, 2012). Most healthcare maturity models are in the form of white papers, websites, or presentations which hinders researchers from validating their validity and procedure.

Another important weakness of the current maturity models as discussed by Goncalves and Waterson in their 2018 maturity model in safety is that results obtained during one point in time may not prove to be repeatable during another (Goncalves Filho and Waterson, 2018). As some of the limitations of the mentioned study, reliability, validity, 
and overall robustness of using maturity models have been argued. Although in some areas such as safety, industry and related literature can be used to mitigate validation problems (as in (Flin et al., 2000)), there's a lack of methodologies that systematically include validation for their criteria and results.

In sum, in the studied literature around maturity models and specifically maturity models in the healthcare-related topics there are certain types of gaps that can be merged into one gap group. Initially, most maturity models are built based on models such as CMM which merely categorizes performances or initiatives into different levels of maturity stages. In other words, no amount of quantification goes into these models which increases the amount of subjectivity which can be counted as a limitation of these models. Furthermore, many of these maturity models are products of companies, reports, and/or whitepapers which means they have not been through a peer-reviewed process and more importantly validation. Lastly, on top of the need for a quantified and validated model, there is a need for the proposed model to be repeatable for both being implemented on the same health organization more than once and to serve as a tool for evaluation of inter-organizational performances among different health organizations.

Hence the second gap identified in this study is as follows: 


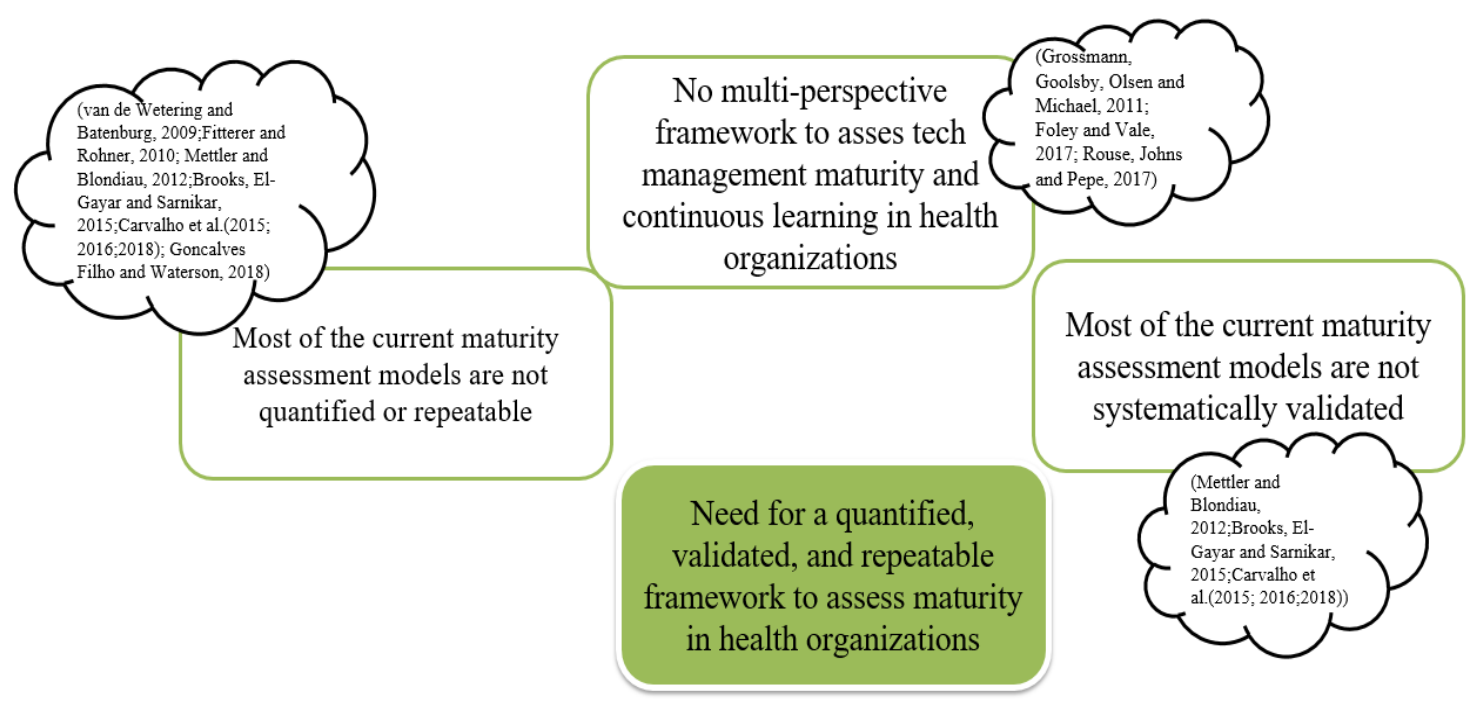

Figure 10: Gap 2

Gap 2: There is a lack of a quantified, validated, and repeatable model for assessing maturity in health organizations in terms of technology management from different perspectives.

\subsubsection{Gap III}

Recent progress in healthcare-related fields such as information technology has led to the proliferation of volumes of both clinical and financial healthcare data. Despite the fact that healthcare is increasingly dependent upon these fields such as IT, the accumulation of data has outpaced our ability to utilize it to improve operational efficiency, clinical quality, and financial effectiveness (Mettler and Vimarlund, 2009; Ferranti et al., 2010). Moreover, the technological innovations have brought upon some new issues/opportunities that healthcare organizations need to leverage or mitigate. About half of the cost growth in health care over the past 40 years has been caused by technology innovation (Congressional Budget Office (CBO), 2008). Moreover, studies have found causal relationships between technology and management of decisions and performance in fields such as nursing ( $\mathrm{Li}$ and Benton, 2006). Therefore, managing new technologies 
and innovations has become an important issue in healthcare organizations' day-to-day items. Technology management is needed to be developed in health organizations alongside important practices such as patient-centered care, interdisciplinary teams, evidence-based practice, continuous quality improvement, use of new informatics, and integration of public health to enable and bolster policies, management, and leadership (Frenk et al., 2010). At the same time, if healthcare organizations are considered as complex adaptive systems due to the dynamic environment that they exist in, the task of managing them becomes a challenge due to the constant system redesign as an adaption mechanism stressing the importance of specific technology management further (Rouse, 2008). A technology manager, as a leader, in organizations going towards continuous and systematic learning and maturity, needs to act as a designer of the learning process and a steward of the vision and as a teacher assisting the whole health organization in fostering continuous desire to increase maturity (Argyris, 1991; Mohr, 2005). At the same time, managers should advocate local learning and standardized practices while balancing it out with attitudes and structures that encourage exploration and discovery (Carroll, 2002). Some of these issues and items have been explained in detail by Stephen J. Swensen, and James Dilling of Mayo Clinic in the Institute of Medicine report "Engineering a Learning Healthcare" in 2011 (Grossmann, Goolsby, Olsen and McGinnis, 2011). In this part, the main issues around technology management in healthcare and continuous learning will be briefly reviewed. Moreover, the usefulness of maturity models in assisting with some of these issues will be highlighted. One of the important aspects of technology management is Policies. Public policy and health insurance programs are important 
drivers of technology management. Decisions regarding what is incentivized and paid for dictates a critical role in deciding what is done and prescribed for the patients. Public policy and health insurance programs are culprits of high expenditures related to technology. The fact that the fee for service to fee for value in healthcare systems in the United States has not been completely adopted yet causes the healthcare system that has not transitioned yet to pay for more exams, which in turn drive technology use and subsequently cost. Appropriate technology management can expedite and finalize this adoption and diffusion of value for service. In the current system, instead of paying for outcomes, safety, or service which is provided through time, the service fee is considered. When merely service fee is paid, things such as safety, accuracy, reliability are not considered. In other words, patients/payors are charged a certain amount of dollars for procedures with different accuracy or complication rates as use is being paid for not the value of care. Inefficient technology management in healthcare organizations may cause some providers to charge more as in the case of Hillman et al.'s research. According to Hillman et al.'s research, physicians/providers that own their equipment may incline to order more exams and charging more for less quality (Hillman et al., 1990, 1992, 1995). These are only some of the reasons why policy and programs play such an important role in technology purchase and management in the United States.

Another issue that is important from the technology management aspect of continuous learning and maturity in healthcare organizations is the appropriateness of the technology use. Health organizations must make sure that a patient receives no more and no less than the right amount of care. Based on Thrall (2004), 30\% to 40\% of imaging procedures in 
the United States are estimated to be unnecessary while it is estimated that poor quality costs a company like General Electric $\$ 127$ million per year, which about $\$ 60$ million that amount is caused at by overuse of technology in the radiology department (de Brantes, 2003; Thrall, 2004; Tosczak, 2004). There are many other examples of technology misuse across many departments in health organizations which can be mitigated by standard evidence-based work rooted in best-practice order sets and decision support. In the case of imaging and pharmaceuticals specifically, due to patient's conceived value due to commercials and advertisements, more is desired which leads to higher cost while not necessarily being more effective in diagnoses and treatments in health organizations (Wennberg, Fisher, et al., 2007; Wennberg, O’Connor, et al., 2007).

Also, technology management encapsulates managing technology in terms of not only volume but also reliability in terms of accuracy and safety (Grossmann et al., 2010). In other words, healthcare providers, including residents and fellows, should be placed in environments their rate of medical errors will decrease with training and management. Training providers to work in teams is critical to ensure an increase in reliability and safety to make sure optimal technology management is flowing in the organization.

Another important issue is the effective diffusion of best practices as well as safety nets to support high-reliability patient care through Effective and efficient technology management which has been slow and inconsistent in the healthcare industry (Wennberg, Fisher, et al., 2007; Wennberg, O'Connor, et al., 2007; Ting et al., 2008). Some of the enablers of effective diffusion is for health organizations to know what their people know through smooth communication, dissemination of best practices, lessons learned, and 
importantly transparency. Swensen and Cortese (2008) argue that enterprise-quality dashboards showcasing outcomes, safety, and service using common definitions and processes as effective transparency efforts (Swensen and Cortese, 2008).

Technology itself can have a critical place in technology management. Information technology may take important roles in the optimization of technology appropriateness. It can also aid organizations to bridge expected knowledge gaps in healthcare delivery for points that providers may not know some information about the care they need to provide (not knowing what they don't know) through knowledge repositories (Grossmann, Goolsby, Olsen and McGinnis, 2011). Technologies such as "Ask Mayo Expert" that demonstrate the agreed-upon standard best practice, salient risks, and references, and frequently asked questions, and appropriate medical specialty contact information to providers for assisting them in providing care.

Finally, Stephen J. Swensen, and James Dilling of Mayo Clinic pinpoint the importance of sentient investment in social capital as an important part of effective technology management strategy with goals of high-reliability patient care. Social capital investment encapsulates the goals of transforming an organization from a collection of individuals into an agile, coherent, and collective mind (Lynch et al., 2000; Gopee, 2002; Grossmann, Goolsby, Olsen and McGinnis, 2011). This can be done by utilizing research engagement, administrations, and education. Swensen and Dilling emphasize on areas like transparency, teamwork training, horizontal infrastructure, and cross-functional, teambased simulation training as important social engineering topics. An example of this can be utilized in health organizations' training teams to train health medical and nursing 
students and residents, together in cross-functional teams to enforce a more effective transformation culture.

In the past couple of decades, maturity models have been introduced as reference frameworks for fields like Information System management in organizations across a myriad of industries (Carvalho et al., 2018). As maturity models are instruments to assess and continually improve organizational processes, they can play an important role in managing these new technologies or technologically enabled changes that have been happening and significantly affecting the healthcare landscape. In the healthcare domain, maturity models have also been used to pinpoint a wide spectrum of challenges and the areas such as business intelligence, information system, safety, calibration, and capability assessment (Harigopal and Satyadas, 2001; Liu, Hwang and Chang, 2011; Zephir, Minel and Chapotot, 2011; Batenburg, Neppelenbroek and Shahim, 2014; Brooks, El-Gayar and Sarnikar, 2015; Tarhan, Turetken and van den Biggelaar, 2015; Goncalves Filho and Waterson, 2018; Carvalho et al., 2019). Maturity models in healthcare have been generally used to pinpoint strengths and opportunities of maturity in different aspects of health and subsequently, paving the path for future improvement and evolution. However, there has been minimal focus on the management and technology management side of maturity models in healthcare as how a health organization as a system would be assessed in terms of maturity from different perspectives and dimensions to give management better insight into health organization's opportunities and strengths. Maturity models can give technology managers a clearer picture and a sense of self-awareness on where their organizations are currently, where they want to be, and how they can manage their 
resources to get to their desired maturity level from different aspects. Technology management maturity goals can be reached and reliable patient care can be provided on the condition that the healthcare industry fosters systems changes to drive continuous learning (Grossmann, Goolsby, Olsen and McGinnis, 2011).

There is a definite need to study the maturity of health organizations in terms of adoption and implementation of continuous learning and technology maturity from technology management aspect as this field is significantly important in the healthcare system in the United States due to the importance of:

- Policies, especially those which create incentives such as payment can be central motivators of activities and performance.

- Managing the appropriate use of technology and ensuring the high reliability of the technologies applied

- Effective diffusion of best practices and safety nets is crucial for efficient and effective technology management as it allows for the optimization of technology use.

- Social engineering strategies such as transparency, teamwork training, horizontal infrastructure, and cross-functional team-based simulations, can contribute to moving an organization toward integrated care coordination in which the decision is made with an organizational perspective. (Grossmann, Goolsby, Olsen and McGinnis, 2011) 
The initial action in the improvement of the business processes in terms of effectiveness and efficiency is to be on the same page and understand the current state of the organization or business by assessing that organization's processes from a different dimension. Maturity models have successfully assisted in this matter in different areas. One of the areas that there has been a lack of such models is in the healthcare domain (Tarhan, Turetken and van den Biggelaar, 2015). Although there are some hints at the importance of leadership in healthcare systems maturity and implementation and adoption of continuous and systematic learning, there is no literature focusing mainly or solely on the management side of the healthcare organizations' technology management maturity and its socio-technological infrastructure (Morain, Kass and Grossmann, 2017; Pronovost et al., 2017). There's a gap that needs to be filled in studying technology management maturity in healthcare organizations due to the importance of policies, managing the appropriate use of technology, effective diffusion of best practices and safety nets, and social engineering strategies (Yong, Saunders and Olsen, 2010; Grossmann, Goolsby, Olsen and Michael, 2011). All in all, although technology management is a growing issue that continues to require significant attention in healthcare, there is a lack of studies on the technology management side of maturity models. 


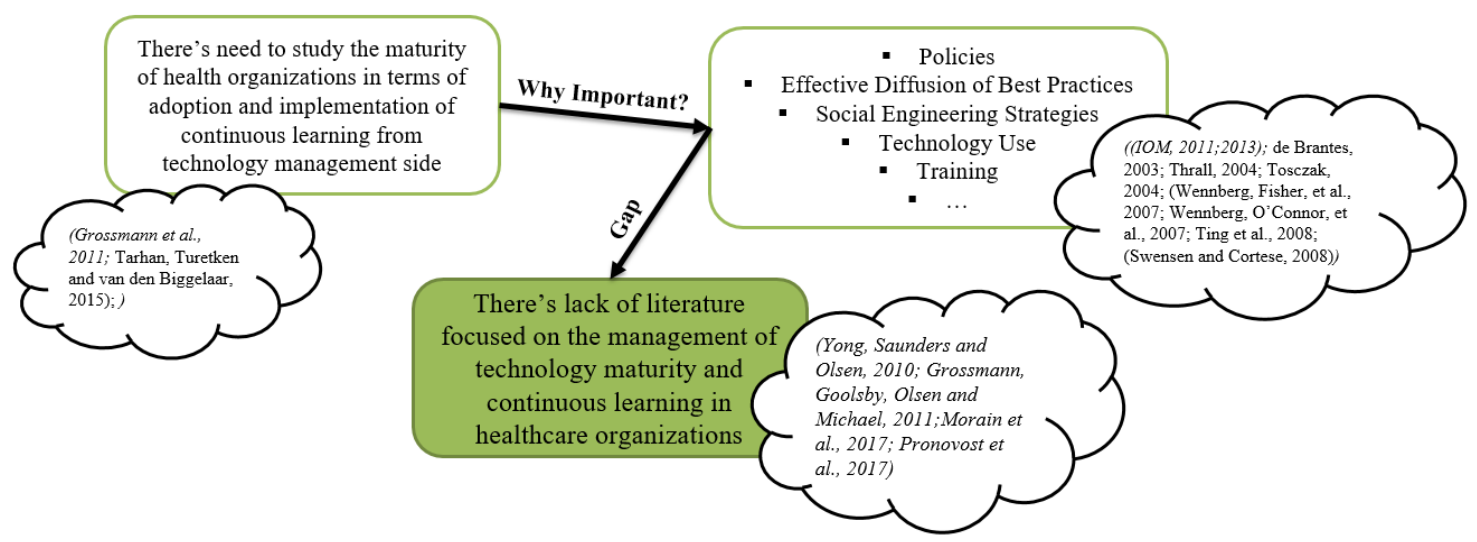

Figure 11: Gap 3

Gap 3: Despite the fact that technology management is a growing issue that continues to require significant attention in healthcare organizations, there is a lack of studies on the assessment of technology management maturity in healthcare organizations.

\subsubsection{Gap IV}

The fourth and final gap identified in this research is the lack of literature on research centers and institutes within university hospitals. This need came after the identification of the study unit of this research. The unit of study in this research is research centers and institutes within university hospitals in the United States as focusing on healthcare organizations as the "unit" would be too broad and complex to tackle. The results and methodology in this research, however, may apply to broader cases and organizational structures. Usually, different organizations within a hospital may have different management styles and smaller governance silos with certain guidelines being the same across these organizations. These centers focus on specific areas within a bigger health organization and have their directors, managers, and staff with different budget sources such as grants and donations, among others. Although they operate under the guidelines 
and goals of the university hospital, they have their own goals and missions which are aligned with their parent organization but more specific. These units also have a certain amount of autonomy that allows them to operate aligned with their mission and goals. During researching and studying about 100 health research centers in around 15 university hospitals in around 12 states in the United States, the lack of literature especially peer-reviewed articles and conference proceedings was identified. Almost all the information from these research centers had to be obtained using either their parent hospital's website or their website. Some of these websites outline detailed information about research areas, history, and funding while others did not offer the same number of details.

In addition to the lack of literature around the funding channels, level of autonomy, and structural details, there is almost no literature on how these health research centers are managed and how these entities manage innovation (specifically continuous improvement and organizational learning).

As mentioned before, in the United States, health systems are shifting from decades of operational systems designed to deliver health care as a reimbursable service into systems that deliver health as a population goal (Goldman, Kumanyika and Shah, 2016). In this direction, both transformation and incremental improvement are called for continuously improving and learning, as in systems that can improve themselves in different areas using the data generated internally and externally meaning clinicians use each patient encounter as an opportunity to make the next one better. This is especially important in the research centers within health organizations as their goal and mission are to learn and improve in a specific area of healthcare to be able to serve the population in need of that specific 
service or treatment better. There needs to study on how these research centers manage the technology, knowledge, and resources to facilitate and maintain continuous improvement and efficient learning and innovations while dealing with and/or taking advantage of multiple multi-perspective factors.

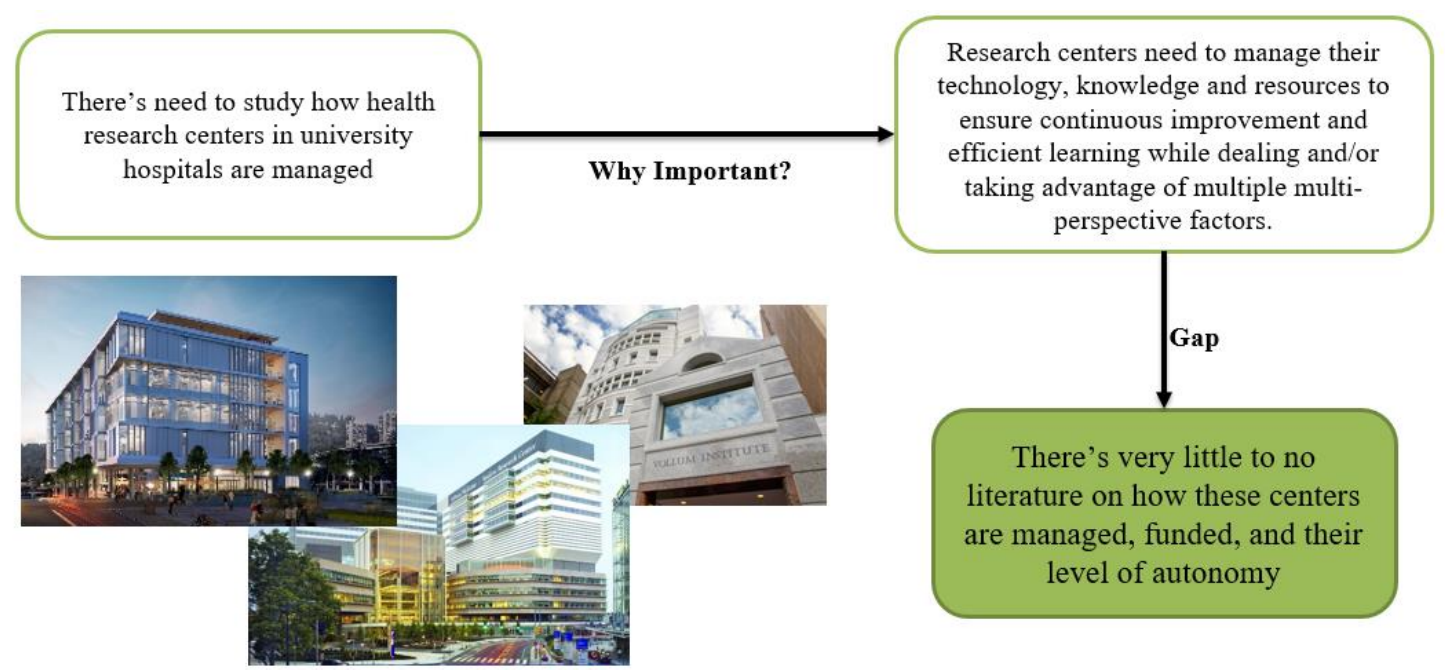

Figure 12: Gap 4

Gap 4: There is very little to no information around the structure and management of research centers within university hospitals.

\subsection{Research Goal}

The objective of this research is to develop a framework for assessing technology management maturity in healthcare organizations in the United States in an area such as continuous learning. The model can help health organizations pinpointing their strengths and weaknesses in the adoption and implementation of continuous and systematic learning and its socio-technical infrastructure while giving them organizational and competitive self-awareness. The model will serve as a much-needed technology 
management tool for health organizations to assess their technology management maturity for human advantage in a more effective way.

\subsection{Research Questions}

RQ1: What are the main perspectives and criteria in the assessment of technology management maturity in healthcare organizations?

RQ2: What are the weights of criteria and sub-criteria related to the assessment of maturity in healthcare organizations?

RQ3: Does the proposed framework offer a validated, quantified, repeatable, and practical way to assess technology management maturity in healthcare organizations?

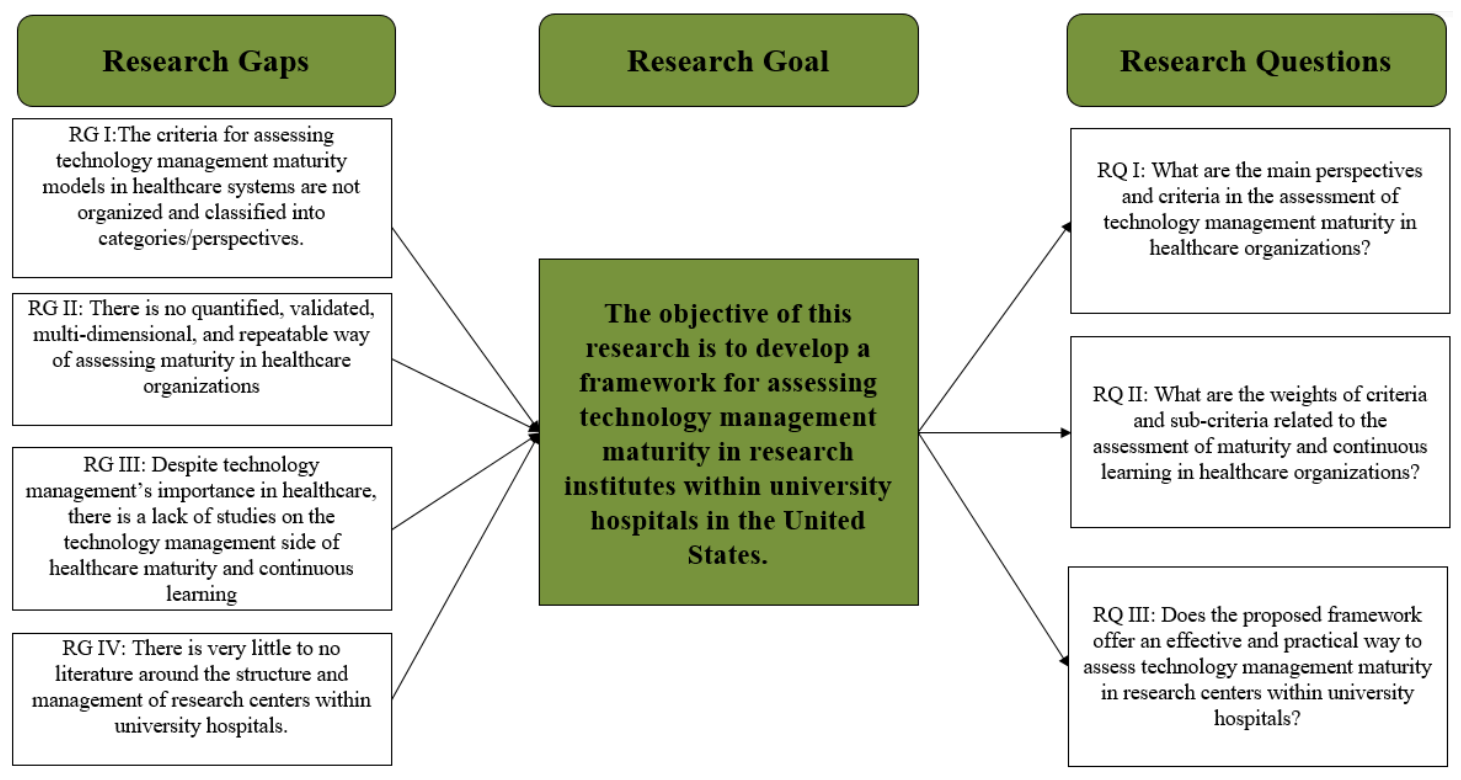

Figure 13: Research Gaps, Goal, and Questions 


\section{Methodology}

This section includes the methodological discussion of this study.

\subsection{Hierarchical Decision Model (HDM) Model}

To better understand the pros and cons of using the Hierarchical Decision Modeling (HDM), there is a need to better understand what it is.

The Hierarchical Decision Model (HDM) was initially proposed by Kocaoglu in 1983. HDM is a methodology to analyze strategic decisions in a hierarchical structure by formulating consensus among participants who are mostly experts in specific areas related to decisions.

HDM represents the problem hierarchically, enabling the decision-makers to visualize the criteria and their sub-criteria affecting the objective/mission. It is mostly applied for evaluating alternatives or selecting best-fitting options to accomplish an objective previously specified (Turan et al., 2009). HDM calculates the relative contribution/weights of perspectives/criteria through a systematic process of eliciting and evaluating subjective judgments of relevant experts in order to assist decision-makers in the decision-making process. HDM may be similar to the Analytic Hierarchy Process (AHP) introduced by Saaty (Saaty, 1977). However, HDM utilizes a different computational approach (Constant Sum calculations as opposed to Eigenvectors).

Munkongsujarit et al. (2009), argues that HDM aids the decision-maker by presenting the decision problem as a hierarchy of problems that are more facilitated in terms of handling (Munkongsujarit et al., 2009). This model breaks the various elements of the problem 
down to simpler sub-problems in a way that the decision problem morphs into a hierarchy (Taha et al., 2007). HDM is a tool used in decision-making to rank and evaluate the available alternative that is available followed by determining the most suitable choice among them (Munkongsujarit et al., 2009). It is a tool that assists decision-makers in quantifying and incorporating quantitative and qualitative judgments into a complex problem (Taha et al., 2007).

In the general form, HDM has five levels named as Mission-Objective-Goal-StrategyAction (MOGSA) (shown in figure 27), yet there is no restriction on the numbers of levels, but elements at the same level have to be "preferentially independent" (Kocaoglu, 1983). As the HDM structure is set, pair-wise comparisons among sub-elements for each branching node are made (figures 28 -29). The weights of each criterion are derived from pair-wise comparisons. Thus, in the generalized form of HDM researchers need to make pair-wise comparisons among objectives, goals under each objective, and strategies under each goal separately (Daim and Kocaoglu, 2015).

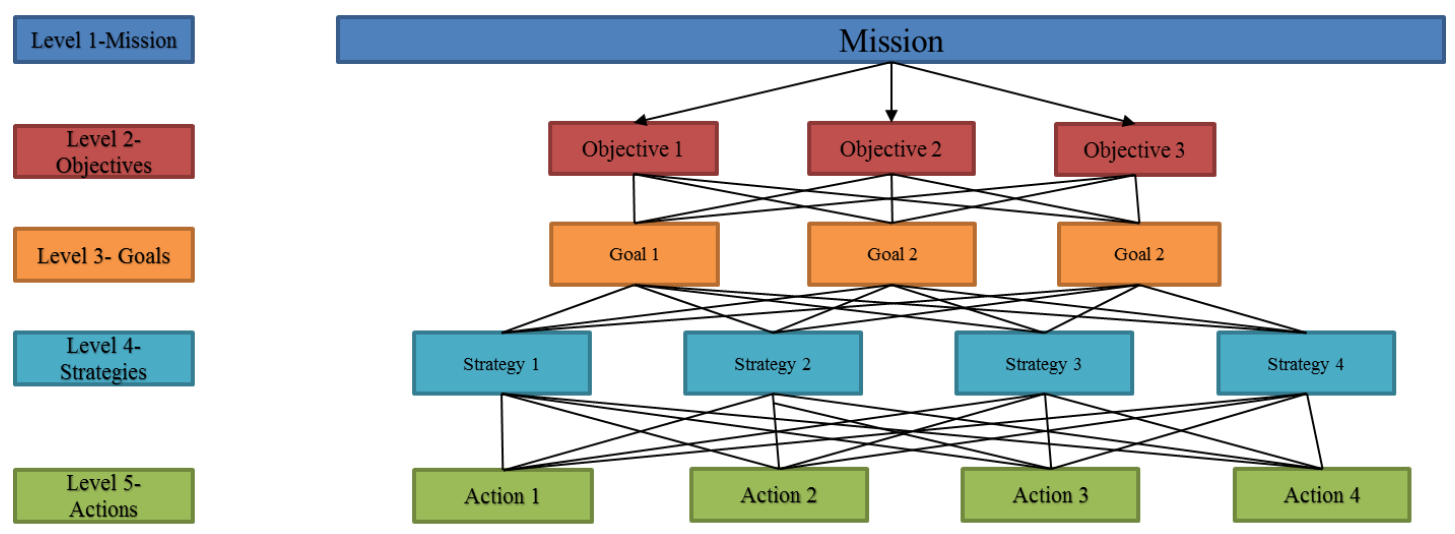

Figure 14: MOGSA HDM Model (Kocaoglu, 1983) 


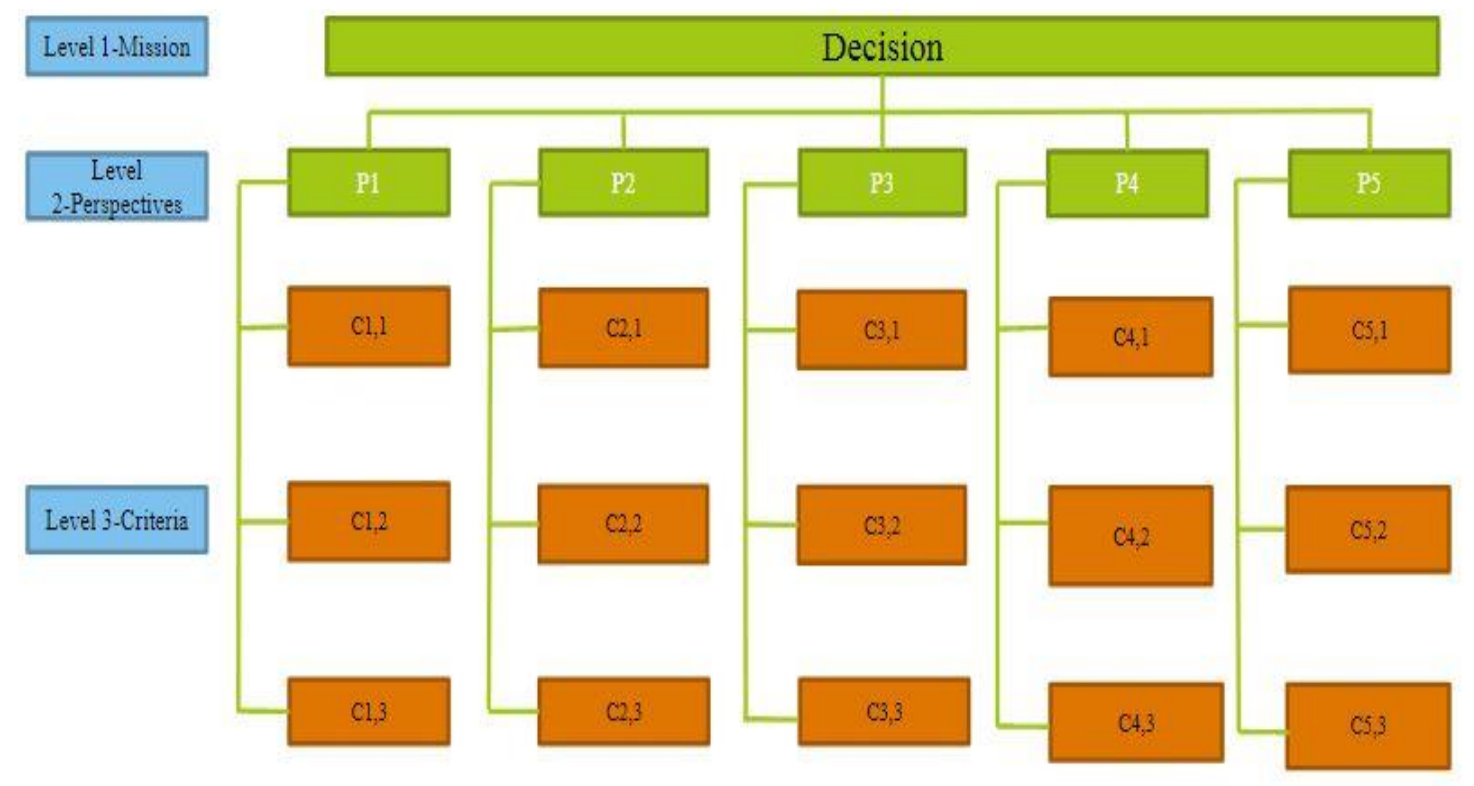

Figure 15: Mission /Perspective/ Criteria Model (Shaygan, Ozdemir-Gungor, D. Kutgun and Daneshi, 2017; Shaygan et al., 2018a)

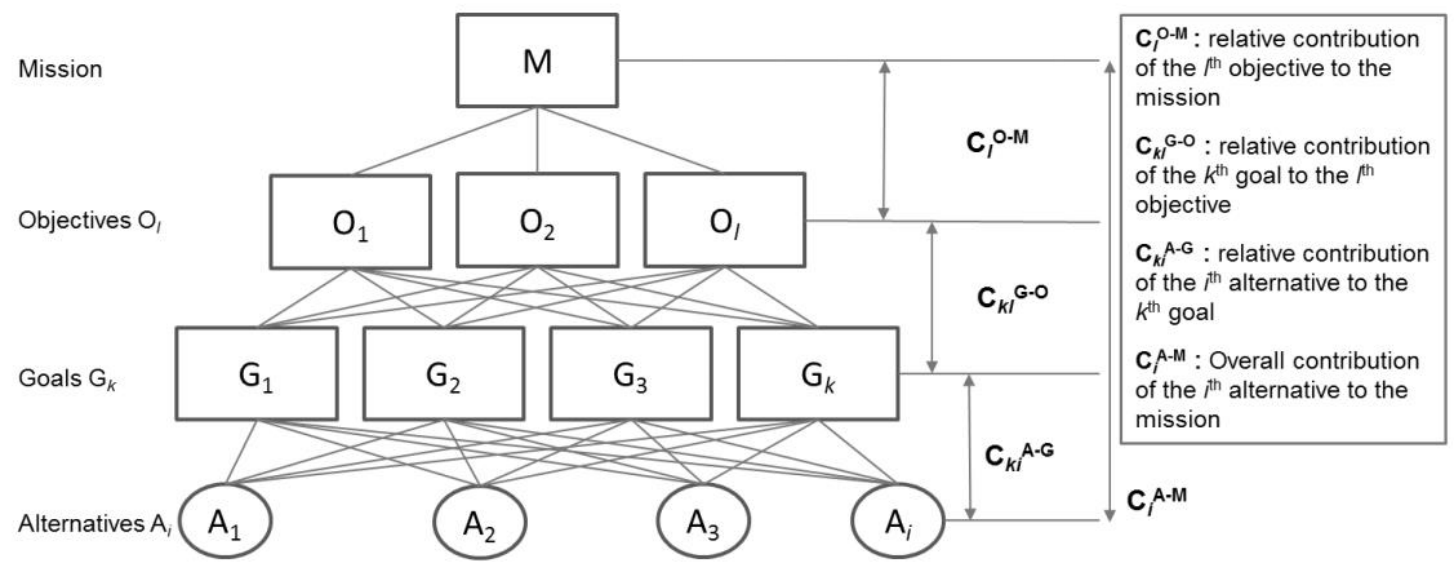

Figure 16: Depiction of the HDM Model (Gibson, 2016; Estep, 2017)

When implementing the HDM model, structured expert panels are asked to validate/quantify the model. The quantification will result in weights for each criterion/perspective in the model. Each item will have a "global" weight against all other 
items in the same level, and a "local" weight within its accommodating category. Then, the alternatives are evaluated against the lowest level of the hierarchy, which is the decision criteria, to find the best decision possible. The comparison is implemented by the distribution of 100 points between the two elements (pairwise comparison). The element with higher priority/importance assigned to it (by the allocation of more points reflecting its degree of priority/importance). In models such as AHP, 1-5 or 1-9 scales are used in the pairwise comparison process. In HDM, the Constant Sum method is used for the aggregation of expert judgments.

To evaluate alternatives, performance scores of alternatives for each criterion are required as well. Performance scores can be determined by using scoring for scalar scores or desirability functions for discrete scores. A desirability function is a transformation function that converts actual performance value to a score ranging from 0 to 100 based on market desirability or expert opinion (Daim et al., 2015).

Simply, HDM breaks down contributing factors to an objective into perspectives and criteria on different hierarchical levels and enables the analysis of the contribution of each factor or criterion to the objective. Then each option is evaluated in terms of the criteria to have a final point of achieving the objective, between 0 and 100 (Kocaoglu, 1983). The final score for each alternative is calculated by using Equation 1.

$$
T V_{n}=\sum_{K=1}^{K} \sum_{J K=1}^{J K} W_{K} \times f_{j k, k} \times V\left(t_{n, j k, k}\right)
$$

Where:

$\mathrm{TV}_{\mathrm{N}}$ : Technology value of alternative (n) 
$\mathrm{W}_{\mathrm{k}:}$ Weight of criterion $(\mathrm{k})$

$\mathrm{F}_{\mathrm{jk}, \mathrm{k}}$ : Relative importance of factor (jk) with respect to criterion $(\mathrm{k})$

$T_{n, j k, k}$ Performance and physical characteristics of technology (n) along with factor (jk) for criterion $(k)$

$\mathrm{V}\left(\mathrm{t}_{\mathrm{n}, \mathrm{j}, \mathrm{k}, \mathrm{s}}\right)$ : Desirability value of the performance and physical characteristics of technology (n) along factor (jk) for criterion (k) (Shaygan, Ozdemir-Gungor, D. Kutgun and Daneshi, 2017).

In the case of this research, some minor changes have been applied to the model which are as follows:

$$
M=\sum_{k=1}^{K} \sum_{j k=1}^{J K} P_{k} \times C_{j k} \times D_{j k}
$$

Where:

M: Maturity Score

$\mathrm{K}=$ Number of Perspectives

$\mathrm{J}=$ Number of Criteria

Pk: Weight of Perspective (k), k=1...k

Cjk: Relative importance of Criterion (jth) for Perspective (kth) (k), $\mathrm{j}=1 \ldots \mathrm{j}$ and $\mathrm{k}=1 \ldots \mathrm{k}$ 
$\mathrm{D}(\mathrm{jk})$ : Desirability value (Maturity Assessment Value) of Criterion (jth) for Perspective (kth)

Each item in the hierarchy is given weights. Each item will have a "global" weight against all other items, and a "local" weight within the category it belongs to. Then, the alternatives are evaluated against the lowest level of the hierarchy, which is the decision criteria, to find the best decision possible.

The experts evaluate criteria hierarchy and alternatives by conducting pairwise comparisons, with a constant-sum measurement scale (1-100 scale) for comparing every two elements. For example, each expert can evaluate the perspectives through pairwise comparison similar to the following example: (P1 40:60 P2), which means, in terms of importance, Perspective 1 is less important than Perspective 2 with the ratio of 40 to 60 . All experts will do the same for perspectives and criteria under each perspective. Then, based on HDM mathematical formulas, the experts' evaluation will be aggregated in order to calculate the weights of perspectives and criteria, with the total sum of 1 , for each level within the hierarchy and on the whole hierarchy. As well as weights for the alternatives against each other for each criterion. And a final score for each alternative in comparison with the other alternatives based on the aggregated evaluations of all experts, to find out the best decision possible (Kocaoglu, 1983; Daim and Kocaoglu, 2016).

Additionally, in instances in which there is a need in having a reusable model, or in instances of having many alternatives, desirability curves can be used. The combination of desirability curves with HDM is used to identify levels/ metrics for each criterion. Each level/metric connected to a criterion acts as a useful value to assist decision-makers. Using 
the desirability curves approach, the experts need to evaluate related levels/metrics for each criterion (desirability matrix) while giving each metric a scaled quantitative value. This enables the normalization of the evaluation results by experts across all the criteria (Phan, 2013; Estep and Daim, 2016; Gibson and Daim, 2016).

HDM also includes the calculations for disagreement, inconsistency, and sensitivity analysis (explained in details in question 3) to validate the reliability and robustness of the final model (Kocaoglu, 1983; Chen and Kocaoglu, 2008; Daim and Kocaoglu, 2015; Estep and Daim, 2016; Gibson and Daim, 2016; Estep, 2017; Shaygan, Ozdemir-Gungor, D. Kutgun and Daneshi, 2017; Shaygan et al., 2018a).

\subsubsection{Merits of the HDM model}

As mentioned in the previous section, the HDM model helps the decision-makers to visualize the criteria and their sub-criteria affecting the objective/mission. This is specifically helpful in understanding more complex problems, and consequently better decision-making under uncertainty. It offers a quantified, validated, and repeatable analysis of the decision elements by considering the expert judgments. By aggregation of diverse yet relevant expert judgments, the decision-makers are able to propose more meaningful and robust solutions. Furthermore, sending the survey/software links to experts individually avoids the risk of a physical meeting being monopolized by more vocal individuals. At the same time by hearing out each expert separately, mitigates the risk of not hearing and considering fewer vocal experts (as the case of focus groups may be). Furthermore, with the analysis of inconsistencies, disagreements, and model sensitivity, and the validation of perspectives and criteria by experts, HDM provides the 
much-needed validation for creating the multi-criteria decision model (in this case, maturity model). The use of desirability curves allows the reusability of the model in a different case (such as different health organizations, departments, etc.). Finally, the HDM model has been used in a vast variety of technology management areas such as technology adoption, strategic planning, technology planning, technology transfer, and technology assessment in a wide variety of industries such as healthcare, energy, semiconductors, transportation, internet of things, wearable devices among others (Munkongsujarit et al., 2009; Fenwick and Daim, 2011; Abotah, 2014; Iskin, 2014; Kocaoglu et al., 2016; Estep, 2017; Shaygan, Ozdemir-Gungor, D. Kutgun and Daneshi, 2017; C. G. Pereira et al., 2018; Shaygan et al., 2018a).

In the case of maturity models in healthcare organizations, the HDM model can bridge several gaps identified in the gaps analyses section. Specifically, the second gap reads as: "There is a lack of a quantified, validated and repeatable model for assessing maturity in health organizations". The validation part is addressed through the validation of perspectives, criteria, and desirability curve metrics by the experts. Moreover, the quantification part is addressed through quantifying the perspectives, criteria, and desirability curve metrics. Finally, through utilizing the desirability curves, the model can be used for different cases without the need to quantify the perspectives and criteria again.

\subsubsection{Limitations of the HDM Model}

When using the HDM model, the decision-makers may face several challenges/limitations that need to be addressed. Some of these limitations are imputed to the expert judgment nature of the HDM model which will be discussed in detail shortly. 
However, some limitations are related to the nature of the model itself. These limitations are discussed in this section.

\subsubsection{Expert related limitations}

HDM model uses experts for validation and quantification of the decision elements. The expert judgment is however subject to potential bias. In cases like healthcare, the experts may have been selected from a wide variety of areas/departments and they may bring some subjectivity and that is not always on purpose. In other words, inherent human bias may be present and may represent a threat to achieving solid results if not treated and mitigated properly. Some ways of coping with this limitation are to be meticulous in selecting the experts and ensuring that different (yet relevant) backgrounds in the research are represented. Other ways to mitigate the risk of bias have been discussed in question 3.

Another group of export-related challenges is the risk of inconsistent, inaccurate judgments by the experts. Furthermore, expert disagreements pose a limitation to the HDM model. Although these challenges are some of the characteristics of human judgment, there is a way to mitigate each of these challenges. As a part of validating the results of the model, the HDM methodology measures the inconsistency and disagreement indexes for the collected judgments. In cases where the obtained index is higher than the acceptable threshold (both for inconsistency and disagreement), remedies can be applied which are explained in question 3 in detail (such as clarification of research goals and careful expert selection). 
The last category of the limitations for the export-related category is related to the amount of work/time needed from the experts in order to validate/quantify the model. In many cases, the experts may be busy people and may not have substantial time to allocate to pairwise comparisons or perspective/criteria validations. For each level of the model with "n" elements, there is a need to pairwise compare elements “ $\frac{n(n-1)}{2}$, times. This can deem very time-consuming for a level that has 8 elements (meaning 28 pairwise comparisons). This may lead to experts getting tired and putting less attention into comparing the decision elements and consequently deteriorate the model's reliability.

\subsubsection{Model Sensitivity}

In instances where the HDM model includes alternative (actions), the addition and/or removal of new alternatives may substantially change the final weights (results). However, in cases that use desirability curves instead of alternatives, this limitation is avoided.

Another issue is the lack of adaptability to changes/time. In the dynamic world that we are living in, rarely will the problems stay the same through time. The dynamic environment may subject the model to changes in the importance of decision elements and even the decision elements themselves. In other words, with time, some elements (criteria) may become less important or even obsolete whereas there may be an increase in the priority of some criteria or even emerging criteria in the model. These dynamics can happen gradually or abruptly and based on HDM's nature; these changes will result in changes in the final results/weights. This may mean that there should be frequent (based 
on the importance and pace of changes) updates for the models, their components, and weights. Sensitivity Analysis (SA) is one of the ways to bolster decision makers' awareness by learning about how robust the model is and how resistant it is to changes in cases where the model should be modified (Tran, 2000; Abotah, 2014; Iskin, 2014; Gibson, 2016; Estep, 2017).

\subsubsection{Criteria Balance among Perspectives/Criteria}

Finally, it is important to point out that when using HDM, it is preferred to have a close number of criteria under each criterion. The reason for this is that when there is a difference (more than 1) between the number of criteria under different perspectives it may lead to smaller weights for the criteria under the perspectives with a higher number of criteria (although it may be the perspective with the highest weight.). The only known remedy for this limitation is trying to keep the criteria under each perspective close to the others.

\subsubsection{HDM Justification}

Earlier, in the research gaps section the need for a multi-perspective, validated, and quantified model was stressed. Carvalho et al.(2016; 2015) discuss the need for a model including the main ingredient factors of healthcare technology maturity and the potential benefits of assigning weights to them in order to understand their relative importance by specifying that there should be a model that includes the main influence factors with different weights depending on their relative importance and its development should be supported by rigorous scientific methods of conceptualization and validation. 
Furthermore, technology management maturity models are complex, and specifically in the case of healthcare organizations, there is a need for a method/model that can simplify some of that complexity. Significant time-gaps in the practical transformation of clinical knowledge into clinical practices, ever-increasing healthcare costs, high rates of medical errors, healthcare institutions' obligations towards improving safety, clinical outcomes, and efficacy of care from one side, and the rise of disruptive innovations such as genomics, wearables, machine learning, and artificial intelligence in health systems, the adoption of EHRs and novel diagnostic tools, and the plethora of data from the other side has made the need for a new approach in managing the U.S healthcare systems an imperative. These issues are multi-perspective and there is a need to take a multiperspective approach when dealing with decision-making problems in this area.

With the gaps identified in the pre-comprehensive exam proposal and more specifically in the research gaps section, there is a need for a model which:

- Considers the criteria for assessing technology management maturity and continuous learning in healthcare organizations in classified categories.

- Takes a quantified, validated, and reusable approach in assessing maturity in healthcare organizations (weights for factors in each level of the hierarchy)

- Allows multi-perspective and hierarchical approach in decision making. 


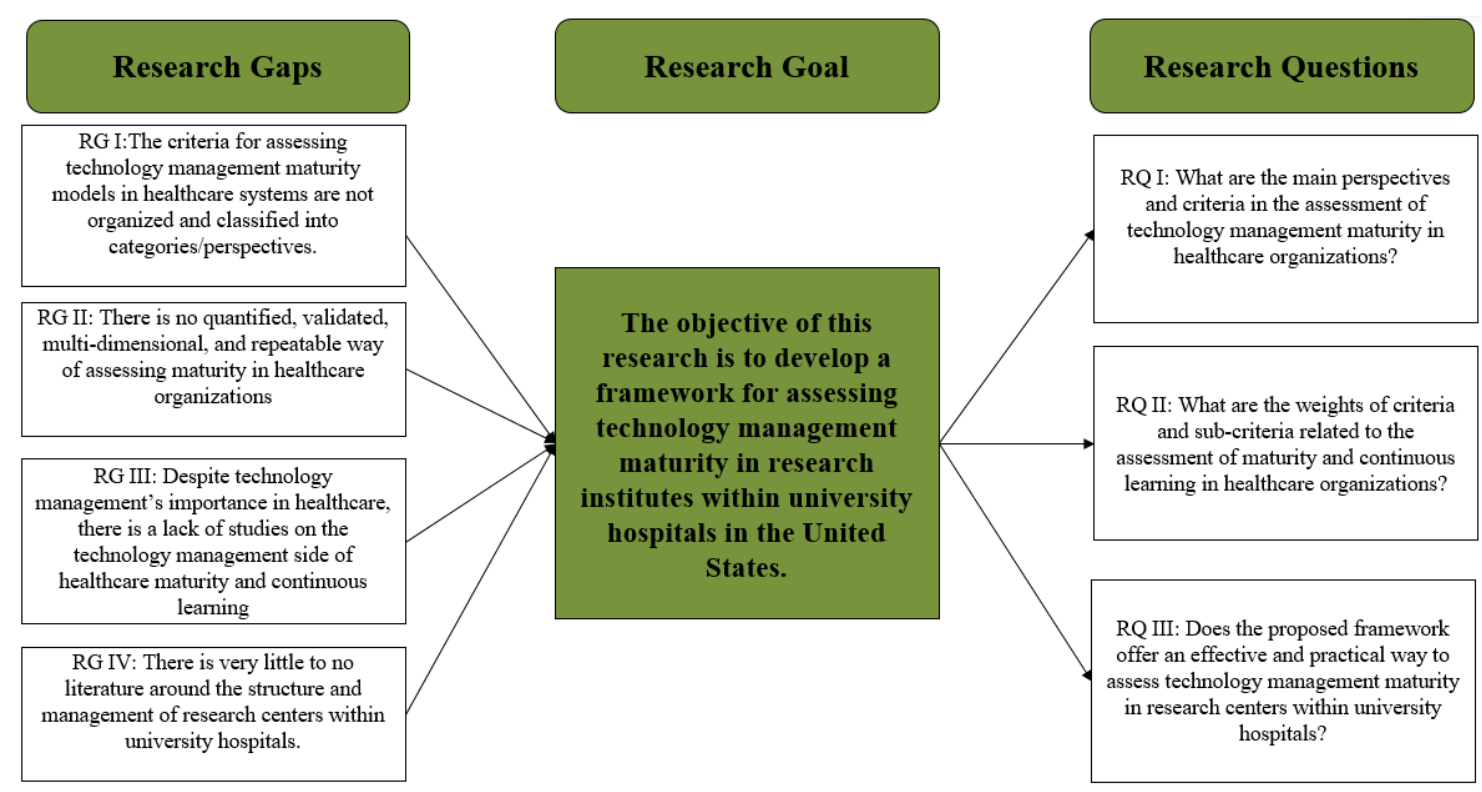

Figure 17: Research Gaps, Goal, and Questions (Reprise)

After reviewing several other models which are used in multi-criteria decision-making, it is found that the HDM methodology can adequately tackle the gaps mentioned above. HDM is indeed a multi-criteria decision-making method with a hierarchical structure that enables a more complex analysis through pairwise comparing the important factors (perspectives/criteria) in a certain problem/decision. Furthermore, HDM captures experts' judgments and turns them into the weights for important factors regarding the problem. This data collection can be done anonymously and individually bolstering the quality of data that can be used towards helping the decision-maker. When using HDM, different kinds of analysis such as Inconsistency analysis and Disagreement analysis can be done to validate expert judgments. Moreover, at each level of the hierarchy, surveys/questionnaires/interviews can be used to validate the selected perspectives/criteria (initially found using literature review). In addition, Sensitivity Analysis provides the decision-makers with a better understanding of the model in terms 
of flexibility while giving them a better idea of when the model will require an update. Finally, although a heavy load of quantification may go into the quantification, validation, and calculation of the results, HDM results are intuitive and not difficult to use/understand by people who have a less academic background.

HDM, as result, will provide weights for each of the decision elements in the model as a prioritization/ resource allocation tool. By learning about the importance of each decision element in each level of the hierarchy, decision-makers can have a better idea and understanding of the situation at hand.

The use of desirability curves in this study will bolster the model through the identification of the desired outcome for each criterion. In the case of this study, the desirability curves will allow each health organization to be measured in terms of predefined metrics for each criterion. Moreover, the desirability curves pave the way for the model to become a reusable one. The reusability of the model is specifically important as health organizations may need to measure their maturity regularly to make sure if they are on the right path or not. The reusability factor will also allow the model to be applied to several healthcare organizations (partners/competitors) to gain a better understanding of their performance.

The HDM model can be used by health organizations to identify their strengths and opportunities. This will give health organizations a sense of self-awareness as "where they are?", "where they need to be?", and "how can they better reach the point they want to be?". The model can serve decision-making assisting tool where health organizations can measure their maturity and better prioritize their projects and initiatives in order to 
reach the goals of continuous improvement and learning in the delivery of care. Although cutting costs may not be an initial goal of this, it can certainly be a result of maturity in healthcare organizations.

The HDM model has been used to tackle many multi-criteria problems in different areas and industries. It has been used to determine the innovativeness of companies (Phan, 2013), evaluation of energy policies (Abotah, 2014), assigning technology transfer scores to research proposals (Estep, 2017), measuring research center performances (Gibson, 2016), measuring readiness for smart city projects (Barham and Daim, 2018), forecasting of emerging therapeutic antibodies patents (Pereira et al., 2018), and development of technology transfer score (Lavoie, Kim and Daim, 2017; Lavoie and Daim, 2020) among others and is, therefore, selected as the methodology for measuring maturity in healthcare organizations with goals of improvement in the quadruple aim of care.

\subsection{Critical Issues in Forming an Expert Panel and Selecting the Experts}

As a part of the methodology in this research, expert panels in the field of the study will help with the validation and quantification of the model. This validation and quantification are in terms of the pairwise comparisons in the model (perspective/criteria) as well as the desirability curves for each criterion's metrics. In order to be able to discuss the critical issues around forming an expert panel and the selection of these experts, there should be some clarification and definitions around what an expert is and what is not. A short literature review has been conducted to shed some light on this issue.

The roots of the word "Expert" comes from Middle English, borrowed from AngloFrench, borrowed from Latin expertus "tested, shown to be true," from past participle 
of experīin "to put to the test, attempt, have experience of, undergo"(Merriam-Webster, 2019a). As for the meaning, based on Merriam Webster's definition, Expert means having, involving, or displaying special skill or knowledge derived from training or experience. As for the panel, it is defined as a group of persons selected for some service (such as investigation or arbitration) (Merriam-Webster, 2019b). Martin et al. (2012) define expert knowledge as important information on a specific topic that is not widely known by others (Martin et al., 2012). Some technical papers refer to expert opinion as informed opinion on a technical problem based on experience and training (Meyer and Booker, 2001). From these definitions, we can conclude that expert panels can be defined as: "A group of people having, involving, or displaying special skill or knowledge derived from training or experience in a field not widely known by others, selected for judgment and decision making of a topic in that specific field.”.

Expert panels are frequently utilized in developing and accessing projects at model development, and interpretation of results (Fazey, Fazey and Fazey, 2005; Runge, Converse and Lyons, 2011; Sutherland et al., 2011; Martin et al., 2012). In the fields of technology and healthcare, expert panels have been used in the quantification and validation of multi-criteria decision models (Daim and Kocaoglu, 2015; Shaygan and Testik, 2017; Shaygan, Ozdemir-Gungor, D. Kutgun and Daneshi, 2017; Testik et al., 2017b; Shaygan et al., 2018b)

Expert panels' judgments, according to some studies, as well be the only or the most, credible source of information available for making management decisions in cases where the topic is not widely known (Martin et al., 2005; Firn et al., 2013). Holman et al. in 
2000 discuss that expert panels are invaluable in terms of addressing the multi-perspective nature of complex problem while it is important to be wary of potential biases (Holman et al., 2000). Moreover, expert panels can play an important role in raising qualitative issues in the creation of conceptual models (Knol et al., 2010).

Kuhnert et al. (2010) signify that expert judgment in ecology is increasing in importance as a tool for conservation decision-making in instances of insufficient data (Kuhnert, Martin and Griffiths, 2010). Expert knowledge assists researchers in the science and practice of conservation due to the complexity of problems, insufficient data, and the imminent nature of many decision-making processes (Martin et al., 2012).

In a seminal study about expert judgments, Dreyfus and Dreyfus (2005) discuss a fivestage phenomenological model of skill acquisition and thus different levels of expertise which will be briefly introduced here to bolster the clarification of the subject (Dreyfus and Dreyfus, 2005).

The first level of skill acquisition is the "Novice" stage which means that although the person has no prior experience or knowledge in the field, he/she has a grasp of the basic rules, conditions, and the environment surrounding the subject at hand. The second stage, "Advanced Beginner", is a novice who has now a wider understanding of the subject matter due to a little experience/exposure. The next level is "Competence" which refers to having more grasp of the subject in terms of elements and prioritization. In other words, the once novice now has a better understanding of the subject and can disintegrate the complex issue into simpler parts to make them less overwhelming and more feasible. The person in the "Competence" level can attend, evolve, and develop the related skills in 
each of these simpler parts one at a time. As the fourth level, "Proficiency" hints at situational subjectivity in the reasoning. In other words, as the level of expertise grows, long and structured lines of reasoning are replaced by faster actions and decisions more intuitively. The final level, based on this study, is "Expertise". The final level of skill acquisition means that the expert has a higher level of understanding and refinement to the previous levels enabling her/him to reach faster intuitive solutions/decisions for complex scenarios. This means that, at this stage, the expert can see what is needed to be done and how it can be done in an intuitive way (Dreyfus and Dreyfus, 2005). Day (2002) however, stresses that decisions based on intuitions do not necessarily turn a person into an expert (Day, 2002). Day continues to emphasize that, in order to become experts, there is a need to have different kinds of practice in terms of cognition and its context as opposed to being solely based on the accumulation of practice.

As for critical issues around forming and selection of expert panels, there are a couple of areas to consider which are discussed below:

\subsubsection{Bias}

The first critical issue discussed in the forming and selection of expert panels for research is biased. Bias is indeed one of the most important challenges faces when expert panels are used in research (in this case, validation, and quantification of the model). At times, the bias from the experts' side can be caused to the overconfidence in the subjects known well by the experts (Nemet, Anadon and Verdolini, 2017). In order to better understand bias, there's a need for a definition for it in the relevant literature. Bias happens when an expert tends to stress and believe his/her own judgment/experience over the ones that 
challenge that judgment (Mahoney, 1977). Also, experts, as humans, are unable to get rid of cognitive bias and overconfidence (Morgan, 2014). In addition to overconfidence, the bias from the experts' side can be due to the expert's tendency to influence the outcome of the study. Research assessment performed by expert panels may be biased because of the factors on the organizational, the panel, or individual evaluator's level (Langfeldt, 2004). Langfeldt also categorizes the bias in research assessment based on cognitive constraints, and interests from one side and scholarly/professional and non-professional side. When in professional and based on cognitive constraints, experts may only view the decision/problem through the own scholarly view (Mullen and Goethals, 1987). At the same time when the scholarly bias happens due to interest, there is a risk of Nepotism. In terms of non-professional bias with cognitive constraints, there is the risk of disregarding data/information because of the sub-optimal information seeking. As for the nonprofessional due to interest, the bias can be motivated by personal interest/Nepotism (Langfeldt, 2004). 


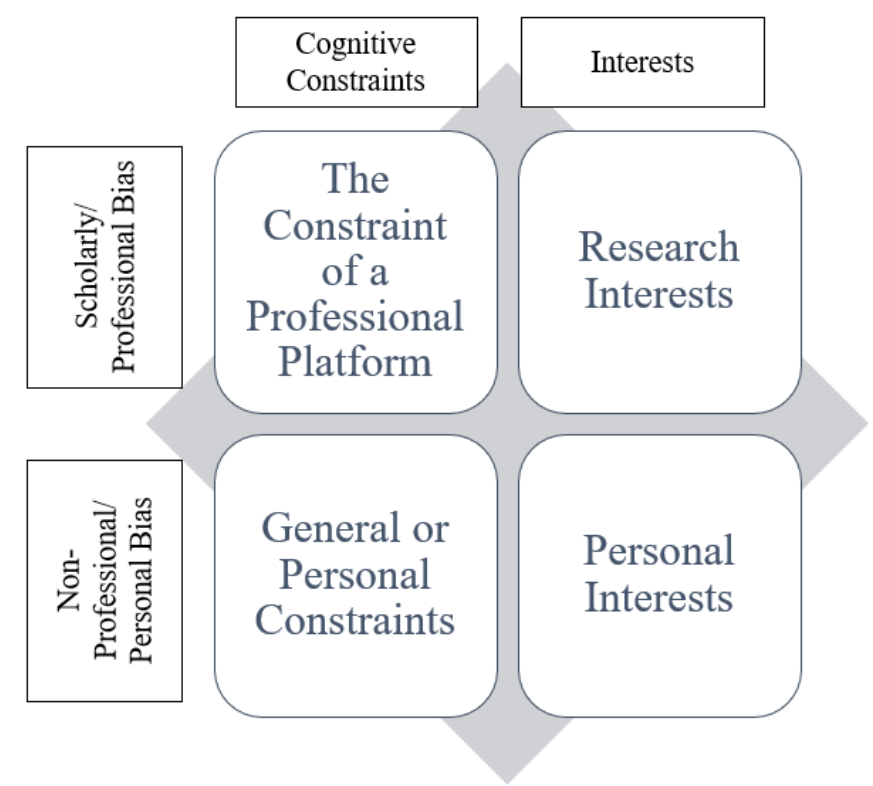

Figure 18: Categories of Bias in Research Evaluation (Langfeldt, 2004)

These issues make the identification and recruitment of proper and reliable experts for the problem a challenging and arduous process (Tran, 2000; Abotah, 2014). Being impartial is important as impartiality in judgment ensures both the consistency and meritocracy of expert judgment/decisions (Lee et al., 2013).

To mitigate some of the problems caused by the potential bias from the experts' side, models like Hierarchical Decision Models, Analytical Hierarchical Processes, and Delphi elicit anonymous judgments from experts. However, these methods are still prone to bias from personal judgment due to personal judgments and/or interests (gains) which may conceal the reality of the subject as a result.

Considering the mentioned issues regarding bias, researchers need to pay extra attention in selecting the expert panel to ensure that there are no reasons for the experts (in terms of personal/organizational interests) to influence their assessment of the subject matter. 


\subsubsection{Size}

The other critical issue around forming expert panels is the size of the panel. In terms of the size of the panel for the research/studies/dissertations using the HDM methodology, a different number of experts have been used. Phan (2013) argues that the ideal size for an expert panel should be around 10-15 experts while Okoli and Pawlowski suggest the panel of between 10 and 18 (Okoli and Pawlowski, 2004; Phan, 2013). However, the size of expert panels has varied for different studies. Tran (2013) and Chan (2013) used a smaller expert panel of 5 and 3 members respectively (Tran, 2000; Chan, 2013). Many research in technology management utilizing expert panels for judgment in terms of validation of quantification of models has used between 6-12 experts as panel members as a manageable range time-wise yet reliable result-wise (Abotah, 2014; Gibson, 2016; Estep, 2017).

\subsubsection{Balance}

Other than the risk of bias and the size of the panel other important factors should be considered when forming an expert panel. As this research revolves around maturity models in healthcare and organizational learning, it is important to have a wide variety of experts covering different perspectives and dimensions of the healthcare environment. There is a definite need to have healthcare experts from the technological, social, organizational, regulatory, and financial sides as one of the gaps of this research is that technology management maturity in healthcare should be studied in a multi-perspective way (Shaygan, Lavoie and Daim, 2018). A balanced expert panel is a more robust and 
significant representation of the information and knowledge in the field of the problem/decision (Abotah, 2014).

\subsubsection{Quality}

Another important issue is the quality and value of the judgment provided by the experts. The expert judgment should reflect the reality of what is happening regarding the problem/decision which is being studied. Factors such as bias can affect this aspect of the expert panels. Looking at this aspect from a different lens, it should be considered that how much of an expert the selected individual is which will directly affect the potential contribution and value of the judgment provided by the expert. This judgment will eventually determine the value and the merit of the study itself. Another side of the expert quality can be imputed to how much time and contribution the expert is willing to allocate to the research. Before selecting the experts, matters such as the time, knowledge, and meticulousness needed for taking part in the expert panel should be discussed (Chan, 2013; Abotah, 2014). Another important aspect that should be ensured beforehand is the expert's willingness to participate in the study.

\subsubsection{Researcher's Burden}

While most of the topics discussed in this section are the issues around experts, there are some points that the person in charge of constructing the expert panel should do. The researcher must communicate the research goals to the experts. In instances where there is poor communication of the goals to experts, judgments will be prone to inaccuracy. A researcher should walk the thin line between under and over-communicating the research 
goals to the experts in order to make sure he/she is neither misleading/influencing the experts nor providing insufficient information to experts.

Furthermore, the researcher should leverage the right communication tools to assure they are clear, fair, and user-friendly for the experts while making sure that the output is usable for the research. Software, survey websites, phone conversations, emails can be used to collect the expert judgment. In the case of study groups, sometimes an expert may monopolize the conversation or influence the other experts that are attending that study group.

Issues such as inconsistency and disagreement and how their judgments can be validated in terms of these issues will be discussed in detail in the following sections.

\subsection{Inconsistency}

One of the ways in which the Hierarchical Decision Model (HDM) ensures the validation of the quantification done by the expert panel is the inconsistency test. The word “inconsistent" means "Acting at variance with one's principles or former behavior" (Oxford Dictionary, 2019b). Based on Estep (2017), inconsistency is the disagreement within an individual's (in this case, expert) assessment (Estep, 2017). Moreover, Abbas (2016) defines inconsistency in the HDM context as: "Inconsistency is a slight or gross, deliberate or unintentional error in the elicited pairwise judgment related to the rank order and mutual preference proportionality of alternatives." (Abbas, 2016). In addition, Abotah (2014), defines inconsistency as "a measure that explains how reliable and homogeneous in his or her answers each expert was through the whole questionnaire" (Abotah, 2014). However, based on the literature, expert judgment inconsistencies are 
quite common in multi-criteria decision models such as AHP, FAHP, and HDM (Leung and Cao, 2000; Aguarón and Moreno-Jiménez, 2003; Grošelj and Zadnik Stirn, 2012; Chan, 2013; Gibson, 2016). This probability is invigorated in cases where the decision models are complicated and have many criteria involved.

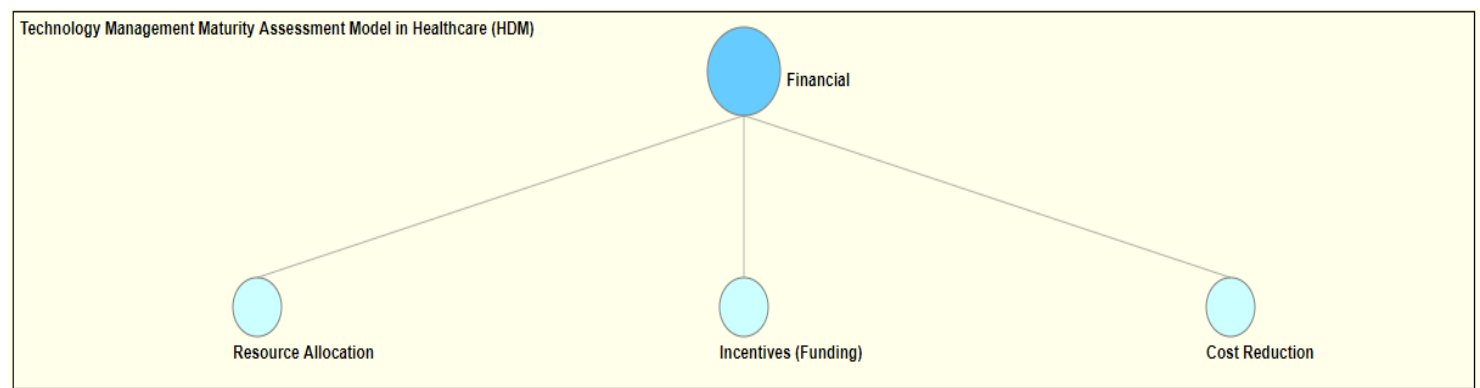

Figure 19: Screenshot of the Financial Pairwise Comparisons

As an example of the kind of inconsistency caused during the experts' assessment and quantification of the model, consider the following hypothetical scenario:

During the quantification of the "Financial" perspective's criteria in this study, an expert has pairwise compared the three criteria within this perspective namely, resource allocation, funding, and cost reduction (shown in figure 26). Let us assume based on our hypothetical expert's judgment, resource allocation is more important than funding while funding is more important compared to cost reduction. Based on this logic, resource allocation must be more important compared to cost reduction based on this expert's judgment. In addition, if based on the expert's judgment, resource allocation is 1.5 times more important than funding and funding is 2 times more important compared to cost reduction, this means that resource allocation should be $3(2 \times 1.5)$ times more important 
than cost reduction based on our hypothetical expert's judgment. This type of consistency is called cardinal consistency.

Another example of expert inconsistency is shown in the following scenario:

Let us assume our hypothetical expert has ranked resource allocation 2 times as important as cost-cutting and funding. Moreover, the expert has ranked funding 2 times as important as cost-cutting. Based on this ranking, resource allocation should be the most important criterion. However, in this case, the ordinal consistency has been violated as based on the expert's judgment, resource allocation and funding should have the same importance. If the cardinal consistency is maintained in a judgment, the ordinal consistency will automatically be guaranteed.

Experts, as humans, are subject to inconsistency in their judgment. This issue is even more frequent in more complex problems. This implies that inconsistencies are expected to occur in the quantification of the HDM model (Gibson, 2016). Therefore, the result of expert judgment in the HDM model will each contain a level of inconsistency which must be measured and controlled to ensure the soundness of the decision (Abbas, 2016).

Here, a summary of the way inconsistency is measure in the HDM model is provided. Inconsistency measures have been calculated for many HDM related literature through the years (Kocaoglu, 1983; Chan, 2000; Phan, 2013; Abbas, 2016; Lingga, 2016; Estep, 2017; Shaygan, Ozdemir-Gungor, D. Kutgun and Daneshi, 2017; Shaygan et al., 2018a). In the HDM model, the inconsistency is calculated using the sum of standard deviations. For $\mathrm{n}$ elements of comparison, $\mathrm{n}$ ! vectors are created using the constant sum calculation $(\mathrm{s} 1, \mathrm{~s} 2 \ldots, \mathrm{sn})$. The Constant Sum Scaling is a technique in which the experts (in this case) 
are asked to allot a constant sum of units, to some items based on a specified criterion (Chayes, 1960). Each of the resulted vectors represents an element combination (Phan, 2013). As an example, for the financial perspective, there are $(3$ ! =6) possible combinations for the criteria as follows:

1- Resource allocation, Funding, Cost cutting

2- Resource allocation, Cost cutting, Funding.

3- Funding, Resource allocation, Cost-cutting.

4- Funding, Cost cutting, Resource allocation.

5- Cost-cutting, Funding, Resource allocation

6- Cost-cutting, Resource allocation. Funding

Consistency in providing the pairwise comparison will result in relative values for each of the mentioned criteria combinations whereas any inconsistency will result in inconsistent values for each combination. Therefore, the inconsistency in HDM is obtained through calculating the standard deviation between the values calculated in the n! combinations (Phan, 2013; Estep, 2017). In this section, the calculation of inconsistency in HDM is presented with referencing to the HDM-related literature (Kocaoglu, 1983; Phan, 2013).

Let:

$C_{i j}=$ relative value of the $\mathrm{i}^{\text {th }}$ element in the $\mathrm{j}^{\text {th }}$ orientation for an expert

$\bar{C}_{l}=$ mean relative value of the $\mathrm{i}^{\text {th }}$ element for that expert 


$$
\frac{1}{n !} \sum_{j=1}^{n !} C_{i j}
$$

Inconsistency in the relative value of the $\mathrm{i}^{\text {th }}$ (for $\mathrm{i}=1,2,3 \ldots, \mathrm{n}$ ) element is calculated as:

$$
\text { Inconsistency }=\sqrt{\frac{1}{n !} \sum_{j=1}^{n !}\left(\bar{C}_{l}-C_{i j}\right)^{2}}
$$

Variance of the expert in providing relative values for the $\mathrm{n}$ elements is calculated using the formula below:

Inconsistency $=\frac{1}{n} \sum_{i=1}^{n} \sqrt{\frac{1}{n !} \sum_{j=1}^{n !}\left(\bar{C}_{l}-C_{i j}\right)^{2}} \alpha$

The acceptable threshold for the inconsistency measure in HDM models has been discussed in the literature. Kocaoglu in 1983 established a maximum of $\mathbf{1 0 \%}$ as the acceptable threshold for the inconsistency in HDM models (Kocaoglu, 1983). In cases where the inconsistency is higher than $10 \%$ or 0.1 , several actions can be taken. It can be requested from the expert to do the pairwise comparisons again. Moreover, a conversation with the expert may clarify the evaluation procedure for the expert and thus, solve the inconsistency problem in the second attempt. In cases where a consensus cannot be reached with the expert or in cases of continued inconsistency, the expert's judgment may be deleted from the results. Another action that can be taken was introduced by Abbas (2016) which argues that the threshold for inconsistency should be based on the criticality 
of the decision (Abbas, 2016). This method uses the root-sum of variances by considering the number of pairwise comparisons made by the expert.

$$
\text { Root Sum of the Variance }=\sqrt{\sum_{i=1}^{n} \alpha_{i}^{2}}
$$

In the above equation, the Root sum of the variance is used as the inconsistency value. $\alpha_{i}^{2}$ represents the variance of the for the $\mathrm{i}^{\text {th }}$ decision element and is calculated using the formula below (Abbas, 2016).

$$
\alpha_{i}=\sqrt{\frac{1}{n !} \sum_{j=1}^{n !}\left(V_{i j}-\bar{V}_{i j}\right)^{2}}
$$

In the equation above, $\mathrm{V}_{\mathrm{ij}}$ is the normalized relative value for the $\mathrm{j}^{\text {th }}$ combination of variable $\mathrm{i}$ in "n!" orientations. The value $\bar{V}_{i j}$ is the mean of the normalized relative values for the $\mathrm{j}^{\text {th }}$ combination of variable $\mathrm{I}$ and is calculated as follows:

$$
\overline{V_{l j}}=\frac{1}{n !} \sum_{j=1}^{n !} x_{i j}
$$

\subsection{Disagreement}

Another important topic in the validation of the quantification of the HDM model is the analysis of the disagreement among experts. The word disagreement means lack of consensus or approval (Oxford Dictionary, 2019a). In the context of HDM, different experts may have different ideas in terms of the assessment of the factors and the most important criteria and their degree of importance may be subjective and different. Thus, 
despite the fact the disagreement among experts is expected and natural, it is critical to measure and analyze the disagreement degree among the model experts as a part of validating the maturity model in healthcare before moving on to the data analysis procedure. As mentioned, disagreement among experts is an expected element of the model. However, it is critical to set a threshold for an acceptable disagreement value. agreement on problem/decision is necessary before an analysis based on quantification of the criteria/perspectives (Mumpower and Stewart, 1996). Disagreement among experts can be sourced to different factors such as different approaches and ways of thinking/ideology, lack of expertise, self-interest, lack of goal communications, insufficient or poor quality data/ feedback (Hammond, 2000). Therefore, although some of these disagreement risks may be mitigated through articulate and clearly defined research goals and user-friendly and intuitive tools, some sources of disagreement may still exist in the model.

What does disagreement entail in the context of HDM? Disagreement is defined by Estep (2017) as "The extent to which an expert panel is in agreement with their judgment quantification is represented by a disagreement value." (Estep, 2017). Moreover, Tran (2000) and Abotah (2013) define disagreement as "the agreement among the experts' judgment is represented by a disagreement value of the expert group in a pairwise comparison procedure." and "the disagreement of experts can be understood as the deviation of their judgments from each other." respectively (Tran, 2000; Abotah, 2014). Disagreement value has been calculated and considered as a part of model/results 
validation for literature using HDM (Tran, 2000; Abotah, 2014; Estep, 2017; Shaygan $e t$ al., 2017, 2018a; Cowan and Daim, 2018).

Similar to the inconsistency threshold, the acceptable disagreement level should not exceed 10\% (0.1) (Chan, 2013; Estep, 2017; Shaygan et al., 2017). In instances where the disagreement value exceeds the threshold, several remedy methods can be performed to alleviate the disagreement among experts. In cases where a small number of experts are contributing to the disagreement value, can be eliminated as outlier after identification through standard deviation analysis. Moreover, in cases where the views provided by the experts which are causing the disagreement are deemed valid and concerning, their ideas should be shared with other experts through methods such as the Delphi method followed which can potentially decrease the disagreement value in the next quantification iterations. Furthermore, the Hierarchical Clustering Method (HCM) can be utilized to categorize similar data points in a cluster (Iskin, 2014). The goal of HCM is to discover natural groupings. Iskin defines this model as " $\mathrm{HCM}$ obtains homogeneous clusters of cases based on measured characteristics. The process starts where each case is considered as a separate cluster; and for each iteration, a new cluster is determined by combining one case with a cluster identified earlier in a fashion that the arithmetic distance between new and old clusters remains the shortest among all possible alternatives. The process continues until one cluster is left." (Iskin, 2014).

\subsubsection{Hierarchical Agglomerative Clustering (HAC)}

The disagreement index was proposed in Kocaoglu's 1983 publication (Kocaoglu, 1983). Assume the research is using "m" experts for " $\mathrm{n}$ " decision variables. The HDM software 
used in this research uses Hierarchical Agglomerative Clustering (HAC) method to calculate the disagreement value for the experts' judgment. The following formulas show the HAC method to calculate the disagreement index.

Let $\mathrm{v}_{\mathrm{ij}}$ be $\mathrm{j}^{\text {th }}$ expert's relative value for the $\mathrm{i}^{\text {th }}$ decision variable. Thus, group relative value for the $\mathrm{i}^{\text {th }}$ decision variable for all " $\mathrm{m}$ " experts can be calculated as (for $\mathrm{i}=1,2 \ldots \mathrm{n}$ ):

$$
V_{i}=\sum_{j=1}^{m} v_{i j} \cdot \frac{1}{m}
$$

The standard deviation for the relative value of the $\mathrm{i}^{\text {th }}$ decision variable can be calculated as:

$$
S t d v_{i}=\sqrt{\frac{1}{m} \sum_{j=1}^{m}\left(V_{i}-v_{i j}\right)^{2}}
$$

Consequently, the disagreement index for the $\mathrm{m}$ experts can be obtained by calculating the average of the standard deviations of " $n$ " decision variables:

$$
\text { Disagreement Index }=\frac{1}{m} \sum_{i=1}^{m} S t d v_{i}
$$

\subsubsection{Intraclass Correlation Coefficient (ICC)}

Another method for calculating the disagreement index (the measurement of the reliability of quantitative scales) is to use Intraclass Correlation Coefficient (ICC) (Fleiss and Cohen, 1973; Sheskin, 2007; Estep, 2017) intra-class correlation coefficients can be used as statistics for measuring homogeneity in cases where there is a need to study the relationship between variables of a common class (in terms of metric and variance) (O. 
McGraw and P. Wong, 1996). McGraw et al. continue to discuss ICC as a measure of the proportion of a variance that is imputed to objects of measurement, often called targets (Shrout and Fleiss, 1979). In the context of this research and for the HDM methodology, ICC calculates the disagreement among experts for a relative number of elements. In this case, ICC determines the agreement measure among experts based on " $n$ " elements' relative importance which is obtained through the pairwise comparisons.

ICC can be calculated as follows (Bartko, 1966; LeBreton and Senter, 2008):

Intraclass Correlation Coefficient $(I C C)=\frac{M S_{D}-M S_{E}}{M S_{D}+(k-1) M S_{E}+\frac{k}{n}\left(M S_{I}-M S_{E}\right)}$ $\mathrm{n}=$ Number of targets (decision elements)

$\mathrm{k}=$ Number of experts

$M S_{D}=$ Mean square for decision elements

$\mathrm{MS}_{\mathrm{I}}=$ Intra-expert mean square

$\mathrm{MS}_{\mathrm{E}}=$ Mean square error (residual) (calculated using two-way ANOVA)

For this case, ICC can take values in the interval $\left(\frac{-1}{(k-1)}<\operatorname{ICC}<+1\right)$ with $(+1)$ showing total intra-expert agreement while any values that are zero or negative would show total disagreement among experts (Bartko and Carpenter, 1976). There are different opinions about the acceptable threshold for disagreement in the ICC method. LeBreton and Senter (2008) argued that an ICC of 0.7 or higher would indicate an acceptable level of 
agreement among experts whereas other studies stress the importance of research questions, objectives, and data in indicating the threshold (LeBreton and Senter, 2008; Trevethan, 2017).

\subsubsection{F-Test}

Another way to determine the significance of expert disagreement is to use the F-test through hypothesis testing (Shrout and Fleiss, 1979). The F-test can be used to justify the disagreement values above the $10 \%$ threshold to decide the significance of the model. In this context, the F-test will hypothesize whether the ICC value is equal to zero:

ICC $=$ Intraclass Correlation Coefficient

$\mathrm{MS}_{\mathrm{D}}=$ Mean square for decision elements

$\mathrm{MS}_{\mathrm{E}}=$ Mean square error (residual)

Hypothesis:

$$
\begin{aligned}
& H_{0}: I C C=0 \\
& H_{\alpha}: I C C>0
\end{aligned}
$$

$\mathrm{H}_{0}$ implies that there is no correlation among the judgment and therefore shows complete disagreement among experts. $\mathrm{H}_{\alpha}$ however, implies that there is no statistically significant disagreement among experts. The $\mathrm{F}$ value is calculated using the following formula:

$$
F=\frac{M S_{D}}{M S_{E}}
$$

With one and two degrees of freedom for decision and error respectively, and with $95 \%$ $(\alpha=0.05)$ confidence level, the obtained $F$ value is compared to the critical $F$ value. If the 
calculated $\mathrm{F}$ is bigger than the critical value, $\mathrm{H}_{0}$ can be rejected concluding that there is no significant disagreement among experts.

$$
\text { If } F>F_{\text {Critical }} \text { Then } H_{0} \text { is rejected Else Fail to reject } H_{0}
$$

\subsection{Sensitivity Analysis}

In the HDM model, in order to analyze the impacts of potential changes in the values at levels of the model, Sensitivity Analysis (SA) is used. SA is a method that can be used to perform analysis on how different perspectives/criteria of the model will change in scenarios where there is a change in value/weight of model elements. In other words, SA helps decision-makers determine how much a given model depends on its input factors (Saltelli, Tarantola and Chan, 1999). It also facilitates the process of understanding model behavior and the extent to which its different factors interplay. Lilburne and Trantola (2008) define SA as "the study of how uncertainty in model predictions is determined by uncertainty in model inputs." (Lilburne and Tarantola, 2009).

Local SA focuses on the impact of change in value one at a time, while a global SA considers the potential impacts of simultaneous variation of model inputs over their finite range of uncertainty. In other words, in the case of local SA, the value other than the one changed is assumed as fixed. In the global SA however, several values would concurrently change and the average of the changing output over the variation of all inputs is simulated (Tian, 2013). The global SA can be performed using different methods such as regression (SRC, SRRC, t-value methods), screen (Morris method), or variance based-

meta models (FAST, Sobol, MARS, ACOSSO, SVM methods), perturbation and 
derivative methods, multiple-start perturbation method, regional SA, and density-based method (Morris, 1991; Park and Ahn, 1994; Storlie et al., 2009; de Wilde and Tian, 2010; Mechri, Capozzoli and Corrado, 2010; Eisenhower et al., 2012; Tian and Choudhary, 2012; Hygh et al., 2012; Spitz et al., 2012; Tian, 2013; Paton, Maier and Dandy, 2013; Pianosi et al., 2016).

In the field of technology management, due to its dynamic and fast-paced nature, SA has been used to determine potential impacts of change in the importance/weights of the perspectives as a way to ensure the robustness of the model/results (Phan, 2013; Estep, 2017). In the case of Multi-Criteria Decision Making (MCDM) models, the importance (weights) may change due to the pairwise comparison matrix's adjustment or change/recalculation of the matrix after changing inputs (Chen, Yu and Khan, 2013). Moreover, SA helps decision-makers in verifying the stability of the optimal solution and validating the used methodology (Muñoz, Romana and Ordóñez, 2016).

Estep (2017) performed SA on her model on technology transfer score for research proposal evaluation with test several analysis scenarios. The SA in Estep's dissertation was performed to better understand the impact of future-based scenarios (rank of proposals in her case), with changes in the importance of difference model perspectives. Moreover, SA determined how sensitive the model was to changes in expert judgment and showed the path the organization can take to improve its technology transfer potential (Estep, 2017).

In the context of the technology management maturity model in healthcare (HDM model), sensitivity analysis will demonstrate the impact of changes in the perspective relevance 
on health organization's final maturity score. In this context, sensitivity analysis can be very helpful in comparing different health organizations, comparing different departments within a health organization, and justification of changes in prioritization due to changes in the importance of different perspectives (technological, social, organizational, regulative, and financial). Furthermore, SA, in the context of this research will demonstrate the changes in the prioritization of factors in different extreme cases (scenarios). In other words, the model's results will be tested in 5 different scenarios where one perspective is utterly dominant compared to the other 4 in terms of relative importance/weight. In summary, SA in the case of HDM, help decision-makers with the following (Chen and Kocaoglu, 2008):

- Assist decision-makers with clarifying the impact of changes at policy, strategy, and operation levels.

- Validating and testing the robustness of results/decisions.

- Providing a wider understanding of possible outcomes based on the generation of different rankings (for perspective/criteria) in different scenarios.

- Facilitation of expert consensus.

- Provide decision-makers with "what if" scenarios and questions.

This approach has been utilized by studies in the field of technology management and multi-criteria decision-making (Abotah, 2014; Estep, 2017). The SA will need to (in this case) determine the extent to which the model can withhold the changes before resulting in new values/weights. 
Chen and Kocaoglu proposed a method in 2008 to determine the allowed range of values changes which would not result in changing the final prioritization of the results (Chen and Kocaoglu, 2008). This method has also been used in the technology management literature (HDM methodology) (Tran, 2000; Phan, 2013; Iskin, 2014; Estep, 2017).

Based on this method, the initial prioritization of the model's output will not be subject to change under the following circumstance:

For the perturbation $P_{l *}^{O}$ (the perturbation affecting one of the objectives $\left(C_{l}^{O}\right)$ ) where:

$$
-C_{l *}^{O} \leq P_{l *}^{O} \leq 1-C_{l *}^{O}
$$

The original maturity score (ranking) $\left(A_{r}, A_{r+n}\right.$ for healthcare organization will not be subject to change if:

$$
\lambda \geq P_{i}^{O} \cdot \lambda^{O}
$$

Where:

$$
\lambda=C_{r}^{A}-C_{r+n}^{A}
$$

And:

$$
\lambda^{O}=C_{r+n, l *}^{A-O}-C_{r l *}^{A}-\sum_{l=1, l \neq l *}^{L} C_{r+n, l *}^{A-O} \cdot \frac{C_{l}^{O}}{\sum_{l=1, l \neq l *}^{L} C_{l}^{O}}+\sum_{l=1, l \neq l * l}^{L} \frac{C_{r l}^{A-O}}{\sum_{l=1, l \neq l *}^{L} C_{l}^{O}}
$$

* indicates that perturbation(s) is induced on the contribution(s) related to that specific decision element. 
The top choice will remain at the top rank if the above condition is satisfied for all $r=1$ and $n=1,2 \ldots I-1$. The rank order of all $A_{i}$ 's will not change if the above condition stands of all $\mathrm{r}=1,2 \ldots \mathrm{I}-1$, and $\mathrm{n}=1$.

The allowance range of perturbations $\left(C_{l}^{O}\right)$ is obtained as:

$$
\left[\delta_{l-}^{C}, \delta_{l+}^{C}\right]
$$

The sensitivity coefficient is obtained as:

$$
\frac{1}{\left|\delta_{l+}^{C}-\delta_{l-}^{C}\right|}
$$

This research will use the "Boost" approach to test the HDM model's reliability and sensitivity. The boosting approach is a scenario-driven method in which the analysis is done around boosting one factor (at a time) to the maximum and observe the impact of that on the final result and other perspectives/criteria's relative importance. In the case of this research, the Boost approach is performed for all of the 5 perspectives in the model. In each scenario, one of the perspectives is given the maximum importance (without removing any perspectives). These scenarios are performed to learn how the overall index score of each alternative is changed, and whether that would result in re-prioritization of alternatives (Chen and Kocaoglu, 2008; Abotah, 2014; Iskin, 2014; Gibson, 2016; Estep, 2017). 


\subsection{Value/Desirability Curves}

In instances in which there is a need in having a reusable model, or in instances of having many alternatives, desirability curves can be used. The combination of desirability curves with HDM is used to identify levels/ metrics for each criterion. Each level/metric connected to a criterion acts as a useful value to assist decision-makers. Using the desirability curves approach, the experts need to evaluate related levels/metrics for each criterion (desirability matrix) while giving each metric a scaled quantitative value. This enables the normalization of the evaluation results by experts across all the criteria (Phan, 2013; Estep and Daim, 2016; Gibson and Daim, 2016).

HDM also includes the calculations for disagreement, inconsistency, and sensitivity analysis (already explained in the methodology section) to validate the reliability and robustness of the final model (Kocaoglu, 1983; Chen and Kocaoglu, 2008; Daim and Kocaoglu, 2015; Estep and Daim, 2016; Gibson and Daim, 2016; Estep, 2017; Shaygan, Ozdemir-Gungor, D. Kutgun and Daneshi, 2017; Shaygan et al., 2018a; Estep, Daim and Shaygan, 2021).

When using the HDM model, each criterion that is being evaluated by an entity (health research centers/institutes in this case), can be assigned a level that best fits it for each criterion in that research center. For example, the research center's current status for each factor affecting technology maturity and continuous learning will be identified by the decision-makers after investigating the research center's capabilities. Taking the perspective and criteria weights already identified by the model, the decision-makers 
within the research center will use the value curves of each criterion to determine which

level in that value curve represents the research center the closest.

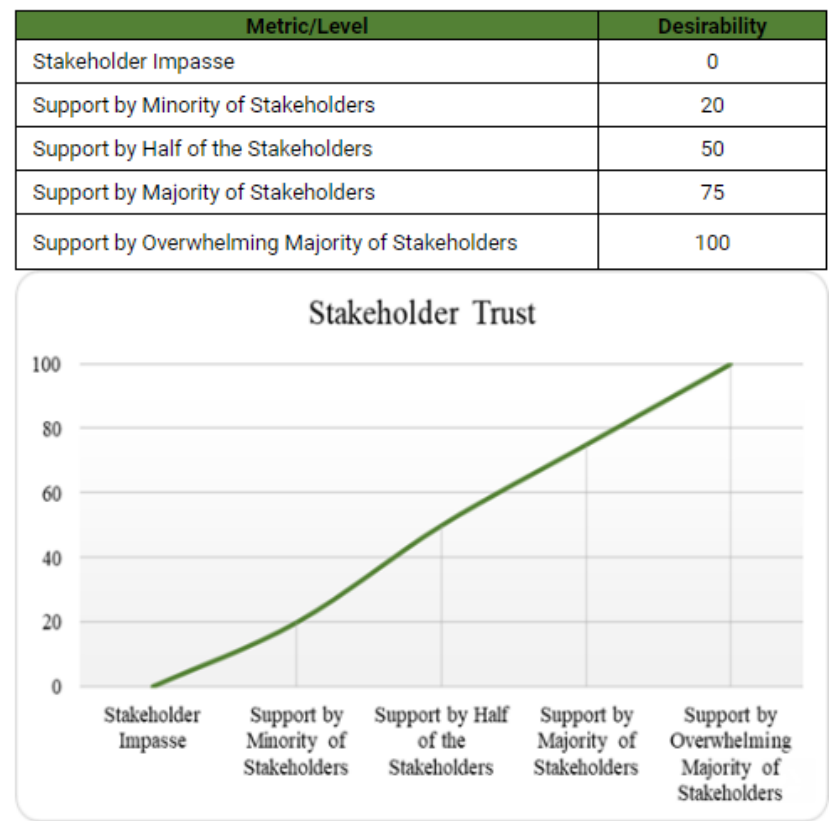

Figure 20: An Example of Desirability Curves 


\section{Research Design}

The research phases in this study will include thorough literature that delves into the background information regarding the healthcare landscape in the US, continuous learning in healthcare, and Maturity Models in healthcare. The knowledge and data gathered in this section will be the basis of gap analysis and bricks of the HDM model in terms of perspectives and criteria.

After the literature review, based on the findings of the literature review, the initial HDM model and desirability curves will be developed. This step will, in parts, will be done simultaneously with the next step which includes experts giving feedback in terms of validating the model to finalize the HDM model.

In the next phase, several panels of experts will be formed with the goals of validating and quantifying the model. Each panel will provide certain expertise that will be used to evaluate respective parts of the model. The experts are initially asked to evaluate the criteria and desirability curve values. Then, they will be asked to perform a pairwise comparison based on the HDM approach to quantify the perspective and criteria.

Next, the pairwise comparisons done by experts will be initially validated by using inconsistency and disagreement analyses. Finally, the business impact of the results and their meaning and implication will be discussed. Leading to the conclusions of the research. The general phases which are going to be implemented in this research are illustrated in the figure below. 
Figure 21: Research Framework (Basic)

A more detailed breakdown of the proposed and implemented steps in this study are demonstrated in the diagram below (Figure 22). As it can be seen the actions fall under three categories which are "model development and validation", "Model quantification and analysis", and "Case study and results".

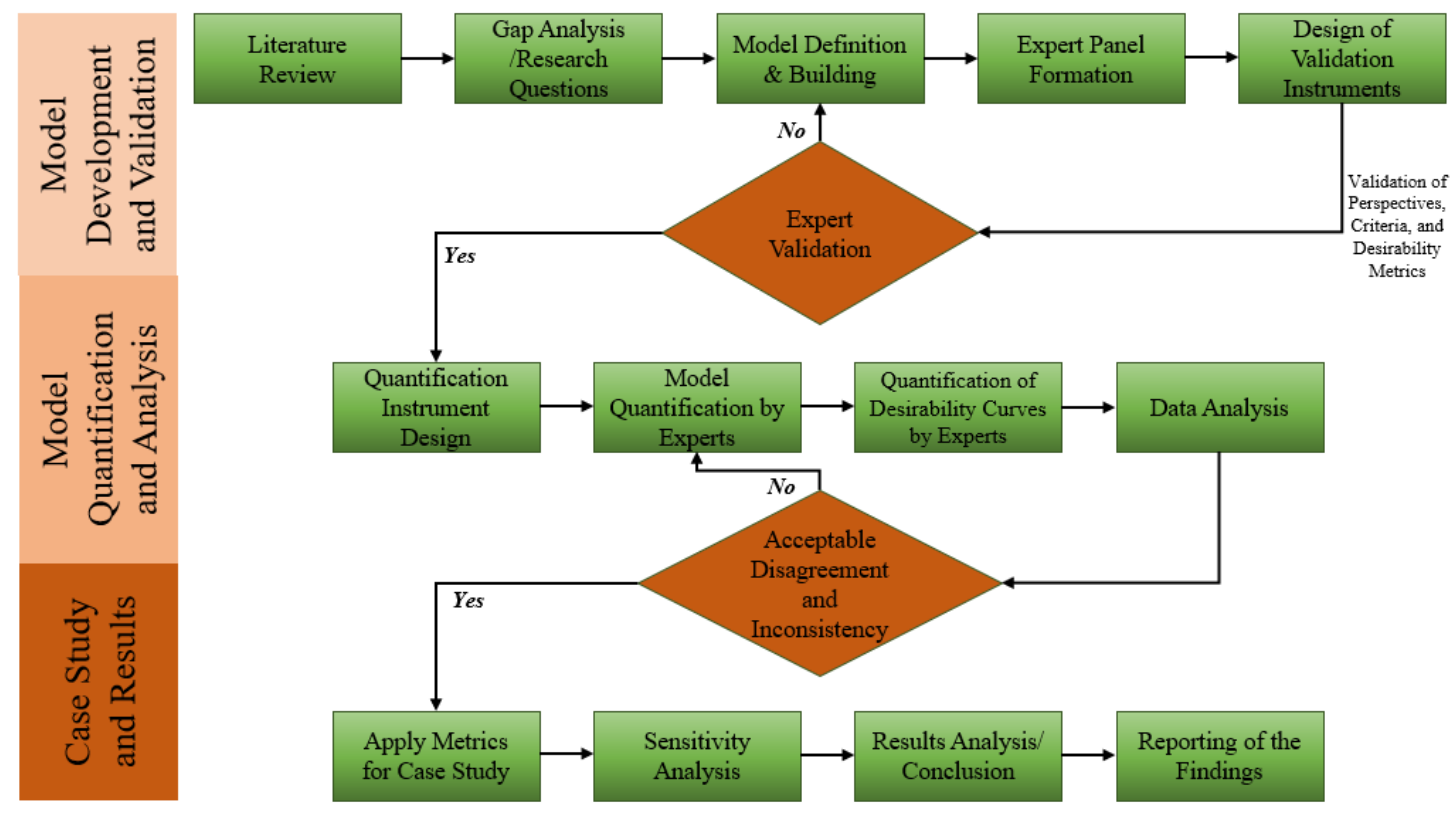

Figure 22: Research Framework (Detailed)

Each step and phase will require a different set of data and data collection sources which are summarized below:

- Literature Review: In the literature review part of this research, academic publications will be the main source of information. These publications can be in the form of papers that are published in peer-reviewed publications, academic 
books related to the research interest areas, and peer-reviewed conference proceedings. In some cases, information available from reputable websites will be used. The knowledge extraction from websites happens mainly in order to provide recent statistics and regulations cited that are hard to get from academic publications. Government websites (such as CMS.gov, NIH.gov), credible business websites (Forbes.com, PWC.com), and healthcare organizations (OHSU.edu, mayoclinic.org). Research gaps, goals, and questions are the results of this step.

- Determination of Critical Factors: Following the clarification of research gaps, questions, and goals, the critical decision elements revolving around the proposed decision should be identified. Since the HDM model is being used, in this step, following the information collected in the literature review section, initial perspectives and their sub-criteria should be identified.

- Model Definition and Build: For this part of the research, the initial model should be designed. The figure below shows the initial proposed model for this research. As it can be seen, the initial model has a hierarchical structure including perspectives and criteria. 


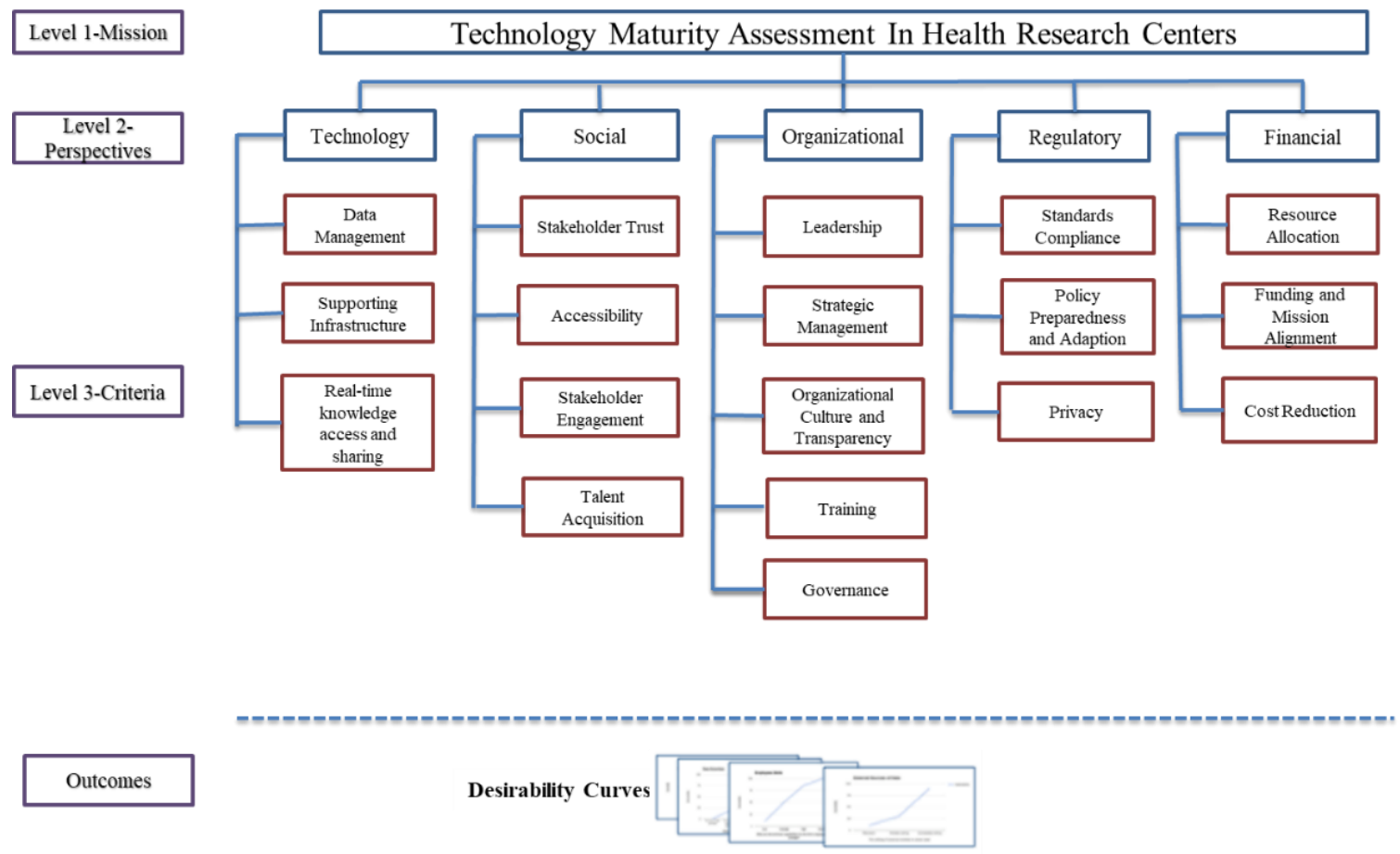

Figure 23: Initial Model Depiction

- Expert panel formation: with the goals of validating and quantifying the model, 5 expert panels were created. This section has already been discussed in detail in the previous section.

- Design of Validation Instruments: This section included creating the Qualtrics surveys for perspectives, criteria, and desirability metrics validation. This section also included creating the invitations, emails, and expert instructions for the validation of the mentioned elements. These emails, instructions, and surveys are completely shown in appendixes $\mathrm{C}$ and $\mathrm{D}$. As for desirability curve metrics validations, the list of metrics was sent to the respective panel of experts, and their feedback was considered in polishing the metrics. 
- Quantification Instrument Design: Following the model validation in terms of decision elements and metrics, this section included creating the HDM model using the ETM HDM software. This section also included creating the invitations, emails, and expert instructions for the quantification of decision elements. These emails, instructions, and surveys are completely shown in appendixes C and D.

- Model Quantification by Experts: In this section, experts quantified the perspectives and criteria using the instructions provided in the previous step. Each expert conducts pairwise comparisons in both perspectives and criteria levels. In addition to the importance weights of decision elements, the inconsistency index, and disagreement index are products of this section.

- Quantification of Desirability Curve Metrics: Using Qualtrics, the experts needed to assign a value (0-100) to each criterion's metric. Sufficient instructions are provided to experts in this section.

- Data Analysis: For this step, as mentioned before, the inconsistency index and the disagreement index are analyzed to check if they are acceptable or not based on the defined thresholds. If any of them are not within the acceptable range, actions should be taken to solve the issue. In case the model has acceptable inconsistency and disagreement, the weights obtained from the expert quantification are finalized.

- Case Study Metrics Assignment: In this step, expert(s) familiar with the research and project (health research center) are asked to assign the proper metrics for each criterion. Metric values are the results of the desirability metrics quantification 
steps. The experts will take the research goals into account. Furthermore, while assigning these numbers the performance of the research center against each criterion considering the goals of technology maturity and continuous learning. The product of this section is the technology maturity score for the healthcare organization.

- Sensitivity Analysis: sensitivity analysis will demonstrate the impact of changes in the perspective relevance on the research center's final maturity score. In this context, sensitivity analysis can be very helpful in comparing different health organizations, comparing different departments within a health organization, and justification of changes in prioritization due to changes in the importance of different perspectives (technological, social, organizational, regulative, and financial). Furthermore, scenario analysis, in the context of this research will demonstrate the changes in the prioritization of factors in different extreme cases (scenarios). In other words, the model's results will be tested in 5 different scenarios where one perspective is utterly dominant compared to others in terms of relative importance/weight.

- Results Analysis/ Conclusion: Following all the validation, quantification, and analysis, the technology maturity score results for healthcare organizations. Healthcare organizations can use this score to gain a sense of self-awareness of where they are, where they want to be, and how to get there. This model's results will provide opportunities and strengths to the healthcare organization. Using this 
model as a decision-making assistant will bolster healthcare organizations' efficiency and effectiveness in resource allocation and productive initiatives.

\subsection{Perspectives and Criteria}

During the initial literature review, using the ATLAS.ti qualitative and research software, 68 potential criteria were mined from the literature. This was done through coding the specific parts of the papers that were being read with the tag "potential criteria". Following this, the criteria that were being repeated in different papers were deemed as more important. Public health and strategic management-related criteria, data security, standards, transparency, and cost-cutting were some of these recurring themes. Through this procedure, as more papers' codes were added, certain patterns and themes were identified which eventually lead to the merge/add/removal of some identified potential criteria. As an example, criteria such as "Public Trust", "Physician Trust", "Public Acceptance", "Respecting Clinical Judgement", "Respecting the rights and dignities of physicians", and "Physician Trust" was later merged into "Stakeholder Trust". Although some criteria did not qualify to the initial model, they were later added to the model through the expert validation of the criteria (such as training). The experts suggested removing/adding some criteria. To verify that these items were aligned with the literature, a deeper literature review which is provided in this exam was implemented for 3 of the added or moved criteria (training, governance, privacy). Following the literature review and then expert validation, the model was finalized before being quantified. Based on this

question, the criteria and perspectives were in the range of 4 to 5 decision elements at 
each stage. This research has 5 perspectives, and each perspective holds between 4 to 5 items as its criteria.

Some of the reasons for this numerical range have been discussed in the HDM literature. When the number of criteria under a perspective increases it may turn the pairwise comparison procedure into an arduous one. This may be a big advantage as many of the experts may be busy professionals that may not be able to allocate a significant amount of time to quantifying and validating the model. The number of pairwise comparisons at each perspective is $\left(n^{*}(n-1) / 2\right)$ with $n$ being the number of sub-criteria. If there are 3 subcriteria there will be 3 pairwise comparisons while if there are 6 sub-criteria there will be 15 pairwise comparisons. However, if the number of criteria exceeds 6 it will need a minimum of 21 pairwise comparisons at that perspective which may be too timeconsuming and lead to significant deterioration in terms of reliability. Looking at the number of criteria from the other side, if the number of criteria is less than 3 , basically there would be no pairwise comparisons and it would merely be "a" comparison. Hence the numerical range of 4-5 has been used in the HDM related dissertations and research for each perspective node (Munkongsujarit et al., 2009; Phan, 2013; Iskin, 2014; Gibson, 2016; Mudavadi, Hogaboam and Daim, 2016; Estep, 2017; Shaygan et al., 2017, 2018a). 


\begin{tabular}{|c|c|}
\hline & Potential Criteria \\
\hline 1 & Analytics and Data Management \\
\hline 2 & Data Governance(Appropriate Security and Privacy Controls (Confidentiality), Collection,Identification \\
\hline 4 & Patient and Physician Partnership \\
\hline 5 & Supporting Infrastructure \\
\hline 7 & Dedicated programming resources and expertise \\
\hline 8 & Leadership instilled culture of learning \\
\hline 9 & Principles Adherence (Standards) \\
\hline 10 & Health IT \\
\hline 11 & Meaningful Use \\
\hline 12 & Technologies \\
\hline 14 & Public and physicianTrust (acceptance) (Respect clinical Judgement)(Respecting the right and dignity) \\
\hline 15 & Patient Consent \\
\hline 16 & Comparative Effectiveness \\
\hline 17 & Public Health (Accessablity) Address health inequalities, ethics and oversight \\
\hline 19 & External Funding \\
\hline 20 & Regulative and legislative influences \\
\hline 21 & Strategic Management (Mergers, Competing prioriteis,RESEARCH Partnerships,Problem Identification and Solution Prioritization) \\
\hline 22 & Organizational Culture (training,inclusiveness) \\
\hline 23 & Skilled Individuals \\
\hline 27 & Data systems design \\
\hline 28 & Internal Transpareny of quality metrics \\
\hline 29 & Payer provider intergration \\
\hline 30 & Academic/Clinical integration \\
\hline 32 & Process Improvement,reengineering,QI \\
\hline 34 & Balancing resource investment \\
\hline 36 & Incentive Alignment \\
\hline 38 & Patient an Family and coomunity and stakeholder Engagement \\
\hline 39 & Evaluate \\
\hline 40 & Adjuts \\
\hline 41 & Disseminate \\
\hline 42 & Culture of Learning supported by leadership \\
\hline 43 & Real time knowledge access and sharing \\
\hline 44 & Governance \\
\hline 45 & Cooperative and Participatory Leadership \\
\hline 46 & Transparency \\
\hline 48 & Adaptability \\
\hline 50 & Understanding the Vision \\
\hline 56 & Decision Support Systems \\
\hline 59 & Patient Centered Care \\
\hline 61 & Payment Policies \\
\hline 62 & National Assesment of Technologies \\
\hline 66 & Scientific Integrity \\
\hline 67 & QI and Healthcare Delivery Evaluation \\
\hline 68 & Cost Cutting \\
\hline
\end{tabular}

Figure 24: Snapshot of the Initial Selected Criteria

\subsubsection{Technology Perspective}

Systematic assessment of technology management maturity needs a socio-technical infrastructure and therefore, the technological side of the adoption of assessment of goals such as systematic and continuous learning has significant importance. The adoption of EHRs in the US from one side and the development in fields such as genomics and 
wearable devices from the other side are paving the path for the collection of great amounts of data for health systems. Especially with all the demographic and demand changes, healthcare organizations are forced to leverage the value of technology advancements to be cost-effective, competitive, and responsive. Concurrent to the mentioned advances, acquiring experienced data scientists and leveraging the benefits of data integration, sharing with different stakeholders, and acquisition and curation of data are some of the areas that can help healthcare organizations with their goals of increasing quality care, patient satisfaction, and public health while driving down the costs (Krumholz, Terry and Waldstreicher, 2016; Barham, 2017).

Information technology (IT) which is the transformation of data into useful information involves identifying data needs, collecting the right data, storing and analyzing it, and turn it into a useful and ideally automatic reporting system in a format desired by its end users. Several types of information exist for specialized uses by health care professionals, managers, payers, patients, researchers, and the government. IT departments in health care organizations play a significant part in decisions to adopt new information technologies to improve health care delivery, increase organizational efficiency, and comply with various laws and regulations (In terms of clinical information systems, administrative information systems, and decision support systems) (Shi and Singh, 2019). IT departments in healthcare organizations use medical records systems to collect, transcribe, and store clinical data; radiology and clinical laboratory reporting systems; pharmacy data systems to track medication use and mitigate the risks of errors, medical/drug reactions/interactions; scheduling systems for patients (both in terms of room and participating professionals); and financial systems for billing and collections, 
materials management, among others. Moreover, Health informatics (application of information science to improve the efficiency, accuracy, and reliability of health care services) necessitates the use of IT but transcends it by stressing the improvement of health care delivery (such as design and assessment of the effectiveness of clinical decision support systems). As mentioned among the goals of learning healthcare systems and continuous learning in those organizations, using health data beyond their primary use can play a pivotal part in turning health organizations into learning ones. Some of the topics covered in this perspective are:

- Management of big data resources in terms of security, privacy, governance, and ethics.

- The socio-technical infrastructure needed to improve and provide the capacity to capture, compile, and protect clinical and financial data, which enables the evaluation, adjustment, analysis, and dissemination, and integration of learned knowledge into clinical care processes to secure the promised improvements.

- Data systems and tools are used to capture, share, and integrate data, information, visualizations, and knowledge gained from research into the organization in real-time.

- Ensuring that technologies and IT products are user-friendly (e.g., considering health literacy and technology competence) to all users (stakeholders).

This perspective covers topics such as data management and handling of technology in terms of infrastructure, security, privacy, and knowledge flow and sharing in healthcare organizations.

\subsubsection{Social Perspective}

This perspective encapsulates topics such as public acceptance, trust, accessibility, equity, and engagement. 
The mission of public health has been defined as fulfilling "society's interest in assuring conditions in which people can be healthy." by the institute of medicine in 1988 dealing with a spectrum of concerns to make sure that optimal health is disseminated for society (Institute of Medicine (US) Committee for the Study of the Future of Public Health, 1988). Enabling this mission and goal requires support and engagement from the involved stakeholders. The stakeholders can take the shape of patients, providers, policymakers, payers, and physicians.

These social and public health-related issues may involve the application of scientific and technological know-how to avoid, mitigate, or cope with any public health and safety threats. The wide scope of topics in terms of the social aspect of technology in healthcare can have effects on topics varying from nutrition issues to health policy. Shi and Singh (2019) discuss public health's activities that of which include dissemination, both to the public and to health professionals, of timely information about important health issues, particularly when communicable diseases pose potential threats too large segments of a population.

Some of the topics covered in this perspective are:

- Key components of establishing stakeholder (patients, providers, payors, policymaker, purchasers, families) trust include:

- Garnering buy-in and trust from stakeholders so they contribute to a culture of continuous improvement and learning.

○ Transparency with stakeholders regarding current limitations and plans to address and mitigate them through a system that supports clinical and translational research, public health information, and comparative effectiveness. 
○ Maintaining trustworthiness as a research center by following through on promised commitments and ensuring new knowledge is used to improve the care of those who contributed to its generation via an enhanced use of their data.

- The degree to which the stakeholders are benefiting from the clinical and research advantages of continuous learning. Health research centers must carry out consistent and innovative outreach efforts to ensure that services are accessible to diverse populations to reduce racial, ethnic, sexual orientation/gender, and other disparities.

- The degree to which a health research center can engage stakeholders (patients, providers, payors, policymaker, purchasers, families) to participate in continuous improvement and learning projects and initiatives.

- Research centers' ability to acquire and retain talented and diverse staff in different areas (physicians, nurses, researchers, data scientists, public health professionals, managers, epidemiologists, administrative staff, etc.)

\subsubsection{Organizational Perspective}

This perspective covers certain organizational aspects of technology management in healthcare such as leadership, change management, organizational culture and transparency, strategic management, and quality improvement.

The topics covered in this perspective are:

- Broad leadership can expand and guide stakeholders' commitment to the goals of continuous learning and increased technology maturity.

- Research centers' ability to improve through strategic decisions, management of competing priorities, internal and external partnerships/collaborations, problem identification, and finding solutions. 
- The extent to which a research center's employees have adopted and are committed to a culture of continuous learning and practice transparency to safeguard stakeholder trust to improve the health of individuals, communities, and diverse populations.

- Training employees and stakeholders on the latest technologies and best practices within the health research center.

- Having the necessary governance to support a sustainable operation, uphold required standards, build, and maintain trust with stakeholders, and continuously innovate.

\subsubsection{Regulatory Perspective}

Operating in a highly regulated environment, health care managers must comply with government regulations, such as standards of participation in government programs, licensing rules, and security and privacy laws regarding patient information, while operationalizing the organization within the constraints of reimbursement rates. From the public standpoint, the Medicare and Medicaid programs have made significant modifications to their reimbursement methodologies that have caused the need for operational changes in the way services are organized and delivered. On the other hand, Private agencies, such as the Joint Commission, also play an indirect regulatory role especially in the monitoring of the quality of services. Health organizations must obey the rules set by the various public and private agencies that regulate the health care marketplace. Therefore, it is critical for health organizations to constantly keep abreast of the rules and regulations governing at state and federal levels.

The implications of health policy and new reform proposals are better reacted to when health organizations sense and comprehend the issues and their connection to the delivery of health services in the establishments they manage. This knowledge bolsters 
organizations' ability to be flexible and dynamic in terms of being proactive or timely reactive to the regulatory changes in healthcare. Many of these regulations or policies may be affected by the political climate as government policy in the United States plays a significant role in deciding which drugs, devices, and biologics are made available to Americans. The U.S. government is also one of the largest sources of funding for biomedical research. By controlling the amount of funding, public policy indirectly influences medical innovation (Shi and Singh, 2019).

This perspective includes regulatory and legal aspects needed to assess the maturity of technology management in healthcare organizations such as governance, regulative and legal influences, and standard compliance.

The topics covered in this perspective are:

- Research centers' compliance with standards and regulations to ensure transparency with stakeholders, data interoperability, and commitment to meaningful use and joint commissions.

- Research centers' flexibility and agility in responding and adapting to changes (new regulations, legislations, and policies) in terms of anticipation and readiness (resources, policies, strategies, and management).

- Research centers' adherence to regulations and policies to ensure privacy in terms of information technology, medical data, patient access, third party interactions, and ethical use of information (IRBs), among others.

- Research centers' ability to inform policies by participating in expert panels, providing evidence and comments regarding policies under consideration, and securing contracts 
with the state and the federal government to complete analyses regarding policy development, implementation, and impact.

\subsubsection{Financial Perspective}

In terms of technology management and organizations' maturity in this area. Technological innovations are argued as one of the most significant factors in healthcare cost inflation. This issue has been more highlighted in the past couple of decades, accounting for about half of the total increase in health care spending (Sorenson, Drummond and Khan, 2013). These technological costs can be due to the acquisition of new technology or equipment, training, setting, logistics, and maintaining it. Therefore, health organizations need to balance the tradeoffs using new technologies as (concurrent to increasing costs) it may benefit them greatly in terms of incentives, attracting patients, and competition. Hence, widespread adoption of technology has a multiplier effect. Moreover, changes in areas such as financing, insurance, payment, and delivery can present new threats or opportunities in the health care market. On top of the mentioned factors, the external factors discussed in the literature section can make this task of balancing even more difficult. Health organizations can be more effective when they proactively deal with any threats to their institution's profitability and viability.

This perspective encapsulates the financial side of assessing the maturity of technology management in healthcare organizations. Topics such as investment resource allocation, funding, and cost reduction fall under this category.

The topics covered in this perspective are: 
- Research centers' ability to prioritize and decide to invest its resources (financial, human, space...) in alignment with the goals of increased technology maturity and continuous learning.

- The extent to which incentives and funding are aligned with the encouragement of technology maturity, continuous learning and improvement, waste elimination, and rewarding high care value.

- Research centers' ability to cut costs as a result of increased maturity, learning, continuous improvement, and waste eliminations without compromising quality.

- Research centers' success in meeting reimbursement programs' goals (policy incentives, value-based purchasing, etc.) and

- accessing government, foundation, and other grants (NIH, DARPA, DOD, various private funds, etc.) 


\subsection{Initial Identified Perspectives and Criteria}

In this section, the tables for the initially identified perspectives and criteria and their definitions are provided. All criteria were mined from the literature or discussion with healthcare experts validated by literature.

Table 4:Model Perspectives

\begin{tabular}{|c|c|}
\hline Perspective & Definition \\
\hline Technology & $\begin{array}{l}\text { This perspective covers topics such as data management and handling of } \\
\text { technology in terms of infrastructure, security, privacy, knowledge flow, and } \\
\text { sharing in healthcare organizations. }\end{array}$ \\
\hline Social & $\begin{array}{l}\text { This perspective encapsulates topics such as public acceptance, trust, broad } \\
\text { accessibility, equity, and engagement. Stakeholders can take the shape of } \\
\text { patients, providers, policymakers, payers, and clinicians. This perspective also } \\
\text { includes the ability of healthcare organizations to attract and acquire a skilled } \\
\text { and diverse workforce. }\end{array}$ \\
\hline Organizational & $\begin{array}{l}\text { This perspective covers certain organizational aspects of technology } \\
\text { management in healthcare such as leadership, change management, } \\
\text { organizational culture and transparency, strategic management, and governance. }\end{array}$ \\
\hline Regulatory & $\begin{array}{l}\text { This perspective includes regulatory and legal aspects needed to assess the } \\
\text { maturity of technology management in health research centers, such as the } \\
\text { ability to adapt to and comply with changing regulations and informing policies. }\end{array}$ \\
\hline Financial & $\begin{array}{l}\text { This perspective encapsulates the financial side of assessing the maturity of } \\
\text { technology management in health research centers. Topics such as investment } \\
\text { resource allocation, appropriate funding, and cost reduction without } \\
\text { compromising quality fall under this category. }\end{array}$ \\
\hline
\end{tabular}


Table 5: Initial Technology Criteria

\begin{tabular}{|c|c|c|c|}
\hline Perspective & Criteria & Definition & References \\
\hline \multirow{3}{*}{ Technology } & $\begin{array}{c}\text { Data } \\
\text { Management }\end{array}$ & $\begin{array}{l}\text { Management of big data resources in terms } \\
\text { of security, privacy, and ethics }\end{array}$ & $\begin{array}{l}\text { (Saunders and Smith, } \\
\text { 2013; Bernstein } \text { et al., } \\
\text { 2015; English } \text { et al., } \\
\text { 2016; Rumsfeld, Joynt } \\
\text { and Maddox, 2016; } \\
\text { Shah et al., 2016; } \\
\text { Rubin, 2017) }\end{array}$ \\
\hline & $\begin{array}{l}\text { Supporting } \\
\text { Infrastructure }\end{array}$ & $\begin{array}{l}\text { The socio-technical infrastructure needed to } \\
\text { improve and provide the capacity to capture, } \\
\text { compile, and protect clinical and financial } \\
\text { data, which enables the evaluation, } \\
\text { adjustment, analysis, and dissemination of } \\
\text { learned knowledge }\end{array}$ & $\begin{array}{l}\text { (Saunders and Smith, } \\
\text { 2013; Mandl et al., } \\
\text { 2014; English et al., } \\
\text { 2016; Johnson et al., } \\
\text { 2017; Kraft et al., } \\
\text { 2017) }\end{array}$ \\
\hline & $\begin{array}{l}\text { Real-time } \\
\text { Knowledge } \\
\text { Access and } \\
\text { Sharing }\end{array}$ & $\begin{array}{l}\text { Data systems and tools are used to capture, } \\
\text { share, and integrate data, information, } \\
\text { visualizations, and knowledge gained from } \\
\text { research into the organization in real-time. }\end{array}$ & $\begin{array}{l}\text { (Saunders and Smith, } \\
\text { 2013; Bernstein et al., } \\
\text { 2015; Cahan and } \\
\text { Cimino, 2017; Morain, } \\
\text { Kass and Grossmann, } \\
\text { 2017) }\end{array}$ \\
\hline
\end{tabular}


Table 6: Initial Social Criteria

\begin{tabular}{|c|c|c|c|}
\hline Perspective & Criteria & Definition & References \\
\hline \multirow{4}{*}{ Social } & $\begin{array}{l}\text { Stakeholder } \\
\text { Trust }\end{array}$ & $\begin{array}{l}\text { Key components of establishing stakeholder (patients, } \\
\text { providers, payors, policymaker, purchasers, families) } \\
\text { trust include: } \\
\text { - Garnering buy-in and trust from stakeholders } \\
\text { so they contribute to a culture of continuous } \\
\text { improvement and learning. } \\
\text { Transparency with stakeholders regarding } \\
\text { current limitations and plans to address and } \\
\text { mitigate them through a system that supports } \\
\text { clinical and translational research, public } \\
\text { health information, and comparative } \\
\text { effectiveness. }\end{array}$ & $\begin{array}{l}\text { (Friedman, Wong } \\
\text { and Blumenthal, } \\
\text { 2010; Faden et } \\
\text { al., 2013; Kelley } \\
\text { et al., 2015; Kraft } \\
\text { et al., 2017) }\end{array}$ \\
\hline & Accessibility & $\begin{array}{l}\text { The degree to which the public is benefiting from the } \\
\text { advantages of continuous learning. Health research } \\
\text { centers must carry out consistent and innovative } \\
\text { outreach efforts to ensure that services are accessible to } \\
\text { diverse populations to reduce racial, ethnic, sexual } \\
\text { orientation/gender, and other disparities. }\end{array}$ & $\begin{array}{l}\text { (Fiscella et al., } \\
\text { 2000; Faden } \text { et al., } \\
\text { 2013; Bernstein } \text { et } \\
\text { al., 2015; Kraft et } \\
\text { al., 2017; Rubin, } \\
\text { 2017) }\end{array}$ \\
\hline & $\begin{array}{l}\text { Stakeholder } \\
\text { Engagement }\end{array}$ & $\begin{array}{l}\text { The degree to which a health research center can } \\
\text { engage stakeholders (patients, providers, payors, } \\
\text { policymaker, purchasers, families) to participate in } \\
\text { continuous improvement and learning projects and } \\
\text { initiatives. }\end{array}$ & $\begin{array}{l}\text { (Saunders and } \\
\text { Smith, 2013; } \\
\text { Mandl et al., } \\
\text { 2014; Price- } \\
\text { Haywood, 2015; } \\
\text { Kraft et al., 2017) } \\
\end{array}$ \\
\hline & $\begin{array}{c}\text { Talent } \\
\text { Acquisition }\end{array}$ & $\begin{array}{l}\text { Research centers' ability to acquire talented and diverse } \\
\text { staff in different areas (physicians, nurses, researchers, } \\
\text { data scientists, public health professionals, managers, } \\
\text { epidemiologists, administrative staff, etc.) }\end{array}$ & $\begin{array}{l}\text { (Grossmann, } \\
\text { Powers, et al., } \\
\text { 2011; Faden et } \\
\text { al., 2013; } \\
\text { Pronovost et al., } \\
\text { 2017; Schmittdiel } \\
\text { et al., 2017) }\end{array}$ \\
\hline
\end{tabular}


Table 7: Initial Organizational Criteria

\begin{tabular}{|c|c|c|c|}
\hline Perspective & Criteria & Definition & References \\
\hline \multirow{5}{*}{ Organizational } & Leadership & $\begin{array}{l}\text { Broad leadership which can expand and guide } \\
\text { stakeholders' commitment to the goals of } \\
\text { continuous learning and increased technology } \\
\text { maturity. }\end{array}$ & $\begin{array}{l}\text { (Bernstein et al., } \\
\text { 2015; Graban, } \\
\text { 2016; Morain, Kass } \\
\text { and Grossmann, } \\
\text { 2017; Rubin, 2017) } \\
\end{array}$ \\
\hline & $\begin{array}{l}\text { Strategic } \\
\text { Manageme } \\
\text { nt }\end{array}$ & $\begin{array}{l}\text { Research centers' ability to improve through } \\
\text { strategic decisions, management of competing } \\
\text { priorities, research partnerships, problem } \\
\text { identification, and finding solutions. }\end{array}$ & $\begin{array}{l}\text { (English } \text { et al., } \\
\text { 2016; Morain, Kass } \\
\text { and Grossmann, } \\
\text { 2017; Shaygan, } \\
\text { 2018) } \\
\end{array}$ \\
\hline & $\begin{array}{l}\text { Organizati } \\
\text { onal } \\
\text { Culture } \\
\text { and } \\
\text { Transparen } \\
\text { cy }\end{array}$ & $\begin{array}{l}\text { The extent to which a research center's } \\
\text { employees have adopted and are committed to } \\
\text { a culture of continuous learning and practice } \\
\text { transparency to safeguard stakeholder trust in } \\
\text { order to improve the health of individuals, } \\
\text { communities, and diverse populations. }\end{array}$ & $\begin{array}{c}(I O M, \\
\text { 2011)(Institute of } \\
\text { Medicine (IOM), } \\
\text { 2007; McClellan } e t \\
\text { al., 2008; Yong, } \\
\text { Olsen and } \\
\text { Mcginnis, 2010; } \\
\text { Yong, Saunders } \\
\text { and Olsen, 2010; } \\
\text { Curcin, 2016) }\end{array}$ \\
\hline & Training & $\begin{array}{l}\text { Training employees and stakeholders on the } \\
\text { latest technologies and best practices within } \\
\text { the health research center. }\end{array}$ & $\begin{array}{l}\text { (Lynch et al., 2000; } \\
\text { Gopee, 2002; } \\
\text { Grossmann et al., } \\
\text { 2010; Grossmann, } \\
\text { Goolsby, Olsen and } \\
\text { McGinnis, 2011) }\end{array}$ \\
\hline & $\begin{array}{c}\text { Governanc } \\
\mathrm{e}\end{array}$ & $\begin{array}{l}\text { Having the necessary governance to support a } \\
\text { sustainable operation, uphold required } \\
\text { standards, build and maintain trust with } \\
\text { stakeholders, and continuously innovate. }\end{array}$ & $\begin{array}{c}\text { (Bevan and } \\
\text { Cornwell, 2006; } \\
\text { Institute of } \\
\text { Medicine (IOM), } \\
\text { 2007; Chambers, } \\
\text { 2012; Hastings et } \\
\text { al., 2014) }\end{array}$ \\
\hline
\end{tabular}


Table 8: Initial Regulatory Criteria

\begin{tabular}{|c|c|c|c|}
\hline Perspective & Criteria & Definition & References \\
\hline \multirow{3}{*}{ Regulatory } & $\begin{array}{l}\text { Standards } \\
\text { Compliance }\end{array}$ & $\begin{array}{l}\text { Research centers' compliance with } \\
\text { standards and regulations to ensure } \\
\text { transparency with stakeholders, data } \\
\text { interoperability, and commitment to } \\
\text { meaningful use and joint commissions. }\end{array}$ & $\begin{array}{l}\text { (Institute of Medicine } \\
\text { (IOM), 2007; Blumenthal } \\
\text { and Tavenner, 2010; } \\
\text { Friedman, Wong and } \\
\text { Blumenthal, 2010; } \\
\text { Schmittdiel } \text { et al., 2017) }\end{array}$ \\
\hline & $\begin{array}{l}\text { Policy } \\
\text { Preparedness } \\
\text { and } \\
\text { Adaption }\end{array}$ & $\begin{array}{l}\text { Research centers' flexibility in adapting } \\
\text { to new regulations, legislations, and } \\
\text { policies in terms of anticipation and } \\
\text { adaption (resources, policies, strategies, } \\
\text { and management). }\end{array}$ & $\begin{array}{l}\text { (Blumenthal and Tavenner, } \\
\text { 2010; Grossmann, Goolsby, } \\
\text { Olsen and McGinnis, 2011; } \\
\text { Morain and Kass, 2016; } \\
\text { Shaygan, 2018) }\end{array}$ \\
\hline & Privacy & $\begin{array}{l}\text { Research centers' adherence to } \\
\text { regulations and policies to ensure privacy } \\
\text { in terms of information technology, } \\
\text { medical data, patient access, third party } \\
\text { interactions, and ethical use of } \\
\text { information, among others. }\end{array}$ & $\begin{array}{l}\text { (Curran, Stearns and } \\
\text { Kaplan, 1969; Gostin et al., } \\
\text { 1993; Prentnieks and Qual, } \\
\text { 1996; Melton III, 1997; } \\
\text { National Research Council, } \\
\text { 1997; Rothstein and Talbott, } \\
\text { 2006; Sharyl J Nass et al., } \\
\text { 2009; Vandenbroucke, } \\
\text { 2011) }\end{array}$ \\
\hline
\end{tabular}

Table 9: Initial Financial Criteria

\begin{tabular}{|c|c|c|c|}
\hline Perspective & Criteria & Definition & References \\
\hline \multirow{3}{*}{ Financial } & $\begin{array}{l}\text { Resource } \\
\text { Allocation }\end{array}$ & $\begin{array}{l}\text { Research centers' ability to prioritize and } \\
\text { decide to invest its resources in alignment } \\
\text { with the goals of increased technology } \\
\text { maturity and continuous learning. }\end{array}$ & $\begin{array}{l}\text { (Rouse, 2001; Pronovost et } \\
\text { al., 2017; Rouse, Johns and } \\
\text { Pepe, 2017; Shaygan, } \\
\text { Lavoie and Daim, 2018) }\end{array}$ \\
\hline & $\begin{array}{l}\text { Funding } \\
\text { and } \\
\text { Mission } \\
\text { Alignment }\end{array}$ & $\begin{array}{l}\text { The extent to which incentives and funding } \\
\text { are aligned with encouragement of } \\
\text { technology maturity, continuous learning } \\
\text { and improvement, waste elimination, and } \\
\text { rewarding high care value. }\end{array}$ & $\begin{array}{l}\text { (Institute of Medicine } \\
\text { (IOM), 2007; Blizinsky and } \\
\text { Bonham, 2017; Morain, } \\
\text { Kass and Grossmann, } \\
\text { 2017; Pronovost et al., } \\
\text { 2017; Shaygan, 2018) }\end{array}$ \\
\hline & $\begin{array}{c}\text { Cost } \\
\text { Reduction }\end{array}$ & $\begin{array}{l}\text { Research centers' ability to cut costs } \\
\text { through increased maturity, learning, } \\
\text { continuous improvement, and waste } \\
\text { eliminations without compromising quality. }\end{array}$ & $\begin{array}{l}\text { (McClellan et al., 2008; } \\
\text { Yong, Olsen and Mcginnis, } \\
\text { 2010; IOM, 2011, 2013; } \\
\text { Saunders and Smith, 2013) }\end{array}$ \\
\hline
\end{tabular}




\subsection{Model Description for Data Collection}

The following actions were taken to help the experts with their judgment:

In each expert panel email that was sent out to the relevant panel, a concise and clear summary of what is expected and what needs to be done at each level was provided. Furthermore, files containing a more detailed research summary and perspectives/criteria information was attached to each level's emails and shown in detail in Appendix C.

\section{ETM}

Amir Shaygan's Research Model Evaluation

Technology Management Maturity Assessment in Health Research Centers

Thank you for participating in evaluating my research model as an expert!

My research goal is to develop a model that can assess technology management maturity and continuous learning in health research centers in university hospitals. This will help health research centers to:

-Assess their technology management maturity and give them a sense of self-awareness from different perspectives.

-Pinpoint their strengths and opportunities in order to strategize and allocate resources more efficiently and effectively.

The first step is to validate the perspectives of the factors affecting technology maturity and continuous learning in health research centers.

Figure 25:Qualtrics Snapshot (Intro)

Please identify the perspectives important to technology maturity and continuous learning in health research centers.

\begin{tabular}{l|cc} 
& \multicolumn{2}{|c}{ Validation } \\
& Yes & No \\
\hline Technology & 0 & 0 \\
Social & 0 & 0 \\
Organizational & 0 & 0 \\
Regulatory & 0 & 0 \\
Financial & 0 & 0
\end{tabular}

If you think that there are other additional perspectives or have any comments, please add them below. 


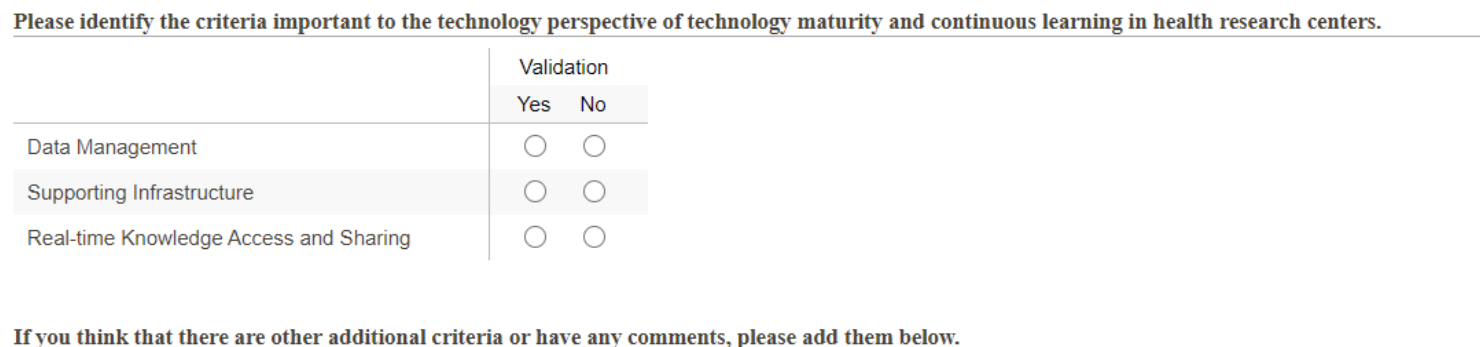

If you think that there are other additional criteria or have any comments, please add them below.

Figure 26: Qualtrics Snapshot (Continued)

- Enough information was provided to experts in each of the validation/quantification steps in Qualtrics. In each survey, description summaries were provided to experts so they could use it as a quick reference while doing the survey. Appendix D shows the surveys along with the related descriptions.

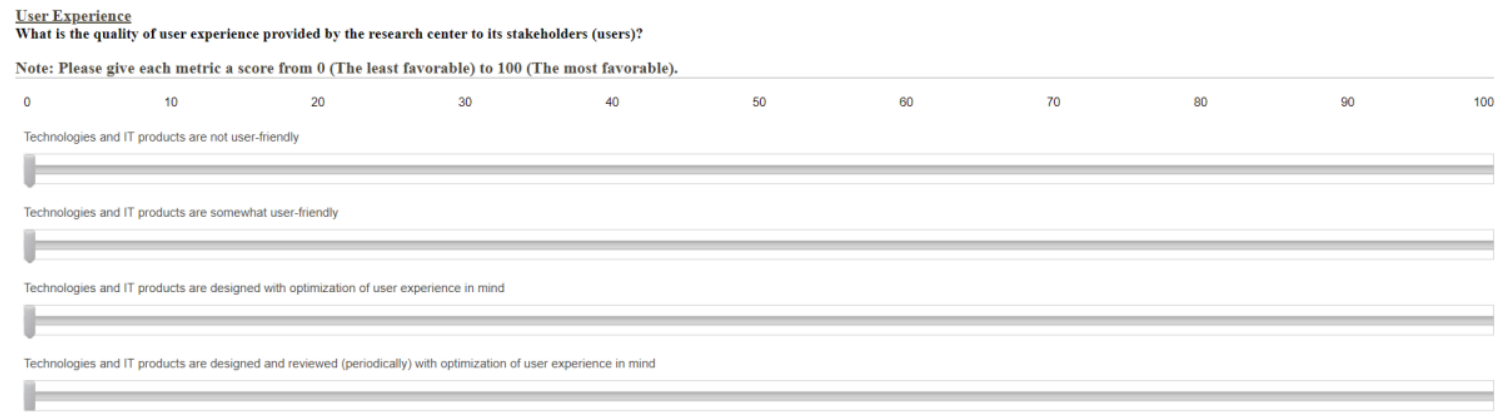

Figure 27: Qualtrics Snapshot (Continued II)

- In the HDM software tool (also, in the invitation email for the perspectives/criteria quantification), Clear descriptions appear as tooltips for each item being quantified. Appendix D shows screenshots from the HDM software tool. 

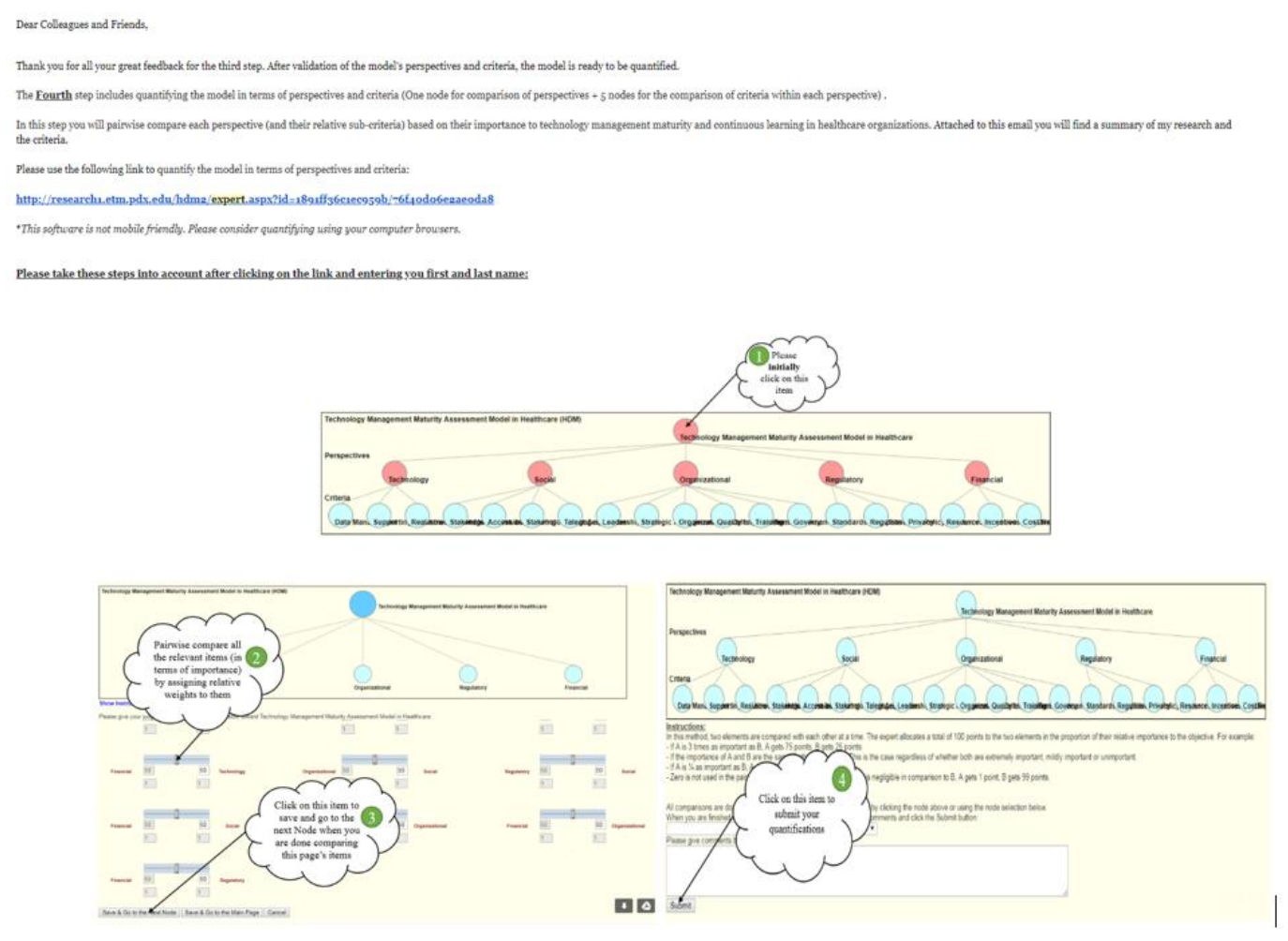

Figure 28: ETM HDM Snapshot

\subsection{Justification of the Selected Elements}

As mentioned in the perspectives/criteria section, different levels of review and validation will go into finalizing the model. Relative papers were reviewed followed by coding them in ATLAS.ti and following pattern identification some criteria were merged or removed from the list. Next, the initial model will be sent to an expert panel. In my comprehensive exam run, there were 6 real experts in the field and after getting their feedback on the model, privacy and training (separating it from talent acquisition) were recommended. It was also recommended to while moving governance from the regulatory perspective to the organizational perspective. The summary of the steps taken to finalize the model for quantification is shown below: 


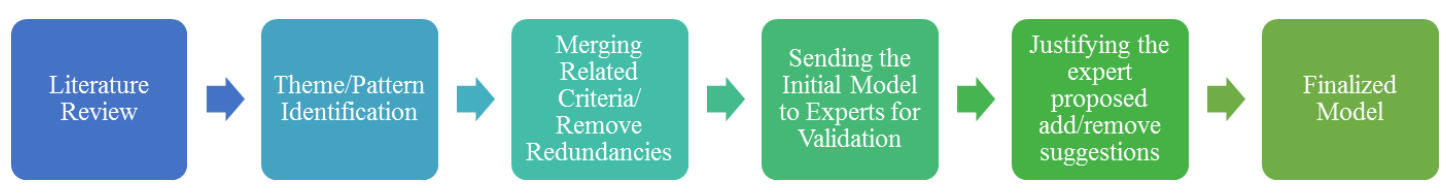

Figure 29: Criteria Selection Stages

In this section the further literature review to verify/validate the added/moved criteria is provided. Privacy, training, and governance are reviewed in a deeper sense in the context of maturity models in healthcare and continuous improvement. These three criteria were added after the advice of the experts in my panel. A further literature review was conducted to make sure their recommendations were aligned with the existing literature.

\subsubsection{Privacy}

One of the results of the criteria validation stage in this research was Privacy. Although privacy was considered as a part of the "Data Management" criterion, some of the experts pointed out the importance of a "Privacy" criterion in the regulatory perspective of the model. Privacy is important in the data management sense but due to its sensitive nature needs to be evaluated in regulatory terms as well. This can be due to fact that many health organizations have growing concerns about patient privacy for their clinical and epidemiological research data (Gostin et al., 1993; Prentnieks and Qual, 1996; Melton III, 1997; National Research Council, 1997). Clinical and medical data has been stored and used for research in health organizations repositories (paper or electronic-based) since

the early 1900s starting with Mayo Clinic in Rochester Minnesota (Kurland and Molgaard, 1981). However, in recent years, because of privacy concerns, using previous 
data to build upon has become trickier and as Vanderbroucke argues in 2011, a jeopardizing element in the use of patient data for research (Vandenbroucke, 2011). For that reason, The Mayo Clinic Foundation started to obtain broad informed consent from patients to use their data for future research as one way to ensure the continuation of research while making sure that all the data being used in by consented patients. At the same time, health organizations should be able to use personal health information to teach, train, conduct research, deliver care, and ensure quality while making sure their employers who get health information to pay claims would not use it for any non-health purposes, like hiring, firing, and promotions (Sharyl J. Nass et al., 2009). Health organizations should also make sure that there are proper safeguards to protect the real interests of patients and increased perceived understanding and benefit of learning from the historical data by society through positive promotion (Vandenbroucke, 2011). Because of the "disruptive" transformation of health records from papers to electronic records health organizations have the arduous task of managing both paper-based and electronic data. This task becomes even more difficult due to the fragmentation of paperbased records especially for the older records (Rothstein and Talbott, 2006). Although Rothstein and Talbott (2006) argue that most individuals can be confident that old, sensitive health information that may have no current clinical usefulness is unlikely to be disclosed when they authorize release of their medical records for employment or insurance purposes, health organizations should still make sure that they are aligned and compliant with privacy regulations. In past couple of years, with the adoption of electronic health records, patient data from different health organizatoins, providers, and geographical areas can be centered through central repositories such as "Epic" with 
privacy and confidentiality protections as a part of the architecture of the nationwide or geographical health Information repositories. As more health organizations are sharing patient data among them for the goals of contninuous learning and improvement, privacy and confidentialty of patient information can be threteaned. For that reason in the United States, laws to protect health information privacy and confidentiality are largely designed to protect against unauthorized access to, use of, and disclosure of personal health information. Myriad of state and federal laws attempt to make health information secure from hackers or misuse or abuse of health data by health care employees (Dwyer III, Weaver and Hughes, 2004). Some of these laws specify the form in which health records may be stored or transmitted; others are focused on the penalties of unauthorized access through civil or criminal sanctions. As the protection of privacy and confidentiality of clinical and medical data is a major issue, state laws focus on health data disclosure are concentrated on specific information while federal laws focus only on the data gathered by federal agencies (Curran, Stearns and Kaplan, 1969). These concerns make health organizations obligated to adopt a code of ethics in addition to clarifying and defining rules and regulation which govern information protection. In other words, when a group of health organizations is sharing their data towards the goals of maturity and continuous improvement, proper standards of ethics and law should be ensured in the collection, storage, disclosure, and use of sensitive data. One way of mitigating the risks revolving around patient privacy due to centralized and shared healthcare information is to include the participating organizations and agencies in the development of the privacy systems and operational policies (Curran, Stearns and Kaplan, 1969). 
Hence, this criterion was added to the regulatory perspective and is defined as:

Organization's adherence to privacy regulations and policies to ensure the privacy of information technology, medical data, patient access, information disclosure, third party interactions, and ethical use of information among others in terms of collection, storage, disclosure, and use of information.

\subsubsection{Governance}

Another change in the criteria was "Governance" which was moved from a regulatory perspective to an organizational. Additional literature review in the context of healthcare was performed for the governance criterion. Corporate governance emerged as a solution to unbalanced relations between companys' main stakeholders (Verdeyen and Buggenhout, 2003). In 1932, Brele Jr and Daniels argued that United State's corporate structure was inefficient as shareholders owned insignificant shares to have the power to monitor and audit companies effectively(Berle Jr and Means Daniel James, 1932). This meant that stakeholders were only investors as opposed to being owners of firms. The creation of boards and governance would allow share or stakeholders to get a return on their investment on top of controlling and influencing the management in effective ways. In the stakeholder type organization such as hospitals, governance structures need to be desgined in such manner that there would be clear ideas about power, responsibilites, and checks and balances and this accountability and control can protect health organizations when there is sufficient amount of disclosure and transparency (Berle Jr and Means Daniel James, 1932; Van Den Berghe and Levrau, 2003). 
Van som (2014) defines clinical governance as: “A governance system for healthcare organizations that promotes an integrated approach towards management of inputs, structures and process to improve the outcome of health-care service delivery where health staff work in an environment of greater accountability for clinical quality" (Vanu Som, 2004). In the United States, hospitals are overseen by at least one board of directors (trustees). While non-profit hospitals normally have a single overseeing board, for-profit hospitals may have different boards and governance systems overseeing their matters such as corporate, regional, and local ones (Jha and Epstein, 2010). The mentioned study argues that the big gap in board activities between high-performing and low-performing hospitals highlights the importance of board policymakers hoping to improve care in U.S. hospitals. There is an emerging argument that boards, including in healthcare, need to incorporate a culture of high trust across the executive and non-executive divide. This should be implemented along with a robust challenge, and a tight grasp on the business of delivering high-quality care for customers (patients in healthcare context) in a financially viable manner (high trust - high challenge - high engagement) (Chambers, 2012).

There have been works in the literature focusing on the impacts of governance in healthcare organizations. One important aspect that governance facilitates is multidisciplinary teamwork, partnerships, and cooperation practices which can have great implications for healthcare delivery and organizational culture organizations (Vanu Som, 2004). Taleb-Bndiab et al. (2006) present a modeling approach for medial guidelines in 
an electronic format using an agent-based method in order to facilitate modularization of clinical governance concerns from the aspect of a system (Taleb-Bendiab et al., 2006).

Bevan (2008) proposes the CHI model which takes into account learning, innovation, and improvement for clinical governance (Bevan, 2008). This model takes into account strategic capacities such as patient focus, leadership, direction, and planning which feeds into resources and processes while utilizing the use of information for patient experience and outcomes. The resources and processes in the $\mathrm{CHI}$ model are quality improvement processes, focus on staff, and use of information (Bevan and Cornwell, 2006).

Hastings et al. (2014) look into the interrelations of health system governance and workforce outcomes (Hastings et al., 2014). In other words, this paper looked into how governance bolsters change in the workforce to guarantee effective use by healthcare providers. Although the results in this study do not show a significant correlation between workforce and improvement in patient outcomes, it emphasizes on key strategies to support change management in healthcare organizations. These key strategies are built trust through the articulation of organizational goals, taking the workforce into account in terms of planning, implementation, and evaluation, and finally making sure of the existence of strong leadership. The governance mechanisms may include shared clinical governance, funding schemes, and professional development with enablers such as engagement, communication, change management, leadership, and vision. These mechanisms while being focused on patient, financial, or clinical systems, should also encompass workforce and human resources to guarantee that employees can and are carrying their work as needed. Ultimately, boards in healthcare organizations have the 
responsibility to ensure the quality of care is provided by their hospital through determining strategy (direction), evaluation of performance (control), and forming organizational culture (values, rules, and tone) (Chambers, 2012). Chmabers (2012) Stresses the importance of avoiding faith-based and exhortative approaches and move towards guidance, training, and development and calls for more research and effort into the composition, structure, processes, and dynamics of healthcare boards for the sake of patient safety (Chambers, 2012). Garratt (2010) stresses on short-term focus on conformance and a more long-term concentration on performance (Garratt, 2010). In other words, although accountability and supervision are really important conformance issues, there should be a long-term focus on policy formation and strategic thinking in terms of external and internal focuses respectively. For this purpose (literature and expert validation), it was concluded that governance is more of an organizational issue than a regulatory matter.

Health organizations may have different governance systems across different and some may work better than others. Regulatory governance is a part of the whole organizational governance that focuses on compliance and accountability of the health organization when it comes to regulations and policy.

As an example, one kind of governance may be data governance within health organizations' information technology groups. Data governance can be a program that creates clear and functioning Standards, Policies, Procedures, Roles, Responsibilities, and Accountabilities, in order to create an environment of trusted and high-quality data that leads to accurate reporting. 
Data governance is important to health organizations as it deals with topics such as data stewardship, data quality management, data access, and privacy among others. This type of governance is critical in healthcare organizations as data can be treated a strategic asset by these organizations since:

- There is clear ownership within the health organizations' business community, and Data Stewards are accountable and responsible for the management of data assets.

- Data is secure and protected, and there are clear pathways to who grants access to data.

- Clear documentation exists to help provide users additional context and meaning to the data they use for decision-making.

- The quality of critical data is constantly monitored and measured, and defects are corrected in the source system.

- There is a clear understanding of the critical data flow of elements throughout the organization.

- Data is easily accessible to reach a potential audience, and data users can easily identify data assets within the environment that can be leveraged.

To sum up, in terms of data governance, health organizations need to pay attention to stewardship (to ensure ownership), processes (to ensure consistency), and governed tools (to deliver access and reports. These three items will provide trusted data and reports for continuous improvement and decision-making around it. In addition to data stewardship 
(accountability and responsibility), topics such as metadata management (business glossaries, report catalogs, application banks,...), data quality (completeness, conformity, consistency, accuracy, timeliness,...), and data access/protection are the key functional area in data governance for a healthcare organization.

The other important aspects of governance in a health organization include but are not limited to, leadership, strategies, financial matters, decision making for resources and investments, policymaking, risk management, planning, training, and evaluation of quality across different departments of a healthcare organization.

\subsubsection{Training}

As for training, it was decided that it would be separated from talent acquisition from the social perspective to the organizational perspective. Technology management encapsulates managing technology in terms of not only volume but also reliability in terms of accuracy and safety (Grossmann et al., 2010). In other words, healthcare providers, including residents and fellows, should be placed in environments their rate of medical errors will decrease with training and management. Training providers to work in teams is critical to ensure an increase in reliability and safety to make sure optimal technology management is flowing in the organization.

Stephen J. Swensen, and James Dilling of Mayo Clinic pinpoint the importance of sentient investment in social capital as an important part of effective technology management strategy with goals of high-reliability patient care. Social capital investment encapsulates the goals of transforming an organization from a collection of individuals into an agile, coherent and collective mind (Lynch et al., 2000; Gopee, 2002; Grossmann, Goolsby, 
Olsen and McGinnis, 2011). This can be done by utilizing research engagement, administrations, and education. Swensen and Dilling emphasize areas like transparency, teamwork training, horizontal infrastructure, and cross-functional, team-based simulation training as important social engineering topics. An example of this can be utilized in health organizations' training teams to train health medical and nursing students and residents, together in cross-functional teams to enforce a more effective transformation culture.

By the help of experts validating the criteria and further literature review, especially on the importance of technology management in continuous learning, the importance of training was stressed in technology maturity of healthcare organizations and the fact the continuous training is needed as an enabler of increased maturity and continuous learning from the organizations' point of view, initiated the move from social to organizational perspective. Figure 14 shows the perspectives and criteria post validation.

\subsection{Initial Model}

After implementing the mentioned approach and justifying the added/moved elements, the model was finalized for the proposal and the initial model in the main dissertation study. The model has between 3 and 6 elements in each perspective with a total of 5 perspectives and 18 criteria. The

next section shows an iteration of running the model and validating/quantifying the decision elements with the expert panels which were identified. 


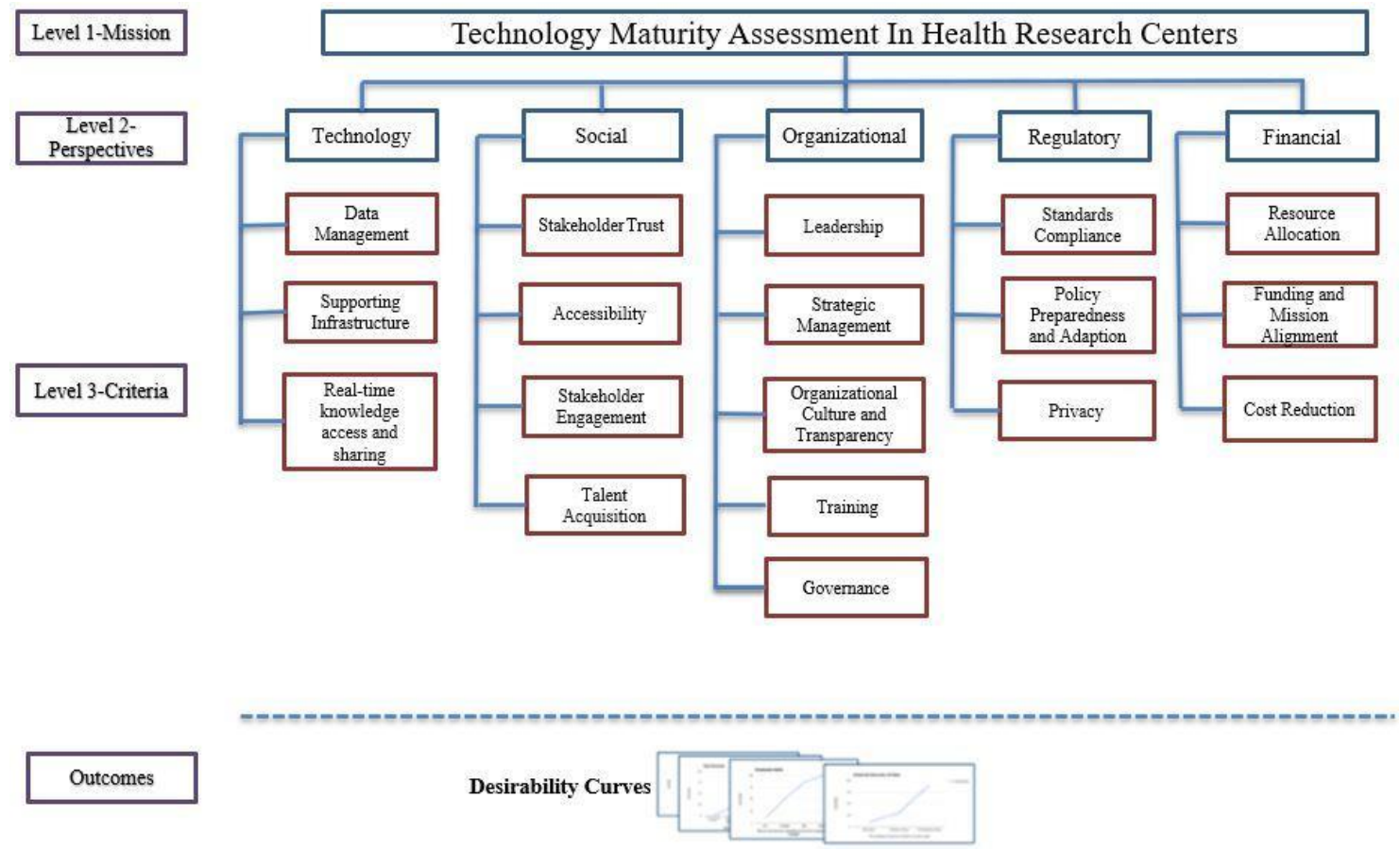

Figure 30: Initial Model

\subsection{Experts Identification and Selection}

Several methods are used to identify and select the potential experts for validation and quantification of the model.

1- Researcher's Connections: the researcher may have several connections related to learning health systems, the healthcare industry, and hospital management, based on previous work experience and current engagement with committees related to this research topic.

2- Advisor and Committee Connections: Help provided by advisor and committee members in terms of the identification of local and national experts related to technology management in healthcare and learning health systems will be 
requested. This is because these people are highly probable to be engaged directly or indirectly in a related field of research and application.

3- Social network analysis (SNA): This process can be used to investigate social structures by identifying networks and people in the centers of those networks (Daim and Kocaoglu, 2015; Kocaoglu et al., 2016).SNA will be used to identify experts related to healthcare technology management and learning health systems which can potentially lead them to be a part of the expert panels based on their expertise.

It should be considered that all experts may not be in all panels. In other words, experts will be divided into panels and each expert will be matched and assigned to a certain area of the proposed model of this research in terms of validation and/or quantification. This means that each expert is able to join more than one panel based on relevant expertise. Here is a table summarizing the involvement of each panel in different steps of validation and quantification of the model.

Table 10: Expert Panel Summary

\begin{tabular}{|c|c|c|c|}
\hline Panel & Task & \# of Experts & Tool \\
\hline P1 & Perspective Validation & 24 & Qualtrics \\
\hline P2 & Criteria Validation & 26 & Qualtrics \\
\hline P3 & Perspectives/Criteria Quantification & 30 & ETM HDM \\
\hline P4 & Desirability Metrics Validation & 11 & Qualtrics \\
\hline P5 & Desirability Metrics Quantification & 13 & Qualtrics \\
\hline
\end{tabular}


Table 11: Panel 1 Participants

\begin{tabular}{|c|c|}
\hline \# & Position \\
\hline 4 & Project Manager \\
\hline 5 & Chief Information Officer \\
\hline 6 & Project Manager \\
\hline 7 & Health Application Analyst \\
\hline 8 & Health Application Analyst \\
\hline 11 & Research Center Director \\
\hline 12 & Professor of Public Health \\
\hline 15 & Public Health Researcher \\
\hline 16 & $\begin{array}{l}\text { Vice President of Nursing } \\
\end{array}$ \\
\hline 17 & Assistant Professor in Medical Ethics \& Health Policy \\
\hline 19 & Chair of Engineering Division in Hospital \\
\hline 20 & Professor of Health Informatics \\
\hline 22 & Research Center Director \\
\hline 24 & Industrial Engineering Professor (Healthcare) \\
\hline 25 & Quality Engineering Professor \\
\hline 26 & Professor at the Department of Healthcare Institutions Management \\
\hline 29 & Research Center Director \\
\hline 30 & Research Center Director \\
\hline 34 & Project Manager \\
\hline 36 & Health Management Researcher \\
\hline 40 & Department of Industrial Engineering (Chair) \\
\hline 41 & Health Informatics Analyst \\
\hline 42 & Health Data Scientist \\
\hline 45 & Project Manager \\
\hline
\end{tabular}


Table 12: Panel 2 Participants

\begin{tabular}{|l|c|}
\hline$\#$ & Positions \\
\hline 5 & Chief Information Officer \\
\hline 6 & Project Manager \\
\hline 8 & Project Manager \\
\hline 11 & Research Center Director \\
\hline 12 & Professor of Public Health \\
\hline 15 & Project Manager \\
\hline 16 & Public Health Researcher \\
\hline 17 & VP of Nursing \\
\hline 19 & Assistant Professor in Medical Ethics \& Health Policy \\
\hline 20 & Chair of Engineering Division \\
\hline 22 & Professor of Health Informatics \\
\hline 24 & Research Center Director \\
\hline 25 & IE Professor (Healthcare) \\
\hline 26 & Quality Engineering Professor \\
\hline 29 & Research Center Director \\
\hline 30 & Research Center Director \\
\hline 32 & Department of Healthcare Institutions Management. Professor \\
\hline 34 & Technology Faculty Research Coordinator \\
\hline 36 & Project Manager \\
\hline 40 & Industrial Engineering Researcher \\
\hline 41 & Health Informatics Analyst \\
\hline 42 & Project Manager \\
\hline 45 & of Industrial Engineering (Department Chair) \\
\hline & \\
\hline
\end{tabular}


Table 13: Panel 3 Participants

\begin{tabular}{|c|c|}
\hline \# & Position \\
\hline 1 & $\begin{array}{c}\text { Data Scientist } \\
\end{array}$ \\
\hline 2 & Senior Information Systems Project Leader \\
\hline 3 & Director of finance and Operations \\
\hline 4 & BI Project Manager \\
\hline 6 & Project Manager \\
\hline 10 & Research Center Director \\
\hline 12 & Professor at Department of Public Health \\
\hline 13 & Professor at Department of Public Health \\
\hline 14 & Project Manager \\
\hline 15 & Public Health Researcher \\
\hline 16 & Vice President of Nursing \\
\hline 18 & Assistant Professor of Learning Health Sciences \\
\hline 19 & Division of Engineering Chair \\
\hline 20 & Professor of Health Informatics \\
\hline 21 & Deputy Director for Public Health \\
\hline 22 & Research Center Director \\
\hline 23 & Learning Health Systems Researcher \\
\hline 26 & Professor at the Department of Healthcare Institutions Management \\
\hline 30 & Research Center Director \\
\hline 32 & Lecturer and Faculty Research Coordinator \\
\hline 33 & $\begin{array}{l}\text { Professor } \\
\end{array}$ \\
\hline 37 & Professor at the Department of Healthcare Institutions Management \\
\hline 39 & Performance Improvement Manager \\
\hline 40 & Department of Industrial Engineering (Department Chair) \\
\hline 41 & Health Data Analyst \\
\hline 42 & Data Scientist \\
\hline 43 & Associate Vice President for Information Systems \\
\hline 44 & Senior Project Manager \\
\hline 45 & Project Manager \\
\hline 46 & Professor, Director, Center for Health Policy and Research \\
\hline
\end{tabular}


Table 14: Panel 4 Participants

\begin{tabular}{|c|c|}
\hline$\#$ & Position \\
\hline 2 & Senior IS Project Leader (Regulatory) \\
\hline 6 & Healthcare Consultant \\
\hline 14 & Project Manager \\
\hline 17 & Assistant Professor in Medical Ethics \& Health Policy \\
\hline 22 & Director of Research Center \\
\hline 26 & Faculty member at Department of Healthcare Institutions Management \\
\hline 33 & Professor of Internal Medicine \\
\hline 35 & Senior Policy Analyst \\
\hline 38 & Chief Technology Officer \\
\hline 41 & Health Data Analyst \\
\hline 43 & Associate Vice President for Information Systems \\
\hline 46 & Professor, Director, Center for Health Policy and Research \\
\hline
\end{tabular}

Table 15: Panel 5 Participants

\begin{tabular}{|c|c|}
\hline Expert \# & Position \\
\hline 13 & Associate Professor and Program Director, MPH Health Management and Policy \\
\hline 14 & Project Manager \\
\hline 15 & Public Health Researcher \\
\hline 16 & Vice President of Nursing \\
\hline 19 & Chair, Division of Engineering \\
\hline 20 & Professor of Health Informatics \\
\hline 22 & Director of Research Center \\
\hline 26 & Faculty member at Department of Healthcare Institutions Management \\
\hline 33 & Professor of Internal Medicine \\
\hline 35 & Senior Policy Analyst \\
\hline 41 & Health Data Analyst \\
\hline 43 & Associate Vice President for Information Systems \\
\hline 46 & Professor, Director, Center for Health Policy and Research \\
\hline
\end{tabular}




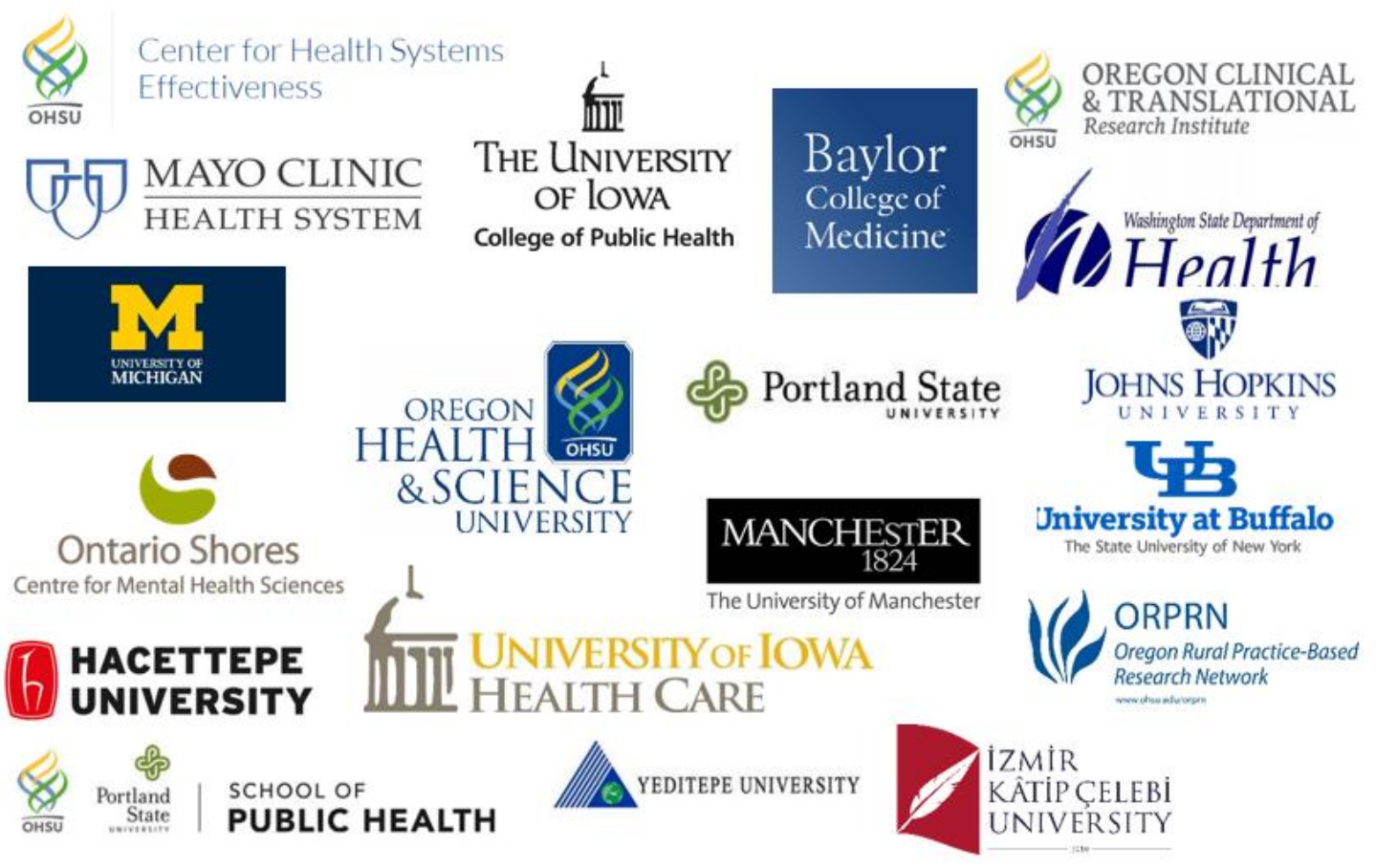

Figure 31: Participating Experts' Affiliations 


\section{Results of Model Validation and Quantification}

\subsection{Model Validation}

In this section, to test the proposed model in theoretic and practical terms, the model was validated and quantified using a panel of experts. In order to test the practicality of this model, it has been applied to a department in a health organization in the United States. As result valuation tools, the inconsistency and disagreement values will be tested with regards to the acceptable threshold and sensitivity analysis will be performed to test the model in different situations based on different prioritizations.

As the first of the model implementation, the perspectives of the model were validated. All 5 different perspectives were validated by 24 experts with $100 \%$ agreeing that the perspectives were sufficient, and no changes were needed to be made. The threshold of $80 \%$ percent was considered for passing for each perspective and criteria. The summary of the prospective validation is shown in Table 16.

Table 16: Perspective Validation

\begin{tabular}{|c|c|c|c|c|}
\hline Perspective & \# of Experts & Yes & No & Validation \\
\hline Technology & 24 & 24 & 0 & $100 \%$ \\
\hline Social & 24 & 24 & 0 & $100 \%$ \\
\hline Organizational & 24 & 24 & 0 & $100 \%$ \\
\hline Regulatory & 24 & 24 & 0 & $100 \%$ \\
\hline Financial & 24 & 24 & 0 & $100 \%$ \\
\hline
\end{tabular}




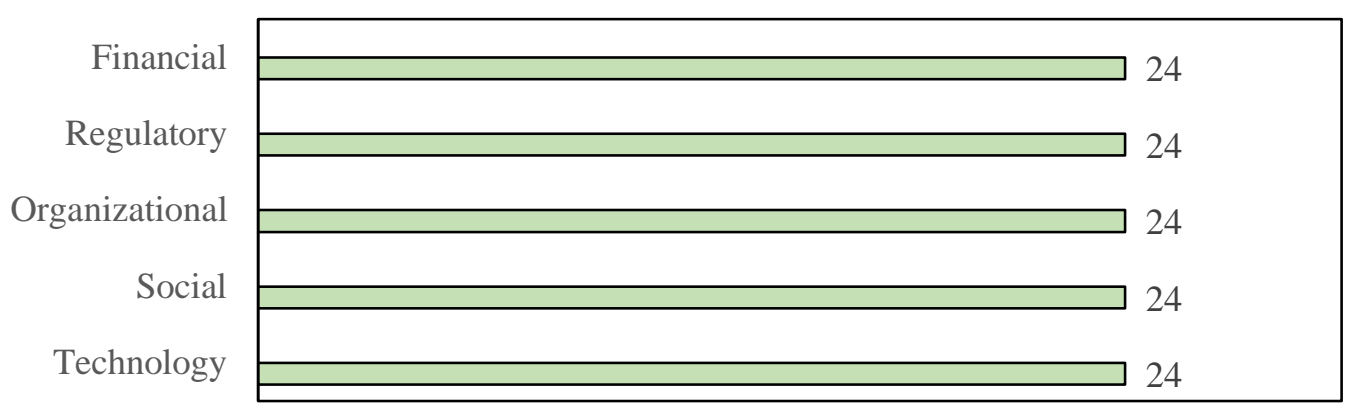

Figure 32: Perspective Validation

After validating the perspectives, each perspective's criteria were validated by each of the 26 experts in panel 2. In this section, I received great feedback from my experts leading to the addition of 2 criteria and reword two criteria. Although none of the criteria failed in terms of getting a validation percentage of under threshold, the changes made in wording and definitions were based on the comments and feedback provided by experts and validated and verified by further literature review. The summary of the criteria validation is shown in the Table 17-21.

Table 17: Technology Criteria Validation

\begin{tabular}{|c|c|c|c|c|}
\hline Technology & \# of Experts & Yes & No & Validation \\
\hline Data Management & 26 & 26 & 0 & $100 \%$ \\
\hline Supporting Infrastructure & 26 & 26 & 0 & $100 \%$ \\
\hline Real-time Knowledge Access and Sharing & 26 & 25 & 1 & $96 \%$ \\
\hline
\end{tabular}


Real-time Knowledge Acess and Sharing

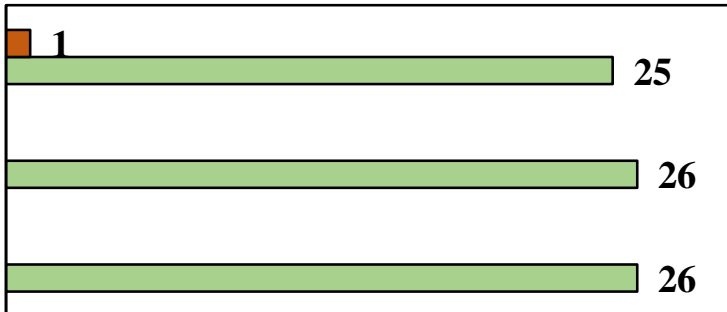

$\square$ No $\square$ Yes

Supporting Infraustructure

Data Management

Figure 33: Technology Criteria Validation

Table 18: Social Criteria Validation

\begin{tabular}{|c|c|c|c|c|}
\hline Social & \# of Experts & Yes & No & Validation \\
\hline Stakeholder Trust & 26 & 23 & 3 & $88 \%$ \\
\hline Accessibility & 26 & 26 & 0 & $100 \%$ \\
\hline Stakeholder Engagement & 26 & 25 & 1 & $96 \%$ \\
\hline Talent Acquisition & 26 & 26 & 0 & $100 \%$ \\
\hline
\end{tabular}

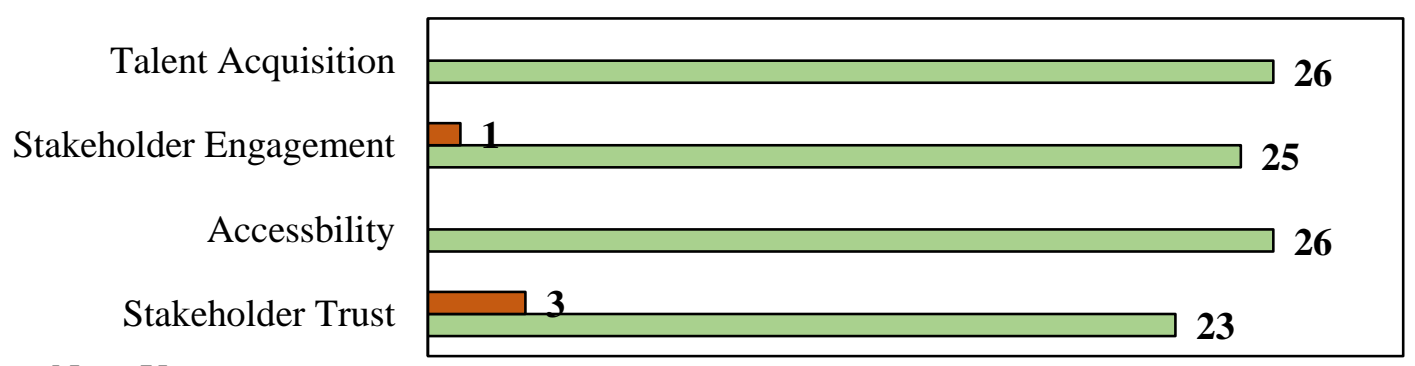

$\square$ No $\square$ Yes

Figure 34: Social Criteria Validation 
Table 19: Organizational Criteria Validation

\begin{tabular}{|c|c|c|c|c|}
\hline Organizational & \# of Experts & Yes & No & Validation \\
\hline Leadership & 26 & 25 & 1 & $96 \%$ \\
\hline Strategic Management & 26 & 25 & 1 & $96 \%$ \\
\hline Culture and Transparency & 26 & 25 & 1 & $96 \%$ \\
\hline Training & 26 & 26 & 0 & $100 \%$ \\
\hline Governance & 26 & 25 & 1 & $96 \%$ \\
\hline
\end{tabular}

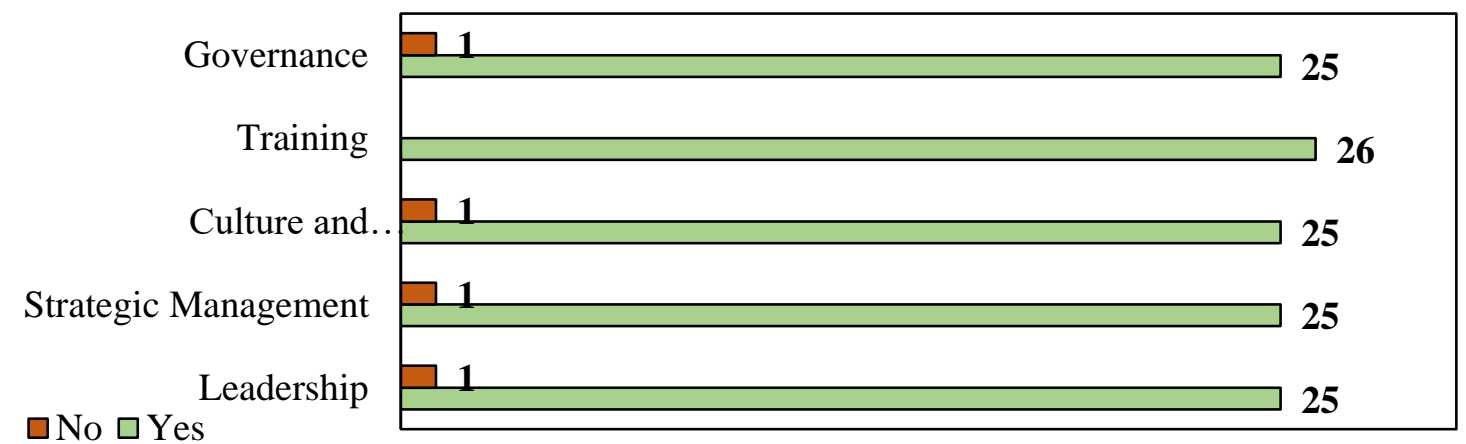

Figure 35: Organizational Criteria Validation

Table 20: Regulatory Criteria Validation

\begin{tabular}{|c|c|c|c|c|}
\hline Regulatory & \# of Experts & Yes & No & Validation \\
\hline Standards Compliance & 26 & 26 & 0 & $100 \%$ \\
\hline $\begin{array}{c}\text { Policy Preparedness and } \\
\text { Adaption }\end{array}$ & 26 & 25 & 1 & $96 \%$ \\
\hline Privacy & 26 & 24 & 2 & $92 \%$ \\
\hline
\end{tabular}

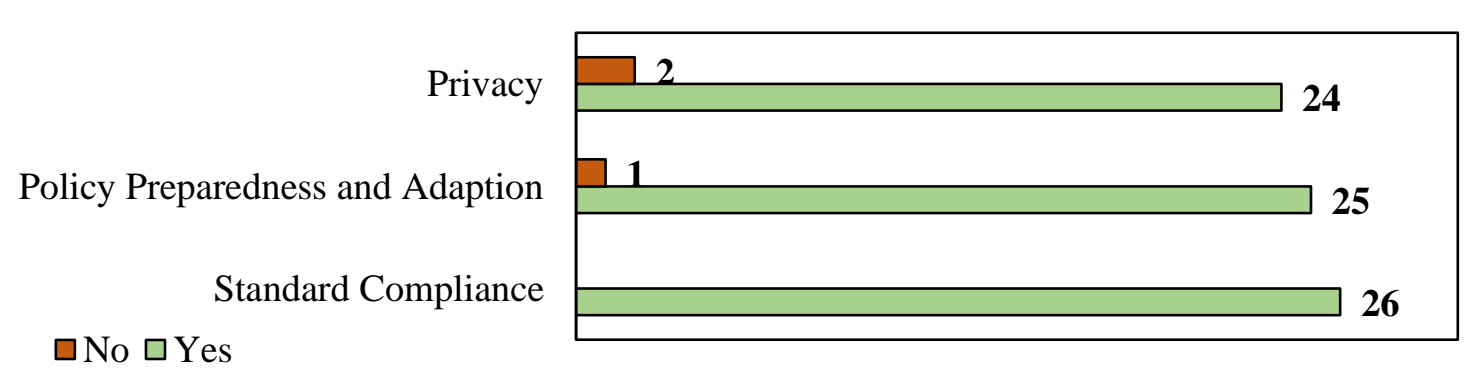

Figure 36: Regulatory Criteria Validation 
Table 21: Financial Criteria Validation

\begin{tabular}{|c|c|c|c|c|}
\hline Financial & \# of Experts & Yes & No & Validation \\
\hline Resource Allocation & 26 & 26 & 0 & $100 \%$ \\
\hline Funding and Mission Alignment & 26 & 25 & 1 & $96 \%$ \\
\hline Cost Reduction & 26 & 25 & 1 & $96 \%$ \\
\hline
\end{tabular}

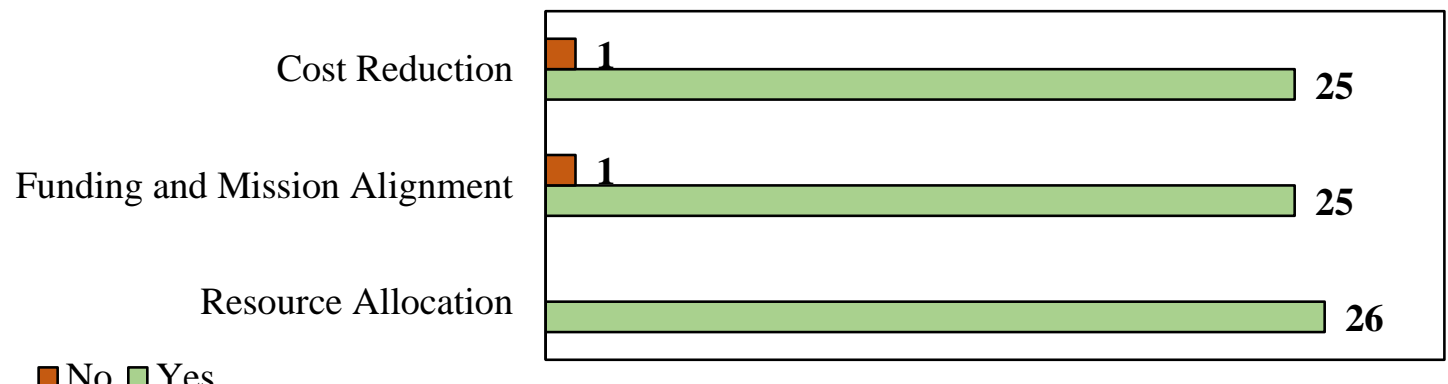

Figure 37: Financial Criteria Validation

\subsection{Validated Model}

After getting the feedback from expert panels 1 and 2, three new criteria were added which are user experience, informing policy, and financial synergy with policies and priorities under technology, regulatory, and financial perspectives, respectively. Furthermore, two criteria were edited in their wording. Organizational culture and transparency were shortened to "Culture and Transparency" and the word retention was added to talent acquisition as the importance of talent retention was realized during the validation process. Finally, based on the feedback, some criteria definitions were reworded or rephrased for clarity and accuracy. Here is an example of expert feedback on "Supporting Infrastructure" which led to definition revision: 
"I think "supporting infrastructure" and/or real-time knowledge access \& sharing need to be conceived more broadly to include not only DISSEMINATION of learned knowledge, but systems to ensure said knowledge is subsequently integrated into clinical care processes to secure the promised improvements. It's not enough just to put it out in the world and hope someone will do something with it."

Another example of expert feedback on "Stakeholder Trust":

"It must also be ensured that the institution is TRUSTWORTHY. Part of this trustworthiness is following through on promised commitments and ensuring new knowledge is used to improve care of those who contributed to its generation via enhanced use of their data)."

In this section each of the added criteria will be briefly explained.

\subsubsection{User Experience}

Defined as "Ensuring that technologies and IT products are user-friendly (e.g., considering health literacy and technology competence) to all users (stakeholders) (Lehoux, 2004; Rudd, 2010; Vehko et al., 2019)", this criterion was added to the model under the technology perspective based on the expert panel's feedback. User Experience (UX) can enhance users' motivation and engagement (Zaharias and Pappas, 2016). As Lehoux (2004) argues, it is enough to only transfer the technology to the user or stakeholders but to make sure the intended knowledge to be transferred as well. The same study looks into the importance of user-friendliness in this transfer. Moreover, Giansanti, Castrichella and Giovagnoli (2007) found that user-friendliness has the biggest effect on user satisfaction with e-learning in healthcare professionals. 
One of the comments that were received during the validation phase stressed the need for involving a criterion that touches on technological user-friendliness and literacy:

"You can think of something around how easy is it for the patients or consumers of the service or research the center is providing can benefit from it in terms of technological user-friendliness and literacy."

To this end, User Experience was added to the technology perspective in the model.

\subsubsection{Informing Policy}

Defined as "Research centers' ability to inform policies by participating in expert panels, providing evidence and comments regarding policies under consideration, and securing contracts with the state and the federal government to complete analyses regarding policy development, implementation, and impact (Douglas et al., 2009; Bendavid and Miller, 2010; Behrns, 2015; McMahon et al., 2020)", this criterion was added during discussions and feedback by the regulatory focused experts in the validation expert panels. It is important for healthcare organizations and research centers to keep abreast and improve policies by:

- Participating in expert panels.

- Providing evidence and comments regarding policies that are under consideration.

- Securing federal and state contracts based on policy development, implementation, and impact. 
An example would be for a research center to define the questions that society, patients, and healthcare need and work towards providing evidence that will help inform policy. Health research centers should reach outside of their organization to connect with policymakers and the community and by engaging in meaningful conversations, gain a better understanding of the existing problems and how they can address them. Moreover, centers need to improve their ability to communicate their findings back to policymakers leading to a more effective policy generation (Pomeroy, no date). This criterion is very important as stakeholders collaborate to generate evidence that informs health policy, the results can be an effective way to push research.

To sum up, academic health research centers can have a significant impact on research and evidence-informed health policy through taking part in new collaborative models of research.

It should be noted that research centers should remain unbiased and keep the aims of healthcare as their moral compass and make sure their research is not misused in politically charged policy shaping.

\subsubsection{Financial Synergy with Policies and Priorities}

The third added criterion is defined as "Research centers' success in meeting reimbursement programs' goals (policy incentives, value-based purchasing, etc.) and accessing government, foundation, and other grants (NIH, DARPA, DOD, various private funds, etc.) (Weeks and Pardee, 2019)". This criterion had been partially realized during the literature review phase for health research centers and this realization was invigorated in the validation phase based on expert feedback. The importance of research grants has 
been explained in section 2.7. Here are some of the comments that hinted at the inclusion of this criterion:

"It is important to include the center's success in accessing government and other grants. NIH, DARPA, DOD, various private funds, etc."

Another expert's comment stressed the importance of policy incentives:

"Greater environmental incentives: consider policy incentives, value-based purchasing, and other reimbursement programs; also, reputation and accreditations and the financial benefit of meeting those requirements."

As the result of the literature review on research centers and the feedback received from the experts at the validation phase, this item was added under the financial perspective.

\subsubsection{Finalized Model}

After the completion of the validation of model perspectives and criteria, the final model consists of 5 perspectives, each of them containing from 4 to 5 criteria. The total number of criteria in this model is 21. The final validated model is shown in Figure 38. Moreover, the refined criteria and their definitions are shown in Tables 22-26. 

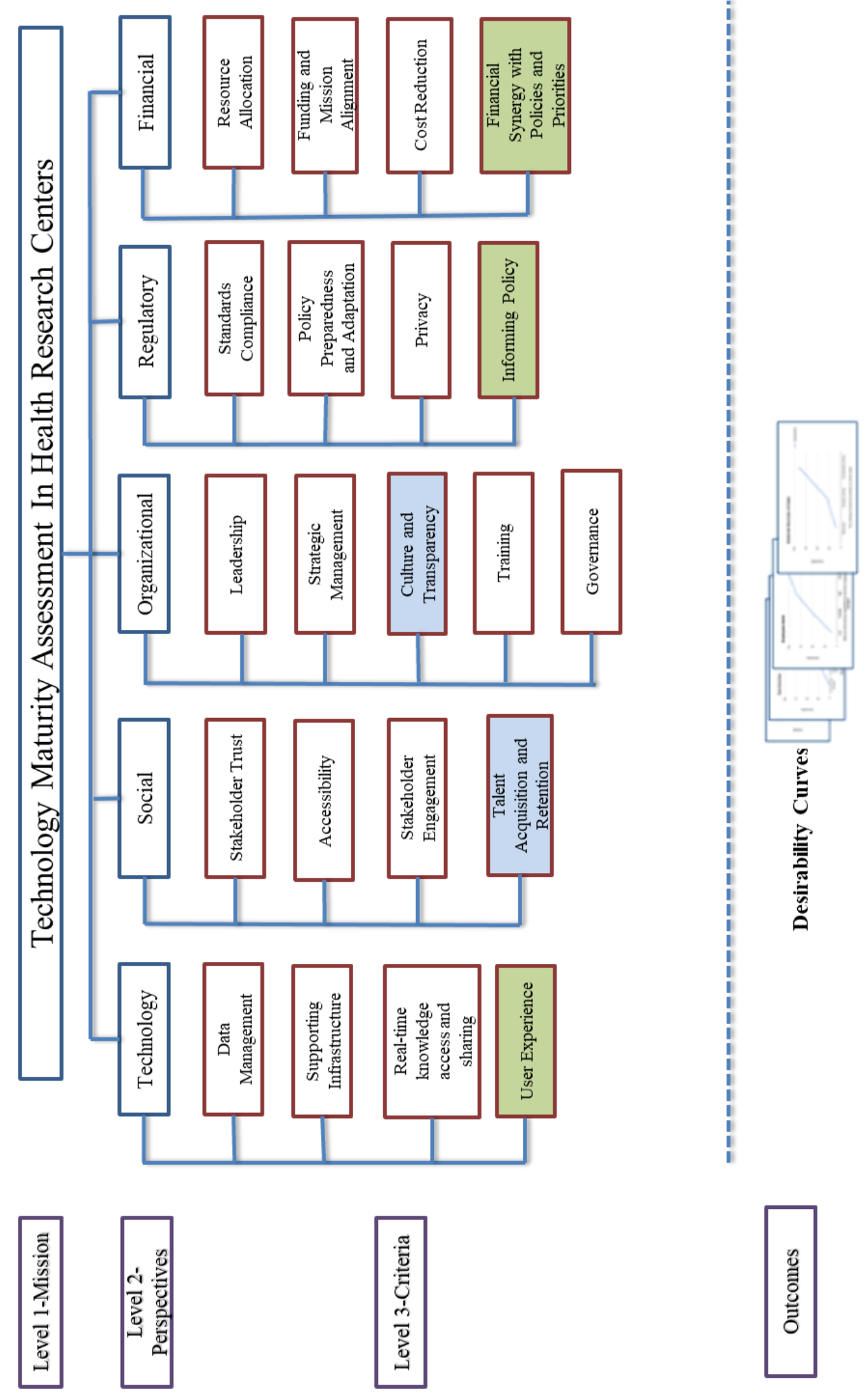

Figure 38:Validated Model

182 
Table 22: Validated Technology Criteria

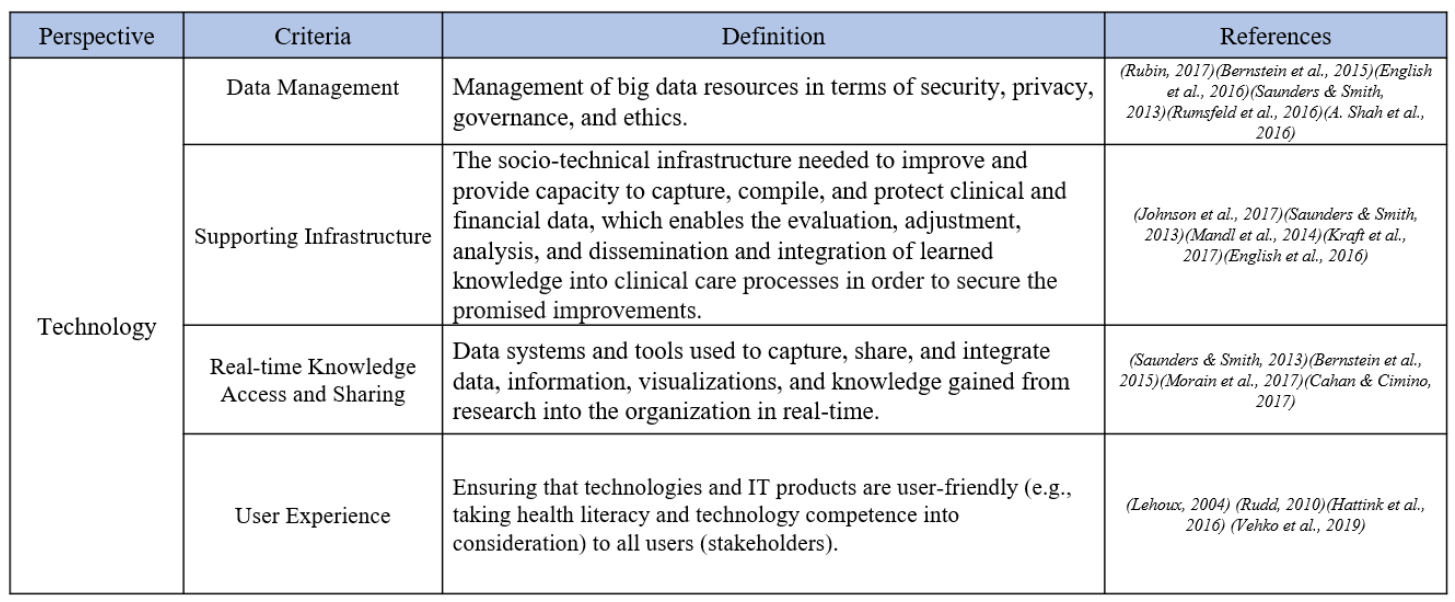

Table 23: Validated Social Criteria

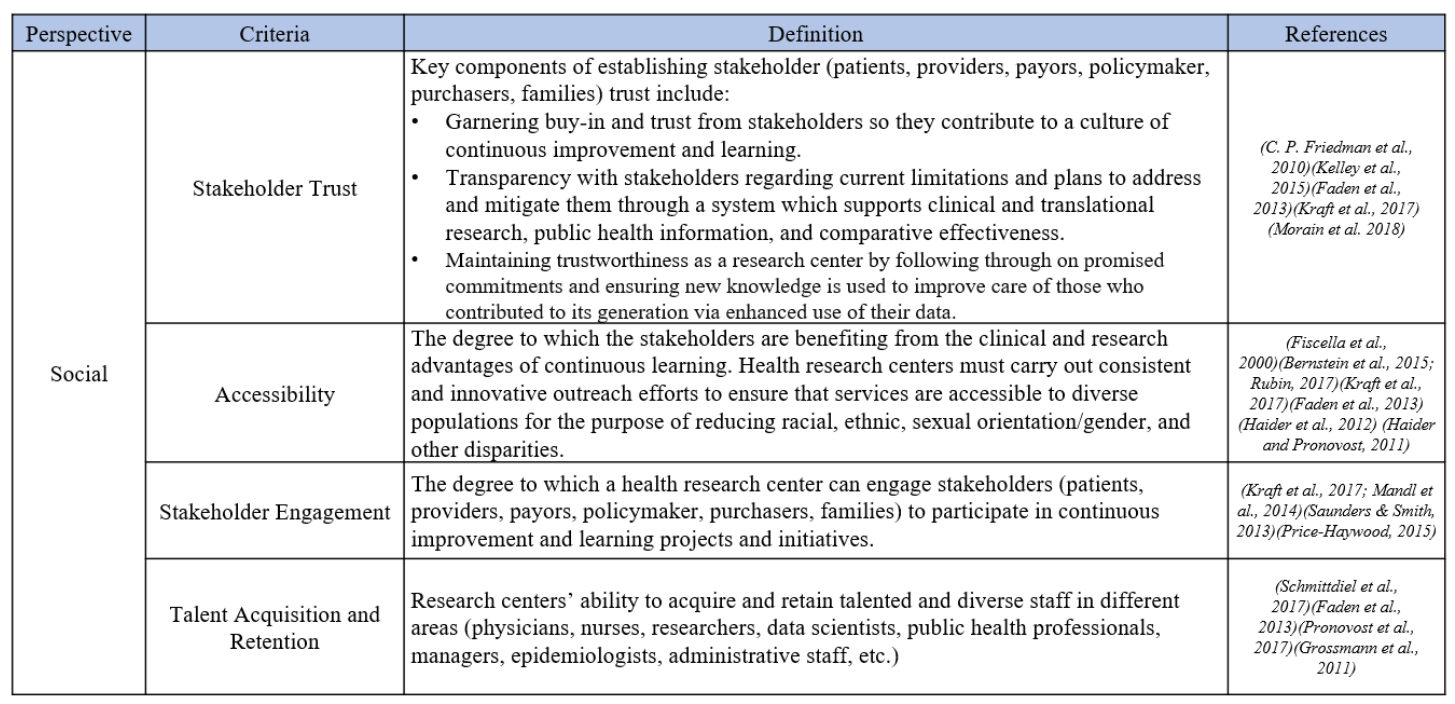


Table 24: Validated Organizational Criteria

\begin{tabular}{|c|c|c|c|}
\hline Perspective & Criteria & Definition & References \\
\hline \multirow{3}{*}{ Organizational } & Leadership & $\begin{array}{l}\text { Broad leadership which can expand and guide stakeholders' commitment } \\
\text { to the goals of continuous learning and increased technology maturity. }\end{array}$ & $\begin{array}{l}\text { (Saunders \& Smith, 2013) (Bernstein et al., } \\
\text { 2015)(Schmittdiel et al., 2017)(Morain et al., } \\
\text { 2017)(Rubin, 2017)(Graban, 2016) }\end{array}$ \\
\hline & Strategic Management & $\begin{array}{l}\text { Research centers' ability to improve through strategic decisions, } \\
\text { management of competing priorities, internal and external } \\
\text { partnerships/collaborations, problem identification, and finding solutions. }\end{array}$ & $\begin{array}{l}\text { (English et al., 2016)(Morain et al, 2017)(Kraft et } \\
\text { al., 2017)(Bernstein et al., 2015) }\end{array}$ \\
\hline & Training & $\begin{array}{l}\text { Training employees and stakeholders on the latest technologies and best } \\
\text { practices within the health research center. }\end{array}$ & $\begin{array}{l}\text { (Schmittdiel et al., 2017)(Faden et al, } \\
\text { 2013)(Pronovost et al., 2017)(Grossmann et al, } \\
\text { 2011) }\end{array}$ \\
\hline
\end{tabular}

Table 25: Validated Regulatory Criteria

\begin{tabular}{|c|c|c|c|}
\hline Perspective & Criteria & Definition & References \\
\hline \multirow{4}{*}{ Regulatory } & Standards Compliance & $\begin{array}{l}\text { Research centers' compliance with standards and } \\
\text { regulations to ensure transparency with stakeholders, data } \\
\text { interoperability, and commitment to meaningful use and } \\
\text { joint commissions. }\end{array}$ & $\begin{array}{l}\text { (C. P. Friedman et al., 2010)(IOM, } \\
\text { 2011)(Care, 2014)(Schmittdiel et al, } \\
\text { 2017)(Institute of Medicine (IOM), } \\
\text { 2007)(Blumenthal \& Tavenner, 2010) }\end{array}$ \\
\hline & Policy Preparedness and Adaption & $\begin{array}{l}\text { Research centers' flexibility and agility in responding and } \\
\text { adapting to changes (new regulations, legislations, and } \\
\text { policies) in terms of anticipation and readiness (resources, } \\
\text { policies, strategies, and management). }\end{array}$ & $\begin{array}{l}\text { (Blumenthal \& Tavenner, 2010) } \\
\text { (Grossmann et al., 2011) (Amir Shaygan, } \\
\text { 2018)(Shaygan \& Daim, 2019) }\end{array}$ \\
\hline & Privacy & $\begin{array}{l}\text { Research centers' adherence to regulations and policies to } \\
\text { ensure privacy in terms of information technology, medical } \\
\text { data, patient access, third party interactions, and ethical use } \\
\text { of information (IRBs), among others. }\end{array}$ & $\begin{array}{l}\text { (Curran, Stearns and Kaplan, 1969; Gostin } \\
\text { et al, 1993; Prentnieks and Qual, 1996; } \\
\text { Melton III, 1997; National Research } \\
\text { Council, 1997, Rothstein and Talbott, 2006; } \\
\text { Nass et al., 2009; Vandenbroucke, 2011) }\end{array}$ \\
\hline & Informing Policy & $\begin{array}{l}\text { Research centers' ability to inform policies by participating } \\
\text { in expert panels, providing evidence and comments } \\
\text { regarding policies under consideration, and securing } \\
\text { contracts with the state and federal government to complete } \\
\text { analyses regarding policy development, implementation, } \\
\text { and impact. }\end{array}$ & $\begin{array}{l}\text { (Douglas et al., 2009) } \\
\text { (Bendavid \& Miller, 2010) (Behrns, 2015) } \\
\text { (McMahon et al., 2020) }\end{array}$ \\
\hline
\end{tabular}




\begin{tabular}{|c|c|c|c|}
\hline Perspective & Criteria & Definition & References \\
\hline \multirow{3}{*}{ Financial } & Resource Allocation & $\begin{array}{l}\text { Research centers' ability to prioritize and decide to invest its } \\
\text { resources (financial, human, space,...) in alignment with the } \\
\text { goals of increased technology maturity and continuous learning. }\end{array}$ & $\begin{array}{c}\text { (Rouse et al., 2017)(Kraft et al., 2017)(Rouse, } \\
\text { 2001)(Pronovost et al., 2017) }\end{array}$ \\
\hline & $\begin{array}{c}\text { Funding and Mission } \\
\text { Alignment }\end{array}$ & $\begin{array}{l}\text { The extent to which incentives and funding are aligned with } \\
\text { encouragement of technology maturity, continuous learning and } \\
\text { improvement, waste elimination, and rewarding high care value. }\end{array}$ & $\begin{array}{l}\text { (Blizinshy \& Bonham, 2017)(Morain et al., } \\
\text { 2017)(Amir Shaygan, 2018)(Institute of Medicine } \\
\text { (IOM), 2007)(Pronovost et al., 2017) }\end{array}$ \\
\hline & $\begin{array}{l}\text { Financial Synergy with } \\
\text { Policies and Priorities }\end{array}$ & $\begin{array}{l}\text { Research centers' success in meeting reimbursement programs' } \\
\text { goals (policy incentives, value-based purchasing, etc.) and } \\
\text { accessing government, foundation, and other grants (NIH, } \\
\text { DARPA, DOD, various private funds, etc.) }\end{array}$ & $\begin{array}{l}\text { (White et al., 2019)(Weeks and Pardee, 2019) } \\
\text { (Menear et al., 2019) }\end{array}$ \\
\hline
\end{tabular}

\subsection{Desirability Curves}

The metrics provided for each criterion were validated. they were tweaked and slightly edited based on the great comments and feedback provided by the expert panel. For the validation of the metrics for each criterion, the initials metrics were sent to the relative panel. Each criterion's metric validation is shown in the tables below (Tables 27-31).

Furthermore, the experts quantified the desirability curve metrics. In other words, each metric for each criterion now has a different quantified amount associated with it. Each criterion's metric/state values. These values are shown in Tables 32-52 while the curves are shown in Figures 39-59. The quantification data for the desirability curve values were collected using Qualtrics in the winter of 2020.

The quantified metrics will be used in the case study section which will add the unique extra layer based on the context of the studied research centers on top of the weighted criteria and perspectives. This is especially helpful in instances in which there is a need in having a reusable model, or in instances of having many alternatives, desirability 
curves can be used. The combination of desirability curves with HDM is used to identify levels/ metrics for each criterion. Each level/metric connected to a criterion acts as a useful value to assist decision-makers. Using the desirability curves approach, the experts need to evaluate related levels/metrics for each criterion (desirability matrix) while giving each metric a scaled quantitative value. This enables the normalization of the evaluation results by experts across all the criteria (Phan, 2013; Estep and Daim, 2016; Gibson and Daim, 2016; Estep, Daim and Shaygan, 2021) 


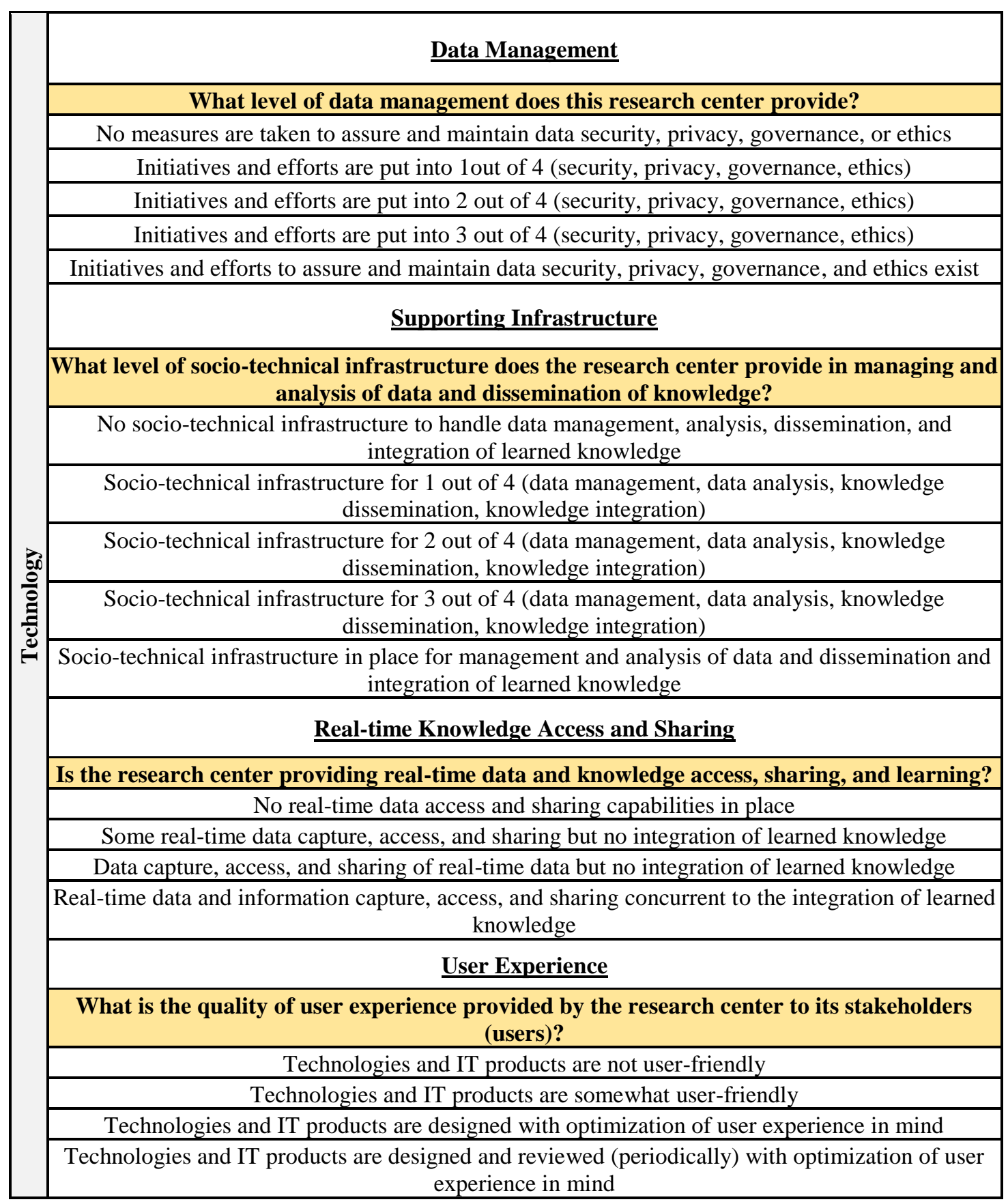


Table 28: Validated Social Metrics

\begin{tabular}{|c|c|}
\hline & $\underline{\text { Stakeholder Trust }}$ \\
\hline & $\begin{array}{l}\text { What is the level of stakeholder trust in the research center's initiatives and projects } \\
\text { (continuous learning)? }\end{array}$ \\
\hline & Not trusted by the overwhelming majority of stakeholders \\
\hline & Trusted by the minority of stakeholders \\
\hline & Trusted by half of the stakeholders \\
\hline & Trusted by the majority of stakeholders \\
\hline & Accessibility \\
\hline & What is the level of benefit from and accessibility to new technologies? \\
\hline & Stakeholders are not benefiting from the clinical and research advantages of the research center \\
\hline & $\begin{array}{c}\text { Only a minority of stakeholders are benefiting from the clinical and research advantages of the } \\
\text { research center }\end{array}$ \\
\hline & $\begin{array}{c}\text { Half of the stakeholders are benefiting from the clinical and research advantages of the research } \\
\text { center }\end{array}$ \\
\hline & $\begin{array}{l}\text { The majority of stakeholders are benefiting from the clinical and research advantages of the } \\
\text { research center }\end{array}$ \\
\hline & All stakeholders are benefiting from the clinical and research advantages of the research center \\
\hline 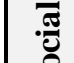 & Stakeholder Engagement \\
\hline & What is the level of stakeholder engagement in research center's projects and initiatives? \\
\hline & $\begin{array}{c}\text { Stakeholders are opposed to participating in the research center's continuous improvement and } \\
\text { learning projects and initiatives }\end{array}$ \\
\hline & $\begin{array}{c}\text { Stakeholders are reluctant to participate in the research center's continuous improvement and } \\
\text { learning projects and initiatives }\end{array}$ \\
\hline & $\begin{array}{c}\text { Some of the stakeholders are eager to participate in the research center's continuous improvement } \\
\text { and learning projects and initiatives }\end{array}$ \\
\hline & $\begin{array}{c}\text { The majority of the stakeholders are eager to participate in the research center's continuous } \\
\text { improvement and learning projects and initiatives }\end{array}$ \\
\hline & $\begin{array}{c}\text { All stakeholders are eager to participate in the research center's continuous improvement and } \\
\text { learning projects and initiatives }\end{array}$ \\
\hline & Talent Acquisition and Retention \\
\hline & $\begin{array}{l}\text { How is the research center performing in acquiring and retaining skilled and relevant } \\
\text { talents? }\end{array}$ \\
\hline & No acquisition and retention of skilled and diverse talent \\
\hline & Acquisition of skilled and diverse talent only in some areas \\
\hline & Acquisition and retention of skilled and diverse talent only in some areas \\
\hline & Acquisition and retention of skilled and diverse talent in all areas \\
\hline
\end{tabular}




\begin{tabular}{|c|c|}
\hline & $\begin{array}{ll}\text { Leadership } \\
\end{array}$ \\
\hline & $\begin{array}{c}\text { What level of leadership support is bolstering technology maturity and continuous learning in } \\
\text { the research center? }\end{array}$ \\
\hline & There is leadership opposition to technology maturity and organizational learning \\
\hline & The leadership is indifferent to technology maturity and organizational learning \\
\hline & The leadership provides some support to technology maturity and organizational learning \\
\hline & $\begin{array}{c}\text { The leadership provides good support and advocacy to technology maturity and organizational } \\
\text { learning }\end{array}$ \\
\hline & $\begin{array}{c}\text { The leadership is enthusiastic about the support and advocacy of technology maturity and } \\
\text { organizational learning }\end{array}$ \\
\hline & $\begin{array}{ll}\text { Strategic Management } \\
\end{array}$ \\
\hline & What level of strategic management is being implemented in the research center? \\
\hline & $\begin{array}{c}\text { There is no strategic vision, plan, or management and implementation of technology maturity and } \\
\text { organizational learning }\end{array}$ \\
\hline & $\begin{array}{c}\text { There are strategic vision and plan but no management and implementation of technology maturity } \\
\text { and organizational learning }\end{array}$ \\
\hline & $\begin{array}{c}\text { There is some clear strategic vision/plan and management/implementation of technology maturity and } \\
\text { organizational learning }\end{array}$ \\
\hline & $\begin{array}{c}\text { There is a clear and calculated strategic vision/plan and managed implementation of technology and } \\
\text { organizational learning }\end{array}$ \\
\hline জ & Culture and Transparency \\
\hline & $\begin{array}{c}\text { What level of organizational culture (in terms of maturity and learning) has been adopted by } \\
\text { the employees of the research center? }\end{array}$ \\
\hline & Organizational Inertia \\
\hline & Support by the minority of employees \\
\hline & Support by half of the employees \\
\hline & Support by the majority of employees \\
\hline & Governance \\
\hline & What level of governance exists in the research center? \\
\hline & No governance \\
\hline & Simply documented governance with no communications among departments \\
\hline & $\begin{array}{c}\text { Documented and Updated governance in separate departments towards the management of inputs, } \\
\text { structures, processes with goals of improving outcomes }\end{array}$ \\
\hline & $\begin{array}{l}\text { Documented and periodically updated governance with some communication among departments } \\
\text { towards the management of inputs, structures, processes with goals of improving outcomes }\end{array}$ \\
\hline & $\begin{array}{c}\text { Central governance promoting an integrated approach/best practices towards the management of } \\
\text { inputs, structures, processes to improve the outcome }\end{array}$ \\
\hline & Training \\
\hline & $\begin{array}{c}\text { What level of training with goals of organizational maturity and continuous learning exists in } \\
\text { the research center? }\end{array}$ \\
\hline & There is no relevant or dedicated training in the research center \\
\hline & There are some non-multidisciplinary informal trainings the research center \\
\hline & There are some multidisciplinary informal trainings in the research center \\
\hline & There are multidisciplinary formal trainings in the research center \\
\hline & There are routine multidisciplinary formal trainings in the research center \\
\hline
\end{tabular}




\begin{tabular}{|c|c|}
\hline & Standards Compliance \\
\hline & $\begin{array}{c}\text { What is the level of the research center's adherence to and compliance with } \\
\text { standards and regulations? }\end{array}$ \\
\hline & There is no compliance with existing regulations \\
\hline & There is low compliance with existing regulations \\
\hline & The health organization is somewhat compliant with existing relevant regulations \\
\hline & The health organization is highly compliant with existing relevant regulations \\
\hline & The health organization is completely compliant with existing relevant regulations \\
\hline & \begin{tabular}{|l} 
Policy Preparedness and Adaption \\
\end{tabular} \\
\hline & $\begin{array}{c}\text { To what extent is the research center flexible and ready in responding and } \\
\text { adapting to policy changes? }\end{array}$ \\
\hline & $\begin{array}{l}\text { The research center has no readiness plans in terms of response and adaptation to } \\
\text { policy changes and only reacts post-change. }\end{array}$ \\
\hline & $\begin{array}{l}\text { The research center aspires to increase flexibility and preparedness for policy changes } \\
\text { but has no plans for different scenarios yet. }\end{array}$ \\
\hline & $\begin{array}{l}\text { The research center has plans for different policy change scenarios in terms of } \\
\text { readiness and adaptation. }\end{array}$ \\
\hline & $\begin{array}{l}\text { The research center is proactive in terms of readiness and adaptation to policy changes } \\
\text { with a systematic plan which is periodically reviewed and modified. }\end{array}$ \\
\hline & \begin{tabular}{|l} 
Privacy \\
\end{tabular} \\
\hline & \begin{tabular}{|c|} 
What level of privacy measures exists in the research center? \\
\end{tabular} \\
\hline & No privacy measures, metrics, or procedures \\
\hline & $\begin{array}{l}\text { Privacy drove by regulatory framework including Ad-hoc vulnerability scanning, basic } \\
\text { metrics and processes. }\end{array}$ \\
\hline & $\begin{array}{l}\text { Privacy protection is driven by the regulatory framework, risk management, IRBs, and } \\
\text { processes }\end{array}$ \\
\hline & $\begin{array}{l}\text { Robust and continuous privacy protection driven by the regulatory framework, risk } \\
\text { management, IRBs, and efficient metrics-based processes }\end{array}$ \\
\hline & Informing Policy \\
\hline & What level of policy informing power existing in the research center? \\
\hline & Research center is not involved with informing policy \\
\hline & $\begin{array}{l}\text { Research center is involved with } 1 \text { out of } 3 \text { (participating in expert panels, providing } \\
\text { evidence and comments regarding policies under consideration, securing contracts) }\end{array}$ \\
\hline & $\begin{array}{l}\text { Research center is involved with } 2 \text { out of } 3 \text { (participating in expert panels, providing } \\
\text { evidence and comments regarding policies under consideration, securing contracts) }\end{array}$ \\
\hline & $\begin{array}{l}\text { Research center is involved by participating in expert panels, providing evidence and } \\
\text { comments regarding policies under consideration, and securing contracts. }\end{array}$ \\
\hline
\end{tabular}


Table 31: Validated Financial Metrics

\section{Resource Allocation}

How well is the research center performing in terms of resource allocation and their alignment with technology maturity and continuous learning?

Prioritization of resources are not aligned with technology maturity and continuous organizational learning

Prioritization of resources are minimally aligned with technology maturity and continuous organizational learning

Prioritization of resources are somewhat aligned with technology maturity and continuous organizational learning

Prioritization of resources are highly aligned with technology maturity and continuous organizational learning

Prioritization of resources are completely aligned with technology maturity and continuous organizational learning

\section{Funding and Mission Alignment}

What level of alignment exists between incentives and technology maturity and continuous organizational learning?

Incentives and funding are not aligned with missions of technology maturity and continuous organizational learning

Incentives and funding are minimally aligned with missions of technology maturity and continuous organizational learning

Incentives and funding are somewhat aligned with missions of technology maturity and

continuous organizational learning

Incentives and funding are highly aligned with missions of technology maturity and

continuous organizational learning

Incentives and funding are completely aligned with missions of technology maturity and continuous organizational learning

\section{Cost Reduction}

What level of success has the research center had in cutting costs?

The research center has had no success in cutting costs

The research center has had success in cutting costs through layoffs and waste elimination

The research center has had success in cutting costs as a result of waste eliminations with compromising quality

The research center has had success in cutting costs as a result of waste eliminations without compromising quality

The research center has had success in cutting costs as a result of increased maturity, learning, continuous improvement, and waste eliminations without compromising quality

\section{Financial Synergy with Policies and Priorities}

What level of financial synergy does the research center have with policies and priorities?

The research center has had no success in meeting reimbursement programs' goals or accessing government, foundation, and other grants

The research center has had little success in meeting reimbursement programs' goals or accessing government, foundation, and other grants

The research center has had success in 1 out of 2 (meeting reimbursement programs' goals, accessing government, foundation, and other grants)

The research center is successful in meeting reimbursement programs' goals and accessing government, foundation, and other grants 


\section{Data Management}

\section{Desirability}

No measures are taken to assure and maintain data security, privacy, governance, or ethics

Initiatives and efforts are put into 1out of 4 (security, privacy, governance, ethics)

Initiatives and efforts are put into 2 out of 4 (security, privacy, governance, ethics)

Initiatives and efforts are put into 3 out of 4 (security, privacy, governance, ethics)

Initiatives and efforts to assure and maintain data security, privacy, governance, and ethics exist

\section{Data Management}

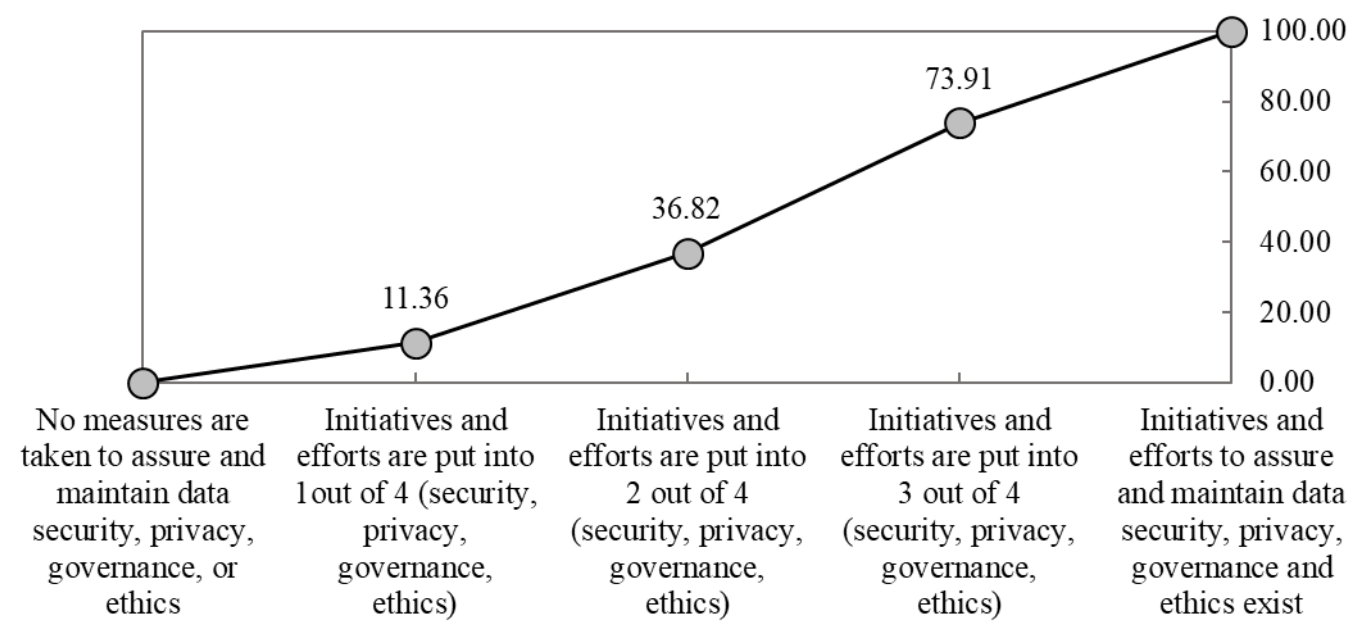

Figure 39: Data Management Metrics Value

Table 33: Supporting Infrastructure Metrics Value

\section{Supporting Infrastructure}

Desirability

No socio-technical infrastructure to handle data management, analysis, dissemination, and integration of learned knowledge

Socio-technical infrastructure for 1 out of 4 (data management, data analysis, knowledge dissemination, knowledge integration)

Socio-technical infrastructure for 2 out of 4 (data management, data analysis, knowledge dissemination, knowledge integration)

Socio-technical infrastructure for 3 out of 4 (data management, data analysis, knowledge dissemination, knowledge integration)

Socio-technical infrastructure in place for management and analysis of data and dissemination and integration of learned knowledge

100.00 


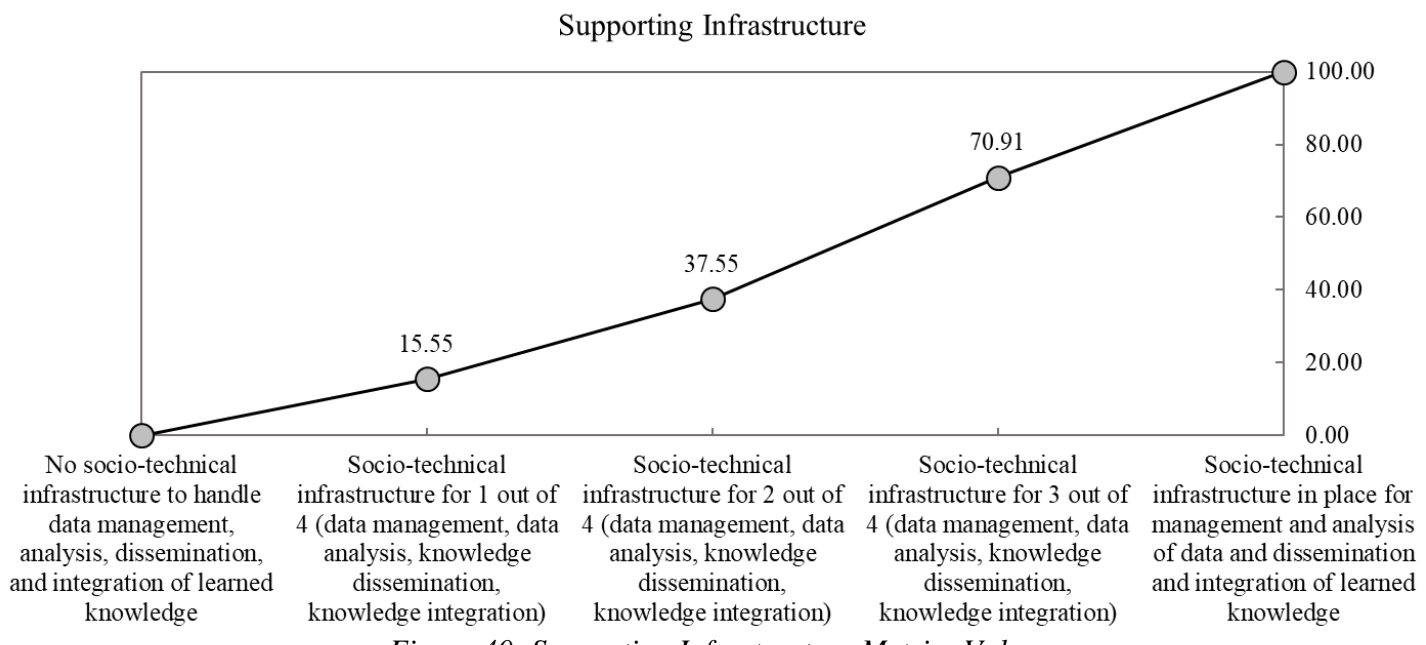

Table 34: Real-Time Knowledge Access and Sharing Metrics Value

\section{Real-Time Knowledge Access and Sharing}

No real-time data access and sharing capabilities in place

Some real-time data capture, access, and sharing but no integration of learned knowledge

Data capture, access, and sharing of real-time data but no integration of learned knowledge

Real-time data and information capture, access, and sharing concurrent to integration of learned knowledge
Desirability

0.00

25.55

59.18

100.00

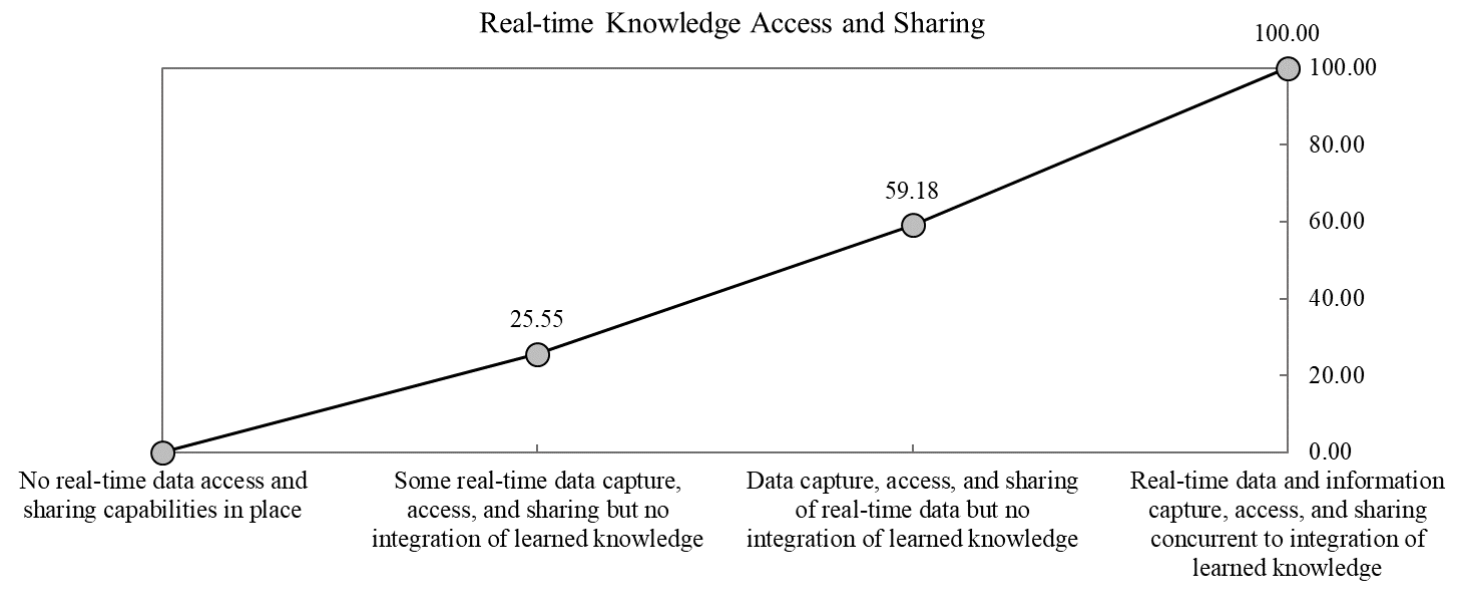

Figure 41: Real-Time Knowledge Access and Sharing Metrics Value 
Technologies and IT products are somewhat user-friendly

Technologies and IT products are designed and reviewed (periodically) with optimization of user experience in mind

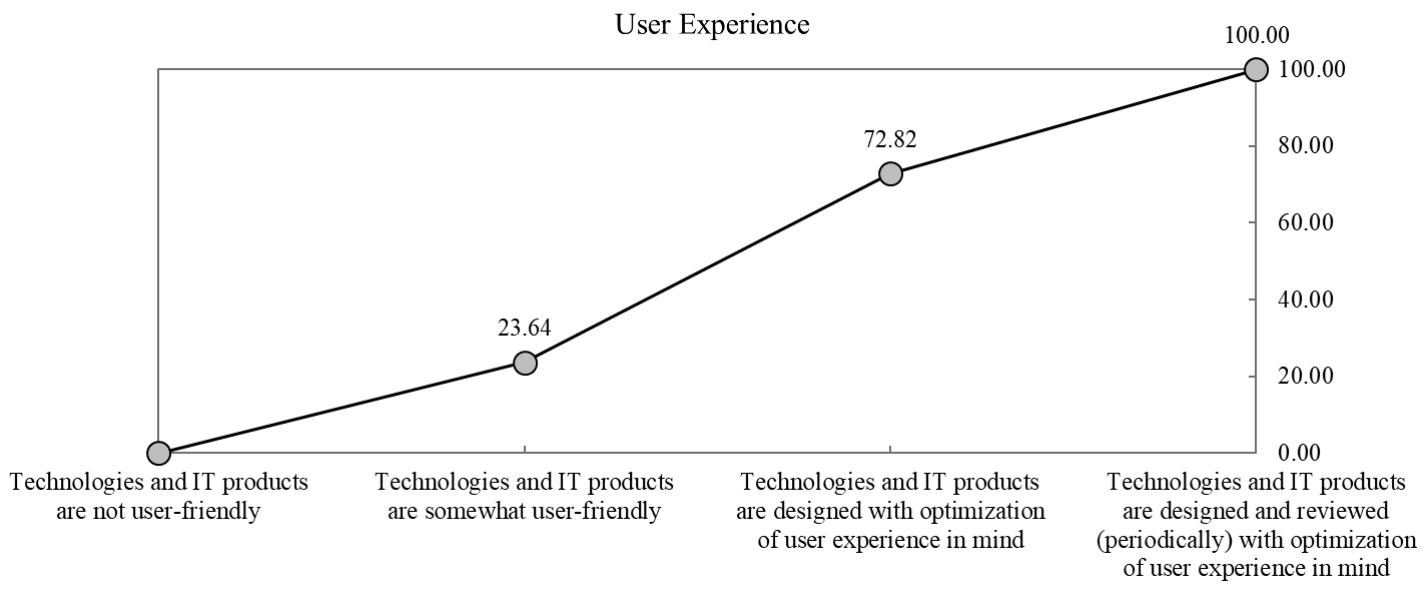

Figure 42:User Experience Metrics Value

Table 36: Stakeholder Trust Metrics Value

\section{Stakeholder Trust}

Not trusted by the overwhelming majority of stakeholders

Trusted by the minority of stakeholders

Trusted by half of the stakeholders

Trusted by the majority of stakeholders

\section{Desirability}

0.00

11.36

45.73

100.00 


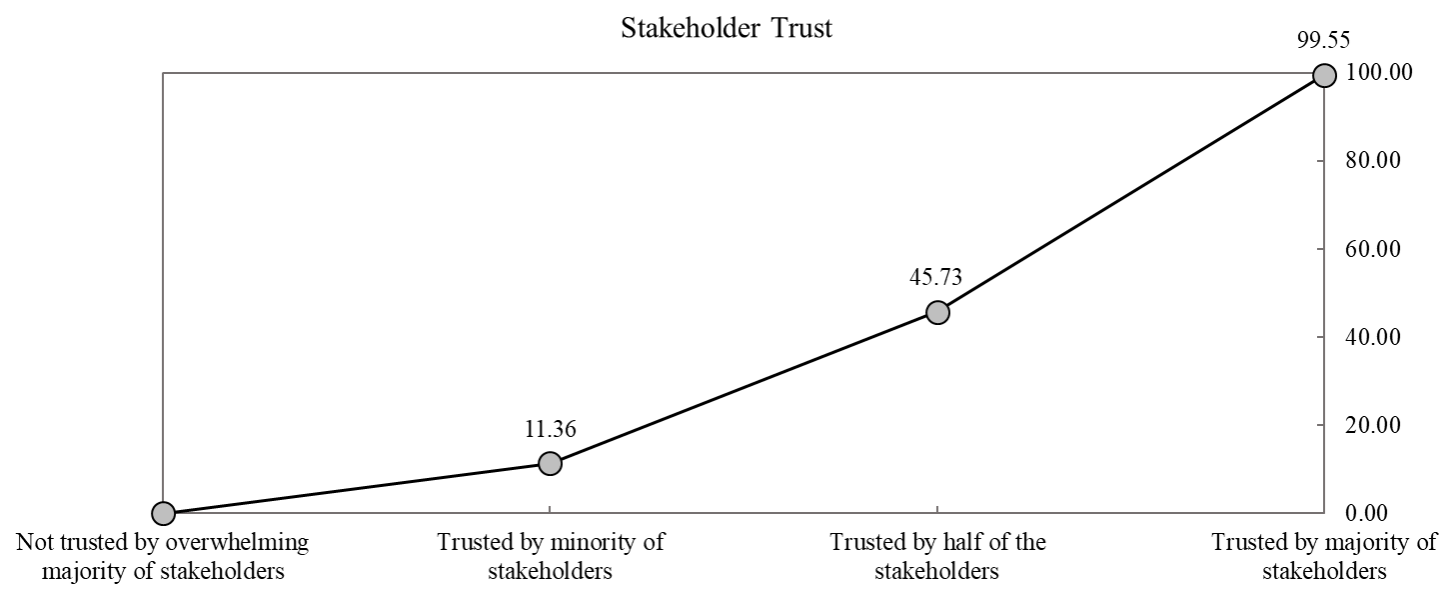

Figure 43: Stakeholder Trust Metrics Value

Table 37: Accessibility Metrics Value

Accessibility

Stakeholders are not benefiting from the clinical and research advantages of the research center

Only a minority of stakeholders are benefiting from the clinical and research advantages of the research center

Half of the stakeholders are benefiting from the clinical and research advantages

\section{of the research center}

The majority of stakeholders are benefiting from the clinical and research advantages of the research center

All stakeholders are benefiting from the clinical and research advantages of the research center

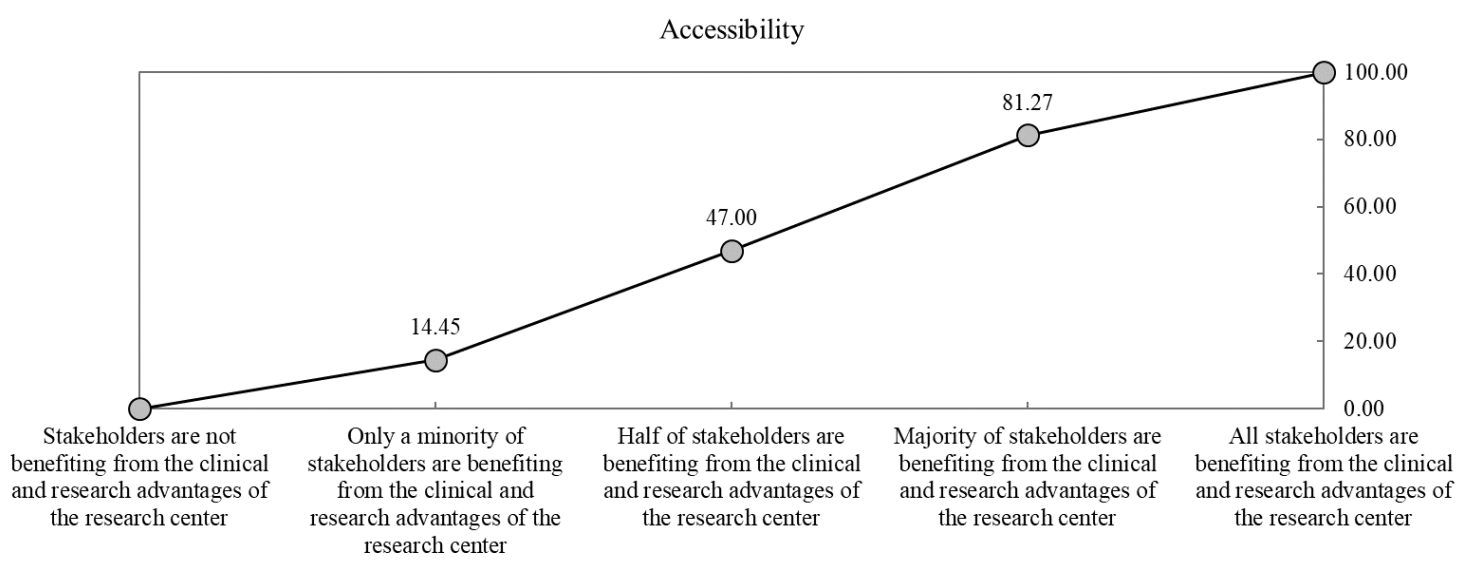

Figure 44: Accessibility Metrics Value 
Table 38: Stakeholder Engagement Metrics Value

\section{Stakeholder Engagement}

Desirability

Stakeholders are opposed to participate in research center's continuous improvement and learning projects and initiatives

Stakeholders are reluctant to participate in research center's continuous improvement and learning projects and initiatives

Half of the stakeholders are eager to participate in research center's continuous improvement and learning projects and initiatives

Majority of the stakeholders are eager to participate in research center's continuous improvement and learning projects and initiatives

All stakeholders are eager to participate in research center's continuous improvement and learning projects and initiatives

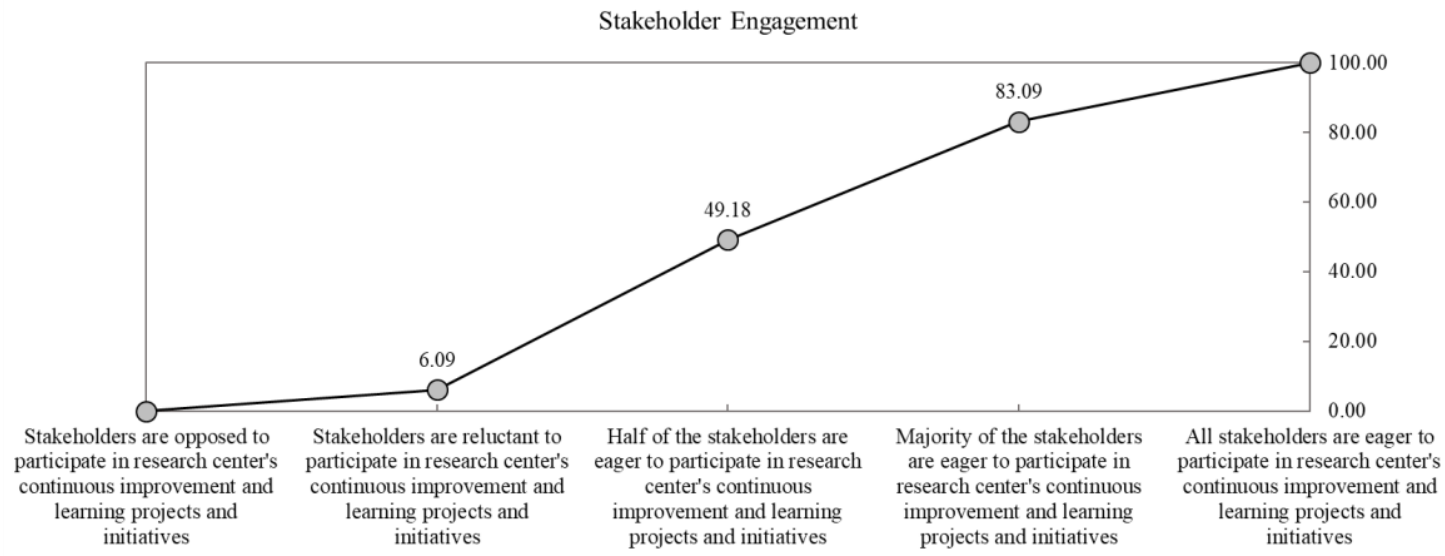

Figure 45: Stakeholder Engagement Metrics Value

Table 39: Talent Acquisition and Retention Metrics Value

\section{Talent Acquisition and Retention}

No acquisition and retention of skilled and diverse talent

Acquisition of skilled and diverse talent only in some areas

Acquisition and retention of skilled and diverse talent only in some areas

Acquisition and retention of skilled and diverse talent in most areas
Desirability

0.00

27.73

55.73

96.82 


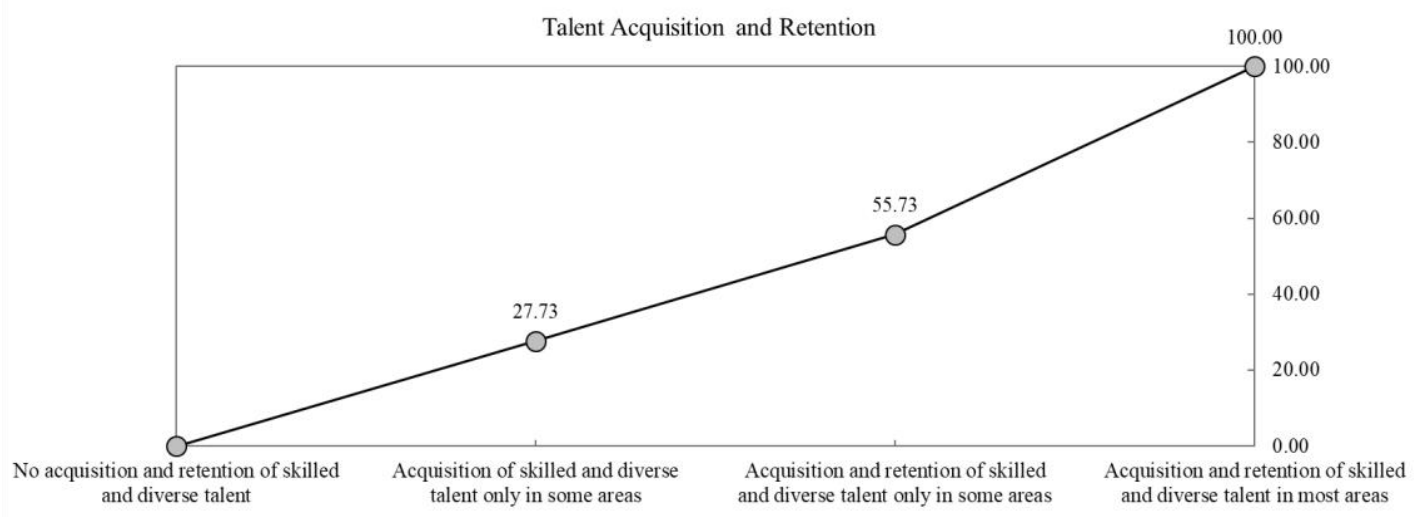

Figure 46: Talent Acquisition and Retention Metrics Value

Table 40: Leadership Metrics Value

\section{Leadership \\ Desirability}

There is leadership opposition to technology maturity and organizational learning 0.09

The leadership is indifferent to technology maturity and organizational learning

The leadership provides some support to technology maturity and organizational

$$
\text { learning }
$$

The leadership provides good support and advocacy to technology maturity and

$$
\text { organizational learning }
$$

The leadership is enthusiastic about the support and advocacy of technology maturity

$$
\text { and organizational learning }
$$

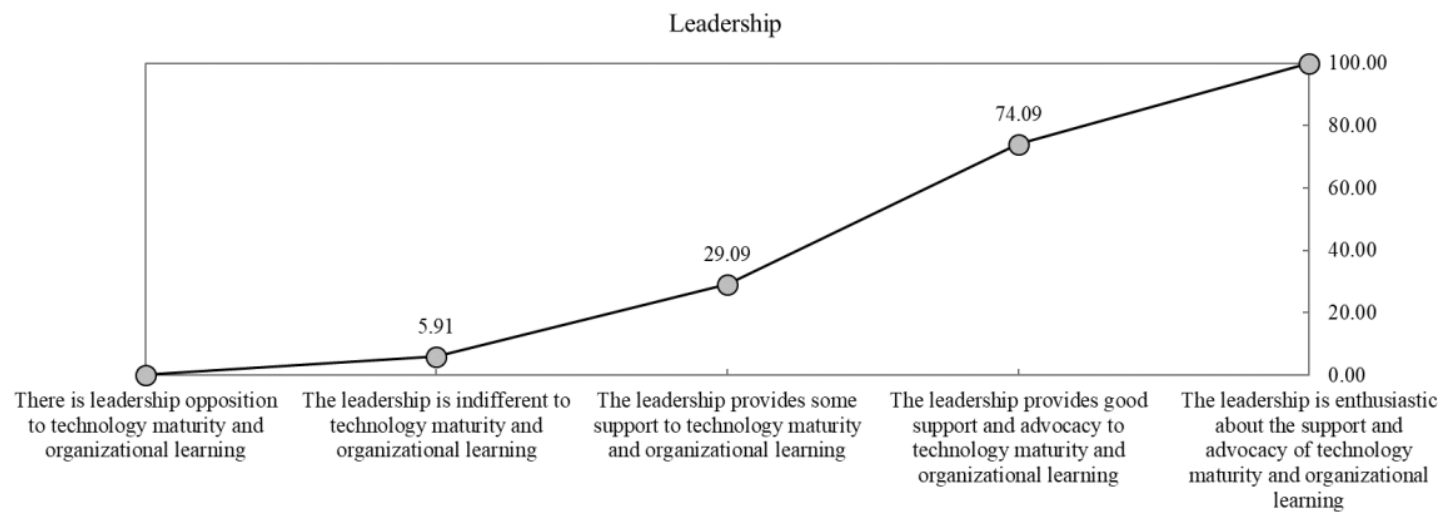

Figure 47: Leadership Metrics Value 
Strategic Management

Desirability

There is no strategic vision, plan, or management and implementation of technology maturity and organizational learning

There are strategic vision and plan but no management and implementation of technology maturity and organizational learning

There is some clear strategic vision/plan and management/implementation of technology maturity and organizational learning

There is a clear and calculated strategic vision/plan and managed implementation of technology and organizational learning

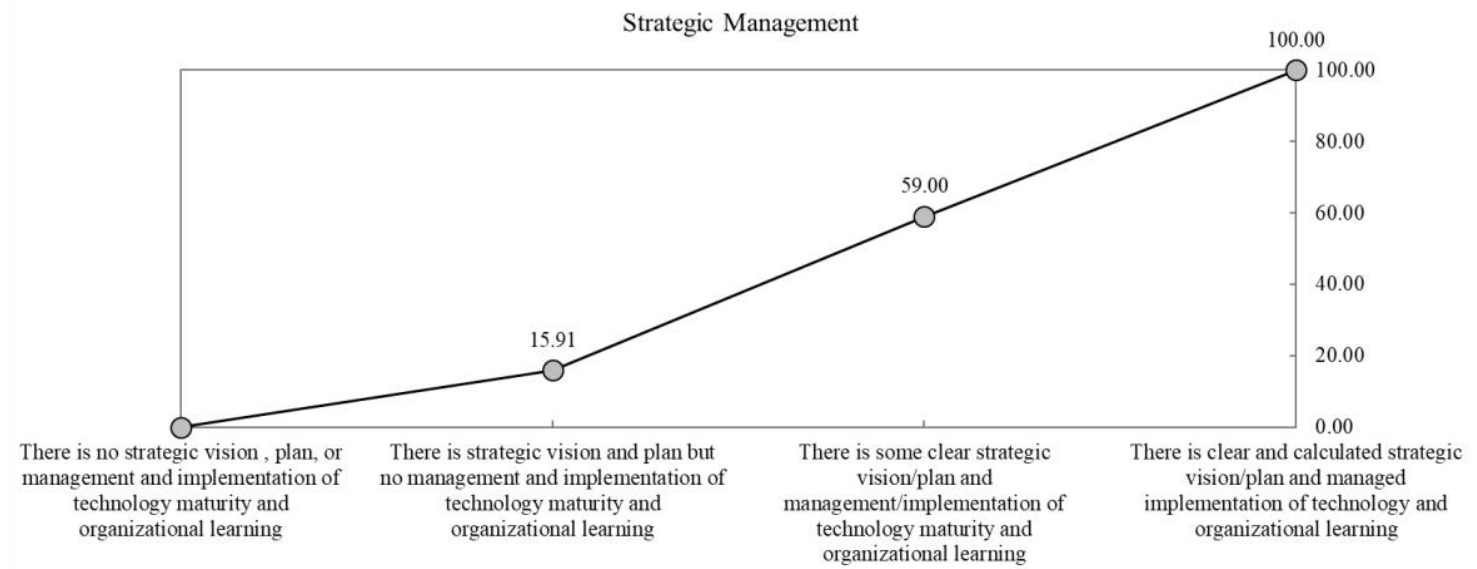

Figure 48: Strategic Management Metrics Value

Table 42: Culture and Transparency Metrics Value

\section{Culture and Transparency}

Organizational Inertia

Support by minority of employees

Support by half of employees

Support by majority of employees

\section{Desirability}

0.45

17.36

51.45

100.00 


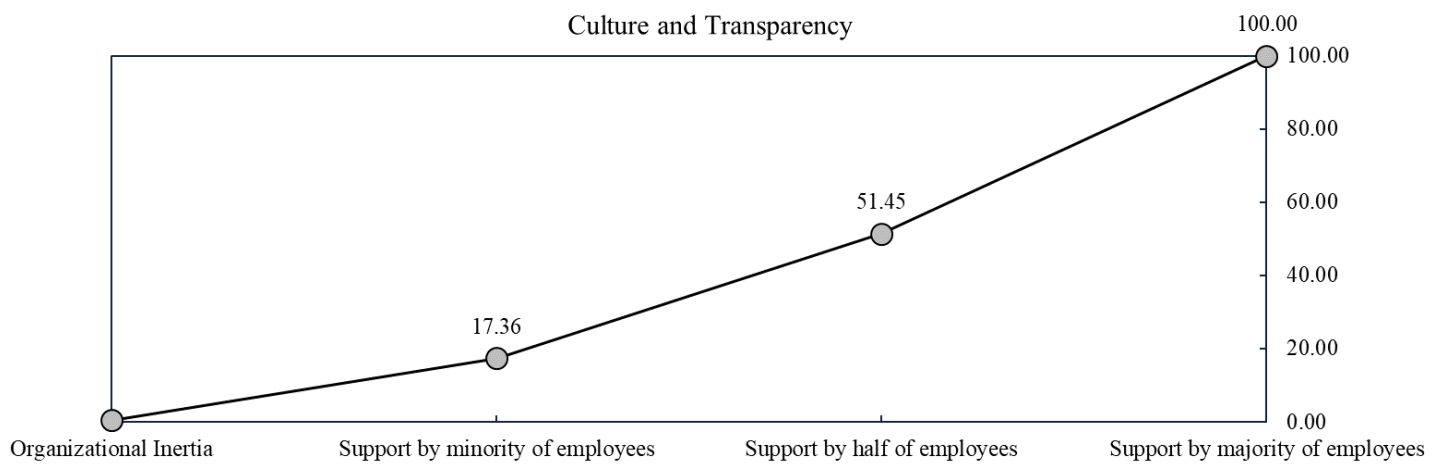

Figure 49: Culture and Transparency Metrics Value

Table 43: Training Metrics Value

Training

There is no relevant or dedicated training in the research center

There are some non-multidisciplinary informal trainings the research center

There are some multidisciplinary informal trainings in the research center

There is multidisciplinary formal training in the research center

There is routine multidisciplinary formal training in the research center
Desirability

0.00

15.27

40.36

76.36

100.00

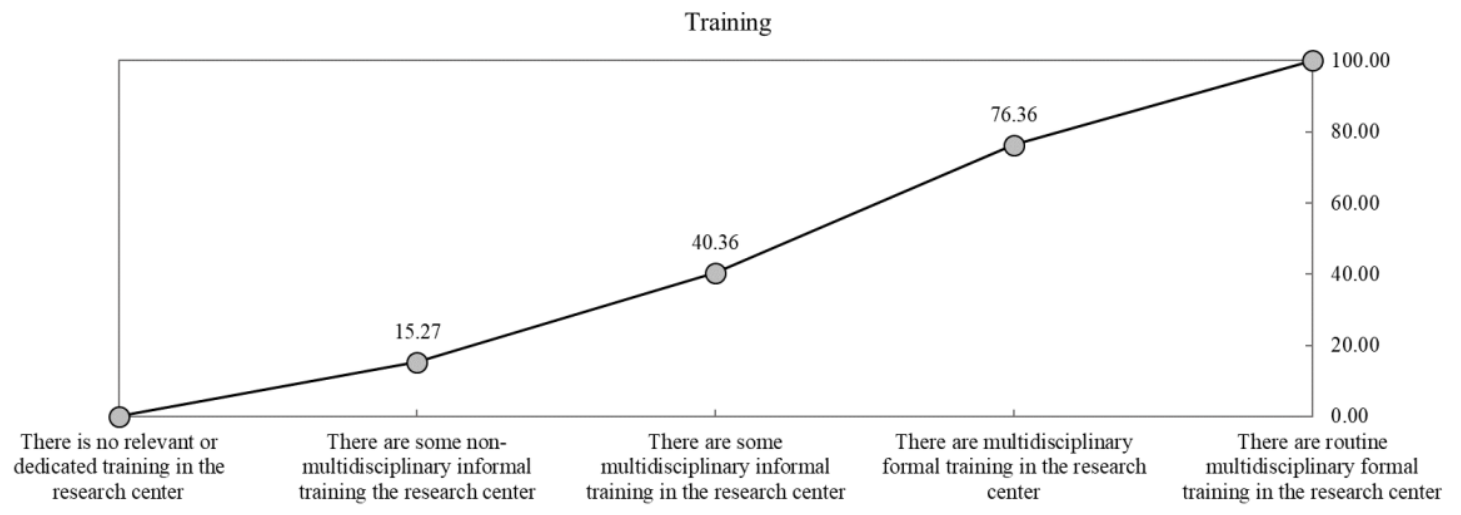

Figure 50: Training Metrics Value 
Table 44: Governance Metrics Value

\section{Governance}

\section{Desirability}

No governance

0.00

Simply documented governance with no communications among departments

Documented and Updated governance in separate departments towards the management of inputs, structures, processes with goals of improving outcomes

Documented and periodically updated governance with some communication among departments towards the management of inputs, structures, processes

with goals of improving outcomes

Central governance promoting an integrated approach/best practices towards the management of inputs, structures, processes to improve the outcome

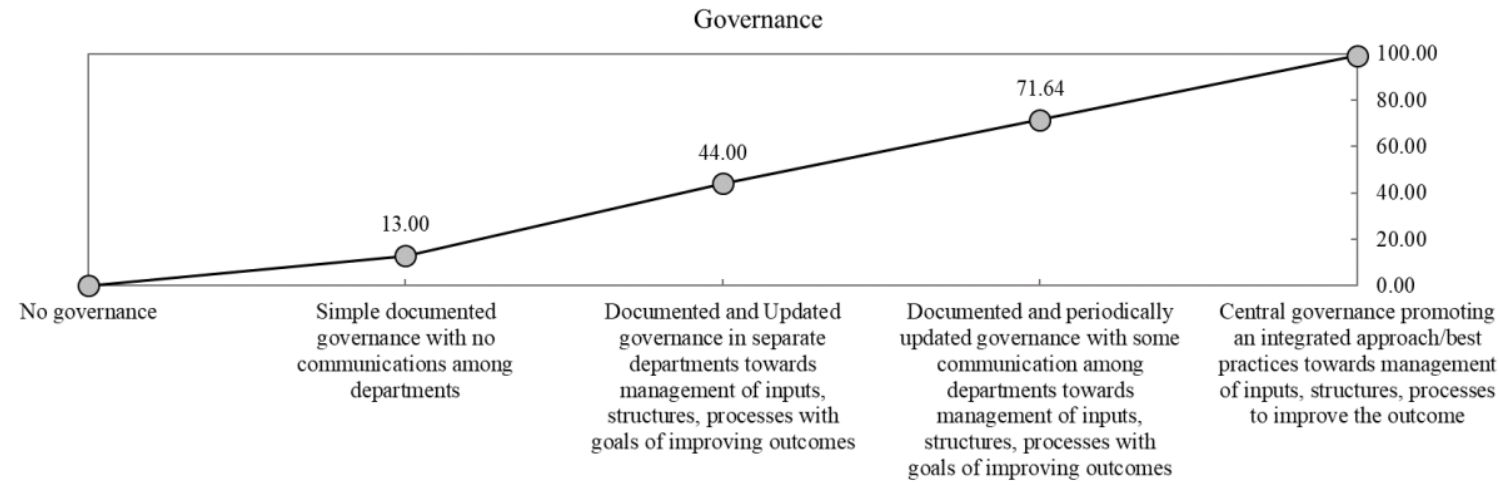

Figure 51: Governance Metrics Value

Table 45: Standards Compliance Metrics Value

Desirability

There is no compliance with existing regulations

There is low compliance with existing regulations

10.27

The research center is somewhat compliant with existing relevant regulations

The research center is highly compliant with existing relevant regulations

The research center is completely compliant with existing relevant regulations

100.00 


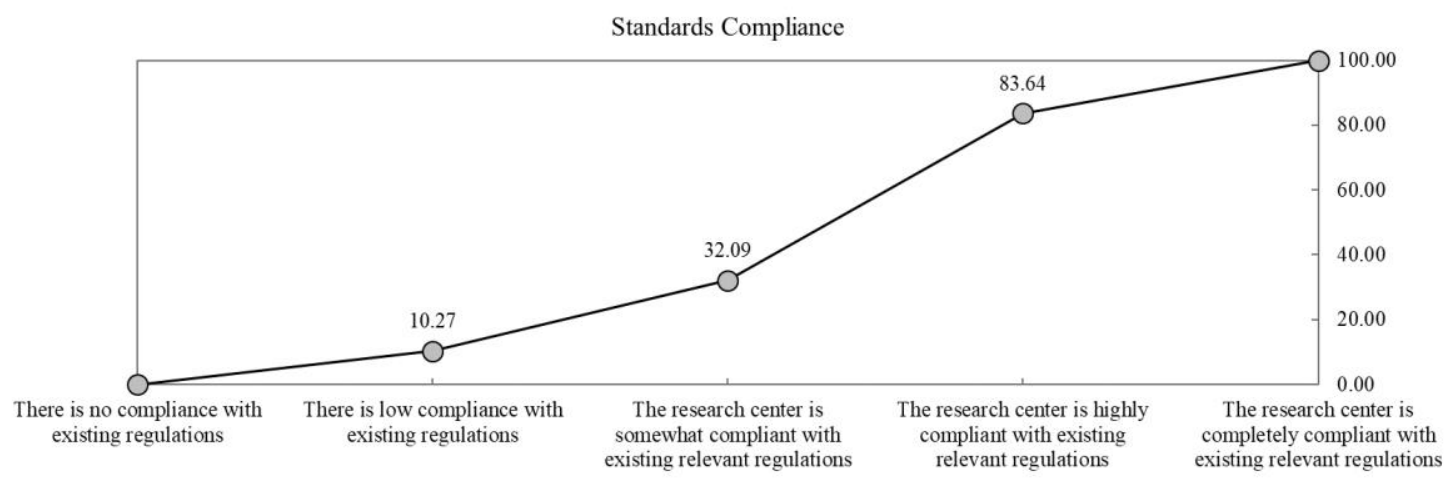

Figure 52: Standards Compliance Metrics Value

Table 46: Policy Preparedness and Adaptation Metrics Value

Policy Preparedness and Adaptation

Desirability

The research center has no readiness plans in terms of response and adaptation

to policy changes and only reacts post-change.

The research center aspires to increase flexibility and preparedness for policy changes but has no plans for different scenarios yet.

The research center has plans for different policy change scenarios in terms of readiness and adaptation.

The research center is proactive in terms of readiness and adaptation to policy changes with a systematic plan which is periodically reviewed and modified.

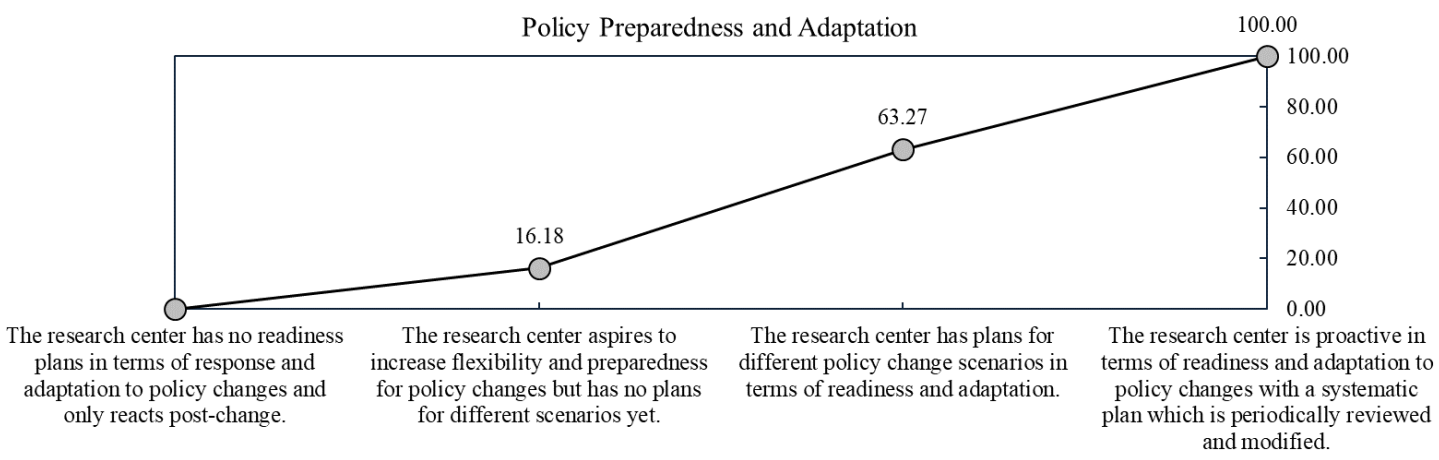

Figure 53: Policy Preparedness and Adaptation Metrics Value 
No privacy measures, metrics, or procedures $\quad 0.00$

Privacy driven by regulatory framework including Ad-hoc vulnerability scanning, basic metrics and processes.

Privacy protection is driven by the regulatory framework, risk management, IRBs, and processes

Robust and continuous privacy protection driven by the regulatory framework, risk management, IRBs, and efficient metrics-based processes

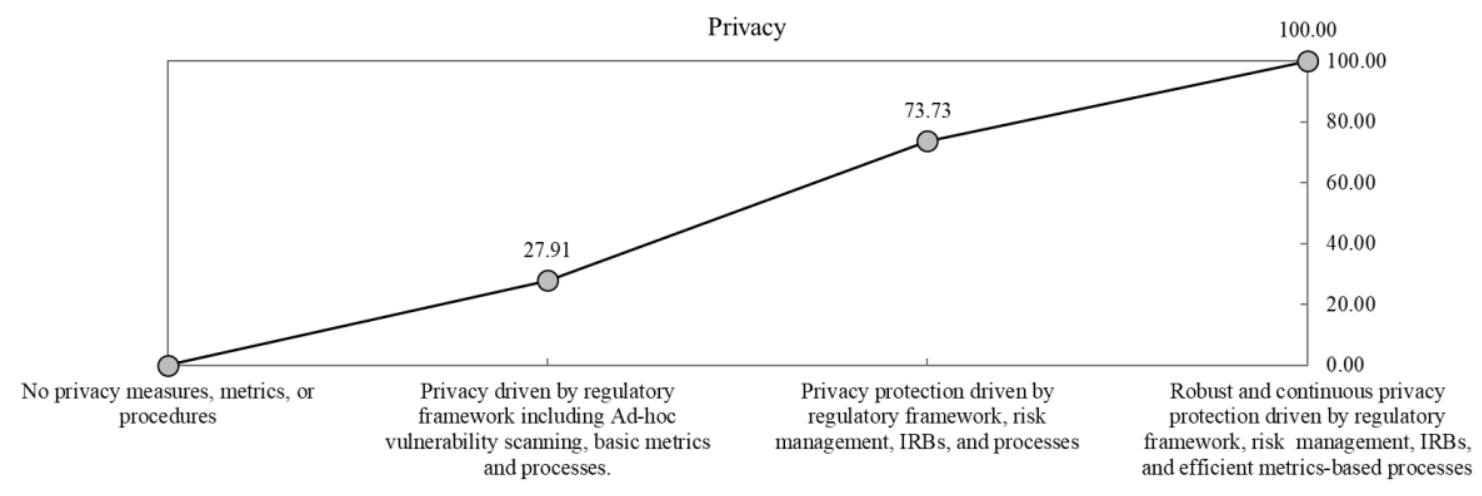

Figure 54: Privacy Metrics Value

Table 48: Informing Policy Metrics Value

\section{Informing Policy}

The research center is not involved with informing policy

The research center is involved with 1 out of 3 (participating in expert panels, providing evidence and comments regarding policies under consideration, securing contracts)

The research center is involved with 2 out of 3 (participating in expert panels, providing evidence and comments regarding policies under consideration, securing contracts)

The research center is involved by participating in expert panels, providing evidence and comments regarding policies under consideration, and securing contracts.
Desirability

0.00

100.00 


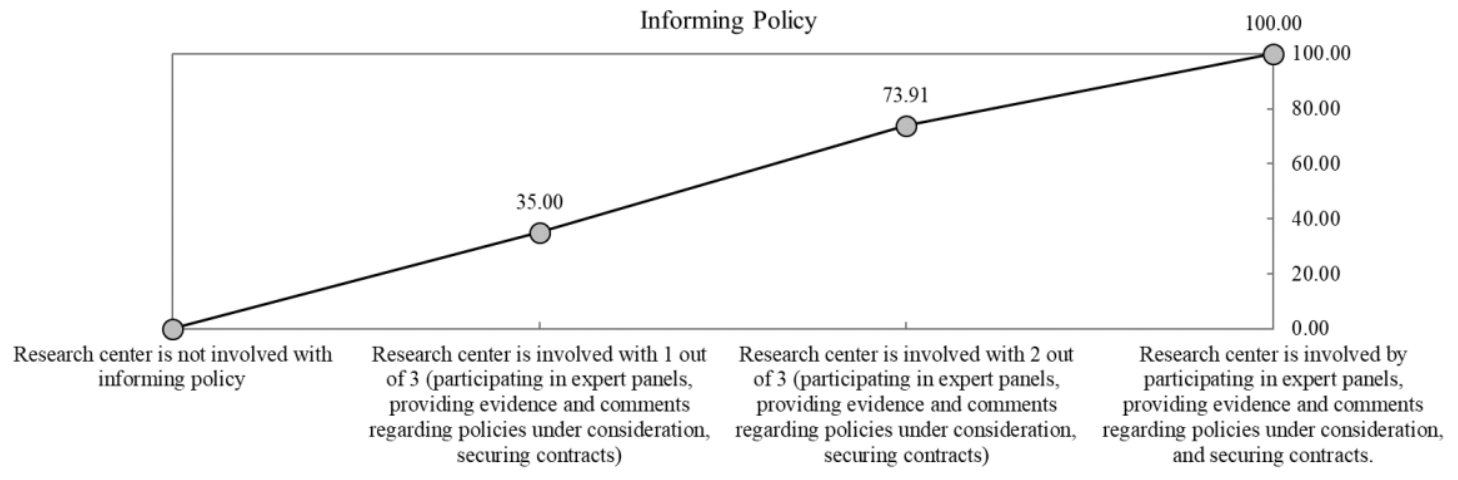

Figure 55: Informing Policy Metrics Value

Table 49:Resource Allocation Metrics Value

Resource Allocation

Prioritization of resources are not aligned with technology maturity and continuous organizational learning

Prioritization of resources are minimally aligned with technology maturity and continuous organizational learning

Prioritization of resources are somewhat aligned with technology maturity and continuous organizational learning

Prioritization of resources are highly aligned with technology maturity and continuous organizational learning

Prioritization of resources are completely aligned with technology maturity and continuous organizational learning

\section{Desirability}

0.00

11.82

100.00

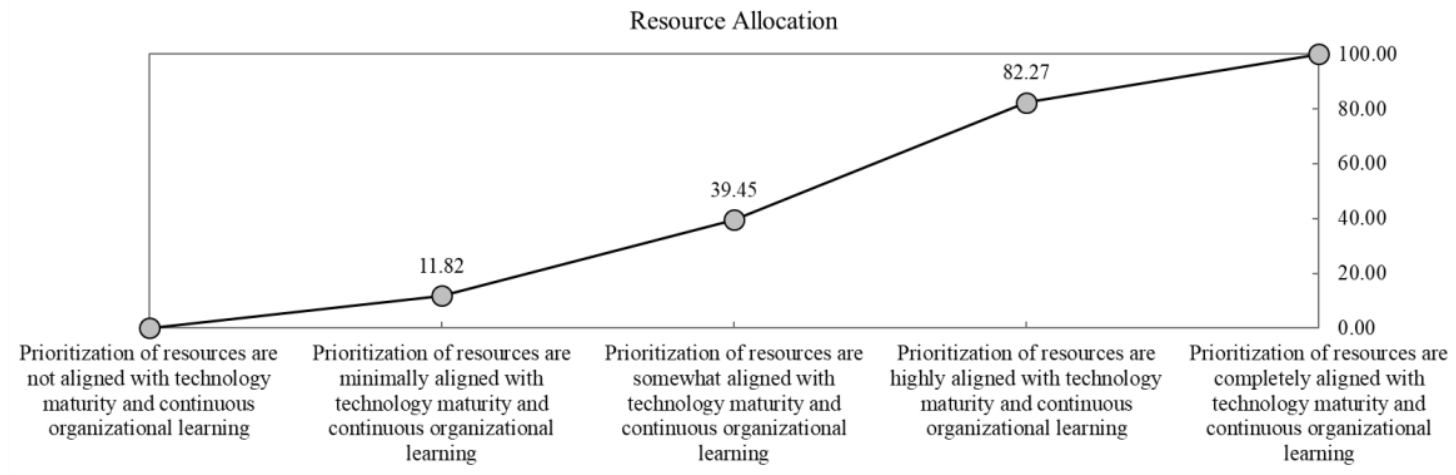

Figure 56: Resource Allocation Metrics Value 


\section{Funding and Mission Alignment}

Desirability

Incentives and funding are not aligned with missions of technology maturity and continuous organizational learning

Incentives and funding are minimally aligned with missions of technology maturity and continuous organizational learning

Incentives and funding are somewhat aligned with missions of technology maturity and continuous organizational learning

Incentives and funding are highly aligned with missions of technology maturity and continuous organizational learning

Incentives and funding are completely aligned with missions of technology maturity and continuous organizational learning

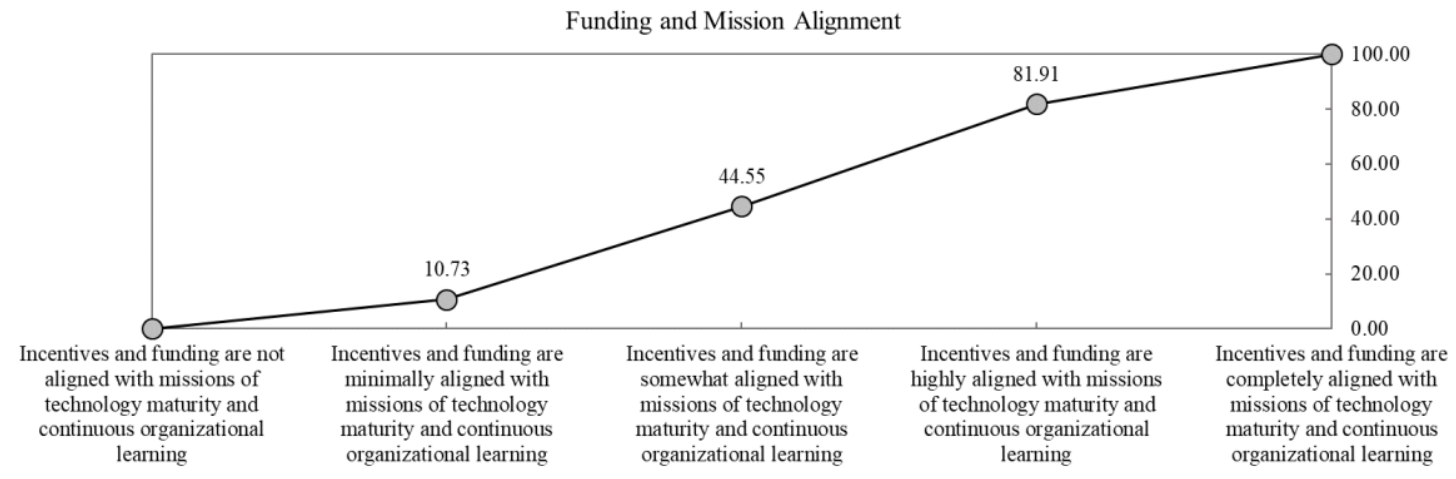

Figure 57: Funding and Mission Alignment Metrics Value

Table 51: Cost Reduction Metrics Value

\section{Cost Reduction}

Desirability

Research center has had no success in cutting costs

Research center has had success in cutting costs through layoffs and waste elimination

Research center has had success in cutting costs as a result of waste eliminations with compromising quality

Research center has had success in cutting costs as a result of waste eliminations without compromising quality

Research center has had success in cutting costs as a result of increased maturity, learning, continuous improvement, and waste eliminations without 


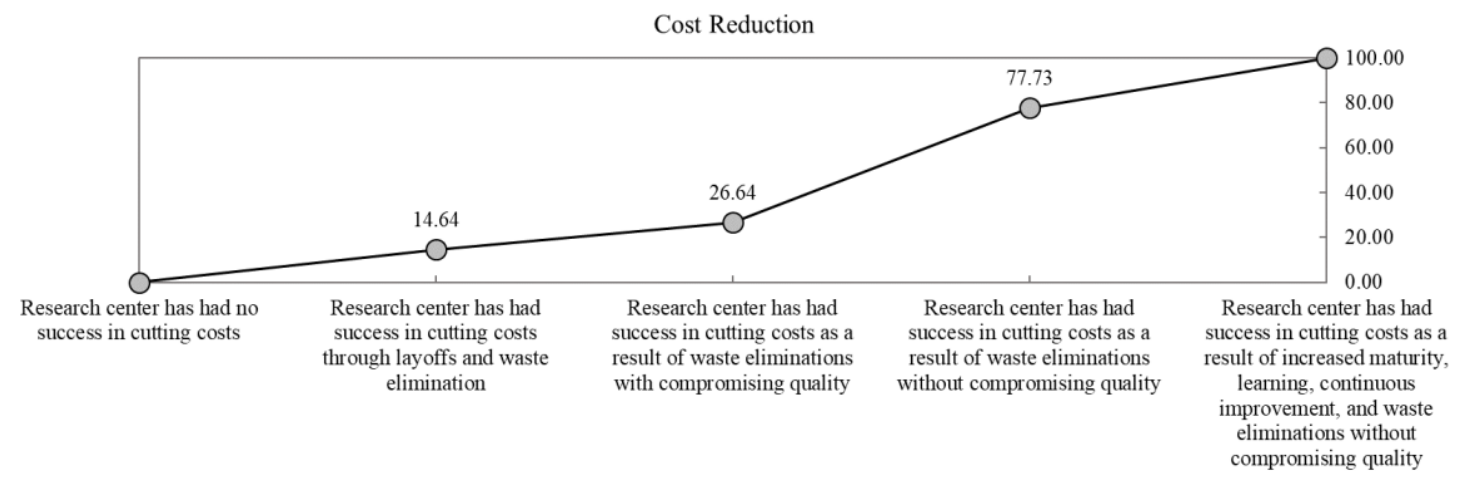

Figure 58: Cost Reduction Metrics Value

Table 52: Financial Synergy with Policies and Priorities Metrics Value

Financial Synergy with Policies and Priorities

Desirability

Research center has had no success in meeting reimbursement programs' goals or accessing government, foundation, and other grants

Research center has had little success in meeting reimbursement programs' goals or accessing government, foundation, and other grants

Research center has had success in 1 out of 2 (meeting reimbursement programs' goals, accessing government, foundation, and other grants)

Research center is successful in meeting reimbursement programs' goals and accessing government, foundation, and other grants

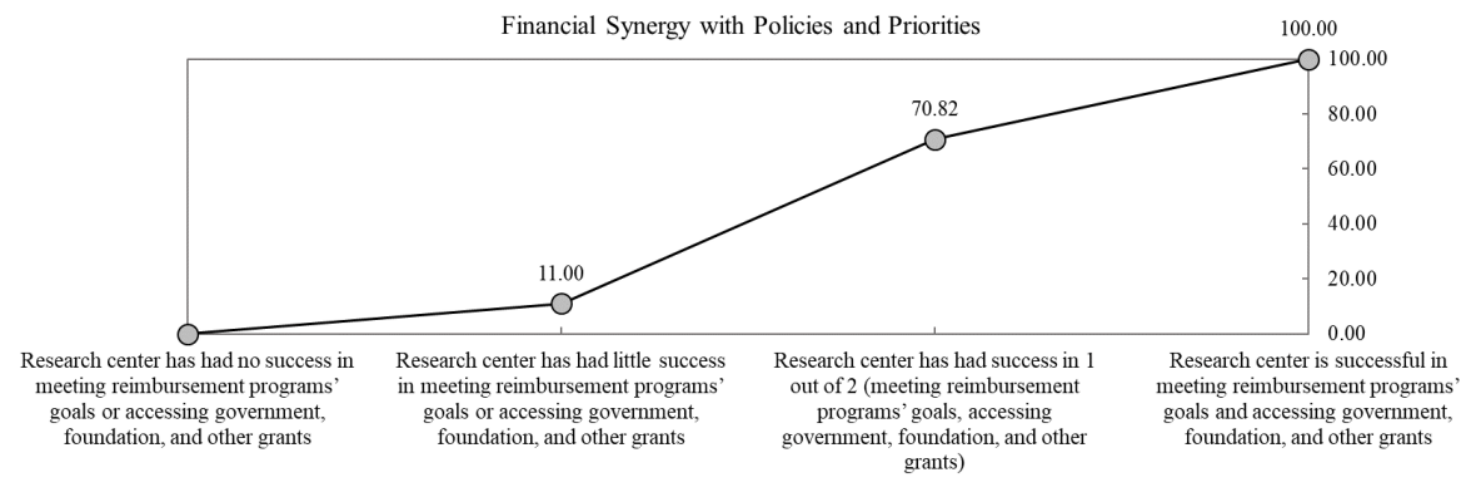

Figure 59: Financial Synergy with Policies and Priorities Metrics Value 


\subsection{Model Quantification Results}

The next part is quantifying the criteria and perspectives based on the pairwise comparison done by the expert panel in the ETM HDM tool software. Each expert performed the pairwise comparisons between the perspective and then each of the underlying criteria for the respective perspective. The results were partly generated by ETM HDM software and partly by manual calculation in Microsoft Excel in order to obtain a better breakdown of the results and validation measurements. The perspective and criteria weights are shown in Tables 49 and 50, respectively.

Table 53: Perspective Weights

\begin{tabular}{|c|c|c|c|}
\hline Perspectives & Mean & $\%$ & Ranking \\
\hline Technology & 0.200 & 20 & 3 \\
\hline Social & 0.217 & 22 & 2 \\
\hline Organizational & 0.226 & $\mathbf{2 3}$ & $\mathbf{1}$ \\
\hline Regulatory & 0.181 & 18 & 4 \\
\hline Financial & 0.175 & 17 & 5 \\
\hline Inconsistency & $\mathbf{0 . 0 1 3}$ & \multicolumn{2}{|l}{} \\
\cline { 2 - 4 }
\end{tabular}




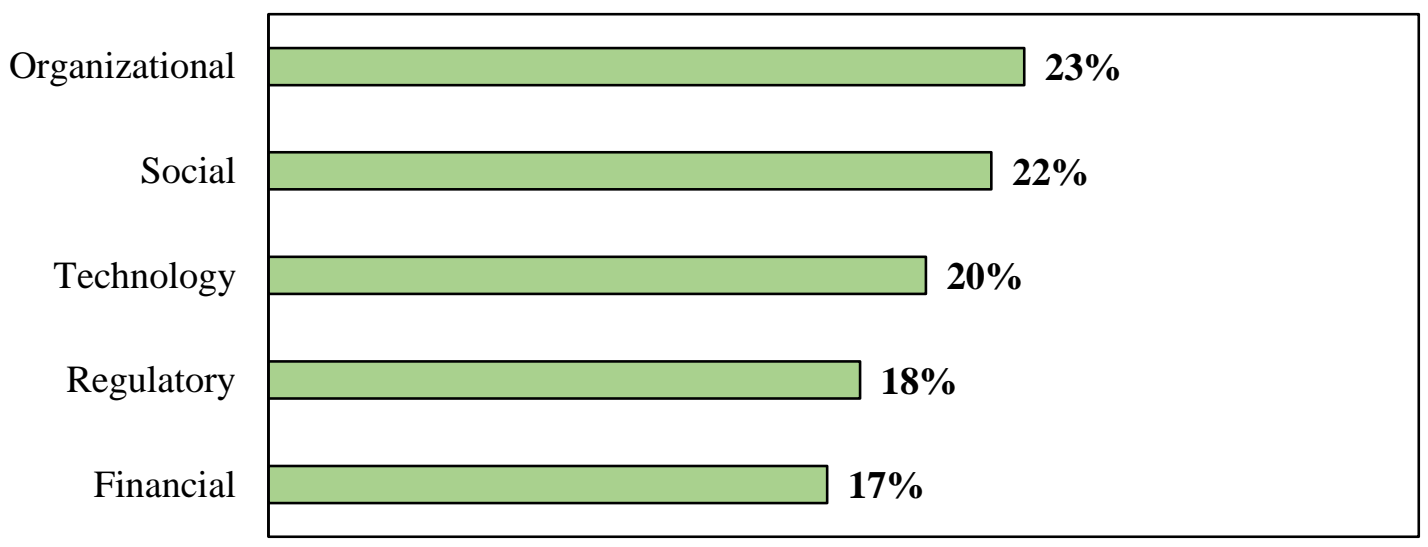

Figure 60: Perspective Weights

Table 54: Technology Criteria Local Weights

\begin{tabular}{|c|c|c|c|}
\hline Perspective & Criteria & Average & $\%$ \\
\hline \multirow{3}{*}{} & Data Management & 0.264 & 27 \\
\cline { 2 - 4 } & Supporting Infrastructure & 0.223 & 22 \\
\cline { 2 - 4 } & Real-time Knowledge Access and Sharing & 0.275 & 28 \\
\cline { 2 - 4 } & User Experience & 0.235 & 23 \\
\hline
\end{tabular}

\section{Technology}

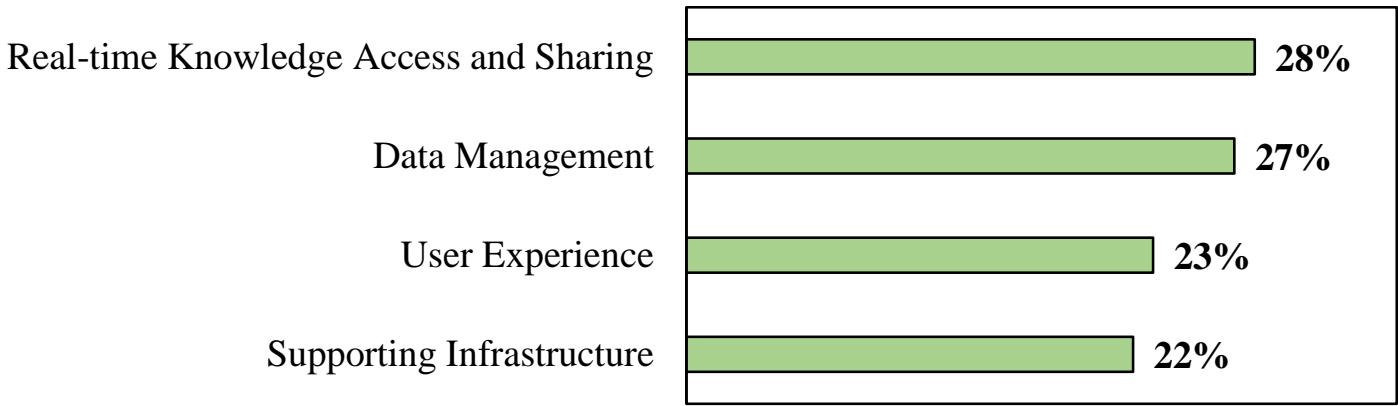

Figure 61: Technology Criteria Local Weights

Table 55: Social Criteria Local Weights 


\begin{tabular}{|c|c|c|c|}
\hline Perspective & Criteria & Average & $\%$ \\
\hline \multirow{3}{*}{$\bar{*}$} & Stakeholder Trust & 0.226 & 23 \\
\cline { 2 - 4 } & Accessibility & 0.196 & 20 \\
\cline { 2 - 4 } & Stakeholder Engagement & 0.295 & 30 \\
\cline { 2 - 4 } & Talent Acquisition and Retention & 0.281 & 28 \\
\hline
\end{tabular}

\section{Social}

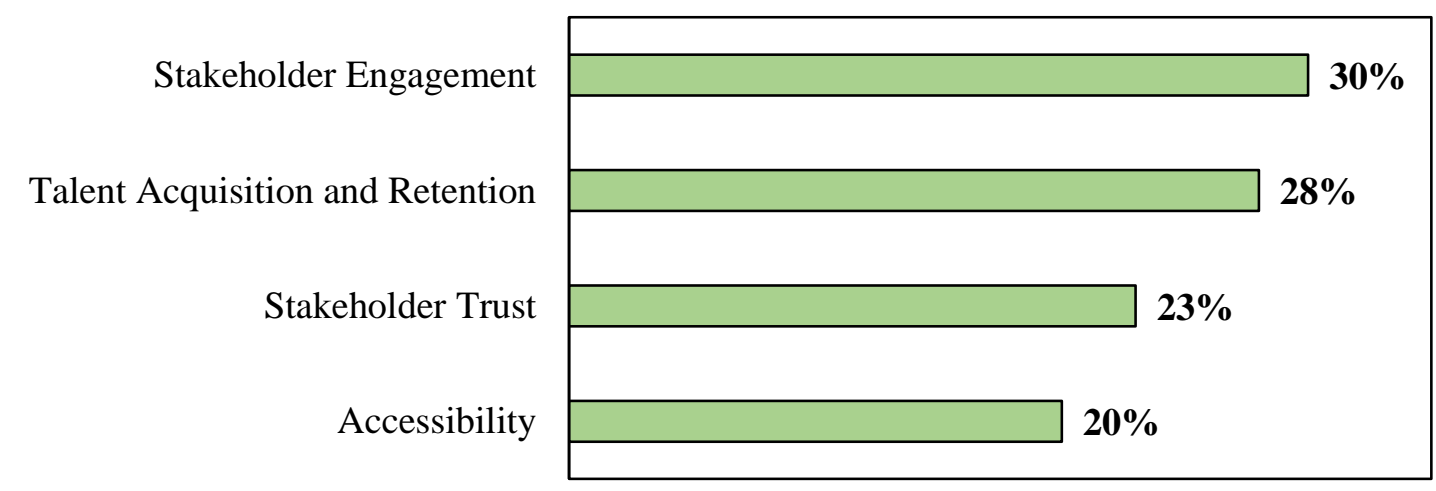

Figure 62: Social Criteria Local Weights

Table 56: Organizational Criteria Local Weights

\begin{tabular}{|c|c|c|c|}
\hline Perspective & Criteria & Average & $\%$ \\
\hline \multirow{3}{*}{} & Leadership & 0.188 & 19 \\
\cline { 2 - 4 } & Strategic Management & 0.238 & 24 \\
\cline { 2 - 4 } & Culture and Transparency & 0.200 & 20 \\
\cline { 2 - 4 } & Training & 0.191 & 19 \\
\cline { 2 - 4 } & Governance & 0.181 & 18 \\
\hline
\end{tabular}




\section{Organizational}

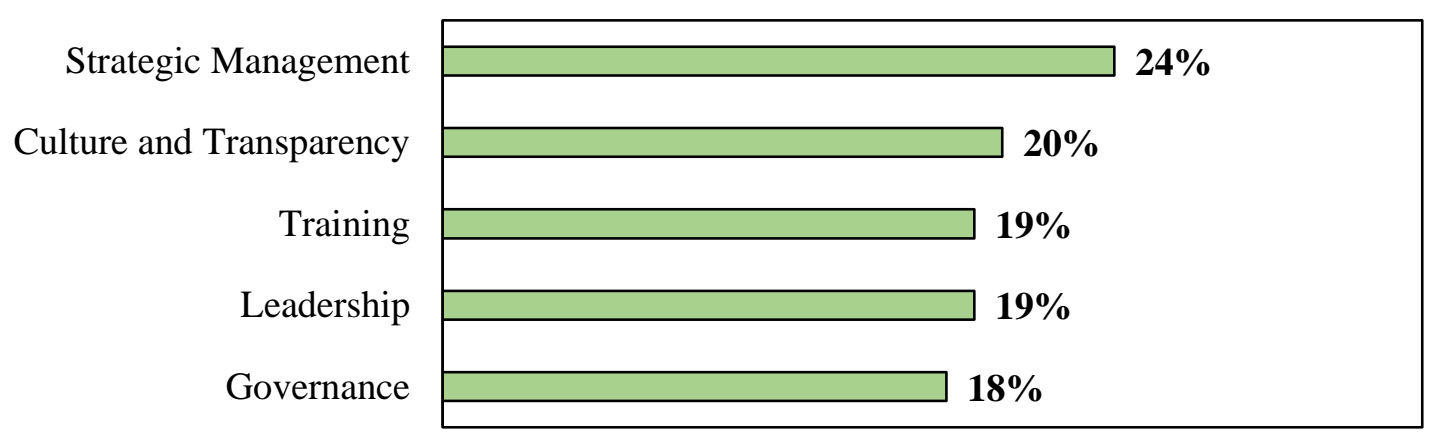

Figure 63: Organizational Criteria Local Weights

Table 57: Regulatory Criteria Local Weights

\begin{tabular}{|c|c|c|c|}
\hline Perspective & Criteria & Average & $\%$ \\
\hline \multirow{2}{*}{} & Standards Compliance & 0.240 & 24 \\
\cline { 2 - 4 } & Policy Preparedness and Adaptation & $\mathbf{0 . 2 8 9}$ & 29 \\
\cline { 2 - 4 } & Privacy & $\mathbf{0 . 2 3 2}$ & 23 \\
\cline { 2 - 4 } & Informing Policy & $\mathbf{0 . 2 3 7}$ & 24 \\
\hline
\end{tabular}

\section{Regulatory}

Policy Preparedness and Adaptation

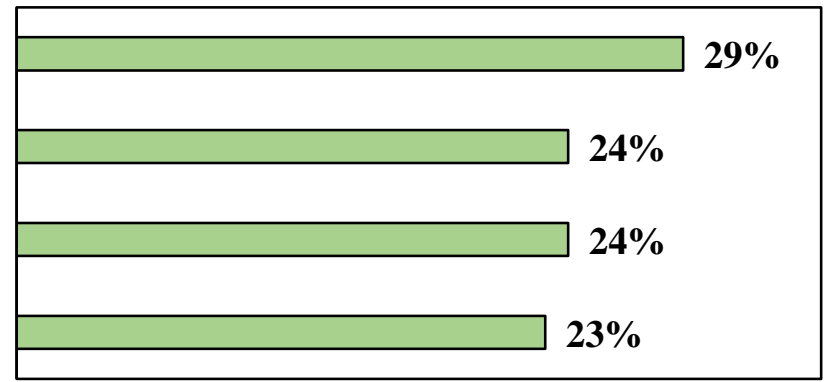

Figure 64: Regulatory Criteria Local Weights 
Table 58: Financial Criteria Local Weights

\begin{tabular}{|c|c|c|c|}
\hline Perspective & Criteria & Average & $\%$ \\
\hline \multirow{3}{*}{} & Resource Allocation & 0.217 & 22 \\
\cline { 2 - 4 } & Funding and Mission Alignment & 0.302 & 30 \\
\cline { 2 - 4 } & Cost Reduction & 0.190 & 19 \\
\cline { 2 - 4 } & Financial Synergy with Policies and & 0.290 & 29 \\
\hline
\end{tabular}

Financial

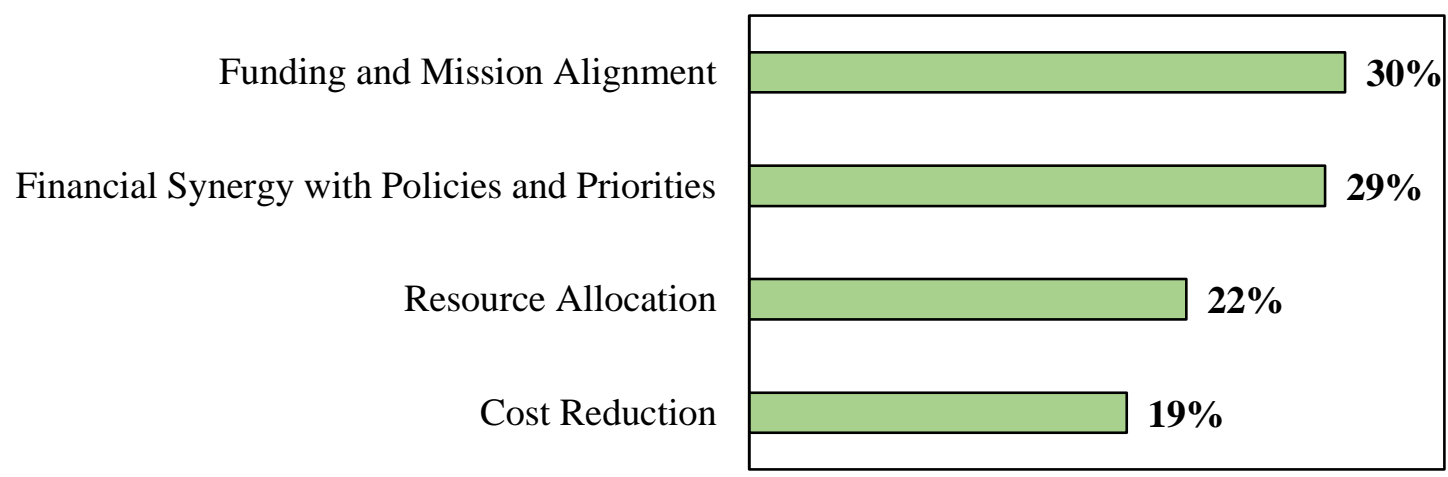

Figure 65: Financial Criteria Local Weights

Table 59: Inconsistency and Disagreement Results

\begin{tabular}{|l|l|l|}
\hline Validation Test & Value & Threshold \\
\hline Disagreement & $\mathbf{0 . 0 2 0}$ & $<=0.1$ \\
\hline Inconsistency & $\mathbf{0 . 0 1 7}$ & $<=0.1$ \\
\hline
\end{tabular}

As it can be seen from the table above the inconsistency for both perspective and criteria are in the acceptable ratio being 0.013 and 0.017 respectively which are below $10 \%$ as Kocaoglu established in 1983 as the acceptable threshold for the inconsistency in HDM models (Kocaoglu, 1983). 
Moreover, the disagreement measure is in the acceptable range $(0.020)$ (below 10\% $(0.1)$ (Chan, 2013; Estep, 2017; Shaygan et al., 2017)). The inconsistency and disagreement thresholds have been used as indicators for validating the HDM results according to the literature (Iskin, 2014; Abbas, 2016; Estep, 2017; Shaygan, Ozdemir-Gungor, D. Kutgun and Daneshi, 2017).

By looking at the universal weights (each criterions' weights in the context of the whole model), "Stakeholder Engagement" is the criteria with the highest weight as determined by the expert panel followed by "Talent Acquisition and Retention", and "Strategic Management" with $0.064(6.4 \%), 0.060$ (6.0\%), and $0.057(5.7 \%)$ respectively. The global weights for the criteria and the sorted global weights are shown in Figure 67-69.

Based on the results of the experts' pairwise comparisons, the Organizational perspective plays the most important role in technology management in research centers with $23 \%$ followed by the social aspect with $22 \%$. By taking a deeper look into these two perspectives in the criteria weights (local weights), we can see that "Strategic Management" plays a very important role (24\% of the organizational perspective) in the maturity of research centers. This is aligned with the literature as it stresses the importance of strategic management in healthcare systems maturity and implementation and adoption of continuous learning (Bernstein et al., 2015; Brooks, El-Gayar and Sarnikar, 2015; Demir, 2018) (This result can alleviate the gap that was mentioned the gap analysis section about lack of literature on literature focusing mainly or solely on the management side of the healthcare system maturity, continuous learning, and its socio-technological infrastructure (Frenk et al., 2010;Pronovost et al., 2017)). In Second place, there is culture 
and transparency followed by training take place with $20 \%$ and $19 \%$ respectively. As for culture and transparency which is defined as "The extent to which a research center's employees have adopted and are committed to a culture of continuous learning and practice transparency to safeguard stakeholder trust in order to improve the health of individuals, communities, and diverse populations.", the resulted weight is in agreement with the literature demonstrating this criterion as an important factor on the maturity of healthcare organizations (Institute of Medicine (IOM), 2007; Saunders and Smith, 2013; Bernstein et al., 2015; Curcin, 2016; Kraft et al., 2017). Examples of the importance of training can be seen in health organizations training teams to train health medical and nursing students and residents, together in cross-functional teams to enforce a more effective transformation culture (Lynch et al., 2000; Gopee, 2002; Grossmann, Goolsby, Olsen and McGinnis, 2011).

In terms of the Social perspective, Stakeholders' engagement was found to be the most important one with $30 \%$ of the local weight in the social aspect. This aligns with the literature emphasizing the importance of healthcare organizations ability to engage stakeholders (patients, providers, payers, policymaker, purchasers, and families) with continuous learning and maturity initiatives with goals of improving patient satisfaction, quality of care, reducing the costs (Mandl et al., 2014; Price-Haywood, 2015; Kraft et al., 2017). Closely following is the talent acquisition and retention with $28 \%$.

From the regulatory perspective, "Policy Preparedness and Adaptation" takes the top spot with $29 \%$. As expected, this criterion is very important as it is related to research centers' flexibility and agility in responding and adapting to changes such as new regulations, 
legislations, and policies in terms of anticipation and readiness. This readiness and anticipation can be in terms of resources, policies, strategies, and management. Moreover, side by side to complying with policies and regulations (24\%), academic health research centers can have a significant impact on research and evidence-informed health policy through taking part in new collaborative models of research $(24 \%)$. In the second place, due to the diffusion of meaningful use at state and federal levels, compliance with standards is a big part of health organizations endeavors in continuous improvement and maturity as the Quality Payment Program creates a new framework for rewarding organizations/clinicians who provide higher-value care (HealthIT.gov, 2018). The close range of percentages in perspectives such as regulatory shows how important each of the criteria is for pursuing goals of maturity and continuous learning. As an example, although the privacy aspect holds $23 \%$ of the local weight in this perspective (lowest among the four), is crucial for every research center. In the world of healthcare, data scientist and business intelligence professionals should guarantee the privacy of data such as demographic information, medical histories, test and laboratory results, mental health conditions, insurance information among others when analyzing, visualizing, or publishing protected health data. For this reason, for actions such as data analysis, visualization, creating predictive models, etc., there is a need for departments to request those specific protected data/database/servers, etc. Again, as there is a good chance that the data used in healthcare organizations is PHI data, it is critical for health organizations or research centers to make sure that the privacy of data such as demographic information, medical histories, test and laboratory results, mental health conditions, insurance information among others are protected from being breached in email hacks and phishing 
attacks. Penalties for HIPAA violations are tiered which is shown in Figure 66. In conclusion in healthcare organizations, especially the ones which have the aim of increasing their technology management maturity through continuous learning and constant transformation of data into knowledge, privacy plays a pivotal part and a backbone to a functioning knowledge-producing health system.

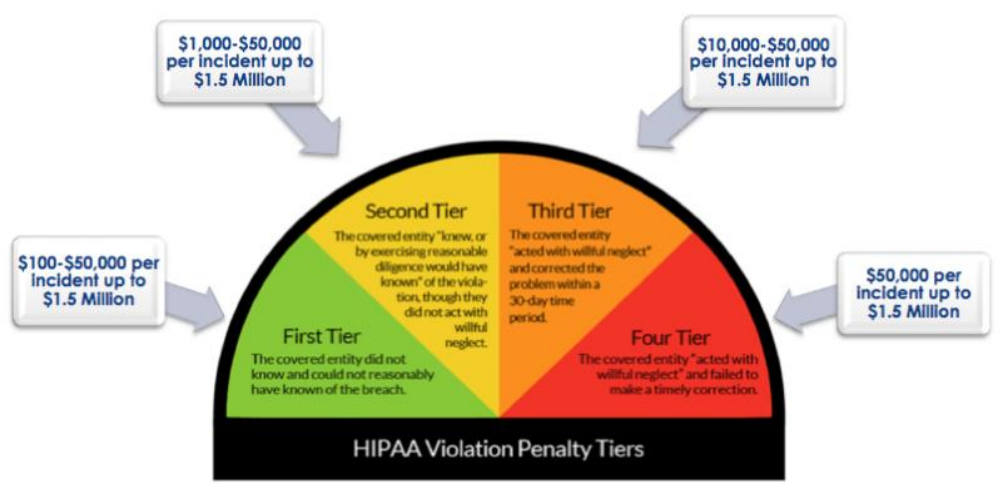

Figure 66: HIPAA Violation Penalty Tiers (compliancy-group, 2018)

In the Financial perspective, "Funding and Mission Alignment" was selected as the most important criterion with $30 \%$ followed very closely by "Financial Synergy with Policies and Priorities with 29\%. Based on these results, the alignment of incentives and funding with the encouragement of continuous learning and technology maturity and rewarding high care value is important while there is a need for research centers to invest time and skills into accessing funds such as grants and ensuring that they meet reimbursement programs' goals.

Last but not least, in the Technology Perspective, Real-time Knowledge Access and Sharing was selected as the most important criterion with $28 \%$. This criterion was mined from the literature review and it is significant especially in the case where the focus of maturity is on continuous learning as means to improve quality continuously. This 
criterion was defined as "Data systems and tools used to capture, share, and integrate data, information, and knowledge gained from biomedical, clinical, and managerial research into the organization in real-time" (Saunders and Smith, 2013; Bernstein et al., 2015; Cahan and Cimino, 2017; Morain, Kass and Grossmann, 2017). The advances in technology (tools and data systems) which expedite the transformation of data into knowledge can bolster this element. In healthcare organizations, since many data elements are patient-related, the access and sharing of these data can be tricky and more research into the opportunities and implications caused by this criterion should be studied further. Protected health information (PHI), also known as personal health information, generally refers to demographic information, medical histories, test and laboratory results, mental health conditions, insurance information, and other data that a healthcare professional collects to identify an individual and determine appropriate care (Rouse, 2018). It should be stressed that a criterion such as data management (27\%) and supporting infrastructure (22\%) are critical to the real-time knowledge access and sharing among others as enablers of this important criterion. 


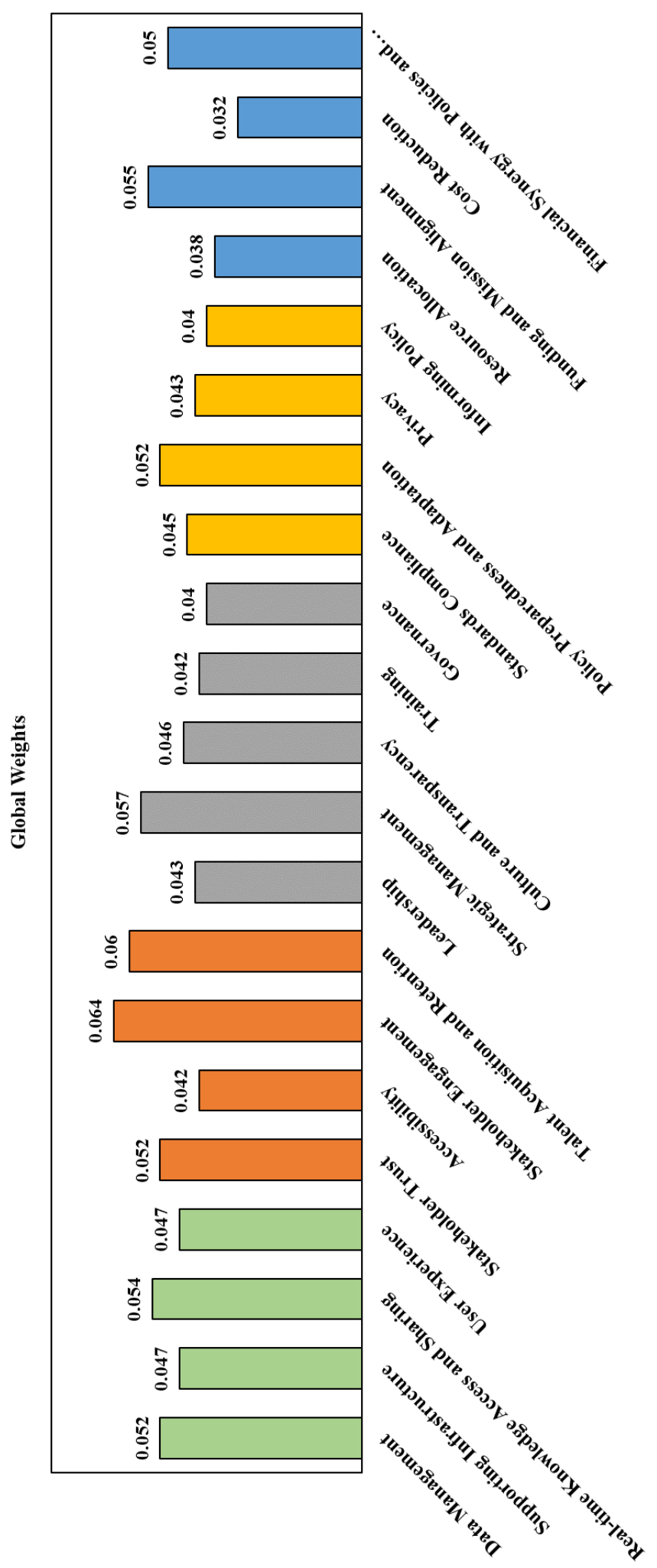

Figure 67: Global Weights 


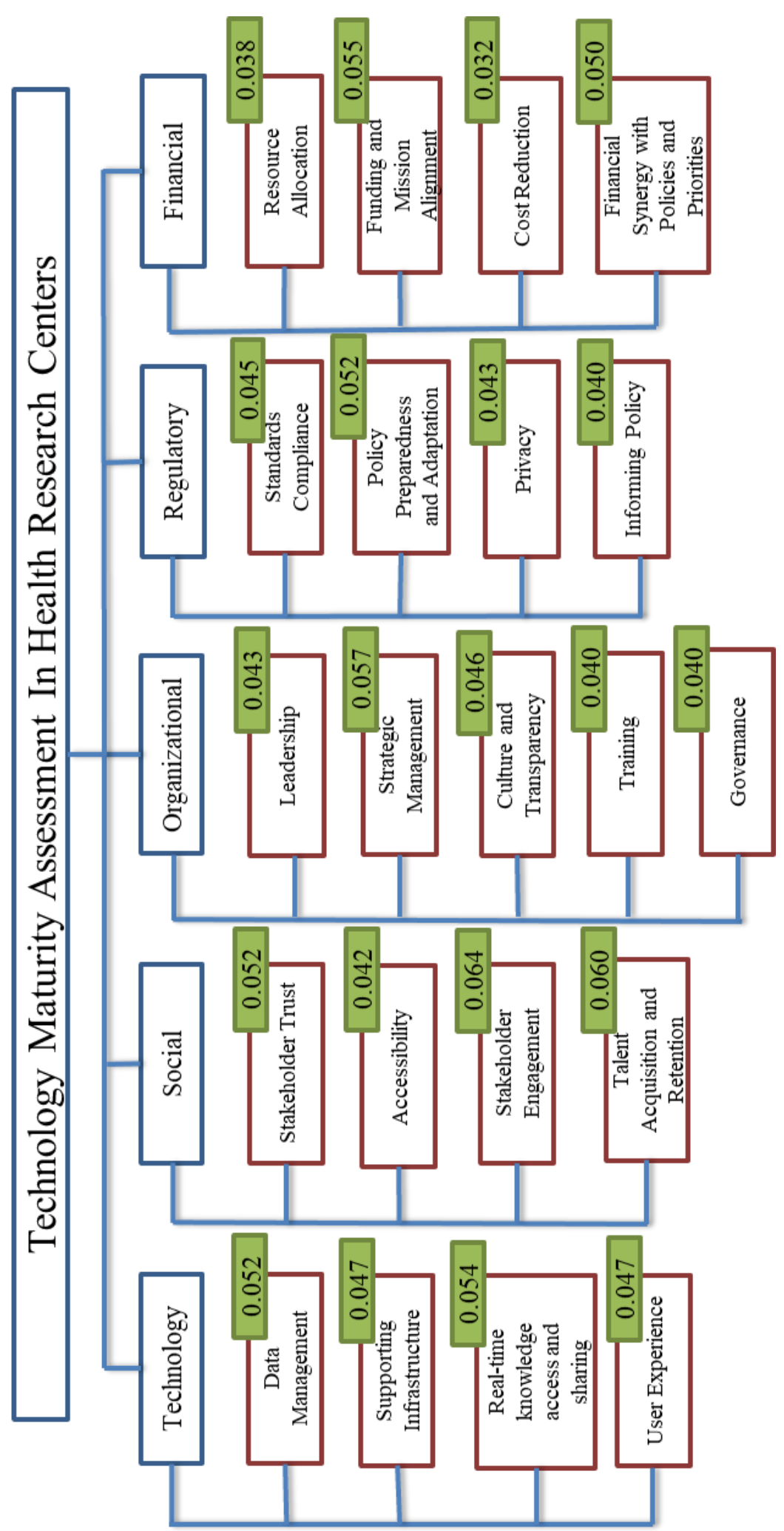

Figure 68: The Model with Global Weights 


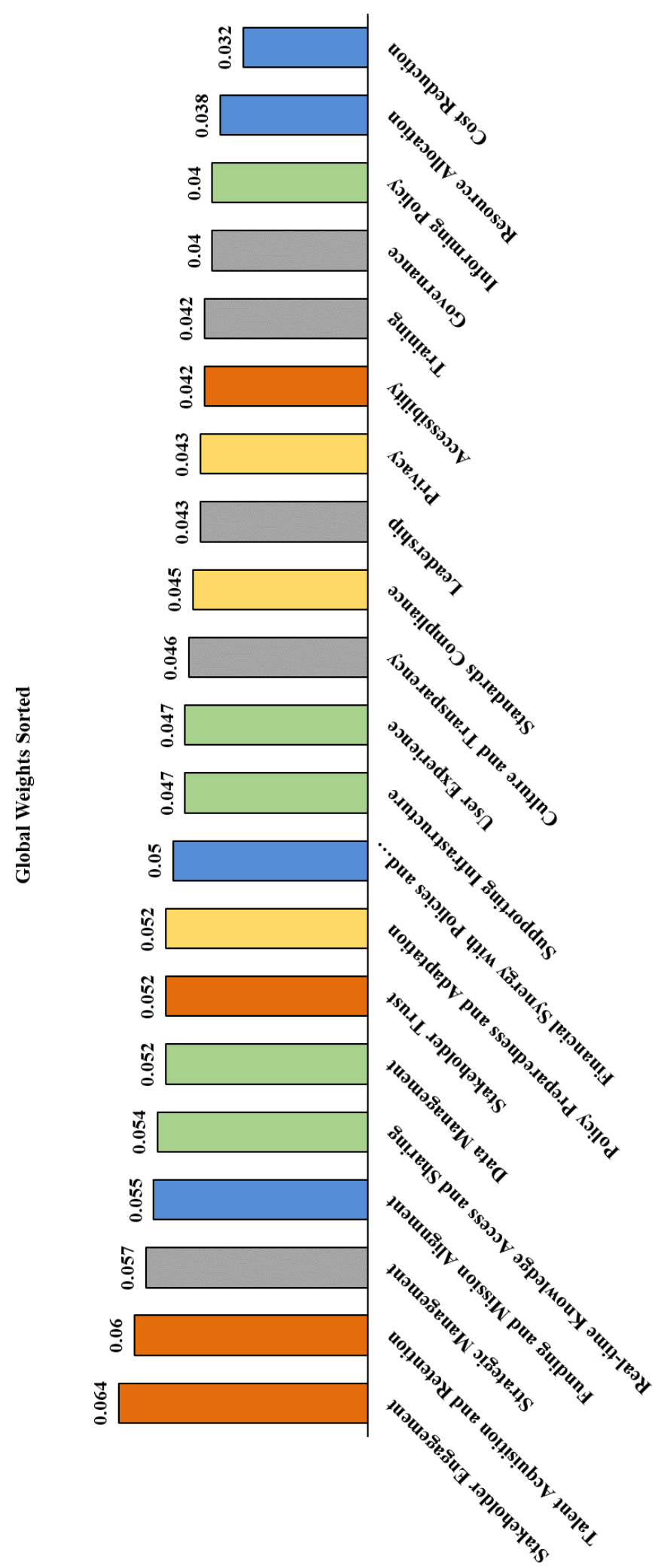

Figure 69: Global Weights Sorted 


\section{Case Studies}

Following the validation, and quantification of the model and its desirability curve/state values, the model is now applied to actual case studies in order to showcase the practical side of the model. Two research centers in the United States were selected to take part in this part of the research. The goal of this section is to show how the model can be applied to real-life health research centers, pinpoint their strengths and opportunities, and look at some ways that they can improve in terms of technology management maturity and continuous learning. Experts within each of these two health research centers helped with the quantification of the desirability scores based on their centers' performance in the relative criteria. Following this, the desirability scores for each criterion is multiplied by the global weight of that criterion and the sum of these calculations for all of the criteria would result in the center's maturity score. This score is important in the sense of the dialogues and initiatives it starts within the organization with goals of improving the center's maturity and continuous learning. These organizations will remain anonymous to respect the privacy of these centers. Both centers are research centers within university hospitals.

Meetings occurred with the people in these centers that had sufficient knowledge about their centers' performance in these specific criteria. The metrics within each criterion helped these experts to be able to quantify where their center stands. These experts included titles such as research center director and project managers.

The research gaps, questions, and goals were explained during these meetings and the perspectives and criteria definitions, the hierarchical model, and the desirability metrics 
and their values were given to them in advance as well in order to give them more time to think about them concerning the centers they are involved in. Figure 69 shows the way these experts could quantify each criterion's desirability value/state.

\begin{tabular}{|c|c|c|c|c|c|c|c|}
\hline Perspective & P Weight & Criteria & Local W & Global W & D score & Score $=G^{*} D$ & Comments \\
\hline \multirow{3}{*}{ Technology } & \multirow{3}{*}{0.200} & Data Management & 0.264 & 0.052 & & 0.000 & \\
\hline & & Supporting Infrastructure & 0.223 & 0.047 & \multirow{3}{*}{\begin{tabular}{|l|}
0.00 \\
11.36 \\
36.82 \\
73.91 \\
100.00 \\
\end{tabular}} & 0.000 & \\
\hline & & Real-time Knowledge Access and Sharing & 0.275 & 0.054 & & 0.000 & \\
\hline \multirow{2}{*}{ Social } & \multirow{2}{*}{0.217} & Stakeholder Trust & 0.226 & 0.052 & & 0.000 & \\
\hline & & Accessibility & 0.196 & 0.042 & & 0.000 & \\
\hline \multirow{5}{*}{ Organizational } & \multirow{5}{*}{0.226} & Leadership & 0.188 & 0.043 & & 0.000 & \\
\hline & & Strategic Management & 0.238 & 0.057 & & 0.000 & \\
\hline & & Culture and Transparency & 0.200 & 0.046 & & 0.000 & \\
\hline & & Training & 0.191 & 0.042 & & 0.000 & \\
\hline & & Governance & 0.181 & 0.040 & & 0.000 & \\
\hline \multirow{4}{*}{ Financial } & \multirow{4}{*}{0.175} & Resource Allocation & 0.217 & 0.038 & & 0.000 & \\
\hline & & Funding and Mission Alignment & 0.302 & 0.055 & & 0.000 & \\
\hline & & Cost Reduction & 0.190 & 0.032 & & 0.000 & \\
\hline & & Financial Synergy with Policies and Priorities & 0.290 & 0.050 & & 0.000 & \\
\hline & & & & & & 0.000 & \\
\hline
\end{tabular}

Figure 70:Snapshot of the Desirability Value Selection as a Part of the case study for testing the results.

The table below shows the results for the first studied case: 
Table 60: Case Study I Results

\begin{tabular}{|c|c|c|c|c|c|c|c|}
\hline Perspective & P Weight & Criteria & Local W & Global W & D score & Score $=\mathrm{GW}^{*} \mathrm{D}$ & Comments \\
\hline \multirow{4}{*}{ Technology } & \multirow{4}{*}{0.200} & Data Management & 0.264 & 0.052 & 36.82 & 1.904 & \\
\hline & & Supporting Infrastructure & 0.223 & 0.047 & 37.55 & 1.748 & \\
\hline & & Real-time Knowledge Access and Sharing & 0.275 & 0.054 & 25.55 & 1.392 & \\
\hline & & User Experience & 0.235 & 0.047 & 72.82 & 3.390 & \\
\hline \multirow{4}{*}{ Social } & \multirow{4}{*}{0.217} & Stakeholder Trust & 0.226 & 0.052 & 45.73 & 2.381 & \\
\hline & & Accessibility & 0.196 & 0.042 & 47 & 1.961 & \\
\hline & & Stakeholder Engagement & 0.295 & 0.064 & 49.18 & 3.154 & \\
\hline & & Talent Acquisition and Retention & 0.281 & 0.060 & 55.73 & 3.363 & \\
\hline \multirow{5}{*}{ Organizational } & \multirow{5}{*}{0.226} & Leadership & 0.188 & 0.043 & 74.09 & 3.168 & \\
\hline & & Strategic Management & 0.238 & 0.057 & 15.91 & 0.900 & \\
\hline & & Culture and Transparency & 0.200 & 0.046 & 51.45 & 2.360 & \\
\hline & & Training & 0.191 & 0.042 & 40.36 & 1.698 & \\
\hline & & Governance & 0.181 & 0.040 & 13 & 0.524 & \\
\hline \multirow{4}{*}{ Regulatory } & \multirow{4}{*}{0.181} & Standards Compliance & 0.240 & 0.045 & 83.64 & 3.778 & \\
\hline & & Policy Preparedness and Adaptation & 0.289 & 0.052 & 0 & 0.000 & \\
\hline & & Privacy & 0.232 & 0.043 & 73.73 & 3.178 & \\
\hline & & Informing Policy & 0.237 & 0.040 & 35 & 1.388 & \\
\hline \multirow{4}{*}{ Financial } & \multirow{4}{*}{0.175} & Resource Allocation & 0.217 & 0.038 & 39.45 & 1.483 & \\
\hline & & Funding and Mission Alignment & 0.302 & 0.055 & 39.45 & 2.163 & \\
\hline & & Cost Reduction & 0.190 & 0.032 & 44.55 & 1.444 & \\
\hline & & $\begin{array}{l}\text { Financial Synergy with Policies and } \\
\text { Priorities }\end{array}$ & 0.290 & 0.050 & 70.82 & 3.516 & \\
\hline & & & & & & 44.89 & \\
\hline
\end{tabular}

The second studied project's maturity score and desirability values is shown in the table below: 
Table 61: Case Study II Results

\begin{tabular}{|c|c|c|c|c|c|c|}
\hline Perspective & P Weight & Criteria & Local W & Global W & D score & Score $=G^{*}$ D \\
\hline \multirow{4}{*}{ Technology } & \multirow{4}{*}{0.200} & Data Management & 0.264 & 0.052 & 73.91 & 3.823 \\
\hline & & Supporting Infrastructure & 0.223 & 0.047 & 37.55 & 1.748 \\
\hline & & Real-time Knowledge Access and Sharing & 0.275 & 0.054 & 25.55 & 1.392 \\
\hline & & User Experience & 0.235 & 0.047 & 72.82 & 3.390 \\
\hline \multirow{4}{*}{ Social } & \multirow{4}{*}{0.217} & Stakeholder Trust & 0.226 & 0.052 & 45.73 & 2.381 \\
\hline & & Accessibility & 0.196 & 0.042 & 47 & 1.961 \\
\hline & & \begin{tabular}{|l|} 
Stakeholder Engagement \\
\end{tabular} & 0.295 & 0.064 & 49.18 & 3.154 \\
\hline & & Talent Acquisition and Retention & 0.281 & 0.060 & 27.73 & 1.673 \\
\hline \multirow{5}{*}{ Organizational } & \multirow{5}{*}{0.226} & Leadership & 0.188 & 0.043 & 29.09 & 1.244 \\
\hline & & \begin{tabular}{|l} 
Strategic Management \\
\end{tabular} & 0.238 & 0.057 & 15.91 & 0.900 \\
\hline & & Culture and Transparency & 0.200 & 0.046 & 17.36 & 0.796 \\
\hline & & Training & 0.191 & 0.042 & 15.27 & 0.642 \\
\hline & & Governance & 0.181 & 0.040 & 13 & 0.524 \\
\hline \multirow{4}{*}{ Regulatory } & \multirow{4}{*}{0.181} & Standards Compliance & 0.240 & 0.045 & 83.64 & 3.778 \\
\hline & & Policy Preparedness and Adaptation & 0.289 & 0.052 & 63.27 & 3.294 \\
\hline & & Privacy & 0.232 & 0.043 & 27.91 & 1.203 \\
\hline & & Informing Policy & 0.237 & 0.040 & 35 & 1.388 \\
\hline \multirow{5}{*}{ Financial } & \multirow{4}{*}{0.175} & Resource Allocation & 0.217 & 0.038 & 39.45 & 1.483 \\
\hline & & Funding and Mission Alignment & 0.302 & 0.055 & 39.45 & 2.163 \\
\hline & & Cost Reduction & 0.190 & 0.032 & 44.55 & 1.444 \\
\hline & & $\begin{array}{l}\text { Financial Synergy with Policies and } \\
\text { Priorities }\end{array}$ & 0.290 & 0.050 & 11.00 & 0.546 \\
\hline & & & & & & 38.93 \\
\hline
\end{tabular}

As can be seen from the table above, the maturity scores were calculated at 44.89 and 32.27 for cases I and II, respectively. This score, however, on its own is not the value of this model. This initially calculated score can serve as a reference point for improving the maturity of this organization/group. By referring to these values, organizations can get a sense of self-awareness of where they are and what needs to be done in order for them to increase their technology management maturity and continuous learning focus.

\subsection{Strengths and Opportunities}

In this section, the strengths, and opportunities for the two studied cases will be briefly reviewed. 


\subsubsection{Case Study I}

In this case, study, based on the obtained results, the performance in the social and financial perspectives are higher compared to the technology and organizational perspectives. There is a particularly high improvement for this center in terms of criteria such as policy preparedness and adaptation and governance. At the same time, this center is performing well based on financial synergy with policies and priorities as they have a good record with accessing government and state grants for their research. Moreover, their products and research conduct tools are designed with user-friendliness in mind leading to a good performance from the user experience criterion. Their leadership provides good support and advocacy for goals of continuous learning and increasing organizational maturity. The leadership in this center has even encouraged the use of third-party firms to help with the assessment and improvement of organizational and technology maturity in order to navigate the complex and multi-disciplinary nature of healthcare organizations to mitigate challenges in terms of process and people management, and information technology and systems.

One of the biggest opportunities for this center is to focus on its strategic management. Currently, they are interested and aspire to strengthen their strategic management and have a vision of positioning themselves as a top research center in their field in terms of state and federal recognition. They are, however, lacking in an action plan and a roadmap to take them from where they are to where they want to be. Initiatives to improve the implementation, realization, and management of these steps can increase the technology management maturity of this center as strategic management is the third highest-ranking 
criterion based on the model's outputs. Setting strategic plans for different scenarios in cases of policy changes can improve the center's policy preparedness and adaptation as well.

Another field that this center can increase its maturity score is through increasing its stakeholder trust and engagement. Scientists, clinicians, funders, journals, academics, regulators, and professional societies can make research more trustworthy and useful through collaborations (Strech, Weissgerber and Dirnagl, 2020). As it can be seen improvement in the strategic management section can improve the social aspect of this research center through increased trustworthiness and engagement. Moreover, it is important for research centers and healthcare organizations to realize that merely providing access may not be enough, and efforts are needed to be in place to earn the trust of communities (trust in vaccine racial and ethnic minorities as an example (Sinha, 2021). From the patient's side as one of the stakeholders, some of the engagement hesitancy regarding accessing healthcare or partaking in research can be imputed to lack of information, fear of discrimination, and legal problems (such as undocumented immigrants) which can be mitigated through ensuring that sufficient, transparent, and comprehensive information about the research or health services are provided and are accessible to patients. As it was discussed in the literature review section of this research the United States is facing an increase in racial, ethnic, and socio-economic diversity which makes these issues even more important. Health institutions should commit to identifying ways to make sure that research learnings are used to improve care for all the communities of patients who agreed to contribute data in addition to generating 
knowledge from their research. When the stakeholders such as patients are ensured that their contribution and engagement will lead to findings that will benefit them and their community, they will deem the research center more worthy of their trust and engagement (Morain, Kass and Faden, 2018).

Lastly addressing their governance shortages can improve their maturity scores by having documented and periodically updated governance and stewardship in the research center. Moreover, engaging the right stakeholders, being on the same page in terms of objectives, aligning incentives, engage in continuous improvement, and strategic management can improve the governance of the center as it can set the grounds for better decision making, resource allocation, and prioritization (LeSueur, 2017).

Here we will simulate the model to see what will happen if the organization addresses some of the opportunities explained here. Improvements in strategic management, governance, policy preparedness, while improving on their stakeholder trust and engagement are provided to mitigate the center's weaker points while improving on some opportunities. The results increase the maturity score to almost 58 which is around 14 points increase (around 30\%). 
Table 62: Recommended Improvements for Case Study I Simulation I

\begin{tabular}{|c|c|}
\hline Criteria & Potential Improvement Action \\
\hline Stakeholder Trust & $\begin{array}{r}\text { Increasing the trustworthiness of the research center (majority } \\
\text { trusting). This trustworthiness can increase through research } \\
\text { results, dissemination, transparency, informing policy, } \\
\text { partnerships, ... }\end{array}$ \\
\hline Stakeholder Engagement & $\begin{array}{c}\text { Planning and implementation of initiatives aimed at increasing } \\
\text { the engagement of stakeholders. The stakeholders can range from } \\
\text { patients, providers, payors, policymaker, purchasers, families. }\end{array}$ \\
\hline Policy Preparedness and & $\begin{array}{c}\text { The research center has plans for different policy change } \\
\text { scenarios in terms of readiness and adaptation. }\end{array}$ \\
\hline Adaptation & $\begin{array}{c}\text { Developing clear strategic vision/plan and } \\
\text { management/implementation of technology maturity and } \\
\text { organizational learning }\end{array}$ \\
\hline Governance & $\begin{array}{c}\text { Documented and periodically updated governance with some } \\
\text { communication among departments towards management of } \\
\text { inputs, structures, processes with goals of improving outcomes }\end{array}$ \\
\hline
\end{tabular}

Table 63:Case Study I, Simulation I

\begin{tabular}{|c|c|c|c|c|c|c|}
\hline Perspective & P Weight & Criteria & Local W & Global W & D score & Score $=\mathrm{GW}^{*} \mathrm{D}$ \\
\hline \multirow{4}{*}{ Technology } & \multirow{4}{*}{0.200} & Data Management & 0.264 & 0.052 & 36.82 & 1.904 \\
\hline & & Supporting Infrastructure & 0.223 & 0.047 & 37.55 & 1.748 \\
\hline & & Real-time Knowledge Access and Sharing & 0.275 & 0.054 & 25.55 & 1.392 \\
\hline & & User Experience & 0.235 & 0.047 & 72.82 & 3.390 \\
\hline \multirow{4}{*}{ Social } & \multirow{4}{*}{0.217} & Stakeholder Trust & 0.226 & 0.052 & 99.55 & 5.183 \\
\hline & & Accessibility & 0.196 & 0.042 & 47 & 1.961 \\
\hline & & Stakeholder Engagement & 0.295 & 0.064 & 83.09 & 5.329 \\
\hline & & Talent Acquisition and Retention & 0.281 & 0.060 & 55.73 & 3.363 \\
\hline \multirow{5}{*}{ Organizational } & \multirow{5}{*}{0.226} & Leadership & 0.188 & 0.043 & 74.09 & 3.168 \\
\hline & & \begin{tabular}{|l|} 
Strategic Management \\
\end{tabular} & 0.238 & 0.057 & 59 & 3.337 \\
\hline & & Culture and Transparency & 0.200 & 0.046 & 51.45 & 2.360 \\
\hline & & Training & 0.191 & 0.042 & 40.36 & 1.698 \\
\hline & & Governance & 0.181 & 0.040 & 71.64 & 2.890 \\
\hline \multirow{4}{*}{ Regulatory } & \multirow{4}{*}{0.181} & Standards Compliance & 0.240 & 0.045 & 83.64 & 3.778 \\
\hline & & Policy Preparedness and Adaptation & 0.289 & 0.052 & 63.27 & 3.294 \\
\hline & & Privacy & 0.232 & 0.043 & 73.73 & 3.178 \\
\hline & & Informing Policy & 0.237 & 0.040 & 35 & 1.388 \\
\hline \multirow{5}{*}{ Financial } & \multirow{4}{*}{0.175} & Resource Allocation & 0.217 & 0.038 & 39.45 & 1.483 \\
\hline & & Funding and Mission Alignment & 0.302 & 0.055 & 39.45 & 2.163 \\
\hline & & Cost Reduction & 0.190 & 0.032 & 44.55 & 1.444 \\
\hline & & $\begin{array}{l}\text { Financial Synergy with Policies and } \\
\text { Priorities }\end{array}$ & 0.290 & 0.050 & 70.82 & 3.516 \\
\hline & & & & & & 57.97 \\
\hline
\end{tabular}

In another simulation, only the top five weighted criteria are improved by one curve value

and as it can be seen it will take the maturity score to from 45 to 55.5 ( $23 \%$ increase).

Some of the proposed improvements for simulation II are shown in Table 64. 
Table 64:Recommended Improvements for Case Study I Simulation II

\begin{tabular}{|c|c|}
\hline Criteria & Potential Improvement Action \\
\hline $\begin{array}{c}\text { Real-time Knowledge Access } \\
\text { and Sharing }\end{array}$ & $\begin{array}{c}\text { Initiatives and projects towards enabling of data capture, access, } \\
\text { and sharing of real-time data. There is still room for improvement } \\
\text { towards the integration of learned knowledge. }\end{array}$ \\
\hline Stakeholder Engagement & $\begin{array}{c}\text { Planning and implementation of initiatives aimed at increasing } \\
\text { the engagement of stakeholders. The stakeholders can range from } \\
\text { patients, providers, payors, policymaker, purchasers, families. }\end{array}$ \\
\hline $\begin{array}{c}\text { Talent Acquisition and } \\
\text { Retention }\end{array}$ & $\begin{array}{c}\text { Working with other departments and leaders to find diverse skills, } \\
\text { looking internally for candidates, assigning mentors, recognition } \\
\text { programs, and supporting advancement opportunities. }\end{array}$ \\
\hline $\begin{array}{c}\text { Strategic Management } \\
\text { Financial Synergy with Policies } \\
\text { and Priorities }\end{array}$ & $\begin{array}{c}\text { Planning and implementation of initiatives aimed at increasing } \\
\text { the engagement of stakeholders. The stakeholders can range from } \\
\text { patients, providers, payors, policymaker, purchasers, families. }\end{array}$ \\
$\begin{array}{c}\text { Initiatives aiming at grants (training, grants writing workshops, } \\
\text { partnerships...) or reimbursement programs and incentives } \\
\text { (closely monitoring the metrics and planning for hitting the } \\
\text { goals). }\end{array}$ \\
\hline
\end{tabular}

Table 65: Case Study I, Simulation II

\begin{tabular}{|c|c|c|c|c|c|c|}
\hline Perspective & P Weight & Criteria & Local W & Global W & D score & Score $=G^{*} * \mathrm{D}$ \\
\hline \multirow{4}{*}{ Technology } & \multirow{4}{*}{0.200} & Data Management & 0.264 & 0.052 & 36.82 & 1.904 \\
\hline & & Supporting Infrastructure & 0.223 & 0.047 & 37.55 & 1.748 \\
\hline & & Real-time Knowledge Access and Sharing & 0.275 & 0.054 & 59.18 & 3.224 \\
\hline & & User Experience & 0.235 & 0.047 & 72.82 & 3.390 \\
\hline \multirow{4}{*}{ Social } & \multirow{4}{*}{0.217} & Stakeholder Trust & 0.226 & 0.052 & 45.73 & 2.381 \\
\hline & & Accessibility & 0.196 & 0.042 & 47 & 1.961 \\
\hline & & \begin{tabular}{|l|} 
Stakeholder Engagement \\
\end{tabular} & 0.295 & 0.064 & 83.09 & 5.329 \\
\hline & & Talent Acquisition and Retention & 0.281 & 0.060 & 100 & 6.034 \\
\hline \multirow{5}{*}{ Organizational } & \multirow{5}{*}{0.226} & Leadership & 0.188 & 0.043 & 74.09 & 3.168 \\
\hline & & Strategic Management & 0.238 & 0.057 & 59 & 3.337 \\
\hline & & Culture and Transparency & 0.200 & 0.046 & 51.45 & 2.360 \\
\hline & & Training & 0.191 & 0.042 & 40.36 & 1.698 \\
\hline & & Governance & 0.181 & 0.040 & 13 & 0.524 \\
\hline \multirow{4}{*}{ Regulatory } & \multirow{4}{*}{0.181} & Standards Compliance & 0.240 & 0.045 & 83.64 & 3.778 \\
\hline & & Policy Preparedness and Adaptation & 0.289 & 0.052 & 0 & 0.000 \\
\hline & & Privacy & 0.232 & 0.043 & 73.73 & 3.178 \\
\hline & & \begin{tabular}{|l|} 
Informing Policy \\
\end{tabular} & 0.237 & 0.040 & 35 & 1.388 \\
\hline \multirow{4}{*}{ Financial } & \multirow{4}{*}{0.175} & Resource Allocation & 0.217 & 0.038 & 39.45 & 1.483 \\
\hline & & Funding and Mission Alignment & 0.302 & 0.055 & 39.45 & 2.163 \\
\hline & & Cost Reduction & 0.190 & 0.032 & 44.55 & 1.444 \\
\hline & & $\begin{array}{l}\text { Financial Synergy with Policies and } \\
\text { Priorities }\end{array}$ & 0.290 & 0.050 & 100.00 & 4.966 \\
\hline & & & & & & 55.46 \\
\hline
\end{tabular}

As it was shown in the simulations, it is important to not only focus on the high weighted

criteria based on the model, but it is also important to address the weakest areas in the

research center to reach a higher maturity score. In this model especially, since the criteria 
weights are closer to each other, this becomes more important. At the same time, it shows how connected are many of these criteria and addressing some may bolster other criteria as well (the case of stakeholder trust and engagement or strategic management and policy preparedness). The decision makers need to initiate conversations based on the results of the model when creating a roadmap as the model can give them a better sense of organizational self-awareness of where they, where they want to be, and how to better get there.

\subsubsection{Case Study II}

In this case, study, based on the obtained results, this center is performing with less success in criteria such as financial synergy with policies and priorities, privacy, policy preparedness, and talent acquisition and retention, and with their organizational criteria in general. This research center can increase its maturity score by starting projects and improvement initiatives around training, governance, and strategic management. The creation of routine multidisciplinary training in the research center around continuous learning and encouraging its culture not only will improve the center's curve/state value on training but also it will potentially improve it in the culture and transparency section. From another aspect, training around grant writing and grant writing seminars can increase the center's success in terms of financial synergy with policies and priorities. Financial synergy with funding and policies in this center can increase with initiatives aiming at grants or reimbursement programs and incentives. As an example, the Oregon Health Authority is using quality health metrics to show how well Coordinated Care Organizations (CCOs) are improving care, making quality care accessible, eliminating 
health disparities, and curbing the rising cost of health care in different areas such as immunization, depression screening, drug, and alcohol use, and diabetes among others (Oregon Health Authority, 2021). When participating health organizations meet certain thresholds on specific quality measures, they will be eligible for incentives. These different quality measures are around different data sources, patient populations, and have different measure stewards. Health organizations and centers aiming for any of these measure incentives can plan to reach the thresholds through relevant strategies and initiatives. As an example, for the childhood immunization status quality measures, initiatives such as checking immunization records at each visit, using benchmark report in ALERT data system to monitor the immunization rates for all patients, scheduling the next well visit before the patient leaving the office, implementing a well-visit reminder/recall system, and immunizing as part of a sick visit in order to improve that measure. Another example may be improving the depression screening and follow-up measure by screening patients for depression at least once a year with standardize depression screening tools and capturing structured data in their electronic health records (Wooden, 2021). Although these kinds of initiatives are more common in hospitals and health centers, health research centers may find the relevant reimbursement programs and plan to meet the incentive thresholds.

In terms of talent acquisition and retention, this research center can improve its performance by strengthening the culture and reputation based on the values and missions of the center. Some of the practices within healthcare organizations for improving acquisition and retention include working with other departments and leaders to find 
diverse skills, looking internally for candidates, assigning mentors, recognition programs, and supporting advancement opportunities.

In general, based on the organizational perspective performance of this research, more excitement from the leadership and management can lead to improvements in this section while encouraging planning and vision for strategic management. These actions can significantly improve the center's outlook on the culture of continuous improvement and learning.

The simulation below will look at the results of maximizing the top highest-ranking criteria for this research center. As it can be seen, the maturity score increases by around $54 \%$ alone when the top 5 criteria are maximized (maturity score of 39 to almost 60 ).

Table 66: Case Study II Simulation

\begin{tabular}{|c|c|c|c|c|c|c|}
\hline Perspective & P Weight & Criteria & Local W & Global W & D score & Score $=G^{*} * \mathrm{D}$ \\
\hline \multirow{4}{*}{ Technology } & \multirow{4}{*}{0.200} & Data Management & 0.264 & 0.052 & 73.91 & 3.823 \\
\hline & & Supporting Infrastructure & 0.223 & 0.047 & 37.55 & 1.748 \\
\hline & & Real-time Knowledge Access and Sharing & 0.275 & 0.054 & 100 & 5.448 \\
\hline & & User Experience & 0.235 & 0.047 & 72.82 & 3.390 \\
\hline \multirow{4}{*}{ Social } & \multirow{4}{*}{0.217} & Stakeholder Trust & 0.226 & 0.052 & 45.73 & 2.381 \\
\hline & & Accessibility & 0.196 & 0.042 & 47 & 1.961 \\
\hline & & Stakeholder Engagement & 0.295 & 0.064 & 100 & 6.414 \\
\hline & & Talent Acquisition and Retention & 0.281 & 0.060 & 100 & 6.034 \\
\hline \multirow{5}{*}{ Organizational } & \multirow{5}{*}{0.226} & Leadership & 0.188 & 0.043 & 29.09 & 1.244 \\
\hline & & Strategic Management & 0.238 & 0.057 & 100 & 5.655 \\
\hline & & Culture and Transparency & 0.200 & 0.046 & 17.36 & 0.796 \\
\hline & & Training & 0.191 & 0.042 & 15.27 & 0.642 \\
\hline & & Governance & 0.181 & 0.040 & 13 & 0.524 \\
\hline \multirow{4}{*}{ Regulatory } & \multirow{4}{*}{0.181} & Standards Compliance & 0.240 & 0.045 & 83.64 & 3.778 \\
\hline & & Policy Preparedness and Adaptation & 0.289 & 0.052 & 63.27 & 3.294 \\
\hline & & Privacy & 0.232 & 0.043 & 27.91 & 1.203 \\
\hline & & Informing Policy & 0.237 & 0.040 & 35 & 1.388 \\
\hline \multirow{4}{*}{ Financial } & \multirow{4}{*}{0.175} & Resource Allocation & 0.217 & 0.038 & 39.45 & 1.483 \\
\hline & & Funding and Mission Alignment & 0.302 & 0.055 & 39.45 & 2.163 \\
\hline & & Cost Reduction & 0.190 & 0.032 & 44.55 & 1.444 \\
\hline & & Financial Synergy with Policies and Priorities & 0.290 & 0.050 & 100.00 & 4.966 \\
\hline & & & & & & 59.780 \\
\hline
\end{tabular}


Table 67: Recommended Improvements for Case Study II Simulation I

\begin{tabular}{|c|c|}
\hline Criteria & Potential Improvement Action \\
\hline $\begin{array}{c}\text { Real-time Knowledge Access } \\
\text { and Sharing }\end{array}$ & $\begin{array}{c}\text { Real-time data and information capture, access, and sharing } \\
\text { concurrent to integration of learned knowledge }\end{array}$ \\
\hline Stakeholder Engagement & $\begin{array}{c}\text { Planning and implementation of initiatives aimed at increasing } \\
\text { the engagement of stakeholders. The stakeholders can range from } \\
\text { patients, providers, payors, policymaker, purchasers, families. }\end{array}$ \\
\hline $\begin{array}{c}\text { Talent Acquisition and } \\
\text { Retention }\end{array}$ & $\begin{array}{c}\text { Working with other departments and leaders to find diverse skills, } \\
\text { looking internally for candidates, assigning mentors, recognition } \\
\text { programs, and supporting advancement opportunities. }\end{array}$ \\
\hline $\begin{array}{c}\text { Strategic Management } \\
\text { Financial Synergy with Policies } \\
\text { and Priorities }\end{array}$ & $\begin{array}{c}\text { Planning and implementation of initiatives aimed at increasing } \\
\text { the engagement of stakeholders. The stakeholders can range from } \\
\text { patients, providers, payors, policymaker, purchasers, families. }\end{array}$ \\
$\begin{array}{c}\text { Initiatives aiming at grants (training, grants writing workshops, } \\
\text { partnerships...) or reimbursement programs and incentives } \\
\text { (closely monitoring the metrics and planning for hitting the } \\
\text { goals). }\end{array}$ \\
\hline
\end{tabular}

It is important to note that even by achieving the maximum curve values for the five top factors in the model, case study II is still far from the desired 100 points. Two lessons can be learned from this:

- As mentioned in the first case study, it is not sufficient to focus solely on the most important criteria and expect to achieve excellence in the whole process. This is even more important in this model and in the world of healthcare where criteria are more connected and are closer to each other in terms of weights in the model output.

- The model can be seen as a maturity model, as it shows the maximum level of performance for each factor as the most desirable outcome. Reaching the maximum level of curve value for every single criterion may not be feasible, realistic, or even worthwhile (in terms of resources and efforts) for research centers. Having said that, this information is still informative and beneficial to 
research centers in terms of leading and guiding them towards continuous improvement and learning.

It is important to note that this model assists decision makers in their decision-making process and it should not be used as the sole decision-making factor. Each research center should have discussion and dialogues based on the results of this model and choose the path and create the roadmap they see to be the best fitting based on the research center's resources, improvements' feasibility, and other unique factors pertaining to that specific healthcare research center. This research used arbitrary simulations to showcase how the model works.

\section{Scenario Analysis}

In this model, in order to analyze the impacts of potential changes in the values at levels of the model, Scenario Analysis is used. The scenario-focused sensitivity analysis used here is a method that can be used to perform analysis on how different perspectives/criteria of the model will change in scenarios where there is a change in value/weight of model elements. In other words, this type of analysis helps decisionmakers determine how much a given model depends on its input factors (Saltelli, Tarantola and Chan, 1999). It also facilitates the process of understanding model behavior and the extent to which its different factors interplay. Lilburne and Trantola (2008) define sensitivity analysis as "the study of how uncertainty in model predictions is determined by uncertainty in model inputs." (Lilburne and Tarantola, 2009). 
For this purpose, using the maturity scores calculated in the case study section as the baseline score, five different extreme situations are analyzed for both case studies in this section. In each of these five extreme scenarios, the maximum available weight is given to one perspective and the maturity score is calculated based on the desirability scores obtained by the studied health department. In other words, in each of these 5 scenarios, one perspective gets $96 \%$ of the importance while the other four only get $1 \%$ each.

For "Case Study I", the research center will yield better results in social and financial extreme scenarios since they had a better average performance in those perspectives. In “Case Study II", the center's maturity score will decrease in the organizational focused extreme scenario since the center had the biggest opportunities in that perspective while it seems to be most ready for regulatory and technology-heavy extreme scenarios due to having more strengths in those areas. Tables 65 to 67 demonstrate the detailed results for each of the mentioned scenarios.

Table 68: Scenario Analysis Allocated Weights

\begin{tabular}{|c|c|c|c|c|c|}
\hline Scenarios & Technology & Social & Organizational & Regulatory & Financial \\
\hline Baseline & 0.20 & 0.22 & 0.23 & 0.18 & 0.18 \\
\hline Tech & $\mathbf{0 . 9 6}$ & 0.01 & 0.01 & 0.01 & 0.01 \\
\hline Social & 0.01 & $\mathbf{0 . 9 6}$ & 0.01 & 0.01 & 0.01 \\
\hline Org & 0.01 & 0.01 & $\mathbf{0 . 9 6}$ & 0.01 & 0.01 \\
\hline Reg & 0.01 & 0.01 & 0.01 & $\mathbf{0 . 9 6}$ & 0.01 \\
\hline Fin & 0.01 & 0.01 & 0.01 & 0.01 & $\mathbf{0 . 9 6}$ \\
\hline
\end{tabular}


Table 69: Scenario Analysis for Case I

\begin{tabular}{|c|c|c|c|c|c|c|c|}
\hline Scenarios & Technology & Social & Organizational & Regulatory & Financial & $\begin{array}{l}\text { Maturity } \\
\text { Score }\end{array}$ & Change \\
\hline Baseline & 0.20 & 0.22 & 0.23 & 0.18 & 0.18 & 44.89 & 0 \\
\hline Tech & 0.96 & 0.01 & 0.01 & 0.01 & 0.01 & 58.82 & 13.92 \\
\hline Social & 0.01 & 0.96 & 0.01 & 0.01 & 0.01 & 65.25 & 20.35 \\
\hline Org & 0.01 & 0.01 & 0.96 & 0.01 & 0.01 & 55.23 & 10.34 \\
\hline $\operatorname{Reg}$ & 0.01 & 0.01 & 0.01 & 0.96 & 0.01 & 61.60 & 16.71 \\
\hline Fin & 0.01 & 0.01 & 0.01 & 0.01 & 0.96 & 65.05 & 20.15 \\
\hline
\end{tabular}

Table 70: Scenario Analysis for Case II

\begin{tabular}{c|c|c|c|c|c|c|c|} 
Scenarios & Technology & Social & Organizational & Regulatory & Financial & $\begin{array}{c}\text { Maturity } \\
\text { Score }\end{array}$ & Change \\
\hline \multirow{2}{*}{$\begin{array}{c}\text { Baseline } \\
\text { Tech }\end{array}$} & 0.20 & 0.22 & 0.23 & 0.18 & 0.18 & 38.93 & 0 \\
\hline $\begin{array}{c}\text { Social } \\
\text { Org }\end{array}$ & 0.96 & 0.01 & 0.01 & 0.01 & 0.01 & 64.45 & 25.52 \\
\hline $\begin{array}{c}\text { Reg } \\
\text { Fin }\end{array}$ & 0.01 & 0.96 & 0.01 & 0.01 & 0.01 & 55.70 & 16.77 \\
\hline
\end{tabular}

The biggest value these scenario analyses offer the decision-makers is to inform them on how to proceed with their efforts towards improving their path towards technology management and continuous learning goals. These will also help health centers with better preparation in terms of criteria such as strategic management and policy preparedness and adaptation, and resource allocation. 


\section{Discussions}

As mentioned in the problem statement of this research, there is a need for a tool that gives health organizations a sense of self-awareness in terms of continuous integration, implementation, and management of the learned knowledge and new technologies. This tool needs to be able to identify potential common factors that have a major impact on the maturity of continuous learning while being able to assess the health organizations' performance against each of those factors. Moreover, this model can help these organizations in identifying the opportunities while giving the organization a sense of self-awareness and direction. This direction can eventually assist decision-makers in their strategic management and roadmaps. As a result of the conversation and dialogues based on the results of the model, health organizations (health research centers in the case of this study) can get a better sense of direction in order to prioritize their improvement paths and agenda while having a better grip on their performance and maturity in terms of continuous learning leading them to be more mature organizations.

As part of the gap analysis (gaps I and II specifically), it was discussed that the criteria for assessing technology management maturity and continuous learning in healthcare organizations are not organized and classified into categories/perspectives while there is no quantified, validated, multi-dimensional, and repeatable way of assessing maturity in healthcare organizations. Discussion with experts from each of the validation and quantification panels confirmed these findings as many of them confirmed the need for such a model for assessing technology management maturity and continuous learning in healthcare organizations. These experts were from different backgrounds related to 
healthcare organizations, health-related academic institutes, and research centers, including directors, officers, vice presidents, project managers, data scientists, academic researchers, public health experts, and academicians.

From the usability perspective of this model in other sectors, although the criteria and perspectives identified in this research may apply to measuring maturity and continuous learning in other fields, the literature review and subject matter experts in this model are heavily focused on the field of healthcare. However, this model can be used as a base of research for other areas with more discussions (validation) and pair-wise comparison of the model elements with the relative expert domain based on the research goal at hand.

\subsection{Research and Practical Implications}

One of the interesting findings of this research is the fact that it shows although there are some perspectives and criteria that ranked higher than the rest, it shows that each identified criterion is very important to the maturity of the center and its goal towards continuous learning. This confirms that not only the external factors in different perspectives related to healthcare organizations are interconnected (chapter 2.2.2), but also the internal factors affecting the success and maturity of healthcare organizations are interconnected as well. This interconnectedness was briefly discussed in the case studies analyses. Although these criteria are in different perspectives, initiatives around one improvement opportunity may improve the center's performance in other areas (either within the same perspective or not). As an example. increased stakeholder trust may trigger an increase in their engagement and the increased stakeholder engagement (patients as an example) may increase health center or health organization's success in 
their regulatory and financial goals. An example of this can be a scenario where the increase in stakeholder engagement bolsters the collaboration to generate evidence that informs health policy. This can create creates a very powerful and exciting research path forward. As a result of the research centers and health organizations being part of these new collaborative models of research, there will be an inevitable rise in the impact of healthcare research entities in optimizing evidence-informed health policy. As the result of this bolstered impact, stakeholder trust and health center's trustworthiness can increase completing one of the many possible continuous improvement circles.

In this section, each of the top five criteria will be reviewed in the context of this research. Many of these findings align with the literature review done in this research. Among the top-ranking criteria, some are the factors that were introduced to the model during the validation process which shows the importance of perspective and criteria validation as a part of the research framework of this study. The elements of this model were fine-tuned through different validation phases and filters through the literature review and validation in the comprehensive exam, proposal, and dissertation phases.

\subsubsection{Stakeholder Engagement}

The most important criterion based on the expert panel is "Stakeholder engagement" which was defined based on the literature defined as: "The degree to which a health research center can engage stakeholders (patients, providers, payors, policymaker, purchasers, families) to participate in continuous improvement and learning projects and initiatives." (Price-Haywood, 2015; Kraft et al., 2017). As much public health-related research is going on in the world of continuous learning in healthcare organizations, this 
criterion's importance of expected. There was a strong emphasis from the experts on the social aspect of technology management maturity in healthcare. Although some of the experts participating in validating and quantifying this model were public health professionals, the stress on the stakeholders' role was not solely based on their results. In contrast, many technology management professionals and project managers gave higher weights to stakeholders' engagement as a critical factor in health organization's maturity and continuous learning initiatives.

\subsubsection{Talent Acquisition and Retention}

The Second highest weighted criterion was "Talent acquisition and Retention". The word retention was added to the title and the definition of this criterion in the validation process. This

criterion was surprising as there is not a lot of literature around it. A limited supply of skilled individuals was mentioned as one of the six challenges discussed by (Morain, Kass and Grossmann, 2017) as origins of continuous learning and learning health systems transformations for healthcare organizations. Moreover, it is very important for health research centers and health organizations, in general, to not only acquire skilled and diverse workforce but also to retain them in the organizations. Based on the results of this research, centers' ability to acquire and retain talented and diverse staff in different areas can play an important role in their technology management maturity and adoption of continuous learning. 


\subsubsection{Strategic Management}

The Third important weight shown by the data is strategic management. Nowadays, hospitals and healthcare organizations are operating in an extremely competitive environment, with ever-increasing pressure to better their quality and reduce expenses. In response to this dynamic environment, organizations require to have the drive and plan to organize delivery around the needs of patients (Speziale, 2015). Successful health care organizations have leaders who understand the nature and implications of external change, the ability to develop effective strategies that account for change, and the will as well as the ability to actively manage the momentum of the organization. Activities such as strategic decisions, management of competing priorities, internal and external partnerships/collaborations, problem identification, and finding solutions are collectively referred to as "strategic management." Strategic management is pivotal in leading organizations in volatile environments. Strategic management provides the momentum needed for change (Ginter, Duncan and Swayne, 2013).

\subsubsection{Funding and Mission Alignment}

The fourth important criterion is from the financial perspective and is defined as "The extent to which incentives and funding are aligned with the encouragement of technology maturity, continuous learning and improvement, waste elimination, and rewarding high care value.". Based on the literature, securing grants that are aligned with the encouragement of continuous learning is important in the realization of learning health systems rather than inspiring it (Morain, Kass and Grossmann, 2017). Furthermore, funding agencies and programs are also key components of this kind of encouragement 
from the scientific pillar, with more flexible, rapid cycle and applied research funding programs serving as accelerators (Menear et al., 2019). External funding and its alignment with goals of continuous learning were also mentioned in the literature as one of the themes related to the creation of learning health systems.

\subsubsection{Real-time Knowledge Access and Sharing}

The fifth-highest ranking is from the technology perspective. It is defined as "Data systems and tools used to capture, share, and integrate data, information, visualizations, and knowledge gained from research into the organization in real-time". As reviewed in the literature review section about learning health systems, the technology side, and especially the real-time capabilities that allow best practice knowledge in different areas to be captured, compiled, protected, learned from, integrated, and disseminated. As revised in the validation phase, health centers need to ensure the learned knowledge is subsequently integrated into practices and processes to bolster the path towards aimed and promised improvements. Although the learned knowledge is valuable, it is not enough for research centers to put it out in the world and hope someone will do something with it. This sharing of knowledge can be within a healthcare organization or with external groups of research clusters in order to strengthen efforts in instances such as infection prevention efforts during viral outbreaks such as Covid-19 (Nathavitharana et al., 2020).

\subsection{Generalizability}

This external validation determines whether the created model in this research can be used for different types of healthcare departments. In other words, although this research focused on assessing the maturity and continuous learning in healthcare research centers 
within university hospitals and used two research centers as case studies, is it possible to use this for other research centers or other types of healthcare departments?

Generalizability was addressed in two ways:

- Panels of subject matter experts from different types of healthcare organizations, in a spectrum of positions, took part in the validation of this model's perspectives, criteria, and desirability/value curves.

- During some communications with some of the participating experts, they confirmed that this model may be used for any type of healthcare organization. The use of desirability/value curves allows for the model to be reused without the need for subject matter experts as these values allow the model to be reusable.

This assessment tool can be taught in academic settings (or in form of organizational training within centers) to be used in different healthcare settings instead of using thirdparty firms for maturity assessment.

It is important to note that while the model is validated and reusable due to subject matter experts and value curves, it is important to update the model's criteria and their weights after a certain time to ensure its timeliness and appropriateness. 


\section{Conclusion}

Following the research results, case studies, and discussions, this chapter will focus on addressing the research objective, questions, and gaps while discussing the research and practice contributions. Moreover, the limitations and future research will be reviewed.

\subsection{Conclusion and Contributions}

The objective of this research is to develop a framework for assessing technology management maturity in research institutes within university hospitals in the United States. Initially, literature research was performed in areas ranging from performing a landscape analysis on the external forces affecting the healthcare organizations in the United States, Learning Health Systems, and Maturity Models, to Healthcare Research Centers. As a result of these reviews, an initial hierarchical model was created consisting of the elements which have an impact on technology management maturity and continuous learning in health research centers. This model was later validated, finalized, and quantified by healthcare experts. Desirability curves/values were used as an extension to the HDM model to allow for the model to be used multiple times without the need to recreate the expert panels and re-quantify the model. Moreover, two case studies are provided to see to the model in practice followed by scenario analyses to observe the model's sensitivity to extreme perspective conditions.

This research contributes to the technology management body of knowledge on technology management maturity assessment in the healthcare industry while delving deeper into how healthcare research centers can achieve a sense of self-awareness in terms 
of where they are and where they need to be in dealing with their technology management and continuous learning issues. More specifically, this research aims at increasing the knowledge on how healthcare research centers assess their maturity and maintain continuous learning in the whole organization by proposing a technology management maturity model assessment tool. According to the literature review and gap analysis conducted in this study, there is a lack of structured and comprehensive understanding of the managerial issues around the maturity assessment of technology management in healthcare generally and more specifically in the growing and imminent field of learning health systems which can bolster the continuous quality improvement goals of the healthcare organizations. This research will provide a quantified, validated, repeatable, and multi-perspective tool for measuring technology management maturity in healthcare research centers with a focus on continuous learning. This maturity model may result in better decision-making in healthcare research centers (and healthcare organizations) and can be used as a step in the right direction in reaching better results regarding patient satisfaction, quality care, cost of care, and resource allocation. This model can assist healthcare managers and decision-makers in identifying strength and opportunity areas within the firm (in the context of maturity in continuous learning), while helping health organizations in classifying and organizing their priorities and bolsters their judgment in terms of proactiveness in achieving the goals of continuous learning and improvement. Knowledge of the health care system and its development is essential for the effective management of health care organizations. By keeping up to date on community needs, technological progress, stakeholder demands, and economic prospects, managers can be 
in a better position to fulfill their organizational missions to enhance access, improve service quality, and achieve efficiency in the delivery of services.

COVID-19 is a great example that shown how important mature continuously learning healthcare systems are as they need to rapidly collect data, learn from it and for communities to address the problems they are facing through combining discovery, data analysis, and taking action (Ellis, 2021).

Table 68 shows how this research has addressed the gaps identified by the literature review. 


\begin{tabular}{|c|c|}
\hline Research Gaps & Addressed By \\
\hline $\begin{array}{l}\text { RG I: The criteria for assessing technology } \\
\text { management maturity and continuous learning } \\
\text { healthcare research centers are not organized and } \\
\text { classified into categories/perspectives. }\end{array}$ & $\begin{array}{l}\text { This study identified and categorized } \\
\text { the most important factors influencing } \\
\text { technology management maturity and } \\
\text { continuous learning while } \\
\text { demonstrating their relative } \\
\text { importance/weights. }\end{array}$ \\
\hline $\begin{array}{l}\text { RG II: There is no quantified, validated, multi- } \\
\text { perspective, and repeatable way of assessing } \\
\text { maturity in healthcare research centers. }\end{array}$ & $\begin{array}{l}\text { The proposed hierarchical model } \\
\text { offers a quantified, validated, multi- } \\
\text { perspective, and repeatable tool for } \\
\text { assessing maturity and continuous } \\
\text { learning in healthcare research } \\
\text { centers. }\end{array}$ \\
\hline $\begin{array}{l}\text { RG III: Despite technology management's } \\
\text { importance in healthcare, there is a lack of studies } \\
\text { on the technology management side of healthcare } \\
\text { maturity and continuous learning }\end{array}$ & $\begin{array}{l}\text { The literature review, model } \\
\text { components, identified weights, and } \\
\text { case studies add to the literature } \\
\text { surrounding the technology } \\
\text { management side of healthcare } \\
\text { maturity and continuous learning, } \\
\text { especially in research centers. }\end{array}$ \\
\hline $\begin{array}{l}\text { RG IV: There is very little to no literature around } \\
\text { the structure and management of research centers } \\
\text { within university hospitals. }\end{array}$ & $\begin{array}{l}\text { This study's focus on health research } \\
\text { centers, its literature review, and } \\
\text { results, add to the body of knowledge } \\
\text { on the structure and management of } \\
\text { these research centers. }\end{array}$ \\
\hline
\end{tabular}

This study's responses to the research questions posed earlier in this dissertation are shown in Table 69. 


\begin{tabular}{|c|c|}
\hline Research Questions & Addressed By \\
\hline $\begin{array}{l}\text { RQ I: What are the main perspectives and } \\
\text { criteria in the assessment of technology } \\
\text { management maturity in healthcare research } \\
\text { centers? }\end{array}$ & $\begin{array}{l}\text { The literature review followed by the } \\
\text { hierarchical decision-making model created } \\
\text { in this research identifies the main } \\
\text { perspectives and criteria in the assessment } \\
\text { of technology management maturity in } \\
\text { healthcare research centers. }\end{array}$ \\
\hline $\begin{array}{l}\text { RQ II: What are the weights of criteria and } \\
\text { sub-criteria related to the assessment of } \\
\text { maturity and continuous learning in } \\
\text { healthcare research centers? }\end{array}$ & $\begin{array}{l}\text { The results of the HDM model demonstrate } \\
\text { the weights of criteria and sub-criteria } \\
\text { related to the assessment of maturity and } \\
\text { continuous learning in healthcare research } \\
\text { centers. }\end{array}$ \\
\hline $\begin{array}{l}\text { RQ III: Does the proposed framework offer } \\
\text { an effective and practical way to assess } \\
\text { technology management maturity in } \\
\text { research centers within university hospitals? }\end{array}$ & $\begin{array}{l}\text { The validated model and the demonstration } \\
\text { of its use in the form of two case studies and } \\
\text { the following analysis offer an effective and } \\
\text { practical way to assess technology } \\
\text { management maturity in healthcare research } \\
\text { centers. }\end{array}$ \\
\hline
\end{tabular}

In summary, this research offers both research and practical implications. From the research side, this research contributes to the technology management body of knowledge and maturity models in healthcare while focusing on the management of continuous learning and focusing on a more specific unit of study (healthcare research centers). Furthermore, from the practical aspect, this study offers a framework and tool to healthcare research centers in particular and healthcare organizations in general to assess their maturity and continuous learning. The results of this research and the framework it offers can help healthcare managers to pinpoint their center's strengths points and opportunities and improve their vision in creating roadmaps and allocating resources. 
As mentioned before, this tool can serve as a helping tool to decision-makers and should not be treated as the main decision-making factor. The results of this model can create and spark dialogues between the decision-makers, managers, and stakeholders on the organization's next steps.

\subsection{Limitations}

The first limitation of this study is imputed to the fact that this model was created, validated, and quantified with a focus on healthcare organizations (healthcare research centers specifically) and the help of healthcare experts. This means that, if there is an intent to use the model in other fields, the model elements and their weights need to be re-validated and re-quantified with experts from intended sectors and fields.

The Seconds limitation is caused by the use of expert panels. Although the experts who participated in this research were selected systematically and carefully to make sure that their expertise is relevant, experts are humans and as such, their judgment could be affected by bias and subjectivity that cannot always be detected. In order to address this, the results of the model were validated in terms of disagreement and inconsistency analysis. Furthermore, user-friendly instructions and further clarification (where needed) were provided to the experts to minimize this potential limitation.

Thirdly, as mentioned in the methodology section, when using HDM, in scenarios that there is a difference of more than 1 between the number of criteria under different perspectives it may lead to smaller weights for the criteria under the perspectives with a higher number of criteria. A remedy for this limitation is to try to keep the criteria under 
each perspective close to the others. The perspectives in this research contain 4-5 criteria which minimize this limitation. The methodology-related limitations of this study have been discussed in detail in the HDM limitations section (4.1.2).

Lastly, although this model can detail the weights related to technology management and continuous learning based on the specific perspective they are in, it is not able to numerically address the effect of changes in one criterion on other criteria. This issue is discussed in both the case study and discussion sections of this research. Although the consequences of changes in criterion may be hard to pinpoint numerically, decisionmakers and managers can address this potential change in their strategic plans and roadmaps during the important conversations following the results of this model. Further studies can focus on addressing this research opportunity.

\subsection{Future Research}

The discussions around the limitations of this study pose research opportunities for future research. Firstly, the creation of this maturity assessment model can be iterated in other fields and sectors with a focus on continuous learning and technology management maturity. Following this, it will be interesting to compare the resulting models with the model created in this research and discuss the similarities and differences. Even without changing industries, there is a great opportunity to study this maturity assessment tool in the context of other healthcare entities such as different departments, administrative, and information technology groups within hospitals and compare the results and model elements with this research. 
As mentioned before, the healthcare environment in the United States is a very dynamic one and there will be a need to refresh this model's elements and weights periodically to maintain its usefulness and relevance.

From the methodology standpoint, there are opportunities to investigate and mitigate some of the methodology limitations which were discussed in the previous section. As an example, the creation of a mathematical coefficient based on the number of criteria within each perspective may mitigate that limitation in cases where there are big differences $( \pm 2)$ in the number of criteria within perspectives.

Moreover, additional research with a focus on each of the identified perspectives and their criteria can shed additional light on the nuances of each of these perspectives, and their impact on continuous learning and centers' maturity. Another route to pursue with future research in the context of this research is to study the impact of the model's criteria on each other. One possible opportunity may be to create the mental landscape of continuous learning in healthcare organizations (or more specifically, healthcare research centers) and understand more about the strength of the impact of these criteria on each other regardless of the perspective group they are included. 


\section{References}

Abbas, M. S. (2016) Consistency Analysis for Judgment Quantification in Hierarchical Decision Model. Portland State University.

Abotah, R. (2014) "Evaluation of Energy Policy Instruments for the Adoption of Renewable Energy: Case of Wind Energy in the Pacific Northwest U.S. Portland State Univeristy. Available at: https://doi.org/10.15760/etd.2126.

Aguarón, J. and Moreno-Jiménez, J. M. (2003) 'The geometric consistency index:

Approximated thresholds', European Journal of Operational Research. doi: 10.1016/S03772217(02)00255-2.

Ainsworth, J. and Buchan, I. (2015) 'Combining health data uses to ignite health system learning', Methods of Information in Medicine, 54(6), pp. 479-487. doi: 10.3414/ME15-010064.

Altman, R. B. et al. (2013) 'Data re-identification: societal safeguards.', Science (New York, N.Y.), 339(6123), pp. 1032-3. doi: 10.1126/science.339.6123.1032-c.

Antheunis, M. L., Tates, K. and Nieboer, T. E. (2013) 'Patients' and health professionals' use of social media in health care: Motives, barriers and expectations', Patient Education and Counseling, 92(3), pp. 426-431. doi: 10.1016/j.pec.2013.06.020.

Argyris, C. (1991) 'Teaching Smart People How to Learn', Harvard business review, 69(3). Armen, S. B. et al. (2016) 'Improving Outcomes in Patients With Sepsis', American Journal of Medical Quality, 31(1), pp. 56-63. doi: 10.1177/1062860614551042.

AXELOS (2018) Portfolio, Programme, and Project Management (P3M3). Available at: https://www.axelos.com/best-practice-solutions/p3m3 (Accessed: 4 May 2018).

Baker, A. (2001) 'Crossing the quality chasm: a new health system for the 21 st century', British Medical Journal Publishing Group, 323(7322), p. 1192. doi: 10.1136/bmj.323.7322.1192.

Baker, L. (2014) Competition keeps health-care costs low, researchers find |News Center $\mid$ Stanford Medicine. Available at: https://med.stanford.edu/news/all-news/2014/10/competitionkeeps-health-care-costs-low--researchers-find.html (Accessed: 11 March 2018).

Balas, E. and Boren, S. (2000) Managing clinical knowledge for health care improvement, Yearbook of Medical Informatics 2000. doi: 10.2196/ijmr.2811.

Barham, H. (2017) 'Achieving Competitive Advantage Through Big Data : A Literature Review', Portland International Conference on Management of Engineering and Technology (PICMET). doi: 10.23919/PICMET.2017.8125459. 
Barham, H. and Daim, T. (2018) 'Identifying Critical Issues in Smart City Big Data Project Identifying Critical Issues in Smart City Big Data Project Implementation', (November). doi: 10.1145/3236461.3241967.

Barry, M. J. and Edgman-Levitan, S. (2012) 'Shared Decision Making — The Pinnacle of Patient-Centered Care', New England Journal of Medicine, 366(9), pp. 780-781. doi: 10.1056/NEJMp1109283.

Bartko, J. J. (1966) 'The Intraclass Correlation Coefficient as a Measure of Reliability', Psychological Reports, 19(1), pp. 3-11. doi: 10.2466/pr0.1966.19.1.3.

Bartko, J. J. and Carpenter, W. T. (1976) 'On the methods and theory of reliability', Journal of Nervous and Mental Disease. doi: 10.1097/00005053-197611000-00003.

Batenburg, R., Neppelenbroek, M. and Shahim, A. (2014) 'A maturity model for governance, risk management and compliance in hospitals', Journal of Hospital Administration, 3(4). doi: 10.5430/jha.v3n4p43.

Bauer, M. S. et al. (2015) 'An introduction to implementation science for the non-specialist', BMC Psychology, 3(1), pp. 1-12. doi: 10.1186/S40359-015-0089-9.

Becher, E. C. and Chassin, M. R. (2001) 'Improving quality, minimizing error: Making it happen', Health Affairs, 20(3), pp. 68-81. doi: 10.1377/hlthaff.20.3.68.

Beckles, G. L. et al. (1998) 'Population-based assessment of the level of care among adults with diabetes in the U.S.', Diabetes care, 21(9), pp. 1432-8. doi: 10.2337/DIACARE.21.9.1432. Behrns, K. E. (2015) 'Big Data And New Knowledge In Medicine: The Thinking, Training, And Tools Needed For A Learning Health System: Krumholz HM (Yale Univ School of Medicine, in New Haven, CT) Health Aff 33:1163-1170, 2014§', Yearbook of Surgery, 2015, pp. 13-14. doi: 10.1016/j.ysur.2014.10.001.

Van de Belt, T. H. et al. (2012) 'Use of social media by Western European hospitals: longitudinal study.', Journal of medical Internet research, 14(3), p. e61. doi: 10.2196/jmir.1992. Bendavid, E. and Miller, G. (2010) 'The US global health initiative: Informing policy with evidence', JAMA - Journal of the American Medical Association. American Medical Association, pp. 791-792. doi: 10.1001/jama.2010.1189.

Van Den Berghe, L. A. A. and Levrau, A. (2003) 'Measuring the Quality of Corporate Governance: In Search of a Tailormade Approach?', Journal of General Management, 28(3), pp. 71-86. doi: 10.1177/030630700302800305.

Berle Jr, A. A. and Means Daniel James, G. C. (1932) The Modern Corporation and Private Property, by Adolf A. Berle Jr. and Gardiner C. Means, Indiana Law Journal. Available at: 
https://www.repository.law.indiana.edu/cgi/viewcontent.cgi?referer=https://www.google.com/\& httpsredir=1\&article=5120\&context=ilj (Accessed: 7 March 2019).

Bernstein, J. A. et al. (2015) 'Ensuring public health's future in a national-scale learning health system', American Journal of Preventive Medicine, 48(4), pp. 480-487. doi:

10.1016/j.amepre.2014.11.013.

Berwick, D. M., Nolan, T. W. and Whittington, J. (2008) 'The triple aim: Care, health, and cost', Health Affairs, pp. 759-769. doi: 10.1377/hlthaff.27.3.759.

Bevan, G. (2008) 'Changing paradigms of governance and regulation of quality of healthcare in England', Health, Risk and Society, 10(1), pp. 85-101. doi: 10.1080/13698570701782494.

Bevan, G. and Cornwell, J. (2006) 'Structure and logic of regulation and governance of quality of health care: was OFSTED a model for the Commission for Health Improvement?', Health Economics, Policy and Law, 1(04), p. 343. doi: 10.1017/S1744133106005020.

Bhandari, R. P. et al. (2016) 'Pediatric-Collaborative Health Outcomes Information Registry (Peds-CHOIR): A learning health system to guide pediatric pain research and treatment', Pain, 157(9), pp. 2033-2044. doi: 10.1097/j.pain.0000000000000609.

Blizinsky, K. D. and Bonham, V. L. (2017) 'Leveraging the learning health care model to improve equity in the age of genomic medicine', Learning Health Systems, (September), pp. 19. doi: 10.1002/lrh2.10046.

Bloomrosen, M. and Detmer, D. E. (2010) 'Informatics, evidence-based care, and research; implications for national policy: a report of an American Medical Informatics Association health policy conference', Journal of the American Medical Informatics Association, 17(2), pp. 115-123. doi: 10.1136/jamia.2009.001370.

Blume-Kohout, M. E. and Adhikari, D. (2016) 'Training the scientific workforce: Does funding mechanism matter?', Research Policy, 45(6), pp. 1291-1303. doi: 10.1016/j.respol.2016.03.011. Blumenthal, D. (2010) 'Launching HITECH', New England Journal of Medicine, 362(5), pp. 382-385. doi: 10.1056/NEJMp0912825.

Blumenthal, D. and Tavenner, M. (2010) 'The "Meaningful Use" Regulation for Electronic Health Records', New England Journal of Medicine, 363(6), pp. 501-504. doi:

10.1056/NEJMp1006114.

Bodenheimer, T. and Sinsky, C. (2014) 'From triple to Quadruple Aim: Care of the patient requires care of the provider', Annals of Family Medicine, 12(6), pp. 573-576. doi:

10.1370/afm.1713.

Borrell, C. et al. (2007) 'Politics and health.', Journal of epidemiology and community health, 252 
61(8), pp. 658-9. doi: 10.1136/jech.2006.059063.

Bosslet, G. T. et al. (2011) 'The Patient-Doctor Relationship and Online Social Networks:

Results of a National Survey', Journal of General Internal Medicine, 26(10), pp. 1168-1174.

doi: 10.1007/s11606-011-1761-2.

de Brantes, F. (2003) 'Bridges to Excellence: A Program to Start Closing the Qualitv Chasm in Healthcare', Journal for Healthcare Quality, 25(2), p. 11. Available at:

https://onlinelibrary.wiley.com/doi/pdf/10.1111/j.1945-1474.2003.tb01037.x (Accessed: 25

February 2019).

Brooks, D. et al. (2017) 'Developing a framework for integrating health equity into the learning health system', Learning Health Systems, 1(3), p. e10029. doi: 10.1002/lrh2.10029.

Brooks, P., El-Gayar, O. and Sarnikar, S. (2013) 'Towards a business intelligence maturity model for healthcare', Proceedings of the Annual Hawaii International Conference on System Sciences, pp. 3807-3816. doi: 10.1109/HICSS.2013.560.

Brooks, P., El-Gayar, O. and Sarnikar, S. (2015) 'A framework for developing a domain specific business intelligence maturity model: Application to healthcare', International Journal of Information Management, 35(3), pp. 337-345. doi: 10.1016/j.ijinfomgt.2015.01.011.

Budrionis, A. and Bellika, J. G. (2016) 'The Learning Healthcare System: Where are we now? A systematic review', Journal of Biomedical Informatics, 64, pp. 87-92. doi:

10.1016/j.jbi.2016.09.018.

Buntin, M. B. et al. (2011) 'The Benefits Of Health Information Technology: A Review Of The Recent Literature Shows Predominantly Positive Results', Health Affairs, 30(3), pp. 464-471. doi: 10.1377/hlthaff.2011.0178.

Bureau, U. C. (no date) 'Census.gov'. Available at: https://www.census.gov/ (Accessed: 11 March 2018).

Butler, J. M. et al. (2017) “"it feels like a lot of extra work”: Resident attitudes about quality improvement and implications for an effective learning health care system', Academic

Medicine, 92(7), pp. 984-990. doi: 10.1097/ACM.0000000000001474.

Caballero, I. et al. (2008) 'IQM3: Information Quality Management Maturity Model', J-Jucs, 14(22), pp. 3658-3685. doi: 10.1016/j.chroma.2012.06.044.

Cahan, A. and Cimino, J. J. (2017) 'A learning health care system using computer-aided diagnosis', Journal of Medical Internet Research, 19(3), pp. 1-17. doi: 10.2196/jmir.6663.

Care, S. H. (2014) Partnering with Patients to Drive Shared Decisions, Better Value, and Care Improvement. doi: 10.17226/18397. 
Carline, J. D. (2004) 'Funding medical education research: Opportunities and issues', Academic Medicine, 79(10), pp. 918-924. doi: 10.1097/00001888-200410000-00004.

Carroll, J. S. (2002) 'Leading organisational learning in health care', Quality and Safety in Health Care, 11(1), pp. 51-56. doi: 10.1136/qhc.11.1.51.

Carvalho, J. V. et al. (2018) 'A health data analytics maturity model for hospitals information systems', International Journal of Information Management, (July), pp. 1-8. doi:

10.1016/j.ijinfomgt.2018.07.001.

Carvalho, J. V. et al. (2019) 'A Maturity model for hospital information systems', Journal of Business Research, 94(December 2017), pp. 388-399. doi: 10.1016/j.jbusres.2017.12.012. de Carvalho, J. V., Rocha, Á. and Vasconcelos, J. (2015) 'Towards an Encompassing Maturity Model for the Management of Hospital Information Systems', Journal of Medical Systems, 39(9), p. 99. doi: 10.1007/s10916-015-0288-1.

Carvalho, J. V., Rocha, Á. and Abreu, A. (2016) 'Maturity Models of Healthcare Information Systems and Technologies: a Literature Review', Journal of Medical Systems, 40(6), pp. 1-10. doi: 10.1007/s10916-016-0486-5.

Casalino, L. et al. (2003) 'External Incentives, Information Technology, and Organized Processes to Improve Health Care Quality for Patients With Chronic Diseases', JAMA, 289(4), p. 434. doi: 10.1001/jama.289.4.434.

Chambers, D. A., Feero, W. G. and Khoury, M. J. (2016) 'Convergence of implementation science, precision medicine, and the learning health care system: A new model for biomedical research', Jama, 315(18), pp. 1941-1942. doi: 10.1186/1748-5908-1-1.5.

Chambers, N. (2012) 'Healthcare board governance', Journal of Health Organization and Management, 26(1), pp. 6-14. doi: 10.1108/14777261211211133.

Chan, L. (2000) Developing a Strategic Policy Choice Framework for Technological Innovation: Case of Chinese Pharmaceuticals, Dissertations and Theses. Portland, OR. doi: 10.15760/etd.1041.

Chan, L. (2013) Developing a Strategic Policy Choice Framework for Technological Innovation: Case of Chinese Pharmaceuticals. Portland State University.

Charles, D., Gabriel, M. and Searcy, T. (2015) Adoption of Electronic Health Record Systems among U.S. Non-Federal Acute Care Hospitals: 2008-2014.

Chassin, M. R., Galvin, R. W. and Quality, and the N. R. on H. C. (1998) 'The Urgent Need to Improve Health Care Quality:Institute of Medicine National Roundtable on Health Care Quality', JAMA, 280(11), p. 1000. doi: 10.1001/jama.280.11.1000. 
Chayes, F. (1960) 'On correlation between variables of constant sum', Journal of Geophysical Research, 65(12), pp. 4185-4193. doi: 10.1029/JZ065i012p04185.

Chen, H. and Kocaoglu, D. F. (2008) 'A sensitivity analysis algorithm for hierarchical decision models', European Journal of Operational Research, 185(1), pp. 266-288. doi:

10.1016/J.EJOR.2006.12.029.

Chen, Y., Yu, J. and Khan, S. (2013) 'The spatial framework for weight sensitivity analysis in AHP-based multi-criteria decision making, Environmental Modelling and Software, 48, pp. 129-140. doi: 10.1016/j.envsoft.2013.06.010.

Chou, W. S. et al. (2009) 'Social media use in the United States: implications for health communication.', Journal of medical Internet research, 11(4), p. e48. doi: 10.2196/jmir.1249. Christensen, C. M., Bohmer, R. and Kenagy, J. (2000) 'Will disruptive innovations cure health care?', Harvard Business Review, 78(5), pp. 102-112.

Clair, L., Brown, E. and Moore, C. (2010) 'Electronic Medical Records Need More To Support “Meaningful Use'.

Cleven, A. K. et al. (2014) 'Process management in hospitals: an empirically grounded maturity model', Business Research, 7(2), pp. 191-216. doi: 10.1007/s40685-014-0012-x.

CMS.gov (2016) National Health Expenditure Data (Centers for Medicare \& Medicaid Services). Available at: https://www.cms.gov/research-statistics-data-and-systems/statisticstrends-and-reports/nationalhealthexpenddata/nationalhealthaccountshistorical.html. CMS.gov (2019a) Bundled Payments for Care Improvement (BPCI) Initiative: General Information | Center for Medicare \& Medicaid Innovation. Available at: https://innovation.cms.gov/initiatives/bundled-payments (Accessed: 6 November 2019). CMS.gov (2019b) Hospital Readmissions Reduction Program (HRRP) - Centers for Medicare \& Medicaid Services. Available at: https://www.cms.gov/medicare/medicare-fee-for-servicepayment/acuteinpatientpps/readmissions-reduction-program.html (Accessed: 6 November 2019).

CMS.gov (2020) Prospective Payment Systems . Available at: https://www.cms.gov/Medicare/Medicare-Fee-for-ServicePayment/ProspMedicareFeeSvcPmtGen/index?redirect=/prospmedicarefeesvcpmtgen/ (Accessed: 31 January 2021).

CMS.GOV (2017) Electronic Health Records (EHR) Incentive Programs, Centers for Medicare \& Medicaid Services. Available at: https://www.cms.gov/Regulations-andGuidance/Legislation/EHRIncentivePrograms/index.html?redirect=/EHRIncentivePrograms 
(Accessed: 1 January 2017).

Coiera, E. (2017) 'The forgetting health system', Learning Health Systems, 1(4), p. e10023. doi: 10.1002/lrh2.10023.

Colaco, M. et al. (2013) 'Is there a relationship between national institutes of health funding and research impact on academic urology?', Journal of Urology, 190(3), pp. 999-1003. doi: 10.1016/j.juro.2013.02.3186. compliancy-group (2018) HIPAA Violation \&amp; Breach Fines | List of HIPAA Violations, HIPAA Done Right. Available at: https://compliancy-group.com/hipaa-fines-directory-year/ (Accessed: 30 April 2019).

Congressional Budget Office (CBO) (2008) Technological Change and the Growth of Health Care Spending CONGRESS OF THE UNITED STATES CONGRESSIONAL BUDGET

OFFICE. Available at: https://www.cbo.gov/sites/default/files/110th-congress-20072008/reports/01-31-techhealth.pdf (Accessed: 25 February 2019).

Conklin, T. P. (2002) 'Health Care in the United States: An Evolving System', Michigan Family Review, 07(1). doi: http://dx.doi.org/10.3998/mfr.4919087.0007.102.

Consoli, D. and Mina, A. (2009) 'An evolutionary perspective on health innovation systems', Journal of Evolutionary Economics, 19(2), pp. 297-319. doi: 10.1007/s00191-008-0127-3.

Consoli, D. and Ramlogan, R. (2008) 'Out of sight: Problem sequences and epistemic boundaries of medical know-how on glaucoma', Journal of Evolutionary Economics, 18(1), pp. 31-56. doi: 10.1007/s00191-007-0074-4.

Corrigan, D. et al. (2017) 'Requirements and validation of a prototype learning health system for clinical diagnosis', Learning Health Systems, (April), p. e10026. doi: 10.1002/lrh2.10026. Corrigan, J. (2001) 'Crossing the Quality Chasm: A New Health System for the 21st Century', in Building a Better Delivery System: A New Engineering/Health Care Partnership. National Academy Press, Washington, DC: Institute of Medicine, National Academy of Engineering, p. 95.

COVID-19 Hospitalization and Death by Race/Ethnicity | CDC (no date). Available at: https://www.cdc.gov/coronavirus/2019-ncov/covid-data/investigationsdiscovery/hospitalization-death-by-race-ethnicity.html (Accessed: 30 August 2020). Cowan, K. and Daim, T. U. (2018) 'Technology Planning for Aligning Emerging Business Models and Regulatory Structures - The Case of Electric Vehicle Charging and the Smart Grid', in 2018 Portland International Conference on Management of Engineering and Technology (PICMET). IEEE, pp. 1-10. doi: 10.23919/PICMET.2018.8481977. 
Curcin, V. (2016) 'Embedding data provenance into the Learning Health System to facilitate reproducible research', Learning Health Systems, (August), p. e10019. doi: 10.1002/lrh2.10019. Curran, W. J., Stearns, B. and Kaplan, H. (1969) 'Privacy, Confidentiality and Other Legal Considerations in the Establishment of a Centralized Health-Data System', New England Journal of Medicine, 281(5), pp. 241-248. doi: 10.1056/nejm196907312810505.

CVS Health (2015) CVS Health Opens Digital Innovation Lab in Boston. Available at: https://cvshealth.com/newsroom/press-releases/cvs-health-opens-digital-innovation-lab-boston (Accessed: 7 May 2018).

Daim, T. U. et al. (2015) 'Technology assessment: case of the wearable computing for fitness', International Journal of Medical Engineering and Informatics, 7(4), p. 321. doi:

10.1504/IJMEI.2015.072319.

Daim, T. U. and Kocaoglu, D. F. (2015) Hierarchical decision modeling : essays in honor of Dundar F. Kocaoglu.

Daim, T. U. and Kocaoglu, D. F. (2016) Hierarchical Decision Modeling. (C) Springer International Publishing Switzerland.

Daniel, C. et al. (2017) 'Cross border semantic interoperability for learning health systems: The EHR4CR semantic resources and services', Learning Health Systems, 1(1), p. e10014. doi: 10.1002/lrh2.10014.

Day, J. (2002) 'What is an expert?', Radiography. doi: 10.1053/radi.2002.0369.

Day, J. C. (1992) Population projections of the United States, by age, sex, race, and Hispanic origin: 1992 to 2050. Available at: https://www.google.com/books?hl=en\&lr=\&id=DPMxFHfqNbAC\&oi=fnd\&pg=PR7\&dq=J.+C .+Day, $+\% 22$ Population+projections+of+the+United+States,,+ by+age,+ sex,+ race,,+ and + Hispanic +origin:+1992+to+2050.No.+1092,\%22+S+Department+of+Commerce,+Economics+and+Stati stics+Adminis (Accessed: 6 June 2018).

Deeny, S. R. and Steventon, A. (2015) 'Making sense of the shadows: Priorities for creating a learning healthcare system based on routinely collected data', BMJ Quality and Safety, 24(8), pp. 505-515. doi: 10.1136/bmjqs-2015-004278.

Demir, C. and Kocabas, I. (2010) 'Project Management Maturity Model ( PMMM ) in educational organizations', 9, pp. 1641-1645. doi: 10.1016/j.sbspro.2010.12.379.

Demir, F. (2018) ‘A Strategic Management Maturity Model for Innovation', Technology Innovation Management Review, 8(11), pp. 13-21. doi: 10.22215/timreview/1196.

DeNisco, S. (2019) Advanced practice nursing: Essential knowledge for the profession. Jones \& 
Bartlett Publishers.

Derrington, D. (2017) 'Artificial Intelligence for Health and Health Care', Commisioned report by the Office of the National Coordinator for Health IT (ONC) and the Agency for Healthcare

Research and Quality (AHRQ). Available at: https://www.healthit.gov/sites/default/files/jsr-17task-002_aiforhealthandhealthcare12122017.pdf (Accessed: 19 January 2018).

Dimick, J. B. et al. (2003) 'Complications and costs after high-risk surgery: Where should we focus quality improvement initiatives?', Journal of the American College of Surgeons, 196(5), pp. 671-678. doi: 10.1016/S1072-7515(03)00122-4.

Douglas, K. A. et al. (2009) 'Australia's primary health care workforce — research informing policy', Medical Journal of Australia, 191(2), pp. 81-84. doi: 10.5694/j.1326-

5377.2009.tb02698.x.

Dranove, D. and Lindrooth, R. (2003) 'Hospital consolidation and costs: another look at the evidence', Journal of Health Economics, 22(6), pp. 983-997. doi:

10.1016/J.JHEALECO.2003.05.001.

Dreyfus, H. L. and Dreyfus, S. E. (2005) 'Peripheral Vision: Expertise in Real World Contexts', Organization Studies. doi: 10.1177/0170840605053102.

Dunbrack, L. and Hand, L. (2013) ‘A Maturity Model for Mobile in Healthcare', IDC Health Insights: Business Strategy, Doc \# HI24.

Dwyer III, S. J., Weaver, A. C. and Hughes, K. K. (2004) Health Insurance Portability and Accountability Act Health Insurance Portability and Accountability Act. Available at: http://www.cs.virginia.edu/ acw/security/HIPAA.pdf (Accessed: 4 March 2019).

Van Dyk, L. and Schutte, C. S. L. (2013) 'The Telemedicine Service Maturity Model: A Framework for the Measurement and Improvement of Telemedicine Services BT Telemedicine', Telemedicine, (10), pp. 217-238. doi: 10.5772/56116.

Eisenhardt, K. M. and Martin, J. A. (2000) 'Dynamic capabilities: what are they?', Strategic Management Journal, 21(10-11), pp. 1105-1121. doi: 10.1002/1097-

0266(200010/11)21:10/11<1105::AID-SMJ133>3.0.CO;2-E.

Eisenhower, B. et al. (2012) 'Uncertainty and sensitivity decomposition of building energy models', Journal of Building Performance Simulation, 5(3), pp. 171-184. doi:

10.1080/19401493.2010.549964.

Ellis, L. (2021) Developing Learning Health Systems to Navigate the COVID-19 Pandemic, Harvard Medical School-Trends in Medicine. Available at: https://postgraduateeducation.hms.harvard.edu/trends-medicine/developing-learning-health- 
systems-navigate-covid-19-pandemic (Accessed: 26 April 2021).

Elsaid, M. et al. (2019) 'Chemotherapy Wait Times in a Network of Pediatric Oncology Clinics.', Journal of pediatric hematology/oncology. doi: 10.1097/MPH.0000000000001466.

English, M. et al. (2016) 'Building Learning Health Systems to Accelerate Research and Improve Outcomes of Clinical Care in Low- and Middle-Income Countries', PLoS Medicine, 13(4), pp. 1-8. doi: 10.1371/journal.pmed.1001991.

Epstein, R. M. et al. (2010) 'Why The Nation Needs A Policy Push On Patient-Centered Health Care', Health Affairs, 29(8), pp. 1489-1495. doi: 10.1377/hlthaff.2009.0888.

Estep, J. (2017) Development of a Technology Transfer Score for Evaluating Research Proposals: Case Study of Demand Response Technologies in the Pacific Northwest. Portland State University.

Estep, J. and Daim, T. (2016) 'A framework for technology transfer potential assessment', in 2016 Portland International Conference on Management of Engineering and Technology (PICMET). IEEE, pp. 2846-2852. doi: 10.1109/PICMET.2016.7806626.

Estep, J., Daim, T. and Shaygan, A. (2021) 'R\&D project evaluation: Technology transfer focus', Electricity Journal, 34(2), p. 106904. doi: 10.1016/j.tej.2020.106904.

Faden, R. R. et al. (2013) 'An Ethics Framework for a Learning Health Care System: A Departure from Traditional Research Ethics and Clinical Ethics', Hastings Center Report, 43(SUPPL. 1). doi: 10.1002/hast.134.

Faden, R. R. et al. (2014) 'Informed Consent, Comparative Effectiveness, and Learning Health Care', New England Journal of Medicine, 370(8), pp. 776-8. doi: 10.1056/NEJMhle1313674. Fazey, I. R. A., Fazey, J. A. and Fazey, D. M. (2005) 'Learning more effectively from experience', Ecology and Society. Available at: https://research-repository.standrews.ac.uk/handle/10023/1627 (Accessed: 16 March 2019).

Fenwick, D. and Daim, T. U. (2011) 'Choosing a hybrid car using a hierarchical decision model Adoption Factors of Mobile Services View project Choosing a hybrid car using a hierarchical decision model', Int. J. Sustainable Society, X, No. X(1). doi: 10.1504/IJSSOC.2011.041266. Ferranti, J. M. et al. (2010) 'Bridging the gap: Leveraging business intelligence tools in support of patient safety and financial effectiveness', Journal of the American Medical Informatics Association, 17(2), pp. 136-143. doi: 10.1136/jamia.2009.002220.

Finkelstein, J. and Jeong, I. cheol (2017) 'Machine learning approaches to personalize early prediction of asthma exacerbations', Annals of the New York Academy of Sciences, 1387(1), pp. 153-165. doi: 10.1111/nyas.13218. 
Firn, J. et al. (2013) Priority threat management of invasive plant species in the Lake Eyre Basin Project report Priority Threat Management of invasive plants species in the Lake Eyre Basin. Available at: http://www.lakeeyrebasin.gov.au/sitecollectionimages/resources/b3083f7fb9b3-47c5-ba0e-c5ad47a0e3e4/files/leb-invasive-plant-prioritisation-project-report.pdf.

Fiscella, K. et al. (2000) 'Inequality in quality: Addressing socioeconomic, racial and ethnic disparities in health care', Journal of the American Medical Association. American Medical Association, pp. 2579-2584. doi: 10.1001/jama.283.19.2579.

Fisher, T. (2005) 'How mature is your data management environment?', Business Intelligence Journal, 10(3), p. 20.

Fitterer, R. and Rohner, P. (2010) 'Towards assessing the networkability of health care providers : a maturity model approach', pp. 309-333. doi: 10.1007/s10257-009-0121-9. Fleiss, J. L. and Cohen, J. (1973) 'The Equivalence of Weighted Kappa and the Intraclass Correlation Coefficient as Measures of Reliability', Educational and Psychological Measurement. doi: 10.1177/001316447303300309.

Flin, R. et al. (2000) 'Measuring safety climate: Identifying the common features', Safety Science, 34(1-3), pp. 177-192. doi: 10.1016/S0925-7535(00)00012-6.

Foley, T. J. and Vale, L. (2017) 'What role for learning health systems in quality improvement within healthcare providers?', Learning Health Systems, (April), pp. 1-6. doi: 10.1002/lrh2.10025.

Frank-Stromborg, M. (1991) 'Changing demographics in the united states: Implications for health professionals', Cancer, 67(S6), pp. 1772-1778. doi: 10.1002/cncr.2820671814. French, K. E. et al. (2019) 'Continuous Quality Improvement Measured With Time-Driven Activity-Based Costing in an Outpatient Cancer Surgery Center', Journal of Oncology Practice, 15(2), pp. e162-e168. doi: 10.1200/jop.18.00394.

Frenk, J. et al. (2010) 'The Lancet Commissions Health professionals for a new century: transforming education to strengthen health systems in an interdependent world Executive summary', www.thelancet.com Lancet, 376(376), pp. 1923-58. doi: 10.1016/S01406736(10)61854-5.

Fried, J. et al. (2011) 'Applying a Lean Improvement Process to a Successful Sepsis Protocol Further Improves Processes and Outcomes', Chest, 140(4), p. 960A. doi:

10.1378/chest.1117735.

Friedman, C. et al. (2014) 'Toward a science of learning systems: a research agenda for the high-functioning Learning Health System', Journal of the American Medical Informatics 260 
Association, (December), pp. 43-50. doi: 10.1136/amiajnl-2014-002977.

Friedman, C. P. et al. (2017) 'The science of Learning Health Systems: Foundations for a new journal', Learning Health Systems, 1(1), p. e10020. doi: 10.1002/lrh2.10020.

Friedman, C. P., Wong, A. K. and Blumenthal, D. (2010) 'Achieving a Nationwide Learning', 2(57), pp. 8-10.

Furukawa, M. F. et al. (2014) 'Despite Substantial Progress In EHR Adoption, Health Information Exchange And Patient Engagement Remain Low In Office Settings', Health Affairs, 33(9), pp. 1672-1679. doi: 10.1377/hlthaff.2014.0445.

Galliers, R. D. and Sutherland, A. R. (1991) 'Information systems management and strategy formulation: the "stages of growth" model revisited', Information Systems Journal, 1(2), pp. 89-114. doi: 10.1111/j.1365-2575.1991.tb00030.x.

Garets, D. and Mike, D. (2006) 'Electronic Medical Records vs . Electronic Health Records : Yes, There Is a Difference By Dave Garets and Mike Davis Updated January 26, 2006 HIMSS Analytics , LLC 230 E . Ohio St ., Suite 600 Chicago , IL 60611-3270 EMR vs . EHR :

Definitions The marke', Health (San Francisco), pp. 1-14. doi: 10.3233/978-1-60750-044-5-26. Garratt, B. (2010) The fish rots from the head: developing effective boards. Profile Books. Giansanti, D., Castrichella, L. and Giovagnoli, M. R. (2007) 'New models of e-learning for health-care professionals: A training course for biomedical laboratory technicians', Journal of Telemedicine and Telecare, 13(7), pp. 374-376. doi: 10.1258/135763307782215334.

Gibson, E. (2016) A Measurement System for Science and Engineering Research Center Performance Evaluation. Portland State Univeristy. Available at: https://doi.org/10.15760/etd.3276.

Gibson, E. and Daim, T. U. (2016) 'A measurement system for science and engineering research center performance evaluation', in 2016 Portland International Conference on Management of Engineering and Technology (PICMET). IEEE, pp. 2782-2792. doi:

10.1109/PICMET.2016.7806624.

Ginter, P., Duncan, W. and Swayne, L. (2013) Strategic Management of Health Care Organizations. John Wiley \& Sons.

Glass, N. E. et al. (2020) 'Disproportionally low funding for trauma research by the National Institutes of Health: A call for a National Institute of Trauma', Journal of Trauma and Acute Care Surgery, 88(1), pp. 25-32. doi: 10.1097/TA.0000000000002461.

Goldman, L. R., Kumanyika, S. K. and Shah, N. R. (2016) 'Putting the health of communities and populations first', JAMA - Journal of the American Medical Association. American Medical 
Association, pp. 1649-1650. doi: 10.1001/jama.2016.14800.

Goncalves Filho, A. P. and Waterson, P. (2018) 'Maturity models and safety culture: A critical review', Safety Science, 105(December 2017), pp. 192-211. doi: 10.1016/j.ssci.2018.02.017. Gopee, N. (2002) 'Human and social capital as facilitators of lifelong learning in nursing', Nurse Education Today, 22(8), pp. 608-616. doi: 10.1016/S0260-6917(02)00139-9.

Gospodarowicz, M. K. et al. (2004) 'The process for continuous improvement of the TNM classification', Cancer, 100(1), pp. 1-5. doi: 10.1002/cncr.11898.

Gostin, L. O. et al. (1993) 'Privacy and Security of Personal Information in a New Health Care System', JAMA: The Journal of the American Medical Association, 270(20), p. 2487. doi: 10.1001/jama.1993.03510200093038.

Graban, M. (2016) 'Lean Hospitals: Improving Quality, Patient Safety, and Employee Engagement', International Standard Book Number-13: 978-1-4987-4326-6 (eBook - PDF), Third Edit. Available at: http://www.taylorandfrancis.com.

Grant, R. W. et al. (2016) 'Pre-Visit Prioritization for complex patients with diabetes: Randomized trial design and implementation within an integrated health care system', Contemporary Clinical Trials, 47, pp. 196-201. doi: 10.1016/j.cct.2016.01.012.

Grošelj, P. and Zadnik Stirn, L. (2012) 'Acceptable consistency of aggregated comparison matrices in analytic hierarchy process', European Journal of Operational Research, 223(2), pp. 417-420. doi: 10.1016/j.ejor.2012.06.016.

Grossmann, C. et al. (2010) Clinical Data as the Basic Staple of Health Learning. doi: $10.17226 / 12212$.

Grossmann, C., Powers, B., et al. (2011) Digital Infrastructure for the Learning Health System. doi: $10.17226 / 12912$.

Grossmann, C., Goolsby, W. A., Olsen, L. and Michael, J. (2011) Engineering a Learning Healthcare System. doi: 10.17226/12213.

Grossmann, C., Goolsby, W. A., Olsen, L. and McGinnis, J. . (2011) Engineering a Learning Healthcare System, Engineering a Learning Healthcare System. doi: 10.17226/12213. Guterman, S. and Dobson, A. (1986) 'Impact of the Medicare prospective payment system for hospitals.', Health care financing review, 7(3), pp. 97-114. doi: hcfr-7-3-97 [pii].

Haider, A. H. et al. (2012) 'Association between hospitals caring for a disproportionately high percentage of minority trauma patients and increased mortality: A nationwide analysis of 434 hospitals', Archives of Surgery, 147(1), pp. 63-70. doi: 10.1001/archsurg.2011.254.

Haider, A. H. and Pronovost, P. J. (2011) 'Health information technology and the collection of 
race, ethnicity, and language data to reduce disparities in quality of care', Joint Commission Journal on Quality and Patient Safety, 37(10), pp. 435-436. doi: 10.1016/S15537250(11)37054-7.

Halper, F. and Stodder, D. (2014) 'TDWI BENCHMARK GUIDE TDWI Analytics Maturity Model Guide'. Available at: https://www.microstrategy.com/getmedia/9b914607-084f-4869ae64-e0b3f9e003de/TDWI_Analytics-Maturity-Guide_2014-2015.pdf (Accessed: 18 February 2019).

Hammond, K. (2000) Human Judgment and Social Policy: Irreducible Uncertainty, Inevitable Error, Unavoidable Injustice. Oxford University Press on Demand.

Harigopal, U. and Satyadas, A. (2001) 'Cognizant enterprise maturity model (CEMM)', IEEE Transactions on Systems, Man and Cybernetics Part C: Applications and Reviews, 31(4), pp. 449-459. doi: 10.1109/5326.983928.

Hart, S. L. and Milstein, M. B. (2003) 'Creating sustainable value.', Academy of Management Executive, 17(2), pp. 56-67. doi: 10.5465/AME.2003.10025194.

Hastings, S. E. et al. (2014) 'Exploring the relationship between governance mechanisms in healthcare and health workforce outcomes: a systematic review', BMC Health Services Research, 14(1), pp. 1-14. doi: 10.1186/1472-6963-14-479.

Hawn, C. (2009) 'Take Two Aspirin And Tweet Me In The Morning: How Twitter, Facebook, And Other Social Media Are Reshaping Health Care', Health Affairs, 28(2), pp. 361-368. doi: 10.1377/hlthaff.28.2.361.

HealthIT.gov (no date) Meaningful Use and MACRA, 2018. Available at: https://www.healthit.gov/topic/meaningful-use-and-macra/meaningful-use-and-macra (Accessed: 7 May 2018).

Hegarty, F. et al. (2017) Healthcare Technology Management-A Systematic Approach. CRC Press. Available at: https://content.taylorfrancis.com/books/download?dac=C2014-0-334863\&isbn=9781498703550\&format=googlePreviewPdf (Accessed: 7 November 2019). Henry, J. et al. (2014) Adoption of Electronic Health Record Systems among U.S. Non-Federal Acute Care Hospitals: 2008-2015, Healthit.gov. Available at:

https://dashboard.healthit.gov/evaluations/data-briefs/non-federal-acute-care-hospital-ehradoption-2008-2015.php (Accessed: 6 November 2019).

HHS.gov (2018) FY 2018 Budget in Brief, The U.S. Department of Health and Human Services (HHS). Available at: https://www.hhs.gov/about/budget/fy2018/budget-in-brief/index.html (Accessed: 29 April 2020). 
Hillman, B. J. et al. (1990) 'Frequency and Costs of Diagnostic Imaging in Office Practice - A Comparison of Self-Referring and Radiologist-Referring Physicians', New England Journal of Medicine, 323(23), pp. 1604-1608. doi: 10.1056/NEJM199012063232306.

Hillman, B. J. et al. (1992) 'Physicians' Utilization and Charges for Outpatient Diagnostic Imaging in a Medicare Population', JAMA: The Journal of the American Medical Association, 268(15), p. 2050. doi: 10.1001/jama.1992.03490150102032.

Hillman, B. J. et al. (1995) 'Responses to a Payment Policy Denying Professional Charges for Diagnostic Imaging by Nonradiologist Physicians', JAMA: The Journal of the American Medical Association, 274(11), p. 885. doi: 10.1001/jama.1995.03530110047034. HIMSS (no date) HIMSS Usability Maturity Model | HIMSS. Available at: http://www.himss.org/himss-usability-maturity-model (Accessed: 6 June 2018).

HIMSS Analytics (2018) Electronic Medical Record Adoption Model. Available at: https://www.himssanalytics.org/emram (Accessed: 3 May 2018).

Hobbs, F. D. R. (2012) 'Envisioning a Learning Health Care System ':, Ann Fam Med, 10, pp. 54-59. doi: 10.1370/afm.1313.Department.

Holman, D. et al. (2000) 'Change and innovation in modern manufacturing practices: An expert panel survey of U.K. companies', Human Factors and Ergonomics in Manufacturing, 10(2), pp. 121-137. doi: 10.1002/(SICI)1520-6564(200021)10:2<121::AID-HFM1>3.0.CO;2-5.

Hygh, J. S. et al. (2012) 'Multivariate regression as an energy assessment tool in early building design', Building and Environment, 57, pp. 165-175. doi: 10.1016/J.BUILDENV.2012.04.021. Institute of Medicine (IOM) (2007) The Learning Healthcare System: Workshop Summary (IOM Roundtable on Evidence-Based Medicine), Roundtable on Evidence-Based Medicine. doi: $10.17226 / 11903$.

Institute of Medicine (US) Committee for the Study of the Future of Public Health (1988) The future of public health. National Academy Press (US). doi: 10.17226/1091.

Introna, V. et al. (2014) 'Energy Management Maturity Model: an organizational tool to foster the continuous reduction of energy consumption in companies', Journal of Cleaner Production, 83, pp. 108-117. doi: 10.1016/J.JCLEPRO.2014.07.001.

IOM (2011) Engineering a Learning Healthcare System. Washington, D.C.: Institute of Medicine and National Academy of Engineering National Academies Press. doi: $10.17226 / 12213$.

IOM (2013) Delivering High-Quality Cancer Care. Edited by L. Levit et al. Washington, D.C.: National Academies Press. doi: 10.17226/18359. 
Irony, T. et al. (2016) 'Incorporating Patient Preferences into Medical Device Benefit-Risk Assessments', Statistics in Biopharmaceutical Research, 8(3), pp. 230-236. doi:

10.1080/19466315.2015.1135820.

Iskin, I. (2014) An Assessment Model for Energy Efficiency Program Planning in Electric Utilities: Case of the Pacific of Northwest U.S.A., Dissertations and Theses. Portland, OR. doi: 10.15760/etd.1850.

Jacob, B. A. and Lefgren, L. (2011) 'The impact of research grant funding on scientific productivity', Journal of Public Economics, 95(9-10), pp. 1168-1177. doi:

10.1016/j.jpubeco.2011.05.005.

Jee, K. and Kim, G.-H. (2013) 'Potentiality of Big Data in the Medical Sector: Focus on How to Reshape the Healthcare System', Healthcare Informatics Research, 19(2), p. 79. doi:

10.4258/hir.2013.19.2.79.

Jha, A. and Epstein, A. (2010) 'Hospital Governance And The Quality Of Care', Health Affairs, 29(1), pp. 182-187. doi: 10.1377/hlthaff.2009.0297.

Johnson, L. C. et al. (2017) 'Fostering Collaboration Through Creation of an IBD Learning Health System', American Journal of Gastroenterology, 112(3), pp. 406-408. doi: 10.1038/ajg.2017.9.

Kappel, T. A. (2001) 'Perspectives on roadmaps: how organizations talk about the future', Journal of Product Innovation Management, 18(1), pp. 39-50. doi: 10.1016/S07376782(00)00066-7.

Kass, N. E. et al. (2013) 'The Research-Treatment Distinction: A Problematic Approach for Determining Which Activities Should Have Ethical Oversight', Hastings Center Report, 43(s1), pp. S4-S15. doi: 10.1002/hast.133.

Kayyali, B., Van Kuiken, S. and Knott, D. (2013) The big-data revolution in US health care: Accelerating value and innovation, McKinsey \& Company. Available at:

https://www.mckinsey.com/industries/healthcare-systems-and-services/our-insights/the-bigdata-revolution-in-us-health-care (Accessed: 7 May 2018).

Kelley, M. et al. (2015) 'Patient Perspectives on the Learning Health System: The Importance of Trust and Shared Decision Making', American Journal of Bioethics, 15(9), pp. 4-17. doi: 10.1080/15265161.2015.1062163.

Kerzner, H. (2001) Strategic planning for project management using a project management maturity model. John Wiley. Available at: https://www.wiley.com/enus/Strategic+Planning+for+Project+Management+Using+a+Project+Management+Maturity+M 
odel+-p-9780471436645 (Accessed: 4 May 2018).

Khurshid, A. (2017) 'A tale of two cities: Developing health information platforms for a learning health system in Austin and in New Orleans', Learning Health Systems, 1(2), p. e10017. doi: 10.1002//rh2.10017.

Kilbourne, A. M. et al. (2017) 'Accelerating Research Impact in a Learning Health Care System: VA's Quality Enhancement Research Initiative in the Choice Act Era.', Medical care, 55 Suppl 7(7 Suppl 1), pp. S4-S12. doi: 10.1097/MLR.0000000000000683.

Kinsella, G. and Gist, Y. (1995) Older Workers, Retirement, and Pensions: A Comparative International Chartbook - Kevin G. Kinsella, Yvonne J. Gist - Google Books. Bureau of the Census.

Kitson, A. L. and Harvey, G. (2016) 'Methods to Succeed in Effective Knowledge Translation in Clinical Practice', Journal of Nursing Scholarship, 48(3), pp. 294-302. doi:

10.1111/jnu.12206.

Knol, A. B. et al. (2010) 'The use of expert elicitation in environmental health impact assessment: a seven step procedure', Environmental Health, 9(1), p. 19. doi: 10.1186/1476069X-9-19.

Kocaoglu, D. F. (1983) 'A Participative Approach to Program Evaluation. IEEE Transactions on Engineering Management', IEEE Transactions on Engineering Management, 3:112-18. Kocaoglu, D. F. et al. (2016) 'Technology Assessment: Criteria for Evaluating a Sustainable Energy Portfolio', in, pp. 3-34. doi: 10.1007/978-3-319-18558-3_1.

Kohn, L., Corrigan, J. and Donaldson, M. (2002) 'To err is human: building a safer health system', National Academy of Science, Institute of Medicine, 6.

Kolodzey, L. et al. (2017) 'Wearable technology in the operating room : a systematic review', BMJ Innov, 3, pp. 55-63. doi: 10.1136/bmjinnov-2016-000133.

Kraft, S. et al. (2017) 'Building the learning health system: Describing an organizational infrastructure to support continuous learning', Learning Health Systems, (April), pp. 1-9. doi: 10.1002/lrh2.10034.

Kripalani, S. et al. (2014) 'Reducing Hospital Readmission Rates: Current Strategies and Future Directions', Annual Review of Medicine, 65(1), pp. 471-485. doi: 10.1146/annurev-med022613-090415.

Krumholz, H. M., Terry, S. F. and Waldstreicher, J. (2016) 'Data acquisition, curation, and use for a continuously learning health system', JAMA - Journal of the American Medical Association, 316(16), pp. 1669-1670. doi: 10.1001/jama.2016.12537. 
Kuhnert, P. M., Martin, T. G. and Griffiths, S. P. (2010) 'A guide to eliciting and using expert knowledge in Bayesian ecological models', Ecology Letters. doi: 10.1111/j.14610248.2010.01477.x.

Kurland, L. T. and Molgaard, C. A. (1981) 'The Patient Record in Epidemiology', Scientific American. Scientific American, a division of Nature America, Inc., pp. 54-63. doi: $10.2307 / 24964578$.

Kwon, S. et al. (2012) 'Creating a learning healthcare system in surgery: Washington State's Surgical Care and Outcomes Assessment Program (SCOAP) at 5 years', Surgery, 151(2), pp. 146-152. doi: 10.1016/j.surg.2011.08.015.

Lambin, P. et al. (2013) "“Rapid Learning health care in oncology" - An approach towards decision support systems enabling customised radiotherapy', Radiotherapy and Oncology, 109(1), pp. 159-164. doi: 10.1016/j.radonc.2013.07.007.

Langfeldt, L. (2004) 'Expert panels evaluating research: Decision-making and sources of bias', Research Evaluation. doi: 10.3152/147154404781776536.

Lavoie, J. R. and Daim, T. (2020) 'Towards the assessment of technology transfer capabilities: An action research-enhanced HDM model', Technology in Society, 60, p. 101217. doi: 10.1016/j.techsoc.2019.101217.

Lavoie, J. R., Kim, J. and Daim, T. U. (2017) 'A Technology Transfer Framework: A Case Study from the Energy Sector’, 8(4). doi: 10.18178/ijimt.2017.8.4.737.

LeBreton, J. M. and Senter, J. L. (2008) 'Answers to 20 Questions About Interrater Reliability and Interrater Agreement', Organizational Research Methods, 11(4), pp. 815-852. doi: $10.1177 / 1094428106296642$.

Lee, C. J. et al. (2013) 'Bias in peer review', Journal of the American Society for Information Science and Technology, 64(1), pp. 2-17. doi: 10.1002/asi.22784.

Lee, J., Lee, D. and Kang, S. (2007) 'An Overview of the Business Process Maturity Model (BPMM)', in Advances in Web and Network Technologies, and Information Management. Berlin, Heidelberg: Springer Berlin Heidelberg, pp. 384-395. doi: 10.1007/978-3-540-729099_42.

Lee, S. B. (2013) 'A continuous quality improvement project to reduce medication error in the emergency department', World Journal of Emergency Medicine, 4(3), p. 179. doi: 10.5847/wjem.j.issn.1920-8642.2013.03.004.

Lehoux, P. (2004) 'Patients' perspectives on high-tech home care: A qualitative inquiry into the user-friendliness of four technologies', BMC Health Services Research, 4. doi: 10.1186/1472- 
6963-4-28.

LeSueur, D. (2017) Governance in Healthcare: The Biggest Factor in Success, HealthCatalyst . Available at: https://www.healthcatalyst.com/governance-in-healthcare-the-biggest-factor-insuccess/ (Accessed: 7 February 2021).

Leung, L. C. and Cao, D. (2000) 'On consistency and ranking of alternatives in fuzzy AHP', European Journal of Operational Research, 124(1), pp. 102-113. doi: 10.1016/S03772217(99)00118-6.

Levitan, B., Phillips, L. D. and Walker, S. (2014) 'Structured Approaches to Benefit-Risk Assessment', Therapeutic Innovation \& Regulatory Science, 48(5), pp. 564-573. doi: $10.1177 / 2168479014536500$.

Li, L. and Benton, W. C. (2006) 'Hospital technology and nurse staffing management decisions', Journal of Operations Management, 24(5), pp. 676-691. doi:

10.1016/j.jom.2005.06.001.

Lilburne, L. and Tarantola, S. (2009) 'Sensitivity analysis of spatial models', International Journal of Geographical Information Science, 23(2), pp. 151-168. doi:

10.1080/13658810802094995.

Lingga, M. (2016) Developing a Hierarchical Decision Model to Evaluate Nuclear Power Plant Alternative Siting Technologies. Portland State University. doi:

https://doi.org/10.15760/etd.2938.

Liu, C.-F., Hwang, H.-G. and Chang, H.-C. (2011) 'E-Healthcare Maturity in Taiwan', Telemedicine and e-Health, 17(7), pp. 569-573. doi: 10.1089/tmj.2010.0228.

Lowes, L. P. et al. (2017) “'Learn From Every Patient”: implementation and early results of a learning health system', Developmental Medicine and Child Neurology, 59(2), pp. 183-191. doi: 10.1111/dmen.13227.

Lukowicz, P., Kirstein, T. and Tröster, G. (2004) 'Wearable systems for health care applications.', Methods of information in medicine, 43(3), pp. 232-8. doi:

10.1267/METH04030232.

Lynch, J. et al. (2000) 'Social capital--is it a good investment strategy for public health?', Journal of epidemiology and community health, 54(6), pp. 404-8. doi: 10.1136/JECH.54.6.404. Lyons, E. J. et al. (2014) 'Behavior change techniques implemented in electronic lifestyle activity monitors: a systematic content analysis.', Journal of medical Internet research, 16(8), p. e192. doi: 10.2196/jmir.3469.

Mahoney, M. J. (1977) 'Publication prejudices: An experimental study of confirmatory bias in 268 
the peer review system', Cognitive Therapy and Research, 1(2), pp. 161-175. doi: 10.1007/BF01173636.

Makary, M. A. and Daniel, M. (2016) 'Medical error-the third leading cause of death in the US.', BMJ (Clinical research ed.), 353, p. i2139. doi: 10.1136/BMJ.I2139.

Malenfant, J. M. et al. (2019) 'Cross-Network Directory Service: Infrastructure to enable collaborations across distributed research networks', Learning Health Systems, (October 2018), p. e10187. doi: 10.1002/lrh2.10187.

Mallard, S. et al. (2004) 'Same-Day Scheduling in a Public Health Clinic: A Pilot Study: Journal of Public Health Management and Practice', Journal of Public Health Management and Practice, 10(2), pp. 148-155. Available at:

https://journals.lww.com/jphmp/Abstract/2004/03000/Same_Day_Scheduling_in_a_Public_Hea lth_Clinic_A.9.aspx (Accessed: 11 March 2018).

Mandl, K. D. et al. (2014) 'Scalable collaborative infrastructure for a learning healthcare system (SCILHS): Architecture', Journal of the American Medical Informatics Association, 21(4), pp. 615-620. doi: 10.1136/amiajnl-2014-002727.

Mardis, E. R. (2008) 'The impact of next-generation sequencing technology on genetics', Trends in Genetics, 24(3), pp. 133-141. doi: 10.1016/J.TIG.2007.12.007.

Martin, T. G. et al. (2005) 'THE POWER OF EXPERT OPINION IN ECOLOGICAL MODELS USING BAYESIAN METHODS: IMPACT OF GRAZING ON BIRDS', Ecological Applications, 15(1), pp. 266-280. doi: 10.1890/03-5400.

Martin, T. G. et al. (2012) 'Eliciting Expert Knowledge in Conservation Science', Conservation Biology. doi: 10.1111/j.1523-1739.2011.01806.x.

McClellan, M. B. et al. (2008) Evidence-Based Medicine and the Changing Nature of Health Care: Meeting Summary (IOM Roundtable on Evidence-Based Medicine). doi: 10.17226/12041. McGlynn, E. A. et al. (2003) 'The Quality of Health Care Delivered to Adults in the United States', New England Journal of Medicine, 348(26), pp. 2635-2645. doi:

10.1056/NEJMsa022615.

McKinsey\&Comapny (no date) The big-data revolution in US health care: Accelerating value and innovation | McKinsey \&amp; Company. Available at:

https://www.mckinsey.com/industries/healthcare-systems-and-services/our-insights/the-bigdata-revolution-in-us-health-care (Accessed: 18 January 2018).

McMahon, M. et al. (2020) 'Informing Canada's health system response to COVID-19: Priorities for health services and policy research', Healthcare Policy. Longwoods Publishing 269 
Corp., pp. 1-27. doi: 10.12927/HCPOL.2020.26249.

Mechri, H. E., Capozzoli, A. and Corrado, V. (2010) 'USE of the ANOVA approach for sensitive building energy design', Applied Energy, 87(10), pp. 3073-3083. doi:

10.1016/J.APENERGY.2010.04.001.

Melton III, L. . (1997) 'The threat to medical-records research', New England Journal of Medicine, 337(1466-1470).

Menear, M. et al. (2019) 'A framework for value-creating learning health systems', Health Research Policy and Systems, 17(1), pp. 1-13. doi: 10.1186/s12961-019-0477-3.

Merriam-Webster (2019a) Expert | Definition of Expert by Merriam-Webster. Available at: https://www.merriam-

webster.com/dictionary/expert?utm_campaign=sd\&utm_medium=serp\&utm_source=jsonld\#lea rn-more (Accessed: 16 March 2019).

Merriam-Webster (2019b) Panel | Definition of Panel by Merriam-Webster. Available at: https://www.merriam-webster.com/dictionary/panel (Accessed: 16 March 2019).

Mettler, T. and Blondiau, A. (2012) 'HCMM - a maturity model for measuring and assessing the quality of cooperation between and within hospitals', in 2012 25th IEEE International Symposium on Computer-Based Medical Systems (CBMS). IEEE, pp. 1-6. doi:

10.1109/CBMS.2012.6266397.

Mettler, T. and Rohner, P. (2009) 'Situational maturity models as instrumental artifacts for organizational design', Proceedings of the 4th International Conference on Design Science Research in Information Systems and Technology - DESRIST '09, p. 1. doi:

$10.1145 / 1555619.1555649$.

Mettler, T. and Vimarlund, V. (2009) 'Understanding business intelligence in the context of healthcare', Health Informatics Journal, 15(3), pp. 254-264. doi: 10.1177/1460458209337446. Van Der Meulen, J. P. (1994) 'Can Academic Health Centers Survive Health Care Reform?', Neurosurgery, 35(4), pp. 725-731. doi: 10.1227/00006123-199410000-00020.

Meyer, M. A. and Booker, J. M. (2001) Eliciting and analyzing expert judgment : a practical guide. Society for Industrial and Applied Mathematics and American Statistical Association. Milstein, A. (2013) 'Code Red and Blue - Safely Limiting Health Care's GDP Footprint', The NEW ENGLAND JOURNAL of MEDICINE, 368(1), pp. 1-3. doi: 10.1056/NEJMp1002530. Min-Hooi Chuah, M.-H. and Wong, K.-L. (2012) 'A framework for accessing an enterprise business intelligence maturity model (EBI2M): Delphi study approach', AFRICAN JOURNAL OF BUSINESS MANAGEMENT, 6(23), pp. 6880-6889. doi: 10.5897/AJBM11.2819. 
Mohr, J. J. (2005) ‘Creating a Safe Learning Organization', Frontiers of Health Services Management, 22(1), pp. 41-44. doi: 10.1097/01974520-200507000-00006.

De Moor, G. et al. (2015) 'Using electronic health records for clinical research: The case of the EHR4CR project', Journal of Biomedical Informatics, 53, pp. 162-173. doi:

10.1016/j.jbi.2014.10.006.

Morain, S. R. and Kass, N. E. (2016) 'Ethics Issues Arising in the Transition to Learning Health Care Systems: Results from Interviews with Leaders from 25 Health Systems.', EGEMS (Washington, DC), 4(2), p. 1212. doi: 10.13063/2327-9214.1212.

Morain, S. R., Kass, N. E. and Faden, R. R. (2018) 'Learning Is Not Enough: Earning Institutional Trustworthiness Through Knowledge Translation', American Journal of Bioethics. Routledge, pp. 31-34. doi: 10.1080/15265161.2018.1431708.

Morain, S. R., Kass, N. E. and Grossmann, C. (2017) 'What allows a health care system to become a learning health care system: Results from interviews with health system leaders', Learning Health Systems, 1(1), p. e10015. doi: 10.1002/lrh2.10015.

Morgan, M. G. (2014) 'Use (and abuse) of expert elicitation in support of decision making for public policy.', Proceedings of the National Academy of Sciences of the United States of America, 111(20), pp. 7176-84. doi: 10.1073/pnas.1319946111.

Morris, M. D. (1991) 'Factorial Sampling Plans for Preliminary Computational Experiments', Technometrics, 33(2), pp. 161-174. doi: 10.1080/00401706.1991.10484804.

Morris, Z. S., Wooding, S. and Grant, J. (2011) 'The answer is 17 years, what is the question: understanding time lags in translational research', Journal of the Royal Society of Medicine, 104(12), pp. 510-520. doi: 10.1258/jrsm.2011.110180.

Mottes, T. A., Goldstein, S. L. and Basu, R. K. (2019) 'Process based quality improvement using a continuous renal replacement therapy dashboard', BMC Nephrology, 20(1), pp. 1-10. doi: 10.1186/s12882-018-1195-8.

Mudavadi, C., Hogaboam, L. and Daim, T. U. (2016) 'A hierarchical decision model (HDM) for exploring the adoption of electronic health records', in 2016 Portland International Conference on Management of Engineering and Technology (PICMET). IEEE, pp. 2770-2781. doi:

10.1109/PICMET.2016.7806627.

von Muhlen, M. and Ohno-Machado, L. (2012) 'Reviewing social media use by clinicians', Journal of the American Medical Informatics Association, 19(5), pp. 777-781. doi:

10.1136/amiajnl-2012-000990.

Mullen, B. and Goethals, G. R. (1987) Theories of group behavior. Springer-Verlag. Available 
at:

https://books.google.com/books/about/Theories_of_group_behavior.html?id=ZrfaAAAAMAAJ (Accessed: 16 March 2019).

Mumpower, J. L. and Stewart, T. R. (1996) 'Expert Judgement and Expert Disagreement', Thinking \& Reasoning. doi: 10.1080/135467896394500.

Munkongsujarit, S. et al. (2009) 'Decision model for a place to live at PSU: The case of international graduate students', in PICMET '09 - 2009 Portland International Conference on Management of Engineering \& Technology. IEEE, pp. 513-534. doi:

10.1109/PICMET.2009.5262104.

Muñoz, B., Romana, M. G. and Ordóñez, J. (2016) 'Sensitivity Analysis of Multicriteria Decision Making Methodology Developed for Selection of Typologies of Earth-retaining Walls in an Urban Highway', Transportation Research Procedia, 18(June), pp. 135-139. doi: 10.1016/j.trpro.2016.12.019.

Nambiar, R. et al. (2013) 'A look at challenges and opportunities of Big Data analytics in healthcare', in 2013 IEEE International Conference on Big Data. IEEE, pp. 17-22. doi: 10.1109/BigData.2013.6691753.

Nass, Sharyl J. et al. (2009) Beyond the HIPAA privacy rule : enhancing privacy, improving health through research. National Academies Press.

Nass, Sharyl J et al. (2009) Beyond the HIPAA Privacy Rule, Beyond the HIPAA Privacy Rule: Enhancing Privacy, Improving Health Through Research. National Academies Press (US). doi: $10.17226 / 12458$.

Nathavitharana, R. R. et al. (2020) 'Innovation and knowledge sharing can transform COVID19 infection prevention response', Journal of Hospital Medicine. Frontline Medical Communications, pp. 299-301. doi: 10.12788/jhm.3439.

National Health Service (NHS) (2014) 'Five Year Forward View', (October). Available at: https://www.england.nhs.uk/wp-content/uploads/2014/10/5yfv-web.pdf.

National Institutes of Health (NIH) (2020) Impact of NIH Research, NIH. Available at: https://www.nih.gov/about-nih/what-we-do/impact-nih-research (Accessed: 13 April 2020). National Research Council (1997) For the Record: Protecting Electronic Health Information. The National Academies Press. Available at: https://www.nap.edu/initiative/committee-onmaintaining-privacy-and-security-in-health-care-applications-of-the-national-informationinfrastructure (Accessed: 4 March 2019).

Neff, A. A. et al. (2014) 'Developing a maturity model for service systems in heavy equipment 
manufacturing enterprises', Information and Management, 51(7), pp. 895-911. doi:

10.1016/j.im.2014.05.001.

Nehta (2007) 'National E-Health Transition Authority Interoperability Framework', (August 2007). Available at: https://www.ghdonline.org/uploads/NEHTA_2007_-

_InteroperabilityFramework.pdf.

Nemet, G. F., Anadon, L. D. and Verdolini, E. (2017) 'Quantifying the Effects of Expert

Selection and Elicitation Design on Experts' Confidence in Their Judgments About Future

Energy Technologies', Risk Analysis, 37(2), pp. 315-330. doi: 10.1111/risa.12604.

NHS (2011) Infrastructure Maturity Model, NHS Digital. Available at:

https://digital.nhs.uk/services/nhs-infrastructure-maturity-model (Accessed: 3 May 2018).

Nicolucci, A. et al. (2008) 'Five-year impact of a continuous quality improvement effort

implemented by a network of diabetes outpatient clinics', Diabetes Care, 31(1), pp. 57-62. doi:

$10.2337 / \mathrm{dc} 07-1515$.

Nolan, R. L. (1973) 'Managing the computer resource: a stage hypothesis', Communications of the ACM, 16(7), pp. 399-405. doi: 10.1145/362280.362284.

Nordo, A. H. et al. (2019) 'Use of EHRs data for clinical research: Historical progress and current applications', Learning Health Systems, 3(1), p. e10076. doi: 10.1002/lrh2.10076. Nunes, J. W. et al. (2016) 'Continuous quality improvement in nephrology: a systematic review', BMC Nephrology, 17(1), pp. 1-9. doi: 10.1186/s12882-016-0389-1.

O. McGraw, K. and P. Wong, S. (1996) 'Forming inferences about some intraclass correlation coefficients', Psychological Methods, 1(1), pp. 30-46. doi: Doi 10.1037//1082-989x.1.4.390.

Okoli, C. and Pawlowski, S. D. (2004) 'The Delphi method as a research tool: An example, design considerations and applications', Information and Management. doi:

10.1016/j.im.2003.11.002.

Okun, S. and Goodwin, K. (2017) 'Building a learning health community: By the people, for the people', Learning Health Systems, 1(3), p. e10028. doi: 10.1002/lrh2.10028.

Olsen, L., Saunders, R. S. and Mcginnis, J. M. (no date) 'THE LEARNING HEALTH SYSTEM SERIES PATIENTS CHARTING THE COURSE Citizen Engagement and the Learning Health System Workshop Summary'. Available at: https://www.ncbi.nlm.nih.gov/books/NBK91496/pdf/Bookshelf_NBK91496.pdf.

Olsen, L., Saunders, R. and Yong, P. (2010) The Healthcare Imperative: Lowering Costs and Improving Outcomes: Workshop ... - Institute of Medicine, Roundtable on Evidence-Based Medicine - Google Books. Edited by Institute of Medicine. National Academies Press. 
Oregon Health Authority (2021) CCO Metrics : Office of Health Analytics : State of Oregon, Oregon Health Authority. Available at:

https://www.oregon.gov/oha/HPA/ANALYTICS/Pages/CCO-

Metrics.aspx?wp2897=se:\%22child+imm\%22 (Accessed: 8 February 2021).

Oxford Dictionary (2019a) disagreement | Definition of disagreement in English by Oxford

Dictionaries. Available at: https://en.oxforddictionaries.com/definition/disagreement (Accessed:

18 March 2019).

Oxford Dictionary (2019b) inconsistent | Definition of inconsistent in English by Oxford

Dictionaries. Available at: https://en.oxforddictionaries.com/definition/inconsistent (Accessed:

17 March 2019).

Pak, J. and Song, Y.-T. (2016) 'Health Capability Maturity Model: Person-centered Approach in Personal Health Record System', in Twenty-second Americas Conference on Information Systems, . San Diego.

Park, C. K. and Ahn, K.-I. (1994) 'A new approach for measuring uncertainty importance and distributional sensitivity in probabilistic safety assessment', Reliability Engineering \& System Safety, 46(3), pp. 253-261. doi: 10.1016/0951-8320(94)90119-8.

Paton, F. L., Maier, H. R. and Dandy, G. C. (2013) 'Relative magnitudes of sources of uncertainty in assessing climate change impacts on water supply security for the southern Adelaide water supply system', Water Resources Research, 49(3), pp. 1643-1667. doi: 10.1002/wrcr.20153.

Paulk, M. (2002) 'Capability Maturity Model for Software', in Encyclopedia of Software Engineering. Hoboken, NJ, USA: John Wiley \& Sons, Inc. doi: 10.1002/0471028959.sof589. Paulk, M. C. (2009) 'A History of the Capability Maturity Model for Software', The Software Quality Profile, 1(1), pp. 5-19.

Paulzen, O. et al. (2002) 'A Maturity Model for Quality Improvement in Knowledge Management', (December).

Pereira, C. et al. (2018) 'Assessment of biotechnologies: a therapeutic monoclonal antibody emerging score', in 2018 IEEE Technology and Engineering Management Conference (TEMSCON). IEEE, pp. 1-6. doi: 10.1109/TEMSCON.2018.8488437.

Pereira, C. G. et al. (2018) 'Forecasting of emerging therapeutic monoclonal antibodies patents based on a decision model', Technological Forecasting and Social Change, (September). doi: 10.1016/j.techfore.2018.11.002.

Petrick, I. J. (2008) 'Developing and Implementing Roadmaps - A Reference Guide', White 
Paper, pp. 1-11. Available at: https://www.sopheon.com/wp-content/uploads/WhitePaperPetrick-RoadmappingReferenceGuide.pdf.

Phan, K. (2013) 'Innovation Measurement: A Decision Framework to Determine Innovativeness of a Company', Dissertations and Theses, Portland State University. doi: https://doi.org/10.15760/etd.1017.

Pianosi, F. et al. (2016) 'Sensitivity analysis of environmental models: A systematic review with practical workflow', Environmental Modelling \& Software, 79, pp. 214-232. doi: 10.1016/J.ENVSOFT.2016.02.008.

Pinto-valverde, J. M. et al. (2013) 'HDQM2 : Healthcare Data Quality Maturity Model', Transactions of the International Conference on Health Information Technology Advancement, pp. 199-207. doi: 10.1016/j.clay.2010.10.025.

Poksinska, B., Swartling, D. and Drotz, E. (2013) 'The daily work of Lean leaders - lessons from manufacturing and healthcare', Total Quality Management and Business Excellence, 24(78), pp. 886-898. doi: 10.1080/14783363.2013.791098.

Pomeroy, C. (no date) How Research Can and Should Inform Public Policy, Association of Academic Health Centers. Available at: https://www.aahcdc.org/Publications-

Resources/Author-Commentaries/How-Research-Can-and-Should-Inform-Public-Policy (Accessed: 1 February 2021).

Porter, M. E. and Teisberg, E. O. (2006) Redefining Health Care: Creating Value-based Competition on Results. Harvard Business Press.

Prentnieks, M. and Qual, S. (1996) 'Protecting the Privacy of Computerized Health Information: The Kassebaum-Kennedy Act', Minnesota Medicine, 79(11), pp. 47-51.

Price-Haywood, E. G. (2015) 'Clinical Comparative Effectiveness Research Through the Lens of Healthcare Decisionmakers.', The Ochsner journal, 15(2), pp. 154-161.

Priestman, W. (2007) ICT Strategy 2007-2011 for The Royal Liverpool and Broadgreen University Hospitals NHS Trust. Trust Board Meeting 6th November 2007.

Priestman, W. et al. (2019) 'Lessons learned from a comprehensive electronic patient record procurement process - Implications for healthcare organisations', BMJ Health and Care Informatics, 26(1), pp. 1-6. doi: 10.1136/bmjhci-2019-000020.

Project Management Institute (PMI) (2013) Organizational Project Management Maturity Model (OPM3) - Knowledge Foundation, Third Edition.

Pronovost, P. J. et al. (2017) 'Creating a purpose-driven learning and improving health system: The Johns Hopkins Medicine quality and safety experience', Learning Health Systems, 1(1), p. 
e10018. doi: 10.1002//rh2.10018.

Quintegra Solutions Limited (2008) Electronic Healthcare Maturity Model (eHMM). Chennai . Available at: http://www.quintegrasolutions.com/eHMM White Paper.pdf (Accessed: 23 February 2019).

Raber, D., Winter, R. and Wortmann, F. (2012) 'Using quantitative analyses to construct a capability maturity model for Business Intelligence', Proceedings of the Annual Hawaii International Conference on System Sciences, pp. 4219-4228. doi: 10.1109/HICSS.2012.630.

Ren, Y., Pazzi, R. and Boukerche, a (2010) 'Monitoring patients via a secure and mobile healthcare system', Wireless Communications, IEEE, 17(February), pp. 59-65. doi:

10.1109/MWC.2010.5416351.

Rocha, A. (2011) 'Evolution of information systems and technologies maturity in healthcare', in Tan, J. (ed.) Healthcare Information Technology Innovation and Sustainability: Frontiers and Adoption. McMaster University, p. 238.

Rodríguez Perera, F. de P. and Peiró, M. (2012) 'Strategic Planning in Healthcare Organizations', Revista Española de Cardiología (English Edition), 65(8), pp. 749-754. doi: 10.1016/J.REC.2012.04.004.

Roth, M. G. et al. (2016) 'The Learning Health-System: A New Frontier for Human Factors', Proceedings of the International Symposium on Human Factors and Ergonomics in Health Care, 5(1), pp. 141-144. doi: 10.1177/2327857916051020.

Rothstein, M. A. and Talbott, M. K. (2006) 'Compelled disclosure of health information: Protecting against the greatest potential threat to privacy', Journal of the American Medical Association, 295(24), pp. 2882-2885. doi: 10.1001/jama.295.24.2882.

Rouse, M. (2018) What is protected health information (PHI) or personal health information?, TechTarget. Available at: https://searchhealthit.techtarget.com/definition/personal-healthinformation (Accessed: 30 April 2019).

Rouse, W. B. (2001) Essential challenges of strategic management. Wiley. Available at: https://www.wiley.com/en-us/Essential+Challenges+of+Strategic+Management-p9780471389248 (Accessed: 6 April 2018).

Rouse, W. B. (2008) 'Health care as a complex adaptive system: implications for design and management', The Bridge (National Academy of Engineering), 38(1), pp. 17-25. doi: $10.1177 / 1538574411407082$.

Rouse, W. B., Johns, M. M. E. and Pepe, K. M. (2017) 'Learning in the health care enterprise', Learning Health Systems, (February), p. e10024. doi: 10.1002/lrh2.10024. 
Rubin, J. (2017) 'Patient empowerment and the Learning Health System', Learning Health Systems, 1(3), p. e10030. doi: 10.1002/lrh2.10030.

Rudd, R. E. (2010) 'Improving Americans' Health Literacy', New England Journal of Medicine, 363(24), pp. 2283-2285. doi: 10.1056/nejmp1008755.

Rumsfeld, J. S., Joynt, K. E. and Maddox, T. M. (2016) 'Big data analytics to improve cardiovascular care: Promise and challenges', Nature Reviews Cardiology, 13(6), pp. 350-359. doi: $10.1038 /$ nrcardio.2016.42.

Runge, M. C., Converse, S. J. and Lyons, J. E. (2011) 'Which uncertainty? Using expert elicitation and expected value of information to design an adaptive program', Biological Conservation, 144(4), pp. 1214-1223. doi: 10.1016/J.BIOCON.2010.12.020.

Saaty, T. L. (1977) 'A scaling method for priorities in hierarchical structures', Journal of Mathematical Psychology, 15(3), pp. 234-281. doi: 10.1016/0022-2496(77)90033-5.

Saltelli, A., Tarantola, S. and Chan, K. P. S. (1999) 'A quantitative model-independent method for global sensitivity analysis of model output', Technometrics. doi:

10.1080/00401706.1999.10485594.

Sanders, D. and Burton, D. A. (2016) 'The Healthcare Analytics Adoption Model: A

Framework and Roadmap', Health Catalyst Whitepaper.

Santilli, J. and Randy Vogenberg, F. (2015) 'Key strategic trends that impact healthcare decision-making and stakeholder roles in the new marketplace', American Health and Drug Benefits, 8(1), pp. 15-20.

Santilli, J. and Vogenberg, F. R. (2015) 'Key Strategic Trends that Impact Healthcare DecisionMaking and Stakeholder Roles in the New Marketplace.', American health \& drug benefits, 8(1), pp. 15-20. Available at: http://www.ncbi.nlm.nih.gov/pubmed/25945154 (Accessed: 7 May 2018).

Saunders, R. and Smith, M. D. (2013) The path to continuously learning health care, Issues in Science and Technology. doi: 10.17226/13444.

Schmittdiel, J. A. et al. (2017) 'The Delivery Science Rapid Analysis Program: a research and operational partnership at Kaiser Permanente Northern California', Learning Health Systems, 1(4), p. e10035. doi: 10.1002/lrh2.10035.

Sekar, K. (2020) National Institutes of Health (NIH) Funding: FY1994-FY2020. Available at: www.crs.gov (Accessed: 29 April 2020).

Seoane-Vazquez, E. et al. (2008) 'Incentives for orphan drug research and development in the United States', Orphanet Journal of Rare Diseases, 3(1), p. 33. doi: 10.1186/1750-1172-3-33. 
Seoane, L. et al. (2013) 'Using quality improvement principles to improve the care of patients with severe sepsis and septic shock', Ochsner Journal, 13(3), pp. 359-366.

Shah, A. et al. (2016) 'Building a rapid learning health care system for oncology: Why cancerlinq collects identifiable health information to achieve its vision', Journal of Clinical Oncology, 34(7), pp. 756-763. doi: 10.1200/JCO.2015.65.0598.

Shah, N. (2016) EMR Data mining.

Shanafelt, T. D. et al. (2012) 'Burnout and satisfaction with work-life balance among US physicians relative to the general US population', Archives of Internal Medicine, 172(18), pp. 1377-1385. doi: 10.1001/archinternmed.2012.3199.

Sharma, B. (2008) 'Electronic Healthcare Maturity Model', HIMSS AnalyticsTM White Paper, (June).

Shaygan, A. et al. (2017) 'Adoption criteria evaluation of activity tracking wristbands for university students', in PICMET 2017 - Portland International Conference on Management of Engineering and Technology: Technology Management for the Interconnected World, Proceedings. doi: 10.23919/PICMET.2017.8125443.

Shaygan, A. et al. (2018a) 'Decision Making Model for Choosing Voice-Operated Intelligent Speakers for Graduate Students', in 2018 IEEE International Conference on Engineering, Technology and Innovation, ICE/ITMC 2018 - Proceedings. doi: 10.1109/ICE.2018.8436273. Shaygan, A. (2018) 'Landscape Analysis: What Are the Forefronts of Change in the US Hospitals?', in Daim, T., Chang, L., and Estep, J. (eds) Infrastructure and Technology Management. Springer International Publishing AG, pp. 213-243. doi: 10.1007/978-3-31968987-6_6.

Shaygan, A. et al. (2018b) 'Operated Intelligent Speakers for Graduate Students', in 2018 IEEE International Conference on Engineering, Technology and Innovation (ICE/ITMC). IEEE, pp. $1-9$.

Shaygan, A. and Daim, T. (2019) 'Technology Management Maturity Assessment Model : An Exploratory Multi-criteria Approach for Healthcare Organizations', in Portland International Conference on Management of Engineering and Technology (PICMET). IEEE.

Shaygan, A., Lavoie, J. R. and Daim, T. (2018) 'Learning Health Systems : A Multi-perspective Analysis', in IEEE Technology and Engineering Management Conference (TEMSCON), pp. 16.

Shaygan, A., Ozdemir-Gungor, D. Kutgun, H. and Daneshi, A. (2017) 'Adoption Criteria Evaluation of Activity Tracking Wristbands for University Students', Picmet. 
Shaygan, A. and Testik, Ö. M. (2017) 'A fuzzy AHP-based methodology for project prioritization and selection', Soft Computing. doi: 10.1007/s00500-017-2851-9.

Shellum, J. L. et al. (2017) 'Knowledge management in the era of digital medicine: A programmatic approach to optimize patient care in an academic medical center', Learning Health Systems, 1(2), p. e10022. doi: 10.1002/lrh2.10022.

Sheskin, D. J. (2007) Handbook of PARAMETRIC and NONPARAMETRIC STATISTICAL PROCEDURES. Available at: http://www.biruni.tn/gw_2009_4_3/thumbs/contentstable/73/TM.739196.pdf (Accessed: 18 March 2019).

Shi, L. and Singh, D. (2019) Delivering health care in America: a system approach. 7th Edition. Burlington, MA: Jones \& Bartlett Learning.

Shrout, P. E. and Fleiss, J. L. (1979) 'Intraclass correlations: Uses in assessing rater reliability.', Psychological Bulletin, 86(2), pp. 420-428. doi: 10.1037/0033-2909.86.2.420.

Singer, S. J., Benzer, J. K. and Hamdan, S. U. (2015) 'Improving health care quality and safety: The role of collective learning', Journal of Healthcare Leadership. Dove Medical Press Ltd., pp. 91-107. doi: 10.2147/JHL.S70115.

Sinha, A. (2021) Reducing COVID-19 vaccine hesitancy among racial and ethnic minorities, Baylor College of Medicine. Available at: https://blogs.bcm.edu/2021/01/22/reducing-covid-19vaccine-hesitancy-among-racial-and-ethnic-minorities/ (Accessed: 7 February 2021). Solberg, L. I. et al. (1997) 'Using continuous quality improvement to improve diabetes care in populations: the IDEAL model. Improving care for Diabetics through Empowerment Active collaboration and Leadership.', The Joint Commission journal on quality improvement, 23(11), pp. 581-592. doi: 10.1016/S1070-3241(16)30341-8.

Sorenson, C., Drummond, M. and Khan, B. B. (2013) 'Medical technology as a key driver of rising health expenditure: Disentangling the relationship', ClinicoEconomics and Outcomes Research, 5(1), pp. 223-234. doi: 10.2147/CEOR.S39634.

Speziale, G. (2015) 'Strategic management of a healthcare organization: Engagement, behavioural indicators, and clinical performance', European Heart Journal, Supplement, 17, pp. A3-A7. doi: 10.1093/eurheartj/suv003.

Spitz, C. et al. (2012) 'Practical application of uncertainty analysis and sensitivity analysis on an experimental house', Energy and Buildings, 55, pp. 459-470. doi:

10.1016/J.ENBUILD.2012.08.013.

Stall, J. (2010) Aging and the Health Care Workforce - Population Reference Bureau. Available at: https://www.prb.org/agingandhealthcare/ (Accessed: 6 December 2019). 
Storlie, C. B. et al. (2009) 'Implementation and evaluation of nonparametric regression procedures for sensitivity analysis of computationally demanding models', Reliability Engineering \& System Safety, 94(11), pp. 1735-1763. doi: 10.1016/J.RESS.2009.05.007. Strech, D., Weissgerber, T. and Dirnagl, U. (2020) 'Improving the trustworthiness, usefulness, and ethics of biomedical research through an innovative and comprehensive institutional initiative', PLOS Biology, 18(2), p. e3000576. doi: 10.1371/journal.pbio.3000576.

Suchman, A. L. (2001) 'The influence of health care organizations on well-being.', The Western journal of medicine, 174(1), pp. 43-7. Available at: http://www.ncbi.nlm.nih.gov/pubmed/11154668 (Accessed: 11 March 2018).

Sutcliffe, K. M. and Weber, K. (2003) 'The high cost of accurate knowledge.', Harvard business review, 81(5), pp. 74-82, 129. Available at: http://www.ncbi.nlm.nih.gov/pubmed/12747164 (Accessed: 11 March 2018).

Sutherland, W. J. et al. (2011) 'Horizon scan of global conservation issues for 2011', Trends in Ecology \& Evolution, 26(1), pp. 10-16. doi: 10.1016/J.TREE.2010.11.002.

Swensen, S. J. and Cortese, D. A. (2008) 'Transparency and the "End Result Idea", Chest, 133(1), pp. 233-5. doi: 10.1378/chest.07-2101.

Swinehart, K. D. and Smith, A. E. (2005) 'Internal supply chain performance measurement: A health care continuous improvement implementation', International Journal of Health Care Quality Assurance, 18(7), pp. 533-542. doi: 10.1108/09526860510627210.

Taha, R. A. et al. (2007) 'Application of Hierarchical Decision Modeling for Selection of Laptop', in PICMET '07 - 2007 Portland International Conference on Management of Engineering \& Technology. IEEE, pp. 1160-1175. doi: 10.1109/PICMET.2007.4349440. Takenouchi, K. et al. (2019) 'Development of a new seamless data stream from EMR to EDC system using SS-MIX2 standards applied for observational research in diabetes mellitus', Learning Health Systems, 3(1), p. e10072. doi: 10.1002/lrh2.10072.

Taleb-Bendiab, A. et al. (2006) 'A principled approach to the design of healthcare systems: Autonomy vs. governance', Reliability Engineering and System Safety, 91(12), pp. 1576-1585. doi: 10.1016/j.ress.2006.01.011.

Tarhan, A., Turetken, O. and van den Biggelaar, F. J. H. M. (2015) 'Assessing Healthcare Process Maturity: Challenges of Using a Business Process Maturity Model', Proceedings of the 9th International Conference on Pervasive Computing Technologies for Healthcare, pp. 339342. doi: 10.4108/icst.pervasivehealth.2015.259105.

Teece, D. J. (2007) 'EXPLICATING DYNAMIC CAPABILITIES: THE NATURE AND 280 
MICROFOUNDATIONS OF (SUSTAINABLE) ENTERPRISE PERFORMANCE', Strategic

Management Journal Strat. Mgmt. J, 28, pp. 1319-1350. doi: 10.1002/smj.640.

Tegenege, M. et al. (2017) 'Advancing the science of patient input throughout theregulatory decision-making process.pdf’, Learning Health Systems, 1(3).

Testik, O. M. et al. (2017a) 'Selecting Health Care Improvement Projects : A Methodology Integrating Cause-and-Effect', Quality Management in Healthcare, 26(1), pp. 40-48. doi: 10.1097/QMH.0000000000000119.

Testik, O. M. et al. (2017b) 'Selecting health care improvement projects: A methodology integrating cause-and-effect diagram and analytical hierarchy process', Quality Management in Health Care. doi: 10.1097/QMH.0000000000000119.

The World Bank (no date) Health expenditure, total (\% of GDP) | Data. Available at: https://data.worldbank.org/indicator/SH.XPD.TOTL.ZS?view=chart\&year_high_desc=true (Accessed: 18 January 2018).

Thomas, L. and Woodside, J. M. (2016) 'Social media maturity model', International Journal of Healthcare Management, 9(1), pp. 67-73. doi: 10.1080/20479700.2015.1101940.

Thrall, J. H. (2004) 'The Emerging Role of Pay-for-Performance Contracting for Health Care Services', Radiology, 233(3), pp. 637-640. doi: 10.1148/radiol.2333041412.

Tian, W. (2013) 'A review of sensitivity analysis methods in building energy analysis', Renewable and Sustainable Energy Reviews, 20, pp. 411-419. doi: 10.1016/j.rser.2012.12.014. Tian, W. and Choudhary, R. (2012) 'A probabilistic energy model for non-domestic building sectors applied to analysis of school buildings in greater London', Energy and Buildings, 54, pp. 1-11. doi: 10.1016/J.ENBUILD.2012.06.031.

Tiep, B. L. et al. (2018) 'Management of COPD Via a Clinical Guidance System: 16th Year of a Continuous Improvement Model', PULMONARY REHABILITATION: CLINICAL STUDIES IN COPD, American Thoracic Society, A66, pp. A2161-A2161.

Ting, H. H. et al. (2008) 'Implementation and Integration of Prehospital ECGs Into Systems of Care for Acute Coronary Syndrome', Circulation, 118(10), pp. 1066-1079. doi:

10.1161/CIRCULATIONAHA.108.190402.

Tosczak, M. (2004) UnitedHealth trying to limit imaging use - Triad Business Journal, bizjournals.com. Available at: https://www.bizjournals.com/triad/stories/2004/12/27/story2.html (Accessed: 25 February 2019).

Tran, T. (2000) Strategic Evaluation of University Knowledge and Technology Transfer

Effectiveness, Dissertations and Theses. Portland, OR. doi: 10.15760/etd.1059. 
Travis, P. et al. (2004) 'Overcoming health-systems constraints to achieve the Millennium Development Goals', The Lancet, 364(9437), pp. 900-906. doi: 10.1016/S0140-6736(04)169870 .

Trevethan, R. (2017) 'Intraclass correlation coefficients: clearing the air, extending some cautions, and making some requests', Health Services and Outcomes Research Methodology, 17(2), pp. 127-143. doi: 10.1007/s10742-016-0156-6.

Turan, T. et al. (2009) 'Use of Hierarchal Decision Modeling (HDM) for selection of graduate school for master of science degree program in engineering', in PICMET '09 - 2009 Portland International Conference on Management of Engineering \& Technology. IEEE, pp. 535-549. doi: 10.1109/PICMET.2009.5262107.

Turley, C. B. (2016) 'Leveraging a Statewide Clinical Data Warehouse to Expand Boundaries of the Learning Health System', eGEMs (Generating Evidence \& Methods to improve patient outcomes), 4(1), p. 25. doi: 10.13063/2327-9214.1245.

U.S. Department of Health and Human Services (HHS) (2020) U.S. Department of Health and Human Services (HHS) | GRANTS.GOV. Available at: https://www.grants.gov/learngrants/grant-making-agencies/department-of-health-and-human-services.html (Accessed: 29 April 2020).

Understanding Science at Berkeley (2020) Who pays for science?, Berkeley. Available at: https://undsci.berkeley.edu/article/who_pays (Accessed: 13 April 2020).

US Census Bureau (2012) Statistical Abstract of the United States: 2012.

US Census Bureau Public Information Office (2012) 'Most Children Younger Than Age 1 are Minorities, Census Bureau Reports - Population - Newsroom - U.S. Census Bureau'. Available at: https://www.census.gov/newsroom/releases/archives/population/cb12-90.html (Accessed: 6 June 2018).

Vance, K., Howe, W. and Dellavalle, R. P. (2009) 'Social internet sites as a source of public health information.', Dermatologic clinics, 27(2), pp. 133-6, vi. doi: 10.1016/j.det.2008.11.010. Vandenbroucke, J. P. (2011) 'Maintaining privacy and the health of the public', Bmj, 316(7141), pp. 1331-1332. doi: 10.1136/bmj.316.7141.1331.

Vanu Som, C. (2004) 'Clinical governance: A fresh look at its definition', Clinical Governance: An International Journal, 9(2), pp. 87-90. doi: 10.1108/14777270410536358.

Vehko, T. et al. (2019) 'Experienced time pressure and stress: Electronic health records usability and information technology competence play a role', BMC Medical Informatics and Decision Making, 19(1), pp. 1-9. doi: 10.1186/s12911-019-0891-z. 
Verdeyen, V. and Buggenhout, B. Van (2003) 'Social governance: Corporate governance in institutions of social security, welfare and healthcare', International Social Security Review, 56(2), pp. 45-64. doi: 10.1111/1468-246X.00157.

Vidal Carvalho, J., Rocha, Á. and Abreu, A. (2017) 'Maturity of hospital information systems: Most important influencing factors', Health Informatics Journal, p. 146045821772005. doi: $10.1177 / 1460458217720054$.

Walshe, K. and Rundall, T. G. (2001) 'Evidence-based Management: From Theory to Practice in Health Care', The Milbank Quarterly, 79(3), pp. 429-457. doi: 10.1111/1468-0009.00214.

Watson, H., Ariyachandra, T. and Matyska, R. J. (2001) 'Data Warehousing Stages of Growth', Information Systems Management, 18(3), pp. 42-50. doi:

10.1201/1078/43196.18.3.20010601/31289.6.

Weeks, J. and Pardee, R. (2019) 'Learning to Share Health Care Data: A Brief Timeline of Influential Common Data Models and Distributed Health Data Networks in U.S. Health Care Research', eGEMs (Generating Evidence \& Methods to improve patient outcomes), 7(1), p. 4. doi: 10.5334/egems.279.

Weiss, G. and Lonnquist, L. (2000) The Sociology of Health, Healing, and Illness - Gregory L. Weiss, Lynne E. Lonnquist - Google Books. Upper Saddle River,NJ: Prentice Hall.

Wennberg, J. E., O’Connor, A. M., et al. (2007) 'Extending The P4P Agenda, Part 1: How Medicare Can Improve Patient Decision Making And Reduce Unnecessary Care', Health Affairs, 26(6), pp. 1564-1574. doi: 10.1377/hlthaff.26.6.1564.

Wennberg, J. E., Fisher, E. S., et al. (2007) 'Extending The P4P Agenda, Part 2: How Medicare Can Reduce Waste And Improve The Care Of The Chronically Ill', Health Affairs, 26(6), pp. 1575-1585. doi: 10.1377/hlthaff.26.6.1575.

West, C. P. (2016) 'Physician Well-Being: Expanding the Triple Aim', Journal of General Internal Medicine, 31(5), pp. 458-459. doi: 10.1007/s11606-016-3641-2.

van de Wetering, R. and Batenburg, R. (2009) 'A PACS maturity model: A systematic metaanalytic review on maturation and evolvability of PACS in the hospital enterprise', International Journal of Medical Informatics, 78(2), pp. 127-140. doi:

10.1016/j.ijmedinf.2008.06.010.

White, C. M. et al. (2019) 'AHRQ Series on Improving Translation of Evidence: Linking Evidence Reports and Performance Measures to Help Learning Health Systems Use New Information for Improvement', Joint Commission Journal on Quality and Patient Safety, 45(10), pp. 706-710. doi: 10.1016/j.jcjq.2019.05.002. 
de Wilde, P. and Tian, W. (2010) 'Predicting the performance of an office under climate change: A study of metrics, sensitivity and zonal resolution', Energy and Buildings, 42(10), pp. 1674-1684. doi: 10.1016/J.ENBUILD.2010.04.011.

Wolf, M. S. et al. (2009) 'Literacy and Learning in Health Care: FIGURE 1', Pediatrics, 124(Supplement 3), pp. S275-S281. doi: 10.1542/peds.2009-1162C.

Wolinsky, F. (1988) The sociology of health: Principles, practitioners, and issues. Belmont, CA: Wadsworth.

Wooden, A. (2021) Quality Incentive Measures - Advanced Health, AdvancedHealth. Available at: http://advancedhealth.com/providers/quality-incentive-measures/ (Accessed: 8 February 2021).

Worley, Christopher G. and Mohrman, S. A. (2014) 'Is change management obsolete?', Organizational Dynamics, 43(3), pp. 214-224. doi: 10.1016/J.ORGDYN.2014.08.008. Worley, Christopher G and Mohrman, S. A. (2014) 'Is Change Management Obsolete?', CEO Special Issue of Organizational Dynamics. Available at: http://ceoprogram.usc.edu/Is_Change_Management_Obsolete-Org_Dynamics_2014.pdf (Accessed: 18 January 2018).

Yeo, K. T. and Ren, Y. (2008) 'Risk Management for Complex Product Systems ( CoPS ) Projects', 12(4), pp. 275-294. doi: 10.1002/sys.

Yong, P. L., Olsen, L. and Mcginnis, J. M. (2010) Value in Health Care. doi: 10.17226/12566. Yong, P. L., Saunders, R. S. and Olsen, L. (2010) The Healthcare Imperative. doi: 10.17226/12750.

Zaharias, P. and Pappas, C. (2016) Quality Management of Learning Management Systems: A User Experience Perspective, Current Issues in Emerging eLearning. Available at: https://scholarworks.umb.edu/cieeAvailableat:https://scholarworks.umb.edu/ciee/vol3/iss1/5 (Accessed: 1 February 2021).

Zephir, O., Minel, S. and Chapotot, E. (2011) 'A maturity model to assess organisational readiness for change', International Journal of Technology Management, 55(3/4), p. 286. doi: 10.1504/IJTM.2011.041954.

Zook, C. (2007) 'Finding Your Next Core Business', Harvard Business Review, 85(4), p. 66. Available at: www.hbrreprints.org (Accessed: 11 March 2018). 


\section{Appendices}

Appendix A- Perspectives and Criteria Definitions
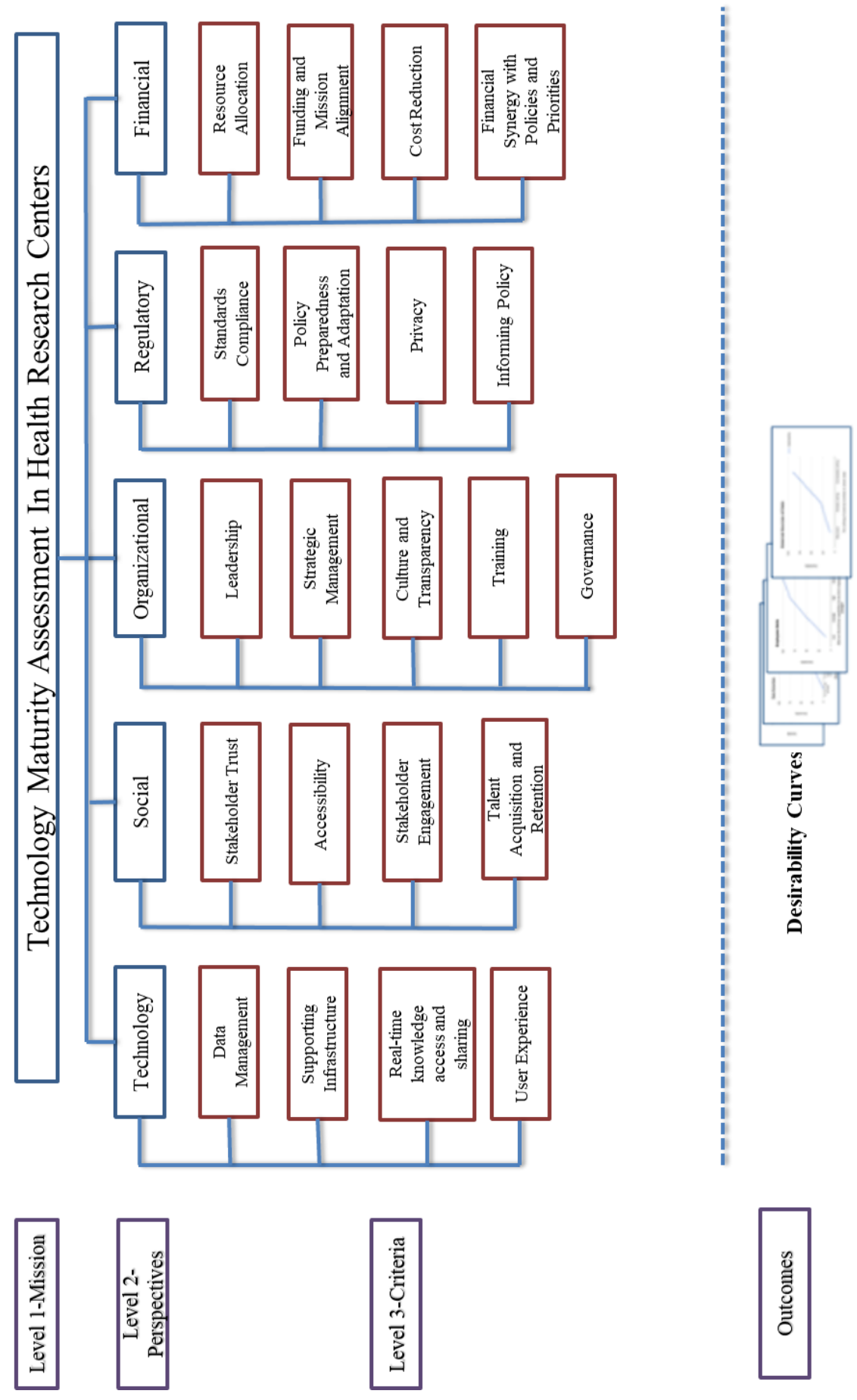


\begin{tabular}{|c|c|}
\hline Perspective & Definition \\
\hline Social & $\begin{array}{l}\text { This perspective encapsulates topics such as public acceptance, trust, broad accessibility, equity, and engagement. Stakeholders } \\
\text { can take the shape of patients, providers, policy makers, payers, and clinicians. This perspective also includes the ability of } \\
\text { healthcare organizations to attract and acquire a skilled and diverse workforce. }\end{array}$ \\
\hline Organizational & $\begin{array}{l}\text { This perspective covers certain organizational aspects of technology management in healthcare such as leadership, change } \\
\text { management, organizational culture and transparency, strategic management, and governance. }\end{array}$ \\
\hline Financial & $\begin{array}{l}\text { This perspective encapsulates the financial side of assessing the maturity of technology management in health research centers. } \\
\text { Topics such as investment resource allocation, appropriate funding, and cost reduction without compromising quality fall under } \\
\text { this category. }\end{array}$ \\
\hline
\end{tabular}

\begin{tabular}{|c|c|c|c|}
\hline Perspective & Criteria & Definition & References \\
\hline \multirow{4}{*}{ Technology } & Data Management & $\begin{array}{l}\text { Management of big data resources in terms of security, privacy, } \\
\text { governance, and ethics. }\end{array}$ & $\begin{array}{c}\text { (Rubin, 2017)(Bernstein et al., 2015)(English } \\
\text { et al., 2016)(Saunders \& Smith, } \\
\text { 2013)(Rumsfeld et al., 2016)(A. Shah et al., } \\
\text { 2016) }\end{array}$ \\
\hline & $\begin{array}{l}\text { Supporting } \\
\text { Infrastructure }\end{array}$ & $\begin{array}{l}\text { The socio-technical infrastructure needed to improve and } \\
\text { provide capacity to capture, compile, and protect clinical and } \\
\text { financial data, which enables the evaluation, adjustment, } \\
\text { analysis, and dissemination and integration of learned } \\
\text { knowledge into clinical care processes in order to secure the } \\
\text { promised improvements. }\end{array}$ & $\begin{array}{l}\text { (Johnsonet al., 2017)(Saunders \& Smith, } \\
\text { 2013)(Mandl et al., 2014)(Kraft et al., } \\
\text { 2017)(English et al., 2016) }\end{array}$ \\
\hline & $\begin{array}{l}\text { Real-time Knowledge } \\
\text { Access and Sharing }\end{array}$ & $\begin{array}{l}\text { Data systems and tools used to capture, share, and integrate } \\
\text { data, information, visualizations, and knowledge gained from } \\
\text { research into the organization in real-time. }\end{array}$ & $\begin{array}{l}\text { (Saunders \& Smith, 2013)(Bernstein et al., } \\
\text { 2015)(Morain et al., 2017)(Cahan \& Cimino, } \\
\text { 2017) }\end{array}$ \\
\hline & User Experience & $\begin{array}{l}\text { Ensuring that technologies and IT products are user-friendly (e.g., } \\
\text { taking health literacy and technology competence into } \\
\text { consideration) to all users (stakeholders). }\end{array}$ & $\begin{array}{c}\text { (Lehoux, 2004) (Rudd, 2010)(Hattink et al., } \\
\text { 2016) (Vehko et al., 2019) }\end{array}$ \\
\hline
\end{tabular}




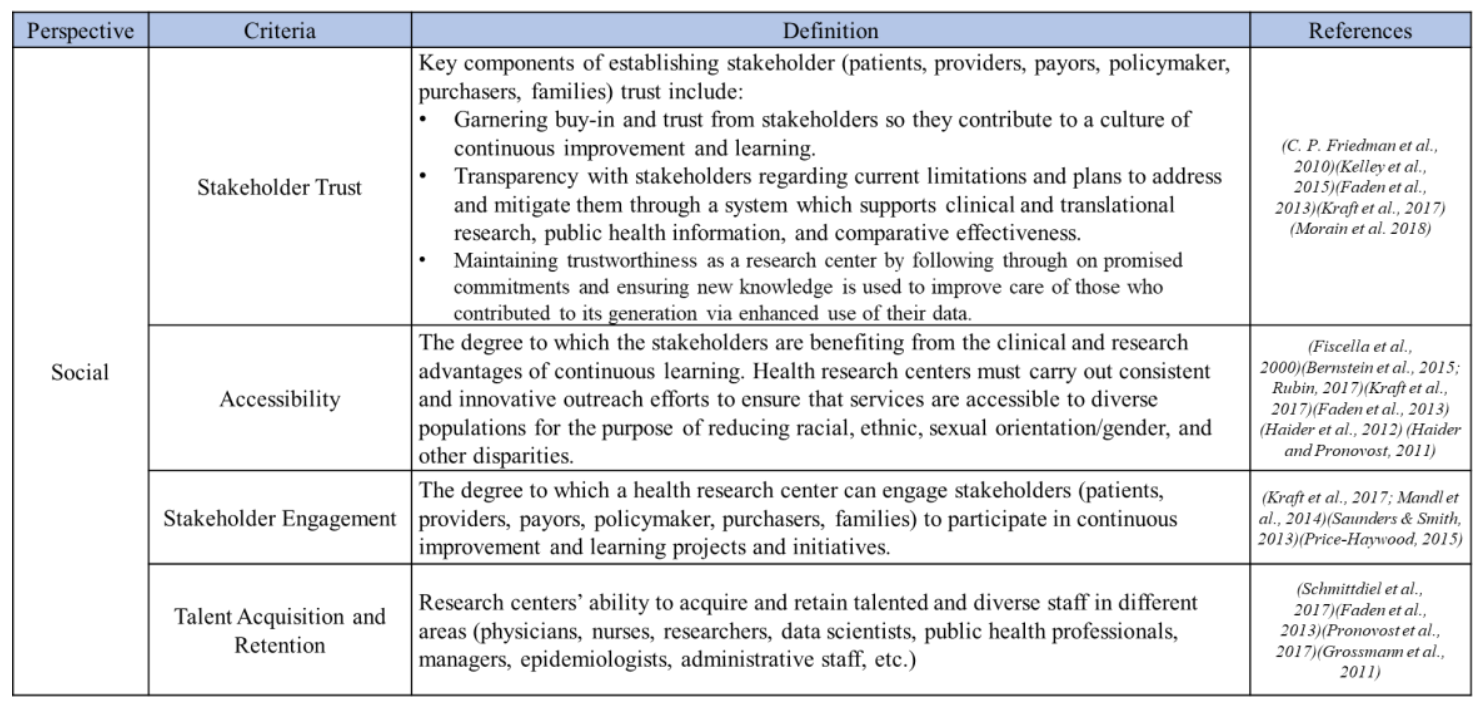

\begin{tabular}{|c|c|c|c|}
\hline Perspective & Criteria & Definition & References \\
\hline \multirow{5}{*}{ Organizational } & Leadership & $\begin{array}{l}\text { Broad leadership which can expand and guide stakeholders' commitment } \\
\text { to the goals of continuous learning and increased technology maturity. }\end{array}$ & $\begin{array}{l}\text { (Saunders \& Smith, 2013)(Bernstein et al., } \\
\text { 2015)(Schmittdiel et al., 2017)(Morain et al., } \\
\text { 2017)(Rubin, 2017)(Graban, 2016) }\end{array}$ \\
\hline & Strategic Management & $\begin{array}{l}\text { Research centers' ability to improve through strategic decisions, } \\
\text { management of competing priorities, internal and external } \\
\text { partnerships/collaborations, problem identification, and finding solutions. }\end{array}$ & $\begin{array}{l}\text { (English et al., 2016)(Morain et al., 2017)(Kraft et } \\
\text { al., 2017)(Bernstein et al., 2015) }\end{array}$ \\
\hline & $\begin{array}{l}\text { Organizational Culture } \\
\text { and Transparency }\end{array}$ & $\begin{array}{l}\text { The extent to which a research center's employees have adopted and are } \\
\text { committed to a culture of continuous learning and practice transparency } \\
\text { to safeguard stakeholder trust in order to improve the health of } \\
\text { individuals, communities, and diverse populations. }\end{array}$ & $\begin{array}{c}\text { (Kraft et al., 2017)(Institute of Medicine (IOM), } \\
\text { 2007)(IOM, 2011)(Bernstein et al., 2015) (Rubin, } \\
\text { 2017)(Curcin, 2016)(Saunders \& Smith, } \\
\text { 2013)(Institute of Medicine (IOM), 2007)(Yonget } \\
\text { al., 2010) }\end{array}$ \\
\hline & Training & $\begin{array}{l}\text { Training employees and stakeholders on the latest technologies and best } \\
\text { practices within the health research center. }\end{array}$ & $\begin{array}{l}\text { (Schmittdiel et al., 2017)(Faden et al., } \\
\text { 2013)(Pronovostet al., 2017)(Grossmamnet al., } \\
\text { 2011) }\end{array}$ \\
\hline & Governance & $\begin{array}{l}\text { Having the necessary governance to support a sustainable operation, } \\
\text { uphold required standards, build and maintain trust with stakeholders, } \\
\text { and continuously innovate. }\end{array}$ & $\begin{array}{l}\text { (Rubin, 2017)(Bernstein et al., 2015)(English et al,, } \\
\text { 2016)(Institute of Medicine (IOM), 2007) }\end{array}$ \\
\hline
\end{tabular}




\begin{tabular}{|c|c|c|c|}
\hline Perspective & Criteria & Definition & References \\
\hline \multirow{4}{*}{ Regulatory } & Standards Compliance & $\begin{array}{l}\text { Research centers' compliance with standards and } \\
\text { regulations to ensure transparency with stakeholders, data } \\
\text { interoperability, and commitment to meaningful use and } \\
\text { joint commissions. }\end{array}$ & $\begin{array}{l}\text { (C. P. Friedman et al., 2010)(IOM, } \\
\text { 2011)(Care, 2014)(Schmittdiel et al., } \\
\text { 2017)(Institute of Medicine (IOM), } \\
\text { 2007)(Blumenthal \& Tavemer, 2010) }\end{array}$ \\
\hline & Policy Preparedness and Adaptation & $\begin{array}{l}\text { Research centers' flexibility and agility in responding and } \\
\text { adapting to changes (new regulations, legislations, and } \\
\text { policies) in terms of anticipation and readiness (resources, } \\
\text { policies, strategies, and management). }\end{array}$ & $\begin{array}{l}\text { (Blumenthal \& Tavenner, 2010) } \\
\text { (Grossmann et al., 2011) (Amir Shaygan, } \\
\text { 2018)(Shaygan \& Daim, 2019) }\end{array}$ \\
\hline & Privacy & $\begin{array}{l}\text { Research centers' adherence to regulations and policies to } \\
\text { ensure privacy in terms of information technology, medical } \\
\text { data, patient access, third party interactions, and ethical use } \\
\text { of information (IRBs), among others. }\end{array}$ & $\begin{array}{l}\text { (Curran, Stearns and Kaplan, 1969; Gostin } \\
\text { et al., 1993; Prentnieks and Qual, 1996; } \\
\text { Melton III, 1997; National Research } \\
\text { Council, 1997; Rothstein and Talbott, 2006; } \\
\text { Nass et al., 2009; Vandenbroucke, 2011) }\end{array}$ \\
\hline & Informing Policy & $\begin{array}{l}\text { Research centers' ability to inform policies by participating } \\
\text { in expert panels, providing evidence and comments } \\
\text { regarding policies under consideration, and securing } \\
\text { contracts with the state and federal government to complete } \\
\text { analyses regarding policy development, implementation, } \\
\text { and impact. }\end{array}$ & $\begin{array}{l}\text { (Douglas et al., 2009) } \\
\text { (Bendavid \& Miller, 2010) (Behrns, 2015) } \\
\text { (McMahonet al., 2020) }\end{array}$ \\
\hline
\end{tabular}

\begin{tabular}{|c|c|c|c|}
\hline Perspective & Criteria & Definition & References \\
\hline \multirow{4}{*}{ Financial } & Resource Allocation & $\begin{array}{l}\text { Research centers' ability to prioritize and decide to invest its } \\
\text { resources (financial, human, space,...) in alignment with the } \\
\text { goals of increased technology maturity and continuous learning. }\end{array}$ & $\begin{array}{c}\text { (Rouse et al., 2017)(Kraft et al., 2017)(Rouse, } \\
\text { 2001)(Pronovostet al., 2017) }\end{array}$ \\
\hline & $\begin{array}{l}\text { Funding and Mission } \\
\text { Alignment }\end{array}$ & $\begin{array}{l}\text { The extent to which incentives and funding are aligned with } \\
\text { encouragement of technology maturity, continuous learning and } \\
\text { improvement, waste elimination, and rewarding high care value. }\end{array}$ & $\begin{array}{l}\text { (Bli:insky \& Bonham, 2017)(Morainet al., } \\
\text { 2017)(Amir Shaygan, 2018)(Institute of Medicine } \\
\text { (IOM), 2007)(Pronovost et al., 2017) }\end{array}$ \\
\hline & Cost Reduction & $\begin{array}{l}\text { Research centers' ability to cut costs as a result of increased } \\
\text { maturity, learning, continuous improvement, and waste } \\
\text { eliminations without compromising quality. }\end{array}$ & $\begin{array}{l}\text { (Saunders \& Smith, 2013)(Health, 2013)(IOM, } \\
\text { 2011)(Institute of Medicine (IOM), } \\
\text { 2007)(McClellanet al., 2008)(Yonget al., 2010) }\end{array}$ \\
\hline & $\begin{array}{l}\text { Financial Synergy with } \\
\text { Policies and Priorities }\end{array}$ & $\begin{array}{l}\text { Research centers' success in meeting reimbursement programs' } \\
\text { goals (policy incentives, value-based purchasing, etc.) and } \\
\text { accessing government, foundation, and other grants (NIH, } \\
\text { DARPA, DOD, various private funds, etc.) }\end{array}$ & $\begin{array}{l}\text { (White et al., 2019)(Weeks and Pardee, 2019) } \\
\text { (Menear et al., 2019) }\end{array}$ \\
\hline
\end{tabular}




Cochnology Management Maturity Assessment Model in Healthcare
Amir Shaygan
Committee
Dr. Tugrul Daim (Chair)
Dr. Mark Ahn
Dr. Peter Graven
Dr. Kelly Clifton

\section{(1) Introduction}

(2) Gap Analysis

3

Research Approach

\section{(4) Methodology}

5 (5)

Systematic Challenges in US Healthcare "we do a lot, without hnowing what works" *

The US care spending has grown significantly in the last decades.Makary and Daniel, 2016:IOM,2011,Shah,2010, Shi \& Singh, 2019)

US has world class doctors and treatments parallel to a broken system (Graban,2016)

(] Concerns with public health concern (Friedman et al.,2014; Bhandari et al.,2016:Shi \& Singh, 2019)

- Medical errors are the third leading cause of death in US (NHS, 2014)

L Long delay times for the transfer of clinical knowledge to clinical practice in the US (about 17 years) (Ballas and Boren, 2000;Morris et al., 2011:Kitson \& Haney, 2010)
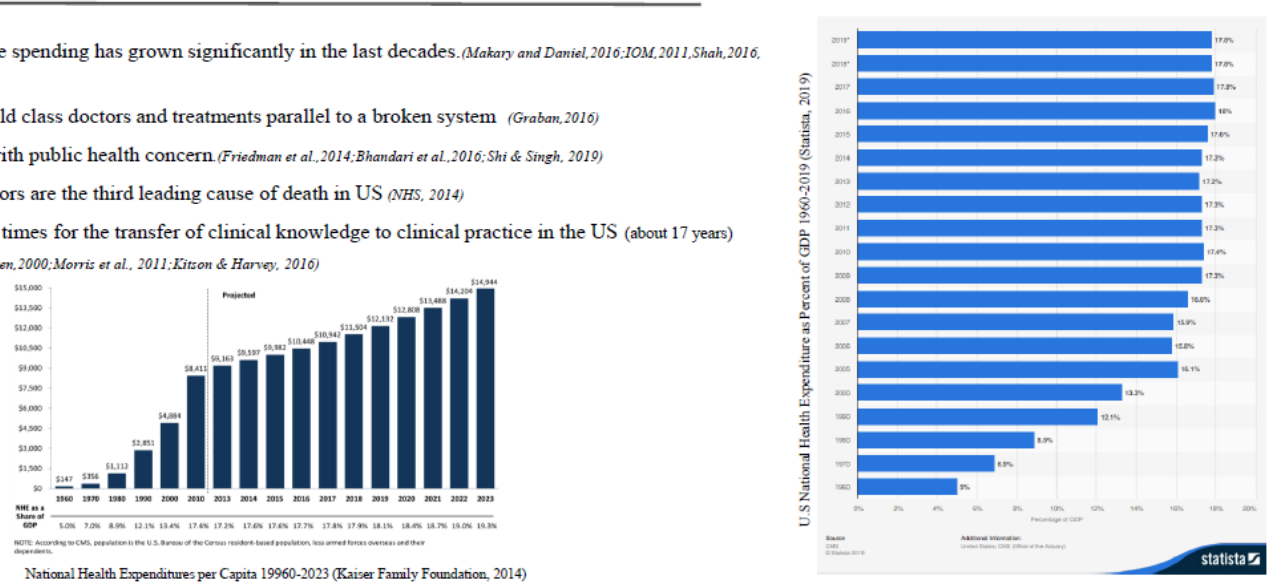

There are systematic issues in healthcare organizations leading to problems in different areas 


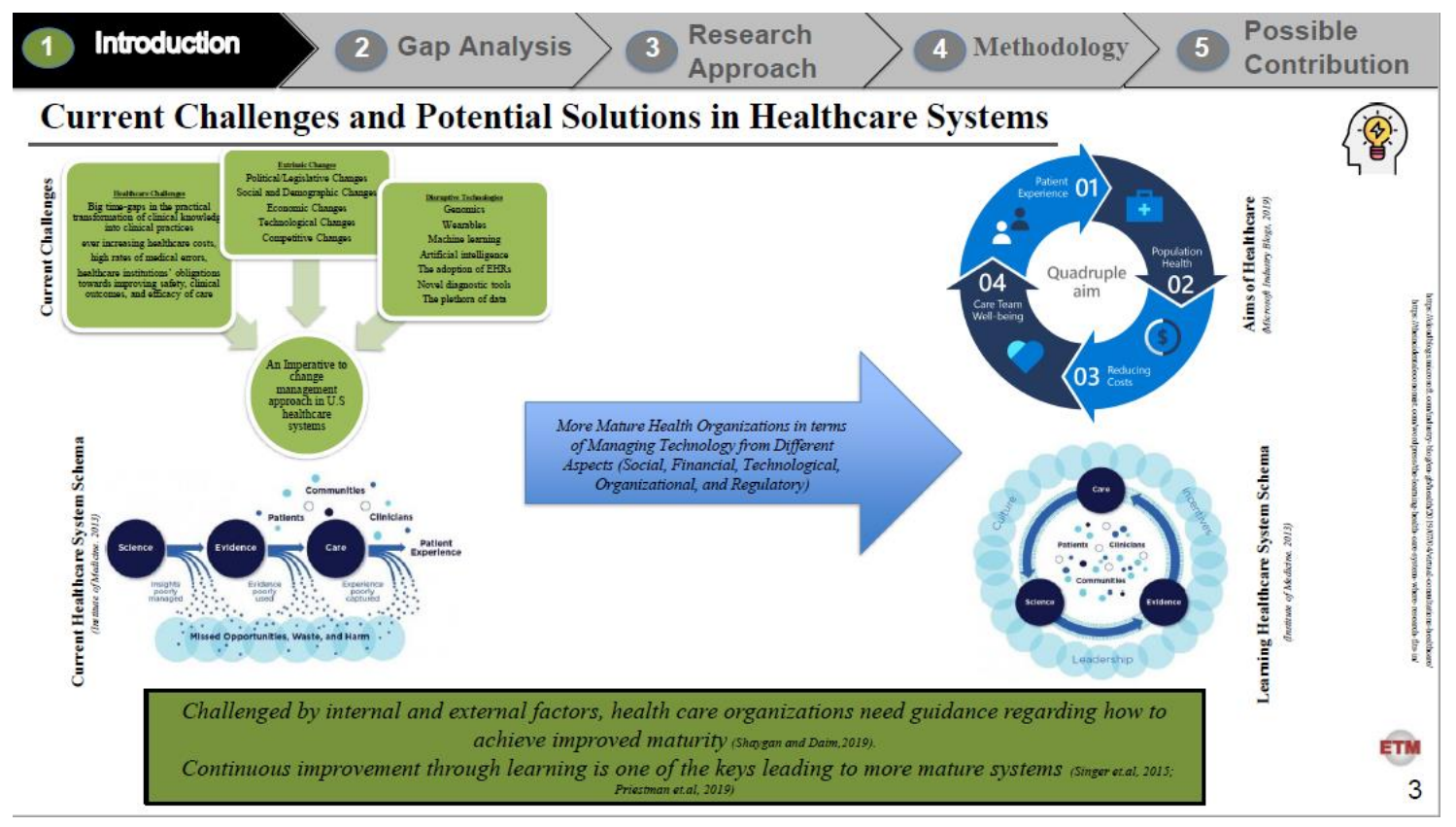

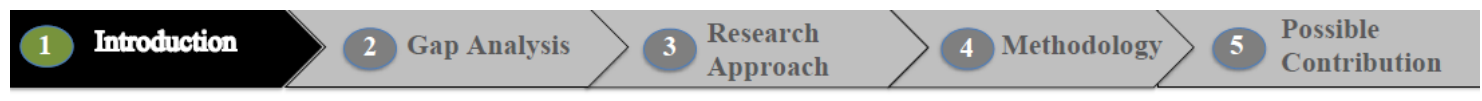

\section{Maturity Models}

Maturity is a measurement of the ability of an organization for continuous improvement in a particular discipline. (Paulk, 2009)*

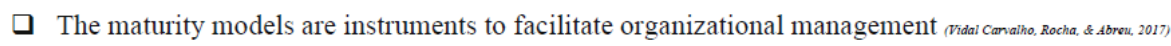

Maturity models have also been used in the healthcare domain specifically in the information system

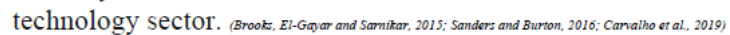

\section{As for health organizations, a mature system is defined as a system that} generates timely actions to the information that it derives from internal and external data in order to create meaningful measurement regarding systematic learning and increase effectiveness in health outcomes.

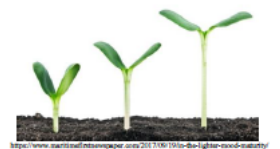




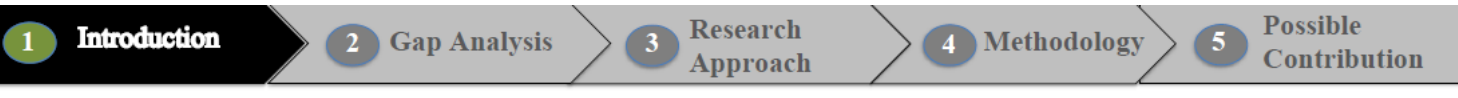

\section{Unit of Study}

- Healthcare organizations and hospitals (including university hospitals) are large institutions and consist of very complex structure of management.

University hospitals undergo significant cost to develop the ability to treat serious illnesses at the high end and in doing so they need to cope with a larger population with needs around different disorders as more and more types of patients are now being treated in more specialized care centers (Christensen, Bohmer and Kenag), 2000,

a For this reason, this research will focus on a smaller entity within university hospitals which are University Hospital Research Centers as the unit of study for this research.

These centers focus on specific areas within bigger health organizations and have their own directors, managers, and staff with varying budget sources such as grants and donations, among others.

This research will focus on a smaller entity inside university hospitals which are University Hospital Research Centers as the unit of study for this research.

\section{Introduction 2 Gap Analysis}

\section{Research Gaps, Goals, and Questions}

\begin{tabular}{|c|c|c|}
\hline Research Gaps & Research Goal & Research Questions \\
\hline $\begin{array}{l}\text { RG I:The criteria for assessing } \\
\text { technology management maturity and } \\
\text { continuous learning in healthcare } \\
\text { organizations are not organized and } \\
\text { classified into categories/perspectives. }\end{array}$ & & $\begin{array}{l}\text { RQ I: What are the main perspectives } \\
\text { and criteria in the assessment of } \\
\text { technology management maturity and } \\
\text { continuous learning in healthcare } \\
\text { organizations? }\end{array}$ \\
\hline $\begin{array}{l}\text { RG II: There is no quantified, validated, } \\
\text { multi-dimensional, and reusable way of } \\
\text { assessing maturity in healthcare } \\
\text { organizations }\end{array}$ & $\begin{array}{l}\text { The objective of this } \\
\text { research is to develop a } \\
\text { framework for assessing } \\
\text { technology management }\end{array}$ & $\begin{array}{l}\text { RQ II: What are the weights of criteria } \\
\text { and sub-criteria related to the }\end{array}$ \\
\hline $\begin{array}{l}\text { RG III: Despite technology } \\
\text { management's importance in healthcare, } \\
\text { there is lack of studies on the } \\
\text { technology management side of } \\
\text { healthcare maturity and continuous } \\
\text { learning }\end{array}$ & $\begin{array}{l}\text { learning in research } \\
\text { institutes within university } \\
\text { hospitals in the United } \\
\text { States. }\end{array}$ & $\begin{array}{l}\text { assessment of maturity and contunuous } \\
\text { learning in healthcare organizations? }\end{array}$ \\
\hline $\begin{array}{l}\text { RG IV: There is very little to no } \\
\text { literature around the structure and } \\
\text { management of research centers within } \\
\text { university hospitals. }\end{array}$ & & $\begin{array}{l}\text { offer an effective and practical way to } \\
\text { assess technology management maturity } \\
\text { and continuous learning in research } \\
\text { centers within university hospitals? }\end{array}$ \\
\hline
\end{tabular}




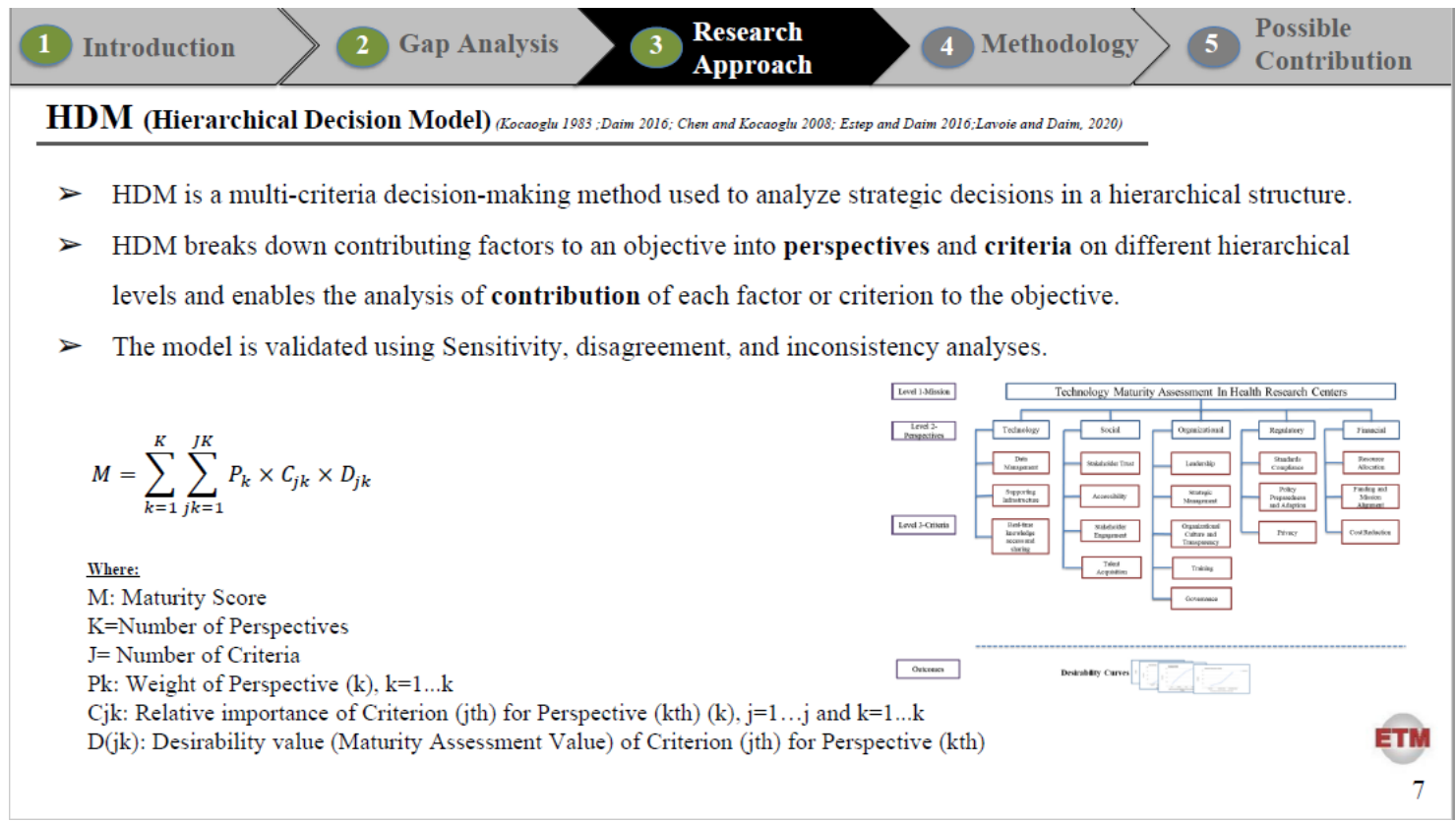

\section{(1) Introduction 2 Gap Analysis Research
Approach

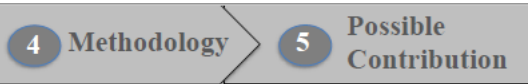

Desirability Curves (Estep 2017; Gibson 2016: Phan 2013)

- In instances where there is a desire to reuse a model, or in instances of having many alternatives, desirability curves can be used.

- The combination of desirability curves with HDM is used to identify levels/metrics for each criterion as a useful value to assist decision makers.

U Using the desirability curves approach, the experts evaluate related levels/metrics for each criterion while giving each metric a scaled quantitative value. This enables the normalization of the evaluation results by experts across all the criteria

Desirability curves allow for better understanding of the dynamics of the criterion as they quantify different states of health organizations regarding a specific criterion.
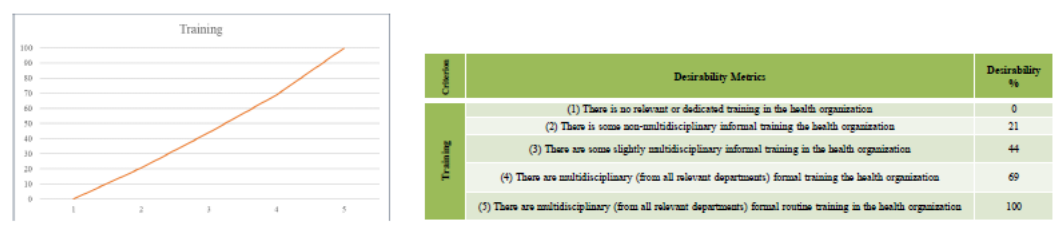


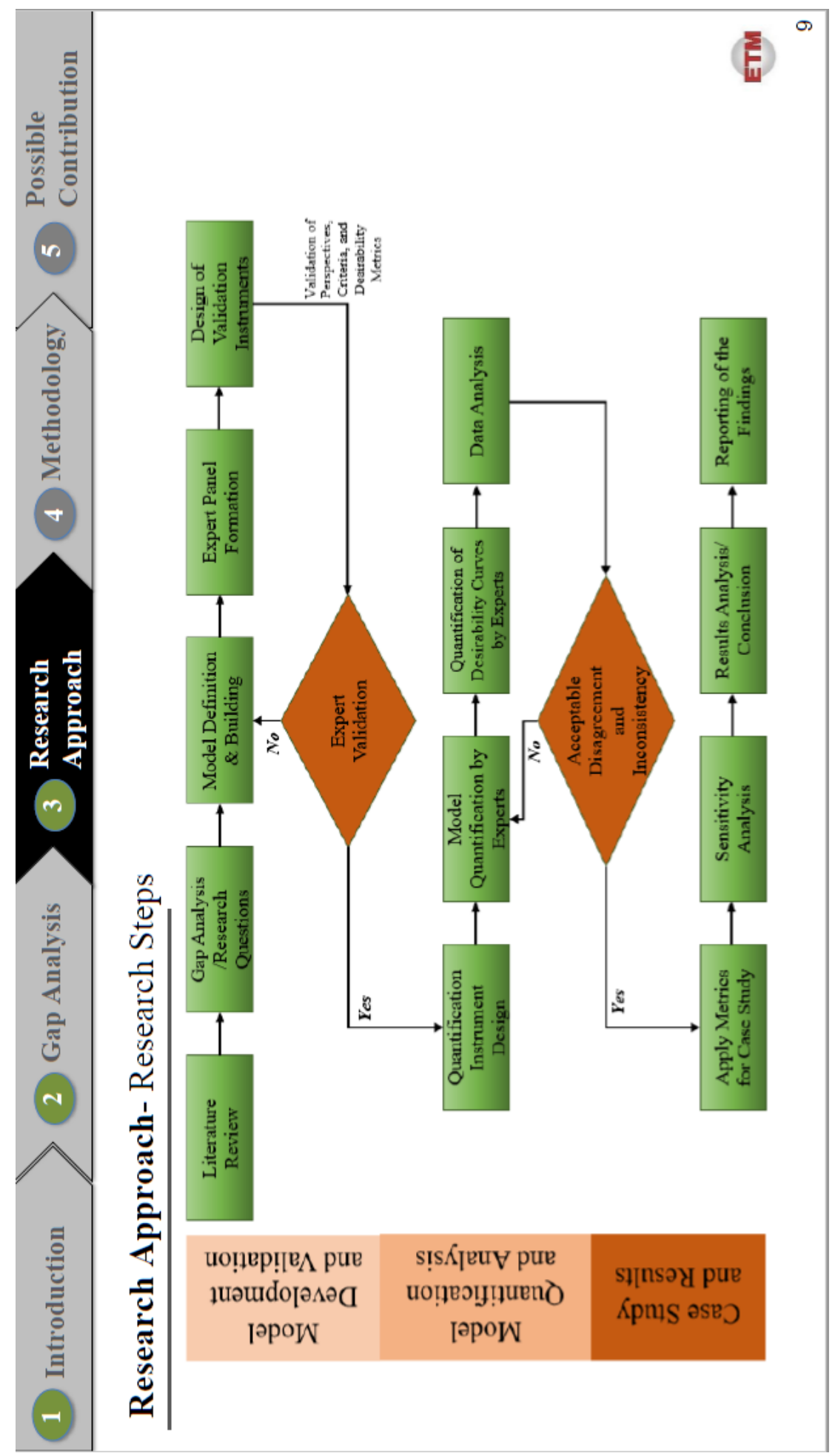




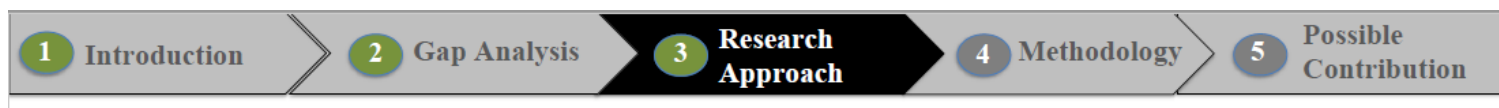

\section{Data Collection Steps}

\begin{tabular}{|c|c|c|}
\hline Step & Task & Tool \\
\hline 1 & Perspective Validation & Qualtrics \\
\hline 2 & Criteria Validation & Qualtrics \\
\hline 3 & Perspectives Quantification & ETM HDM Software \\
\hline 4 & Criteria Quantification & ETM HDM Software \\
\hline 5 & Desirability Metrics Validation & Qualtrics \\
\hline 6 & Desirability Metrics Quantification & Qualtrics \\
\hline
\end{tabular}

\section{HDM Model- The Initial Model}

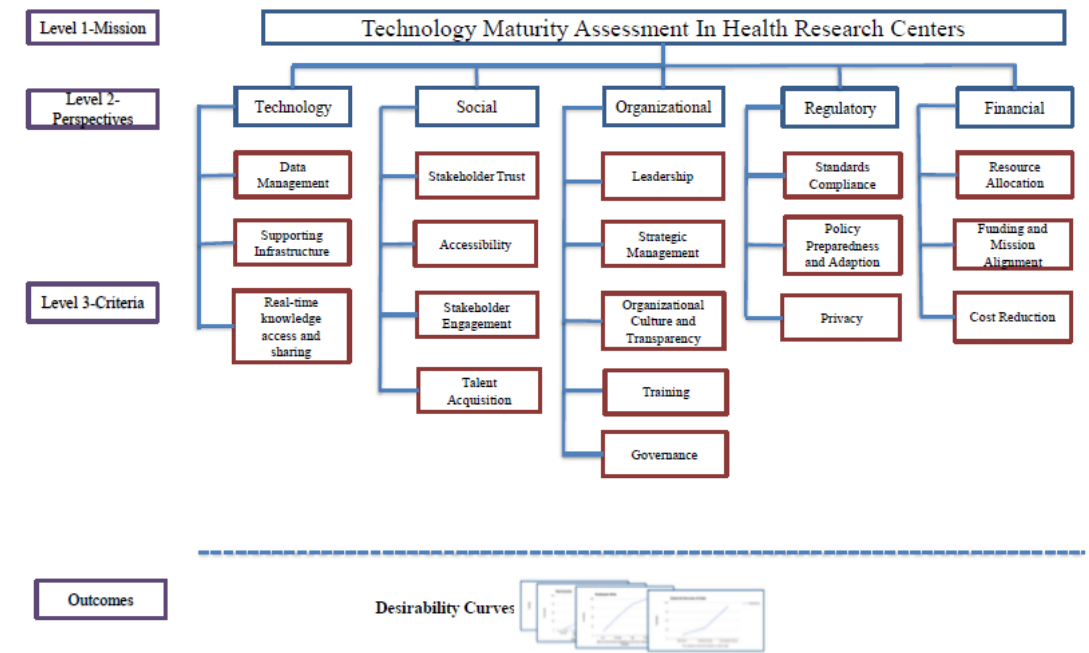




(1) Introduction $\ 2$ Gap Analysis $>3$ Research $\begin{aligned} & \text { Approach }\end{aligned} 4$ Model $>\begin{aligned} & \text { Possible } \\ & \text { Contribution }\end{aligned}$

\section{Research Contribution}

- This research contributes to the technology management body of knowledge on technology management maturity assessment in the healthcare industry while delving deeper into how healthcare research centers can achieve a sense of self awareness in terms of where they are and where they need to be in terms of technology management maturity (strengths and opportunities).

- This research will provide a quantified, validated, repeatable, and multi-dimensional tool for measuring the maturity in health organizations and health research centers with goals of facilitating continuous improvement and learning.

- According to the literature review and gap analysis conducted in this study, there is a lack of structured and comprehensive understanding of the managerial issues around maturity in healthcare organizations especially around research centers and institutes, and further research can bolster the continuous quality improvement goals of these institutions.

- This maturity model can lead to better decision making in health organizations and can be used as a step in the right direction in reaching better results regarding patient and provider satisfaction, quality care, cost of care, and resource allocation.

- Managers and stakeholders can initiate dialogues around what the results of the model mean to them and how to take further actions aligned with their goals and mission and create more effective roadmaps. 


\section{Appendix C- Expert Emails and Instructions}

2/22/2021

Portland State University Mail - Expert Panel Step 1\& 2: Validating the Perspectives and Criteria

Portland State

Amir Shaygan <ashaygan@pdx.edu>

Expert Panel Step 1\& 2: Validating the Perspectives and Criteria

Amir Shaygan <ashaygan@pdx.edu>

Sun, Oct 11, 2020 at 10:43 PM

Dear expert,

Thank you for participating in the validation and quantification of my research model.

1-The first step is to validate the perspectives (categories) of the factors affecting technology maturity and continuous learning in health research centers. You can go to the questionnaire by clicking on the link below:

https://portlandstate.qualtrics.com/jfe/form/SV_8k176azmX3lkOLr

2-The second step is to validate the criteria affecting technology maturity and continuous learning in health research centers. The following link will take you to the questionnaire:

https://portlandstate.qualtrics.com/ife/form/SV_3Kmltb2RPWLRC3H

I'm planning to send the quantification steps (parts $3 \& 4$ ) in the last week of October. I would appreciate it if responses for parts $\underline{1} \& \underline{\underline{2}}$ are sent by October $\mathbf{2}$ nd. This way I will have enough time to finish validating the model and to modify the quantification tools based on the received feedback.

Attached to this email you will find a summary of my research, perspective definitions, and criteria definitions.

If you have any questions please don't hesitate to contact me.

Best Regards,

$-$

Amir Shaygan

Ph.D. Candidate and Graduate Assistant

Engineering \& Technology Management Department

Portland State University

Student Worker

Business Intelligence and Advanced Analytics

Oregon Health and Science University

Graduate Assistant

Portland State University Office of Institutional Research and Planning (OIRP) 


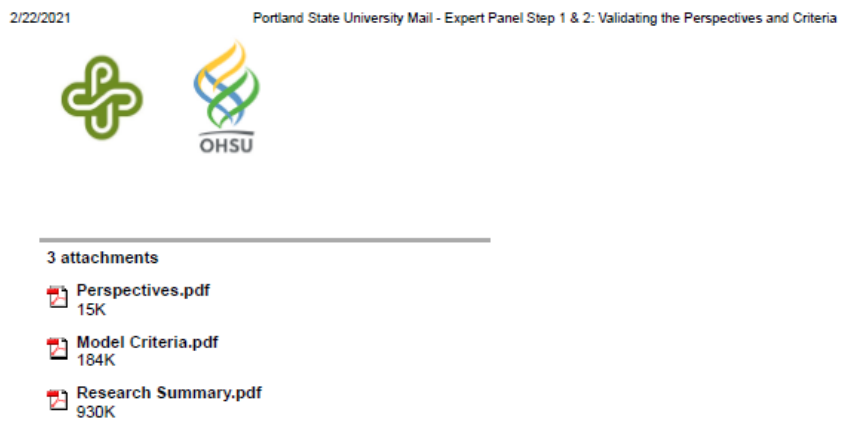




\section{Expert Panel Steps 3 \& 4: Quantification of the Perspectives and Criteria \\ 7 messages}

Amir Shaygan <ashaygan@pdx.edu>

Mon, Nov 2, 2020 at 7:08 AM

Dear Expert,

Thank you for your great feedback on the validation of my model. The Third and Fourth steps include quantifying the model in terms of perspectives and criteria, respectively.

In these steps, you will pairwise compare each perspective (and their relative criteria) based on their importance to technology management maturity and continuous learning in health research centers. Your feedback in this step is very valuable even if you have not participated in the validation of the model.

Attached to this email you will find:

- Instructions for the quantification steps (please use the attached "HDM Quantification Instructions" document as a guide).

-A summary of my research.

-The validated model, and the definitions for the model elements.

Please use the following link to quantify the model in terms of perspectives and criteria (Steps $3 \& 4$ ): http://research1.etm.pdx.edu/hdm2/expertaspx?id=1891ff36c/ec.959b/cb35670aa7fe. $2 \mathrm{fb} 8$

*This software is not mobile-friendly. Please consider quantifying using your computer browsers.

I would appreciate it if responses for quantification are sent by November 13th. If you have any questions please don't hesitate to email me.

Thank you so much for your time and input!

Best Regards,

Amir Shaygan

$-$

Amir Shaygan

PhD Candidate and Graduate Assistant

Engineering \& Technology Management Department

Portland State University

Student Worker

Business Intelligence and Advanced Analytics

Oregon Health and Science University

Graduate Assistant

Portland State University Office of Institutional Research and Planning (OIRP) 


\section{Expert Panel Steps 6: Quantification of the Desirability Curves \\ 2 messages}

Amir shavoan esshavoanilodidr.edu>

Dear Expert,

Thank you for your fendback on the previous steps.

The final step is the quantification of the desirability curves associated with the criteria affoting technology maturity and continuous learning in bealth research centers

The desirability curves represent metrics/levels that could be assigned to a center's status/performance against each criterion in the model.

Please use the following link:

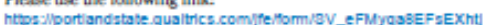

Here is a sample of how the final desirability curves would look like:
\begin{tabular}{|l|c|}
\hline \multicolumn{2}{|c|}{ Training } \\
\hline $\begin{array}{c}\text { What level of training with goals of organizational maturity and } \\
\text { continuous learning exists in the research center? }\end{array}$ & Desirability \\
\hline There is no relevant or dedicated training in the research center & 0 \\
\hline There are some non-multidisciplinary informal training the research center & 10 \\
\hline There are some multidisciplinary informal training in the research center & 50 \\
\hline There are multidisciplinary formal training in the research center & 85 \\
\hline There are routine multidisciplinary formal training in the research center & 100 \\
\hline
\end{tabular}

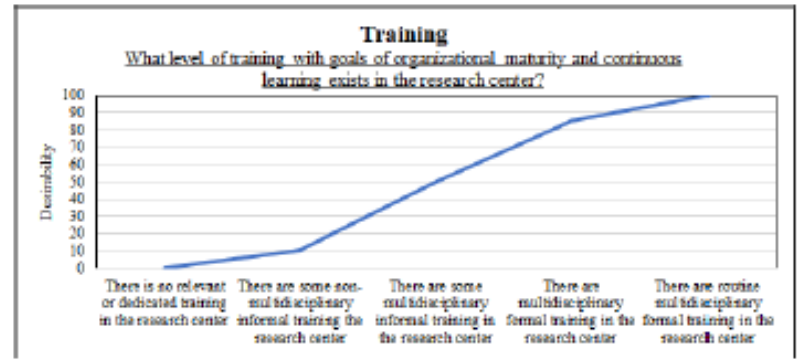

For each metric quantification, please assign a level of desirability (o-100). An example has been provided below:

Tharroctines:

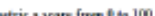

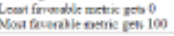

Salatidecting

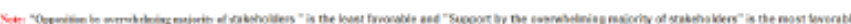

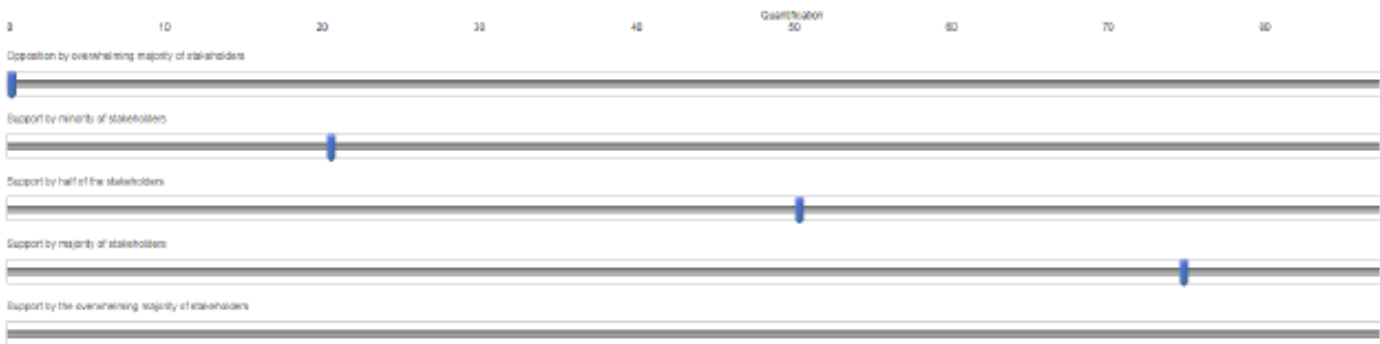

Attached to this emall you will find a summary of my research and the criteris.

I would appreciate it if responses for quantification are sent by November 13th. If you have any questions please don't hesitate to email me.

Thank you so much for your time and inpur! 
Best Regards,
Amir Shaygan
-

Amir Shaygan

Ph.D. Candidate and Graduate Assistant

ment Department

Portland State Unlversity

Student Worker

Business Inteliligence and Advanoed Analytics

Oregon Health and Sclence University

Craduate Assistant
Portland State University Offlice of Institutional Research and Planning (OLRP)

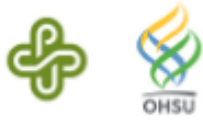

A. Virus-free, www.avastcom

2 attaohmente

ت] Valldated model, perepeotivec, and oriteria.pdt

기 $\begin{aligned} & \text { Recearoh a ummary.pdt } \\ & 942 \mathrm{~K}\end{aligned}$

Amir shaygan <ashaygan glpdr.edus

Sun, Dec 13,2020 at 1002 AM

Bce: Amir 3hsygan <amir.ghaypan. 89 gagmal.com?

jouted teet hidder|

2 attaohmente

ஏ Valldated model, perepeotivec, and oriteria.pdt

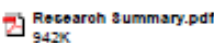




\title{
Technology Management Maturity Assessment in Health Research Centers
}

\author{
Amir Shaygan \\ Instructions for \\ Expert Panel Steps 3 \& 4: Quantification of the Perspectives and Criteria
}

Please use the following link to quantify the model in terms of perspectives and criteria (Steps 3 \& 4 ): http://research1.etm.pdx.edu/hdm2/expert.aspx?id=1891ff36c1ec059b/cb35670aa 7 fe2fb8
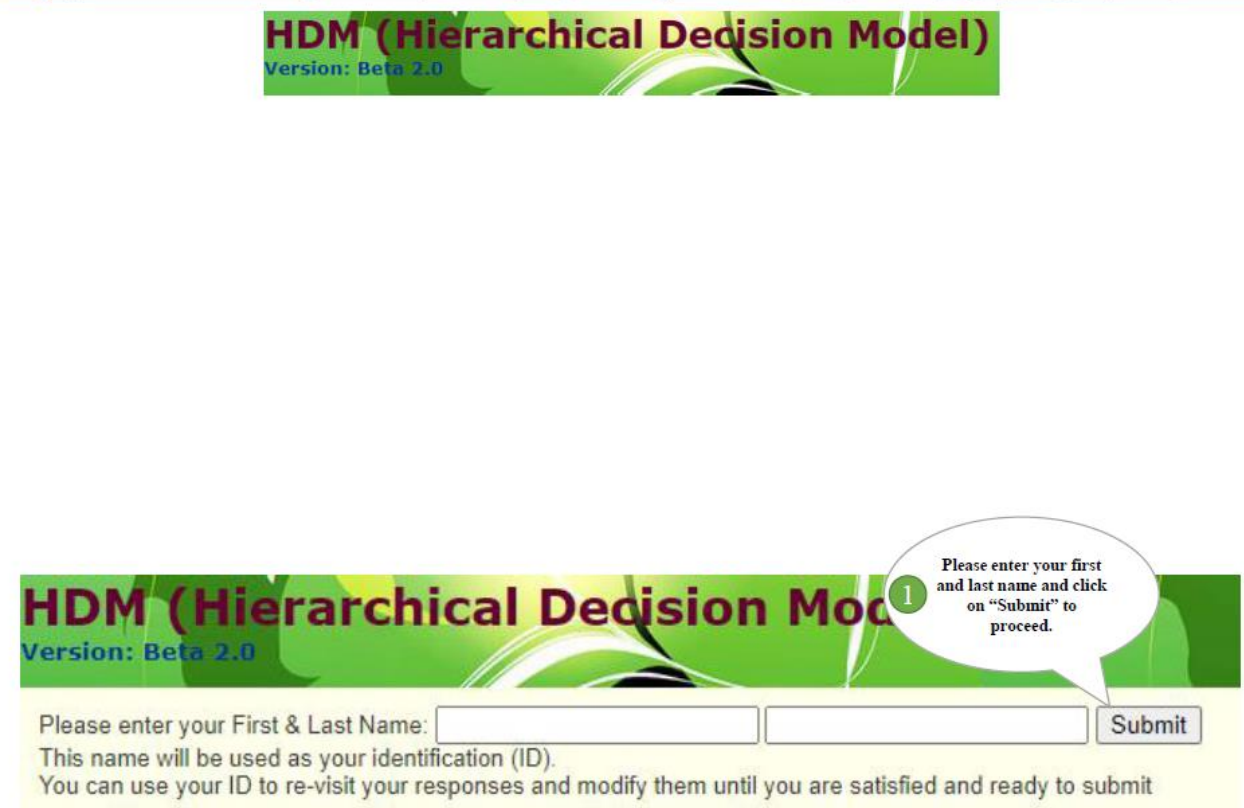


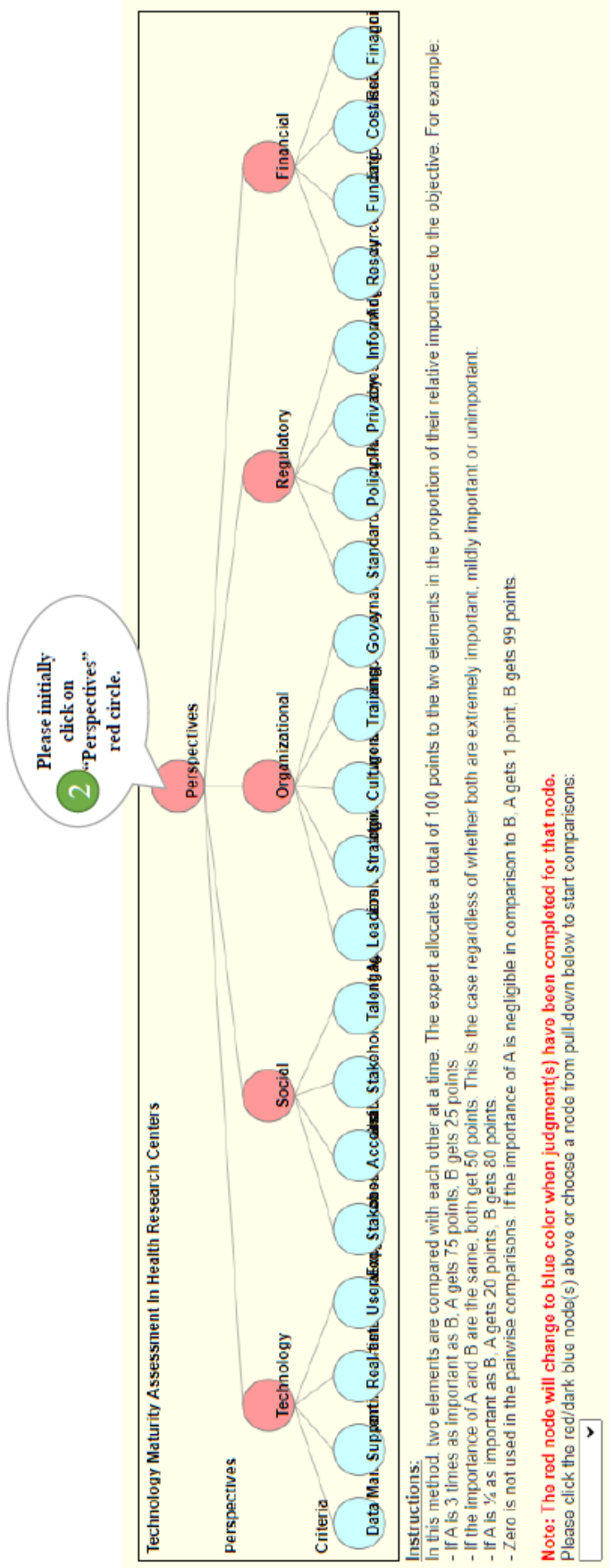

302 
$\checkmark$

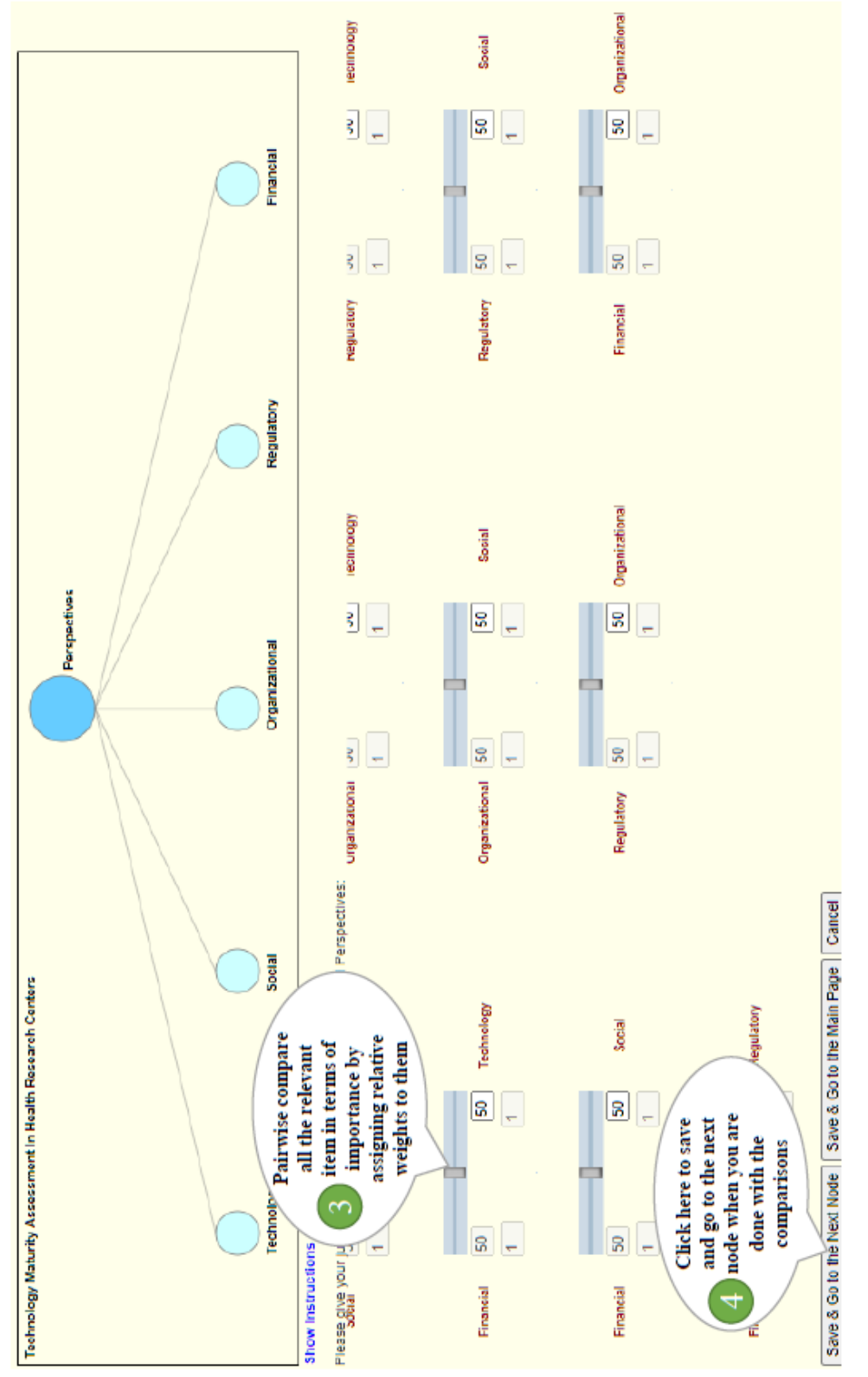




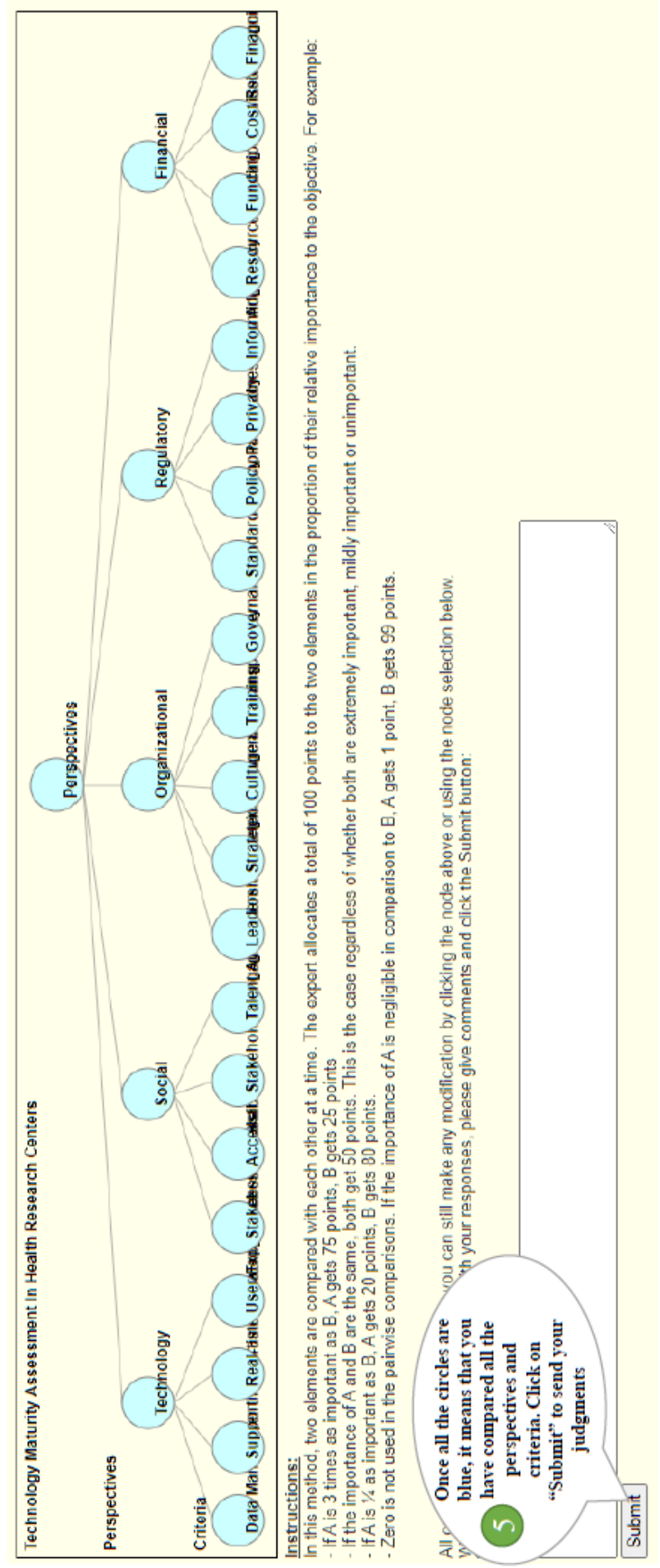




\section{Appendix D- Qualtrics Surveys and ETM HDM Software}

Block 1

ETM

NGINEERING \& TECHNOLOG MANAGEMENT

Amir Shaygan's Research Model Evaluation

Technology.Management Maturity Assessment in Health Reseanch Centers.

Thank you for participating in evaluating my research model as an expert!

My research goal is to develop a model that can assess technology management maturity and continuous learning in health research centers in university hospitals.

This will help bealth research centers to:

-Assess their technology management maturity and give them a sense of self-awareness from different perspectives.

-Pinpoint their strengths and opportumities in order to strategize and allocate resources more efficiently and effectively.

The first step is to validate the perspectives of the factors affecting technology maturity and continuous learning in health research centers.

\section{Medel Perspectives}

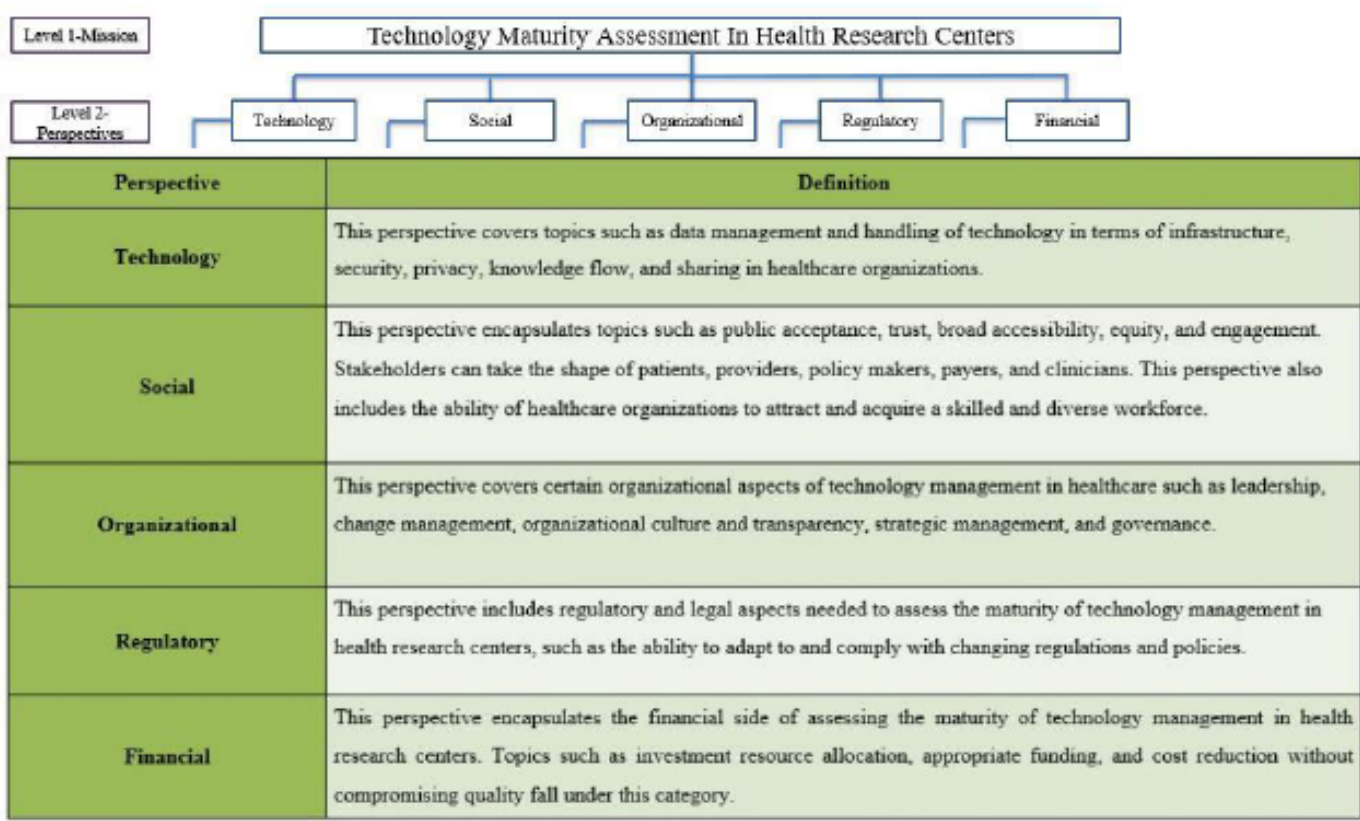

Default Question Block

Name

Last Name 


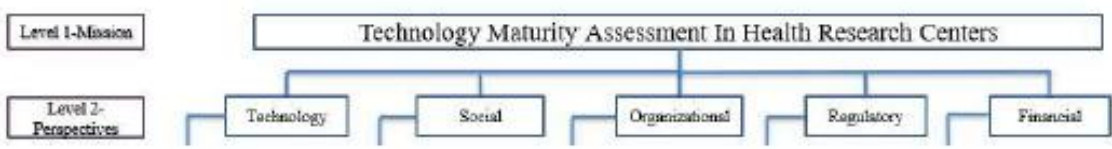

Techeology Maturity Assesment in Health Research Centers Perepective:

\begin{tabular}{|c|l|}
\hline Perspective & \multicolumn{1}{c|}{ Definition } \\
\hline Terhnology & $\begin{array}{l}\text { This perspective covers topics such as data management and handling of technology in terms of infrastructure, } \\
\text { security, privacy, knowledge flow, and sharing in healthcare organizations. }\end{array}$ \\
\hline Social & $\begin{array}{l}\text { This perspective encapsulates topics such as public acceptance, trust, broad accessibility, equity, and engagement. } \\
\text { Stakeholders can take the shape of patients, providers, policy makers, payers, and climicians. This perspective also } \\
\text { includes the ability of healthcare organizations to attract and acquite a skilled and diverse workforce. }\end{array}$ \\
\hline Organizational & $\begin{array}{l}\text { This perspective covers certain organizational aspects of technology management in healthicare such as leadership. } \\
\text { change management, organizational culture and transparency, strategic management, and govemance }\end{array}$ \\
\hline Regulatory & $\begin{array}{l}\text { This perspective includes regulatory and legal aspects needed to assess the maturity of technology management in } \\
\text { bealth research centers, such as the ability to adapt to and comply with changing regulations and policies. }\end{array}$ \\
\hline Financial & $\begin{array}{l}\text { This perspective encapsulates the financial side of assessing the maturity of technology management in health } \\
\text { rescarch centers. Topics such as investment resource allocation, appropriate funding, and cost reduction without } \\
\text { compromising quality fall under this category. }\end{array}$ \\
\hline
\end{tabular}

Plesse Identify the perspectives Important to technology maturity and continuous learning in health research centers.

\begin{tabular}{l|cc}
\hline & \multicolumn{2}{|c}{ Valdasion } \\
& Yes & No \\
\hline Technoiogy & 0 & 0 \\
Socla & 0 & 0 \\
Organizational & 0 & 0 \\
Reguatory & 0 & 0 \\
Finandal & 0 & 0
\end{tabular}

If you think that there are ocher additionsl perspectives or have any comments, please add them below. 
Block 1

\section{ETM}

NMGINEERING \& TECHNOL OQY MANAGEMENT

Amir Shargan's Research Model Evalunion

Technology_Management Maturity. Assessment in Health Research Centers.

Thank you for participsting in evaluating my research model as an expert?

My research goal is to develop a model that can assess technology management maturity and continuous learning in bealth research centers in university hospitals.

This will belp health research centers to:

-Assess their technology management maturity and give them a sense of self-awareness from different perspectives.

-Pinpoint their strengths and opportunities in order to strategize and allocate resources more efficiently and effectively.

The second step is to validate the criteria for each of the perspectives affecting technology maturity and continuous learning in health research centers.

Sedel Criteria

Lerel 1-Mission

Lerel 1-Mission

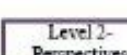

Perspectives

Level 3-Criteris

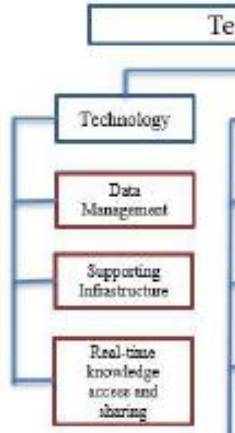

Technology Maturity Assessment In Health Research Centers

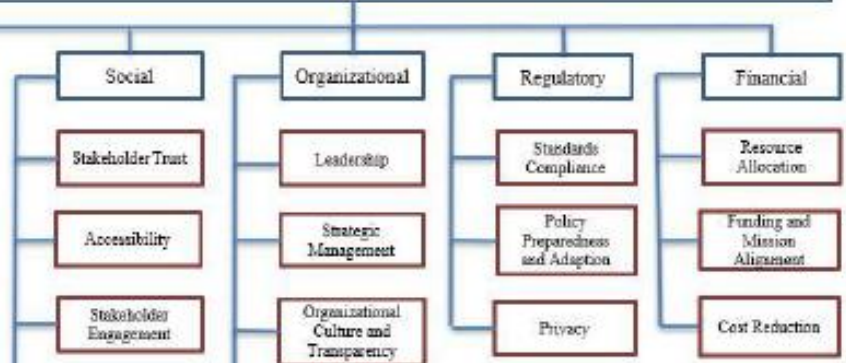

Outcomes

Desirability Curves

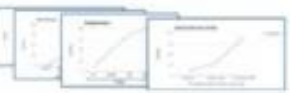

\section{Default Question Block}

Please eater your name:

Name

Last Name 

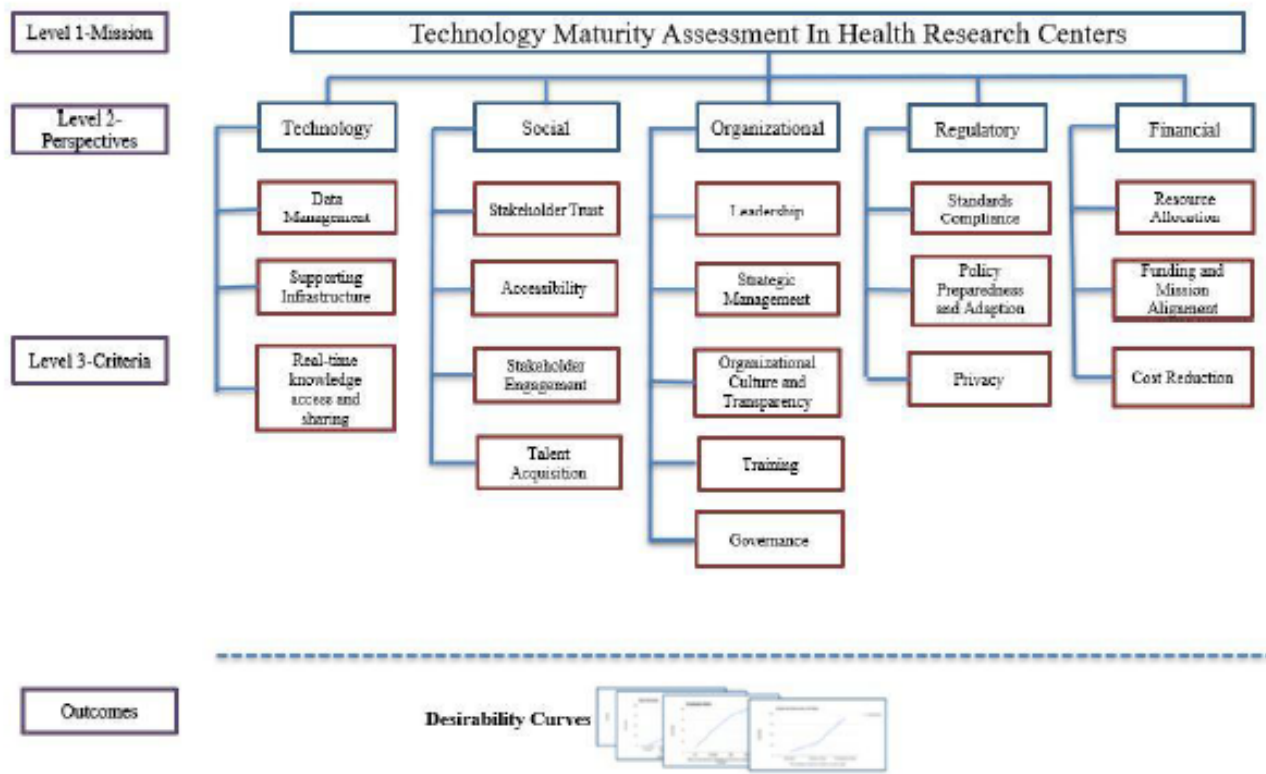

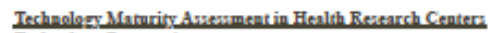
Teshnolegr Perresstive:

\begin{tabular}{|c|c|c|c|}
\hline Perspeetive & Criteria & Definition & References \\
\hline \multirow{3}{*}{ Technology } & Data Management & $\begin{array}{l}\text { Management of big data resources in terms of security, privacy, } \\
\text { governance, and ethics. }\end{array}$ & 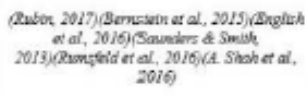 \\
\hline & $\begin{array}{l}\text { Supporting } \\
\text { Infrastructure }\end{array}$ & $\begin{array}{l}\text { The socio-technical infrastructure needed to improve and } \\
\text { provide capacity to capture, compile, and protect clinical and } \\
\text { finaneial data, which enables the evaluation, adjustment, } \\
\text { analysis, and dissemination of learned lonowledge. }\end{array}$ & 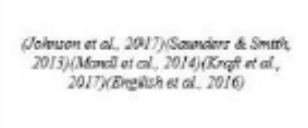 \\
\hline & $\begin{array}{l}\text { Real-time Knowledge } \\
\text { Access and Sharing }\end{array}$ & $\begin{array}{l}\text { Data systems and tools used to capture, share, and integrate } \\
\text { data, information, visualizations, and knowledge gained from } \\
\text { research into the organization in real-time. }\end{array}$ & 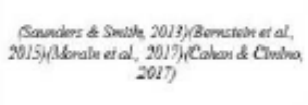 \\
\hline
\end{tabular}

Plesse idencify the criteria important to the technology perspective of technology maturity and continuous learning in health research centers.

\begin{tabular}{l|cc}
\hline & \multicolumn{2}{|c}{ Valdation } \\
& Yes & No \\
\hline Data Managemert & 0 & 0 \\
Supporting Irffastructure & 0 & 0 \\
Real-tme Knowfedge Access and Sharing & 0 & 0
\end{tabular}

If you think that there are other additional criteria or have any comments, please add them below. https://portlandstate.ca1.qualitrics.com/Q/EditSection/Blocks/Ajax/GetSurveyPrintPreview?ContextSurveylD=SV_3Kmltb2RPWLRC3H\&ContextLibrary... 2 


\begin{tabular}{|c|c|c|c|}
\hline \multicolumn{2}{|l|}{$2 / 22 / 2021$} & Qualtrics Survey Software & \\
\hline \multicolumn{4}{|l|}{ Block 2} \\
\hline \multicolumn{4}{|c|}{$\begin{array}{l}\text { Technolegy Management Maturity in Health Regearch Centers } \\
\text { Seclal Pergnective: }\end{array}$} \\
\hline Perspective & Criteria & Definition & References \\
\hline \multirow{4}{*}{ Soxial } & Stakebolder Trust & $\begin{array}{l}\text { Key components of establishing stakeholder (patients, providers, payors, policymaker, } \\
\text { purchasers, families) trust include: } \\
\text { - Gamering buy-in and trust from stakebolders so they contribute to a culture of } \\
\text { continuous improvement and leaming. } \\
\text { Transparency with stakeholders regarding current limitations and plans to address } \\
\text { and mitigate them through a system which supports clinical and translational } \\
\text { research, public health information, and comparative effectiveness. }\end{array}$ & 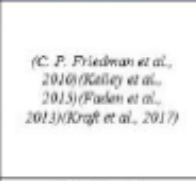 \\
\hline & Accessibility & $\begin{array}{l}\text { The degree to which the public is benefiting from the advantages of continuous } \\
\text { learning. Health research centers must carry out consistent and innovative outreach } \\
\text { efforts to ensure that services are accessible to diverse populations for the purpose of } \\
\text { reducing racial, ethnic, sexual orientation'gender, and other digparitics. }\end{array}$ & 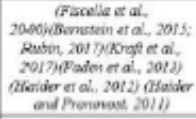 \\
\hline & Stakeholder Engagement & $\begin{array}{l}\text { The degree to which a bealth research center can engage stakeholders (patients, } \\
\text { providers, payors, policymaker, purchasers, families) to participate in continuous } \\
\text { improvement and learning projects and initiatives. }\end{array}$ & 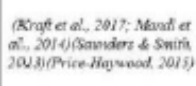 \\
\hline & Talent Acquisition & $\begin{array}{l}\text { Research centers' ability to acquire talented and diverse staff in different areas } \\
\text { (physicians, nurses, researchers, data scientists, public bealth professionals, managers, } \\
\text { epidemiologists, administrative staff, etc.) }\end{array}$ & 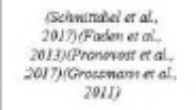 \\
\hline
\end{tabular}

Please Identify the criterla Important to the soclal perspective of technology maturity and continuous learning in health research centers in university hospitals.

\begin{tabular}{l|cc}
\hline & \multicolumn{2}{|c}{ Valdation } \\
& Yes & No \\
\hline Stakehoider Trust & 0 & 0 \\
Accessibility & 0 & 0 \\
Stakehoider Engagement & 0 & 0 \\
Talent Acquistition & 0 & 0
\end{tabular}

If you think that there are other additional criteria or have any comments, please add them below.

Block 3

Technology Management Maturity in Health Research Centers:

Organizational Perspective: 


\begin{tabular}{|c|c|c|c|}
\hline Perspective & Criteria & Definition & References \\
\hline \multirow{5}{*}{ Organizational } & Leadership & $\begin{array}{l}\text { Broad leadership which can expand and guide stakeholders' commitment } \\
\text { to the goals of continuous learning and iacreased technology manurity. }\end{array}$ & 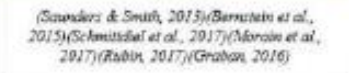 \\
\hline & Strategic Management & $\begin{array}{l}\text { Research centers' ability to improve through strategic decisions, } \\
\text { management of competing griorities, rescarch partnerships, problern } \\
\text { identification, and finding solutions. }\end{array}$ & 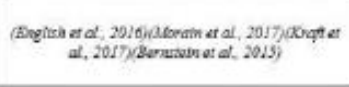 \\
\hline & $\begin{array}{l}\text { Organizational Culture } \\
\text { and Transparency }\end{array}$ & $\begin{array}{l}\text { The extent to which a research center's employees have adopted and are } \\
\text { committed to a culture of continuous learning and practice transparency } \\
\text { to safeguard stakeholder trust in order to improve the health of } \\
\text { indrviduals, communaties, and diverse populations. }\end{array}$ & 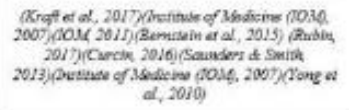 \\
\hline & Training & $\begin{array}{l}\text { Training employees and stakeholders on the latest technologies and best } \\
\text { practices within the health research center. }\end{array}$ & 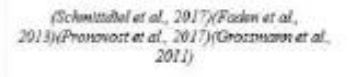 \\
\hline & Governance & $\begin{array}{l}\text { Having the necessary governasce to support a sustainable operation, } \\
\text { uphold required standards, build and maintain trust with stakeholders, } \\
\text { and continuously innovate. }\end{array}$ & 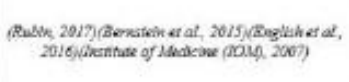 \\
\hline
\end{tabular}

Please Identify the criterla important to the organizational aspect of technology maturity and comtinuous learning in healthcare organizations

\begin{tabular}{l|cc}
\hline & Valdation \\
& Yes & No \\
\hline Leadership & 0 & 0 \\
Strdeglc Management & 0 & 0 \\
Organizational Culture and Transparency & 0 & 0 \\
Trining & 0 & 0 \\
Govemance & 0 & 0
\end{tabular}

If you think that there are other additiosal criteria or hare any comments, plesse add them below.

Block 4

Techeology Management Maturity in Health Research Centers: Regelatory Perspective:

\begin{tabular}{|c|c|c|c|}
\hline Perspective & Criteria & Definition & References \\
\hline \multirow{3}{*}{ Regulatory } & Standards Compliance & $\begin{array}{l}\text { Research centers" compliance with standards and } \\
\text { regulations to ensure transparency with stakeholders, data } \\
\text { interoperability, and commitment to meaningful use and } \\
\text { joint commissions. }\end{array}$ & 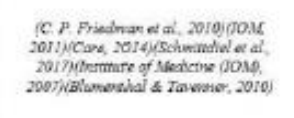 \\
\hline & Policy Preparedness and Adaption & $\begin{array}{l}\text { Research centers" flexibility in adapting to new regulations, } \\
\text { legislations, and policies in terms of anticipation and } \\
\text { adaption (resources, policies, strategies, and management), }\end{array}$ & 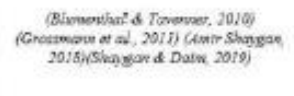 \\
\hline & Privacy & $\begin{array}{l}\text { Research centers' adherence to regulations and policies to } \\
\text { eusure privacy in terms of information technology, medical } \\
\text { data, patient access, third party interactions, and ethical use } \\
\text { of information, among others. }\end{array}$ & 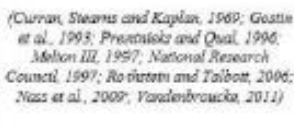 \\
\hline
\end{tabular}

https://portlandstate.ca1.qualtrics.com/Q/EditSection/Blocks/Ajax/GetSurveyPrintPreview?ContextSurveylD=SV_3Kmltb2RPWLRC3H\&ContextLibrary... 
Please Identify the criterla important to the regulatory perspective of technology maturity and comtinuous learning in heslth research centers in wniversity hospitals.

\begin{tabular}{l|cc}
\hline & \multicolumn{2}{|c}{ Valldation } \\
& Yes & No \\
\hline Standards Complance & 0 & 0 \\
Polcy Preparectiness and Adaption & 0 & 0 \\
Privacy & 0 & 0
\end{tabular}

If you think that there are other additional criteria or have any comments, please add them below.

\section{Block 5}

Technology. Management Maturity in Health Rerearch Centers Financial Perspestime:

\begin{tabular}{|c|c|c|c|}
\hline Perspective & Criteria & Definition & References \\
\hline \multirow{3}{*}{ Financial } & Resource Allocation & $\begin{array}{l}\text { Research centers' ability to prioritize and decide to invest its } \\
\text { resources in alignment with the goals of inereased technology } \\
\text { maturity and continuous learning. }\end{array}$ & 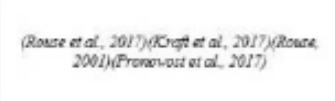 \\
\hline & $\begin{array}{l}\text { Funding and Mission } \\
\text { Alignment }\end{array}$ & $\begin{array}{l}\text { The extent to which incentives and funding are aligned with } \\
\text { encouragement of technology maturity, continuous learning and } \\
\text { improvement, waste elimination, and rewarding high care value. }\end{array}$ & 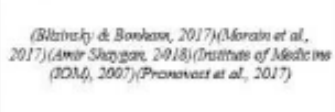 \\
\hline & Cost Reduction & $\begin{array}{l}\text { Research centers' ability to cut costs through increased maturity, } \\
\text { learning, continuous improvement, and waste eliminations } \\
\text { without compromising quality. }\end{array}$ & 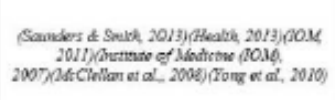 \\
\hline
\end{tabular}

Please Identify the criterla important to the financial aspect of technology matarity and contimsows learning in bealth research centers in university hospitals.

\begin{tabular}{l|cc}
\hline & \multicolumn{2}{|c}{ Validation } \\
Yes & No \\
\hline Resource Alocation & 0 & 0 \\
Funding and Mission Allgnment & 0 & 0 \\
Cost Reduction & 0 & 0
\end{tabular}

If you think that there are other additionsl criteria or have any comments, please add them below. 
Block 1

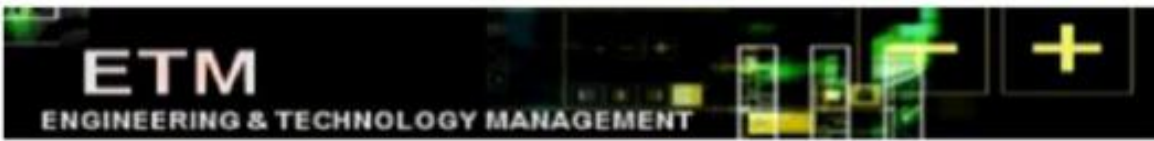

Technology Management Matwrity Assessment in Health Research Centers

Thank you for participating in evaluating my research model as an expert!

My research goal is to develop a model that can assess technology management maturity and continuous learning in health research centers in university hospitals.

This will help health research centers:

-Assess their technology management maturity and give them a sense of self-awareness from different perspectives.

-Pinpoint their strengths and opportunities in order to strategize and allocate resources more efficiently and effectively.

The third step is to identify and evaluate the desirability curves associated with the important factors affecting technology maturity and continuous learning in health research centers.

In this model. desirability curves represent the metrics/levels that can be assigned to a health organization's current status against each criterion in the model for technology management maturity assessment.

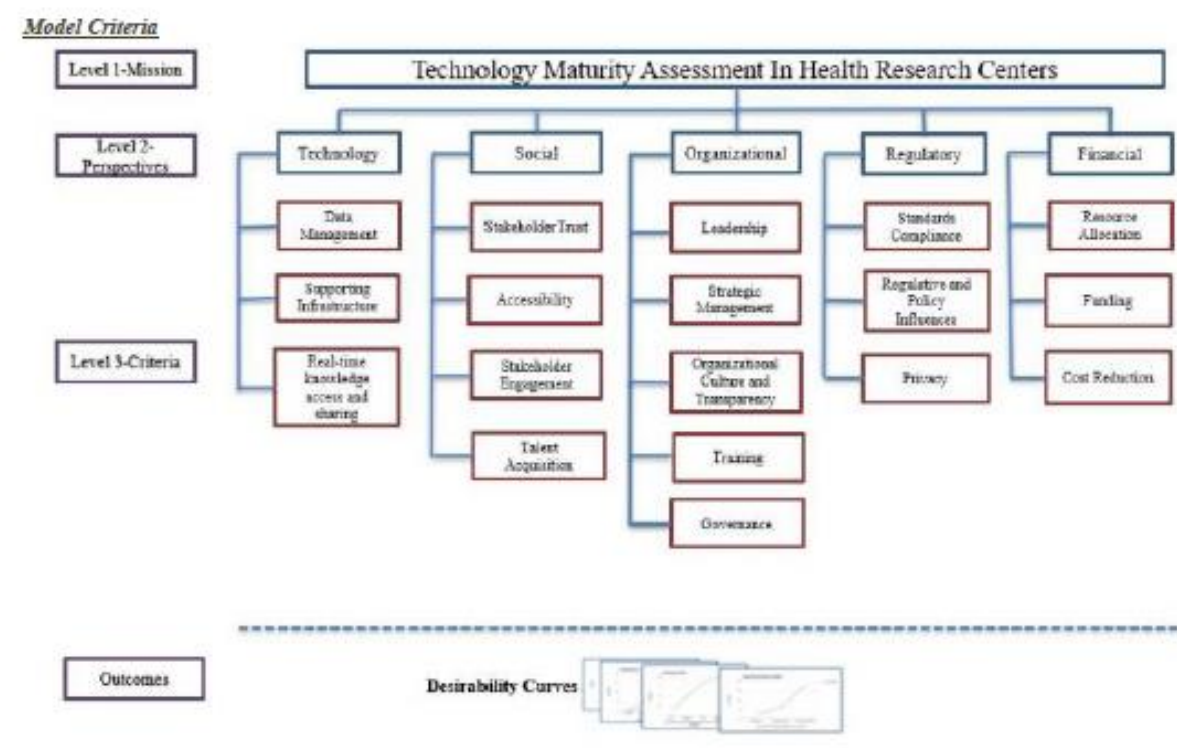

\section{Default Question Block}

Please enter your name: 
Block 1

\section{ETM}

ENGINEERING \& TECHMOL OGY MAMAGEMENT

Amir Shaygan's Research Model Evaluntion

Iechaolegr-Management Matariv. Arresument in Health Rerearch Ceater

Thank fou for participnting in evalustiug my research model as an expert!

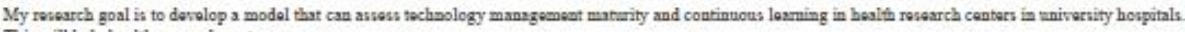

This will belp besith reisarch conters

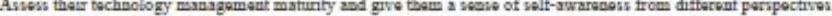

-Pinpoint their strengths as opportaities in order to stratogive and allocate recources more efficiestly and effectively.

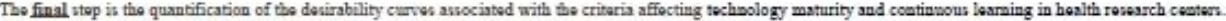

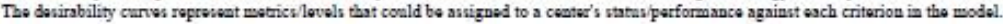

Nodel Criteria

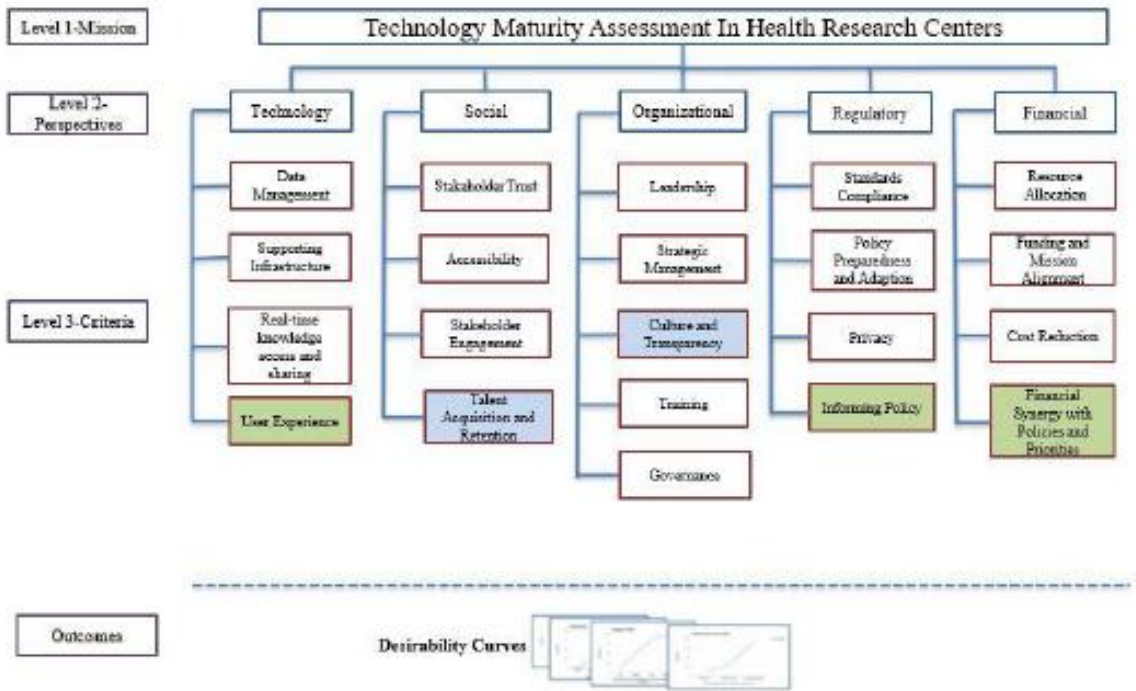

Default Question Block

Plasse eater your name:

Name

Last Name

https://portlandstate.ca1.qualtrics.com/Q/EditSection/Blocks/Ajax/GetSurveyPrintPreview?ContextSurveylD=SV_eFMyqa8EFsEXhtj\&ContextLibraryl... $1 / 16$ 


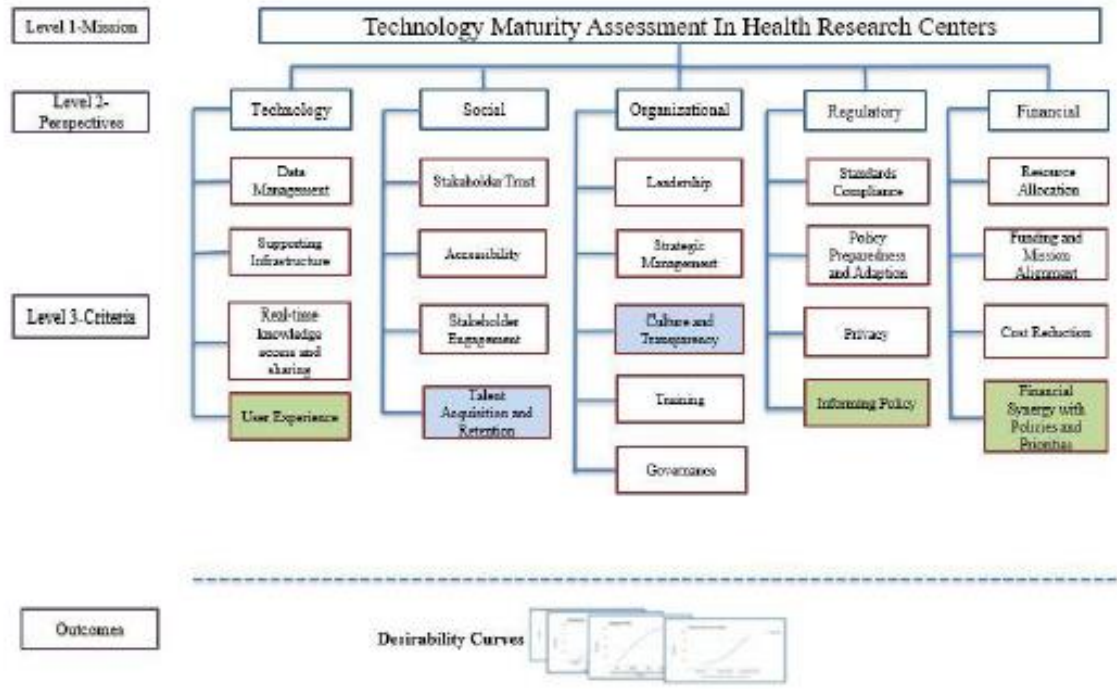

Iechnology Perspective:

\begin{tabular}{|c|c|c|c|}
\hline Perspective & Criteria & Definition & References \\
\hline \multirow{4}{*}{ Technology } & Data Management & $\begin{array}{l}\text { Management of big data resources in terms of security, privacy. } \\
\text { governance, and ethics. }\end{array}$ & 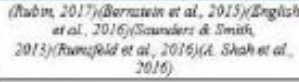 \\
\hline & $\begin{array}{l}\text { Supporting } \\
\text { Infrastructure }\end{array}$ & $\begin{array}{l}\text { The socio-technical infrastructure needed to improve and } \\
\text { provide capacity to capture, compile, and protect clinical and } \\
\text { financial data, which enables the evaluation, adjustment. } \\
\text { analysis, and dissemination and integration of learned } \\
\text { knowledge into clinical care processes in order to secure the } \\
\text { promised improvements. }\end{array}$ & 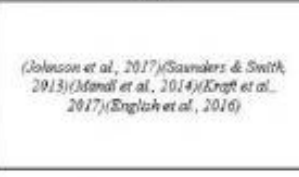 \\
\hline & $\begin{array}{l}\text { Real-time Knowledge } \\
\text { Access and Sharing }\end{array}$ & $\begin{array}{l}\text { Data systems and tools used to capture, share, and integrate } \\
\text { data, information, visualizations, and knowledge gained from } \\
\text { research into the organization in real-time. }\end{array}$ & 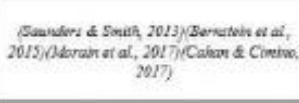 \\
\hline & User Experience & $\begin{array}{l}\text { Ensuring that technologies and IT products are user-friendly (e.g.- } \\
\text { taking health literacy and technology competence into } \\
\text { consideration) to all users (stakeholders). }\end{array}$ & 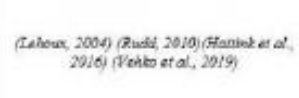 \\
\hline
\end{tabular}

Instructions:

Please give each metric a score from 0 (The least favorable) to 100 (The most favorable).

Data Manogement

What level of data management does this research center provide?

Note: Please give each metric a score from 0 (The least favorable) to 100 (The most favorable).

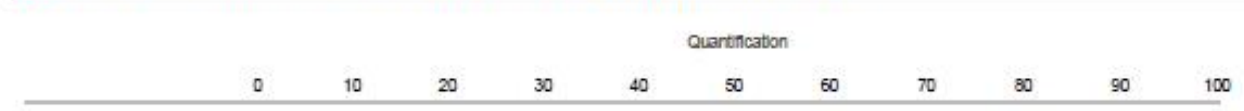

https://portandstate.ca1.qualtrics.com/Q/EditSection/Blocks/Ajax/GetSurveyPrintPreview?ContextSurveylD=SV_eFMyqa8EFsEXhtj\&ContextLibraryl... 2/16 
Quartitication

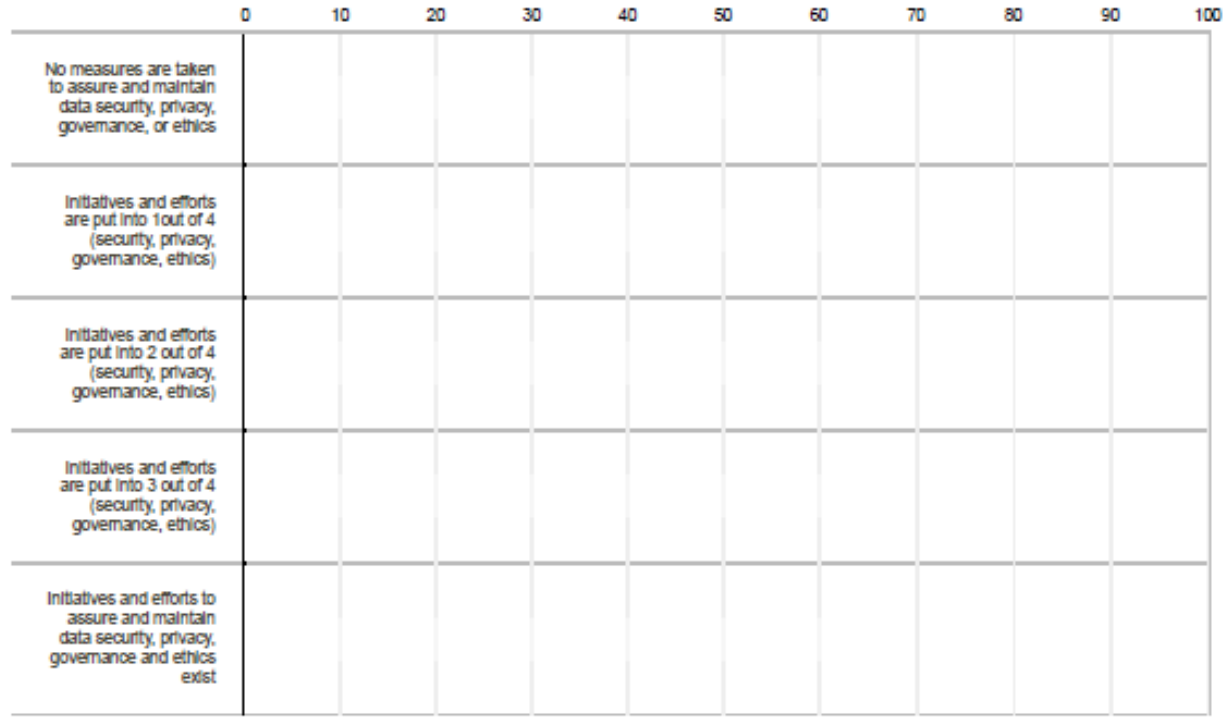

Supporting Infrastructure

What level of socio-technical infrastructure does the research center provide in managing and analysis of data and dissemination of knowledge?

Note: Please give each metric a score from 0 (The least favorable) to 100 (The most favorable).

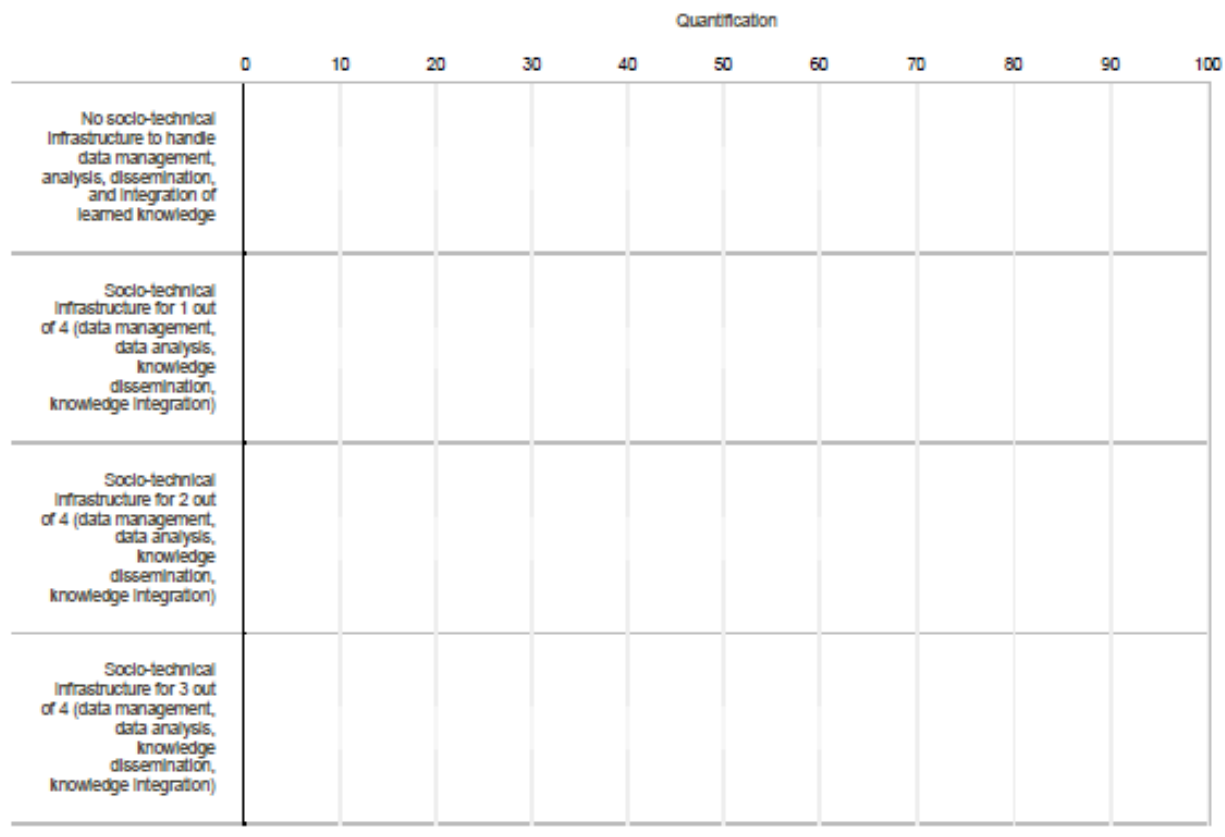




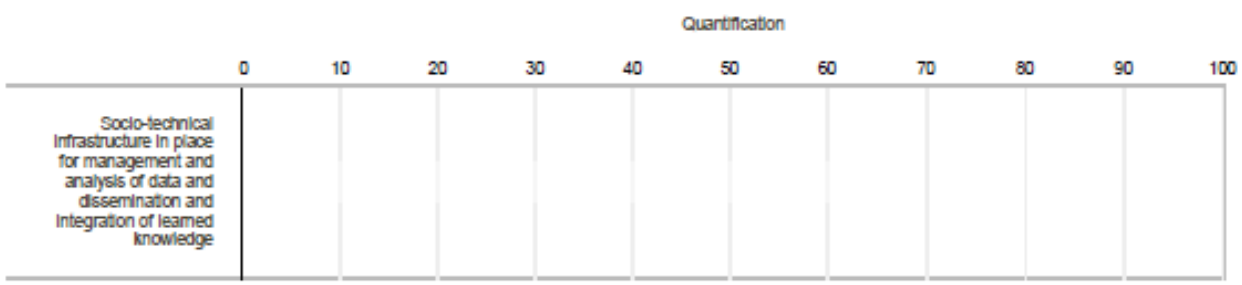

Real-time Knowledge-Access and Sharing

Is the research center providing real-time data and knowledge access, sharing, and learning?

Note: Please give each metric a score from 0 (The least favorable) to 100 (The most favorable).

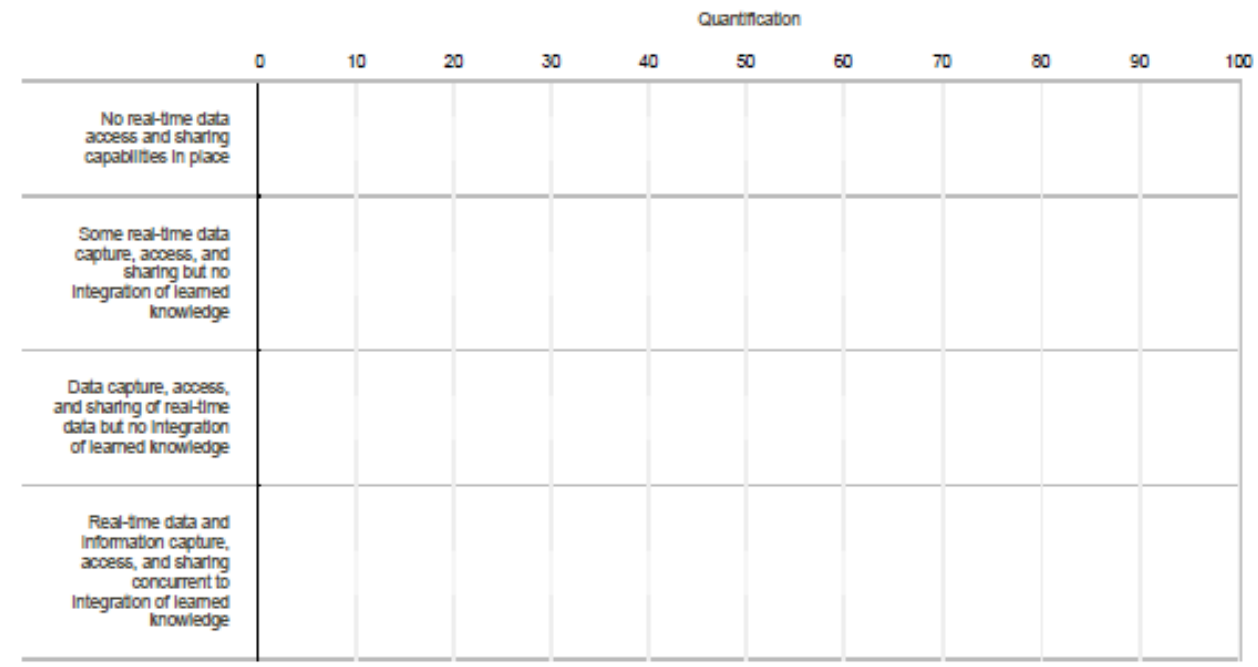

\section{User Erperience}

What is the quality of user experience provided by the research center to its stalkeholders (users)?

Note: Please give each metric a score from 0 (The least favorable) to 100 (The most favorable).

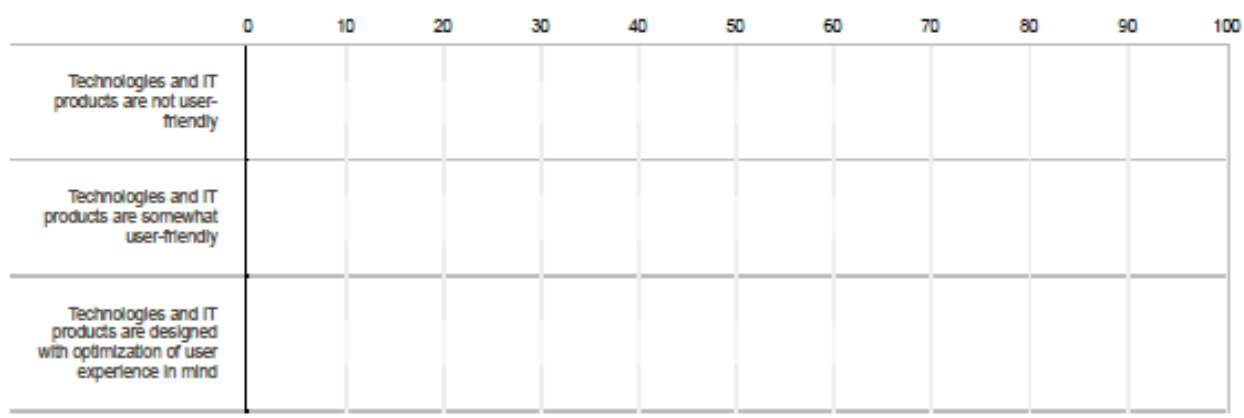




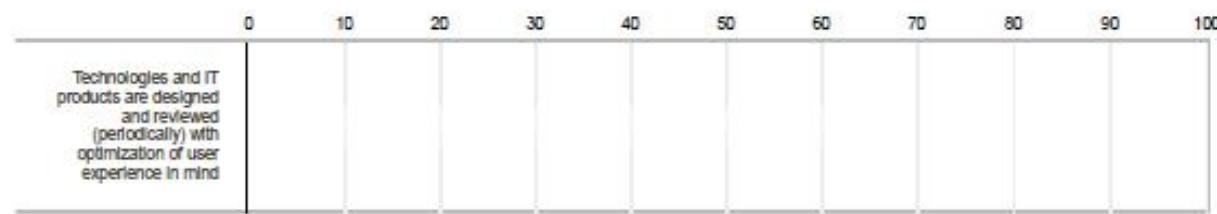

Block 2

\begin{tabular}{|c|c|c|c|}
\hline Perspective & Criteria & Definition & References \\
\hline \multirow{4}{*}{ Social } & Stakeholder Tnust & $\begin{array}{l}\text { Key components of establishing stakeholder (patients, providers, payors, policymaker. } \\
\text { purchasers, families) truat include: } \\
\text { Garnering buy-in and trust from stakeholders so they contribute to a culture of } \\
\text { continuous improvement and learning. } \\
\text { Transparency with stakcholders regarding current limitations and plans to address } \\
\text { and mitigate them throngh a system which stpports clinical and translational } \\
\text { research, public health information, and comparative effectiveness. } \\
\text { Maintaining trustworthiness as a research center by following through on promised } \\
\text { commitments and ensuring new knowledge is used to improve care of those who } \\
\text { contributed to its generation via enhanced use of their dats. }\end{array}$ & 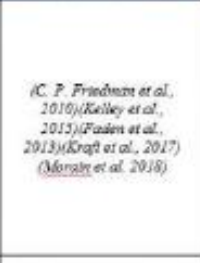 \\
\hline & Accessibility & $\begin{array}{l}\text { The degree to which the stakeholders are benefiting from the clinical and research } \\
\text { advantages of continuous leaming. Health research centers must carry out consistent } \\
\text { and innovative outreach efforts to casure that services are accessible to diverse } \\
\text { populations for the purpose of reducing racial, ethnic, sexual erientation'gender, and } \\
\text { other disparities. }\end{array}$ & 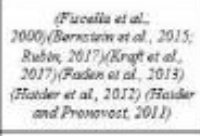 \\
\hline & Stakeholder Engagement & $\begin{array}{l}\text { The degree to which a health research center can engage stakeholders (patients, } \\
\text { providers, payors, policymaker, purchasers, families) to participate in continuons } \\
\text { improvement and learning projects and initiatives. }\end{array}$ & 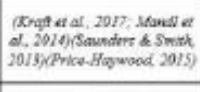 \\
\hline & $\begin{array}{l}\text { Talent Acquisition and } \\
\text { Retention }\end{array}$ & $\begin{array}{l}\text { Research centers' ability to acquire and retain talented and diverse staff in different } \\
\text { areas (physicians, nurses, researchers, data scientists, public health professionals, } \\
\text { managers, epidemiologists, administrative staff, etc.) }\end{array}$ & 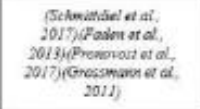 \\
\hline
\end{tabular}

Instructions:

Please give each metric a score from 0 (The least favorable) to 100 (The most favorable).

Stakeholder Trust

What is the level of stakeholder trust in the research center's initiatives and projects (increased maturity and continuous learning)?

Note: Please give each metric a score from 0 (The least favorable) to 100 (The most favorable).

Quartincation

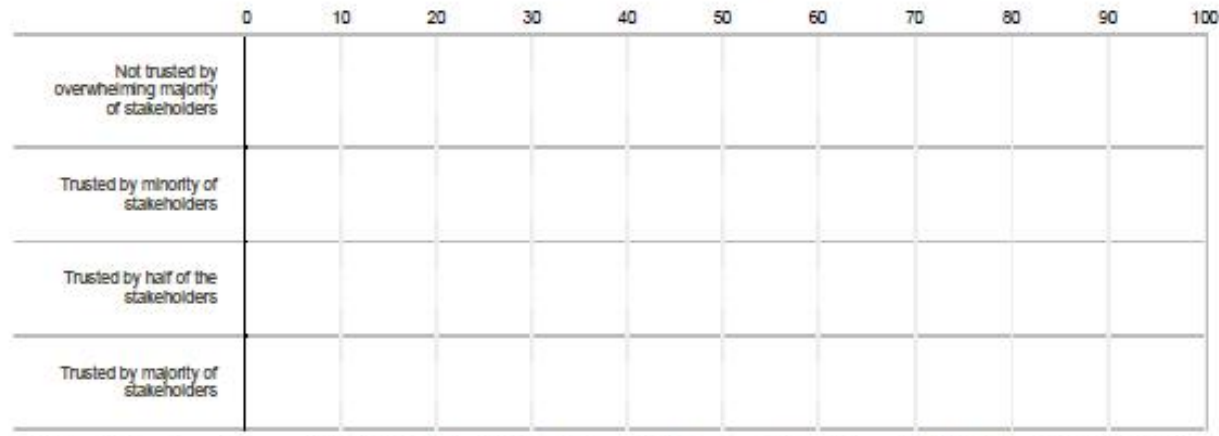

https://portlandstate.ca1.qualtrics.com/Q/EditSection/Blocks/Ajax/GetSurveyPrintPreview?ContextSurveylD=SV_eFMyqa8EFsEXhtj\&ContextLibraryl... 5/16 
Accessibility

What is the level of benefit from and accessibility to new technologies?

Note: Please give each metric a score from 0 (The least favorable) to 100 (The most favorable).

Stakehoiders are not
beneting fom the
clinical and research
advantages of the
research centier

Stakeholder Engagement

What is the level of stakeholder engagement in the research center's projects and initiatives?

Note: Please give each metric a score from 0 (The least favorable) to 100 (The most favorable).

\begin{tabular}{|c|c|c|c|c|c|c|c|c|c|c|c|}
\hline & \multicolumn{11}{|c|}{ Quartincation } \\
\hline & 0 & 10 & 20 & 30 & 40 & 50 & 60 & 70 & 80 & 90 & 100 \\
\hline $\begin{array}{l}\text { Stakehoiders are } \\
\text { opposed to partidipate } \\
\text { in research centers } \\
\text { conthnous } \\
\text { improvement and } \\
\text { learning projects and } \\
\text { intlititives }\end{array}$ & & & & & & & & & & & \\
\hline 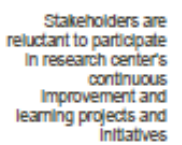 & & & & & & & & & & & \\
\hline
\end{tabular}

https://portlandstate.ca1.qualtrics.com/Q/EditSection/Blocks/Ajax/GetSurveyPrintPreview?ContextSurveylD=SV_eFMyqa8EFsEXhtj\&ContextLibraryl... 6/16 


\begin{tabular}{|c|c|c|c|c|c|c|c|c|c|c|c|}
\hline & \multicolumn{11}{|c|}{ Quartincation } \\
\hline & 0 & 10 & 20 & 30 & 40 & 50 & 60 & 70 & 80 & 90 & 100 \\
\hline $\begin{array}{l}\text { Half of the stakenoiders } \\
\text { are eager to partipalpate } \\
\text { in research centers } \\
\text { continuous } \\
\text { improvement and } \\
\text { leaming projects and } \\
\text { Initiatives }\end{array}$ & & & & & & & & & & & \\
\hline $\begin{array}{l}\text { Majortly or the } \\
\text { stakehoiders are eager } \\
\text { to partipate in } \\
\text { research center's } \\
\text { continuous } \\
\text { improvement and } \\
\text { learning projects and } \\
\text { initiatives }\end{array}$ & & & & & & & & & & & \\
\hline $\begin{array}{l}\text { Al stakehoiders are } \\
\text { eager to partipate in } \\
\text { research center's } \\
\text { continuous } \\
\text { improvement and } \\
\text { learning projects and } \\
\text { initlatives }\end{array}$ & & & & & & & & & & & \\
\hline
\end{tabular}

\section{Talent Acquisition}

How is the research center performing in acquiring and retaining slilled and relevant talents?

Note: Please give each metric a score from 0 (The least favorable) to 100 (The most favorable).

\begin{tabular}{|c|c|c|c|c|c|c|c|c|c|c|c|}
\hline & \multicolumn{11}{|c|}{ Quartincation } \\
\hline & 0 & 10 & 20 & 30 & 40 & 50 & 60 & 70 & 80 & 90 & 100 \\
\hline $\begin{array}{l}\text { No acquisiton and } \\
\text { retention of skilled and } \\
\text { diverse talent }\end{array}$ & & & & & & & & & & & \\
\hline $\begin{array}{r}\text { Acquisition of skilled } \\
\text { and diverse talent only } \\
\text { in some areas }\end{array}$ & & & & & & & & & & & \\
\hline $\begin{array}{l}\text { Acquisiton and } \\
\text { retention of skilled and } \\
\text { diverse talent only in } \\
\text { some areas }\end{array}$ & & & & & & & & & & & \\
\hline $\begin{array}{l}\text { Acquisition and } \\
\text { retention of skilled and } \\
\text { dlverse talent in most } \\
\text { areass }\end{array}$ & & & & & & & & & & & \\
\hline
\end{tabular}

Block 3

Technology. Management Maturity in Health Research Centers:

organizational Persoectlve:

https://portlandstate.ca1.qualtrics.com/Q/EditSection/Blocks/Ajax/GetSurveyPrintPreview?ContextSurveylD=SV_eFMyqa8EFsEXhtj\&ContextLibraryl... 7/18 


\begin{tabular}{|c|c|c|c|}
\hline Perspective & Criteria & Definition & References \\
\hline \multirow{5}{*}{ Organizational } & Leadership & $\begin{array}{l}\text { Broad leadership which can expand and guide stakeholders' commitment } \\
\text { to the goals of continuous learning and increased technology maturity. }\end{array}$ & 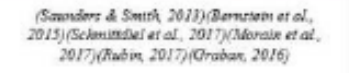 \\
\hline & Strategic Management & $\begin{array}{l}\text { Research centers' ability to improve through strategic decisions, } \\
\text { management of competing priorities, internal and external } \\
\text { partnerships'collaborations, problem identification, and finding solutions. }\end{array}$ & 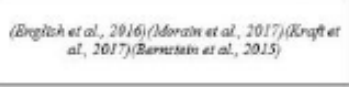 \\
\hline & $\begin{array}{l}\text { Organizational Culture } \\
\text { and Transparency }\end{array}$ & $\begin{array}{l}\text { The extent to which a resaarch center's employees have adopted and are } \\
\text { committed to a culture of continuous leaming and practice transparency } \\
\text { to safeguard stakeholder trust in order to improve the bealth of } \\
\text { individuals, communities, and diverse populations. }\end{array}$ & 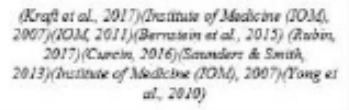 \\
\hline & Training & $\begin{array}{l}\text { Training employees and stakeholders on the latest technologies and best } \\
\text { practices within the health research center. }\end{array}$ & 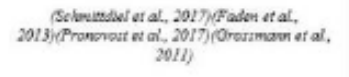 \\
\hline & Governance & $\begin{array}{l}\text { Having the necessary governance to support a sustainable operation, } \\
\text { uphold required standards, build and maintain trust with stakeholders, } \\
\text { and continuously innovate. }\end{array}$ & 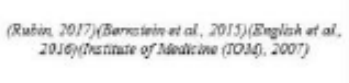 \\
\hline
\end{tabular}

Instructions:

Please give each metric a score from 0 (The least favorable) to 100 (The most favorable).

\section{Leadership}

What level of leadership support is bolstering technology maturity and continuous learning in the research center?

Note: Please give each metric a score from 0 (The least favorable) to 100 (The most favorable).

\begin{tabular}{|c|c|c|c|c|c|c|c|c|c|c|c|}
\hline & \multicolumn{11}{|c|}{ Quartincation } \\
\hline & 0 & 10 & 20 & 30 & 40 & 50 & 60 & 70 & 80 & 90 & 100 \\
\hline $\begin{array}{r}\text { There is leaderstip } \\
\text { opposition to } \\
\text { technology maturty and } \\
\text { organizationa leaming }\end{array}$ & & & & & & & & & & & \\
\hline $\begin{array}{l}\text { The leadership is } \\
\text { Indiferent to technology } \\
\text { maturty and } \\
\text { organizationa leaming }\end{array}$ & & & & & & & & & & & \\
\hline $\begin{array}{l}\text { The leadership provides } \\
\text { some support to } \\
\text { technology maturty and } \\
\text { organizational leaming }\end{array}$ & & & & & & & & & & & \\
\hline $\begin{array}{l}\text { The leadership provides } \\
\text { good support and } \\
\text { advocacy to technology } \\
\text { maturty and } \\
\text { organizational leaming }\end{array}$ & & & & & & & & & & & \\
\hline $\begin{array}{l}\text { The lezorship is } \\
\text { enthuslisatic about the } \\
\text { support and advocacy } \\
\text { of technology maturtty } \\
\text { and orgarizationa } \\
\text { leaming }\end{array}$ & & & & & & & & & & & \\
\hline
\end{tabular}

\section{Strategic Management}

What level of strategic management is being implemented in the research center?

https://portlandstate.ca1.qualtrics.com/Q/EditSection/Blocks/Ajax/GetSurveyPrintPreview?ContextSurveylD=SV_eFMyqa8EFsEXhtj\&ContextLibraryl... 8/16 
Note: Please give each metric a score from 0 (The least favorable) to 100 (The most favorable).

\begin{tabular}{|c|c|c|c|c|c|c|c|c|c|c|c|}
\hline & \multicolumn{11}{|c|}{ Cuantitication } \\
\hline & 0 & 10 & 20 & 30 & 40 & 50 & 60 & 70 & 80 & 90 & 100 \\
\hline $\begin{array}{l}\text { There is no strategic vision, } \\
\text { plan, or management and } \\
\text { Implementation of } \\
\text { technology maturtin and } \\
\text { organtzatonal learming }\end{array}$ & & & & & & & & & & & \\
\hline $\begin{array}{l}\text { There is strategic vision and } \\
\text { plan but no management } \\
\text { and Implementation of } \\
\text { technology maturty and } \\
\text { organizatonal leaming }\end{array}$ & & & & & & & & & & & \\
\hline $\begin{array}{l}\text { There is some clear strategic } \\
\text { visionjplan and } \\
\text { managementimplementation } \\
\text { of fechnology mathrinty and } \\
\text { organizatonal leaming }\end{array}$ & & & & & & & & & & & \\
\hline $\begin{array}{l}\text { There is dear and caiculated } \\
\text { strategc visioniplan and } \\
\text { managed implementation of } \\
\text { feccinology and } \\
\text { organizational learing }\end{array}$ & & & & & & & & & & & \\
\hline
\end{tabular}

Organizational Culture and Transparency

What level of organizational culture (in terms of maturity and learning) has been adopted by the employees of the research center? Note: Please give each metric a score from 0 (The least favorable) to 100 (The most favorable).

Quartincation

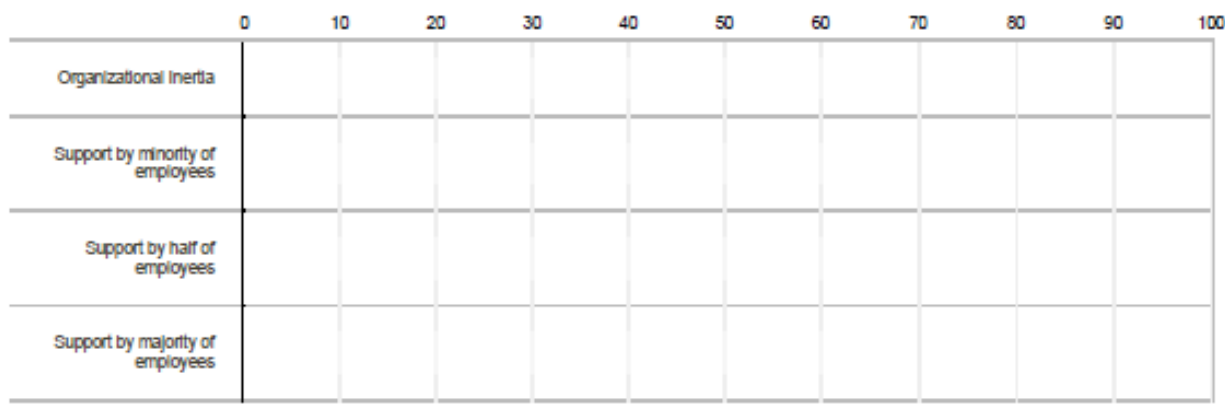

\section{Training}

What level of training with goals of organizational maturity and continuous learning erists in the research center?

Note: Please give each metric a score from 0 (The least favorable) to 100 (The most favorable).

Quartincation

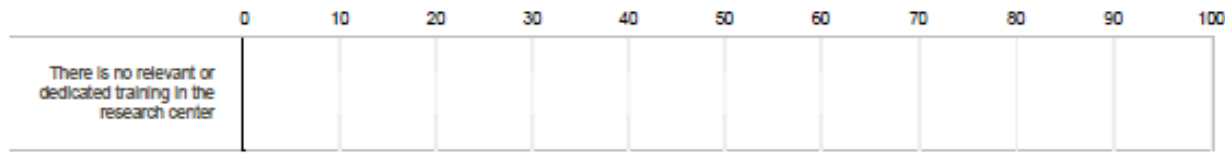




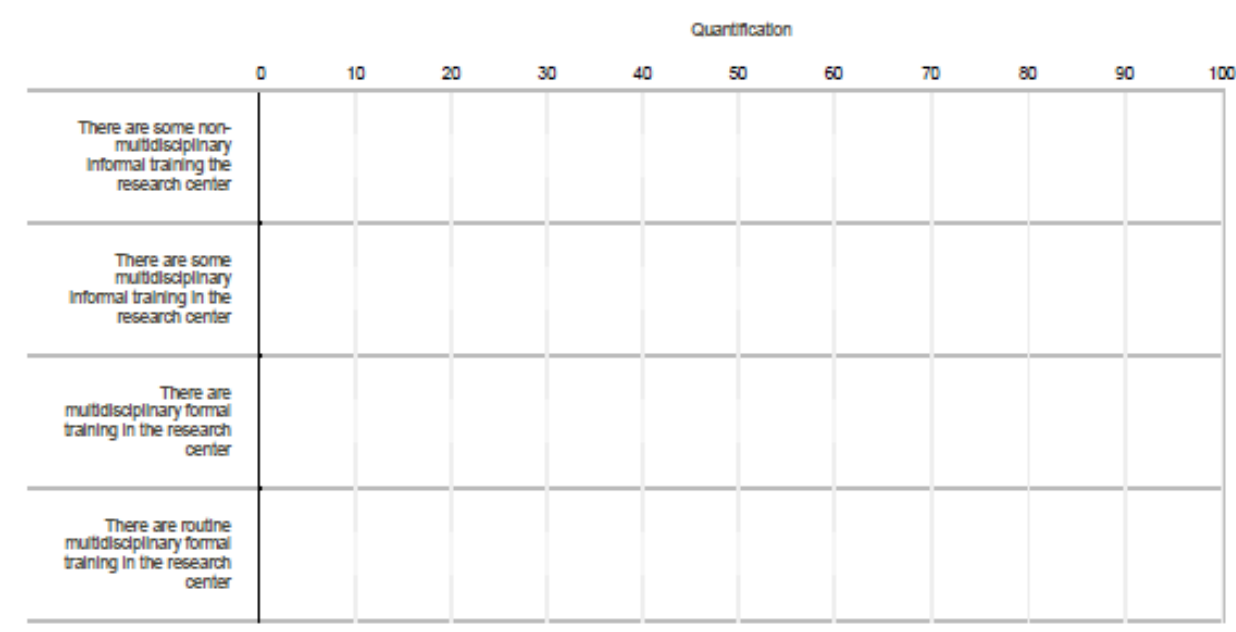

\section{Governance}

What level of governance erists in the research center?

Note: Please give each metric a score from 0 (The least favorable) to 100 (The most favorable).

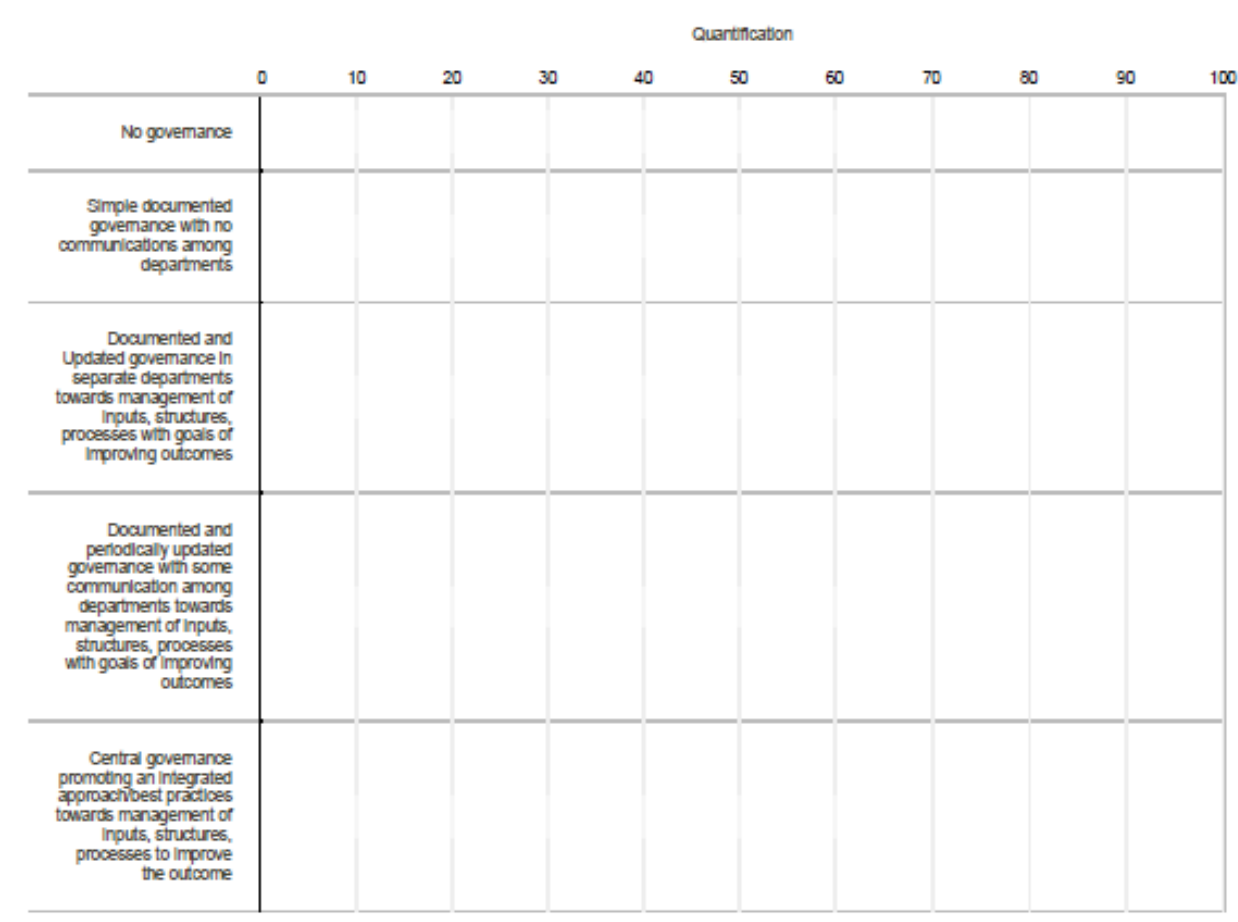

Block 4

https://portlandstate.ca1.qualtrics.com/Q/EditSection/Blocks/Ajax/GetSurveyPrintPreview?ContextSurveylD=SV_eFMyqaBEFsEXhtj\&ContextLibrary... 10/16 
Technology Management Maturty in Health Research Centers: Requlator Perspective:

\begin{tabular}{|c|c|c|c|}
\hline Perspective & Criteria & Definition & References \\
\hline \multirow{4}{*}{ Regulatory } & Standards Compliance & $\begin{array}{l}\text { Research centers' compliance with standards and } \\
\text { regulations to ensure transparency with stakeholders, data } \\
\text { interoperability, and commitment to meaning ful use and } \\
\text { joint commissions. }\end{array}$ & 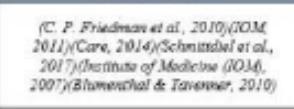 \\
\hline & Policy Preparedness and Adaption & $\begin{array}{l}\text { Research centers' flexibility and agility in responding and } \\
\text { adapting to changes (new regulations, legislations, and } \\
\text { policies) in terms of anticipation and readiness (resources, } \\
\text { policies, strategies, and management). }\end{array}$ & 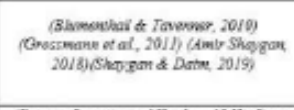 \\
\hline & Privacy & $\begin{array}{l}\text { Research centers' adherence to regulations and policies to } \\
\text { ensure privacy in terms of information technology, medical } \\
\text { data, patient access, third party interactions, and ethical use } \\
\text { of information (IRBs), among others. }\end{array}$ & 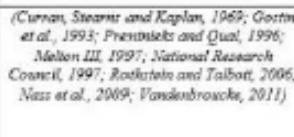 \\
\hline & Informing Policy & $\begin{array}{l}\text { Research centers' ability to inform policies by participating } \\
\text { in expert panels, providing evidence and comments } \\
\text { regarding policies under consideration, and securing } \\
\text { contracts with the state and federal government to complete } \\
\text { analyses regarding policy development, implementation, } \\
\text { and impact. }\end{array}$ & 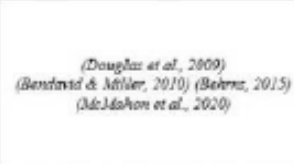 \\
\hline
\end{tabular}

Instructions:

Please give each metric a score from 0 (The least favorable) to 100 (The most favorable).

Standards Compliance

What is the level of the research center's adherence to and compliance with standards and regulations?

Note: Please give each metric a score from 0 (The least favorable) to 100 (The most favorable).

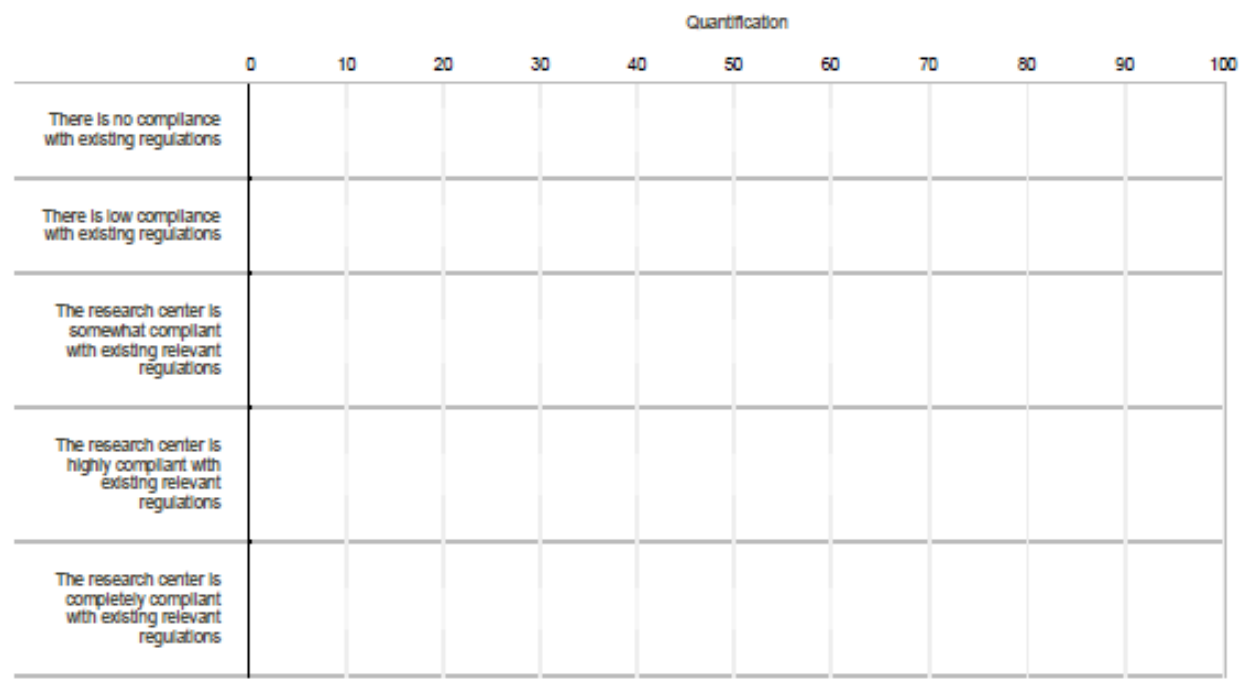

Policy Preparedness and Adaptation

To what extent is the research center flerible and ready in responding and adapting to policy changes?

Note: Please give each metric a score from 0 (The least favorable) to 100 (The most favorable).

Quantiflcation

https://portlandstate.ca1.qualtrics.com/Q/EditSection/Blocks/Ajax/GetSurveyPrintPreview?ContextSurveylD=SV_eFMyqa8EFsEXhtj\&ContextLibrary... 11/16 


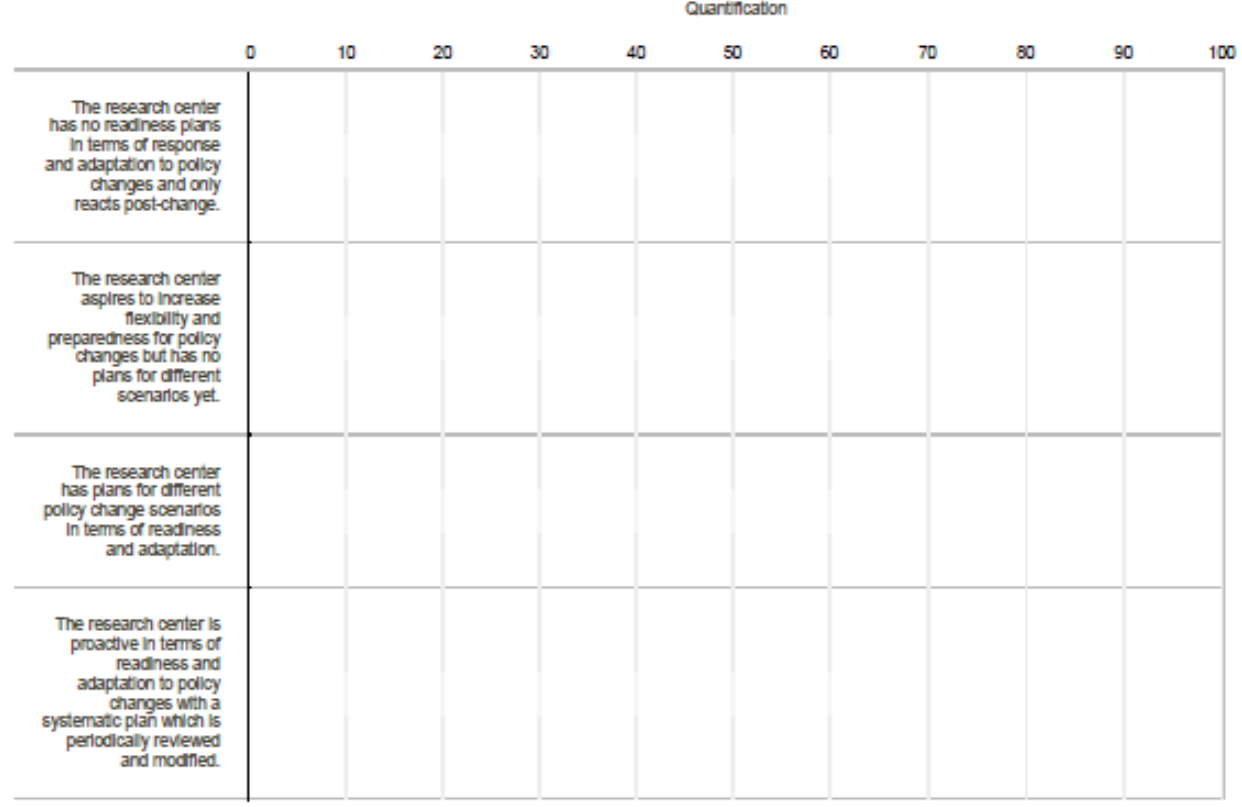

Privacy

What level of privacy measures erists in the research center?

Note: Please give each metric a score from 0 (The least favorable) to 100 (The most favorable).

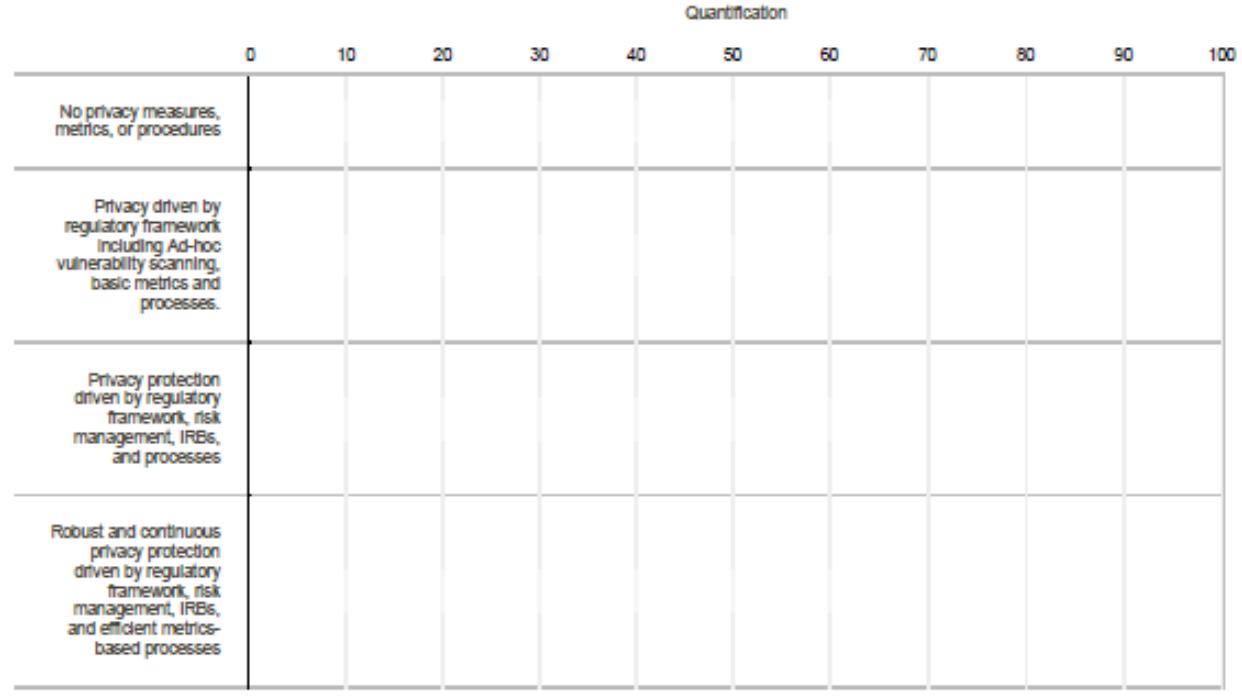

Informing Policy.

What level of policy informing power eristing in the research center?

Note: Please give each metric a score from 0 (The least favorable) to 100 (The most favorable).

https://portlandstate.ca1.qualtrics.com/Q/EditSection/Blocks/Ajax/GetSurveyPrintPreview?ContextSurveylD=SV_eFMyqa8EFsEXhtj\&ContextLibrary... 12/16 


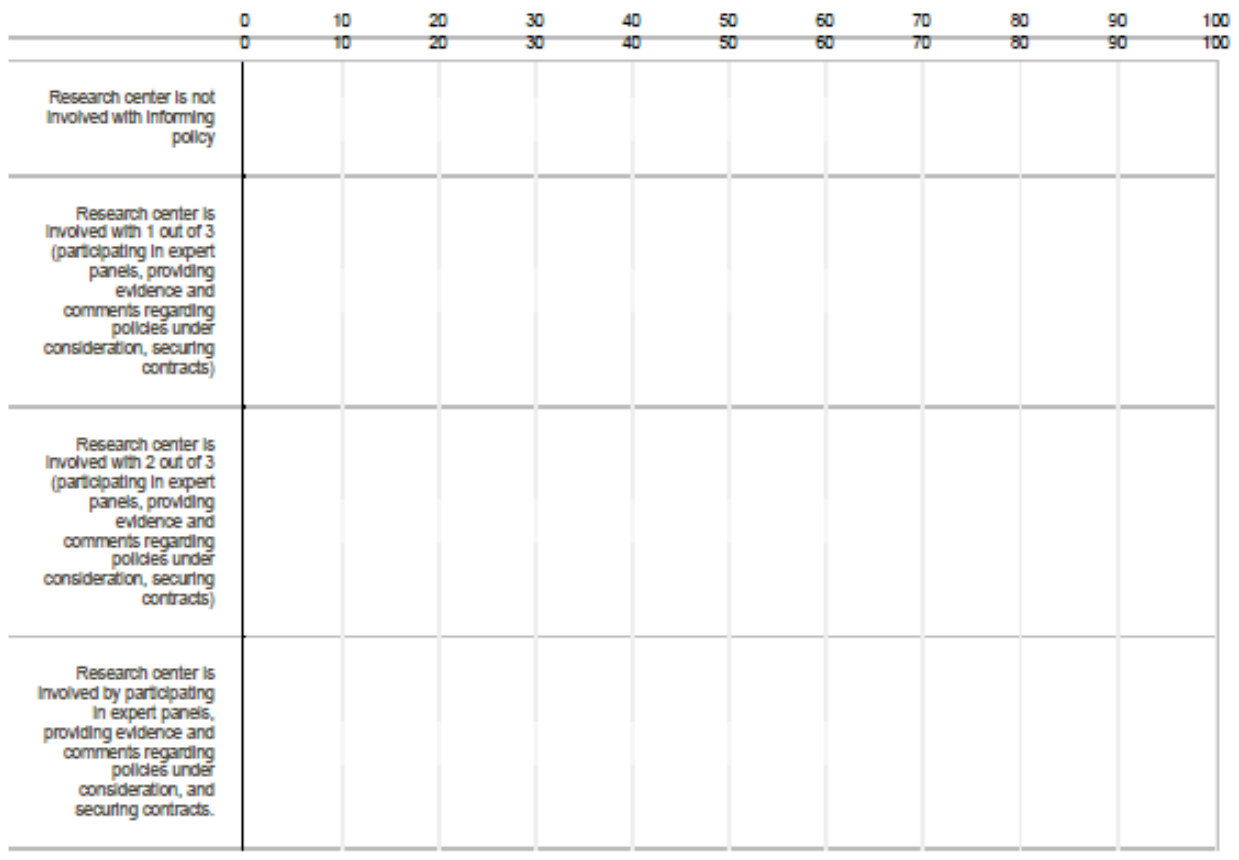

Block 5

Technology Management Maturity. In Health Research Centers

Financlal Perspective:

\begin{tabular}{|c|c|c|c|}
\hline Perspective & Criteria & Definition & References \\
\hline \multirow{4}{*}{ Financial } & Resource Allocation & $\begin{array}{l}\text { Research centers' ability to prioritize and decide to invest its } \\
\text { resources (financial, human, space,...) in alignment with the } \\
\text { goals of increased technology maturity and continuous learning. }\end{array}$ & 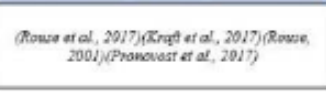 \\
\hline & $\begin{array}{l}\text { Funding and Mission } \\
\text { Alignment }\end{array}$ & $\begin{array}{l}\text { The extent to which incentives and funding are aligned with } \\
\text { encouragement of technology maturity, continuous learning and } \\
\text { improvement, waste elimination, and rewarding high care value. }\end{array}$ & 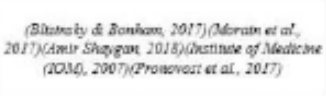 \\
\hline & Cost Reduction & $\begin{array}{l}\text { Research centers' ability to cut costs as a result of increased } \\
\text { maturity, learning, continuous improvement, and waste } \\
\text { eliminations without compromising quality. }\end{array}$ & 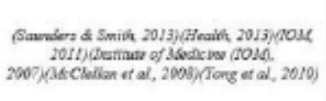 \\
\hline & $\begin{array}{l}\text { Financial Synergy with } \\
\text { Policies and Priorities }\end{array}$ & $\begin{array}{l}\text { Research centers' success in meeting reimbursement programs' } \\
\text { goals (policy incentives, value-based purchasing, etc.) and } \\
\text { accessing government, foundation, and other grants (NIH, } \\
\text { DARPA, DOD, various private funds, etc.) }\end{array}$ & 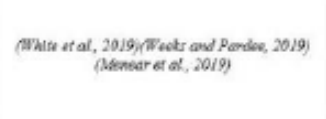 \\
\hline
\end{tabular}

Instructions:

Please give each metric a score from 0 (The least favorable) to 100 (The most favorable).

Resource Allocation

How well is the research center performing in terms of resource allocation and their alignment with technology maturity and continuous

https://portlandstate.ca1.qualtrics.com/Q/EditSection/Blocks/Ajax/GetSurveyPrintPreview?ContextSurveylD=SV_eFMyqa8EFsEXhtj\&ContextLibrary... 13/16 
learning?

Note: Please give each metric a score from 0 (The least favorable) to 100 (The most favorable).

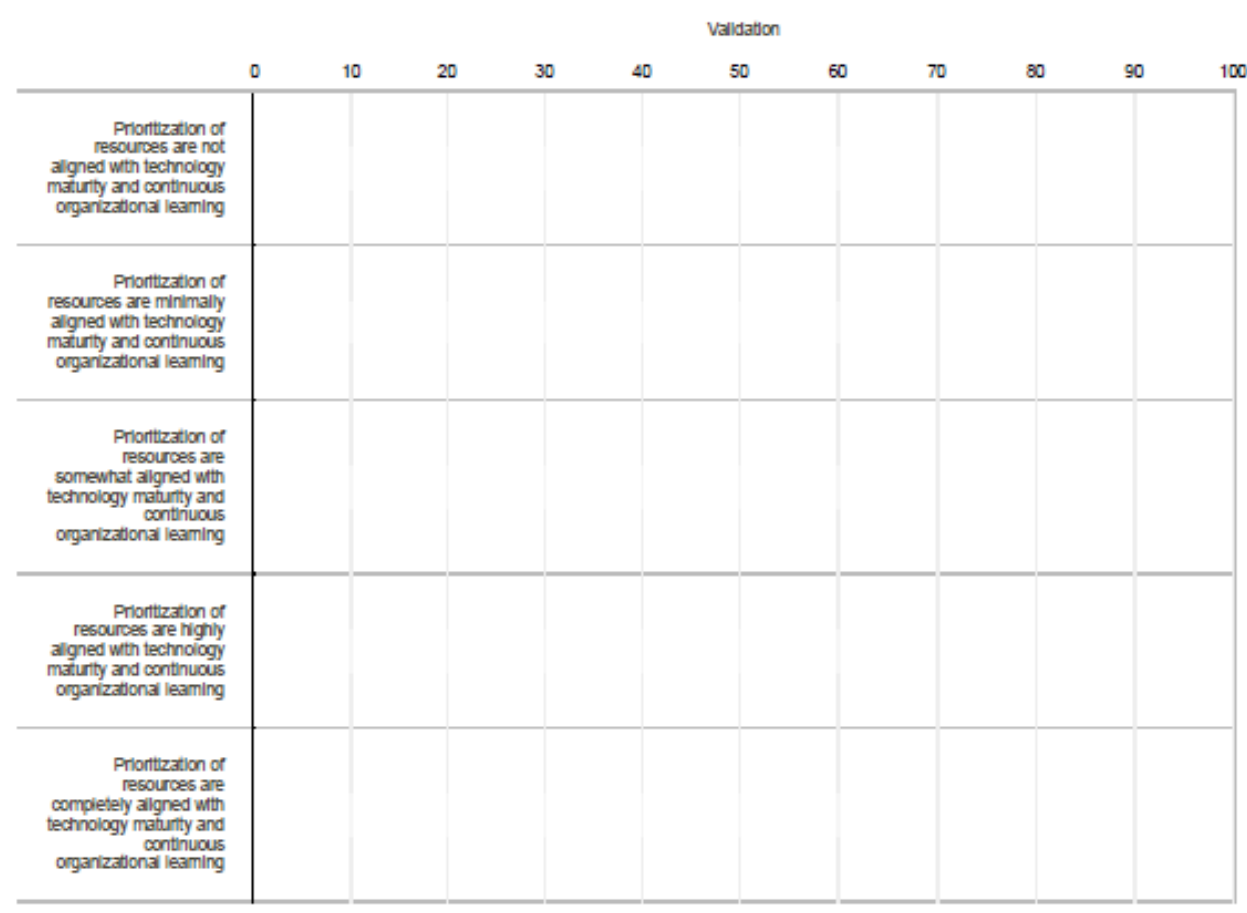

Funding and Mission Alignment

What level of alignment exists between incentives and technology maturity and continuous organizational learning? Note: Please give each metric a score from 0 (The least favorable) to 100 (The most favorable).

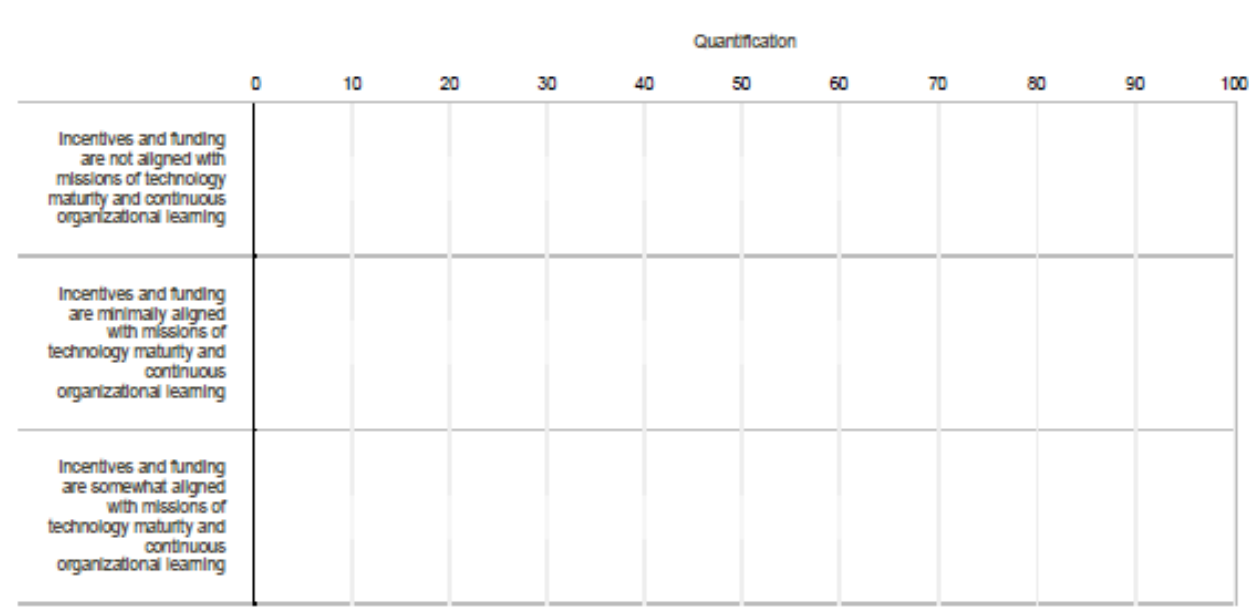




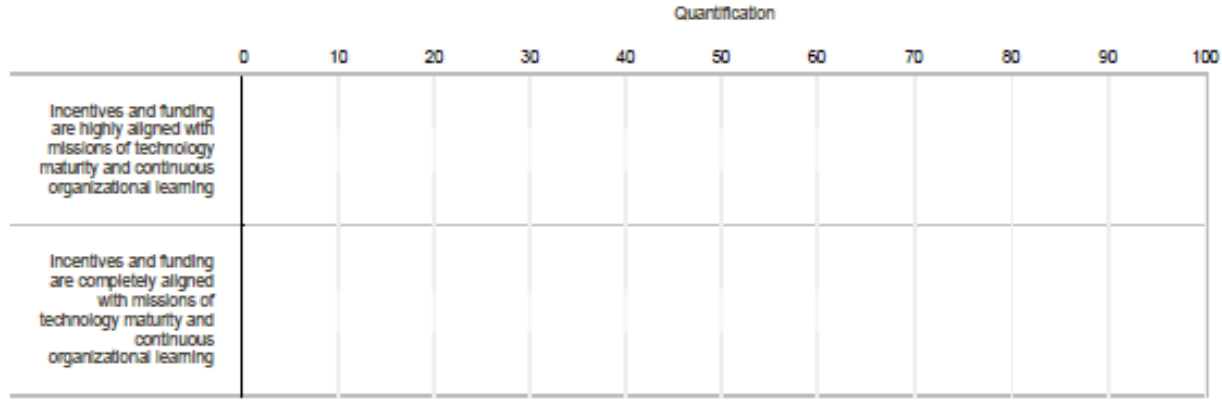

\section{CestReduction}

What level of success has the research center had in cutting costs?

Note: Please give each metric a score from 0 (The least favorable) to 100 (The most favorable).

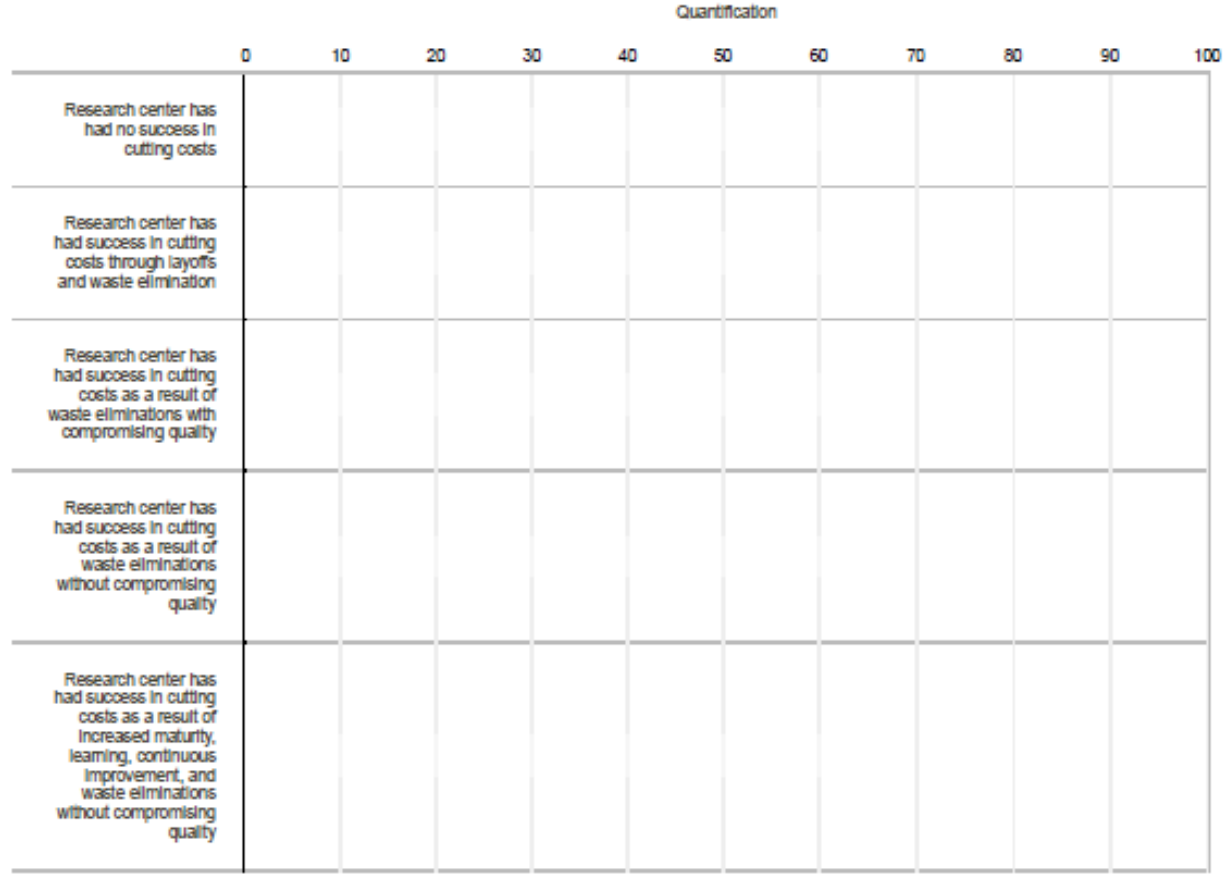

Financial Synergy with Policies and Priorities

What level of financial synergy does the research center have with policies and priorities?

Note: Please give each metric a score from 0 (The least favorable) to 100 (The most favorable).
$10 \quad 20 \quad 30$
40
50
60
70
80
90
100 
2/22/2021

Qualtrics Survey Software

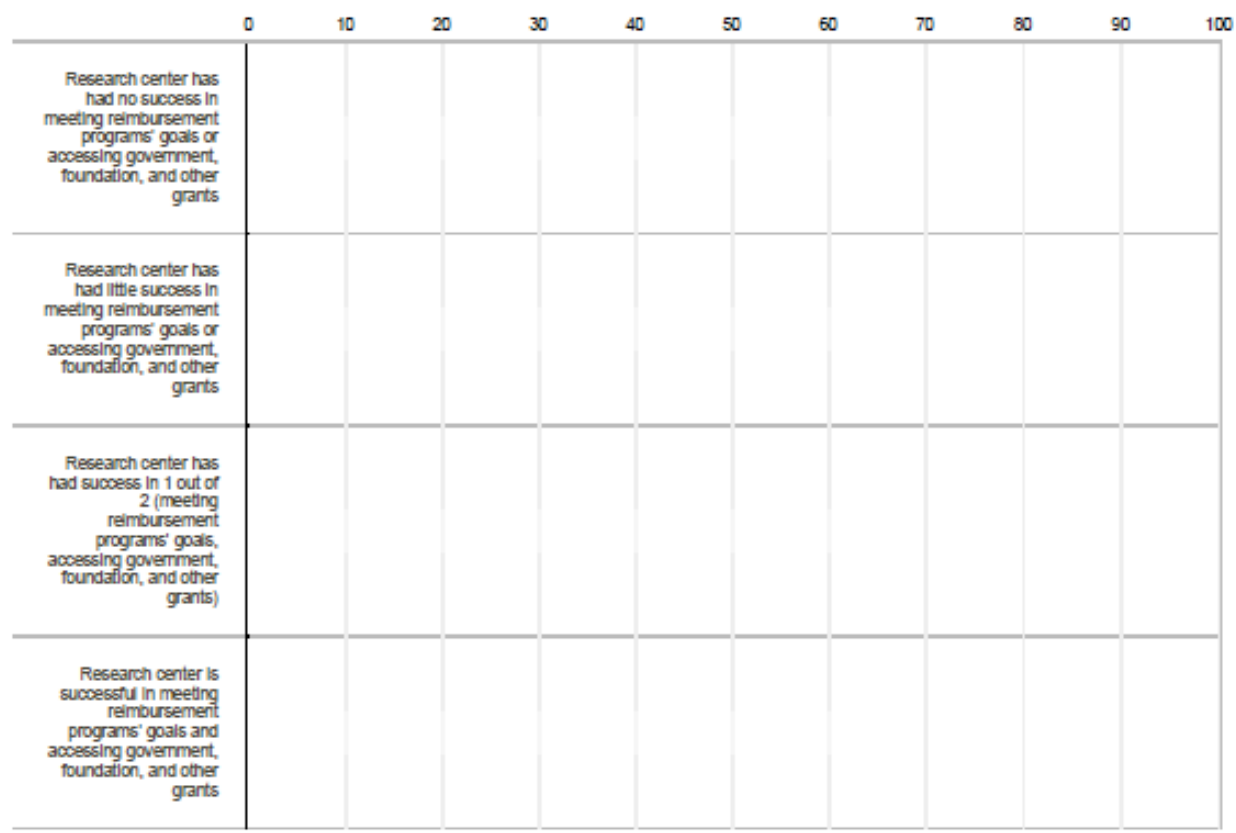

https://portlandstate.ca1.qualtrics.com/Q/EditSection/Blocks/Ajax/GetSurveyPrintPreview?ContextSurveylD=SV_eFMyqa8EFsEXhtj\&ContextLibrary... 16/16 


\section{HDM (Hierarchical Decision Mode() \\ Version: Beta 2.0

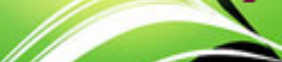

Please enter your First \& Last Name:

This name will be used as your identification (ID).

Submit

You can use your ID to re-visit your responses and modify them until you are satisfied and ready to submit

HDM (Hierarchical Decision Model)

Version: Beta 2.0
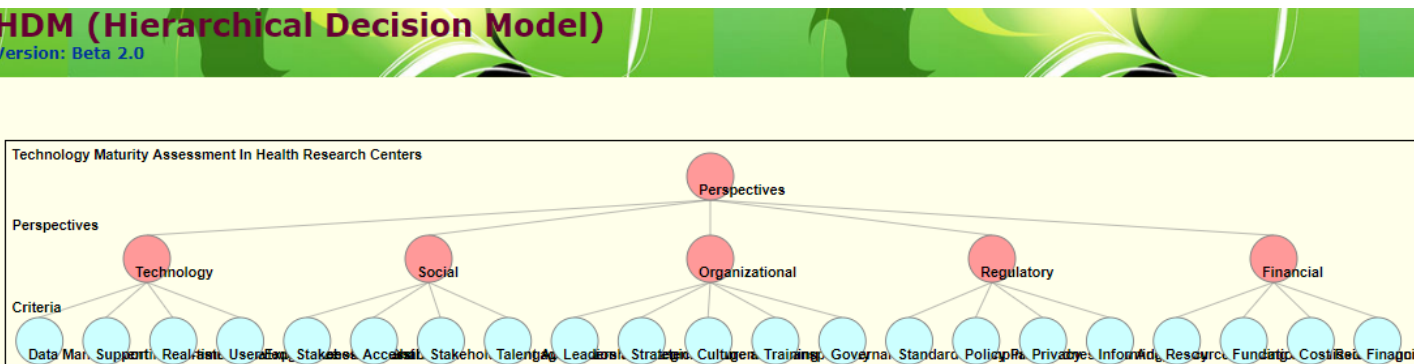

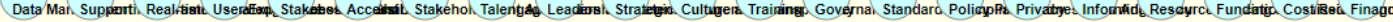

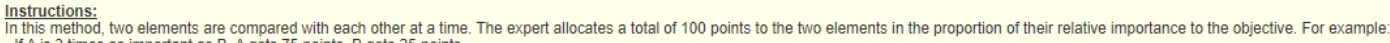

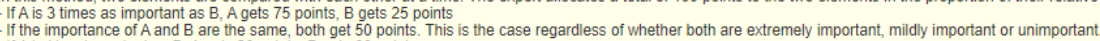
-if A is \% as important as B, A gets 20 points, B gets 80 point

Donce of A is negligible in comparison to B, A gets 1 point, $B$ gets 99 points.

Note: The red node will change to blue color when judgment(s) have been completed for that node.
Please click the red/dark blue node(s) above or choose a node from pull-down below to start comparisons:

$\checkmark$
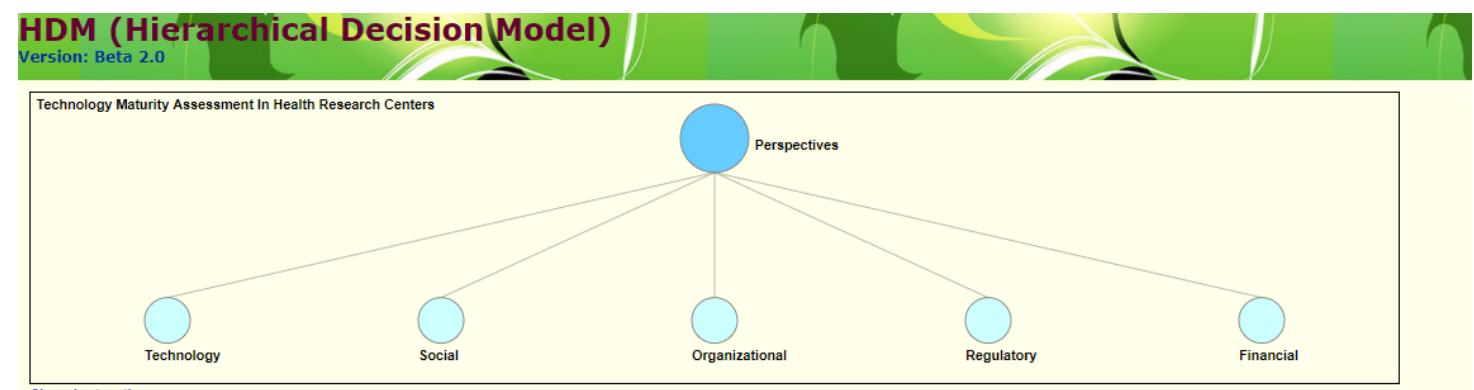

Phow Instructions

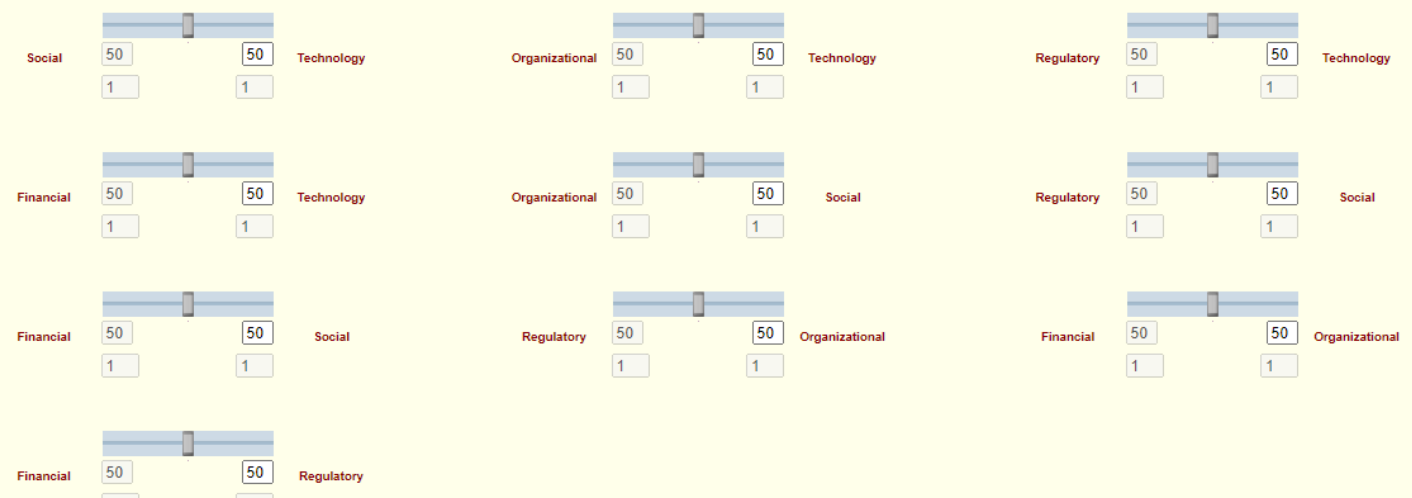




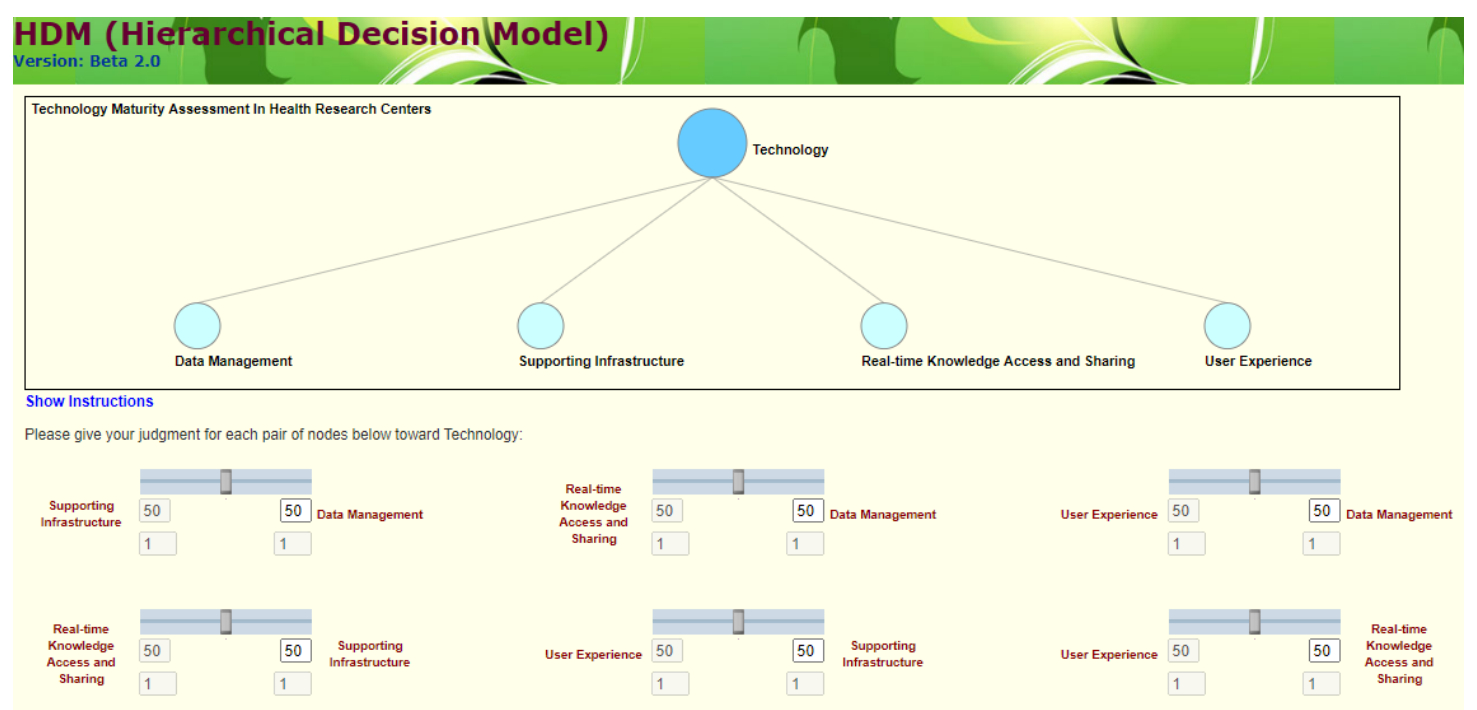

Save \& Go to the Next Node Save \& Go to the Main Page Cancel

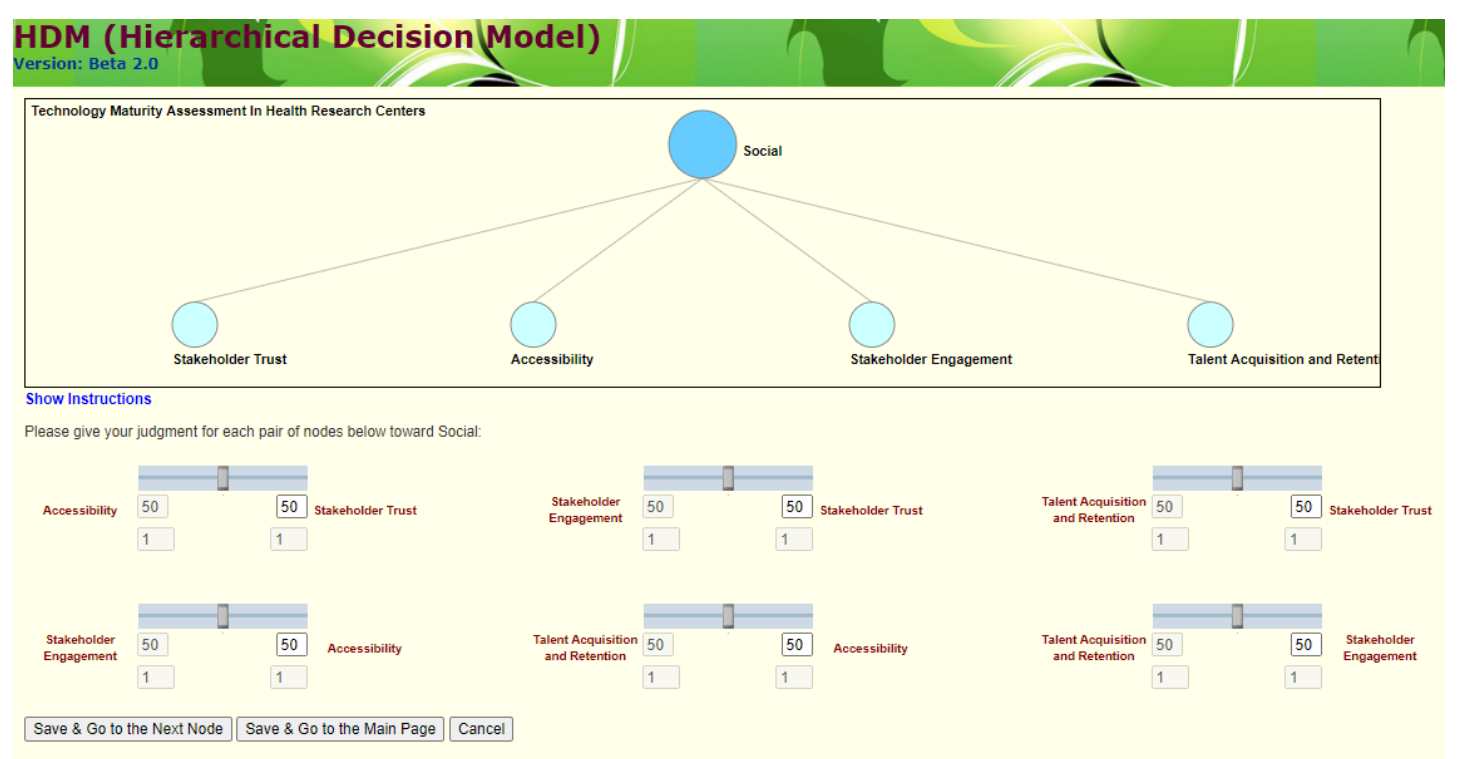



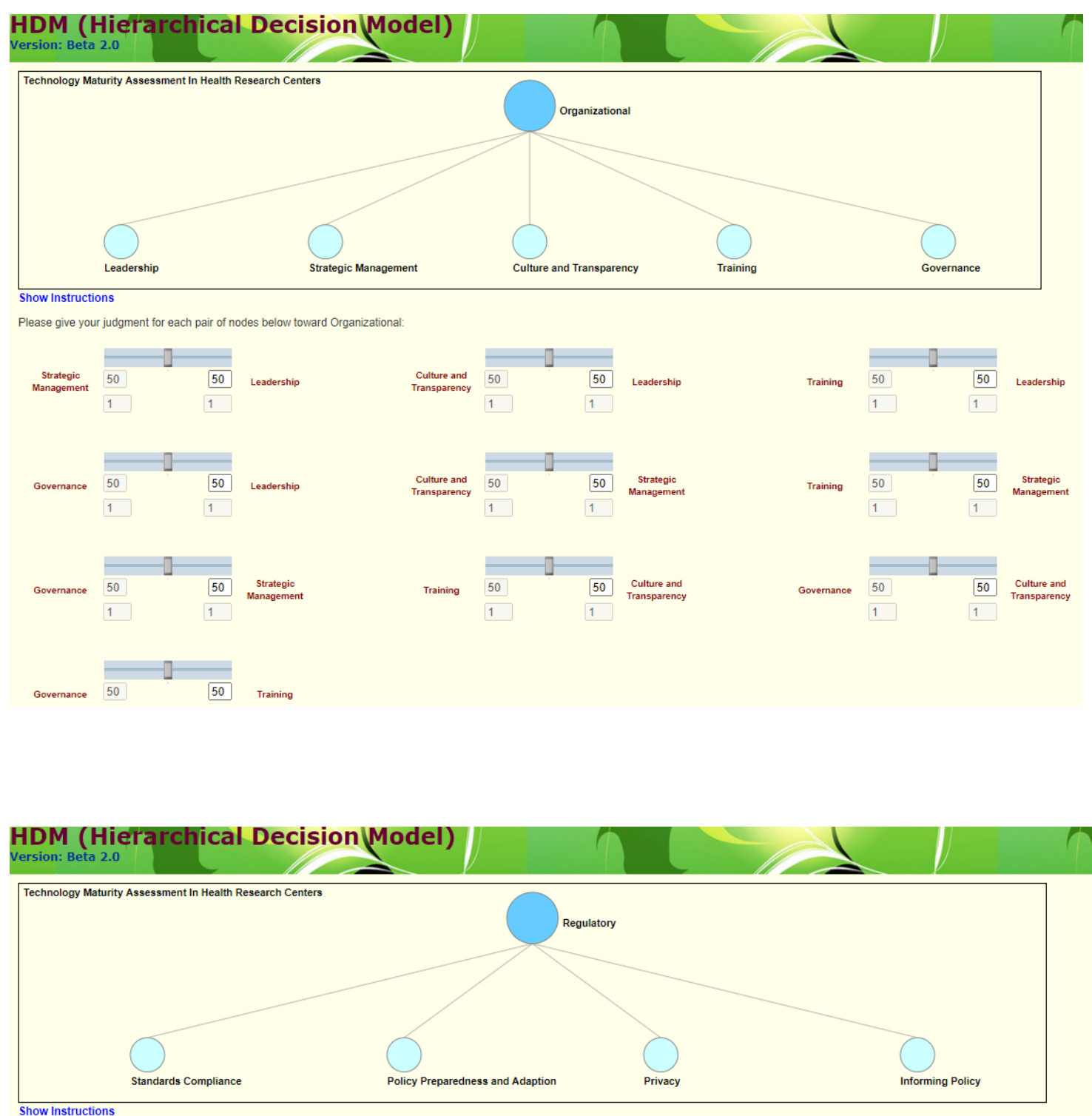

Please give your judgment for each pair of nodes below toward Regulatory:

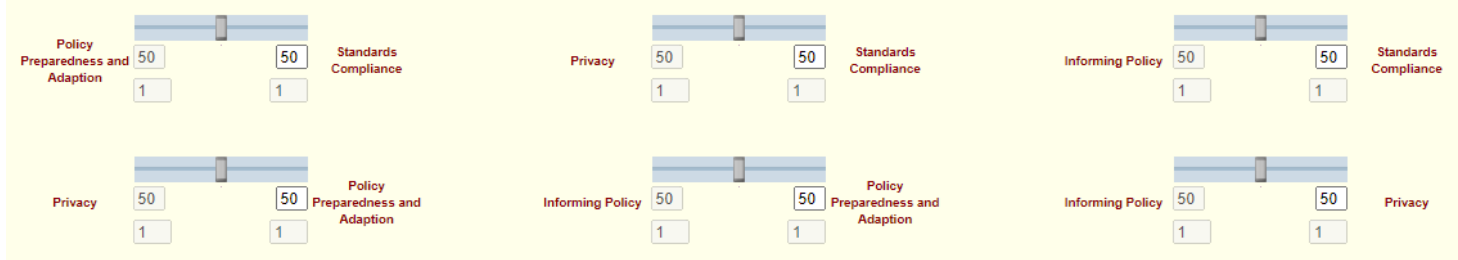

Save \& Go to the Next Node Save \& Go to the Main Page Cancel 

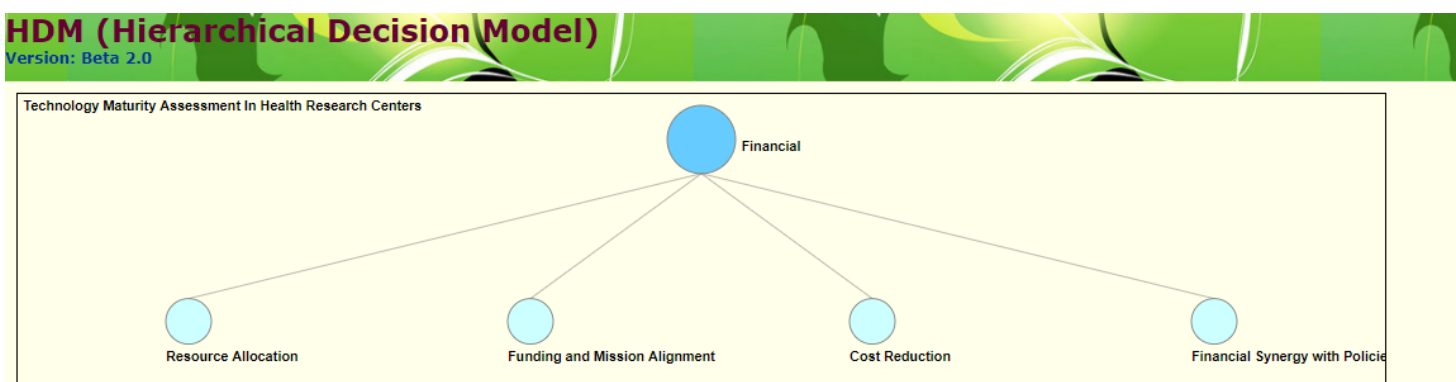

Show Instructions

Please give your judgment for each pair of nodes below toward Financial:

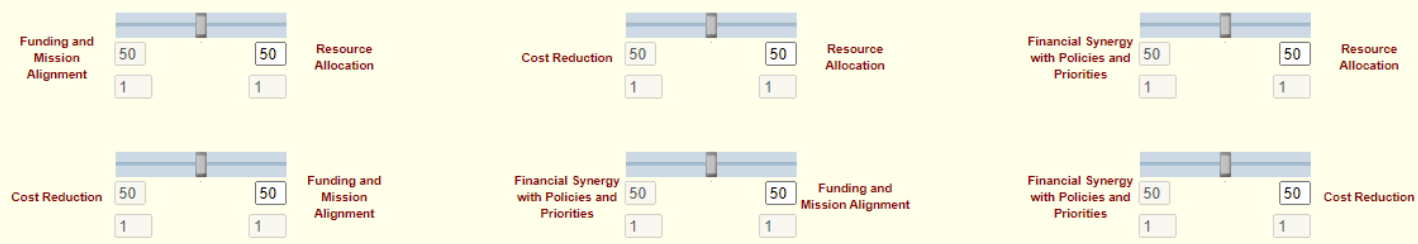

Save \& Go to the Main Page Cancel

HDM (Hierarchícal Decision Model)

version: Beta 2.0

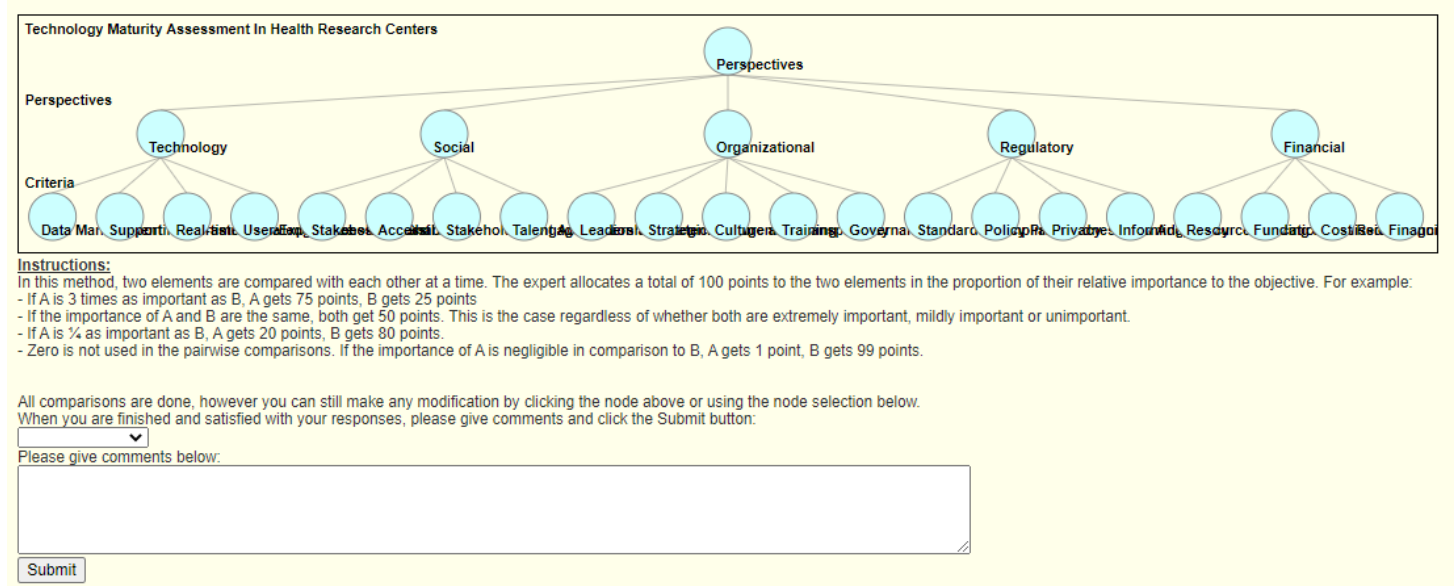

